\title{
Abstract 2018
}

Published online: 14 August 2018

(C) International Society of Behavioral Medicine 2018

\author{
Workshop \#1 \\ CLINICAL IMPACT OF RANDOMIZED CLINICAL TRIALS - DO \\ THEIR DESIGNS MEET EXPECTATIONS? \\ Lynda Powell ${ }^{1}$, Ken Freedland ${ }^{2}$, Peter Kaufmann ${ }^{3}$ \\ ${ }^{1}$ Rush University, USA \\ ${ }^{2}$ Washington University, USA \\ ${ }^{3}$ University of Colorado Denver, College of Nursing, USA
}

Behavioral Medicine has witnessed spectacular successes as well as disappointments. The objective of this workshop is to discuss many of the most important aspects of design that, apart from the intervention itself, can drive the outcome of a randomized trial of a behavioral intervention. During two workshop sessions, speakers will draw on their substantial experience in conducting, reviewing, and monitoring clinical trials to discuss key issues in trial design. Each session will provide unique perspectives and propose approaches to mitigate the risk of conducting an uninformative clinical trial.

\section{Workshop \#2}

DESIGNING INNOVATIVE BEHAVIORAL INTERVENTIONS FOR CHRONIC DISEASES: THE "NUTS AND BOLTS" OF BEHAVIORAL TREATMENT DEVELOPMENT

Susan M. Czajkowski ${ }^{1}$, Sylvie Naar ${ }^{2}$, Lynda Powell ${ }^{3}$, Kenneth Freedland $^{4}$

${ }^{1}$ National Cancer Institute, NIH, USA

${ }^{2}$ Florida State University, USA

${ }^{3}$ Rush University Medical Center, USA

${ }^{4}$ Washington University in St. Louis, USA

This workshop will provide behavioral medicine researchers an opportunity to enhance their skills in designing and optimizing innovative behavioral interventions to prevent and treat chronic diseases. Participants will: (1) learn about the ORBIT model, a new framework for behavioral treatment development (see http://psycnet.apa.org/psycinfo/2015-03938-001/); (2) learn about appropriate study designs and methods for developing behavioral treatments; and (3) apply the ORBIT model and knowledge about relevant methodologies to their own behavioral treatment development projects.

\section{Workshop \#3 \\ MHEALTH INTERVENTIONS IN HARD TO REACH GROUPS: HOW TO ENGAGE WITH LESS ACCESS OR FAMILIARITY WITH MOBILE HEALTH TECHNOLOGY \\ Anna Serlachius ${ }^{1}$ \\ ${ }^{1}$ Department of Psychological Medicine, University of Auckland, New Zealand}

This workshop will 1) provide an overview of both the potential and the challenges of mHealth technology for diverse groups; 2) discuss what makes a well-designed mHealth programme and what factors must be considered when designing mHealth programmes for hard to reach groups; and 3) discuss the strategies that improve the usability and engagement of mhealth interventions for different groups.

Workshop \#4

APPLICATIONS OF LATENT GROWTH MODELING IN LONGITUDINAL BEHAVIORAL MEDICINE RESEARCH

Maria Magdalena Llabre ${ }^{1}$, Stephanie L. Fitzpatrick ${ }^{2}$

${ }^{1} \mathrm{PhD}$, University of Miami, USA

${ }^{2} \mathrm{PhD}$ Kaiser Permanente Center for Health Research, USA

Clinical trials and observational studies in behavioral medicine research often use longitudinal designs where a sample of participants are repeatedly measured over time. Designing and analyzing data from longitudinal designs requires anticipation of critical issues such as invariance of measurement, participant attrition, and models that allow estimation of linear and nonlinear change. Longitudinal designs are also often used for understanding mechanisms of change using mediation. This workshop will start with an introduction to longitudinal designs, including the types of research questions that may addressed with such designs. It will cover the requirements associated with these designs and the optimal timing for data collection. We will examine how measurement models may be incorporated in longitudinal designs to insure equivalence over time and approaches and assumptions related to dealing with missing data. The workshop will focus on the analysis of longitudinal data in the form of latent growth models (LGMs). LGM expands the variety of questions that may be addressed with the data including the type of change (linear or nonlinear). These models also allow for the examination of multiple outcomes simultaneously and, therefore, are particularly suited to study mediation.

\section{Workshop \#5}

CLOSING THE GAP ON ACCESS TO HEALTHCARE AMONG INTERNATIONAL MIGRANTS TO CHILE: EVIDENCE AND SOLUTIONS

Karen Carpio ${ }^{1}$, Claudia Silva ${ }^{2}$, Báltica Cabieses ${ }^{3}$, Macarena Chepo ${ }^{3}$, Daniel Larenas ${ }^{3}$, Jossette Iribarne ${ }^{4}$, Sofia Astorga ${ }^{3}$

${ }^{1}$ International Organization for Migration, Costa Rica

${ }^{2}$ Department of Foreign Affairs, Ministry of Domestic Affairs, Chile

${ }^{3}$ Universidad del Desarrollo, Chile

${ }^{4}$ Ministry of Health, Chile

This workshop aims to describe current evidence and existing solutions for the healthcare gap on access to services for international migrants to Chile compared to natives. Specific objectives are to:

1) characterize international migration flows in Latin America and their access to healthcare, the socioeconomic profile of international migrants to Chile and their access to healthcare, and existing gaps and needs for immigrant adults and children; 2) discuss healthcare gaps of immigrants 
to Chile with regards to the existing national legislation and how it compares to other countries in the region; 3) describe governmental efforts and health policies for reducing healthcare gaps on access to services for international migrants to Chile; and 4) describe local efforts for reducing healthcare gaps on access to services for international migrants to Chile, with focus on early detection of needs and cultural competency.

\section{Workshop \#6}

THIRD GENERATION BEHAVIORAL THERAPIES IN HOSPITAL SETTINGS: ACT FOR CHILDREN AND ADOLESCENTS Cointa Arroyo Jimenez ${ }^{1}$, Lucia Torres Perez ${ }^{2}$, Argelia Lara Puente ${ }^{1}$

${ }^{1}$ National Institute of Pediatrics, Mexico

${ }^{2}$ National Autonomous University of Mexico

Most behavioral interventions with children and adolescents in medical settings have focused in changing contingencies and working with parents as agents of change; however in some critical conditions, implementing other third generation behavioral variables, such as acceptance and values in the clinical treatment have showed promising results in hospital settings and medical conditions like chronic pain, anorexia, autism, diabetes, intellectual disability, obsessive compulsive disorder and anxiety. This workshop will offer an overview of the core concepts of third generation behavioral therapies, specifically acceptance and commitment therapy applied to children and adolescents in medical settings. Objectives are to: 1) describe an overview of third generation behavioral therapies or contextual behavioral approach; 2) explain which contextual therapies are most commonly applied in children and adolescents; 3 ) define and illustrate all ACT (Acceptance and commitment therapy) components and explain their adaptation for use with children and adolescents; and 4) provide skills training for the application of ACT with children and adolescents in hospital settings.

\section{Workshop \#7}

USING QUALITATIVE DATA TO INFORM INTERVENTION DEVELOPMENT, REFINEMENT AND ADAPTATION TO MEET NECESSARY BENCHMARKS BEFORE A FULLY POWERED EFFICACY TRIAL

Ana-Maria Vranceanu ${ }^{1}$

${ }^{1}$ Associate Professor of Psychology, Harvard Medical School; Director, Integrated Brain Health Clinical and Research Program, Department of Psychiatry, Massachusetts General Hospital, USA

This workshop will focus on the early stages of behavioral health intervention development and adaptation. We will discuss the stages of intervention development up to an efficacy trial, including the role of qualitative data (focus groups, dyadic interviews, individual interviews and exit interviews) with patients and stakeholders (e.g., family caregivers, medical team) in ensuring that feasibility and acceptability benchmarks are met prior to efficacy testing. We will present information on how to design a qualitative interview and use the information for intervention development, adaptation, and refinement. We will use specific examples on how to decide between intervention development or adaptation, how to set a priori evidence based benchmarks, and how to revise the intervention (e.g., content, assessment measures, structure, mode of delivery, training procedures) to ensure that benchmarks are met.

\section{Workshop \#8}

TEACHING BEHAVIORAL MEDICINE: AN INSPIRATIONAL WORKSHOP

Anne H Berman ${ }^{1}$, Jamie Bodenlos ${ }^{2}$, Dori Pekmezi ${ }^{3}$, Wong Mee Lian ${ }^{4}$, Konstadina Griva $^{5}$, Pernilla Åsenlöf ${ }^{6}$, Yanjie Yang ${ }^{7}$, Joost Dekker ${ }^{8}$

${ }^{1}$ Karolinska Institutet, Department of Clinical Neuroscience/Center for Psychiatry Research, Sweden

${ }^{2}$ Hobart and William Smith Colleges, Department of Psychology, USA
${ }^{3}$ University of Alabama at Birmingham, Department of Health Behavior, School of Public Health, USA

${ }^{4}$ National University of Singapore, Saw Swee Hock School of Public Health, Singapore

${ }^{5}$ Imperial College \& Nanyang Technological University, Centre for Population Health Sciences (CePHaS), Lee Kong Chian School of Medicine, Singapore

${ }^{6}$ Uppsala University, Department of Neuroscience, Physiotherapy program, Sweden

${ }^{7}$ Harbin Medical University, Department of Medical Psychology, Public Health College, China

${ }^{8}$ VU University Medical Center, Dept Psychiatry and Dept Rehabilitation Medicine, Netherlands

The objectives of this workshop are to: 1) learn about a variety of innovative ways of teaching behavioral medicine at the university level, 2) apply the new information experimentally to your existing curricula/ syllabi for teaching behavioral medicine; and 3) exit the workshop with an idea of your next action to improve your existing curricula/syllabi.

Workshop \#9

BRINGING AN EXPERIMENTAL MEDICINE APPROACH TO BEHAVIOR CHANGE: AN INTRODUCTION TO THE NIH SOBC PROGRAM

Karina W. Davidson ${ }^{1}$, Donald Edmondson ${ }^{1}$, Jennifer A. Sumner ${ }^{1}$

${ }^{1}$ Columbia University Medical Center, USA

Unhealthy behaviors account for a sizeable proportion of risk associated with preventable premature deaths worldwide. However, it is difficult for individuals to initiate and maintain healthy behavior changes, and our interventions are inconsistent and only modestly effective. The NIH Common Fund's Science of Behavior Change (SOBC) program was formed to shed light on how behavior change works using systematic, rigorous, and transparent methods. SOBC's goal is to move behavior change science toward a mechanism-focused experimental medicine approach. This method is now being included in many NIH funding opportunities. Additionally, efforts to improve the conduct and translation of behavior change science that are complementary to SOBC are underway around the world. Together, these initiatives emphasize the importance of better understanding behavior change and the need for international collaboration. In this workshop, we will introduce attendees to SOBC and its method and resources. By the end of the workshop, participants will be able to: 1) Describe the SOBC experimental medicine approach; 2) Apply the SOBC method to a novel research question; and 3) Generate a Specific Aims page for this research.

Workshop \#10

COMPLEX INTERVENTIONS IN HEALTH: GUIDANCE ON DEVELOPMENT, EVALUATION AND THE IMPORTANCE OF CONTEXT

Sharon Simpson ${ }^{1}$, Rona Campbell ${ }^{2}$, Laurence Moore ${ }^{1}$

${ }^{1}$ University of Glasgow, Scotland

${ }^{2}$ University of Bristol, England

The UK Medical Research Council's (MRC) Guidance on Developing and Evaluating Complex Interventions is a key reference point in the field. This workshop is an opportunity to learn more about this and other related guidance and for participants to influence the content of a new version of the guidance currently being developed. The workshop leaders are members of three teams commissioned by the MRC, UK National Institute of Health Research and Canadian Institute for Health Research to update this guidance, and to develop related guidance on feasibility studies and on the importance of context to the design, evaluation and roll-out of complex interventions. The workshop will inform participants about 
the guidance as well as giving opportunities for input. Participants will: 1) learn about the key challenges and methods in developing and evaluating complex interventions; 2) learn about feasibility studies conducted before a full-scale effectiveness RCT, which seek to develop or refine the intervention, and to estimate key trial design parameters; 3 ) learn about the importance of considering contextual issues such as cultural, policy, and service and organizational factors in the design, evaluation and real world delivery of interventions; and 4) have the opportunity to co-produce with the workshop leads a list of key points to be considered in the final version of the overarching guidance and to examine how the guidance can be applied to their own research.

\section{Workshop \#11 \\ INTRODUCTION TO APPLYING NETWORK META-ANALYSIS IN BEHAVIORAL MEDICINE \\ Chris Noone ${ }^{1}$, Gerry Molloy ${ }^{1}$ \\ ${ }^{1}$ NUI Galway, Ireland}

Network meta-analysis has proven to be a useful tool for comparative effectiveness research of medical and pharmacological interventions - it allows researchers to identify not just whether a particular intervention works, but which intervention works best. It has been used relatively less frequently to examine the comparative effectiveness of interventions in behavioral medicine. One key advantage of network meta-analysis relevant to behavioral medicine is its ability to provide estimates of comparisons for which little or no head-to-head data is available. Through practical exercises and demonstrations, this workshop will introduce how network meta-analysis works, important considerations for its use in behavioral medicine and how to conduct a network meta-analysis using R. It will also guide researchers in how to tailor the systematic review process when planning a network meta-analysis. The learning objectives of this workshop are to 1) understand what network meta-analysis is and how it works; 2) understand the assumptions of network meta-analysis and how to check them; and 3) be aware of the challenges of using network metaanalysis on behavioral trials.

Workshop \#12

INTRODUCTION TO MOTIVATIONAL INTERVIEWING: TECHNIQUES, PROCESSES, PROFICIENCY

Anne H Berman ${ }^{1}$

${ }^{1}$ Motivational Interviewing Network of Trainers (MINT); Karolinska Institutet, Sweden

Evidence for the effectiveness of Motivational Interviewing (MI) has grown for a variety of health-related behaviors. Training in MI is essential for delivering brief, effective interventions that engage the client, focus on a particular behavior for strategic change, evoke client motivation to begin taking concrete steps towards change, and formulate a specific plan for carrying out the change. This workshop offers training in MI that will facilitate effectiveness in participants' beginning or continuing MI practice.

\section{Workshop \#13 \\ INTEGRATION OF PHYSIOTHERAPY IN BEHAVIORAL MEDICINE INTERVENTION RESEARCH \\ Pernilla Åsenlöf ${ }^{1}$, Helena Igelström ${ }^{1}$, Maria Sandborgh ${ }^{2}$, Johanna Fritz ${ }^{2}$ ${ }^{1}$ Uppsala University, Sweden \\ ${ }^{2}$ Mälardalen University, Sweden}

This workshop will share the experiences from three prominent Swedish research groups regarding the development and implementation of contemporary physiotherapy, that is physiotherapy integrated with behavioural medicine. Speakers will draw on their scientific experiences of developing a line of research on the integration of physiotherapy and behavioural medicine. The workshop begins with a brief presentation of theoretical assumptions and motives for the integration, followed by a description of a seminal intervention model, and its evaluation in randomised controlled trials in pain, cancer, and obstructive sleep apnea populations. The third part deals with strategies for skills training of physiotherapists to deliver interventions according to a behavioural medicine protocol and challenges associated with implementation into clinical practice.

Workshop \#14

EPISTEMONIKOS (WHAT IS WORTH KNOWING): THE WORLD'S LARGEST DATABASE OF HEALTH EVIDENCE

Gabriel Rada ${ }^{1}$

${ }^{1}$ Epistemonikos Foundation, Chile

Epistemonikos is the largest database of health evidence. It is maintained by systematically searching multiple information sources, including all major electronic databases. It is currently the most comprehensive systematic reviews database $(200,000$ systematic reviews relevant for health decision-making). It will be integrated in the Cochrane Library during the first quarter of 2018. Epistemonikos can be searched in several ways, including a simple 'Google-like' interface and an advanced boolean search. An important feature of Epistemonikos is that it links together systematic reviews, broad syntheses of reviews and primary studies, thus providing a highly efficient method for searching. A unique tool of Epistemonikos is a matrix of evidence: a table comparing the systematic reviews addressing a question, and all the included studies of these reviews. In addition, it includes translations of the titles and abstracts of included records to facilitate searching in different languages. The objectives of this workshop are to 1) introduce Epistemonikos database and 2) demonstrate the tools of Epistemonikos and how they can help translate research into practice.

\section{P001}

PREGNANCY-SPECIFIC ANXIETY INCREASES ODDS OF LOW BIRTHWEIGHT AND PRETERM BIRTH IN A MILITARY SAMPLE Karen Weis ${ }^{1}$, Regina Lederman ${ }^{2}$, Katherine Walker ${ }^{1}$, Wenyaw Chan ${ }^{3}$

${ }^{1}$ University of the Incarnate Word- USA

${ }^{2}$ University of Texas Medical Branch- USA

${ }^{3}$ University of Texas Health Science Center at Houston- USA

Introduction/Purpose: Increasing evidence points to a relationship between prenatal maternal anxiety and poor birth outcomes. One of the strongest predictors of preterm birth is pregnancy-related anxiety. Findings vary by type and timing of the anxiety as well as population. The purpose of this study was to determine the changes across pregnancy for pregnancy-specific measures of anxiety and symptoms of depression and determine the relationship of prenatal anxiety and symptoms of depression to preterm birth and low birth weight in a military sample. Methods: Military women (both pregnant active duty and spouses of active duty) in the first trimester of pregnancy and at least 18 years of age from one of the largest military communities in the United States were invited to participate. Of those approached, 367 were consented. Women completed study questionnaires in each trimester of pregnancy. Birth outcomes and pregnancy complications were obtained from prenatal and delivery records. RESULTS: Of those consented, 246 women completed all study elements. After adjusting for active duty status and ethnicity, for each $1 / 10$ unit increment in the slope for anxiety related to well-being of self and baby, the odds ratio for low birthweight was increased by $83 \%$. Active duty women were significantly more likely to have a low birthweight infant. Each 1/10 unit increase in the slope for anxiety related to acceptance of pregnancy, preparation for labor and fear of pain, helplessness and loss of control in labor, increased the odds ratio for preterm birth by $37 \%, 60 \%$, and $54 \%$ respectively. Active duty status 
was not significant within the preterm models. CONCLUSIONS: Rather than a generalized measure of anxiety, each dimension of pregnancyspecific anxiety represented distinct differences in outcomes. The findings offer details beyond transient anxiety, essential for intervention development. Focused interventions addressing each dimension of pregnancy-specific anxiety could effectively decrease the incidence of low birthweight and preterm infants.

CORRESPONDING AUTHOR: Karen Weis, University of the Incarnate Word,weis@uiwtx.edu

\section{$\mathrm{P} 002$}

CONTINUED SMOKING AFTER A CANCER DIAGNOSIS: A LONGITUDINAL STUDY OF INTENTIONS AND ATTEMPTS TO QUIT

Christine Paul ${ }^{1}$, Flora Tzelepis ${ }^{1}$, Allison Boyes ${ }^{1}$, Afaf Girgis ${ }^{1}$, Catherine DEste $^{2}$, Emma Sherwood ${ }^{1}$

${ }^{1}$ University of Newcastle- Australia

${ }^{2}$ Australian National University- Australia

Introduction: Given the benefits of smoking cessation beyond a cancer diagnosis, this study aimed to identify the stability of smoking status after diagnosis.

Method: Participants were recruited via the cancer registries in NSW and Victoria and completed mailed surveys at T1 (6 months post diagnosis), T2 (1 year ), T3 (2 years) and T4 (3.5 years). Participants were adults diagnosed with a first primary cancer.

Results: 1453 people were recruited. Sixty-six (35.5\%) of the 186 selfreported smokers at diagnosis quit in the 6 months post-diagnosis and 120 $(64.5 \%)$ reported being a smoker at $\mathrm{T} 1$. Of the smokers at $\mathrm{T} 1,40.1 \%$ intended to quit: with $7.5 \%$ having quit by $\mathrm{T} 2 ; 10 \%$ quit by $\mathrm{T} 3$; and $10.8 \%$ quit by $\mathrm{T} 4$. Of the 49 who reported at $\mathrm{T} 1$ that they intended to quit in the next 6 months, $10.2 \%$ or less reported having quit at a subsequent point. A small proportion of non-smokers or former smokers $(<1 \%)$ took up smoking at each study time point.

Conclusion: While a diagnosis of cancer may prompt a quit attempt, the majority of smokers continue to smoke beyond diagnosis despite attempting to stop. There is a need to identify and implement effective cessation strategies for those who need it.

CORRESPONDING AUTHOR: Christine Paul, University of Newcastle,chris.paul@newcastle.edu.au

\section{P003}

\section{PARENTAL ATTACHMENT AND BODY SATISFACTION IN} ADOLESCENTS

Tamás Dömötör Szalai ${ }^{1}$, Edit Czeglédi ${ }^{1}$

${ }^{1}$ Semmelweis University, Institute of Behavioural Sciences- Hungary

Introduction \& Purpose. Insecure attachment contributes to various psychological problems including body dissatisfaction. It is has barely been investigated how far, and through which mechanisms attachment can predict body satisfaction. The role of maternal and paternal anxiety and avoidance has not been tested on adolescents' body satisfaction. The aim was to test these aspects. Methods. A cross-sectional, questionnaire-based survey investigated the mental health of a large randomized sample consisted of Hungarian adolescents $(\mathrm{N}=5214,51.6 \%$ boys, mean age 14.8 years, $\mathrm{SD}=2.6$ years). Measures included self-reported body weight and height data, body satisfaction scale (appearance, weight, shape, muscles, masculinity or femininity), the Experience in Close Relationships Scale, and the Child Depression Inventory. To examine the role of depression in the relationship between attachment and body satisfaction, structural equation modeling was conducted. Results. Boys had significantly higher body satisfaction, and worse maternal attachment than girls, who showed worse paternal attachment and higher depression. Higher paternal anxiety and avoidance, and maternal avoidance predicted lower body satisfaction in both genders adjusted for age and BMI $(\mathrm{R} 2=6.1-$ $12.5 \%)$. Depression fully mediated the relationship between dysfunctional maternal attachment and lower body satisfaction in both genders, between dysfunctional paternal attachment and lower body satisfaction in girls, while only partially mediated between dysfunctional paternal attachment and lower body satisfaction in boys. Conclusions. Dysfunctional parental attachment can play a significant role in adolescents' body dissatisfaction, mediated by the level of depression. Paternal attachment issues can be highlighted in boys' body concerns. Results suggest that handling negative moods, counteracting parental anxiety and avoidance may be useful in the case models and treatments aiming to improve adolescents' body satisfaction. Path analyses on longitudinal, intervention based studies are required to reconfirm these results.

CORRESPONDING AUTHOR: Tamás Dömötör Szalai, Semmelweis University, Institute of Behavioural Sciences, szalai.domotor@gmail.com

\section{P004}

DEVELOPMENT OF A CBT INTERVENTION TO REDUCE FEAR OF HYPOGLYCEMIA AND IMPROVE SELF-MANAGEMENT BEHAVIOR IN INDIVIDUALS WITH T1DM

Jennifer Duffecy ${ }^{1}$, Laurie Quinn ${ }^{1}$, Sue Penckofer ${ }^{2}$, Dan Mihailescu ${ }^{1}$, Chang Park ${ }^{1}$, Pamela Martyn-Nemeth ${ }^{1}$

${ }^{1}$ University of Illinois, Chicago- USA

${ }^{2}$ Loyola University, Chicago- USA

Introduction: Hypoglycemia is one of the major factors impacting optimal blood glucose control in people with type 1diabetes (T1DM). Hypoglycemia is life-threatening and can have long term consequences, which results in fear of hypoglycemia $(\mathrm{FOH})$. FOH can adversely affect self-management behavior, leading to greater glucose variability. Cognitive Behavioral Therapy (CBT) has been demonstrated effective in reducing anxiety in other chronic illness populations but has not been explored in $\mathrm{FOH}$ in individuals with T1DM. The purpose of this study was to pilot test a CBT intervention to improve FOH in young adults with T1DM. Methods: Participants were individuals diagnosed with T1DM aged 18-30 years old who use an insulin pump and have self-reported FOH. They were randomized to receive either the experimental CBT intervention, which consisted of eight weekly one-hour sessions based on principles of cognitive restructuring and exposure treatment for anxiety and wore a continuous glucose monitor (CGM) or an attention control group, which consisted of wearing a CGM and returning weekly for site change. Participants were followed 4 weeks post-treatment. Results: Participants $(\mathrm{N}=10)$ were predominantly white $(100 \%)$ females $(60 \%)$. Average age was 26.1 with diabetes duration of 14.7 years. Descriptive statistics were used to examine the data for this pilot. In the experimental group, FOH decreased from baseline $(\mathrm{M}=35.5)$ to 12 weeks $(\mathrm{M}=21.4)$ while fear levels stayed approximately the same for the control group ( $M=41$ at baseline to $M=42$ at 12 weeks). Similar patterns were demonstrated for anxiety levels as measured by the GAD7 (experimental group baseline $M=7.7$ to $M=5$ at 12 weeks; Control group baseline $M=6.8$ to $M=5.8$ ), and diabetes distress (experimental group baseline $M=2.4$ to $\mathrm{M}=1.6$ at follow up; Control group baseline $\mathrm{M}=2.6$ and $\mathrm{M}=2.5$ at follow up). Diabetes self-efficacy and self-management both increased in the experimental group but showed no change or decreased in the control group. Conclusions: While this was a pilot with a small sample, it appears that measures of $\mathrm{FOH}$, anxiety and diabetes distress showed improvement following the CBT intervention. Next steps include examining the change in glucose variability as related to changes in $\mathrm{FOH}$.

CORRESPONDING AUTHOR: Jennifer Duffecy, University of Illinois - Chicago, jduffecy@uic.edu 
$\mathrm{P} 005$

EDUCATIONAL INTERVENTION BY SALUTOGENETIC APPROACH IN DIABETES MELLITUS TYPE 2

Alfredo Armando Abuín Landin ${ }^{1}$, Yolaine Rodriguez ${ }^{2}$

${ }^{1}$ Medicos Descalzos Latinoamerica- Brazil

${ }^{2}$ Intermeds

Introduction: The Educational/Research/Assistance Unit is a principle of coherent behavioral of our communities. Diabetes Mellitus type 2 is one of the main causes of ascending morbidity and mortality.

Methods: A complete cycle work: community educational capacitation, pre and postgraduate programs, research, specialized healthy food production and assistance could be integrated. Educational intervention is carried out both in the community and in the professional wich dietetics training in Cuban Matanzas city with Ecohealthy Diet (with balance substitution and adequacy principles) in 47 Diabetic adult treated with some type of hypoglycemic and / or insulin, who were trained with a collective and individual salutogenesis approach. Data were taken from dietary survey, clinical evolution, measurement of nutritional status, basic indicators of glucidic metabolism, lipid and drug consumption. A clinical-metabolic evaluation was performed by conventional occidental procedures. Estimation of metabolic control by made by estimation of Unitary Entropy Metabolic Variables Changes (S).

Results: The intervention showed that significantly decreased of glycemia in $27 \%$, cholesterol $15 \%$ and triglycerides $31 \%$. The application of insulin decreased by $40 \%$ and the consumption of oral hypoglycemics decreased by $66 \%$. The body mass index decreased by $8 \%$. Metabolic estimation of Entropy $\Lambda \mathrm{S}$ decreased in 28.36 units.

Conclusions: A greater coherence and decreased entropy as a measure of better order in the energy metabolism was obtained in educational salutogenetic approach in Diabetes Mellitus community intervention.

CORRESPONDING AUTHOR: Alfredo Armando Abuín Landin, Medicos Descalzos Latinoamerica, alfredoaabuinlandin@gmail.com

\section{P006}

AN INTERACTIVE MODEL OF PARENTAL BONDING, PERSONALITY FUNCTIONING AND EATING DISORDER SYMPTOMS

Tamás Dömötör Szalai ${ }^{1}$, Edit Czeglédi ${ }^{1}$

${ }^{1}$ Semmelweis University, Institute of Behavioural Sciences- Hungary

Introduction \& Purpose. Lower parental care and higher overprotection are conceptualized as distinguishing factors of eating disorders. However, their role has not been clarified in several symptoms. No model investigates the interaction of parental bonding characteristics, and the personality traits, the level of depression and body dissatisfaction of the patient in the development of symptoms. The aim was to test these aspects. Methods. The cross-sectional online survey using expert sampling included sociodemographic, anthropometric and anamnestic data, the Eating Disorder Inventory, the Eating Behavior Severity Scale, the Parental Bonding Instrument, the 44-item Big Five Inventory and the CES-D Depression Scale. The sample consisted of 258 females (mean age: 31.6; mean BMI: 23.3), divided into four groups: 95 eating disorder patients; 117 individuals without eating disorder; 28 emotional eaters; and 18 patients in remission. A path analysis was conducted. Results. Levels of parental care and overprotection were not different between eating disorder patients and individuals without eating disorders. However, lower maternal care was related to emotional eating, lower paternal care to purging, and lower maternal and paternal care to impulsive symptoms. According to the structural equation modeling, lower paternal care and higher paternal overprotection predicted more severe eating disorder symptoms mediated by stronger trait neuroticism, higher levels of depression and body dissatisfaction. The model explained $25.5 \%$ of the variance of symptoms. Conclusions. Levels of parental care and overprotection did not distinguish eating disorder patients and individuals without eating disorders; but they were related to the type and intensity of various symptoms, suggesting the importance of the patient's perception of parental bonding characteristics. Results emphasize the relevance of dysfunctional paternal bonding and trait neuroticism as a core mediator personality factor. An interaction between parental bonding characteristics and the patient's personality functioning can be suspected in the development symptoms. The level of paternal care and overprotection, as well as the patient's emotional instability, depression and body dissatisfaction may offer intervention points. Longitudinal, interventionbased studies are required to confirm these results.

CORRESPONDING AUTHOR: Tamás Dömötör Szalai, Semmelweis University, Institute of Behavioural Sciences, szalai.domotor@gmail.com

\section{P007}

SYSTEMATIC REVIEW OF QUESTIONNAIRES MEASURING SOMATIZATION IN PRIMARY CARE PATIENTS

Kate Sitnikova ${ }^{1}$, Sandra Dijkstra-Kersten ${ }^{1}$, Lidwine Mokkink ${ }^{1}$, Berend Terluin $^{1}$, Harm van Marwijk ${ }^{2}$, Stephanie Leone ${ }^{3}$, Henriëtte van der Horst $^{1}$, Johannes van der Wouden ${ }^{1}$

${ }^{1}$ VU University Medical Center- Netherlands

${ }^{2}$ University of Sussex- United Kingdom

${ }^{3}$ Trimbos Institute: Netherlands Institute of Mental Health and AddictionNetherlands

Introduction \& Purpose: The aim of this review is to critically appraise the evidence on measurement properties of self-report questionnaires measuring somatization in adult primary care patients and to provide recommendations about which questionnaires are most useful for this purpose. Methods: We assessed the methodological quality of included studies using the COnsensus-based Standards for the selection of health Measurement INstruments (COSMIN) checklist. To draw overall conclusions about the quality of the questionnaires, we conducted an evidence synthesis using predefined criteria for judging the measurement properties. Results: We found 24 articles on 9 questionnaires. Studies on the Patient Health Questionnaire-15 (PHQ-15) and the Four-Dimensional Symptom Questionnaire (4DSQ) somatization subscale prevailed and covered the broadest range of measurement properties. These questionnaires had the best internal consistency, test-retest reliability, structural validity, and construct validity. The PHQ-15 also had good criterion validity, whereas the 4DSQ somatization subscale was validated in several languages. The Bodily Distress Syndrome (BDS) checklist had good internal consistency and structural validity. Some evidence was found for good construct validity and criterion validity of the Physical Symptom Checklist (PSC-51) and good construct validity of the Symptom Check-List (SCL-90-R) somatization subscale. However, these three questionnaires were only studied in a small number of primary care studies. Conclusions: Based on our findings, we recommend the use of either the PHQ-15 or 4DSQ somatization subscale for somatization in primary care. Questionnaires, such as the BDS checklist, PSC-51 and the SCL-90-R somatization subscale show promising results but have not been studied extensively in primary care.

CORRESPONDING AUTHOR: Kate Sitnikova, VU University Medical Center, e.sitnikova@vumc.nl

\section{P008}

COGNITIVE BEHAVIORAL CANCER STRESS MANAGEMENT WITHIN CANCER CARE DELIVERY: PATIENT ACCEPTABILITY AND FEASIBILITY

Shawna Ehlers ${ }^{1}$, Lisa Gudenkauf ${ }^{1}$, Sherrie Hanna ${ }^{1}$, Jill Snuggerud ${ }^{1}$, Jeff $\mathrm{Staab}^{1}$, Katharine Price ${ }^{1}$, Andrea Wahner-Hendrickson ${ }^{1}$, Kathryn Ruddy ${ }^{1}$ ${ }^{1}$ Mayo Clinic- USA 
Introduction and Purpose: Cognitive behavioral cancer stress management (CBCSM) has been replicated as efficacious in independent NIHfunded RCTs. The current pilot study/quality improvement project was designed to test the feasibility and acceptability of a 5-session CBCSM group intervention, as integrated into cancer health care delivery.

Methods: Participants were referred by several oncology teams representing multiple cancer sites. In 2017, eight CBCSM groups were conducted ( $\mathrm{n}=24$ participants/completers, 1 non-completer; the majority were partnered/married, employed, white, non-Hispanic, and female). In terms of relative content acceptability, 24 intervention content factors were patient-ranked via card-sort at end of the intervention. After the card sort, the most recent 20 patients completed a detailed intervention acceptability and feasibility assessment via self-report.

Results: In terms of overall acceptability, the majority endorsed 4 or greater on a 5-point Likert scale, indicating they would recommend CBCSM to family and friends. None of the intervention factors were suggested to be cut out of the intervention by more than 1 person. The majority of patients indicated feasibility in terms of session number, session duration, and intervention duration; $33 \%$ desired a greater number of sessions, $11 \%$ greater duration of sessions, $27 \%$ greater duration of intervention, and $77 \%$ desired booster sessions in the future. No more than 1 person desired less of any of these categories.

Conclusions: Acceptability and feasibility of a 5-session CBCSM intervention was supported in the context of cancer care delivery.

CORRESPONDING AUTHOR: Shawna Ehlers, Mayo Clinic, shawna.ehlers.wilbur@gmail.com

\section{P009}

CONTEXTUAL INFLUENCES ON SLEEP AND BEDTIME ROUTINES AMONG PRESCHOOL-AGED CHILDREN OF BRAZILIAN IMMIGRANT PARENTS: A QUALITATIVE STUDY CONDUCTED IN THE UNITED STATES

Ana Cristina Lindsay ${ }^{1}$, Carlos Andre Moura Arruda ${ }^{2}$, Marcia Maria Tavares Machado ${ }^{2}$, Gabriela De Andrade ${ }^{1}$, Mary L. Greaney ${ }^{3}$

${ }^{1}$ Univeristy of Massachusetts Boston- USA

${ }^{2}$ Federal University of Ceara- Brazil

${ }^{3}$ University of Rhode Island- USA

CORRESPONDING AUTHOR: Ana Cristina Lindsay, Univeristy of Massachusetts Boston, Ana.Lindsay@umb.edu

\section{P010}

PERCEIVED COGNITIVE IMPAIRMENT IN HAEMATOLOGICAL CANCER PATIENTS WHO HAVE UNDERGONE ALLOGENEIC STEM CELL TRANSPLANTATION

Amanda Hutchinson ${ }^{1}$, Elise Thompson ${ }^{1}$, Alexis Wheeler $^{1}$, Carlene Wilson $^{2}$, Nicole Loft ${ }^{3}$, Agnes Yong ${ }^{3} 4$

${ }^{1}$ University of South Australia- Australia

${ }^{2}$ Flinders University; Olivia Newton John Cancer Wellness and Research Centre; La Trobe University- Australia

${ }^{3}$ Royal Adelaide Hospital- Australia

${ }^{4}$ SAHMRI; University of Adelaide- Australia

Introduction And Purpose: Few studies have examined cognition in haematological cancer patients. This study aimed to determine whether haematological cancer patients with allogeneic stem cell transplantation (SCT) report greater perceived cognitive impairment (PCI) than controls. METHODS: Participants were 30 haematological cancer patients who had undergone allogeneic SCT in the past 1-5 years and 30 agematched healthy controls. Participants completed measures of PCI, mood, sleep quality, fatigue \& premorbid IQ. RESULTS: Patients reported significantly greater PCI than controls; this difference consituted a large effect. Sleep quality, fatigue and mood explained $82 \%$ of the variance in PCI in patients. CONCLUSIONS: There is evidence of PCI in haematological cancer patients with allogeneic SCT, associated with sleep, fatigue \& mood.

CORRESPONDING AUTHOR: Amanda Hutchinson, University of South Australia, amanda.hutchinson@unisa.edu.au

P011

PSYCHOSOCIAL PREDICTORS OF SCREEN TIME ARE CORRELATED TO OBJECTIVELY MEASURED SEDENTARY BEHAVIOR IN BRAZILIAN ADOLESCENTS?

Bruno Costa ${ }^{1}$, Marcus Vinicius Veber Lopes ${ }^{1}$, Luis Eduardo Argenta Malheiros ${ }^{1}$, Kelly Samara da Silva ${ }^{1}$

${ }^{1}$ Federal University of Santa Catarina- Brazil

Introduction \& Purpose: Sedentary behavior (SB) has been associated with decreased levels of cardiorespiratory fitness, unhealthy body composition, and social anxiety in youth. Interventions have been designed to decrease SB among adolescents, and their actions are based on behavior change theories. These theories postulate that in order to change SB, other psychosocial factors must be targeted first. Thus, identifying psychosocial correlates of SB is the first step towards designing effective actions. The aim of this study was to identify psychosocial factors related to SB with habitual objectively-measured SB in a sample of 11-14-year-old Brazilian adolescents. Methods: Adolescents from two schools wore Actigraph GT3x+ and wGT3x+ accelerometers on the right hip for 10 days. Those who provided 10 hours of valid data on at least 4 days ( 1 weekend day) after exclusion of 60 consecutive minutes of zero acceleration had their habitual SB was estimated and were included in the analyses. A standardized questionnaire was answered, containing questions of attitudes, expectations, self-efficacy and family rules related to watching $\mathrm{TV}$ and using the computer. Linear regression analyses were used to test the associations between psychosocial factors and SB. The adjusted model included age and all psychosocial variables. Results: A total of 198 adolescents received accelerometers, but only 62 (31\%) (24 boys, mean age $13.0 \pm 1.0$ years) answered the questionnaire and provided valid accelerometer datasets and were included in the analysis. They spent $70.2 \%$ of their time in SB. No association was found between SB and attitudes ( $\mathrm{p}=0.604)$, self-efficacy $(\mathrm{p}=0.702)$, expectations $(\mathrm{p}=0.198)$, or family rules $(0.486)$ in the crude and adjusted analyses. Conclusions: Screentime-based psychosocial factors were not associated with objectivelymeasured SB in adolescents. This may be explained by the other components of SB measured by the accelerometer like using smartphones and tablets, studying at school and at home while staying still, which these scales are not sensitive to. Instruments to access psychosocial factors could be updated to include other modalities of SB besides the use of television and computers.

CORRESPONDING AUTHOR: Bruno Costa, Federal University of Santa Catarina, bgcosta6@gmail.com

P012

ALTERING THE PLACEMENT OF PRODUCTS WITHIN PHYSICAL MICRO-ENVIRONMENTS: A CONCEPTUAL REVIEW AND PROPOSED FRAMEWORK

Rachel Pechey ${ }^{1}$, Gareth J. Hollands ${ }^{1}$, Patrice Carter ${ }^{2}$, Theresa M. Marteau ${ }^{1}$

${ }^{1}$ University of Cambridge- United Kingdom

${ }^{2}$ University College London- United Kingdom

Introduction and Purpose: There is much research and policy interest in changing unhealthier behaviours. Altering the placement of food, alcohol and tobacco products in environments such as shops, bars and restaurants comprises one possible set of interventions, encompassing product 
availability and position. However, this body of work lacks consistent conceptualisation, hindering systematic and cumulative synthesis. Methods: This conceptual review describes how Availability and Position interventions have been operationalised, revealing inconsistency in terminology and in the targets of change. A conceptual framework is proposed categorising intervention types and summarising constituent components from which interventions can be reliably described and synthesised. Results: The principal distinctions proposed are: Availability: Interventions are divided into those altering: i. Absolute Availability: overall number of options ii. Relative Availability: proportion of items comprised by a subset of products iii. Both Absolute and Relative Availability. Position: Interventions are divided into those altering positions that are: $i$. All within-reach (Reachable vs. Reachable) ii. Some within-reach and some out-of-reach (Reachable vs. Unreachable) iii. All out-of-reach (Unreachable vs. Unreachable) For (i) and (iii), these can be subdivided into interventions which alter the distance of positions (Proximity) from the participants' starting point, and interventions for which investigated positions are Equidistant. Conclusions: The proposed framework has the potential to facilitate future study of two sets of interventions that could contribute significantly to healthier behaviour across populations.

CORRESPONDING AUTHOR: Rachel Pechey, University of Cambridge, rachel.pechey@medschl.cam.ac.uk

\section{$\mathrm{P} 013$}

EFFECTS OF THREE LEVELS OF PSYCHOSOCIAL INTERVENTION ON THE NEED FOR PSYCHIATRIC CARE AMONG PATIENTS HOSPITALIZED FOR A STEM CELL TRANSPLANTATION

Liliana Mey Len Rivera Fong ${ }^{1}$, Liliana Rivera Fong ${ }^{1}{ }^{2}$, Rebeca Robles García $^{3}$, Corina Benjet ${ }^{3}$, Lara Traeger ${ }^{4}$, Josana Rodríguez Orozco ${ }^{1}$, Juan José Sánchez Sosa ${ }^{1}$, Brenda Lizeth Acosta Maldonado², Luis Manuel Valero Saldaña ${ }^{2}$

${ }^{1}$ National University of Mexico- Mexico

${ }^{2}$ National Cancer Institute- Mexico

${ }^{3}$ National Institute of Psychiatry- Mexico

${ }^{4}$ Massachusetts General Hospital; Harvard Medical School- USA

Over half of patients with hematologic malignancies who are treated with hematopoietic stem cell transplantation (HSCT) report clinically significant psychological symptoms which require psychopharmacological management during the HCST hospital stay. AIM: To evaluate the effect of three levels of psychological intervention on the need for psychiatric care during the HSCT hospital stay. METHOD: Quasi-experimental study comparing need for psychiatric care in three groups: (First) HSCT patients in 2016 who completed a semi-structured clinical interview and self-report measures of psychological symptomatology (which could lead to a brief individualized cognitive-behavioral intervention); (Second) Patients in 2012 who participated in a cognitive-behavioral intervention involving the same techniques for everyone (psychoeducation and progressive muscle relaxation training before hospitalization), and (Third) historical matched control patients in 2014 who only completed a unstructured clinical interview. Need for psychiatric care was determined by: (a) psychiatric consultation (the decision was responsibility of the physician or the head nurse) for moderate to high psychological symptoms like anxiety, depression or insomnia interfering with the medical treatment and/or (b) receive pharmacological treatment. PARTICIPANTS: 78 patients were included in the study, 33 in the first group (12 allogeneic HCST and 21 autologous HCST; mean age $=41$ years $[\mathrm{SD}=14]$; $54.5 \%$ male, $63.6 \%$ married), 14 in the second group (2 allogenic HCST and 12 autologous HCST; mean age $=43.6$ years [SD $=15]$; $50 \%$ male, $43 \%$ married), and 31 in the third group (11 allogeneic HSCT and 20 autologous HSCT; mean age $=43$ years $[\mathrm{SD}=13]$; $54.8 \%$ male; $58.1 \%$ married). RESULTS: Fewer patients in the first group required either psychiatric consultation $(21.2 \%$ vs. $35.7 \%$ vs. $54.8 \%$; $2(2)=7.75, \mathrm{p}=.02)$ or psychopharmacological treatment before HSCT $(18.2 \%$ vs. $21.4 \%$ vs $51.6 \%$; $\mathrm{x} 2(2)=9.08, \mathrm{p}=.01)$ or during the hospital stay ( $24.2 \%$ vs $28.6 \%$ vs $61.3 \%$; $\mathrm{x} 2(2)=10.05$, $\mathrm{p}<.01)$ as compared to group two and three. CONCLUSION: Lower need for psychiatric care was observed when patients were systematically evaluated and clinicians expressly identified patient's intervention needs.

CORRESPONDING AUTHOR: Liliana Mey Len Rivera Fong, National University of Mexico, lmeylenf@ hotmail.com

\section{P014}

DOES A CARE MANAGEMENT PROGRAM FOR PATIENTS WITH CHRONIC DEPRESSION BENEFIT THOSE WITH COOCCURRING ALCOHOL OR DRUG USE DISORDERS?

Julie Richards ${ }^{1}$, Evette Ludman ${ }^{1}$, Emily Williams ${ }^{2}$, Greg Simon ${ }^{1}$

${ }^{1}$ Kaiser Permanente Washington- USA

${ }^{2}$ University of Washington Health Services Department- USA

Introduction: Chronic depression and alcohol \& drug use disorders [AODs] frequently co-occur; but it is unclear whether patients with AOD benefit as much from structured treatment programs. Our goal was to evaluate whether presence of AOD at baseline modified the clinical effectiveness of a treatment program for chronic depression. Methods: Randomized trial participants $(n=302)$ age $\geq 18$ were identified by population-based screening \& provider referral from 5 clinics in Seattle WA. The intervention was a flexible program including outreach care management to improve engagement with mental health services \& pharmacotherapy, and a selfmanagement behavioral \& recovery-oriented group program. Repeated measure estimates of the long-term intervention effects demonstrated improvement for 4 patient-reported outcomes: symptom severity (SCL-20), recovery (RAS), \%with major depression (SCID-I), and \% reporting much improvement at $6,12 \& 18$ months. The AOD+ group was identified by baseline Alcohol Use Identification Test and Drug Psychiatric Diagnostic Screening Questionnaire. To evaluate differential effectiveness of the intervention outcomes by AOD status, we used random-effects mixed models for continuous outcomes and GEE for binary outcomes. We included a 3way interaction term for treatment assignment, AOD status and time, 2way interactions, main effects, and controlled for sex, treatment site, \& baseline value of the outcome; and for significant interactions we present stratified results. Results: Participants were $70 \%$ white, $68 \%$ women, with mean age 49.8. At baseline, 106 (35\%) screened AOD+: 31\% alcohol+, $12 \%$ drug+ and $8 \%$ both. Intervention effects at 18 months did not differ across AOD status, except for the SCID outcome (interaction p-values 0.07, 0.61, 0.84, and 0.02, for SCL-20, RAS, improvement, and SCID, respectively). Stratified analyses of SCID outcome at 18 months indicated AOD+ group randomized to the intervention had lower odds of major depression than the control group participants $(\mathrm{OR}=0.10,95 \% \mathrm{CI} .01$, $0.96, \mathrm{p}=0.046$ ), while no intervention effects were observed among AOD- participants $(\mathrm{OR}=0.61,95 \%$ CI $0.32,1.14, \mathrm{p}=0.122)$. Conclusions: Results suggest the program was beneficial for patients with chronic depression irrespective of AOD status.

CORRESPONDING AUTHOR: Julie Richards, Kaiser Permanente Washington, Health Research Institute, richards.je@ghc.org

\section{P015}

INFLUENCE OF THE EMOTIONAL ALTERATIONS IN THE ARTERIAL STIFFNESS INDEX AND CARDIOVASCULAR RISK OF PREHYPERTENSIVE PATIENTS

Miguel Enrique Sánchez-Hechavarría ${ }^{1}$, Agustín Sánchez-Mengana ${ }^{2}$, Alexander Pascau-Simón ${ }^{3}$, María Eugenia García-Céspedes ${ }^{3}$, Jorge Carlos Abad-Araujo ${ }^{1}$, José Antúnez-Coca ${ }^{1}$, Yailén Saavedra-Fajardo ${ }^{2}$

${ }^{1}$ Universidad de Ciencias Médicas de Santiago de Cuba- Cuba

${ }^{2}$ Policlínico "Josué País García" de Santiago de Cuba- Cuba

${ }^{3}$ Hospital General Docente "Dr. Juan Bruno Zayas Alfonso" de Santiago de Cuba- Cuba 
The environmental and psychosocial factors have an important role in the development of cardiovascular diseases, constituting the distress one of the triggers of hypertension and an element present in early stages of it. Objective: To determine the influence of emotional alterations in the index of arterial stiffness and cardiovascular risk of prehypertensive patients. Methodology: A cross-sectional descriptive study was carried out in 48 prehypertensive patients ( 30 women) from the clinic 2 of the "Josué País García" Polyclinic in Santiago de Cuba, from November 2016 to March 2017, where the Cornell Index test, global cardiovascular risk and lower limb arterial stiffness index with ANGIODIN® PD 3000 equipment records were evaluated. Results: In prehypertensive patients, 58.3\% presented a positive Cornell test, $39.6 \%$ of them female, and $18.8 \%$ male. There was a significant relationship $(\mathrm{p}<0.001)$ between the presence of emotional disturbances and moderate cardiovascular risk. The prehypertension patients with emotional alterations presented a significant increase $(p=0.034)$ in the arterial stiffness index $(7.04 \pm 2.59)$ with respect to the patients who did not find emotional alterations $(5.85 \pm 1.00)$. Conclusions: The presence of emotional alterations in prehypertensive patients is associated with an increase in arterial stiffness and an increased global cardiovascular risk.

CORRESPONDING AUTHOR: Miguel Enrique Sánchez-Hechavarría, Universidad de Ciencias Médicas de Santiago de Cuba, miguel.sanchez881119@gmail.com

\section{P016}

GENDER ROLE ORIENTATION AND INTERPERSONAL STRESS IN VITALLY EXHAUSTED MEN - FINDINGS FROM THE MEN STRESS 40+ STUDY

Susanne Fischer

${ }^{1}$ University of Zurich, Institute of Psychology, Clinical Psychology and Psychotherapy- Switzerland

Introduction Vital exhaustion is defined by unusual fatigue, increased irritability, and feelings of demoralisation. It has repeatedly been found a major risk factor for cardiovascular morbidity and mortality. Stress is a commonly assumed cause of vital exhaustion. However, the exact circumstances evoking stress in this population remain unknown. Gender schema theory posits that individuals rigidly conforming to either masculine or feminine gender roles are less flexible in responding to social demands. Following from this, the same individuals should have a higher propensity towards experiencing interpersonal stress when compared to androgynous individuals. The aim of the present study was to investigate for the first time whether gender role orientation is linked with interpersonal stress in vitally exhausted men. Methods A total of $\mathrm{N}=121$ men with vital exhaustion according to the Maastricht Vital Exhaustion Questionnaire (MVEQ) were recruited. An updated (2011) version of the Bem Sex-Role Inventory (BSRI) was administered in a subsample of $\mathrm{N}=79$ heterosexual men, dividing them into four groups: masculine, feminine, androgynous (above-average masculinity and femininity scores), and undifferentiated (below-average scores). Interpersonal stress was measured multi-dimensionally, using the Trier Inventory for the Assessment of Chronic Stress (TICS). A False Discovery Rate was used to adjust for multiple testing. Results Masculine ( $n=23)$, feminine $(n=14)$, androgynous $(n=21)$ and undifferentiated men $(n=21)$ did not differ in terms of work overload, performance pressure, dissatisfaction with work, social overload, lack of social recognition, social isolation, or chronic worrying (all $\mathrm{p}>.375$ ). However, androgynous men reported significantly lower levels of excessive demands at work $(\mathrm{p}=.041)$ and social tension $(\mathrm{p}=.004)$ when compared to the other groups. Conclusions Vitally exhausted men adopting an either masculine or feminine gender role, and those with a low propensity for both roles were more likely to feel overwhelmed with work and to experience social tension when compared to androgynous men. Failure to navigate masculine and feminine behaviours in a context-dependent manner could thus be one of the major drivers of interpersonal stress in vitally exhausted individuals.
CORRESPONDING AUTHOR: Dr. Susanne Fischer, University of Zurich, s.fischer@psychologie.uzh.ch

\section{P017}

IS PSYCHOLOGICAL WELLBEING ASSOCIATED WITH A HEALTHY LIFESTYLE? CROSS-SECTIONAL SURVEY OF MEDITERRANEAN DIET INTAKE, PHYSICAL ACTIVITY, SMOKING AND WELLBEING IN CHILEAN ADULTS

Guadalupe Echeverría ${ }^{1}$, Sonia D'Acuña ${ }^{2}$, Inés Urquiaga ${ }^{2}$, Catalina Mena $^{2}$, María Pía Nitsche ${ }^{2}$, Marcela Bitran ${ }^{3}$, Nuria Pedrals ${ }^{2}$, Attilio Rigotti $^{2} 4$

${ }^{1}$ Pontificia Universidad Católica de Chile- Chile

${ }^{2}$ Center for Molecular Nutrition and Chronic Diseases, School of Medicine, Pontificia Universidad Católica de Chile- Chile

${ }^{3}$ Center of Medical Education, School of Medicine, Pontificia Universidad Católica de Chile- Chile

${ }^{4}$ Department of Nutrition, Diabetes and Metabolism, School of Medicine, Pontificia Universidad Católica de Chile- Chile

Noncommunicable chronic diseases, including ischemic heart disease and stroke, are rapidly increasing worldwide. In developed countries, wellbeing indicators have been consistently associated with healthy lifestyles and reduced cardiovascular risk, however similar information is very limited from developing countries. We performed a cross-sectional internet survey to evaluate psychological wellbeing (including measurements of Mental Health Continuum (MHC), optimism, and subjective vitality) and its relationship with lifestyle habits (diet, physical activity and tobacco use) as well as nutritional status in 1,750 Chilean adults ( $33 \pm$ 12 years-old, $75 / 25 \%$ female/male ratio, $49 \%$ with overweight or obesity). Our results showed that elderly ( $>50$ years-old) subjects exhibited higher wellbeing, optimism and subjective vitality than younger people. Additionally, healthy eating -defined as a high adherence to Mediterranean diet intake- and high physical activity, but not smoking, were significantly associated with all wellbeing measures. We also found a strong inverse association between wellbeing scores and body mass index. In conclusion, our findings support a link between wellbeing measures and different components of a healthy lifestyle behavior in Chile. Interventions aimed to increase wellbeing may lead to a better lifestyle as well as reduced risk of chronic diseases.

CORRESPONDING AUTHOR: Guadalupe Echeverría, Pontificia Universidad Católica de Chile, gecheverria@bio.puc.cl

\section{P018}

SOMATIC SYMPTOM PERCEPTION AND INTEROCEPTION - A PSYCHOMETRIC APPROACH

Michael Witthöft ${ }^{1}$

${ }^{1}$ Johannes Gutenberg-University, Mainz- Germany

Introduction: Chronic somatic symptom distress (as, e.g., characteristic of chronic pain conditions, panic disorder, and pathological health anxiety) represents a widespread and disabling clinical condition of transdiagnostic relevance. Cognitive models assume that abnormalities in interocption might be causally related to the development and maintenance of chronic somatic symptom distress. Different models, however, disagree on the exact nature of the assumed abnormality, i.e., cognitivebehavioral models stress a hypervigilant interoceptive cognitive style (predicting higher interoceptive accuracy) whereas recent models based on the predictive-coding framework assume a less detailed sensory processing (predicting lower interoceptive accuracy). Based on these two theoretical positions, this study aims at empirically testing the relationship between interoceptive accuracy and chronic somatic symptom distress. Methods: Using structural equation modelling (SEM), associations between latent variables of interoception (based on the heartbeat mental 
tracking task) and symptom perception (based on the PHQ-15) were tested in a heterogeneous sample $(\mathrm{N}=340)$ of college students $(n=227)$, healthy members of the general population $(n=50)$, patients with a somatoform disorder $(n=63)$. Affective-motivational and sensory facets of symptom perception were dissociated using a bi-factor model of the PHQ-15. Results: Model fit was excellent (CFI=.99; RMSEA=.02). The affective-motivational component of symptom perception was unrelated to interoceptive accuracy ( $\mathrm{r}=-.04, \mathrm{p}=.66)$. In terms of the symptomspecific factors (most likely reflecting sensory aspects of symptom perception), only the cardio-respiratory factor showed a significant negative association $(r=-.34, p=.02)$ with interoceptive accuracy. None of the other latent variables of symptom perception showed significant associations to symptom perceptions. Discussion: Stronger sensory (but not affective) symptom perception in the cardio-respiratory system appears to be associated with lower interoceptive accuracy. The findings are more in line with the predictive-coding approach of symptom perception, suggesting a less detailed and more biased interoception associated with higher somatic symptom distress (particularly in the cardio-respiratory system). Better paradigms for the assessment of interoceptive accuracy (that are able to distinguish between sensitivity and bias) across multiple domains of interoception are needed for future research.

CORRESPONDING AUTHOR: Michael Witthöft, Johannes GutenbergUniversity, Mainz,witthoef@uni-mainz.de

\section{P019}

INTEROCEPTIVE ACCURACY, SOMATOSENSORY AMPLIFICATION, ABSORPTION, AND SYMPTOMS REPORTS Ferenc Köteles $^{1}$

${ }^{1}$ ELTE Eötvös Loránd University- Hungary

Introduction \& Purpose The role of trait-like characteristics in the perception and reporting of subjective somatic symptoms is an open question. Concerning the direct impact of interoceptive information, usually assessed as the ability to detect heartbeats, the available empirical findings are equivocal. Two self-report characteristics, somatosensory amplification and absorption, have been reliably associated with symptom reports in past studies, however, they joint impact and possible interaction are yet to be explored. This study aimed to investigate the independent contributions of the aforementioned three characteristics to symptoms reports, as well as the interaction of the latter two. Methods 134 college students (52.2\% female) completed a questionnaire consisting of the Somatosensory Amplification Scale (SSAS), Tellegen Absorption Scale (TAS), PANAS Negative Affect Scale (NA), and the Subjective Somatic Symptom Scale of the patient health questionnaire (PHQ-15). Interoceptive accuracy was assessed using the Schandry heartbeat tracking paradigm. Results Interoceptive accuracy was not related to any of the assessed constructs. In a multiple linear regression analysis $(\mathrm{R} 2=0.283, \mathrm{p}<$ $0.001)$, predictors of PHQ-15 were female gender $(\beta=0.259$, $p<$ $0.01)$, NA $(\beta=0.358, p<0.001)$, SSAS $(\beta=0.166, p<0.05)$, and TAS $(\beta=0.154, p<0.05)$ scores. However, contribution of the SSAS $\mathrm{x}$ TAS interaction term was not significant $(\beta=0.011, \mathrm{p}=0.879)$. Conclusions For healthy individuals, interoceptive accuracy does not play a role in symptom reporting. Beyond gender and negative affect, somatosensory amplification and absorption independently contribute to symptom reports. The lack of interaction can be explained by the substantial differences between the two constructs. While absorption refers to episodes of "total" non-evaluative attention paid to external or internal sensory experiences, somatosensory amplification assesses the automatic evaluation of the possible threat the perceived body signals might represent.

CORRESPONDING AUTHOR: Ferenc Köteles, ELTE Eötvös Loránd University, fecuska@gmail.com

\section{P020}

ASSOCIATION BETWEEN SEXUAL PERCEIVED BARRIERS AGAINST SAFER SEX AND PREPARATORY BEHAVIORS AMONG MOZAMBICAN WOMEN AT RISK FOR HIV/AIDS INFECTION

Ana Luísa Patrão ${ }^{1}$, Teresa McIntyre ${ }^{2}$

${ }^{1}$ Institute of Collective Health- Brazil

${ }^{2}$ Department of Psychology, Houston Baptist University- USA

Introduction \& Purpose: Preparatory behaviors are extremely important for sexual protection in African women, because the intention to use and get condoms are the best predictors of effective condom use in some African contexts. However, these preventive behaviors not as successful as desired, because it is often associated with negative meanings attributed to sexual acts. This work aims to identify if perceived barriers against safer sex are associated with sexual preparatory behaviors among Mozambican women at risk for HIV/AIDS infection. Methods: Women (173), patients at a public Hospital and at risk for HIV infection, completed measures of sociodemographic and marital characteristics, perceived barriers against safer sex, and sexual preparatory behaviors. Results: Socio-demographic variables (age and education) explained $16.1 \%$ of variance $(\Delta \mathrm{F}(2,170)=16.30, \mathrm{p}<.001)$, and marital variables, "marital status" and "talking about AIDS with partner" explained $22.3 \%$ of additional variance $(\Delta \mathrm{F}(2,168)=30.36, \mathrm{p}<.001)$. The results of the hierarchical regression analyses for the perceived barriers against safer sex subscales revealed that this set of variables explained $14.3 \%$ of additional variance in sexual preparatory behaviors $(\Delta \mathrm{F}(4,164)=12.82, \mathrm{p}<.001)$. More specifically, the univariate results showed that absence of risk perception is a marginally significant predictor $(\mathrm{t}=-1.80, \mathrm{p}=.074)$, perceived partner negative attitudes against safer sex is significant $(t=-6.17$; $\mathrm{p}=.000$ ), and perceived low communication self-efficacy with partner is a marginally significant predictor $(t=-1.71 ; \mathrm{p}=.090)$. Perceived partner negative attitudes against safer sex was the most important predictor $(\beta$ $=-.40$ ). Perceived negative attitudes against safer sex was not a significant predictor. The overall model explained $50.8 \%$ of variance in sexual preparatory behaviors. Conclusions: These results seem to support an exploratory predictive model of sexual preparatory behaviors that can inform interventions directed at behavioral change among Mozambican women at sexual risk.

CORRESPONDING AUTHOR: Ana Luísa Patrão, Institute of Collective Health, lispatrao@gmail.com

\section{P021}

HANDGRIP STRENGTH IS ASSOCIATED WITH ASPECTS OF COGNITIVE FUNCTION IN CANCER SURVIVORS

Lee Smith ${ }^{1}$, Lin Yang ${ }^{2}$, Graham Colditz ${ }^{3}$, Thomas Waldhor ${ }^{2}$

${ }^{1}$ Anglia Ruskin University- United Kingdom

${ }^{2}$ Medical University of Vienna- Austria

${ }^{3}$ Washington University School of Medicine- USA

Background: Cancer- and cancer treatment-related cognitive impairments are prevalent among cancer survivors. Recently, low serum level of brainderived neurotrophic factor (BDNF) was found to be associated cognitive impairments, and genetic variations in the BDNF gene were found to protect against cognitive impairments in cancer patients receiving chemotherapy. These findings are promising as BNDF is a neurotrophin, secreted in responses to muscle contraction. The present study aimed to evaluate the associations of handgrip strength and cognitive function in cancer survivors over 60 years old using data from the National Health and Nutrition Examination Survey (NHANES). Methods: Data in two waves of NHANES (2011-2014) were aggregated. Handgrip strength in kilogram $(\mathrm{kg})$ was defined as the maximum value achieved using either hand. Two cognitive function tests were conducted among adults 60 years and older. The Animal Fluency Test (AFT) examines categorical verbal 
fluency (a component of executive function), and the Digital Symbol Substitution test (DSST) assesses processing speed, sustained attention, and working memory. Survey analysis procedures were used to account for the complex sampling design of the NHANES. Multiple linear regression models were used to estimate associations of handgrip strength with cognitive test scores, adjusting for confounders (age, gender, race/ethnicity, education, marital status, smoking status, depressive symptoms and leisure time physical activity). Results: Among 383 cancer survivors $(58.5 \%$ women, mean age $=70.9$ years, mean $\mathrm{BMI}=29.3 \mathrm{~kg} / \mathrm{m} 2)$, prevalent cancer types were breast (22.9\%), prostate (16.4\%), colon (6.9\%) and cervix $(6.2 \%)$. In women, each increase $\mathrm{kg}$ of handgrip strength was associated with 0.20 (95\% CI: 0.08 to 0.33 ) higher score on AFT and 0.83 (95\% CI: 0.30 to 1.35 ) higher score on DSST. In men, we observed an inverted U-shape association where cognitive function peaked at handgrip strength of 40-42 kg. Conclusions: Handgrip strength, a modifiable factor, appears to be associated with aspects of cognitive functions in cancer survivors. Prospective studies are needed to address their causal relationship.

CORRESPONDING AUTHOR: Lee Smith, Anglia Ruskin University, lee.smith@anglia.ac.uk

\section{$\mathrm{P} 022$}

HOW DOES OFFERING DISINCLINED PEOPLE CHOICE BETWEEN DIFFERENT SCREENING APPOINTMENTS AFFECT SCREENING INTENTIONS? EVIDENCE FROM AN ONLINE EXPERIMENT

Sandro Tiziano Stoffel ${ }^{1}$, Alex Ghanouni ${ }^{1}$, Yasemin Hirst $^{1}$, Jo Waller ${ }^{1}$, Christian von Wagner ${ }^{1}$

${ }^{1}$ University College London- United Kingdom

Introduction Previous research has demonstrated how increasing access to cancer screening via single timed appointment can increase uptake. The current study investigated whether offering a range of different timed appointment slots would either further enhance this effect or lead to confusion and increased perceived difficulty to make the screening decision. Methods We recruited 9,127 men and women aged 35-54 without previous diagnosis of bowel cancer living in England for this online study. After being informed about bowel scope screening (BSS), 2,125 (23.3\%) stated that they did not intend to participate. These respondents were randomised to receive either 1, 2, 4 or 6 available appointment alternatives. We used multivariable logistic regression adjusting for sociodemographic variables to predict intention by condition and report adjusted odds ratio $(\mathrm{aOR})$ and $95 \%$ confidence intervals (CI). Results 1,908 of 2,125 (89.8\%) respondents successfully completed all comprehension and manipulation checks, most were female (57.8\%), White-British (82.0\%), married/cohabiting $(65.5 \%)$ and working $(76.2 \%)$. Compared to offering a single appointment option, we found that offering people the choice of 2, 4 or 6 appointments decreased their likelihood of confirming one of the given appointments $(25.8 \%, 25.3 \%$ and $24.8 \%$ vs $34.5 \%)$. Giving people the choice between 2 appointments significantly decreased intention (aOR 0.64; CI 0.48-0.85). Similarly, offering 4 (aOR 0.63 ; CI $0.47-0.83$ ) or all 6 alternatives (aOR 0.63 ; CI $0.47-0.85$ ) decreased intentions to a similar extent. Interestingly, we found no evidence for confusion as individuals across the conditions perceived the invitation process as easy $(\mathrm{p}=0.778)$ and the screening decision as not difficult $(\mathrm{p}=0.550)$. Furthermore, individuals in the choice conditions perceived the offered appointments as more convenient $(\mathrm{p}=0.003)$. Conclusions Although we could not find evidence for confusion, the present study supports the notion that offering more than one pre-set appointment may have a detrimental impact on uptake. Reminders for previous non-responders may therefore not benefit from being offered multiple preset appointments.

CORRESPONDING AUTHOR: Sandro Tiziano Stoffel, University College London, s.stoffel@ucl.ac.uk
P023

FACTORS RELATED TO PAIN INTENSITY AND PHYSICAL FUNCTIONING IN THE HYPERMOBILE EHLERS-DANLOS SYNDROME

Carolina Baeza-Velasco ${ }^{12}$, Larissa Kalisch ${ }^{3}$, Caroline Bourdon ${ }^{4}$, Lucile Montalescot ${ }^{1}$, Cécile de Cazotte ${ }^{1}$, Claude Hamonet ${ }^{4} 56$

${ }^{1}$ Laboratory of Psychopathology and Health Processes, University Paris Descartes - Sorbonne Paris Cité, Boulogne Billancourt- France

${ }^{2}$ INSERM U1061, Neuropsychiatry: Epidemiological and Clinical Research, Department of Emergency Psychiatry and Acute Care, CHU Montpellier- France

${ }^{3}$ Anglia Ruskin University, Cambridge- United Kingdom

${ }^{4}$ Functional Rehabilitation Center, Evry Hospital, Evry- France

${ }^{5}$ Department of Physical Medicine and Rehabilitation, Hôtel-Dieu Hospital, APHP Paris, France

${ }^{6}$ University Paris-Est Créteil- France

Introduction: The Ehlers-Danlos syndromes (EDS) are a heterogeneous group of hereditary disorders affecting connective tissue matrix proteins. The most common form of EDS is the hypermobile EDS (hEDS), which represents $80-90 \%$ of EDS cases. Unlike other EDS subtypes, hEDS cannot can be confirmed by biological tests. Thus, its diagnosis remains clinical. This aspect negatively affects the recognition of this pathology, which is currently underresearched and underdiagnosed worldwide. hEDS is characterized by fragility of tissue which leads to multi-systemic symptoms. Pain is nevertheless the most reported. They are often musculoskeletal, resulting from a propensity to micro- and macrotraumatism. Even though there is not life-risk, the variety and accumulation of symptoms and their long duration make hEDS a chronic, painful and highly disabling condition. Unfortunately, factors associated with pain and disability in this condition are poorly known. These merits more attention for clinical and prevention purposes. Purpose: To identify sociodemographic, health and psychological factors associated to pain intensity and physical functioning in hEDS. Method: In this cross-sectional study, pain, fatigue, physical functioning, BMI, diagnosis delay, kinesiophobia, pain catastrophizing, depression, anxiety and sociodemographic characteristics were explored in $75 \mathrm{hEDS}$ patients through interviews and self-questionnaires. Univariate and multivariate analyses were performed to identify associated and predictors variables of pain intensity and physical functioning. Results: Severe pain intensity was significantly associated to diagnosis delay (Mean=22.4 years; $\mathrm{SD}=12.9$ ), helplessness and rumination. Binary logistic regression showed that diagnosis delay and helplessness were variables that predict severe pain. Physical functioning was significantly associated to age, BMI, diagnosis delay, pain, fatigue and depression. These variables except depression were retained by multivariate analyze to predict physical functioning. Conclusion: Results suggest that health and psychological factors influence pain and physical functioning and should be explored in those affected. Dissemination of knowledge on hEDS seem a central issue to promote early detection and avoid/reduce the important diagnosis delay which is detrimental to patients' physical and psychosocial health.

CORRESPONDING AUTHOR: Carolina Baeza-Velasco, University Paris Descartes-Sorbonne, baezacarolina@yahoo.com

\section{P024}

EXPERIENCE OF SIBLING DEATH IN CHILDHOOD AND RISK OF PSYCHIATRIC CARE IN ADULTHOOD: A NATIONAL COHORT STUDY FROM SWEDEN

Mikael Rostila ${ }^{1}$, Lisa Berg ${ }^{1}$, Jan Saarela ${ }^{2}$, Ichiro Kawachi ${ }^{3}$, Anders Hjern ${ }^{1}$

${ }^{1}$ Stockholm University- Sweden

${ }^{2}$ Åbo Akademi University- Finland

${ }^{3}$ Harvard University- USA

Introduction and purpose: Sibling loss is associated with an increased risk of suicide and other external causes, but little is known about the 
association with psychiatric health problems. An understanding of this association may inform the health care system about preventive efforts. We studied the influence of sibling loss during childhood on inpatient and outpatient psychiatric care in young adulthood, adjusting for family related psychosocial covariates shared by siblings in childhood. Methods: A national cohort born in Sweden in 1973-1982 ( $\mathrm{N}=701$ 320) was followed prospectively until 2013. Cox proportional hazards models were used to analyse the association between sibling loss during childhood and psychiatric inpatient and outpatient care identified by the Hospital Discharge Register. Out- and inpatient psychiatric care was defined as at least one visit or hospital admission with a main or complimentary diagnosis with an ICD10 code F00-99 registered in the Hospital Discharge Register. Results: After adjustment for confounders, the HRs of psychiatric care in men who experienced sibling loss were 1.16 (95\% CI 1.01-1.33) for inpatient care and HR $1.16(95 \% \mathrm{CI}$ 1.06-1.27) for outpatient care while the associations were nonsignificant in women. Among women, loss of a sibling during adolescence (i.e. during ages 12-18 years) was significantly associated with psychiatric care; HRs were 1.34 (95\% CI 1.08-1.67) for inpatient care and 1.18 (95\% CI 1.02-1.36) for outpatient care. Among men bereaved in adolescence the HR were 1.25 (95\% CI 1.07-1.46) for outpatient care. Conclusions: The death of a sibling in childhood was associated with increased risk of hospital psychiatric care in adulthood among men and women bereaved in adolescence. Increased psychiatric health problems following bereavement could underlie the previously found association between sibling loss in adolescence and mortality from external causes.

CORRESPONDING AUTHOR: Mikael Rostila, Stockholm University, mikael.rostila@su.se

\section{P025}

PSYCHOSOCIAL PREDICTORS OF HEALTHY LIFESTYLE AMONG BRAZILIAN MEN

Ana Luísa Patrão ${ }^{1}$, Estela Aquino ${ }^{12}$, Maria da Conceição Almeida ${ }^{3}$, Sheila Alvim ${ }^{12}$

${ }^{1}$ Institute of Collective Health- Brazil

${ }^{2}$ Federal University of Bahia- Brazil

${ }^{3}$ Bahia Oswaldo Cruz Foundation- Brazil

Introduction \& Purpose: Individual actions with respect to healthier lifestyles may be limited due to psychosocial factors that influence an individual's decision-making capacity. The present work aimed to identify psychosocial predictors of healthy lifestyle among Brazilian men. Methods: This cross-sectional study was conducted using Brazilian Longitudinal Study of Adult Health (ELSA-Brasil) baseline data $(6,887$ men currently working and retired) collected between 2008-2010. Six Brazilian public higher education and research institutions integrated this research. The lifestyle indicator was constructed by summing the scores attributed to four different behaviors. Results: Among men, a healthy lifestyle is associated with: age 60 years or older $(\mathrm{OR}=1.32$; IC95\% 1.08-1.61); white/Caucasian race/skin color $(\mathrm{OR}=1.20$; IC95\% 1.02$1.41)$ or Asian (OR=1.66; IC95\% 1.05-2.60); a high-school equivalent (secondary) level of education (OR=2.16; IC95\% 1.83-2.54) or university (tertiary) level $(\mathrm{OR}=1.36$; IC95\% 1.17-1.59); being retired $(\mathrm{OR}=1.31$; IC95\% 1.07-1.61); having a housekeeper (OR=1.34; IC95\% 1.16-1.56); self-perception of health status as good or very good (OR=1.20; IC95\% $1.02-1.41)$ and being satisfied with body image (OR=1.23; IC95\% 1.071.41). Conclusions: These results seem to support an exploratory predictive model of healthy lifestyles among Brazilian adult men, a fact which must be addressed when developing programs designed to promote health.

CORRESPONDING AUTHOR: Ana Luísa Patrão, Institute of Collective Health, lispatrao@gmail.com
P026

NICOTINE MISPERCEPTION AND TOBACCO PRODUCTS USE

Robert Feldman ${ }^{1}$, Rui Shi ${ }^{1}$, Pamela Clark ${ }^{1}$

${ }^{1}$ University of Maryland- USA

Introduction \& Purpose: A large proportion of smokers have misperceptions of the health risks associated with nicotine use. Although nicotine causes minimal damage to smokers' health it has continuously been seen as the main carcinogen in cigarettes and the leading cause of diseases. Overestimation of nicotine harm could potentially discourage tobacco users from using nicotine replacement therapy (NRT) and electronic cigarettes (EC) as smoking cessation options. This study aims at evaluating the potential of correcting nicotine misperception to influence tobacco users' use of NRT and EC. Methods: A U.S. nationally representative online sample of 614 tobacco users was recruited to take a survey of their perception of the harm of nicotine per se, and their risk perception, attitude, and intention towards the use of NRT and EC. Two multivariate multiple regression analyses were conducted, one per product, to predict tobacco users' NRT and EC risk perception, attitude, and intention based on their nicotine harm perception after controlling for age, gender, and education level. Results: Over half of the tobacco users in the study $(56 \%)$ overestimated nicotine harm and believed that a large part of health risks in smoking comes from nicotine. For NRT, tobacco users' nicotine harm perception was associated with their NRT perceived risk $(\beta=.21$, $\mathrm{p}<.001)$, but not with their attitude $(\beta=.03, \mathrm{p}=.54)$ or intention $(\beta=$ $.03, \mathrm{p}=.48)$ towards the use of NRT, $\lambda=.92$, multivariate $\mathrm{F}(12,1548.06)$ $=3.92, \mathrm{p}<.001$. For EC, tobacco users' nicotine harm perception was significantly associated with their EC perceived risk $(\beta=.30, p<.001)$, attitude $(\beta=-.16, p<.001)$ and intention $(\beta=-.16, p<.001), \lambda=.87$, multivariate $\mathrm{F}(12,1540.12)=6.85, \mathrm{p}<.001$. Conclusions: Many tobacco users overestimate nicotine harm, and correcting such misperception could heighten their confidence in the safety of NRT, but it may not promote their actual use of NRT. On the other hand, the correct belief that nicotine is of lower harm could lead tobacco users to believe EC is safer, and it would also help them form a more favorable attitude and a higher intention to using electronic cigarettes as a smoking cessation option.

CORRESPONDING AUTHOR: Robert Feldman, University of Maryland, rfeldman@umd.edu

\section{P027}

PATIENT-CENTERED COMMUNICATION RESULTS IN BETTER MEDICATION ADHERENCE: A RANDOMIZED CONTROLLED TRIAL

Julia Wittkowski ${ }^{1}$, Winfried Rief ${ }^{1}$, Frauke Weiß ${ }^{1}$, Bettina K. Doering ${ }^{1}$, Maria Kleinstäuber ${ }^{1}$, Julia Rheker ${ }^{1}$

${ }^{1}$ Philipps University Marburg- Germany

Medication non-adherence not only contributes to deterioration of the original health problem and causes high costs for healthcare systems but even correlates with higher mortality. Despite these consequences, many patients stop pharmacotherapy early. Therefore, enhancing adherence is crucial. One factor thought to influence medication adherence is the physician-patient-communication. However, experiments are required to investigate this assumed causal influence. We carried out a randomized controlled trial with three arms. We hypothesized that participants who receive a doctor's consultation with a patient-centered communication style (PC-group; open questions, validation of feelings, simple language etc.) are more likely to take a medication than participants who receive a doctor's consultation with a doctor-centered communication style (DC-group; closed questions, focus on rational facts, use of technical terms etc.) or participants who do not receive a doctor's consultation at all (control group). Healthy women $(\mathrm{N}=120)$ were allocated randomly to the three groups. In individual sessions, they viewed a film 
that induced a critical attitude towards a supposedly neuroenhancing drug. The PC-group and the DC-group received the respective doctor's consultations. Participants' self-rated intention of taking the drug at the end of the experiment was measured at baseline and after the doctor's consultation via visual analogue scales. Voluntary intake of the drug (a placebo pill) served as a behavioral outcome. Data were analyzed via ANCOVAs and Chi-square-tests. After doctor's consultation, likelihood of taking the drug was higher in the $\mathrm{PC}$-group compared to the control group, (time*group interaction: $\mathrm{F}(4)=3.80 ; \mathrm{p}=.005$; difference PCgroup and control group: $\mathrm{PCG}-\mathrm{CG}=11.28 ; \mathrm{p}=.011$ ). The groups did not differ with regard to the actual drug intake. Our results indicate that patient-centered communication can increase medication adherence. The discrepancy between participants' reported likelihood to take the drug and actual drug intake might be due to the measures' different sensitivities. Future studies should investigate the role of physicians' communication in clinical samples and consider long-term medication intake.

CORRESPONDING AUTHOR: Julia Wittkowski, Philipps University Marburg, julia.wittkowski@staff.uni-marburg.de

\section{P028}

PERCEIVED BARRIERS TO AND RECOMMENDATIONS FOR CESSATION AMONG URBAN YOUNG POLY-USERS IN BALTIMORE: FINDINGS FROM A QUALITATIVE STUDY

Daisy Le ${ }^{1}$, Gypsyamber D'Souza ${ }^{2}$, Meghan Moran ${ }^{3}$

${ }^{1}$ Johns Hopkins University: Bloomberg School of Public Health- USA

${ }^{2}$ Johns Hopkins University, Bloomberg School of Public Health: Department of Epidemiology- USA

${ }^{3}$ Johns Hopkins University, Bloomberg School of Public Health: Department of Health, Behavior and Society- USA

Introduction: Poly-use of tobacco is increasing, with $12 \%$ of current adult tobacco users in 2014 reporting the use of more than one type of tobacco product during the past 30 days. While there is considerable research on cessation generally, use and cessation among poly-users have not been well explored. Effective cessation programs must be informed by an understanding of different use profiles and the obstacles that urban young adults may face in an attempt to quit smoking. Purpose: We sought to study a novel group of tobacco users (urban young adult poly-users) and their perceived barriers to and recommendations for cessation. Methods: 17 focus groups were conducted among 97 tobacco users between the ages of 18 and 26 from Baltimore, Maryland. Qualitative data were analyzed using framework analysis. Results: Most participants $(85 \%, \mathrm{n}=82)$ reported using more than one type of tobacco product during the past 30 days. Poly-use was higher among men than women ( $65 \%$ vs. $35 \%)$, those $18-21$ than $22+$ years $(60 \%$ vs $40 \%$ ), and those with a high school education or less vs. some college ( $58 \%$ vs. $42 \%$ ). Several barriers to tobacco cessation among poly-users emerged including the idea of self-control, the need for constant stress relief, belief that one was not actually addicted, the influence of those within their social networks, and easy access to the variety of tobacco products. However, some services for cessation were viewed favorably by our participants including mobilebased cessation programs, with everyone indicating that these programs would be substantially more appealing than other paper- or phone-based cessation tools. Discussions suggested that the ideal program would be "fun", quick, interactive, and would provide tailored/customized prompts along the way, delivering cues to action such as relatable messages that would assist its' users along their own tobacco cessation journey. Conclusions: Given the higher prevalence of poly-use in comparison to single use for this population, cessation efforts may need to address/ account for this new trend in tobacco use behavior to be successful. Results from this study not only suggest that mobile-based programs (e.g., apps, text-based programs) are potentially useful cessation tools for this population, but that they should also expand their focus from cigarette-specific cessation messages to include other types of tobacco products. Mobile technology and social media allow young adults to manage cessation in their own time, and it fits into their existing patterns of media use and lifestyle, serving as a cue to action.

CORRESPONDING AUTHOR: Daisy Le, Johns Hopkins University: Bloomberg School of Public Health, drdaisyle@gmail.com

\section{P029}

UNCLEAR GUIDELINES AND POOR COMMUNICATION PRACTICES - A QUALITATIVE STUDY OF GESTATIONAL DIABETES MELLITUS CARE IN A DANISH SETTING

Anne Timm ${ }^{1}$, Karoline Kragelund Nielsen ${ }^{1}$, Helle Terkildsen Maindal ${ }^{1}$, Ulla Christensen ${ }^{2}$

${ }^{1}$ Steno Diabetes Center Copenhagen- Denmark

${ }^{2}$ University of Copenhagen- Denmark

Introduction and Purpose: Gestational diabetes mellitus (GDM) is a temporary condition which demands attention from a variety of healthcare professionals across sectors. Women with prior GDM are at high risk of subsequently developing type 2 diabetes; yet, this risk can be reduced through moderate lifestyle changes. However, limited systematic follow up on life style is provided after pregnancy and exploratory studies have documented how women with prior GDM experience the treatment course as fragmented and sub-optimal. This study investigated healthcare professionals' views on the cross-sectoral treatment pathway and care provision to women with GDM during and after pregnancy. Methods: Two focus group discussions with eight visiting nurses and eight semistructured interviews with healthcare professionals (four midwives, two obstetricians, one diabetes nurse and one physiotherapist) in two Danish cities were conducted. Systematic text condensation was used for analyzing the data. Results: The analysis revealed four major themes: 1) the relationship with the women was essential to midwives and visiting nurses, who struggled to address health and adverse risks in the consultations whereas obstetricians and nurses felt more obligated to do so. 2) Lack of resources allocated to the GDM area resulted in knowledge gaps and unclear responsibilities during the treatment course. 3) Cross-sectoral communication was poor and often the woman with GDM acted as a mediator in the knowledge transaction between sectors (hospital, GP and midwife). 4) Lastly, healthcare professionals experienced that women with GDM felt trapped in a medicalization regime sustained by a negatively affected pregnancy and lack of follow up after birth. Securing the baby's health and partner support were pointed out as drivers to engage in healthy lifestyle activities. Conclusions: This study identified inadequate organizational structures as the main barrier for healthcare professionals to deliver optimal care in the complex treatment trajectory offered to women with a GDM diagnosis. More focus should be put on structures that ease cross-sectoral communication practices and advanced uniform guidelines must be employed to unite the antenatal and postpartum care for women with current and prior GDM.

CORRESPONDING AUTHOR: Anne Timm, Steno Diabetes Center Copenhagen, anne.matilde.timm.01@ regionh.dk

\section{P030}

PROBLEMATIC MOBILE PHONE USE IS ASSOCIATED WITH MENTAL AND SOMATIC HEALTH ISSUES IN A SAMPLE OF GERMAN ADULTS

Alexander Winkler ${ }^{1}$, Franziska Jeromin ${ }^{2}$, Bettina Doering ${ }^{2}$, Antonia Barke $^{2}$

${ }^{1}$ Justus-Liebig-Universität Gießen- Germany

${ }^{2}$ Philipps-Universität Marburg- Germnay

Introduction \& Purpose: Problematic mobile phone use, as defined by excessive use of mobile phones that interferes with one's personal life, 
involving social conflicts, loss of interest in other activities, tolerance, craving, and maintaining use despite of adverse consequences, is associated with mental health issues. However, previous studies are predominantly based on student samples, limiting their generalizability. Therefore, the current study aimed at examining problematic mobile phone use in a broad sample of adults of different educational and vocational backgrounds. Since there is insufficient evidence for the association between problematic mobile phone use and somatic symptoms, we also explored somatic symptoms in addition to mental health outcomes. Methods: Participants $(n=978)$ completed an online survey comprising demographic questions, problematic mobile phone use scales, somatic symptom inventories, as well as depression, anxiety, stress and sleep quality scales. Results: The results showed small, but significant associations between problematic mobile phone use and stress $(\mathrm{r}=.37, \mathrm{p}<.01)$, sleep disturbances $(\mathrm{r}=.24, \mathrm{p}<.01)$, symptoms of anxiety $(\mathrm{r}=.29, \mathrm{p}<.01)$, depression $(\mathrm{r}=.20, \mathrm{p}<.01)$, and somatic symptoms $(\mathrm{r}=.28, \mathrm{p}<.01)$. All associations remained stable after controlling for age and gender. Participants classified as high problematic mobile phone users (based on their scores in the MMPUS-10) slept worse and were more stressed, anxious, and depressed than unproblematic mobile phone users $(\mathrm{p}<.01)$. Conclusions: While future studies should assess the causal direction of the association found, this study points to the need to sensitize for the various mental and somatic health problems associated with problematic mobile phone use.

CORRESPONDING AUTHOR: Alexander Winkler, Justus-LiebigUniversität Gießen, alexander.winkler@psychol.uni-giessen.de

\section{P031}

EVERYDAY LIFE IN OLDER MEN LIVING ALONE - A COMPLEX VIEW NEEDING A BIOPSYCHOSOCIAL PERSPECTIVE Petra von Heideken Wågert ${ }^{1}$, Susanne Nygård ${ }^{2}$, Sara Cederbom ${ }^{3}$

${ }^{1}$ Mälardalen University- Sweden

${ }^{2}$ Kungsör Municipality- Sweden

${ }^{3}$ Oslo Metropolitan University- Norway

Introduction and purpose: For older people, it is important to maintain ability to perform everyday activities as well as independence for as long as possible. The living situation of older men living alone is not well studied and studies are needed to enhance the understanding of their life situation and interventions needed to enhance their abilities, health and quality of life. The purpose was to explore how older men living alone describe their everyday activities as well as their abilities, and to determine what could help them perform everyday activities. Methods: An explorative design was used. Qualitative semi-structured interviews were analysed with inductive content analysis. Eight men aged 65+ years, living alone with home help services, being able to walk and with sufficient cognitive ability (MMSE $\geq 24$ ) were included. Results: The analysis resulted in one theme "The driving force of managing social togetherness outside the home requires a broad spectrum of support" and four categories: The importance of performing physical and social activities; Individual prerequisites enable everyday activities; The body and mind inhibit and react to the decreased ability and The importance of the environment. The participants reported that important activities included going outdoors, being physically active, spending time with others and participating in social activities and the community. Their own strategies and environmental factors may facilitate everyday activities as well as support from people in the participants' environment and improved medical conditions. The wish, and longing, to perform everyday activities by themselves are strong among the participants, but there is also an acceptance of not being able to do this anymore because of changed life conditions. Conclusions: The results reflect a complex view of older men's everyday life, and it is clear that men's everyday lives were affected by biological, psychological, behavioural, social and environmental factors that interact, in accordance with the biopsychosocial model. It is important for the men's independence that health care and rehabilitation staff have a comprehensive view and works from a biopsychosocial perspective.

CORRESPONDING AUTHOR: Petra von Heideken Wågert, Mälardalen University, petra.heideken.wagert@mdh.se

\section{P032}

MATERNAL PARENTING SKILLS ARE ASSOCIATED WITH SUBSTANCE USE DISORDER THROUGH SELF-CONTROL IN YOUNG OFFENDERS IN DRUG TREATMENT

Mónica Lobato Concha ${ }^{1}$, Robbert Sanderman ${ }^{1}$, Mariët Hagedoorn ${ }^{1}$

${ }^{1}$ University medical Center Groningen, University of GroningenNetherlands

Introduction. Drug treatment success rate overall is not high (around $20 \%$ ), but with young offenders it tends to be lower. We need more insight of how to help them and involving parents may be helpful. Self-control is a key factor in drug use treatment and, according to self-control theory, parents play an important role in the development of their offspring's selfcontrol. Our aim was to evaluate whether parenting skills were indirectly associated to drug use through self-control in young offenders with substance use disorder who are in treatment. Methods. Ninety-eight young offenders, $(\mathrm{M}=17.5 ; \mathrm{SE}=1.6)$ attending the Chilean National Drug Treatment Program (2016-2017) answered a questionnaire about drugs used, self-control and maternal parenting skills (warm/harsh parenting, involvement, autonomy support, attitude towards drug use and parental drug use). We conducted mediation analyses to test indirect effect of parenting skills on young offenders' number of drugs used through young offenders' self-control, controlling for potential confounders. Results. In mediation analyses, variables that had indirect associations with young offenders' number of drugs used through young offenders' self-control, were maternal warmth parenting ( $b=-0.10,95 \% \mathrm{BCa} C \mathrm{CI}[-0.224,-0.034])$, maternal harsh parenting ( $\mathrm{b}=0.14,95 \% \mathrm{BCa} \mathrm{CI}[0.066,0.260])$, and maternal autonomy support $(\mathrm{b}=-0.13$, BCa CI $[-0.263,-0.058])$, with small to medium effect sizes. Conclusions. Young offenders with Substance use Disorder is a difficult group to treat successfully. The insights of the current study on maternal parenting indicate that it might be fruitful to extend this line of research in order to explore - among others - whether maternal style may be a promising avenue to improve young offenders' outcomes in drug treatment.

CORRESPONDING AUTHOR: Mónica Lobato Concha, University medical Center Groningen, University of Groningen, lobatoc.monica@gmail.com

\section{P033}

"TO KNOW THAT THERE IS A LIGHT AT THE END OF THE TUNNEL": PATIENTS PREFERENCES FOR GYNAECOLOGICAL CANCER FOLLOW-UP CARE

Val Morrison ${ }^{1}$, Rhiannon Tudor Edwards ${ }^{1}$

${ }^{1}$ Bangor University- United Kingdom

Introduction and purpose In the UK, patients traditionally have regular follow-up appointments (of a reducing frequency) in secondary care for five years post treatment, with the aim of detecting possible recurrence and management of any physical or psychological morbidities. During this appointment, patients receive a gynaecological examination and possible tests. There is no NICE guidance or medical consensus as to what form or frequency of gynaecological cancer after care is most effective. The effectiveness of this model of care in detecting asymptomatic recurrence and then improving overall survival is questioned. Recurrence is often symptomatic and patients often present with symptoms in between scheduled follow appointments, and will then generally be seen promptly. Patients report unmet needs during this period, and have asked for 
alternative models of gynaecology follow up to better meet these needs. The study aimed to understand patients preferences for gynaecological cancer follow up based on their experience of this care. Methods Participants were opportunistically recruited by their health care professionals at their aftercare appointments. Seventeen patients took part in a semi-structured cross-sectional focus group $(n=4)$ or interview $(n=5)$. Eight participants were diagnosed with endometrial cancer, five with cervical cancer and four with ovarian cancer. Eight participants had stage 1 disease, one participant stage 2, one participant stage 3 or 4 , but six patients did not know or had forgotten their staging. Results Iterative analysis using the framework approach identified two themes governed by the need for reassurance. Reassurance that: the cancer had not returned; they were recovering as planned; through the emotional support received; and by the answering of any questions. The theme 'Meet my Needs Please' (with sub themes: access to an expert; procedures; holistic care) provided patients with reassurance. The theme 'Barriers to care' (with the sub themes: system failure; low self-efficacy) prevented patients from accessing care and so reassurance. Conclusion The overwhelming need for reassurance governing preferences for care raises ethical implications for the traditional model of care given its lack of evidence base, and highlights the need for alternative models of NHS follow-up care.

CORRESPONDING AUTHOR: Val Morrison, Bangor University, v.morrison@bangor.ac.uk

\section{$\mathrm{P} 034$}

FUNCTIONAL SOMATIC SYNDROMES AND BODILY DISTRESS SYNDROME IN THE GENERAL DANISH POPULATION - THE DANFUND STUDY (DATA FROM A CLINICAL DIAGNOSTIC INTERVIEW)

M.W. Petersen ${ }^{1}$, A. Schröder ${ }^{1}$, T. Jørgensen ${ }^{2} 3^{4}$, T.M. Dantoft ${ }^{2}$, M. Eliasen $^{2}$, L.F. Eplov ${ }^{5}$, P. Fink ${ }^{1}$

${ }^{1}$ The Research Clinic for Functional Disorders and Psychosomatics, Aarhus University Hospital- Denmark

${ }^{2}$ Centre for Clinical Research and Prevention, Bispebjerg and Frederiksberg Hospital, Capital Region of Denmark- Denmark

${ }^{3}$ Department of Public Health, Faculty of Health and Medical Sciences, University of Copenhagen

${ }^{4}$ Faculty of Medicine, Aalborg University- Denmark

${ }^{5}$ Mental Health Centre Copenhagen, Capital Region of DenmarkDenmark

Introduction and purpose: Prevalence estimates based on self-reported screening questionnaires have shown a high prevalence of functional somatic syndromes (FSS) and bodily distress syndrome (BDS) in the Danish population. However, the prevalence of clinically diagnosed cases of FSS and BDS has not been investigated. The aim of this study was to establish prevalence of clinical FSS, i.e. irritable bowel syndrome, fibromyalgia, chronic fatigue syndrome, whiplash associated disorder, multiple chemical sensitivity and BDS in the general Danish population, based on a clinical diagnostic interview.

Methods: A stratified subsample $(n=1604)$ of the part 2 DanFunD general population cohort $(n=7493)$ underwent a brief version of Schedules of Clinical Assessment in Neuropsychiatry conducted by trained family physicians per telephone. Validated diagnostic algorithms were used to assign diagnoses of FSS and BDS. According to diagnostic criteria, physical and mental differential diagnoses were excluded, i.e. participants with physical or mental comorbidities that, according to the clinician, better explained the observed symptom pattern were not assigned a FSS or BDS diagnosis, i.e. moved to the non-case group.

Results: Unweighted results showed a prevalence of FSS on $19.1 \%(95 \%$ CI: $17.2-21.1$ ): $12.0 \%$ (95\% CI: 10.4-13.6) with one FSS, $5.8 \%$ (95\% CI: 4.7-6.9) with two FSS, and $1.3 \%$ (95\% CI: $0.8-1.9$ ) with $\geq 3$ FSS. The most prevalent FSS was chronic fatigue syndrome with $14.2 \%$ (95\% CI: 12.5-15.9). Prevalence of BDS was $21.0 \%$ (95\% CI: 19.1-23.0): $16.8 \%$
(95\% CI: 15.0-18.7) with single-organ BDS and 4.2\% (95\% CI: 3.2-5.2) with multi-organ BDS. The most prevalent BDS subtype was gastrointestinal BDS with 10.9\% (95\% CI: 9.3-12.4). Excluding diagnoses of well-defined physical or mental disease were e.g. asthma, colitis ulcerosa, osteoarthritis, migraine, depression and anxiety.

Conclusions: Unweighted results showed high prevalence-estimates of FSS and BDS. Including a physician's assessment through a diagnostic interview makes it possible to differentiate between symptom patterns caused by FSS or BDS, comorbid disease, or both, and allows for a more clinically relevant case-population.

CORRESPONDING AUTHOR: Marie Weinreich Petersen, The Research Clinic for Functional Disorders and Psychosomatics, Aarhus University Hospital, mawept@ rm.dk

P035

THE EXPERIENCE OF PSYCHOLOGICAL DISTRESS, SYMPTOMS OF TRAUMA, COPING AND POST-TRAUMATIC GROWTH IN PARENTS OF CHILDREN WITH CONGENITAL HEART DISEASE: A SYSTEMATIC REVIEW OF LITERATURE Amy Mullens ${ }^{1}$, Jodie Brown ${ }^{1}$

${ }^{1}$ University of Southern Queensland- Australia

AIM: This systematic review explored the experience of psychological distress, trauma, post-traumatic growth (PTG) and coping in parents of children with congenital heart disease (PCCHD). METHOD: PRSIMA guidelines for systemic reviews were followed to identify 82 studies for analysis. RESULTS: PCCHD experience high levels of distress that manifest in a variety of symptoms (including depression, anxiety, stress and somatization). Generally, this is at levels higher than normative populations and at levels consistent with parents of children with chronic and/or severe health conditions. Psychosocial stressors (commonly but not exclusively found in families of more severe CHD) appear to exacerbate this experience. Diagnosis (and birth) of a child with CHD is deeply distressing for PCCHD. Invasive surgical procedures (open heart surgery) are also a significant source of distress for PCCHD, regardless of CHD severity or the complexity of the procedure, and may serve as a trigger for concerns about child mortality and suffering, and the experiences of role loss, uncertainty and lack of control. Parental descriptions of these experiences are in keeping with those of other trauma survivors and a number of PCCHD meet threshold for diagnosis of Acute Stress Disorder and Post-Traumatic Stress Disorder, with many more experiencing multiple clinically significant symptoms. Ongoing symptoms of trauma (especially hypervigilance), depression and anxiety are also frequently reported. PCCHD employ a variety of coping strategies to deal with their experiences, whilst also appearing to experience the transformational nature of their traumatic experiences in a way that is consistent with the experience of PTG. CONCLUSIONS: Findings are discussed in relation to existing research, and implications for further research and practice.

CORRESPONDING AUTHOR: Amy Mullens, University of Southern Queensland, amy.mullens@usq.edu.au

\section{P036}

DOES FUEL TYPES AND PLACE OF COOKING MATTER FOR ACUTE RESPIRATORY INFECTIONS AMONG AFGHAN CHILDREN? EVIDENCE FROM A CROSS-SECTIONAL SURVEY Rayhan $\mathrm{Sk}^{1}$

${ }^{1}$ Jawaharlal Nehru University- India

Background In Afghanistan, acute respiratory infections (ARI) is a leading cause of under-five years child's death. Many studies predicted the effects of types of cooking fuel on ARI merely which act as 'sources of origin' of smoke and ignored the effects of kitchen room and location of 
cooking place which act as 'place of origin' of smoke. This study mainly focused on estimating the effects of fuel types and place of cooking on ARI among children aged under-five years. Methods We performed descriptive statistics and multilevel logistic regression analyses on 30304 children using data from the first round of Afghanistan Demographic and Health Survey 2015. Results Overall, 13\% of the children suffered from ARI symptoms during the two weeks before survey in Afghanistan varying widely across the country. From multilevel analyses, we found that using clean cooking fuel in house without separate kitchen room has $32 \%$ lower risk [95\% confidence interval $(\mathrm{CI})=0.50-0.91]$ to have $\mathrm{ARI}$; and with separate kitchen room has $16 \%$ lower risk in the same $(95 \%$ $\mathrm{CI}=0.68-1.04$ ); whereas, using polluting cooking fuel in house without kitchen has $11 \%$ higher risk to have ARI (95\% CI=0.89-1.40) than households using clean cooking fuel in outsides. Rather, mother's education and occupational status, community poverty, ethnicity are the more influential factors of ARI. Conclusions For the sake of reducing child mortality, the government should encourage people to use clean fuels for cooking and provide LPG at a subsidised price; promote education, and spread awareness; introduce special policy programs for specific communities.

CORRESPONDING AUTHOR: Rayhan Sk, Jawaharlal Nehru University, rayhangog@gmail.com

\section{$\mathrm{P} 037$}

INCIDENCE OF LOW BIRTH WEIGHT INFANTS AND IT'S RISK FACTORS IN SOUTH ASIA: A MULTILEVEL ANALYSIS

Rayhan $\mathrm{Sk}^{1}$

${ }^{1}$ Jawaharlal Nehru University- India

Background Low birth weight (LBW) is a leading cause of prenatal and neonatal deaths. According to UNICEF and WHO, half of the low birth weight children are in South Central Asia. However, studies on LBW across the region, and contextual variations in it are hardly known. Therefore, studies on LBW are needed especially in South Asian (SA) countries, since sub-optimal birth weight may have consequences in the perinatal period, during infancy, and even in adulthood. Thus, the aim of this study is to examine the risk factors of LBW and to estimate the between-areas variations in it. Methods This cross-sectional study pooled the latest round of nationally representative data from the Demographic and Health Surveys conducted in five SA countries (Myanmar 2015-16; Afghanistan 2015; Pakistan 201213; Nepal 2011; India 2005-06). A sum of 23,698 women aged between 15-49 years who had given last single live birth, lived in 4,925 communities nested in 87 regions have been analysed. Multilevel binary logistic regressions have been employed for data analysis. Results The overall prevalence of LBW is $17.5 \%$, varied from $7.3 \%$ in Myanmar to $24.5 \%$ in Pakistan. The results of the multilevel analysis revealed that the odds of having LBW are higher among female newborn $(\mathrm{OR}=1.53 ; \mathrm{p} 0.001)$ of giving LBW baby than their counterparts. Wealthier women have lower odds of giving LBW baby. Among the contextual factors, countries are the significant risk factor of LBW after controlling for other factors. Myanmar $(\mathrm{OR}=0.29 ; \mathrm{p}<0.001)$ and Afghanistan $(\mathrm{OR}=0.54 ; \mathrm{p}<0.001)$ have the lowest odds of having LBW. The between-regions variance in the log-odds of having LBW is estimated as $18 \%$ (SE 0.068). Conclusions This study revealed that female new-born, inadequate prenatal care, poverty and illiteracy are the major risk factors of LBW. Further, the significant variations in LBW are observed by the contextual factors, e.g., countries (fixed effects) and regions (random effects). Implementing appropriate policies on regional and country level should be adopted to reduce the incidence of LBW infants.

CORRESPONDING AUTHOR: Rayhan Sk, Jawaharlal Nehru University, rayhangog@gmail.com
P038

"AN ASSESSMENT OF ORAL HEALTH STATUS, TOBACCO USE AND CANCER AWARENESS AMONG TEA PLANTATION WORKERS (IRULA TRIBES), NILGIRI HILLS, TAMILNADU, INDIA"

Delfin Lovelina Francis ${ }^{1}$

${ }^{1}$ Dr MGR Medical University- India

Background: Tea is an important agro-industry of India, which contributes immensely to the countries economy. Tea garden population constitutes approximately $1 / 12$ th of tea growing state's population. Poor socioeconomic conditions, ignorance due to illiteracy, over-crowded and unhygienic living conditions in the residential colonies make tea garden population vulnerable to various communicable diseases and malnutrition. Hence this study was contemplated with an aim to assess the oral health status, tobacco use and cancer awareness among tea plantation workers, Nilgiri Hills, Tamil Nadu, India. Methodology: A crosssectional descriptive study was conducted to assess the tobacco use and cancer awareness among tea plantation workers, Nilgiri Hills.Data was collected using a pretested Questionnaire, which included Demographic data, tobacco habits, its frequency and form. The data collected was analysed using SPSS version15. Results: Results showed that among 900 study population, showed $57 \%$ had no formal education, $34.5 \%$ had not visited dentist before. $64.5 \%$ had indigenous brushing habits. $52 \%$ of oral mucosal lesions and $6 \%$ malignant oral tumors were observed. A very high prevalence of periodontal disease, tobacco chewing, deep rooted beliefs and customs regarding dentition and dental treatment was observed in this community. Prevalence of oral mucosal lesions in the study population was due to tobacco usage and lack of awareness regarding the deleterious effects of the products used. Conclusion: The dangers from smoking and chewing tobacco are well documented within the literature but the public's lack of knowledge of the risks is a concern. Health professionals are encouraged to ensure that the public is made aware of these risks, especially those within high-risk groups.

CORRESPONDING AUTHOR: Delfin Lovelina Francis, Dr MGR Medical University, delfin_lovelina@yahoo.co.in

\section{P039}

SMOKELESS TOBACCO USE AND LOW BIRTH WEIGHT CHILD AMONG WOMEN IN A RURAL SETTING OF INDIA

Delfin Lovelina Francis ${ }^{1}$

${ }^{1}$ Dr MGR Medical University- India

Introduction Low birth weight and preterm birth are powerful determinants of morbidity and mortality in newborn babies and infants. It has been known for more than 40 years that babies born to mothers who smoke weigh less than babies whose mothers don't smoke. Smoking during pregnancy also increases the risk for preterm delivery. In South East Asia smoking among women may be rare, but use of smokeless tobacco is common. There are indications that using smokeless tobacco could be as detrimental to fetal health as cigarette smoking. Aim To study the effect of using smokeless tobacco during pregnancy on babies' birth weight at birth. Materials and Methods A prospective cohort study was carried out in a teaching hospital in a rural setup among 54 pregnant women who had used smokeless tobacco product at least once a day for the past six months. The frequency of use was categorized as light (one to four times per day) or heavy (five or more times per day). Commonly used forms of tobacco wasbetel quid (paan) with tobacco, gutka, and paan masala are chewed and generally retained in the mouth for longer periods. They were followed for a period of seven months and birth weight of the newborn was measured. Results Smokeless tobacco use was associated with an average reduction of $105 \mathrm{~g}$ in birth weight (95\% confidence interval $30 \mathrm{~g}$ to $181 \mathrm{~g}$ ). The odds ratio for low birth weight was $1.6(1.1$ to 2.4), adjusted by logistic regression for maternal age, education, 
socioeconomic status, weight, anaemia, antenatal care, and gestational age. The adjusted odds ratio for preterm delivery ( $<37$ weeks) was 1.4 (1.0 to 2.1$)$; for delivery before 32 weeks it was 4.9 (2.1 to 11.8$)$ and before 28 weeks it was 8.0 (2.6 to 27.2 ).

CORRESPONDING AUTHOR: Delfin Lovelina Francis, Dr MGR Medical University, delfin_lovelina@yahoo.co.in

\section{$\mathrm{P} 040$}

\section{A PROPOSITIONAL ACCOUNT OF GENITAL PAIN}

Marieke Dewitte $^{1}$, Jan De Houwer ${ }^{2}$

${ }^{1}$ Maastricht University- Netherlands

${ }^{2}$ Ghent University- Belgium

Introduction: Although sexual behavior is typically described as an instinctive and hormonally-driven process, learning mechanisms are critically implicated in the development and expression of sexual behavior. We present a theoretical framework that challenges traditional learning principles and forwards the novel idea that people learn to behave sexually based on how they attribute meaning to their environment. This implies that sexual learning depends on high-level, propositional processes that involve the formation of beliefs on the basis of instructions and inference instead of stimulus pairings. In the context of genital pain, it is often observed that fear of pain and its physical correlates occur during the very first sexual encounter, thus without having actually experienced the pairing of penetration and pain before. This suggests an important role for learning via instruction or inference. Methods: We illustrate these ideas by examining how women infer contingencies based on the information they receive. We use a vaginal pressure inducer (VPI) to simulate the vaginal sensations women experience during penetration and give manipulated feedback about the nature of the device before applying it (describing it as an invasive (threatening) medical device versus a sex toy). We examine the impact of these instructions on expectations and actual indices of sexual arousal and genital pain upon the first experience of the VPI. In addition, we allow participants to repeatedly experience the VPI either in a positive context (in combination with a sex film) or a mildly negative context (no sexual stimulation). This allows examining the way in which experience-consistent and -inconsistent instructions moderate the impact of actual experiences. Results: The data collection is still pending, but intermediate analyses reveal promising results on the effects of instruction on the appraisal of sexual sensations. Conclusion: We will discuss the clinical implications of these results in light of propositional theory stating that therapy can benefit from combining different sources of information (experiencing events, providing instructions that allow for causal inference such as acceptance of negative sensations) in order to change beliefs that underlie genital pain.

CORRESPONDING AUTHOR: Marieke Dewitte, Maastricht University, Marieke.dewitte@maastrichtuniversity.nl

\section{P041 \\ LEARNING PROCESSES IN CHRONIC PAIN \\ Ann Meulders ${ }^{1}$ \\ 1 KU Leuven- Belgium}

Introduction \& Purpose: Impaired selective fear learning has been advanced as a core mechanism involved in excessive spreading of protective responses such as pain-related fear and avoidance leading to disability in chronic pain conditions. The litmus test for selective learning effects is the blocking procedure. We tested the hypothesis whether fibromyalgia patients (FM) would show less selective threat learning than healthy controls (HC) using a novel contingency learning task based around a clinical scenario. Methods: This study included $27 \mathrm{FM}$ (26 females, mean $\pm \mathrm{SD}$ age $=46 \pm 9$ years), and $27 \mathrm{HC}$ (mean \pm SD age $=48 \pm 9$ years). On a trial-by-trial basis, participants rated whether they expected certain situations $(\mathrm{A}, \mathrm{B}, \mathrm{Z}, \mathrm{X})$ in the diary of a fictive fibromyalgia patient, Kim, to cause pain in that patient. The procedure did not involve any experimental pain induction, but the verbal outcomes "pain" or "no pain" were used. During the elemental acquisition phase, one situation was followed by "pain" (A+, e.g. "Kim slept badly"), whereas another situation was followed by "no pain" (Z-, e.g. "Kim was stressed"). During the compound acquisition phase, another situation $(\mathrm{X})$ referred to as the blocked stimulus, presented in compound previously pain-eliciting event is also paired with "pain" $(\mathrm{AX}+)$ and a novel situation was also followed by "pain" (B+). Results: Within-group comparisons confirmed that pain expectancy judgments for $\mathrm{B}$ were higher than for $\mathrm{X}$ in the $\mathrm{HC}$ group, $\mathrm{F}(1$, $52)=10.38, p<.01$, but not in the FM group, $F(1,52)=2.50, p=.12$. The between-group contrast comparing the difference between $\mathrm{B}$ and $\mathrm{X}$ between the FM group and the $\mathrm{HC}$ group, however, was not significant, $\mathrm{F}(1$, $52)=1.35, p=.25$. Conclusions: Although the two-way interaction was not significant, we observed blocking (i.e. significant difference between $\mathrm{B}$ and $\mathrm{X}$ ) in $\mathrm{HC}$, but not in the FM. This study provides direct, yet partial evidence for impaired selective learning in fibromyalgia patients. We believe that deficits in selective threat appraisal may contribute to the transition from regional pain to widespread pain through the proliferation of anxiety and expectancy biases. Future research should explore how these deficits can be targeted in cognitive-behavioral treatments.

CORRESPONDING AUTHOR: Ann Meulders, KU Leuven, ann.meulders@kuleuven.be

P042

GRATITUDE AND FORGIVENESS IN PREGNANCY: PRELIMINARY FINDINGS FROM AN ONLINE-STUDY IN GERMAN SPEAKING EUROPEAN COUNTRIES

Pearl La Marca-Ghaemmaghami ${ }^{1}$, Roberto La Marca ${ }^{2}$, Franziska Uggowitzer $^{2}$, Nadine Weymerskirch ${ }^{2}$, Mathias Allemand ${ }^{3}$, Ulrike Ehlert ${ }^{2}$ ${ }^{1}$ University of Zurich- Switzerland

${ }^{2}$ Clinical Psychology and Psychotherapy, Department of Psychology, University of Zurich, Zurich- Switzerland

${ }^{3}$ Differential Healthy Aging Research, Department of Psychology, University of Zurich, Zurich- Switzerland

Introduction \& Purpose: Stress over-exposure during pregnancy can negatively impact upon the health and well-being of mother and child. Therefore, it is essential for research and practice to identify stress protective maternal resources. There is accumulating evidence for systematic associations between gratitude and forgiveness and physical and mental health. However, these two constructs have received little attention in prenatal stress research. The aim of the current study is to explore whether gratitude and forgiveness 1) predict maternal psychological symptoms of stress during pregnancy and 2) have a beneficial effect on neonatal birth outcome and maternal wellbeing after birth. Methods: Four-hundred and ninety three healthy pregnant women from different German-speaking countries participated in an online-study (mean age: 31 years, range: 18-45 years). They responded to the Gratitude Questionnaire (GQ-6; McCullough et al., 2002), the Tendency to Forgive Scale (TTF; Brown et al. 2002), the Prenatal Distress Questionnaire (PDQ; Yali \& Lobel, 1999), and the Edinburgh Postnatal Depression Scale (EPDS; Cox et al., 1987). Approximately four weeks after birth, a subsample of these women $(\mathrm{N}=107)$, who had not experienced any medical complications during pregnancy, volunteered to participate in a second online-questionnaire, provided information on neonatal birth outcome, and responded once more to the EPDS. Results: Regression analyses revealed that higher TTF values during pregnancy were associated with lower PDQ scores $(\mathrm{b}=-.16, \mathrm{p}<.05 ; \mathrm{R} 2=.02, \mathrm{~F}(1,130)=4.53, \mathrm{p}<.05)$, and that higher GQ-6 scores were linked to lower EPDS levels $(b=-.14, p<.001 ; R 2=.03$, $\mathrm{F}(1,130)=9.38, \mathrm{p}<.001)$. Pregnant women of more advanced age were more grateful $(\mathrm{r}=.20, \mathrm{p}<.05)$. Prospectively, the TTF and GQ-6 were 
unrelated to EPDS values postpartum. There was a trend towards a significant association between the GQ-6 and pregnancy duration $(\mathrm{r}=.17$, $\mathrm{p}=.08$ ), suggesting that women who were more grateful during pregnancy were longer pregnant. Conclusions: Gratitude and forgiveness may serve as psychological resources against stress and depressive symptoms during pregnancy, though no conclusions can yet be made regarding causality.

CORRESPONDING AUTHOR: Pearl La Marca-Ghaemmaghami, University of Zurich, pearl.lamarca@ psychologie.uzh.ch

\section{P043}

ROLE OF PICTORIAL WARNING ON CIGARETTE PACKETS IN TOBACCO CESSATION- A QUESTIONNAIRE SURVEY AMONG CIGARETTE SMOKERS IN CHENNAI

Clement Joy Francis ${ }^{1}$

${ }^{1}$ Nestle India Limited- India

Background: Warning labels on cigarette packages are meant to communicate such smoking-associated risks. The study is designed to find out the effectiveness of pictorial warnings present on cigarette packets in India for tobacco cessation among cigarette smokers. Methodology: A questionnaire was distributed to 800 current smokers attending an outpatient department of a college. Statistical analysis was done to find association between socioeconomic status and effectiveness of pictures to quit cigarette smoking. Results: $48 \%$ smokers perceive text warning is an efficient method to create awareness. 56\% emphasized the importance of pictorial warning and greater area to be covered. $43 \%$ felt that warning on cigarette packets helped them to quit smoking. Conclusions: Though pictorial warning is an effective method to improve the awareness among smokers on the ill effects of smoking, the size, area covered and the position of the picture on cigarette packets needs to be reviewed to improve the quit rate.

CORRESPONDING AUTHOR: Clement Joy Francis, Nestle India Limited, clement.k12@fms.edu

\section{P044}

SMOKING HABITS AND AWARENESS ABOUT ANTI-SMOKING ACTS AMONG GENERAL PUBLIC IN GURGAON, HARYANA, INDIA

Clement Joy Francis ${ }^{1}$

${ }^{1}$ Nestle India Limited- India

Background: India is the world's third largest tobacco-growing country. The Indian scenario as far as tobacco consumption is concerned is far worse because of the prevalence of the tobacco chewing habit which covers a wide spectrum of socioeconomic and ethnic groups and is spread over urbanized area as well as remote village. Tobacco use is alarming in terms of its current and projected future impact on global mortality. Recent shift in global tobacco consumption to developing countries indicate that an estimated 930 million of the world's 1.1 billon in India alone. Despite the facts, that the harmful effects of tobacco chewing and smoking are widely known, many young people start smoking during adolescence, largely because they believe that smoking will boost their social acceptability and image. This study was contemplated with an aim to assess tobacco / smoking habits and awareness about anti-smoking act among general public in Gurgaon, Haryana, India. Method: A structured questionnaire consisting of 14 questions related to tobacco/smoking habits and awareness about anti-smoking act were asked to general public and their response was recorded. Random sampling method was used and data was collected from a cross-sectional survey. Anti-tobacco counseling was given on the spot and followed. Results: The study population consisted of total 430 individuals, male $364(84.65 \%)$ and females
$66(15.34 \%)$. Then the questionnaires were asked and statistically analyzed. Around 286 (78.57\%) from 364 male were indulged in some form of tobacco usage $($ smoker $=32.86 \%$, tobacco chewer $=16.78 \%$, both $=11.18 \%$, alcohol +tobacco user $=21.67 \%$ ). In the present study, most common cause of tobacco use was pleasure $40.5 \%$, inducing factor were friends $53.1 \%$ followed by parents and siblings. $36.20 \%$ patients used tobacco as second hand exposure in job places. $54.8 \%$ were aware about the anti-smoking act in public places, so only $8.6 \%$ people from all males enrolled, were smoking in public places.

CORRESPONDING AUTHOR: Clement Joy Francis, Nestle India Limited, clement.k12@fms.edu

\section{P045}

SOCIAL ISOLATION IN THE INSTITUTIONALIZED OLDER ADULTS: A CASE REPORT

Carlos Laranjeira ${ }^{1}$, Zaida Azeredo ${ }^{2}$

${ }^{1}$ RECI I\&D - Piaget Institute- Portugal

${ }^{2}$ Piaget Institute/RECI I\&D- Research in Education and Community Intervention, Viseu- Portugal

Objective: This study examined the programs that address the issues of social isolation in the life of the older adults residing in nursing home care services (NHCSs) in Portugal. Method: Data was collected from eight NHCSs through semi-structured qualitative interviews, and was analyzed using interpretative phenomenological analysis. Results: Findings revealed that none of the facilities have any specific constructive strategic program to alleviate social isolation besides the use of companion animals by their residents. Conclusions: The findings from this study indicated that caregivers working with the older adults in NHCSs should embark in setting up meaningful strategic programs such as music, humor, and reminiscence therapy; social support programs; animal assisted therapy; elders-helping-elders programs; recreational therapy; information and communication technology, which will help in combating social isolation among the older adults. For wider society and for services engaged with preventing social isolation and its negative impact the challenge is to support the maintenance and renewal of social connections.

CORRESPONDING AUTHOR: Carlos Laranjeira, RECI I\&D - Piaget Institute, carloslaranjeira@hotmail.com

P046

PERCEPTIONS OF HAPPINESS AND WELL-BEING IN INSTITUTIONALIZED ELDERLY: A PORTUGUESE STUDY

Carlos Laranjeira ${ }^{1}$, Zaida Azeredo ${ }^{2}$

${ }^{1}$ RECI I\&D - Piaget Institute- Portugal

${ }^{2}$ Piaget Institute/RECI I\&D- Research in Education and Community Intervention, Viseu- Portugal

Objective: We sought to know which perceptions the institutionalized elderly have about their happiness and wellbeing. Method: It is a qualitative study, using the semistructure interview. The findings were analyzed according to the content analysis, with a priori categorization (Bardin, 2015). Results: The sample consisted of 13 older people resident in a nursing home care in Viseu (Portugal), aged between 77 and 94 years and institutionalized between 4 and 12 years. The institutionalization is for some arising from their own will and for others a decision of the family (children/nephews). Happiness for most of the elderly depends on a number of factors, such as being healthy, being well with oneself, being cherished in the nursing home, living with others, escaping from loneliness, not starving yourself, being loved and loving, possessing money for yourself and for others and fun. Most older people have confirmed that they feel good about themselves, yet two elderly people do not feel well because of sadness and illness. They memories of the past are related 
to marriage, the constitution of family, the strength to work and the conviviality with friends; in the present, with happiness, a sense of general well-being, not being alone and living with other institutionalized elders. Conclusion: We can therefore conclude that the ability to build and sustain friendship is powerful socio-psychological resource of today's socially active and valuable older people.

CORRESPONDING AUTHOR: Carlos Laranjeira, RECI I\&D - Piaget Institute, carloslaranjeira@hotmail.com

P047

CROHN'S DISEASE: QUALITY OF LIFE, STRESS COPING AND NEGATIVE EMOTIONS

Mariela Gonzalez Tovar ${ }^{1}$

${ }^{1}$ Pontificia Universidad Católica de Chile- Chile

Introduction: Crohn's disease is a non-specific inflammatory process, presenting a chronic evolution, which affects the whole digestive tract. This process has a significant effect on the patient's lives, physically, psychologically, personally and socially. In addition to representing a physical disorder in the intestine, Crohn's disease currently presents several environmental factors which play a key role in its pathogenesis. This description has aroused interest in systemic and precise research over the Crohn's disease itself, from a bio-behavioral point of view. Following this direction, this study aimed to evaluate quality of life, stress coping and negative emotions. Methods: The study has been carried out taking into account 25 patients ( 15 women and 10 men) having a Crohn's disease diagnosis, and receiving treatment in Caracas, Venezuela. For this study purposes, the Coping Stress/Strategies Questionnaire and the Inflammatory Bowel Disease Questionnaire-32, including a subhospital anxiety and depression scale, were applied. Results and discussion: Results show that patients presenting Crohn's disease obtained high levels of anxiety and depression. Depression was associated with a cognitive-dysfunctional coping style. These findings provide clear evidence directed to implement strategies leading to reduction of impaired quality of life in people presenting Crohn's disease, especially those who generate a better psychological adjustment.

CORRESPONDING AUTHOR: Mariela Gonzalez Tovar, Pontificia Universidad Católica de Chile, mgonzalez4@uc.cl

\section{P048}

PERSONAL, SOCIAL, AND ENVIRONMENTAL FACTORS ASSOCIATED WITH LIFEJACKET WEAR IN ADULTS AND CHILDREN: A SYSTEMATIC LITERATURE REVIEW

Kyra Hamilton ${ }^{1}$, Amy Peden ${ }^{2}$, Daniel Demant ${ }^{3}$, Martin Hagger ${ }^{4}$

1 Griffith University- Australia

${ }^{2}$ Royal Life Saving Society- Australia

${ }^{3}$ University of Technology Sydney- Australia

${ }^{4}$ Curtin University- Australia

Introduction and purpose: Drowning claims $7 \%$ of the global burden of injury-related deaths. Lifejackets are routinely recommended as a drowning prevention strategy; however, a review of related factors regarding lifejacket wear has not previously been investigated. Methods: This systematic review examined literature published from inception to December 2016 in English and German languages. The personal, social, and environmental factors associated with lifejacket wear among adults and children were investigated; a quantitative evaluation of the results undertaken; and gaps in the literature identified. Results: Twenty studies were identified. Fifty-five percent were cross-sectional studies. All studies were scored IV or V on the National Health and Medical Research Council (NHMRC) grading system indicating mostly descriptive and cross-sectional levels of evidence. Factors associated with increased wear included age (mostly children), gender (mostly female), boat type (nonmotorised), boat size (small boats), role modelling (children influenced by adult lifejacket wear), and activity (water-skiing, fishing). Factors not associated or inconsistent with lifejacket wear included education, household income, ethnicity, boating ability, confidence in lifejackets, waterway type, and weather and water conditions. Factors associated with reduced lifejacket wear included adults, males, discomfort, cost and accessibility, consumption of alcohol, and swimming ability. Three studies evaluated the impact of interventions. Conclusions: This review identified factors associated with both increased and decreased lifejacket wear. Future research should address the motivational factors associated with individuals' decisions to wear or not wear lifejackets. This, combined with further research on the evaluation of interventions designed to increase lifejacket wear, will enhance the evidence base to support future drowning prevention interventions.

CORRESPONDING AUTHOR: Kyra Hamilton, Griffith University, kyra.hamilton@griffith.edu.au

\section{P049}

THE ACTUAL SITUATION OF SCHOOL CHILDREN WITH DEVELOPMENTAL DISABILITIES IN REGULAR CLASSES OF PUBLIC ELEMENTARY SCHOOLS IN JAPAN

Yumiko Sakata ${ }^{1}$, Yuriko Takata ${ }^{1}$, Yoko Kamio ${ }^{2}$, Ichiko Yoshida ${ }^{3}$

${ }^{1}$ University of Tsukuba, Japan

${ }^{2}$ National Center of Neurology and Psychiatry- Japan

${ }^{3}$ Teikyo University of Science- Japan

Purpose This study aims to elucidate the actual situation of school children with developmental disabilities, or suspected with developmental disabilities, in regular classes of public elementary schools in Japan. Methods Using a stratified two-stage random sampling method for all elementary schools in Japan, we selected 1,000 public elementary schools. Then, we distributed a mail-back questionnaire to the principal of each selected elementary school, who was asked to pass the questionnaire to a school nurse. The surveillance period for this study was from October to December 2017. Results We assessed 212 responses in this study (effective response rate, $21.2 \%$ ). While children suspected with a developmental disability were enrolled in 204 (96.2\%) elementary schools, those diagnosed with a developmental disability were registered in $190(89.6 \%)$ elementary schools. Regarding the special support education system in schools, in $65.3 \%$ of the total schools, individual guidance plans were created for all the children with developmental disorders. In $55.3 \%$ of the total schools, individual education support plans were created for all the children with developmental disorders. Nomination of the special support coordinator was reported in $86.3 \%$, and organizing the special support cooperation meeting was reported in $52.8 \%$ of all schools. Regarding school nurse teachers of more than three generations $(p<0.001)$ or experience of 6 or more years $(p=0.001)$, preparation for children to receive medical check-ups was conducted well. In addition, schools with a higher number of children with or suspected with developmental disabilities presented a high nomination rate of the education support coordinator $(\mathrm{p}=0.002)$ and preparation for children to receive medical check-ups smoothly $(\mathrm{p}=0.041)$. However, in schools with a higher number of children with developmental disabilities, school nurse teachers experienced difficulty in cooperating with the organization, other children, and their guardians $(p=0.023)$. Conclusions This study suggests the need for increasing the social capital and fostering a community that supports the education system, especially in elementary schools, to provide equal education for both children with and without developmental disabilities.

CORRESPONDING AUTHOR: Yumiko Sakata, University of Tsukuba, sakata@md.tsukuba.ac.jp 
P050

ERIC: AN EMOTION REGULATION TREATMENT FOR YOUNG PEOPLE WITH COMPLEX SUBSTANCE USE AND MENTAL HEALTH ISSUES

Elise Sloan ${ }^{1}$, Kate Hall ${ }^{1}$, Richard Moulding ${ }^{1}$, George Youssef ${ }^{1}$, Helen Mildred $^{1}$, Petra Staiger ${ }^{1}$

${ }^{1}$ Deakin University- Australia

Background: Young people seeking treatment in alcohol and other drug (AOD) services present with complex patterns of mental health comorbidity and interrelated psychosocial difficulties. Current evidence based treatments are often disorder specific and thus are an inadequate fit for youth AOD settings. We developed ERIC- a flexible and transdiagnostic intervention that targets emotion regulation and impulse control, two constructs implicated in the development and maintenance of mental health and substance use disorders in young people. Aim: To describe the development of ERIC as a transdiagnostic and modular intervention and present pre-post data from ten young people who received ERIC as part of their AOD treatment. Methods: Participants were ten individuals aged between 16-20 years old who were receiving treatment in a youth AOD residential setting. Participants received 4-6 sessions of ERIC as an adjunct to their existing treatment and completed self report measures of emotion regulation, depression, and anxiety at baseline and two weeks after receiving ERIC. Results: Pre-post measures indicated that $60 \%$ of the young people experienced clinically reliable and significant reductions in emotion dysregulation, measured by their (1) awareness and understanding of emotions; (2) use of effective strategies to control emotions; (3) avoidance of emotions (i.e. use of drugs and alcohol to cope) and (4) ability to engage in goal directed behaviour when distressed. Reliable and clinically significant reductions in depression and anxiety were also observed in $50 \%$ and $60 \%$ of participants respectively. Conclusions: Results highlight the viability of an adjunctive and flexible emotion regulation based treatment for complex and high risk young people whose mental health and substance use difficulties are often unable to be addressed by existing single disorder evidence based treatment protocols.

CORRESPONDING AUTHOR: Elise Sloan, Deakin University, elise.sloan@deakin.edu.au

\section{P051}

CORRELATION BETWEEN THE INVOLVEMENT OF SCHOOL CHILDREN WITH DEVELOPMENTAL DISABILITIES IN REGULAR CLASSES AND SCHOOL NURSE TEACHERS' OCCUPATIONAL STRESS

Yuriko Takata $^{1}$, Yumiko Sakata ${ }^{1}$, Yoko Kamio ${ }^{2}$, Ichiko Yoshida ${ }^{3}$

${ }^{1}$ University of Tsukuba- Japan

${ }^{2}$ National Center of Neurology and Psychiatry- Japan

${ }^{3}$ Teikyo University of Science- Japan

Purpose This study aims to investigate the correlation between involvement with school children with developmental disabilities in regular classes and school nurse teachers' occupational stress. Methods We distributed a mailback questionnaire survey to 1,000 public elementary schools between October and December 2017. The scores of difficulty or correspondence in the involvement with children with developmental disabilities were classified into two groups, the high group and the low group, with the median. We assessed occupational stress scores between the two groups using a ttest. Results The analysis of responses revealed that school nurse teachers who scored higher on "the difficulty of learning instruction" presented higher scores on "quantitative burden of psychological work" ( $\mathrm{p}=0.001)$ but lower scores on "decent work" ( $p=0.038)$, "support from one's coworkers" ( $p=0.012)$, "support from one's a family and friend" $(p=0.045)$, and "satisfaction with occupation or life" ( $\mathrm{p}=0.016)$. The group that scored higher on "the management to the understanding of the children" also had higher scores on "quantitative burden of psychological work" $(p=0.027)$ and "subjective physical burden" ( $\mathrm{p}=0.015)$. In addition, the group that scored higher on "the difficulty of the cooperation with the staff in the school" exhibited higher scores on "the stress of the personal relationships in the workplace" $(p<0.001)$, "working environmental stress" $(p=0.048)$, "irritable feeling" ( $\mathrm{p}=0.035)$, and "anxiety complex" $(\mathrm{p}=0.033)$ but lower scores on "occupational adaptability" $(\mathrm{p}=0.027)$, "suitableness of the job" ( $p=0.022)$, "decent work" ( $p=0.035)$, "support from one's superior" $(p=0.04)$, "support from one's co-workers" $(p<0.001)$, and "satisfaction with occupation or life" $(p=0.013)$. Conclusions The instructions related to dealing with developmentally disabled children posed difficulties to school nurse teachers, which eventually led to a stress reaction. Thus, this study suggests formulating a policy that mandates the enforcement of a training to raise awareness of instructions related to managing children with developmental disabilities and, thereby, reduce the occupation-related stress of school nurse teachers in the future.

CORRESPONDING AUTHOR: Yuriko Takata, University of Tsukuba, takatayuriko@ob.md.tsukuba.ac.jp

\section{$\mathrm{P} 052$}

ALCOHOL USE, SELF-MANAGEMENT, AND HBA1C AMONG UNIVERSITY STUDENTS WITH TYPE 1 DIABETES

Lauren Wisk $^{12}$, Eliza Nelson ${ }^{1}$, Kara Magane ${ }^{1}$, Elissa Weitzman ${ }^{12}$

${ }^{1}$ Boston Children's Hospital- USA

${ }^{2}$ Harvard Medical School- USA

Introduction \& Purpose: Adolescents and young adults (AYA) with Type 1 Diabetes (T1D) struggle to achieve glycemic control and the increase in risk behaviours during university may complicate disease control. We sought to quantify alcohol use and self-management behaviours, and estimate their impact on glycemic control among university students with T1D. Methods: We recruited/consented 138 university students (ages 17-25) with T1D via several social media platforms (e.g., Facebook) and traditional avenues (e.g., newsletter). Participants self-reported their alcohol use, diabetes self-management and burden, recent hemoglobin A1c (HbA1c), and sociodemographics. Multivariable linear and ordinal regression were used to model $\mathrm{HbAlc}$ and $\mathrm{HbAlc}$ targets, adjusting for age, sex, frequency of daily blood glucose testing, and disease burden (illness cognition questionnaire). Results: Participants were from 85 universities across the US and Canada. Participants were 20.5 years old on average ( $\mathrm{SD}=1.5), 80.4 \%$ female, $82.6 \%$ white non-Hispanic, an average of 10.9 years old at diagnosis $(\mathrm{SD}=5.2), 84.1 \%$ were pump users and $60.1 \%$ used continuous glucose monitors, $44.2 \%$ tested their blood sugar $\geq 5$ times per day, $65.9 \%$ had $\geq 3 \mathrm{HbAlc}$ tests in the past year, with an average last $\mathrm{HbAlc}$ of $7.6(\mathrm{SD}=1.3) .81 .9 \%$ reported drinking in the past year, averaging 13.6 drinks in the past month. Multivariable analyses revealed that those who drank three+ days in the past month $(50.7 \%)$ had significantly higher $\mathrm{HbAlc}$ (by $0.63 \%, p<0.01$ ) and were significantly more likely to be above recommended glycemic targets (OR:3.08, 95\%CI:1.59-5.98). Conclusions: Like their peers without T1D, university students with T1D frequently consume alcohol; those who drink more frequently experience higher $\mathrm{HbAlc}$ and are less likely to achieve glycemic targets, independent of blood glucose testing and diabetes burden. AYA may underestimate the effects of alcohol on glycemic control and additional education about how alcohol affects their diabetes may help them to achieve glycemic targets during university.

CORRESPONDING AUTHOR: Lauren Wisk, Boston Children's Hospital and Harvard Medical School, lauren.wisk@childrens.harvard.edu

\section{P053}

MODERATING IMPACT OF PARENTAL RELATIONSHIP QUALITY ON QUALITY OF LIFE FOR YOUTH WITH AND WITHOUT CHRONIC CONDITIONS

Lauren Wisk $^{12}$, Kara Magane ${ }^{1}$, Elissa Weitzman ${ }^{1} 2$

${ }^{1}$ Boston Children's Hospital- USA

${ }^{2}$ Harvard Medical School- USA 
Introduction \& Purpose: Family functioning is an important contributor to quality of life (QoL), particularly for families of youth with chronic conditions (YCC), as chronic illness increases stress \& distress for the affected individual and their family. As family functioning and relationship quality may be particularly dynamic during adolescence and young adulthood, we evaluated trajectories of parental relationship quality (RQ) during this period for YCC compared to their healthy peers, and evaluated how these trajectories impacted individual QoL over time. Methods: Longitudinal data are from 3101 adolescents and young adults (ages 828 years) interviewed for the Panel Study of Income Dynamics, a population-based survey in the US, between 2002 and 2013. We utilized multivariate generalized linear mixed models with natural cubic splines to model trajectories in RQ (MSALT scale, range 1-4) and their effect on QoL (MIDUS scales for emotional, social \& psychological well-being, range 1-6) for youth with and without chronic conditions, adjusting for confounders. Results: Compared to their healthy peers, YCC reported lower RQ with their mothers and fathers, particularly during late adolescence $(\mathrm{p}<0.01)$ and after age $20(\mathrm{p}<0.01)$ respectively. YCC also reported lower QoL than their peers across emotional, social \& psychological domains (all $\mathrm{p}<0.01)$. Better relationships with both mothers and fathers resulted in gains in all three domains of QoL and the impact of RQ on QoL diminished with age. Yet, YCC with lower parental RQ experienced significantly diminished QoL relative to their peers (e.g., average emotional QoL was 0.30 points (standardized) lower for YCC with low maternal RQ) while YCC with higher parental RQ saw smaller disparities in their QoL (e.g., average emotional QoL was 0.15 points lower for YCC with high maternal RQ). Conclusions: Parent-child relationship quality appears to exert a significant effect on youth QoL well into young adulthood, especially so for YCC who may rely on support from their parents over a longer period of time than their peers. Practitioners may want to assess parent-child relationship quality to buttress resilience resources among medically vulnerable youth.

CORRESPONDING AUTHOR: Lauren Wisk, Boston Children's Hospital and Harvard Medical School, lauren.wisk@ childrens.harvard.edu

P054

INTEROCEPTIVE SENSITIVITY FROM A MULTIMODAL PERSPECTIVE

Eszter ferentzi ${ }^{1}$

${ }^{1}$ ELTE Eötvös Loránd University- Hungary

Introduction \& Purpose Although interoception is regarded as a multimodal construct, associations among reported sensitivity/accuracy of the different channels is a scarcely investigated topic. Some findings support the association between the interoceptive accuracy of the gastrointestinal and the respiratory systems. The majority of the studies did not find any correlation; however, only a limited number and type of channels were assessed. The aim of this study is to unfold which interoceptive modalities can be grouped together based on their sensitivity/accuracy. Methods University students ( $\mathrm{N}=118,53 \%$ male) participated in the following six sensory measurements: heartbeat tracking task, water load test, tasks assessing bitterness sensitivity (intensity \& unpleasantness), ischemic pain (threshold \& tolerance), the proprioceptive accuracy of the elbow joint (reproduction with the same arm \& opposite arm) and balancing ability with closed eyes. Results Exploratory factor analyses (principal component analysis and maximal likelihood extraction with oblimin rotation) uniformly revealed three factors: bitter taste sensitivity, distension of the stomach, and pain. Loadings of variables referring to proprioception, balancing, and cardioception were low and inconsistent. Conclusions The present findings do not support the existence of general interoceptive sensitivity. Different aspects of the same modality (i.e. intensity \& unpleasantness for taste; fullness \& discomfort of the stomach; threshold \& tolerance for pain) were loading on the same factor, suggesting that modalities themselves provide congruent information. The three factors represent sensory channels that are associated with negative evaluation and directly serve the survival of the species. Interestingly, none of the sensory channels were associated with pain, the most common body symptom.

CORRESPONDING AUTHOR: Eszter ferentzi, ELTE Eötvös Loránd University, ferentzi.eszter@ppk.elte.hu

\section{P055}

LEARNING TO FEEL TIRED: A CLASSICAL AND OPERANT CONDITIONING APPROACH TO CHRONIC FATIGUE

Bert Lenaert $^{1}$, Rudolf Ponds ${ }^{1}$, Caroline van Heugten ${ }^{1}$

${ }^{1}$ Maastricht University- Netherlands

Introduction and purpose: Chronic fatigue is highly prevalent in the general population as well as in multiple chronic diseases and psychiatric disorders. Its etiology however remains poorly understood and cannot be explained by biological factors. Based on the cognitive-behavioral model we recently developed, chronic fatigue is approached from an associative learning perspective, implicating classical and operant conditioning processes in the transition from acute to chronic fatigue. The current experiment investigated whether fatigue reporting can be brought under operant control. That is, interpersonal interactions may strengthen and perpetuate fatigue reporting through social reinforcement. Methods: Forty-four individuals engaged in prolonged cognitive effort (working memory task) and were asked to rate their current fatigue levels repeatedly throughout several blocks of the task. In the experimental condition, subtle social reward was given when fatigue increased relative to the previous rating; or disapproval when fatigue decreased. In the control condition, only neutral feedback was given. Results: Crucially, there was a significant Block $\times$ Condition interaction, $F(2,84)=4.96, p=$ .009 , partial $\eta 2=.106$, with planned comparisons revealing a greater increase in fatigue in the experimental (reinforced) condition than in the control (non-reinforced) condition throughout the experiment. This effect occurred independently of conscious awareness of the contingency between fatigue ratings and reinforcement. Interestingly, conditions also differed in objective cognitive performance during the task, with participants in the experimental condition performing worse than participants in the control condition. Conclusions: Results indicate that fatigue reporting may become controlled by their consequences, such as social reward. This may explain why - as is seen in chronic fatigue conditions - fatigue complaints may become loosened from its antecedents such as mental or physical effort or acute illness. Moreover, these findings suggest that operant conditioning by the social environment may also influence objective cognitive functioning.

CORRESPONDING AUTHOR: Bert Lenaert, Maastricht University, bert.lenaert@maastrichtuniversity.nl

\section{P056}

SOCIAL DISPARITIES IN FERMENTABLE CARBOHYDRATE INTAKE PROFILE IN CHILDHOOD, METROPOLITAN REGION, CHILE

Maria Jose Monsalves ${ }^{1}$, Iris Espinoza ${ }^{2}$, Josefina Aubert ${ }^{3}$, Patricia Moya ${ }^{3}$, Oscar Arteaga ${ }^{2}$, Doris Duran ${ }^{1}$, Macarena Valdes ${ }^{2}$, Shrikant Bangdiwala ${ }^{4}$ ${ }^{1}$ Universidad San Sebastián- Chile

${ }^{2}$ Universidad de Chile- Chile

${ }^{3}$ Universidad Finis Terrae- Chile

${ }^{4}$ McMaster University- Canada

Objective: To characterize the profile of fermentable carbohydrate intake in children 1-6 years old in the Metropolitan Region (MR), Chile. Methods: A cross-sectional multilevel study (school/communes) was conducted in a sub-sample of the FONIS-SA13I20130 project with a 
questionnaire for caregivers of children 1-6 years old in 13 communes of the MR in Chile during 2014-2015. Intake frequency of 5 food-groups was assessed: biscuits/cakes/cream cakes; sugary juice/beverages; jam/ honey; sweets/candy; and tea/coffee/milk with sugar. Six categories for intake-frequency were considered never; several times a month, once a week, several times a week, at least once a day every day, several times in a day. Social characterization was obtained with educational level of the caregiver and family income. Statistical analysis included uni-bivariate analysis of the proportions (Chi-squared). Coefficients/odds of food intake-frequency for each food-group by education and income in the high intake-frequency category (several times a day) were obtained with ordinal logistic multilevel regression. Results: Sample-size was 1989. Three food-groups had the highest intake-frequency of at least once a day every day: sugary juice/beverages with a $48.92 \%$; tea/coffee/milk with sugar with $38.67 \%$, and biscuits/cakes/cream cakes with $27.77 \%$. In relation to social characterization, educational level was associated with intake frequency of sugary juice/beverages; tea/coffee/milk with sugar; and Sweets/candy $(\mathrm{p}<0.05) .88 \%$ higher odds of having a high intake frequency of tea/coffee/milk with sugar, $35 \%$ for sugary juice/ beverages, $34 \%$ for jam/honey was found for caregivers with primary education. $71 \%$ lower odds of never consuming tea/coffee/milk with sugar, $46 \%$ for sugary juice/beverages and for sweets/candy between the highest family income in relation to the lowest. Conclusions Sugary juice/beverages; tea/coffee/milk with sugar, and biscuits/cakes/cream had the highest frequencies of intake, and the higher differences between socioeconomic groups. Primary educational level and low family income present the higher frequency of intake for food-groups related to caries, evidencing their disadvantaged position to have better oral-health.

CORRESPONDING AUTHOR: Maria Jose Monsalves, Universidad San Sebastián, mjmonsalves@gmail.com

\section{P057}

GREEN PRESCRIPTIONS AND THERAPEUTIC LANDSCAPES: A NEW ZEALAND STUDY

Bruno Marques ${ }^{1}$, Jacqueline McIntosh ${ }^{1}$, Tosin Popoola ${ }^{1}$

${ }^{1}$ Victoria University of Wellington- New Zealand

Physical inactivity has significant implications for overall health status, especially among older adults and poor participation in physical activity can worsen the physical, mental and sociological functioning in old age. As a result, increased participation in physical activity through exercise prescription (Green Prescription) continues to be encouraged since it has proven to be a potent health maintenance tool for combatting the various debilitating health conditions that accompany old age. However, the proportion of the older population participating in physical activity continues to be low because of lack of conducive and supportive environments where exercise activities can be carried out with little or no risks. As such, there is a renewed interest to understand the relationship between the environment and the social context, and the roles they play in the promotion of physical, spiritual and mental wellbeing. It is within this context that this research was conducted to understand the processes of initiation, implementation and feedback that occur in the prescription of exercise and the implication that these have for therapeutic and rehabilitative landscapes. The research was conducted in two phases; an in-depth literature review on Green Prescriptions, followed by a set of interviews with 20 New Zealand General Practitioners, experienced with prescribing nonpharmaceutical treatments. The interview data were thematically analysed. The findings from the research revealed that while there is a positive disposition towards the need for increased exercise among older adults; the schisms between whether interventions should focus on environments that intentionally contribute to health or environments that unintentionally contribute to health raises questions for the future of Green Prescriptions and therapeutic landscapes. This separation, therefore, has implications for the barriers to Green Prescriptions and suggests a need to understand better how therapeutic landscapes contribute to health promotion and maintenance.

CORRESPONDING AUTHOR: Bruno Marques, Victoria University of Wellington, bruno.marques@vuw.ac.nz

\section{P058}

PREDICTING THE SYMPTOM BURDEN OF CHRONIC PAIN FROM PSYCHOLOGICAL PROFILE AND THE MODERATING IMPACT OF PAIN AETIOLOGY

David McNaughton $^{1}$, David McNaughton ${ }^{1}$

${ }^{1}$ Macquarie University- Australia

Background: The relationship between chronic pain and psychological distress remains a complex neuro-psychological process integral to the symptom burden experienced. In addition, the role of pain aetiology in a biopsychosocial formulation of chronic pain is not well understood. One approach to classify chronic pain disorders is to consider whether the condition has an identifiable pathophysiology or not. Such a distinction has led to the use of terms organic and functional conditions, respectively. The aim of the present study is to determine whether the association between psychological profile and chronic pain symptom burden is moderated by a functional or organic pain aetiology. Methods: The present study is a secondary analysis of data collected from an internet-delivered pain management program, known as The Pain Course [1]. Standardized psychological measures of 471 chronic pain sufferers were used in a nonhierarchical cluster analysis, which allocated participants into mutually exclusive profiles. Participants pain aetiology was identified as functional or organic, via interview and self-reported data provided. Non-parametric testing of psychological profiles with chronic pain outcomes of disability and intensity was followed by a formal test of moderation of pain aetiology via an analysis of covariance. Results: The psychological profiles identified through the cluster analysis are described as psychologically healthy, mildly psychologically distressed, highly psychologically distressed and 'normal'. The highly psychologically distressed profile experienced the greatest mean pain intensity $(4.07(\mathrm{SD}=2.1))$ and disability $(17.53(\mathrm{SD}=3.65)$ ), however, this relationship was not moderated by preceding pain aetiology; $2(3)=0.65, p=0.58$ and $2(3)=1.82, p=$ 0.14 , respectively. Discussion/Conclusion: The present study's psychological profiles provide a more realistic representation of the psychological experience in chronic pain suffers. These data suggest that the disability and intensity experienced by people with functional pain disorders is similar to people with organic conditions and that both are not psychologically driven. Future research of a longitudinal nature should focus on how psychological profiles alter treatment response and if moderation of this effect occurs due to the preceding pain aetiology.

CORRESPONDING AUTHOR: David McNaughton, Macquarie University, david.mcnaughton@hdr.mq.edu.au

\section{P059}

\section{A NEW PERSPECTIVE ON SYMPTOM PERCEPTION \\ Omer Van den Bergh ${ }^{1}$ \\ ${ }^{1} \mathrm{KU}$ Leuven, University of Leuven- Belgium}

In recent years, a major shift took place in the view on how the brain works. Rather than passively receiving input, the brain is basically a prediction testing organ that tries to make sense of the stimulation it receives. As a discussant, I will use this framework to elaborate on the results presented in this symposium that show how learning mechanisms may contribute to the development and maintenance of bodily symptoms. In addition, this framework may also help to integrate a other phenomena such as information processing biases, repetitive negative thinking, and reduced autobiographical memory that are often found associated with 
psychopathology and somatization.

CORRESPONDING AUTHOR: Omer Van den Bergh, KU Leuven University of Leuven, omer.vandenbergh@ppw.kuleuven.be

P060

A RANDOMISED FEASIBILITY TRIAL OF OPTIMAL P ER S O N A L IS E D C A R E A F T ERTREAT M EN TGYNAECOLOGICAL CANCER (TOPCAT-G)

Val Morrison ${ }^{1}$, Simon Leeson

${ }^{1}$ Bangor University- United Kingdom

${ }^{2}$ Betsi Cadwaladr University Health Board, Bangor- United Kingdom

Introduction and purpose The follow-up care currently provided in the UK after treatment for gynaecological cancer is underpinned by a largely retrospective evidence-base and tends to be clinic-based and clinician-led. Furthermore, there are no guidelines from the National Institute for Health and Care Excellence (NICE) as to what form or frequency of follow-up is appropriate in relation to either effective recurrence detection or patient wellbeing. The feasibility of completing a randomised controlled trial (RCT) of nurse-led telephone follow-up in the gynaecology cancer setting (OPCAT-G, Optimised Personal Care After Treatment) has been assessed in terms of specific objectives: eligibility, recruitment, retention rates, completion rates of outcome measures. Methods The unblinded trial recruited patients who had completed treatment of cervical, endometrial, epithelial ovarian, or vulval cancer within the previous 3 months. Patients were randomised to either usual hospital follow-up or specialist nurse-led telephone education, empowerment and structured needs assessment follow up. The EORTC (QLQ C30) and EQ5D assessed quality of life. Results Fifty-eight women were screened to take part in the study during a period of six months, 44 were deemed eligible to take part ( $76 \%$ of the screened population). The main reason for ineligibility was that the patient required on-going hospital care (64\%). Of the 44 eligible women, 24 consented to take part in the study, giving a recruitment rate of $55 \%$. The main reason for non-recruitment was that patients did not want to be randomised, primarily due to wanting to see a doctor for follow-up. Patients were recruited from three hospitals in North Wales, UK, by a research nurse $(\mathrm{RN})$ and clinical nurse specialists (CNSs), with some site differences in recruiting rate due to lack of early CNS involvement and research support. Participants had a mean(SD) age of 60 (11.2) years and were approximately 5 months from initial diagnosis (mean 159, SD 58 days). Seventeen (71\%) of participants had an endometrial cancer diagnosis. Outcome measure completion rates exceeded $96 \%$. One participant was lost to follow up (3, 6 months post baseline). Exploratory analysis of outcome measures indicated positive change in quality of life within the intervention arm, and a per patient saving of $£ 27$. Conclusion We demonstrated intervention feasibility with satisfactory eligibility, recruitment and retention, and lessons learned for early nurse specialist involvement and dedicated in-clinic research support. A definitive rct is being prepared.

CORRESPONDING AUTHOR: Val Morrison, Bangor University, v.morrison@bangor.ac.uk

P061

MEDICATION ADHERENCE AMONG STROKE SURVIVORS IN RURAL CHINA: A MIXED METHODS STUDY

Wanbing $\mathrm{Gu}^{1}$, Enying Gong ${ }^{1}$, Lijing Yan $^{1}$

${ }^{1}$ Duke Kunshan University- China

Introduction Stroke is the leading cause of deaths and disabilities in China with about 11.1 million stroke survivors. Despite the growing burden from stroke in rural China, effective secondary prevention of stroke is far below guideline recommendations. Good medication adherence is one of the most important components of secondary prevention, which is associated with better health outcome and lower risk of recurrence and mortality. The objective of this study is to describe the current situation of medication adherence among stroke survivors in Nanhe County, rural China, and to explore factors influencing medication adherence. Methods Stroke survivors from 50 villages of five townships (ten villages in each township) in Nanhe County were recruited through a multistage cluster sampling. Information on history of stroke, use of and adherence to medication was obtained through a standard questionnaire by trained interviewers. Medication adherence were measured by using four-item Morisky Green Levine Scale with score 0-1 represents a good adherence. Stroke survivors were also invited to participated in semi-structured interviews during the intervention. The interview questions include patients' attitude toward medication use and adherence. Descriptive analysis was performed on survey data by using STATA and thematic content analysis was performed for interview data by using Nvivo. Results In total, 1299 stroke survivors in Nanhe County, rural China participated in the survey (57.4\% males, average age: 65.7 years old). Among these participants, 1030 were taking anti-hypertensive medicines, while only $797(77.4 \%)$ adhered to the anti-hypertensive medicines. 852 were taking aspirin with $691(81.1 \%)$ had good adherence. Only 340 patients were taking statins with 274 (80.6\%) stroke survivors adhere to the prescription. The qualitative data showed that forgetting taking medicine, economic difficulty in purchasing medicine, asymptomatic conditions and lack of knowledge of medicine are the most common reasons of poor adherence. In addition, the severity of diseases, having a caregiver and the expertise and authority of healthcare providers are facilitating factors in promoting medication adherence among stroke survivors. Conclusion Improving medication adherence among stroke survivors is essential to promote the secondary prevention of stroke in rural China. In addition to factors related to patients, factors related to caregivers and healthcare providers, which are always overlooked, also have impacts on patients' medication adherence. Future intervention programs should target on patients, caregivers and healthcare providers to promote medication adherence.

CORRESPONDING AUTHOR: Wanbing Gu, Duke Kunshan University, wanbing.gu@duke.edu

\section{P062}

DEVELOPMENT OF PERSONALISED RISK INFORMATION TO PROMOTE SMOKING CESSATION IN A LUNG CANCER SCREENING CONTEXT

Grace McCutchan ${ }^{1}$, Kate Brain ${ }^{1}$, Samantha Quaife ${ }^{2}$, Pamela Smith ${ }^{1}$, Mat Callister $^{3}$, Phillip Crosbie ${ }^{4}$, Rachael Murray ${ }^{5}$

${ }^{1}$ Cardiff University- United Kingdom

${ }^{2}$ University College London- United Kingdom

${ }^{3}$ NHS- United Kingdom

${ }^{4}$ Manchester University- United Kingdom

${ }^{5}$ University of Nottingham- United Kingdom

Background CT screening and smoking cessation have additive effects on reducing lung cancer mortality. Despite widespread adoption of CT screening in North America, several important questions remain about implementation, including how to most effectively embed smoking cessation support into lung cancer screening programmes. This study aimed to explore participant views and preferences regarding different formats for presenting personalised risk information to support smoking cessation in a lung cancer screening context. Methods An online survey was circulated to a panel of current smokers to elicit preferences regarding visual format (bar chart, pictogram, pie chart), time frame (3, 5, 7 or 10 years), and gain versus loss framed written statements relating to personalised risk information. Further, three focus groups with current smokers and recent quitters (within one year) over the age of 55, living in areas of deprivation were conducted to gauge format preferences, comprehension 
(including relative vs absolute risk and time frame) and potential influence of personalised risk information on motivation to quit smoking. Results Findings from the online survey with eight smokers indicated preferences for displaying personalised risk as pictograms, highlighting risk reduction in a contrasting colour, and coupled with gain framed statements. Preliminary focus group findings suggest a preference for a shorter time frame for risk information, and scan images that were more representative of their heart or lungs, and displayed in colour may motivate a quit attempt. Conclusion Pictograms and scan images displaying personalised risk information with positively framed statements were preferred by smokers. This work will inform the development of materials to be tested in a funded trial of personalised risk information vs standard best care as methods to support smoking cessation in a lung cancer screening context.

CORRESPONDING AUTHOR: Grace McCutchan, Cardiff University, mccutchanGM@cardiff.ac.uk

P063

SLEEP, FATIGUE AND QUALITY OF LIFE AMONG TEENAGE AND YOUNG ADULT CANCER SURVIVORS

Abigail Fisher ${ }^{1}$, Judith Fortmann ${ }^{1}$, Gemma Pugh ${ }^{1}$, Rachael Hough ${ }^{2}$, Alice Gregory ${ }^{3}$

${ }^{1}$ University College London- United Kingdom

${ }^{2}$ University College London Hospitals- United Kingdom

${ }^{3}$ Goldsmiths, University of London- United Kingdom

Purpose: Teenage and young adult (TYA) cancer survivors experience a range of health-related problems during and beyond the active disease period. This study examined associations between fatigue, poor sleep quality, and impaired health-related quality of life (HR-QoL) in TYA survivors. Methods: Self-reported data on sleep quality, fatigue and HR-QoL were gathered from UK TYA survivors aged between 13 and 24 years. TYA survivors were stratified into those on $(n=67)$ and off $(n=135)$ treatment. Linear regression analyses were used with HR-QoL as the dependent variable to investigate potential associations. Fatigue and sleep were entered separately, and together in the same model. Age, gender, ethnicity and education were included as covariates. Results: Strong independent associations between sleep $(\mathrm{B}=0.05,95 \% \mathrm{CI}=0.03-0.08, \mathrm{p}<0.001)$, fatigue $(\mathrm{B}=0.02,95 \% \mathrm{CI}=0.01-0.03, \mathrm{p}<0.001)$, and HR-QoL were observed among TYA survivors on treatment. TYAs off treatment showed moderate to strong associations between sleep $(B=0.04,95 \% \mathrm{CI}=0.02-0.05, \mathrm{p}<0.001)$ and fatigue $(\mathrm{B}=0.02,95 \% \mathrm{CI}=0.01-0.02, \mathrm{p}<0.001)$, and HR-QoL, when examined separately. Sleep was not independently associated with HRQoL in TYAs off treatment $(\mathrm{B}=0.01,95 \% \mathrm{CI}=-0.01-0.02, \mathrm{p}=0.248)$. Conclusion: The significant associations reported suggest sleep quality and fatigue are potential modifiable factors associated with HR-QoL. Further research is warranted to understand the direction of associations.

CORRESPONDING AUTHOR: Abigail Fisher, University College London, abigail.fisher@ucl.ac.uk

\section{P064}

BEHAVIORAL MEDICINE IN THE PREVENTION AND REHABILITATION OF ADDICTIVE DISORDERS

Authors: Dr. Justo Fabelo-Roche ${ }^{1}$, Dr. Serguei Iglesias-More ${ }^{1}$

${ }^{1}$ Universidad de Ciencias Médica de La Habana- Cuba

The abuse of drugs constitutes a health problem that is reflected in the morbidity and mortality statistics of the nations. In Cuba, the consumption of tobacco, alcohol and coffee has been consolidated for centuries as part of the national culture. During the last decades, the use of substances such as cannabis, cocaine, psychotropic drugs and synthetic marijuana increased significantly. Among adolescents and young people, addictive behaviors related to information technology and communications are also observed. All this has determined that resources from Behavioral Medicine are used in prevention and rehabilitation programs for addictive disorders. Aversive techniques, positive reinforcement, cognitive behavioral approaches and third generation therapies are used from a multidisciplinary approach in which knowledge from the biomedical and behavioral sciences is integrated. In the present work, studies carried out in the cities of Havana and Santiago de Cuba are reviewed in which addictive disorders are approached from the perspective of behavioral medicine, obtaining encouraging results from the use of training and procedures aimed at empowering healthy and sick people to promote the reduction of consumption or absolute abstinence. Results of research projects that were aimed at the smoking cessation in smokers, the formation of alcohol rejection attitudes in adolescents, training in social skills and solving problems related to youth drug use and rehabilitation, are presented. social reintegration and relapse prevention in addicted patients. It is concluded that Behavioral Medicine provides an integral perspective of special utility to face the problem of addictions and that it constitutes a space of constructive link between Psychology and medical specialties such as Psychiatry, Public Health and Epidemiology.

CORRESPONDING AUTHOR: Justo Fabelo-Roche, Universidad de Ciencias Médica de La Habana, fabelo@infomed.sld.cu

\section{P065}

MATERNAL AND PATERNAL POSTPARTUM MOOD: ASSOCIATIONS WITH EARLY INTERACTIONS WITH THE BABY Rita Amiel Castro ${ }^{1}$, Vivette Glover ${ }^{2}$, Ulrike Ehlert ${ }^{1}$, Martin Kammerer ${ }^{2}$

${ }^{1}$ University of Zurich- Switzerland

${ }^{2}$ Imperial College London- United Kingdom

Whereas the depressive spectrum has been widely researched in postpartum women, postpartum milder hypomanic symptoms ('the highs') have received less attention. There is some indication that together with the blues the experience of euphoria at early postpartum may be a risk factor for later postpartum depression. Evidence suggests that men also experience mood disorders after the birth of a child, which may be linked to maternal behaviours and also have the potential to affect relationships within the family. Aim: To investigate the association between maternal blues and highs with paternal blues and highs at early postpartum; to examine if parents' mood (blues and highs) are associated with the quality of baby-bonding. Methods: A non-clinical sample of women and their male partners $(\mathrm{N}=98)$ were assessed at the 5th postpartum day for mild hypomania, postpartum blues and quality of baby-bonding. Couples filled in separately psychological questionnaires and gave socio-demographic information. Exclusion criteria were psychotic features, current drug or alcohol addiction and poor general medical condition. Results: Maternal blues were significantly associated with paternal blues $(\mathrm{B}=.29, \mathrm{p}=.03$, CI $(95 \%)=.00-.58)$ and maternal highs also significantly predicted paternal highs $(B=.30, p=.01$, CI $(95 \%)=.06-.53$ ) after controlling for covariates (e.g. age and parity). Paternal blues, but not maternal were significantly associated with worse baby bonding $(\mathrm{B}=.17, \mathrm{p}=.00$, CI $(95 \%)=.06-.29)$. Parents' highs were not related to baby bonding ( $\mathrm{p}>.05$ ). Conclusions: The association between paternal blues and highs with maternal blues and highs has important implications for family health and well-being. Also, fathers' blues were associated with poorer early father-baby bonding. Consideration of co-occurrence of early postpartum behaviours in couples, which may further lead to more severe mental disorders is an important step in research and practice involving childbearing families. Keywords: maternal postpartum blues, maternal postpartum highs, paternal postpartum blues, paternal postpartum highs, baby-bonding.

CORRESPONDING AUTHOR: Rita Amiel Castro, University of Zurich, r.castro@psychologie.uzh.ch 
P066

\section{CONVERSATION WHEEL IN THE FAMILY}

Marilda Coelho ${ }^{1}$

${ }^{1}$ Universidade Federal de Uberlândia- Brazil

Introduction: it is a therapeutic technique based on the practice of almost three decades of families, pain, suffering and grieving inherent to the human condition. Parents are concerned with the cognitive training of children and neglect emotional intelligence and interpersonal relationships. Giving names to feelings is a technique used predominantly in therapeutic processes in general. We adapt to the family group very successfully. Objective: To instrument families to develop the ability to name affections, consequently, better management of emotions. This technique tends to result in the prevention of short, medium and long term emotional disorders. That would be prevention in Mental Health. People need people to be human. Methodology: in the conversation circle the whole family, sits weekly, on the time pre-established and talk about everyday experiences. Begins with an adult coordinating and giving the testimony, as a model. All members of the group are invited to speak, if there is resistance, it is respected; over time the coordination is alternated and the themes are chosen by the members of the group. It is necessary, at least initially, to supervise and monitor the process by an experienced mental health professional. Material chosen for reading should be compatible with the chronological age of the children. Titles of philosophy, are suggested initially, because they deal with moral and affective ethical issues. Over time, videos and controversial social media themes are of the utmost importance. Results: We have observed in the families results such as: cohesion of the members, fraternal relationship between siblings, respect for rules, better relationship between parents and children, respect for authority, feeling of inclusion, improvement in the quality of life of the family and social group. Developing the capacity for building a humanity without frontiers.

CORRESPONDING AUTHOR: Marilda Coelho, Universidade Federal de Uberlândia, marildacoelho.psisaude@ gmail.com

\section{P067}

HOSPITAL DELIVERY AND ASSOCIATED FACTORS IN ETHNIC MINORITY COUNTIES OF TIBET AUTONOMOUS PREFECTURE IN CHINA

Li Zhao $^{1}$, Dongling Xie ${ }^{1}$, Xiao Ma ${ }^{1}$, Ruikan Yang ${ }^{2}$, Ying Zheng ${ }^{3}$, Min Yang ${ }^{1}$ ${ }^{1}$ West China School of Public Health/ West China Research Center for Rural Health, Sichuan University, Chengdu- China

${ }^{2}$ Save the Children

${ }^{3}$ West China Women's and children's Hospital, Sichuan University- China

Background: China has achieved huge success in improving hospital delivery to reduce high maternal and infant mortality rates, with the hospital delivery rates of over $95 \%$ in xx out of 34 provinces by $20 \mathrm{xx}$. However, Tibet Autonomous Region lagged behind the average level, which has been severely neglected in previous studies conducted in the developing world. This study aimed to investigate important factors affecting hospital delivery. from different aspects leading to some implication for policy and future studies. Methods: In 20xx, trained interviewers conducted face-to-face questionnaire among 220 Tibetan women hierarchically sampled in Nagchu and Nyirong counties of Tibet to collect information about the sociodemographic attributes, previous reproductive history, knowledge/perception of antenatal care, health behaviors, and maternal health service utilization. Chi-squared tests were conducted to detect the differences between the two counties. Logistic regression models were used to examine effects of potential determinants on hospital delivery. Results: The hospital delivery rate was $62.8 \%$ in the two sampled counties. The numbers of healthy new-born and antenatal care were independently associated with hospital delivery. The odds of hospital delivery were significantly lower for women who did not receive antenatal care, compared to those who did $(\mathrm{OR}=0.28,95 \% \mathrm{CI}=[0.11-$ $0.69])$. Women who had more healthy new born had higher odds of hospital delivery than their counterparts $(\mathrm{OR}=2.13,95 \% \mathrm{CI}=[1.16-$ 3.85]). Conclusion: This study fills gaps in hospital delivery studies in China by focusing on an extremely under representative ethnic group. Findings could help to understand health inequality across China and design population-based interventions for improving the hospital delivery rate, ultimately achieving the overall goal of reducing maternal mortality and infant mortality.

CORRESPONDING AUTHOR: Li Zhao, West China School of Public Health/ West China Research Center for Rural Health, Sichuan University, Chengdu, zhaoli@scu.edu.cn

\section{P069}

CAN PRIESTS ALTER THE CHOICE AND PREFERENCE OF SUGAR-SWEETENED BEVERAGES? A CLUSTER RANDOMIZED CONTROLLED TRIAL IN CATHOLIC PARISHES Janina Bazalar ${ }^{1}$, J. Jaime Miranda ${ }^{2}$, Alvaro Taype-Rondan ${ }^{2}$, Janina Bazalar-Palacios ${ }^{3}$, Antonio Bernabe-Ortiz ${ }^{2}$, Dan Ariely ${ }^{4}$

${ }^{1}$ Universidad Católica los Ángeles de Chimbote- Peru

${ }^{2}$ CRONICAS Center of Excellence in Chronic Diseases, Universidad Peruana Cayetano Heredia, Lima- Peru

${ }^{3}$ Centro de Estudios de Población, Universidad Católica los Ángeles de Chimbote, Chimbote- Peru

${ }^{4}$ Center for Advanced Hindsight, Duke University, Durham, NC- USA

Background: Latin America ranks among the regions with the highest level of intake of sugary beverages in the world. Innovative strategies to reduce the consumption of sugary drinks are necessary. Research question: What is the impact of a sermon given by priests on the protection of health in the preferences of choosing and consuming sugar-sweetened beverages after mass? Objetive: Evaluating the potential of faith-based interventions by assessing the immediate and short-term impact of a oneoff sermon given by priests during a morning service on the choice and preference of sugar-sweetened beverages. Methods: A pragmatic, clusterrandomized trial, was conducted in Chimbote, Peru. Catholic parishes, paired by number of attendees, were randomly assigned to intervention or control. The intervention, delivered by a priest during the mass, was a short message about the importance of protecting one's health. The primary outcome was the proportion of individuals that choose a bottle of soda instead of a bottle of water immediately after the service. Clusterlevel estimates were used to compare primary and secondary outcomes between intervention and control groups utilizing non-parametric tests. Results: Six parishes were allocated to control and 6 to the intervention group. The proportion of soda selection at baseline was $\sim 60 \%$ in the intervention and control groups, and ranged from $56.3 \%$ to $63.8 \%$ in week 1, and from $62.7 \%$ to $68.2 \%$ in week 3 . Accounting for baseline values, the proportion of mass attendees choosing soda at week 1 after baseline was, on average, $8.2 \%$ lower in the intervention group compared to the control group $(\mathrm{p}=0.03)$. At 3 weeks after baseline, the difference between intervention and control group was $6.2 \%(\mathrm{p}=0.15)$. Conclusions: In Catholic churches, a single low-intensity short-duration one-off sermon given by a priest has an immediate effect and a weak short term effect in reducing the choice of sugar-sweetened beverages over water.

CORRESPONDING AUTHOR: Janina Bazalar, Universidad Católica los Ángeles de Chimbote, saoribp@gmail.com

\section{P070}

ONE CASE REPORT OF HYPOKALEMIC PERIODIC PARALYSIS WITH SENSORY ABNORMALITIES

Yuhong Man ${ }^{1}$, Fang Zhang ${ }^{1}$, Yuhong Man ${ }^{1}$, Xiaoli Meng ${ }^{1}$, Tingmin $\mathrm{Yu}^{1}$ ${ }^{1}$ Department of Neurology, 2nd Hospital of Jilin University- China 
Introduction: It is manifested with recurrent flaccid skeletal muscle weakness or paralysis and without sensory abnormalities accompanied by decreased serum potassium of hypokalemic periodic paralysis(HPP). We are misdiagnosed HPP as Guillain Barre syndrome easily for few patients who have motor and sensory defects. Here we report a HPP case with sensory disturbances in order to provide references for clinicians. Methods: We collected clinical manifestations and auxiliary examination of a 55-year-old male. RESULTS: The patient was admitted to the hospital with lower extremities weakness for 3 days and aggravated gradually with upper extremities weakness for 2 days. Physical examination was normal. Neurological examination revealed low muscle tone, absent reflexes in all extremities and stocking-and-glove distribution of sensory loss. The upper extremities were grade IIIand the lower extremities were gradeII. Bilateral pathological signs were negative. Blood biochemistry revealed hypokalemia $(\mathrm{K}+1.44 \mathrm{mmol} / \mathrm{L})$. He was given oral and intravenous potassium treatment. The clinical abnormalities of the patient disappeared completely and neurological examination were normal when serum potassium reached to regular level. CONCLUSIONS: Unlike the typical HPP,this patient had stocking-and-glove distribution of sensory loss. Researches suggested that sensory nerve action potential(SNAP) amplitude was lower than normal value in the onset of muscle weakness. SNAP amplitude increased after serum potassium returned to normal. It meant that the sensory nerve function was related to the metabolism of serum potassium ion. The mechanism might be inactivity of $\mathrm{Na}+\mathrm{K}+\mathrm{ATP}$ pump at dorsal root ganglia which was susceptible to the changes of extracellular fluid composition, for dorsal root ganglia had poor blood nerve barrier

CORRESPONDING AUTHOR: Yuhong Man, Department of Neurology, 2nd Hospital of Jilin University, myh1996@163.com

\section{P071}

REDUCING WAITING TIME AND RAISING OUTPATIENT SATISFACTION IN A CHINESE PUBLIC TERTIARY GENERAL HOSPITAL-AN INTERRUPTED TIME SERIES STUDY

Jing $\operatorname{Sun}^{12}$, Jing Sun ${ }^{12}{ }^{3}$, Qian $\mathrm{Lin}^{4}$, Pengyu Zhao ${ }^{1}{ }^{2} 3$, Qiongyao Zhang $^{4}$, Kai Xu ${ }^{4}$, Huiying Chen ${ }^{4}$, Cecile Jia Hu${ }^{5}$, Mark Stuntz ${ }^{6}$

${ }^{1}$ Chinese Academy of Medical Sciences- China

${ }^{2}$ Peking Union Medical College- China

${ }^{3}$ School of Public Health- China

${ }^{4}$ Fujian Provincial Hospital- China

${ }^{5}$ Deerfield Institute- China

${ }^{6}$ Deerfield Institute- USA

Introduction and Purpose: It is globally agreed that a well-designed health system deliver timely and convenient access to health services for all patients. Many interventions aiming to reduce waiting times have been implemented in Chinese public tertiary hospitals to improve patients' satisfaction. However, few were well-documented, and the effects were rarely measured with robust methods. This study assess changes in levels and trends of waiting times before and after the introduction of waiting time reduction interventions in a tertiary public hospital in Southern China. Methods: We analyzed longitudinal time series data using a segmented linear regression model to assess changes in levels and trends of waiting times before and after the introduction of waiting time reduction interventions. Around an average of 60,000 outpatients and 70,000 prescribed outpatients per month were targeted for the study during Oct 2014-February 2017. Pearson correlation analysis was conducted to indicate the strength of association between waiting times and patient satisfactions. The statistical significance level was set at 0.05 . Results: The monthly average length of waiting time decreased $3.49 \min (\mathrm{P}=0.003)$ for consultations and $8.70 \mathrm{~min}(\mathrm{P}=0.02)$ for filling prescriptions in the corresponding month when respective interventions were introduced. The trend shifted from baseline slight increasing to afterwards significant decreasing for filling prescriptions $(\mathrm{P}=0.003)$. There was a significant negative correlation between waiting time of filling prescriptions and outpatient satisfaction towards pharmacy services $(\mathrm{r}=-0.71, \mathrm{P}=0.004)$. Conclusions: The interventions aimed at reducing waiting time and raising patient satisfaction in Fujian Provincial Hospital are effective. A longlasting reduction effect on waiting time for filling prescriptions was observed because of carefully designed continuous efforts, rather than a onetime campaign, and with appropriate incentives implemented by a taskforce authorized by the hospital managers. This case provides a model of carrying out continuous quality improvement and optimizing management process with the support of relevant evidence.

CORRESPONDING AUTHOR: Jing Sun, Chinese Academy of Medical Sciences and Peking Union Medical College, sunjing@ @ph.pumc.edu.cn

\section{P072}

WIFE'S HAPPINESS OR DISTRESS? WHICH IS AFFECTING THE HUSBAND'S MENTAL HEALTH?

Noboru Iwata $^{1}$, Akihito Shimazu ${ }^{2}$, Takeo Fujiwara ${ }^{3}$, Norito Kawakami ${ }^{4}$, Kyoko Shimada $^{5}$, Masaya Takahashi ${ }^{6}$, Masahito Tokita ${ }^{7}$, Izumi Watai ${ }^{8}$

${ }^{1}$ Hiroshima International University- Japan

${ }^{2}$ Kitasato University- Japan

${ }^{3}$ Tokyo Medical and Dental University- Japan

${ }^{4}$ University of Tokyo- Japan

${ }^{5}$ Toyo University- Japan

${ }^{6}$ National Institute for Occupational Safety and Health- Japan

${ }^{7}$ Gunma Paz University- Japan

${ }^{8}$ Nagoya University- Japan

Purpose: Although it is believed that psychological status of husband and wife interact each other, little is known about the relative impact of spouse's psychological distress and happiness on the one's own mental health through a longitudinal observation. The purposes of this study are to identify the psychological distress pattern of husbands and wives over time, and examine the association of partners' psychological status to their spouses' distress among Japanese dual working couples. Method: In urban area of Tokyo, 379 dual-income families with children aged 0-5 were participated baseline survey in 2011, and they were followed up twice annually. Psychological distress was measured by K6. Group-based trajectory modeling analysis was conducted to identify psychological distress pattern over time. Multinomial logistic regression analysis was used to determine the relative impact of self-reported health and happiness of couples, and spouses' psychological distress on the trajectory groups of the ones' psychological distress. Result: A four-group linear trajectory model best fit husbands' psychological distress over the three years $(\mathrm{n}=175)$; no symptom (21\%), fewer symptoms (55\%), moderately distressed $(22 \%)$, and persistent severely distressed (1\%). A five-group model was detected for wives $(\mathrm{n}=231)$; no symptom $(9 \%)$, fewer symptoms (66\%), symptom relieving (10\%), symptom increasing (14\%), and persistent severely distressed (1\%). Likelihood ratio test revealed that the ones' own health and happiness and wives' happiness were significant predictors of trajectory group membership of husbands, while husbands' health, instead of happiness, was significant for the wives' membership. Husbands who perceived poor health (risk ratio $(\mathrm{RR})=0.45, \mathrm{p}=.04)$ and less happiness $(\mathrm{RR}=0.38, \mathrm{p}<.001)$ at baseline survey were more likely to belong to moderately distressed group than no symptom group. In addition, husbands whose wives felt more happiness at wave 3 were more likely to belong to fewer symptoms group $(\mathrm{RR}=1.61, \mathrm{p}=.03)$ and moderately distressed group $(\mathrm{RR}=2.06, \mathrm{p}<.01)$. Conclusion: Wife's happiness might be affecting the husband's mental health; more happiness the wives felt, more distressed their husbands were.

CORRESPONDING AUTHOR: Noboru Iwata, Hiroshima International University,n0b0ru1wata515@gmail.com 
P073

WILL IT WORK? HAS IT WORKED? A PRIORI EXPECTATIONS AND PERCEIVED A POSTERIORI EFFICACY ESTIMATES IN CHILDREN UNDERGOING PLACEBO HYPOALGESIA

Elisa Kamper ${ }^{1}$, Silke Leifheit ${ }^{1}$, Christiane Hermann ${ }^{1}$

${ }^{1}$ Justus-Liebig-Universität Gießen, Division of Clinical Psychology and Psychotherapy, Department of Psychology- Germany

Introduction Expectations play a significant role for the placebo response. Little is known about the relationship between a priori expectations and the actual placebo response, and how the latter contributes to recalled efficacy of the intervention. Moreover, the role of age and type of placebo induction in shaping a priori expectancy and recalled efficacy is unclear. Methods Participants were 172 children (52.33\% female; age: 6-17yr, $\mathrm{M}=11.62 \pm 3.21 \mathrm{yr})$ and 32 adults (50\% female; age: $19-29 \mathrm{yr} ; \mathrm{M}=21 \pm$ $2.06 \mathrm{yr}$,). Placebo hypoalgesia using heat pain was induced once by verbal information and once by classical conditioning, in counterbalanced sequence across subjects. Participants and their parents (only in children) rated the efficacy of the "analgesic" cream prior to and after (a posteriori) placebo induction. Placebo response was determined based on pain intensity ratings. Results If placebo hypoalgesia was induced by verbal information, but not by conditioning, there was a significant negative correlation between age and a priori expectations. Interestingly, the correlation between children's a priori expectations and parents a priori expectations increased with age. A posteriori effectiveness ratings were higher, when placebo hypoalgesia was induced by classical conditioning compared to verbal instructions, and decreased with age. Interestingly, a posteriori effectiveness ratings were most strongly associated with the actual placebo response in the last trial, regardless of the induction method. Age and the actual placebo hypoalgesia were the best predictors for a posteriori efficacy. Conclusions Findings suggest that age influences a priori expectations. Moreover, estimated a posteriori efficacy depends on age, induction method, and immediacy of the placebo response. Such recalled efficacy may modulate future treatment responses given recent evidence that recalled rather than actual pain strongly influences future pain in children and adolescents.

CORRESPONDING AUTHOR: Elisa Kamper, Justus-Liebig-Universität Gießen, Division of Clinical Psychology and Psychotherapy, Department of Psychology, elisa.kamper@psychol.uni-giessen.de

\section{P074}

\section{QUALITY OF LIFE IN WOMEN WITH BREAST CANCER}

Sylvia María Ramis Asenjo ${ }^{1}$, Lidia Medina ${ }^{2}$, César Sánchez ${ }^{1}$, Paula Repetto $^{1}$

${ }^{1}$ Pontificia Universidad Católica de Chile- Chile

${ }^{2}$ Centro del Cáncer Red Salud UC Christus- Chile

Background and purpose. Quality of life (QoL) has become an important concept that may evaluate the impact of diseases and their treatment on the overall well-being of patients. Aim. The purpose of the present study was to describe QoL in Chilean patients with breast cancer. Patients and Methods. Prospective, not randomized. Forty-four women in treatment for breast cancer aged $17-71$ years were included in the study, all were receiving adjuvant chemotherapy at the Cancer Center UC Christus. They completed the European Organization for Research and Treatment of Cancer (EORTC) QLQ-C30 and the breast cancer module QLQ-BR23 the first day of treatment (M0) and at the third month of adjuvant chemotherapy. Factors predicting changes in global QoL were investigated using logistic regression models. Results. Before starting treatment (M0), physical functioning and pain were predictors of Global QoL in M3. At lower disruption in physical functioning, better Global QoL in $\mathrm{M} 3$; and at a greater pain in M0, the global QoL in M3 is significantly worse. For the M3, physical functioning, role functioning, social functioning and the symptoms of breast cancer were predictors. Higher scores on these factors were associated with a worse global QoL in M3. Conclusions. The results suggest that global QoL among breast cancer patients during adjuvant treatment is compromised. Physical functioning, role functioning, social functioning and breast symptoms are the factors that most significantly impacted in global QoL.

CORRESPONDING AUTHOR: Sylvia María Ramis Asenjo, Pontificia Universidad Católica de Chile, smramis@uc.cl

\section{P075}

ALCOHOL USE BELIEFS AND BEHAVIORS OF PARENTS OF MEDICALLY VULNERABLE YOUTH

Elissa R. Weitzman ${ }^{12}$, Kara Magane ${ }^{2}$, Lauren E. Wisk ${ }^{12}$, Julie Lunstead ${ }^{2}$ ${ }^{1}$ Harvard Medical School- USA

${ }^{2}$ Boston Children's Hospital- USA

Introduction \& Purpose: Parents' alcohol use behaviors may shape their beliefs about their child's alcohol use and its riskiness. These factors may provide an important context within which youth make decisions about drinking. We sought to characterize this "web of influence" among parents of youth with a chronic illness. These youth rely heavily on their parents for health advice and are particularly vulnerable to alcohol's adverse effects. Design/Methods: Parents of youth (ages 13-18) with diabetes or rheumatic conditions were recruited from disease advocacy networks to take an online survey. Heavy alcohol use was defined as consuming $4+$ drinks on $\geq 1$ occasion in the past 30 days. Multivariate logistic regression was used to model parent beliefs in relation to their alcohol use, adjusting for child and parent gender and parent education. Results: Of 374 responses, $61.2 \% / 38.8 \%$ were from parents of youth with diabetes/rheumatic conditions. Most $(81.6 \%)$ parents thought that any level of alcohol consumption was risky/dangerous for their child. Fewer than half $(43.0 \%)$ agreed/strongly agreed that their child would abstain from alcohol because of his/her condition, more than half (54.9\%) agreed/ strongly agreed that their child would abstain for another reason (e.g., sports). Nearly one-quarter $(24.1 \%)$ of parents reported heavy alcohol use. Compared to parents who did not engage in heavy alcohol use, parents who did were: less likely (AOR $=0.39 ; 95 \% \mathrm{CI}: 0.22-0.70)$ to consider any level of alcohol consumption risky/dangerous for their child; less likely $(\mathrm{AOR}=0.31 ; 95 \% \mathrm{CI}: 0.18-0.56)$ to agree/strongly agree that their child would abstain from alcohol due to his/her condition; and less likely (AOR $=0.35 ; 95 \% \mathrm{CI}: 0.20-0.63)$ to agree/strongly agree that their child would abstain for other reasons. Conclusions: Parents' alcohol consumption was associated with their beliefs about their child's alcohol use; parents with lower consumption were more likely to view alcohol use as risky for their child and more likely to believe that their child would abstain from drinking. Parental behaviors and beliefs regarding alcohol are part of the multifactorial environment in which medically vulnerable youth develop, and should be considered in the context of tailored prevention.

CORRESPONDING AUTHOR: Elissa R. Weitzman, Harvard Medical School, Boston Children's Hospital, elissa.weitzman@childrens.harvard.edu

\section{P076}

THE ACCEPTABILITY OF NUDGING FOR HEALTH: WHAT DOES THE PUBLIC SAY?

Stephan Van den Broucke ${ }^{1}$, Laura Albert ${ }^{2}$, Valerie Broers ${ }^{1}$

${ }^{1}$ Université catholique de Louvain- Belgium

${ }^{2}$ Scientific Institute of Public Health- Belgium

Background: Promoting sustainable health behavior can be arrived at in several ways. While the most often-used strategy of health education has been criticized for being too focused on intentional behavior change, structural approaches such as legislative measures are considered as too 
controlling. To address these shortcomings, nudging has been suggested as an alternative. Nudging refers to behavior-change interventions that organize the choice architecture to alter people's behavior in a predictable way, while preserving their freedom of choice and refraining from using economic incentives. While the strategy is welcomed by health policy makers as a novel and effective way to influence healthy lifestyles, it has also met with ethical criticism. This ethical opposition mainly comes from public health professionals, who consider the technique too paternalistic and disempowering. This qualitative study aimed to investigate the acceptability of nudging from the perspective of both decision makers and the public. Methods:. Interviews were conducted among participants in a study investigating the effects of nudging on healthy vegetable choice on a university restaurant (nudges) and among decision-makers (nudgers) who were involved in the study. The interviews were held immediately after the intervention, lasted a few minutes and used open-ended questions asking the participants whether had realized they had been nudged, whether they agreed, and under what conditions they thought nudging was acceptable. Results: Content analysis of the interviews showed that both nudgees and nudgers accept the use of nudges. Common identified conditions of approval were the use of other health promotion techniques such as education and evidence-based practice. Nudgees emphasized the importance of transparency about the methods and objectives, and respect for free choice and legislation to ensure the boundaries of the technique. Nudgers emphasized the importance of a sense of responsibility of beneficiaries, proof of feasibility, and cost-effectiveness of the technique. Conclusion: The elements favoring the adoption of nudging are confirmed. It encourages the implementation of nudging to promote health. The approach must be transparent, complementary, participatory and encourage thoughtful choices.

CORRESPONDING AUTHOR: Stephan Van den Broucke, Université catholique de Louvain, stephan.vandenbroucke@uclouvain.be

\section{P077}

BEING LEFT-BEHIND, MENTAL DISORDER, AND ELDERLY SUICIDE IN RURAL CHINA: A CASE-CONTROL PSYCHOLOGICAL AUTOPSY STUDY

Jiang Xue ${ }^{1}$

${ }^{1}$ Zhejiang University- China

Background: Suicide rate among rural elderly is the highest among all age groups in China, yet little is known about the suicide risks in this rapidly growing vulnerable population. Methods: This matched case-control psychological autopsy study was conducted during June 2014 to September 2015. Consecutive samples of suicides aged 60 or above were identified in three provinces (Shandong, Hunan, and Guangxi) in China. Living comparisons were 1:1 matched with the suicides in age ( \pm 3 years old), gender, and living location. Risk factors included demographic characteristics, being left-behind, mental disorder, depressive symptoms, stressful life events, and social support. Results: A total of 242 suicides and 242 comparisons were enrolled: 135 (55.8\%) were male, mean (SD) age was 74 (8) years. The most frequently used suicide means were pesticides $(125,51.7 \%)$ and hanging $(95,39.3 \%)$. Independent risks of suicide included unstable marital status $(\mathrm{OR}=4.19,95 \% \mathrm{CI}=1.61-10.92)$, unemployed (comparing to employed, $\mathrm{OR}=4.43,95 \% \mathrm{CI}=1.09-17.95$ ), depressive symptoms $(\mathrm{OR}=1.34,95 \% \mathrm{CI}=1.21-1.48)$, and mental disorder $(\mathrm{OR}=6.28,95 \% \mathrm{CI}=1.75-22.54)$. Structural equation model indicated that the association between being left-behind and suicide was mediated by mental disorder, depressive symptoms, stressful life events, and social support. Conclusions: Unstable marital status, unemployed, depressive symptoms and mental disorder are independent risk factors for suicide in rural elderly. Being left-behind can elevate suicide risk through increasing life stresses, depressive symptoms, mental disorder and decreasing social support. Elderly suicide may be prevented by restricting pesticides, training rural physicians, treating mental disorders, mitigating life stress, and enhancing social connection.
CORRESPONDING AUTHOR: Jiang Xue, Zhejiang University, jiang_xue@urmc.rochester.edu

\section{P078}

NARRATIVE- AND IMAGERY-BASED TREATMENT FOR YOUNG PEOPLE WITH CYSTIC FIBROSIS

Jeremy Russell $^{1}$, David Kavanagh ${ }^{1}$, Esben Strodl ${ }^{1}$, Jennifer Connolly ${ }^{1}$

${ }^{1}$ Queensland University of Technology- Australia

Introduction and Purpose: Young people with cystic fibrosis (CF) are 3 times more likely to develop psychiatric disorders than healthy children. Improved medical treatments now allow people with $\mathrm{CF}$ to live much longer and healthier lives. However, psychological treatments to help young people cope with the stress of CF remain limited. An integration of narrative therapy, meta-cognitive therapy and mental imagery offer exciting new prospects. Young people often report feeling that $\mathrm{CF}$ is the predominant narrative in their lives. By placing $\mathrm{CF}$ within a larger narrative consisting of their values, goals and social supports, the power of $\mathrm{CF}$ over the individual is diminished. Negative feelings can be addressed by finding meaning in their experience, addressing negative metacognitions, regaining control, and strengthening self-esteem. Mental imagery has closer links to emotion than verbal cognition, so the new therapy used imagery in its delivery. The integrated treatment helps young people separate their identity from the illness and build upon their existing resources to limit negative cognitive and emotional effects of the illness. Methods: This paper describes an initial test of this new treatment- the Structured Narrative and Imagery-Based Intervention (SNIBI) - in young people (ages 10-17) with CF. The SNIBI intervention consisted of 3, 1hour sessions over 4 weeks. The first 2 sessions followed a structured framework designed to assist participants in building a richer narrative of their experience of CF. In session 3, participants shared their stories with their parents with the goal of enlisting their support into the future. Both participants and their parents were assessed at baseline, 4 and 8 weeks. Results: Results are presented of single-case studies testing SNIBI among 13 young people with CF. Conclusion: A further study is foreshadowed, where the feasibility of delivery of SNIBI by a social robot is tested. This mode of delivery is expected to enhance engagement, allow participants to feel free to tell their stories without perceived judgement, and allow wider dissemination of the approach.

CORRESPONDING AUTHOR: Jeremy Russell, Queensland University of Technology, jkirkrussell@gmail.com

\section{P079}

CONFIDENCE IN CHILDHOOD VACCINATION

Karena Burke1, Debra Rolfe ${ }^{1}$

${ }^{1}$ Central Queensland University- Australia

Introduction \& Purpose: In Australia, the childhood vaccination program is considered the most significant, and effective disease prevention method available. Vaccination can inhibit the spread of many infectious diseases once the majority of a population is immunised. Despite its success with disease prevention, in ever growing numbers parents are expressing hesitancy to vaccinate their children, citing vaccine safety, efficacy and ingredients as the most prominent concerns. This study explored the factors that may be influencing confidence in childhood vaccinations in an Australian context. Method: An online survey was distributed using various social media platforms, targeting a general population response via snowball sampling. The final sample comprised 211 participants aged 20 to 69 years, of whom $11 \%$ were male $(n=24)$. The majority of respondents reported having children $(n=181)$. Participants responded to a range of scales assessing Vaccine Intention, Disease and Vaccination knowledge, Preferences and attitudes towards the use of complementary and alternative medicines, and an assessment of Health Risk perception. 
Results: Participants with significantly higher confidence in vaccinations were those who had higher intention to vaccinate $\mathrm{t}(185.13)=7.981, \mathrm{p}=$ $<.001$, higher health related risk perceptions $\mathrm{t}(118.84)=8.172, \mathrm{p}=<.001$, and unexpectedly were more likely to report using complementary and alternative therapies $\mathrm{t}(87.67)=10.549, \mathrm{p}=<.001$. Objective knowledge of disease did not influence confidence in vaccination. Conclusions: Surprisingly the current study found that participants who were more frequent users of complementary and alternative therapies had greater levels of confidence in childhood vaccinations. However, the context that forms individual choice to utilise an alternative or a complementary medicine could have impacted on the results, and thus requires further study. Somewhat intuitively, the study confirmed that those with higher intention to vaccinate, were more confident in the efficacy of vaccinations. Actual objective understandings however, did not influence confidence, suggesting a much more complex cognitive process underpinning the reasons why individuals may choose, or choose not, to participate in vaccination schedules.

CORRESPONDING AUTHOR: Karena Burke, Central Queensland University, k.j.burke@cqu.edu.au

\section{P080}

INFLUENCE OF A THEORY-BASED EDUCATION INTERVENTION ON OSTEOPOROSIS TREATMENT INITIATION IN OLDER ADULTS

Katherine McLeod ${ }^{1}$, Shanthi Johnson ${ }^{2}$, Drona Rasali ${ }^{3}$, Ashok Verma ${ }^{4}$

${ }^{1}$ Department of Medical Sciences, Frank H. Netter MD School of Medicine, Quinnipiac University, Connecticut- USA

${ }^{2}$ Faculty of Kinesiology and Health Studies, University of Regina, Saskatchewan- Canada

${ }^{3}$ Provincial Health Services Authority, British Columbia- Canada

${ }^{4}$ Regina Qu'Appelle Health Region, Regina, Saskatchewan- Canada

Introduction \& Purpose: Despite practice guidelines, management of osteoporosis remains sub-optimal. Patient education is an important component in osteoporosis management, including treatment initiation and adherence. The purpose of this study was to examine the influence of theory-based education on osteoporosis pharmacotherapy initiation in older adults after undergoing initial bone mineral density (BMD) testing. Methods: Men and women $\geq 50$ years of age who had no prior diagnosis of or treatment for osteoporosis and whom their health care provider $(\mathrm{HCP})$ referred for initial BMD testing were eligible for the study. After BMD testing, patients were randomized to either an osteoporosis education intervention group $(\mathrm{n}=102)$ or usual care group (no education; $\mathrm{n}=101$ ) and completed a series of questionnaires. The intervention group received one-time, one-on-one multifaceted osteoporosis education based on constructs of the Revised Health Belief Model. Multivariable logistic regression was used to identify baseline predictors of pharmacotherapy initiation at 6-month follow-up. Results: At 6-months, 93\% completed the study. Of the 103 (54.8\%) of patients who discussed their BMD results with their HCP, 31 (30.4\%) were prescribed osteoporosis pharmacotherapy and $25(80.1 \%)$ reported treatment initiation, of whom $16(64.0 \%)$ had received the education intervention. Patients who initiated osteoporosis pharmacotherapy were more likely to have lower BMD results, be over 65 years of age, or advancing age (per year increase), and have a male HCP, and were less likely to have a post-secondary education. In adjusted models, having lower BMD results was independently associated with initiating osteoporosis treatment (OR per unit increase $=0.28, \mathrm{p}<.01,95 \%$ CI $[0.13,0.59])$. Conclusions: A theory-based education intervention was not associated with osteoporosis pharmacotherapy initiation at 6 months; however, the design and uptake of this intervention may have implications for future studies. Comprehensive interventions that include education and personalized feedback of BMD test results may have potential for increasing treatment initiation among at-risk patients.

CORRESPONDING AUTHOR: Katherine McLeod, Quinnipiac University, Katherine.McLeod@qu.edu
P081

THE HEALTHY LIFESTYLES PROGRAM: STUDY PROTOCOL OF AN ONLINE MULTIPLE HEALTH BEHAVIOUR INTERVENTION TO PREVENT COMMON AND EMERGING RISK FACTORS FOR CHRONIC DISEASE AMONG AUSTRALIAN ADOLESCENTS

Louise Thornton $^{1}$

${ }^{1}$ University of New South Wales- Australia

Introduction and Purpose: Chronic diseases are a leading cause of death and disability worldwide. Six behavioural risk factors (smoking, alcohol use, poor diet, physical inactivity, sedentary behaviour and poor sleep) have been identified as strong determinants of chronic disease risk. Alarmingly, $74 \%$ of young Australians (aged 12-17 years) have consumed alcohol, $23 \%$ have used tobacco, $85 \%$ do not meet physical activity guidelines, $80 \%$ do not meet guidelines for sedentary behaviour, $25 \%$ do not get adequate sleep and $95 \%$ do not eat enough fruit and vegetables. Not only are these behaviours risk factors for disease and mortality, in the short-term they are associated with obesity and mental health problems. These six risk factors (the "Big 6") commonly emerge early in life and cooccur as clusters. Therefore, early and effective prevention is critical. This study seeks to develop and evaluate a two-tiered eHealth multiple health behaviour intervention to concurrently target the Big 6 risk behaviours among Australian adolescents. Methods: A cluster randomised controlled trial (RCT) will be run in 80 schools (8000 13year-olds) across three Australian states in 2019. The RCT will evaluate the effectiveness of the two-part intervention: 1) A 'universal', online school-based prevention program and accompanying smartphone application ('app') targeting the Big 6 behaviours delivered to Year 8 students (Healthy Lifestyles program - primary prevention), and 2) An early intervention booster app delivered to students who remain at-risk of chronic disease as they progress through high school (Healthy Lifestyles program - secondary prevention). Results: The co-development process of the interventions and the study design will be outlined. Results from initial end-user consultation and focus testing will also be presented. Conclusions: This will be the first evaluation of a two-tiered eHealth intervention that concurrently targets six key lifestyle risk factors among adolescents, prior to the onset of chronic disease. Addressing the Big 6 during adolescence will not only improve the health of young Australians in the short term, but also carries enormous potential to enhance their capacity to lead healthy adult lives.

CORRESPONDING AUTHOR: Louise Thornton, University of New South Wales, L.Thornton@unsw.edu.au

\section{P082}

OVERVIEW OF CHILDREN'S PSYCHIATRIC AND MEDICAL ISSUES IN A TERTIARY LEVEL HOSPITAL IN MEXICO

Geraldine Leticia Zuñiga Chacón AND SECOND AUTHOR AS Ms. Cointa Arroyo Jiménez.

Geraldine Leticia Zúñiga ${ }^{1}$, Cointa Arroyo Jímenez ${ }^{2}$, Lucía Aurora Torres $^{1}$, Argelia Lara ${ }^{1}$, Karina Torres ${ }^{2}$

${ }^{1}$ Universidad Nacional Autónoma de México- Mexico

${ }^{2}$ Instituto Nacional de Pediatría- Mexico

It is kwown that psychiatric disorders are more common in children with chronic and acute pediatric disorders (Jesmin A., Mullick MSI., Rahman KM. \& Muntasir MM., 2016). There is evidence that shows that in the last 10 years there has been a rise in pediatric hospitalizations for children with, versus without a psychatric diagnosis, and the most common psychiatric disorders are developmental disorders, attention-deficit / hyperactivity disorder, and anxiety disorders (Zima et. al., 2016). OBJECTIVE: The purpose of this study was to describe the most common psychiatric disorders among children and adolescents attending at the National Institute of Pediatrics in Mexico City, and determine the main subspecialties that serve pediatric patients with psychiatric comorbility. 
METHOD: We conducted a retrospective cohort study, analyzing the National Institute of Pediatrics files from January 2017 to December 2017. We identified all children who had at least one consultation in the Mental Health Department in 2017. For each patient who had assisted to a Mental Health appointment, we searched the psychiatric diagnoses and the other subspecialties that attended this child or adolescent. When the database was complete, we grouped similar disorders in order to gain a better grasp of the diagnoses' frequencies. RESULTS: Last year the mental health department attended 2345 patients, of whom 1026 were girls, 1306 were boys, and 13 were intersex children. Nearly twenty percent of the patients were in the age range of 0 to 6 years; from 6 to 18 years the frequency of patients is distributed in a similar way (20\% aprox per group). Patients over 18 years old are non frequent (3.58\%). The main subspecialties that served pediatric patients with psychiatric comorbility in 2017 at the National Institute of Pediatrics were the emergency department (808), human communication and audiology department (451), endocrinology department (435), neurology department (396), and outpatient pediatric consultation (318). The most common psychiatric disorder was mental disability with a frequency of $14.79 \%$ of all diagnoses made in the year, followed by developmental disorders at $8.01 \%$. These, along with problems in cognitive function and academic abilities (3.27\%) and the disruption of activity and attention group (7.14\%), indicate that most patients have a condition that interferes with academic achievement. Adaptation disorders and depressive disorders and episodes were also high, with $7.93 \%$ and $7.81 \%$ respectively. Anxiety disorders were $3.98 \%$ of diagnoses made, although with a lower percentage, these three groups could point to the experience of hospitalization and coping with one's condition. Furthermore, emotional difficulty is reflected in the emotional regulation group $(3.11 \%)$, which can lead to self-inflicted injuries $(0.63 \%)$. CONCLUSIONS: The findings of this study go in line with studies that state developmental disorders as the one of the most common diagnoses in pediatric hospitalizations, as are the perturbation of activity and attention and emotional disorders. It is noteworthy that adaptation, depression and anxiety disorders held high percentages, which could be indeed a result of the hospitalization experience and coping with illness or diverse conditions, however, chronic degenerative illnesses could compromise their quality of life. In addition, social and economic status as well as adverse family situations could be at play, as seen in the problems related to the child's upbringing and problems related to inadequate family support groups.

CORRESPONDING AUTHOR: Cointa Arroyo Jímenez, Instituto Nacional de Pediatría, cointarroyo@gmail.com

\section{P083}

FOOTBALL TO RUGBY: TRANSFERABILITY OF A SUCCESSFUL MEN'S WEIGHT MANAGEMENT PROGRAMME BETWEEN DIFFERENT PROFESSIONAL SPORTS CLUB SETTINGS

Cindy Gray ${ }^{1}$, Greig Logan ${ }^{2}$, Craig Donnachie ${ }^{1}$, Sally Wyke ${ }^{1}$, Kate Hunt ${ }^{1}$ ${ }^{1}$ University of Glasgow- United Kingdom

${ }^{2}$ University of Stirling- United Kingdom

Introduction and Purpose: Despite rising levels of male obesity, men are underrepresented in commercial and NHS weight management programmes. Professional sports clubs can overcome men's disengagement with weight management; in Scotland, Football Fans in Training (FFIT) was successful in attracting men to attend a weight management programme at their local professional football club. FFIT showed significant weight loss (and physical activity and dietary improvements) at 12 months and 3.5 years, and the programme has now been scaled-up to 33 clubs in Scotland, and eight in both England and Germany. However, football does not have universal appeal. To extend the impact of FFIT beyond football, we aimed to explore the programme's transferability to another sports club setting, professional rugby union in the UK. Methods: A pilot study of FFIT (adapted to the rugby setting) at five English
Premiership Rugby clubs, including qualitative telephone interviews $(n=24)$ with club community managers and coaches at three timepoints (start; end; and three months after the 12-week programme). Interviews were analysed thematically. Results: There was variability in the extent to which the active ingredients of FFIT were delivered. Coaches at one club provided more (rugby-based) physical activity than education, and admitted the classroom activities were 'a bit daunting'. Other clubs welcomed the challenge of dealing with a new target group (adult men) and 'wanted to follow the [FFIT] model as closely as we could'. Most interviewees felt the programme was a good fit with their personal aspirations and the community department's core activities of 'trying to promote grassroots sport, health and wellbeing'. However, some felt under-supported by the wider club, and under some pressure because of a mismatch between what they had been asked to deliver in terms of recruitment, and what was achievable 'the pressure on us getting the numbers has been quite intense... it should be more about the result [weight loss]'. Conclusions: An adapted version of FFIT can work well in a professional rugby club setting. However, closer partnership working and more training could help coaches feel more supported and confident about recruitment and delivering the programme's active ingredients.

CORRESPONDING AUTHOR: Cindy Gray, University of Glasgow, cindy.gray@glasgow.ac.uk

\section{P084}

FEASIBILITY RCT OF A WORKBOOK INTERVENTION TO SUPPORT CANCER SURVIVORS BACK TO WORK

Elizabeth Grunfeld ${ }^{1}$, Lauren Schumacher ${ }^{2}$, Steven Sadhra ${ }^{3}$, Anjali Zarkar $^{4}$, Andrew Sutton ${ }^{5}$

${ }^{1}$ Birkbeck College, London- United Kingdom

${ }^{2}$ Coventry University- United Kingdom

${ }^{3}$ University of Birmingham- United Kingdom

${ }^{4}$ University Hospital Birmingham- United Kingdom

${ }^{5}$ University of Leeds- United Kingdom

Introduction and purpose: Returning to and staying at work following illness is associated with better physical and psychological functioning whereas not working has been shown to be associated with reduced selfesteem, lowered self-efficacy, and decreased belief in one's ability to return to the workplace. This study aimed to assess the feasibility and acceptability of a theoretically led workbook intervention designed to support cancer patients in returning to work. Methods: A parallel-group randomised controlled trial with embedded qualitative interviews was undertaken. Patients who had received a diagnosis of breast, gynecological, prostate or colorectal cancer and who were at least 2 weeks post treatment initiation were recruited from oncology clinics within four English National Health Service Trusts. The intervention comprised a self-guided "WorkPlan" workbook, which was used during the introductory session, at home during the 4 weeks of the intervention period and as a reminder during the return to work process. Telephone support calls will be made by the researchers at 2 and 4 weeks during the intervention period to discuss progress. The control group received treatment as usual, and were offered the workbook at the end of their 12-month follow-up. As part of the feasibility trial we assessed aspects of feasibility including eligibility, recruitment, data collection, attrition, feasibility of the methodology, acceptability of the intervention and potential to calculate costeffectiveness. Results: The recruitment rate of eligible patients was $44 \%$ and the target of 60 participants was met. 68 participants consented and $58(85 \%)$ completed baseline measures. Randomization procedures were acceptable, however, there was an over recruitment to the intervention arm due to researcher error using the online randomization programme in the early stages of the study. Data collection materials (including those for cost-effectiveness data) were feasible and the intervention was acceptable to participants. Retention rates at six and 12 months follow-up were $75 \%$ and $69 \%$ respectively. At 6-month follow-up 17\% of participants in the 
usual care group had returned to full or part-time work compared to $31 \%$ of the intervention group. At 12 months the percentages were $47 \%$ (usual care) and 53\% (intervention). Conclusions: The intervention was acceptable and the study design and materials were judged to be feasible and acceptable. Given the higher attrition in the control arm there is a need to consider engagement during the trial of control participants. The findings confirm the feasibility of a definitive trial, although further consideration needs to be given to increasing the participation rates among men diagnosed with cancer.

CORRESPONDING AUTHOR: Elizabeth Grunfeld, Birkbeck College,e.grunfeld@bbk.ac.uk

\section{P085}

A STUDY ON THE RELATIONSHIP BETWEEN QUALITY OF LIFE AND SELF- MANAGEMENT BEHAVIOR IN DIABETIC PATIENTS Chun $\mathrm{CHANG}^{1}$, Shenglan LIU ${ }^{1}$, Heya NA ${ }^{1}$, Xin ZHANG ${ }^{1}$, Weihao LI' ${ }^{1}$, Mo YE ${ }^{1}$

${ }^{1}$ Peking University Health Science Center- China

Introduction \& Purpose The prevalence of diabetes mellitus was $11.6 \%$ in 2010 among Chinese adults. The quickly increasing of diabetes prevalence brings a big burden to the people living with diabetes, their family and the country both in health and economics. The evidence all over the world show that the adoption of self-management behavior can improve blood sugar control, delay or reduce the occurrence of complications. But there are also some studies indicate diabetes complicated selfmanagement behavior may produce negative emotions and boredom. This study aims at exploring the quality-of-life in patients with diabetes mellitus and its relationship with self-management behavior, and to provide scientific basis to improve their quality of life by promoting selfmanagement behavior. Methods: A cross-sectional survey was conducted with a face-to-face questionnaire interview among a convenient sample of diabetic patients managed by community health service centers or stations in the urban area in Beijing. The data of demographic characteristics, the brief version of the diabetes self-management behavior assessment scale, diabetes specific quality-of-life scale (DSQL) were collected. Selfmanagement behavior and quality-of-life were analyzed as continuous variables, multiple linear regression analysis was applied to find out the relationship between quality-of-life and self-management behavior among the respondents. Results: The average score of quality-of-life was $60.44 \pm 16.81$, and the physical dimension score was $26.14 \pm 9.34$, the psychological dimension score was $21.32 \pm 5.74$, the interpersonal relationship dimension score was $7.72 \pm 2.65$, the treatment dimension score was $5.65 \pm 2.58$, the self-management behavior score was 10.46 \pm 2.10 . The quality-of-life was influenced by the length of illness, number of complications and self-management behavior scores, the longer the illness years, the more numbers of complications, the worse quality-oflife. The quality-of-life increased with the increase of self-management behavior score. Conclusions: The quality-of-life was affected by selfmanagement behavior, efforts should be made to promote and maintain the self-management behavior, so as to improve the diabetes mellitus patients' quality-of-life.

CORRESPONDING AUTHOR: Chun CHANG, Peking University Health Science Center, changchun@bjmu.edu.cn

\section{P086}

IMPACT OF COGNITIVE BEHAVIORAL THERAPY (CBT) ON INTERVENTION OF BREAST CANCER PATIENTS' RELATED PSYCHOLOGICAL BEHAVIOUR

Tianyi $\mathrm{Bu}^{1}$, Yanjie Yang ${ }^{1}$, Xiuxian Yang ${ }^{1}$, Xiaohui Qiu ${ }^{1}$, Zhengxue Qiao $^{1}$, Xuejia Song ${ }^{1}$, Lin Wang ${ }^{1}$, Erying Zhao ${ }^{1}$, Jiawei Zhou ${ }^{1}$

${ }^{1}$ Harbin Medical University- China
Introduction \& Purpose: The purpose of the study is to explore the impact of CBT on emotional status, quality of life and quality of sleep of breast cancer patients. Method: There were 93 breast cancer patients meeting the study criteria have been sampled by convenience in the survey. Moreover, all the participants have been divided into three groups randomly. Participants who underwent 3 months CBT were organized into a group called psychological intervention group $(n=23)$, participants only accepted postoperative health care education were organized into a group called clinical management group $(n=24)$ and participants carried out any intervention activities were organized into a group called the comparison group $(n=46)$. In this study, Hamilton Depression Rating Scale, Hamilton Anxiety Rating Scale, Athens Insomnia Scale and Functional Assessment of Cancer Therapy-Breast were utilized during each intervention. All the data have been assessed by repeated measures analysis of variance. Result: After psychological intervention, the level of depression and anxiety in psychological intervention group is lower than clinical management group and comparison group. The QoL in psychological intervention group is higher than clinical management group and comparison group. Insomnia symptoms in psychological intervention group relieve better than clinical management group and comparison group. All the results have statistics significance $(\mathrm{P}<0.05)$. Conclusion: $\mathrm{CBT}$ can efficiently improve depressive and anxious status emotion, insomnia issue and QoL of breast cancer patients.

CORRESPONDING AUTHOR: Tianyi Bu, Harbin Medical University, 994005158@qq.com

\section{P087}

ASSESSING PHYSICAL AND BEHAVIORAL HEALTH OUTCOMES FROM A PEDIATRIC WEIGHT MANAGMENT GROUP: A PILOT STUDY

Timothy Zeiger ${ }^{1}$

${ }^{1}$ Penn State Health- USA

Objective: Pediatric healthcare providers (e.g.- pediatricians and pediatric subspecialties) seeing children and adolescents who are overweight often face many challenges in the treatment of these youth which in turn requires collaboration between many systems (e.g.-healthcare, family, school and community) for successful outcomes and treatment. As a result, our institution like many across the country has developed a collaborative care model to include psychologists to treat the behavioral health needs of these children and adolescents with weight management issues.

Methods: The Division of Child and Adolescent Psychiatry in collaboration with the Department of Pediatrics at Penn State Hershey Medical Center developed an 8 week weight management program for overweight or obese children and adolescents (ages 6-18) as well as their families. Pediatric patients were initially screened for anxiety disorder (SCARED Child version) and depression (CES-DC) during their clinic visit with the weight management physician. Individuals scoring in a clinically significant range were referred for psychological consultation to determine whether individual versus group treatment would be most appropriate to address the clinical concerns. Six females between the ages of 13-16 were referred by their weight management physician to participate in the piloted weight management group. BMI's (Body Mass Index's) were calculated at each visit.

Results: Average attendance to each group was 4 adolescents. Results yielded positive changes in BMI pre and post group.

Conclusions: Given the staggering rates of youth who are overweight or obese there is increased need for collaborative care in the treatment of these individuals. Psychology can play an integral role in the multidisciplinary care of these youth. Our 8 week weight management group was led by a psychologist and was able to yield a positive changes in the youths' BMI.

CORRESPONDING AUTHOR: Timothy Zeiger, Penn State Health, tzeiger@pennstatehealth.psu.edu 
P088

EXPLAINING VARIABILITY IN CANCER HEALTH LITERACYAS A FUNCTION OF PATIENT CHARACTERISTICS

Levent Dumenci ${ }^{1}$

${ }^{1}$ Temple University- USA

Introduction \& Purpose: Research on cancer health literacy (CHL) has gained momentum in recent years to better understand mechanisms thru which CHL exerts influence on health. The purpose of this study is to identify variables that explain differences in cancer health literacy as a function of individual characteristics.

Methods: The participants consisted of 2,318 individuals recruited from the Mid-Atlantic region. A one-way ANOVA was used to estimate differences in cancer health literacy scores measured by the CHLT-30 between diagnostic status, race/ethnicity (African American versus others), gender, income, and educational attainment groups. Multiple imputation with 50 replications was used to handle missing data. The p-values associated with $\mathrm{F}$ tests was supplemented with partial eta-squared as an effect size estimate.

Result: All five main effects and the race $\mathrm{x}$ income interaction were significant in all 50 multiply imputed datasets $(p<.001)$. Following groups had low cancer health literacy scores than their counterparts: African Americans, education level less than high school, below median income, no cancer diagnosis, and males. Partial eta-squared estimates were $17.85 \%, 14.98 \%$, and $11.72 \%$ for race/ethnicity, education, and income variables, respectively, whereas effect size estimates were $1 \%$ or lower for cancer diagnostic status, gender, and race $\mathrm{x}$ income interaction. Conclusion: Race/ethnicity, income, and education explain about $50 \%$ of the variations in cancer health literacy. Interventions designed to improve health outcomes of individuals need to target individuals who are under educated, low income, and African American.

CORRESPONDING AUTHOR: Levent Dumenci, Temple University, ldumenci@temple.edu

\section{P089}

SYSTEMATIC INTEGRATION OF EMPIRICAL FINDINGS ON COOKING AT HOME USING TRIANGULATION PROTOCOL Susanna Mills ${ }^{1}$, Heather Brown ${ }^{1}$, Wendy Wrieden ${ }^{1}$, Martin White ${ }^{2}$, Jean Adams $^{2}$

${ }^{1}$ Newcastle University- United Kingdom

${ }^{2}$ University of Cambridge- United Kingdom

Introduction and purpose: Diet-related diseases are the greatest cause of morbidity and mortality worldwide. Food preparation methods are linked to diet and health. We aimed to use Triangulation Protocol to integrate findings from a mixed methods programme of study on the determinants and outcomes of home food preparation.

Methods: Firstly, we conducted a systematic review of studies on determinants and outcomes of home cooking ( $\mathrm{n}=38$ studies included). Secondly, we undertook qualitative interviews exploring home food preparation amongst United Kingdom (UK) adults $(\mathrm{n}=18)$, and a comparison with focus group participants from Baltimore, United States $(n=53)$. Thirdly, we analysed data from a large population-based UK cohort study. Analyses investigated relationships between meal consumption patterns and a) diet and cardio-metabolic health $(n=11,326)$ and $b)$ sociodemographic characteristics $(n=11,396)$. We used Triangulation Protocol to integrate multiple perspectives within our programme of work, by identifying key emergent meta-themes and coding themes as agreement, silence and dissonance for each of the three research phases. Results: We identified meta-themes as behaviour, determinants, outcomes and research approach. Overall, we noted several areas of agreement between the research phases and the themes, for example potential dietary benefits from home food preparation and lack of consistency in terminology. The relationship between higher socioeconomic status and greater home cooking was a specific area of disagreement. Several potentially contradictory themes were also highlighted, concerning the importance of cooking skills; cultural influences; change in gender-related roles; and relative emphasis on different putative benefits of cooking and eating meals cooked at home. Comparisons were limited by the nature of the included study designs.

Conclusions: Preparation and consumption of meals cooked at home offers potential diet, health and social benefits, and should be supported through public health policy. However, cooking perceptions and practices are diverse, and relationships with socioeconomic status vary according to the research methods used, requiring further exploration.

CORRESPONDING AUTHOR: Susanna Mills, Newcastle University, susanna.mills@newcastle.ac.uk

\section{P090}

SMOKING PREVALENCE OF MEDICAL STUDENTS AND THE ROLE OF DOCTORS IN PREVENTING AND QUITTING SMOKING

Zoltan Abram ${ }^{1}$

${ }^{1}$ University of Medicine and Pharmacy from Tirgu Mures- Romania

Introduction. Quitting smoking could reduce patients's duration of hospitalization, as such, medical professionals should support this and should show positive example. In spite of this fact, smoking prevalence is higher among Romanian physicians than in the general population. Methods. The "Smoke Free Medical University" project was launched at the University of Medicine and Pharmacy of Tirgu Mures, Romania. The smoking habits and attitudes of students were evaluated using an anonymous questionnaire. An antismoking strategy was implemented, followed by reevaluations after one and two years. Results. Data obtained from over 3000 students every year showed a slight decrease in smoking prevalence from $34.1 \%$ in 2014 to $33.5 \%$ in 2015 , and to $33.2 \%$ in 2016 . A significant decrease in second hand smoking due the smoke-free legislation and strategy was determined. The main majority of the students (over $92 \%$ ) agree that health professional students should get specific training on cessation techniques and consider that doctors should routinely advise their patients to quit smoking. Due this necessity, an elective tabacology course was introduced in the medical teaching curricula with a growing number of participants. Conclusions. Primary smoking prevention and cessation programs should be priority for public health in Romania. A rigorous strategy and long term efforts are needed to achieve a significant reduction in smoking prevalence. The expansion of our project to other medical universities can initiate a smoke-free culture among future doctors who can serve as models for their patients and the general population.

CORRESPONDING AUTHOR: Zoltan Abram, University of Medicine and Pharmacy from Tirgu Mures, abramzoltan@yahoo.com

P091

OVEREATING BEHAVIOR ENHANCED BY STRESS AND ITS UNDERLYING NEUROBEHAVIORAL MECHANISMS

Xiu Liu ${ }^{1}$

${ }^{1}$ University of Mississippi Medical Center- USA

INTRODUCTION: Overeating beyond individuals' energy homeostatic need leads to obesity. Stress is proposed to be a risk factor promoting overeating behavior. This animal study examined effect of stress on the motivation for obtaining calorie-condensed food reward and its underlying neurobehavioral mechanisms. METHODS: Male SD rats with ad libitum _ access to rodent chow in their home cages were trained to press a lever for deliveries of high caloric food pellets containing $35 \%$ fat contents. Rats were challenged with a pharmacological stressor yohimbine following an administration of a corticotropin-releasing factor (CRF) 
receptor antagonist NBI or a glucocorticoid receptor antagonist mifepristone or a glucocorticoid synthasis inhibitor metyrapone. RESULTS: Rats pressed the lever for obtaining the calorie-condensed food pellets. Stress challenge increased lever responses, indicating the enhanced motivation. Pretreatment with NBI prior to stress challenge effectively blocked the behavior-motivating effect of stress. However, neither mifepristone nor metyrapone altered stress effects. CONCLUSIONS: These data demonstrated that stress challenge enhanced the motivation for procuring calorie-condensed food reward beyond rat energy homeostatic need. The enhancing effect of stress required activation of the CRF receptors but not activity of HPA axis, suggesting a role of the extrahypothalamic CRF neurtotransmission in overeating behavior under stressful situations. These findings would shed a light on our understanding of the neurobehavioral mechanisms of overeating/obesity.

CORRESPONDING AUTHOR: Xiu Liu, University of Mississippi Medical Center, xliu@umc.edu

\section{P092}

THE EFFECT OF RECIPROCITY PRIMING ON ORGAN DONOR REGISTRATION INTENTIONS AND BEHAVIOR Christopher Miller ${ }^{1}$, Ronan O'Carroll ${ }^{1}$, Jody Quigley ${ }^{1}$

${ }^{1}$ University of Stirling- United Kingdom

Introduction \& Purpose: Internationally, the demand for organ transplants far exceeds the available supply of donated organs. Development of strategies to increase organ donor registration is therefore vital. We examine if a digital reciprocity prime based on reciprocal altruism can be used to increase organ donor registration intentions and behavior. METHODS: In a randomized controlled trial, 420 participants (223 females) from England and Scotland aged 18+ who were not currently registered organ donors were randomized by block allocation using a 1:1 ratio to receive either a reciprocity prime or control message. After manipulation, they were asked to indicate their organ donation intentions and whether or not they would like to be taken to an organ donation registration and information page. RESULTS: Participants primed with a reciprocity statement reported greater intent to register as an organ donor than controls (using a 7-point Likert scale where higher scores = greater intention; prime mean = 4.3 (1.6) vs. control mean $=3.7(1.4), \mathrm{P}=<.001$, d $\mathrm{d}_{-}=0.4[95 \% \mathrm{CI}=$ $0.21-0.59])$. There was however, no effect on behavior as rates of participants agreeing to receive the donation register web-link were comparable between those primed at $11 \%\left(n \_=23 / 210\right)[95 \% \mathrm{CI}=7.4-16.0]$ and controls at $12 \%(\mathrm{n}=25 / 210)[95 \% \mathrm{CI}=8.1-17.1], \quad \mathrm{X}_{-}^{2}(1)=0.09$, $\mathrm{p}_{-}=.759$. CONCLUSIONS: Reciprocal altruism appears useful for increasing intention towards joining the organ donation register. It does not, however, appear to increase organ donor behavior.

CORRESPONDING AUTHOR: Christopher Miller, University of Stirling, christopher.miller@stir.ac.uk

\section{P093}

DIRÉ CLINICAL PROGRAM FOR THE COMMUNICATION OF THE DIAGNOSIS OF HIV / AIDS TO CHILDREN AND ADOLESCENTS IN BARRANQUILLA-COLOMBIA

Ana María Trejos Herrera ${ }^{1}$

${ }^{1}$ Universidad del Norte- Colombia

Introduction: The objective of this work is to improve psychological adjustment and therapeutic adherence through 16 clinical-educational sessions, involving both the participation of the child and their primary caregiver through playful processes, which seek to prepare them to familiarize themselves with the disease. and facilitate understanding of the diagnosis. Method: By means of quasi-experimental temporal series design, we evaluated whether the "DIRÉ" clinical model had effects on the normalization of psychological adjustment and adherence to treatment in children and young people. 41 professionals, 30 caregivers and 33 children / adolescents participated. Nonparametric analysis was performed for the variable adherence to treatment and parametric analysis for the psychological adjustment variable with the IBM SPSS Software ${ }^{\circledR}$ package. Results: No significant differences were found in the adherence to the Friedman treatment $p=.392$ suggesting that the "DIRÉ" clinical program had no negative effects on the therapeutic compliance of children and adolescents in the evaluations carried out at 2 weeks, 3 and 6 months after the implementation; in the psychological adjustment, significant positive differences were found in the follow-up compared with the baseline: 2 weeks $(\mathrm{p}=0.001) ; 3$ months $(\mathrm{p}=0.000)$ and 6 months $(\mathrm{p}=$ 0.000 ) suggesting normalization of internalized and externalized behavior in affected children after their participation in the "DIRÉ" clinical model. Conclusion: The "DIRÉ" program demonstrated efficacy in the normalization of psychological adjustment and maintenance of therapeutic compliance, favoring the trust of professionals and caregivers in this difficult process.

CORRESPONDING AUTHOR: Ana María Trejos Herrera, Universidad del Norte, atrejos@uninorte.edu.co

\section{P094}

SECONDARY HEALTH CONDITIONS FOLLOWING SPINAL CORD INJURY

Amy Richardson ${ }^{1}$, Jo Nunnerley ${ }^{2}$, Johnny Bourke ${ }^{2}$, Sue McAllister ${ }^{1}$, Ari Samaranayaka $^{1}$, Martin Sullivan ${ }^{3}$, Sarah Derrett ${ }^{1}$

${ }^{1}$ University of Otago- New Zealand

${ }^{2}$ Burwood Academy of Independent Living- New Zealand

${ }^{3}$ Massey University- New Zealand

Introduction \& Purpose: Secondary health conditions are physical or psychological health problems resulting from an underlying impairment. These conditions are frequently experienced by people with spinal cord injury (SCI). However, limited research has investigated secondary health conditions in people with SCI over time. The purpose of this longitudinal study was to describe the secondary health conditions experienced by people with SCI, and to examine relationships with self-rated health, depression, and life satisfaction. Methods: Between 2007 and 2009, 118 people with SCI were recruited during their first admission to one of New Zealand's two spinal units. Participants provided comprehensive health and wellbeing information during interviews conducted 6,18 , and 30 months post-SCI. Secondary health conditions were assessed using 14 items derived from the Secondary Complications Survey. The relative risk of experiencing poor outcomes among those with secondary health conditions was calculated. Results: The most commonly reported secondary health condition was leg spasm, with $64 \%, 66 \%$, and $59 \%$ of participants reporting bother from this condition at 6 , 18 , and 30 months, respectively. Back pain, pain below the level of SCI, constipation, and shoulder pain were also frequently reported. The proportion of participants bothered by secondary health conditions declined only minimally over the 30 months of follow-up. Participants reporting pain at 6 months had a greater likelihood of reporting moderate or extreme depressive symptoms at 18 months ( $R R=6.31,95 \% \mathrm{CI}=1.01-39.40, \mathrm{p}=.05)$ after adjusting for age, gender, impairment, and wheelchair use. Furthermore, participants reporting pain at 18 months were more likely to rate their overall health as poor at 30 months $(\mathrm{RR}=2.09,95 \% \mathrm{CI}=1.11-3.94, \mathrm{p}=.02)$. Conclusions: Secondary health conditions are prevalent among people with SCI. Pain is particularly prevalent and is associated with an increased risk of experiencing poor well-being over time. Further longitudinal research is needed to investigate a broader range of secondary health conditions and outcomes in people with SCI. This would inform the development of interventions designed to prevent secondary health conditions and improve quality of life.

CORRESPONDING AUTHOR: Amy Richardson, University of Otago, amy.richardson110@gmail.com 
P095

COPING ACADEMIC STRESS STRATEGIES AND POSITIVE AFFECT INCREASING STRATEGIES RELATED TO HEALTH LIFE QUALITY IN UNIVERSITY STUDENTS

Susan Galdames ${ }^{1}$, Mauricio Gonzalez ${ }^{1}$

${ }^{1}$ Universidad de La Serena- Chile

Introduction: During university education students face academic and social challenges that bring several changes to their life styles, resulting in an increase of health problems and the beginning of health risk habits. From a positive psychology perspective we studied the strategies that students use to cope with academic stress and the strategies to increase positive affect, and evaluated them as possible precursors of health quality of life.

Methods: Using a random sample stratified by Faculty (5), we evaluated 271 students from a public university located in the north of Chile. Participants, $54 \%$ female and $46 \%$ male, had a mean age of 21.6( \pm 2.5$)$, ranging from 18 to 33 years old. They were from 13 different university courses and had 1 to 5 years from entry. With a cross-sectional design we applied the following measures: Spanish version of EuroQol-5D to evaluate Health Quality of life; Coping Academic Stress Scale (Escala de Afrontamiento del Estres Academico, A-CEA de Cabanach, et al., 2010) and the Chilean version of the Happiness Increasing Strategies (HIS) (Moyano, Páez, \& Torres, 2016).

Results show 25 Euroqol health profiles, from which $35 \%$ students had no health problems profile (11111) versus $75 \%$ students showed problems in 1 to 5 health domains, mostly psychological problems (49.9\%) and pain $(34.7 \%)$. VAS scale showed a mean health quality of life of $76.3 \%$ $( \pm 17)$. Man had higher VAS means than women $(\mathrm{t}=2.28, \mathrm{gl}=265$, $\mathrm{P}=0.023$ ). Logistic regression analysis using the outcome health quality of life as a dummy variable: "complete health" versus "health problems" showed a significant regression model of three precursor strategies: Cognitive Reappraisal, Recreational Sociability (or Partying) and Passive Leisure. This results show that students with better health quality of life use more Cognitive Reappraisal stress coping strategies, and more Recreational Sociability as positive affect increasing strategies than students with lesser health quality of life.

Conclusions: Identified strategies related to better health quality of life give orientation to the promotion of healthy coping styles to university stresses.

CORRESPONDING AUTHOR: Susan Galdames, Universidad de La Serena, susan.galdames@yahoo.com

\section{P096}

MOVEMENT CENTERED PSYCHOTHERAPY FOR THE TREATMENT OF FIBROMYALGIA AND SOMATIC SYMPTOMS DISORDERS

Maria Eugenia Moneta ${ }^{1}$

${ }^{1}$ International Psychoanalytic University- Germany

Aim: This paper presents a novel approach of how the boy and the mind can be reunited in the concept of Ebodiment, wich could be useful as a new approach in Psycho-somatic medicine and the study of somatoform disorders. Embodied cognition refers to the fact that somatosensory, visceral, and conceptual or cognitive elaboration overlap or merge together in the individual process of being ill.

Applying this concept to the treatment of somatoform and associated disorders, we suggest some evidences that the use of movement in psychotherapy can be more effective than verbal psychotherapy and other treatments for symptom reduction and adherence to treatment in fibromyalgia and chronic pain.

Methods: A group of patients diagnosed with chronic pain and some with fibromyalgia syndrome were selected for this study. Patients completed the Symptom Check list Spanish version at the beginning of a treatment consisting of 8-10 group- therapy sessions. When the therapy was finished, patients completed a form about their improvements, difficulties and failures during the group therapy.

Results: Results demosntrated that movement- embodied psychotherapy can be in a short term, more effective than other treatments for symptoms reduction and post treatment results, measured in terms of consultations. Discussion: Biological findings of symtoms in fibrmyalgia appear to be multicausal and do not present a coherent picture (Theadom, Cropley Hankins et al. 2015). Nevertheless, a circuit of cognitive, physiological and behavioral- emotional components seem to be involved in maintaining a maladaptive reponse towards environmental stimuli. Somatoform, as well as fibromyalgia patients, commonly present high proportions of drop-out from the treatments offered, including psychotherapy. We found that patients responded well, in a short term, to body-movement group psychotherapy, confirming the hypothesis that an embodiment frame is needed.

CORRESPONDING AUTHOR: Maria Eugenia Moneta, International Psychoanalytic University, mmoneta1@ gmail.com

\section{P097}

EFFECTS OF ACUTE AEROBIC EXERCISE ON INHIBITORY CONTROL AMONG OBESE AFRICAN AMERICAN ADOLESCENTS

Neha Gothe ${ }^{1}$, Mark Greenwald ${ }^{2}$, Sylvie Naar ${ }^{3}$

${ }^{1}$ Kinesiology and Community Health, College of Applied Health Sciences, University of Illinois at Urbana Champaign- USA

${ }^{2}$ Psychiatry and Behavioral Neurosciences, School of Medicine, Wayne State University- USA

${ }^{3}$ Behavioral Sciences and Social Medicine, School of Medicine, Florida State University- USA

Introduction: Obesity has been increasingly associated with lack of inhibitory control, which leads to a failure in deactivating food reward circuits, uncontrolled and impulsive eating, overeating and unsuccessful dieting. Acute aerobic exercise has been demonstrated to improve inhibitory control among college-aged and healthy populations but its' potential to moderate food choices and weight loss remained to be determined. The purpose of this study was to examine the effect of low and high intensity aerobic exercise among obese minority adolescents. METHODS: Twenty-six (mean age $=14.56 \pm 1.3$, BMI $=34.46 \pm 3.2, \mathrm{n}=17$ female) African American adolescents completed a baseline sedentary measurement and two counterbalanced exercise sessions: low intensity aerobic exercise (50$60 \%$ heart rate reserve) and high intensity aerobic exercise (75-85\% heart rate reserve). The 20 -min exercise involved brisk walking on a treadmill at a self-selected pace and incline that enabled participants to reach their target heart rate zones. Immediately following exercise, participants completed the flanker task to assess attention and inhibitory control. RESULTS: A repeated measures ANOVA showed performance following each exercise session was better than baseline performance. Reaction time for both the congruent (attention; $\mathrm{F}=7.38, \ldots \mathrm{p}_{-}<.003$, partial eta2 $=.38$ ) and incongruent (inhibitory control; $\mathrm{F}=7.02, \mathrm{p}_{-}<.004$, partial eta $2=.37$ ) conditions were significantly shorter than the baseline condition. Pairwise comparisons revealed no significant differences between the two exercise conditions. No speed-accuracy trade-off was observed. Conclusions: These promising study results suggest both low and high intensity aerobic walking can significantly improve attention and inhibitory control among obese African American adolescents. Given the association between inhibitory control and obesity, and the robust evidence for physical activity interventions that improve cognition, designing exercise-based weight loss interventions that specifically target inhibitory control maybe more effective in moderating eating behaviors among obese adolescents.

CORRESPONDING AUTHOR: Neha Gothe, University of Illinois at Urbana Champaign, npg@illinois.edu 
P098

DEVELOPMENT OF PERCEIVED BARRIERS RELATED TO PKU TREATMENT INVENTORY

Katia Irie Teruya ${ }^{1}$, Eduardo Remor ${ }^{1}$

${ }^{1}$ Universidade Federal do Rio Grande do Sul- Brazil

Introduction \& Purpose: Phenylketonuria (PKU) is an inborn error of metabolism characterized by deficiency in phenylalanine (Phe) hydroxylase activity. The treatment consists of a Phe-restrict diet and the ingestion of a specific metabolic formula containing amino acids and micronutrients, but free of Phe. The adherence rate of patients attended at specialized centers around the world has been shown to be below 50\%, demonstrating that there are difficulties adapting to the treatment guidelines. In order to identify the difficulties perceived by patients and caregivers in relation to treatment, an inventory of perceived barriers was developed. Method: Literature review (eg. Scielo, PsycInfo, Medline, IndexPsi) to collect studies on adherence behavior and PKU was performed. The topics found that were considered as potential barriers to adherence were classified according to the five dimensions of adherence behavior defined by WHO. In addition, a social support domain, which was also found in the literature as relevant to adherence behavior, was included. Each dimension was composed of difficulties experienced in different contexts, which required different skills. To ensure uniformity and clarity throughout the inventory, all potential barriers listed were transformed into an affirmative sentence, generating 27 items. Three experts from the field were invited to make suggestions on the relevance and clarity of each item. All evaluations were computed and only those items whose agreement was above $80 \%$ in relevance and clarity were kept unmodified. Changes related to the vocabulary used in the inventory, in order to present a language closer to the literacy levels of the patients and their families, were the main contributions of the specialists consulted. Results: A proxy and self-report inventory of adherence barriers to PKU treatment containing 27 items was developed and ready to use in research. Conclusion: The research allowed the development of an inventory that assess aspects relevant to the disease, with a high probability of understanding and sensitivity to the reality and the needs of the patients. After data collection, we will be able to establish a list of barriers to PKU treatment adherence from the perspective of patients and their families.

CORRESPONDING AUTHOR: Katia Irie Teruya, Universidade Federal do Rio Grande do Sul, teruya.katia@gmail.com

\section{P099}

MEASURING SOCIAL SUPPORT WITHIN THE ILLNESS CONTEXT: A SYSTEMATIC REVIEW OF THE CONTEXTUAL ILLNESS-SPECIFIC SUPPORT SCALE

Irina Mindlis ${ }^{1}$, Tracey A. Revenson ${ }^{2}$

${ }^{1}$ The Graduate Center, City University of New York- USA

${ }^{2}$ Hunter College and The Graduate Center, City University of New York- USA

Introduction \& Purpose: Social support has been shown to improve psychological well being among those facing chronic illness, but effect sizes vary across studies. Many support measures are generic, leading to the need for an illness-specific measure. This systematic review examines the psychometric properties of a self-report measure of received social support, the Contextual Illness Support Scale (CISS; Revenson et al., 1991), and its applicability across illness populations. The CISS was designed with both positive and problematic support scales to assess interpersonal interactions that are both positive and negative.

Methods: A computer search retrieved all publications from1990 to 2017 that cited the scale. Peer-reviewed papers and conference proceedings in any language were included if they used the measure in empirical analyses. Articles were coded for scale modifications, reliability, and relationship to other support measures and mental health variables.

Results: In all, 350 references were retrieved; 31 studies met inclusion criteria. The CISS has been used in 9 countries and translated into 6 languages. It has been used primarily in studies of rheumatoid and osteoarthritis conditions, $(38.7 \%)$, but also with cancer (29\%) and other illnesses. Many researchers modified the scale for their use, using only selected items or only one of the two subscales. The source of received support also varied widely, from spouse/partner to family members to "people"; $30 \%$ of studies did not specify a source, changing the measure from one of received support to one of global support perceptions. Internal consistency reliability (coefficient alpha) was good across studies, ranging from .79-.96 for the positive support subscale and from .55-.94 for the problematic support subscale. Construct validity has been evaluated in relation to over 30 instruments, including depressive symptoms, self-efficacy, relationship quality, disease severity, treatment adherence, and pain.

Conclusions: The CISS scale is a valid, reliable instrument to measure both positive and problematic social support in the illness context. It has been translated into multiple language versions that can be used across different illnesses and cultures and can be modified to suit the specific illness.

CORRESPONDING AUTHOR: Irina Mindlis, The Graduate Center, City University of New York, imindlis@gradcenter.cuny.edu

\section{P100}

Factors Associated with Depression Severity among People Living with HIV and depression in China

Jiaying Qiao ${ }^{1}$, Zhimeng $\mathrm{Xu}^{1}$, Hanxi Zhang ${ }^{1}$, Chengbo Zeng ${ }^{1}$, Yiran $\mathrm{Li}^{1}$, Mengting Zhu ${ }^{1}$, Yan Alicia Hong ${ }^{2}$, Weiping $\mathrm{Cai}^{3}$, Yan Guo ${ }^{1}$

${ }^{1}$ Sun Yat-sen University- China

${ }^{2}$ Texas A\&M University- USA

${ }^{3}$ Guangzhou No.8 People's Hospital- China

Introduction: People living with HIV (PLWH) are often stigmatized and face various stressors. They are more likely than the general population to experience mental health problems such as depression. The more severe depression PLWH has, the more adverse impact it has on PLWH. To improve mental health of people living with HIV and depression (PLWHD), a randomized controlled trial is underway in China. In the stage of baseline, the study explored depression severity among PLWHD and its influencing factors in China. Methods: 1555 PLWH were approached and screened for depression with the Center for Epidemiological Studies Depression Scale (CES-D). In total, 300 PLWHD with CES-D $\geq 16$ were recruited for the randomized clinical trial. Results: At baseline, the rate of depression in PLWH was high (538/ $1555,34.60 \%)$. Among the sample of the 300 participants, $277(92.30 \%)$ were male; the mean age was $28.21(\mathrm{SD}=5.83$ ) year. The rates of mild-to-moderate $(16 \leq \mathrm{CES}-\mathrm{D} \leq 24)$ and severe (CES-D $\geq 25)$ depression were $61.3 \%$ and $38.6 \%$, respectively. Using logistic regression, higher levels of HIV-related stigma $(\mathrm{OR}=2.64,95 \% \mathrm{CI} 1.59 \sim 4.41)$ and perceived stress $(\mathrm{OR}=2.47,95 \% \mathrm{CI}$ 1.48 4.13) were associated with elevated depression. Positive coping $(\mathrm{OR}=0.44$, 95\% CI $0.25 \sim 0.76)$ was negatively associated with depression, while negative coping $(\mathrm{OR}=1.77,95 \% \mathrm{CI} 1.02 \sim 3.08)$ was positively associated with depression. Conclusion: Depression in PLWH was prevalent. Interventions targeted to reduce HIV-related stigma and perceived stress and to enhance the coping skills of PLWH have the potential to reduce depression and improve mental health of PLWH in China.

CORRESPONDING AUTHOR: Jiaying Qiao, Sun Yat-Sen University, ycxx105@163.com

P101

CONSTRUCT VALIDITY, CONTENT VALIDITY AND RELIABILITY OF THE SPANISH VERSION OF THE INDEX OF DENTAL ANXIETY AND FEAR (IDAF- 4C+) IN CHILEAN PREGNANT WOMEN

Andrea Herrera Ronda ${ }^{1}$, Luis Palma ${ }^{1}$, Matías Ríos ${ }^{1}$, Elías Lanyon ${ }^{1}$, Nicole Morales ${ }^{1}$, Camila Farías ${ }^{1}$, Catalina Martínez ${ }^{1}$

${ }^{1}$ Facultad de Odontología, Universidad de Chile- Chile 
Introduction: Dental anxiety is a problem that greatly affects oral health and dental compliance in adults. There are many instruments to assess dental anxiety in general population. IDAF-4C+ has a modular structure that allows to identify dental anxiety levels and feared stimuli related to dental setting. The aim of this study is to determine the construct and content validity, and the reliability of the Spanish version of IDAF-4C+ in pregnant women from primary health care centers in Santiago, Chile. Methods: 72 pregnant women ranged from 18 to 40 years old were recruited at primary health care centers from Santiago, Chile. To determine content validity the agreement among experts was assessed. Confirmatory factor analysis was used to demonstrate the construct validity, meanwhile reliability was determined in terms of internal consistency by undertaken Cronbach's alpha coefficient. Results: The results indicated that the prevalence of dental anxiety is $33.34 \%$ in women. The experts did not modify the Spanish version of IDAF-4C+. Confirmatory factor analysis yielded a single factor that accounted for $61,86 \%$ of variance. A high Cronbach's alpha $(\alpha=0,908)$ confirm the internal consistency of the instrument. Conclusion: IDAF- $4 \mathrm{C}+$ presents an appropriate construct and content validity, and an excellent reliability. It is a proper instrument to be used in pregnant women enrolled in primary health care centers in Chile.

CORRESPONDING AUTHOR: Andrea Herrera Ronda, Facultad de Odontología, Universidad de Chile, aherrera@odontologia.uchile.cl

\section{P102}

\section{SOCIO-ENVIROMENTAL DETERMINANTS OF HEALTH IN THE} SCHOOL CONTEXT

Daniella Carucci ${ }^{1}$, Sierra Bainter ${ }^{1}$, Judith McCalla ${ }^{1}$, Patrice Saab

${ }^{1}$ University of Miami- USA

Introduction \& Purpose One out of every six adolescents is obese, and one out of every three is at risk. As adolescents spend most of their time in school, it is important to examine the influence of school contextual factors on body mass index (BMI). School socioeconomic status (SES) and academic achievement may have the potential to shape weightrelated health behaviors. This study investigated the impact of both school-level contextual and student factors on adolescent weight status. Controlling for physical activity and dietary habits, school-level SES and academic achievement are hypothesized to be inversely associated with BMI while self-reported stress is hypothesized to be positively associated with BMI. Methods This sample consisted of 12,559 (84.1\% minority status) from 38 public high schools in Miami, FL. Student height and weight was used to calculate BMI as $\mathrm{kg} / \mathrm{m} 2$. School SES was defined the percentage of students eligible to receive free/reduced-price lunch. Academic achievement was the percentage of students passing the reading portion of the Florida Comprehensive Assessment Test. Stress was operationalized as the total perceived stress in and out of school over the past week. Diet was number of healthy foods consumed on a typical day. Physical activity was defined as the number of days in the past week students participated in vigorous exercise for at least 20 minutes. Multilevel modeling was used to investigate the contribution of school effects to the variance in adolescent BMI. Analysis at the student level estimated the intercept and slope coefficients describing relationships between the Level 1 predictor (stress) and covariates (diet and physical activity) and BMI. Analysis at the school-level added SES and academic achievement to the model to estimate the impact of these factors on BMI. Results Between-school differences accounted for $3 \%$ of the variance in BMI. After adding both student and school-level variables, .33\% of the student-level variance and $70.7 \%$ of the school-level variance were explained. Although self-reported stress and school-level academic achievement were not significantly associated with BMI ( $p>.05$ ), SES was associated with BMI. For every unit increase in the rate of free/reducedprice lunch, the average student BMI increased by $.02 \mathrm{~kg} / \mathrm{m} 2(\beta=.02, \mathrm{p}=$ .02 ), controlling for covariates. Conclusions The findings suggest that school SES may also contribute to adolescent weight status beyond dietary and physical activity habits, self-reported stress, and school-level academic achievement. Thus, unhealthy weight is not strictly the result of individual action but also a product of environmental factors beyond the adolescent's control. In particular, school SES has the potential to capture more effectively the student's exposure to stress, social environment, and resources that shape health behaviors. This is an important consideration for low-income minority youth, who are disproportionately impacted by obesity.

CORRESPONDING AUTHOR: Daniella Carucci, University of Miami, d.carucci@umiami.edu

\section{P103}

PRELIMINARY RESULTS OF A RANDOMIZED CONTROLLED TRIAL EVALUATING EFFICACY OF PROMOTING HUMAN PAPILLOMAVIRUS (HPV) VACCINATION AMONG CHINESE MEN WHO HAVE SEX WITH MEN

Zixin Wang ${ }^{1}$, Joseph T.F. Lau ${ }^{1}$, Mary $\mathrm{Ip}^{1}$, Polly P.K. Lam ${ }^{1}$, Francois Fong $^{2}$, Paul Chan ${ }^{3}$

${ }^{1}$ Centre for Health Behaviours Research, JC School of Public Health and Primary Care, Faculty of Medicine, The Chinese University of Hong Kong, Hong Kong SAR

${ }^{2}$ NeoHealth, Hong Kong SAR

${ }^{3}$ Department of Microbiology, Faculty of Medicine, The Chinese University of Hong Kong, Hong Kong SAR

Introduction \& purpose: HPV-related disease burden is high among men who have sex with men (MSM). HPV vaccination is highly effective in preventing HPV-related diseases among men but under-utilized by Chinese MSM. The primary objective of the randomized controlled trial (RCT) study is to evaluate the relative efficacies of two online, theorybased and interactive interventions [online health communication with and without motivational interviewing (MI)] in increasing uptake of three required doses of HPV vaccination within a 9-month follow-up period among Hong Kong Chinese MSM, as compared to the control group. Methods: A three-arm parallel-group, non-blinded randomized controlled trial was conducted. Participants were Hong Kong Chinese speaking MSM who had never received HPV vaccination. A total of 624 eligible MSM will be recruited and randomized 1:1:1 into three groups: 1) Group HC (Online Health Communication Only Group): exposure to interactive and theory-based online health communication promoting HPV vaccination among MSM, 2) Group HC-MI (Online Health Communication plus MI): receiving 15-minute MI over phone, administered by a trained fieldworkers, on top of the health promotion given to Group HC, and 3) Control group: exposure to online health communication related to mental health that was unrelated to HPV vaccination. Participants were given coded discount coupons ( $10 \%$ discount of the market price) for taking up the three doses of HPV vaccines at a collaborating private clinic. To date, 578 participants completed the baseline telephone survey and were assigned to Group HC ( $n=207)$, Group HC-MI $(n=191)$ and the control group ( $\mathrm{n}=180)$; 162 participants (56 in Group HC, 63 in Group HC-MI, and 43 in the control group) completed the Month 3 follow-up. Results: Among those who have completed the Month 3 follow-up, the uptake of the first dose of HPV vaccination was $14.3 \%(8 / 56)$ in Group HC, $54.0 \%$ $(34 / 63)$ in Group HC-MI and 23.2\% (10/43) in the control group. The uptake was significantly higher in the Group HC-MI than that of the control group (RR: 2.32, 95\%CI: 1.29, 4.18; ARR: 30.7, 95\%CI: 13.1, 48.3; $\mathrm{p}<0.001)$. Conclusions: The theory-based online health communication plus brief MI is of good potential to increase HPV vaccination uptake among Hong Kong Chinese MSM.

CORRESPONDING AUTHOR: Zixin Wang, Centre for Health Behaviours Research, JC School of Public Health and Primary Care, The Chinese University of Hong Kong, wangzx@ cuhk.edu.hk 
P104

CASUAL SEX: HEALTH AND RISK BEHAVIORS BETWEEN WOMEN UNIVERSITY STUDENTS

Andresa Pinho Soster ${ }^{1}$, Miguel Luis Alves de Souza ${ }^{1}$, Elisa Kern de Castro $^{1}$

${ }^{1}$ Universidade do vale do rio dos sinos

Casual sex is a type of experience in which sexual encounters occur among people who know little, and there is evidence that is related to risks and negative health consequences, especially for women. People who practice casual sex seem to have a decreased perceived health risk. This study aimed at exploring and comparing health and risk behaviors involving casual sex between women university students with (WEX) and without experience of casual sex (WOEX). Method: Comparative cross-sectional design with 1,133 women university students between 18 and 25 years old who answered an online questionnaire with questions about the perception of physical risks, health behaviors, alcohol and drug use, sexual behavior and history. The women university students were divided into two groups, with (WEX) and without experience (WOEX) of casual sex. Results: There was a significant difference in health behaviors and risk perceptions between the groups. The WOEX group perceived more risks in relation to casual sex and lower risk in relation to sex in the formal relationship. The WEX group presented more health behaviors such as condom use (WEX 62.9\% WOEX 54.7\% $\mathrm{x}^{2}=6.185, \mathrm{p}$ $<0.013$ ), gynecological preventive exams (WEX 66,5\% WOEX 56,5\% $\left.\mathrm{x}^{2}=14,117 ; \mathrm{p}<0.003\right)$, screening test for sexually transmitted infections (WEX 70.8\% WOEX 53.8\% $\mathrm{x}^{2}=29.901, \mathrm{p}<0.001$ ), although greater use of alcohol and drugs was performed. Conclusion: Conclusion: Contrary to the literature, WEX women show greater self-care regarding sexual health. Although casual sex is related to risk behaviors, in this study it was associated with health behavior. It can be noticed that the risk is related to the lack of condom use and not to casual sex. Interventions to reduce distortions in the perception of risk of sex without condoms are suggested from these findings.

CORRESPONDING AUTHOR: Andresa Pinho Soster, Universidade do Vale do Rio dos Sinos, apsoster@hotmail.com

\section{P105}

WHAT TO ASSESS WHEN STUDYING THE EFFECTS OF LIGHT THERAPY FOR CANCER-RELATED FATIGUE?

Daniëlle Starreveld ${ }^{1}$, Laurien Daniëls ${ }^{2}$, Flora van Leeuwen ${ }^{1}$, Eveline Bleiker $^{1}$

${ }^{1}$ Netherlands Cancer Institute- Netherlands

${ }^{2}$ Leiden University Medical Center- Netherlands

Introduction and purpose: Since the 1980s, light therapy has been studied and proven to be effective in the treatment of seasonal affective symptoms. The success of this easy-to-deliver and cheap intervention was examined in other conditions as well, e.g. it was found to be effective in the treatment of sleep disorders and depressive symptoms. More recently, pilot studies suggested large effect sizes of light therapy for cancer-related fatigue (CRF). However, replication in large scaled randomized controlled trials (RCT) is necessary. Additionally, potential working mechanisms of light therapy for CRF need to be examined. This may provide more information about causative factors for CRF. The current study reviewed which outcome measures and covariates are important to include in a trial design and offers suggestions for study procedures to assess these variables in light therapy studies. Methods: We searched review papers on factors associated with CRF and factors associated with the efficacy of light therapy to identify important outcome measures. Thereafter, literature was examined to identify reliable and valid study procedures to measure these factors. Results: Twelve reviews on CRF and nine study protocols of light therapy efficacy trials were studied. The following outcome measures were identified: fatigue, depression, quality of life, and cognitive complaints. Potential covariates can be categorized into chronobiological variables (including chronotype, sleep quality, circadian rhythms of melatonin and cortisol), psychosocial variables (including anxiety, fatigue catastrophizing, and self-efficacy to manage fatigue), and biological variables (including biomarkers of inflammation, vitamin D and genotype). A total of 55 questionnaires were reviewed to choose valid and reliable assessments, e.g. the Center of Epidemiological Studies - Depression scale for depression. Experts were consulted to agree on a reliable assessment of melatonin and cortisol. Conclusions: The results of this literature search provides suggestions for outcome measurements, covariates, and assessments in studies on light therapy as a treatment for CRF. Results of RCTs on light therapy for CRF will provide more knowledge about the potential benefit of this treatment for CRF.

CORRESPONDING AUTHOR: Daniëlle Starreveld, Netherlands Cancer Institute, d.starreveld@nki.nl

\section{P106}

HEALTH- RELATED BEHAVIORS AND PERCEIVED HEALTH IN PERUVIAN UNIVERSITY STUDENTS

Patty Vilela Alemán ${ }^{1}$, Mónica Cassaretto Bardales ${ }^{1}$

${ }^{1}$ Pontifical Catholic University of Peru- Peru.

Lifestyles, that is, ways of living according to inner beliefs, expectations, motives, values and behaviors, are recognized as one of the main and key determinants of health (World Health Organization [WHO], 2002). Among their components, habits or behaviors are the ones who produce the greater direct influence on health outcomes. As evidence of it, nowadays, the main causes of death are related, among other factors, to unhealthy behaviors which are associated with increased mortality and morbidity across the globe. Youth and Adolescence are decisive stages for the acquisition and consolidation of health-related behaviors, which in many cases, will be maintained throughout life. This process matches with the beginning of university life, moment which young people experience a feeling of greater freedom and control of their lifestyles. Therefore, this stage is an opportunity to establish healthy behaviors and habits. Nonetheless, according to the literature, it presents a greater alcohol consumption, an inadequate stress management, a decrease in physical activity, a change in eating patterns, and sleep disorganization. For this reason, its study is necessary, as well as identifying which behaviors predict physical and mental health. The purpose of this research is to determine the influence of health-behaviors on perceived physical and mental health in a group of Peruvian university students. For this end, a group of 974 university students, between the ages of 16 and $31(\mathrm{M}=20.32, \mathrm{SD}=$ 2.18), from five universities in Lima, Piura and Arequipa was surveyed. The Lifestyles Questionnaire for Young University Students was applied (Chau \& Saravia, 2016). This test is composed of the following areas: physical activity, leisure time, self-care, eating habits, alcohol and drug consumption, and sleep organization. Also, the SF-36 Health Survey (Ware, Snow, Kosinski \& Gandek, 1993), which considers two components of health, physical and mental, was used. The results of the multiple linear regression analysis indicate that leisure time $(\beta=.18 ; p=.00)$, selfcare $(\beta=.09 ; \mathrm{p}=.01)$, eating habits $(\beta=.09 ; \mathrm{p}=.00)$ and sleep organization $(\beta=.36 ; p=.00)$ are the most important predictors of mental health and explain $22.4 \%$ of the model variance $[\mathrm{F}(4,888)=65.28 ; \mathrm{p}=.00]$. On the other hand, physical activity $(\beta=.09 ; \mathrm{p}=.00)$, leisure time $(\beta=.14 ; \mathrm{p}=.00)$, self-care $(\beta=.07 ; p=.04)$, and sleep organization $(\beta=.38 ; p=.00)$ explain $21.6 \%$ of the physical health's model variance $[\mathrm{F}(4,862)=60.76 ; \mathrm{p}=.00]$. In conclusion, the results of this research provide a solid basis of the importance to establish healthy habits interventions that help promote adequate physical and mental health; likewise, they show that it is relevant to raise this type of initiatives in order to contribute with noncommunicable disease prevention in adult life. Finally, the commitment of educational institutions in the formation of responsible citizens with 
health and their environment is highly necessary for major health changes in society.

CORRESPONDING AUTHOR: Yuliana Patty Vilela Alemán, Pontifical Catholic University of Peru, patty.vilelaa@pucp.pe

P107

THE SOCIALITY AND GENDER EFFECT ON DECISION-MAKING IN MORAL DILEMMA SITUATION

Yoshiyuki Takimoto $^{1}$, Akira Yasumura ${ }^{1}$, Eisuke Nakazawa ${ }^{1}$

${ }^{1}$ The University of Tokyo- Japan

Introduction: In moral psychology research, the mechanism of decisionmaking in moral dilemma situation has recently been illuminated. However, there is few research that the association between sociality and decision-making in moral dilemma situation. This research objective is to estimate the sociality and gender effect on decision-making in moral dilemma situation. Methods: Referring to moral dilemma task made by Foot (1978), moral dilemma questionnaire in Japanese version was developed. Three hundred sixty-four person answered this questionnaire. Exclusion criteria were response variation and time. Finally, eligible subjects were 231 person. The association between utilitarian answer, and gender and social status were analyzed. Results: In moral dilemma situation, non-students more frequently judged utilitarian decision making comparing to students. And, male evaluated utilitarian judgment as more appropriate than female. Conclusion: The tendency of utilitarian in nonstudent might reflect their abundant social experience. And the result suggested that female tend toward emotional decision-making relative to male.

CORRESPONDING AUTHOR: Yoshiyuki Takimoto, The University of Tokyo, taki-tky@umin.ac.jp

\section{P108}

BEHAVIORAL FACTORS MEDIATING BETWEEN SOCIOECONOMIC STATUS AND OBESITY: PATHWAY ANALYSIS FROM CHINA HEALTH AND NUTRITION SURVEY Yuxuan $\mathrm{Gu}^{1}$, Minzhuo Huang ${ }^{1}$, Hengjin Dong ${ }^{1}$

${ }^{1}$ Zhejiang University- China

Introduction \& Purpose China has the largest obese population in the world and its prevalence is increasing faster and faster. The researchers are investigating in the association between the socioeconomic status(SES) and obesity in several ways. However, SES may not only play a direct impact on obesity but influences health behaviors which, in turn, affect obesity. The mediating factors have been rarely studied. This study investigates the association between SES and obesity mediating by behavioral factors among adults in China. Methods The longitudinal data was obtained from the eight waves of the China Health and Nutrition Survey from 1991-2011. The outcome of obesity was measured using Body Mass Index(BMI). The SES factors include education and income (low, medium and high). Mediating factors include alcohol consumption, smoking status, dietary patterns and physical activity. A variety of statistical models were used to investigate the association between SES and obesity. Age/gender-adjusted prevalence of obesity was calculated and multiple-logistic regression was used. Results To some extent, SES influenced BMI directly, positively in men and inversely in women, respectively. SES may also operate through behavioral factors. These associations were not always straightforward, and changes in SES might create some offsetting risks. Behavioral factors including alcohol consumption, smoking status, diet and physical activity were associated with SES indicators in all groups. In addition, the prevalence was higher in urban areas than rural areas in China. Several pathways for different SES groups leading to obesity were simulated. Conclusions Higher SES groups are more likely to have higher BMI compared to lower SES groups. Different SES groups have different significant mediating risk factors. The pathways between SES and obesity are complex. This study suggests that it is necessary to apply different interventions to different SES individuals especially focused on the disadvantaged populations according to their different behaviors and preference.

CORRESPONDING AUTHOR: Yuxuan Gu, Zhejiang University, guyuxuan@zju.edu.cn

P109

THE PERCEPTIONS OF OPPORTUNITIES AND BARRIERS IN PREVENTION OF CARDIOVASCULAR DISEASES AMONGST MANAGING HEALTH WORKERS IN LIMPOPO, SOUTH AFRICA Mbuyiselo Douglas ${ }^{1}$, Tholene Sodi ${ }^{1}$, Tebogo Mothiba ${ }^{1}$

${ }^{1}$ University of Limpopo- South Africa

Introduction \& purpose: South Africa is undergoing a process of epidemiological transition from infectious to non-communicable diseases. Cardiovascular diseases (CVDs) are said to be the major cause of mortality in the world and they rank highly in developing countries. In South Africa, about 33 people die per day because of a heart attack, while about 60 die per day because of stroke. The primary purpose of the study was to explore opportunities and barriers in CVD prevention and management in Limpopo Province, South Africa. One question was asked from the participants: Is there any health promotion activities for prevention of CVD's in your area? Methods: A qualitative approach with exploratory and interpretive components was followed. Purposive sampling was used to select 15 key informants for semi-structured interviews from the selected areas in Limpopo Province. Collected data was appropriately analysed and presented under different themes. Tesch's eight steps data analysis method was used. The data was organised and prepared for analysis by first transcribing the interviews verbatim and then analysing the transcriptions. Results: The overarching themes collated included the following: the low socioeconomic status; poor dietary knowledge; ignorance pertaining to physical activity; as well as individual lifestyle behaviours. Conclusions: The absence of coordinated health promotion activities was perceived as a major challenge in the prevention of CVDs. Preventive actions such as health promotion interventions for the empowerment of the vulnerable groups are recommended.

CORRESPONDING AUTHOR: Mbuyiselo Douglas, University of Limpopo, Mbuyiselo.Douglas@ul.ac.za

P110

INCORPORATING BEHAVIOR CHANGE TECHNIQUES TO FACILITATE THE ADOPTION OF AN INSULIN PATIENT DECISION AID IN MALAYSIA

Wen Ting Tong ${ }^{1}$, Yew Kong Lee ${ }^{1}$, Chirk Jenn $\mathrm{Ng}^{1}$, Ping Yein Lee ${ }^{1}$

${ }^{1}$ University of Malaya- Malaysia

Introduction \& Purpose Despite the established benefits of patient decision aids, its uptake remains poor among healthcare providers (HCPs). Behavior change techniques (BCT) are methods that can be used to target specific HCP-related barriers to adoption of innovations. This study aimed to identify the appropriate BCTs to address barriers to the adoption of an insulin-choice patient decision aid (iPDA), a tool to facilitate shared decision making (SDM) between HCPs and patients about insulin initiation in a primary care clinic in Malaysia. Methods: A previous qualitative study had identified barriers to implementing the iPDA. The research team, comprising implementation scientist, representatives from the primary care clinic, and decision expert, selected BCTs from Michie's Behaviour Change Technique Taxonomy (BCTTv1), which aim to bring about changes in behaviours that could overcome the barriers. The 
delivery methods for the BCTs were also discussed. Results: Four BCTs were chosen to overcome the barriers to adoption of iPDA by HCPs. To address time constraints, HCPs will be shown how the iPDA can be delivered without prolonging a consultation (BCT1: Demonstration of the behavior). This can be delivered using a video through e-learning or workshop. To address HCPs' lack of SDM skills and motivation to adopt new innovation, messages emphasizing that using PDAs and practising SDM as part of patient centered care (BCT2: Information about social and environmental consequences) and will improve patient decision quality and satisfaction (BCT3: Information about emotional consequences) should be delivered to the HCPs during a training workshop. To address patient feeling pushed to use insulin when receiving the iPDA, HCPs will be trained to inform patients that the iPDA is not a tool to convince patient to start insulin but to facilitate SDM and help patients make informed decision (BCT4: Framing/reframing). A combination of workshop and elearning can be used to facilitate the BCTs. Conclusion: The Michie's BCTs may be used to address barriers to the adoption of the iPDA by changing HCPs' and patients' attitude and behaviours. The effectiveness of these strategies should be evaluated in future studies.

CORRESPONDING AUTHOR: Wen Ting Tong, University of Malaya, tongwenting@um.edu.my

\section{P111}

LIFE EXPERIENCE, SEXUAL IDENTITY, AND PERCEPTIONS ON SEX WORK: A QUALITATIVE STUDY ON MALE SEX WORKERS IN ZIMBABWE

Shan Qiao ${ }^{1}$, Eileen Yuk-ha Tsang ${ }^{2}$, Freddy Lipeleke ${ }^{2}$, Xiaoming $\mathrm{Li}^{1}$

${ }^{1}$ University of South Carolina- USA

${ }^{2}$ City University of Hong Kong- Hong Kong

Introduction \& Purpose: Male sex workers (MSWs) are marginalized in most of societies due to intersectional stigma against prostitution and homosexuality. Currently homosexuality is illegal in a number of African countries including Zimbabwe, and limited literature has explored their lives and well-beings. This study aims to demonstrate how the life experiences and sexual identify shape perceptions on sex work among MSWs in Zimbabwe and discuss implications in health promotion for this vulnerable population. Methods: Data comes from indepth interviews among $15 \mathrm{MSWs}$ in Bulawayo recruited through snowball sampling. The interviews took 30-40 minutes on average. All the interviews were conducted in privacy settings (e.g., cars) and in native language. The audio records were transformed verbatim and analyzed in Nvivo 11. Results: The age of the participants ranged from 19 to 38. One participant was married and had one child. The rest of them were currently single. Four had girlfriends and four had been engaged in stable relationship with women before. Six of them received college education, five took middle school, and four did not finish primary school. Nine of the MSWs identified as gay and six ones did not. Before engaging in sex trade, quite a few of them (especially the ones with low education attainment) had experienced traumatic life events including early life adversities (sexual abuse by uncles or son of white farm owner), enforcement into same sex behaviors when in prisons, unemployment, poverty, and homelessness. Some of them had faced social environments that provided opportunities for them to contact men who have sex with men and potential clients such as college campuses, hotels for foreign tourists, and new politics places after short-term immigration (from the rural to the urban or from Zimbabwe to South Africa where homosexuality is legal). These life events and environmental change contributed to their initiating and involving in same sex behaviors or sex trade and triggered their realization, reflection and confirmation of their sexual identities. Non-gay MSMs were intended to view sex work as temperate surviving approach in economic difficulties and even the only way to support family. They heighted money as their work motivation, and showed high internal stigma (feel shamed and dirty, embarrassed for sex work) and hoped to live a traditional family life when their life became stable. Gay MSMs engaged in sex trade for both money and attraction. They enjoyed the sexual experiences with various people and they felt the sex work as sex adventure and way of living in the hostile context. They would not quit from sex trade in future. Meanwhile they were more empowered to protect themselves from abuse or condomless sex. Conclusions: Life experience and sexual identities of the MSWs intertwined with each other, shaping their perceptions on sex work. Non-gay MSWs in Zimbabwe may be more vulnerable in terms of life situation, mental health and negotiation for condom use. Future efforts for MSWs' health promotion need a perspective of life course to understand their perceptions and attitudes for their sex work and provide tailored services.

CORRESPONDING AUTHOR: Shan Qiao, University of South Carolina, shanqiao@mailbox.sc.edu

\section{P112}

THE EFFECT OF FACILITATION WHEN IMPLEMENTING A BEHAVIORAL MEDICINE APPROACH IN PHYSICAL THERAPY PRIMARY HEALTH CARE

Johanna Fritz ${ }^{1}$, Anne Söderlund ${ }^{1}$, Lars Wallin ${ }^{2}$, Lena Almqvist ${ }^{1}$, Maria Sandborgh ${ }^{1}$

${ }^{1}$ Mälardalen University, Västerås- Sweden

${ }^{2}$ University of Dalarna, Falun- Sweden

Introduction \& Purpose A behavioral medicine (BM) approach in physical therapy for patients with persistent musculoskeletal pain is recommended based on evidence. The BM approach is complex and it is challenging to achieve changes in physical therapists' clinical behaviors when implementing the approach. Facilitation may, according to research, be beneficial for implementation. The aim was therefore to explore the effect of external facilitation for supporting implementation of a BM approach in physical therapy primary health care. Methods A quasi-experimental pre-posttest trial was conducted. 15 physical therapists working in primary health care were consecutively included in the intervention group and nine in the control group. A theory based implementation intervention was tailored to the individual physical therapist. Facilitation, consisting of multifaceted methods, were used in the intervention group during a six months implementation period. Data were collected with structured observations of clinical behaviors, more specifically if core BM components were used. Within- and between group differences were analyzed with non-parametric analyses. Effect sizes for use of core BM components were calculated using Pearson's r. Results The intervention group significantly increased their use of the core BM components goal setting, self-monitoring and functional behavior analysis $(p \leq 0.05)$. No changes was observed related to the core BM components assessment, basic and applied skills acquisition and maintenance. No changes over time were found in the control group. The effect sizes were large for changes in using goal setting $(\mathrm{r}=.62)$, selfmonitoring ( $\mathrm{r}=.61)$ and functional behavior analysis $(\mathrm{r}=.53)$, medium for assessment ( $\mathrm{r}=.32)$ and applied skills acquisition ( $\mathrm{r}=.39)$, and small or non for basic skills acquisition $(\mathrm{r}=.23)$ and maintenance $(\mathrm{r}=.00)$. Conclusions Multifaceted facilitation methods resulted in observed changes in several clinical behaviors related to a BM approach, and can therefore be useful when supporting implementation of a BM approach in physical therapy primary health care. The BM approach, however, is a complex intervention and in order to succeed future studies need to consider limiting the number of behaviors that are targeted for change.

CORRESPONDING AUTHOR: Johanna Fritz, Malardalen University, johanna.fritz@mdh.se 
P113

PSYCHOMETRIC PROPERTIES OF THE CHILEAN VERSION OF THE FEAR-AVOIDANCE BELIEFS QUESTIONNAIRE IN ACUTE LOW BACK PAIN

Patricia Basualto $^{1}$, Lydia Gomez-Perez ${ }^{2}$, Eliana Guic ${ }^{2}$

${ }^{1}$ Carrera de Kinesiología, Departamento de Ciencias de la Salud, Pontificia Universidad Católica de Chile- Chile

${ }^{2}$ Escuela de Psicología, Pontificia Universidad Católica de Chile- Chile

Chronic pain is one of the leading worldwide causes of disability [1] and causes high economic and social costs for individuals, families, and society. Psychosocial factors have been shown to intervene in the transition from acute to chronic pain and predict pain patient's prognosis [2,3]. Pain-related fear is one of these psychological factors. According to the Fear and Avoidance Beliefs Model [4], people who tend to evaluate their experience of pain in a negative and catastrophic way (pain catastrophizers) would develop high pain-related fear and perform avoidance behaviors in order to prevent pain occurrence or increase. As so, pain-related fear would hinder their recovery and promote deconditioning, emotional distress, and disability, contributing to pain perpetuation. Since its conceptualization in 2001, the Fear and Avoidance Beliefs Model has received considerable empirical support $[5,6]$ and produces huge amounts of research. The Fear-Avoidance Beliefs Questionnaire (FABQ) [7] is one of the most widely used instruments to measure pain-related fear. It is an 11 items self-report questionnaire that has proven to be reliable and valid. Its original English version comprises two major factors: Fear-Avoidance Beliefs about work (FAB-work) and Fear-Avoidance Beliefs about physical activity (FAB-physical activity). FAB-work assesses people's fearavoidance beliefs about how work affects their pain. FAB-physical activity assesses people's belief about how physical activity affects their pain. Both scales have shown to present adequate internal consistency (Cronbach's alpha $=.88$ and .77 , respectively) as well as high test-retest variability (.95 and .88 , respectively). The total scale has shown a Cronbach's alpha of .82. Convergent validity has been demonstrated between FABQ and other fear avoidance beliefs measures such as the Tampa Scale of Kinesophobia [8-11]. The FABQ has also shown to correlate with scales measuring pain intensity [7,9,12-23], pain disability $[7,12,14-16,19-23]$, emotional distress $[(15,17,18)]$, depression $[12,13,15,19,20,22]$, and anxiety $[12,13,15,19,20,23]$. The FABQ has been translated into several languages, including German [20], French [12], Norwegian [14], Greek [13], Portuguese [9], Turkish [15], Spanish [16], Italian [19], Japanese [18], Persian [21], Chinese [17,23], and German-Swiss [22]. Its psychometric properties have shown to be relatively constant across translations. However, three studies (i.e., the German, the Greek, and the Chinese versions) have failed to replicate the original two-factor structure of the questionnaire $[13,17,20]$ where a three-factor structure was found. The FAB-work was split into two different subscales: FAB-work/cause, which assessed beliefs about work being the cause of pain, and FAB-work/prognosis, which assessed patients' beliefs about their ability to return to work in the future. Therefore, the construct validity of the FABQ should be further examined empirically. To our knowledge, the FABQ has been translated into Spanish and the factorial structure has never been examined [16]. Furthermore, although the negative impact of chronic pain recently reported in Chile and the interest to study this phenomenon, FABQ has never been adapted to be used with Chilean populations. Moreover, to our knowledge, only one study [14] has examined its psychometric properties in patients with acute pain, so more studies using acute pain samples are needed. Therefore, the aim of the present research is to examine the psychometric properties of the Chilean version of the FABQ in a sample of Chilean patients with acute low back pain.

CORRESPONDING AUTHOR: Patricia Basualto Alfaro, Pontificia Universidad Católica de Chile, pvbasualto@uc.cl
P114

CULTURALY COMPETENTS HEALTH WORKERS FROM THE PATIENTS POINT OF VIEW

Margarita Bernales ${ }^{1}$

${ }^{1}$ Universidad del Desarrollo- Chile

Nowadays it is essential to respond to patients' health needs considering their cultural background. Different authors defined cultural competence in health as a series of attitudes, behaviours and care policies that allows delivering care to patients according to their culture. This review aims to explore cultural competence in health care setting and its significance from the point of view of patients. This study establishes a review of reviews, which consists of a systematic review that aims to synthesize the results obtained by other systematic reviews in an area of interest. This approach, initially was developed for quantitative evidence, and had been adapted by the authors to summarize synthesis of qualitative evidence. We made a search for synthesis of qualitative studies in PUBMED database, regardless of design, year and place of publication. We included only revisions of qualitative studies that addressed the theme of communication, incorporating the perceptions of patients on cultural aspects of health care. The specific terms culture and cultural competence, and search equations related to those concepts, were used to search in the database. We collected 550 papers which were evaluated by two independent researchers, 35 were finally selected. Once obtained the final selection, papers were examined using thematic analysis through NVivo 11. The findings were classified into two main themes: (1) Beliefs of patients about their way of understanding the health-disease process, among them, religious/spiritual beliefs, beliefs about habits, including lifestyles, food, physical activity, and beliefs about how their relationship with health workers should be, emphasizing concepts such as sensitivity, flexibility and respect as indispensable characteristics in every health worker. (2) Challenges associated with the disease, especially those linked with the identity, related to how patients named his/her illness, which sometimes can be opposed to the diagnosis given by health workers, and challenges linked with the role assumed by the patient in a social, experienced from the presence of an illness. From the patient's point of view, this study shows those issues which should be considered by the health workers to achieve a culturally competent way of deliver care. The findings of this review provided new information to cultural competency models, commonly used, and it contributes to the operationalization of this theoretical construct.

CORRESPONDING AUTHOR: Margarita Bernales, Universidad del Desarrollo, margarita.bernales@gmail.com

P115

"EVALUATION OF THE HOME VISIT IN THE CHILE CRECE CONTIGO PROGRAM, USING THE HOVRS A + SCALE"

Claudia Aldana ${ }^{1}$, Viviana Tartakowsky ${ }^{1}$, Tali Waingortin ${ }^{1}$, Florencia Rodríguez ${ }^{1}$, Yael Kast ${ }^{1}$, Argyro Guglielmetti ${ }^{1}$, Marcela Aracena ${ }^{1}$, Jon Korfmacher $^{2}$

${ }^{1}$ Pontificia Universidad Católica de Chile- Chile

${ }^{2}$ Erikson Institute- USA

This conference illustrates the main results and reflections about the use of the home visit assessment scale HOVRS A + in home visits, carried out as intervention strategies within the framework of the Chile Grows with You Program in Chile. This Program is part of the public policies of our country, offering comprehensive support to the most vulnerable children and their main caregivers, from their gestation to 5 years of life. Specifically, it looks after the psychosocial well-being of the infant, linking the child and his family with the public health system, based on 4 fundamental concepts: neuronal plasticity, growth and maturation, integral development and equal opportunities. On the other hand, the scale of HOVRS A + has been used in Chile and in the world to evaluate the 
home visit, considering the strategies used in home visits and the interaction and commitment of parents and children during visits. It seeks excellence in conducting home visits and has 7 sub-scales that evaluate the strategies used by the visitor in favor of the early development of the child and the promotion of the interaction of the main caregiver and the child. The method of analysis used is the observation of home visiting videos with professionals trained in the use of this instrument at the Eriksonian Institute in Chicago, United States. Specifically 3 home vision processes (3 mothers) were observed, with an average of 6 sessions for each of them lasting 45 minutes. These videos were analyzed by the five researchers, in order to achieve the triangulation of the information. In addition, fragments of them were shared with the team of the Eriksonian Institute, arriving at common conclusions. The main results show that the visit requires strengthening relational and structural aspects; and that some scales of the HOVRS A + instrument are complex to use, due to the multiple elements that influence the realization of the visit. In this sense, it is concluded that the last two scales of the instrument are not feasible to use in the evaluation of this type of visits.

CORRESPONDING AUTHOR: Claudia Aldana, Pontificia Universidad Católica de Chile, claudisol@gmail.com

\section{P116}

STUDENT AND STAFF PERCEPTIONS OF ALCOHOL AS PART OF STUDENT LIFE IN DENMARK: A Q METHODOLOGY STUDY

Stine Holmegaard Christensen ${ }^{1}$, Bridgette M. Bewick ${ }^{2}$, Mette Vinther Skriver $^{3}$, Louise Bryant ${ }^{2}$, Stephen Jeffares ${ }^{4}$, Rebekka Yule ${ }^{5}$, Mette Terp Høybye $^{16}$

${ }^{1}$ Interdisciplinary Research Unit, Elective Surgery Centre, Silkeborg Regional Hospital- Denmark

${ }^{2}$ Division of Psychological and Social Medicine, School of Medicine, University of Leeds, Leeds- United Kingdom

${ }^{3}$ Department of Public Health, Aarhus University, Aarhus- Denmark

${ }^{4}$ Institute of Local Government Studies, School of Government and Society, University of Birmingham, Birmingham- United Kingdom

${ }^{5}$ North West Boroughs Healthcare NHS Foundation trust, WarringtonUnited Kingdom

${ }^{6}$ Interacting Minds Centre, Department of Clinical Medicine, Aarhus University, Aarhus- Denmark

Abstract Introduction \& Purpose: Intervening effectively to prevent students' harmful use of alcohol remains a challenge, and harmful alcohol use has been noted as the most dominant public health problem facing universities today. The objective of this study was to investigate the diversity in staff and student perceptions of the contribution alcohol makes to student life in a Danish university setting. Increasing understanding of staff and students' perceptions of how alcohol fits into student life is required to amend future public health intervention for this population. Methods: This Q-methodology study included 38 staff members and 105 students from Aarhus University, Denmark. All participants were asked, in an online questionnaire, to rank 40 statements about the perception of the contribution alcohol makes to the university student experience from strongly agree to disagree. To support the interpretation of the factors, qualitative data was collected on the participant's reasons for the ranking of the items they mostly agreed or disagreed with. By using Q-factor analysis five statistically independent viewpoints for students and four for staff were found of the contribution alcohol makes to student life. Results: The findings show that students and staff hold a variety of views on alcohol consumption as a part of university life. Diverse norms and perceptions constitute the viewpoints on students' consumption of alcohol and alcohol behaviour. Conclusions: The findings document distinct views on the role of alcohol consumption as part of the university experience for students and how to prevent harmful alcohol use. Some viewpoints suggest a need for individually tailored interventions, while others suggest the need for structural approaches to accommodate potentially harmful alcohol behaviour. The complexity reflected by these varied viewpoints reveals that creators of public health campaigns need to ensure that interventions targeting harmful alcohol use at universities challenge problematic perceptions and attitudes while bolstering positive norms of some individuals.

CORRESPONDING AUTHOR: Stine Holmegaard Christensen, Elective Surgery Centre, Silkeborg Regional Hospital, sthoch@rm.dk

\section{P117}

SYSTEMATIC INTEGRATION OF EMPIRICAL FINDINGS ON COOKING AT HOME USING TRIANGULATION PROTOCOL

Susanna Mills ${ }^{1}$, Jean Adams ${ }^{2}$, Martin White ${ }^{2}$, Wendy Wrieden ${ }^{1}$, Heather Brown $^{1}$

${ }^{1}$ Newcastle University- United Kingdom

${ }^{2}$ University of Cambridge- United Kingdom

Introduction and purpose Diet-related diseases are the greatest cause of morbidity and mortality worldwide. Food preparation methods are linked to diet and health. We aimed to use Triangulation Protocol to integrate findings from a mixed methods programme of study on the determinants and outcomes of home food preparation. Methods Firstly, we conducted a systematic review of studies on determinants and outcomes of home cooking ( $n=38$ studies included). Secondly, we undertook qualitative interviews exploring home food preparation amongst United Kingdom (UK) adults ( $\mathrm{n}=18)$, and a comparison with focus group participants from Baltimore, United States $(n=53)$. Thirdly, we analysed data from a large population-based UK cohort study. Analyses investigated relationships between meal consumption patterns and a) diet and cardio-metabolic health $(n=11,326)$ and $b)$ sociodemographic characteristics $(n=11,396)$. We used Triangulation Protocol to integrate multiple perspectives within our programme of work, by identifying key emergent meta-themes and coding themes as agreement, silence and dissonance for each of the three research phases. Results We identified meta-themes as behaviour, determinants, outcomes and research approach. Overall, we noted several areas of agreement between the research phases and the themes, for example potential dietary benefits from home food preparation and lack of consistency in terminology. The relationship between higher socioeconomic status and greater home cooking was a specific area of disagreement. Several potentially contradictory themes were also highlighted, concerning the importance of cooking skills; cultural influences; change in gender-related roles; and relative emphasis on different putative benefits of cooking and eating meals cooked at home. Comparisons were limited by the nature of the included study designs. Conclusions Preparation and consumption of meals cooked at home offers potential diet, health and social benefits, and should be supported through public health policy. However, cooking perceptions and practices are diverse, and relationships with socioeconomic status vary according to the research methods used, requiring further exploration.

CORRESPONDING AUTHOR: Susanna Mills, Newcastle University, susanna.mills@newcastle.ac.uk

\section{P118}

CORRECTING MISINFORMATION ABOUT VACCINATION: AN EXPERIMENTAL STUDY

Emily Kothe ${ }^{1}$, Mathew Ling ${ }^{2}$, Eddie Clarke ${ }^{3}$

${ }^{1}$ Deakin University- Australia

${ }^{2}$ Misinformation Lab, School of Psychology, Deakin UniversityAustralia

${ }^{3}$ School of Psychology, Monash University- Australia

Introduction \& Purpose: Misinformation in the public around vaccination threatens to hamper efforts to promote public health and well-being. Attempts to counteract misinformation have had mixed effects, 
occasionally resulting in a "backfire effect", where pre-existing beliefs are exacerbated or entrenched rather than refuted. In light of this, there is a need to understand what strategies that might be implemented in this medium are more effective in combating misinformation, and which are liable to promote these backfire effects. In particular, this study tested for short-term familiarity backfire effects (where repetition of a myth before correction can result in increased belief in the myth). While this effect has been reported in some studies, recent literature has suggested that this effect may not be robust and that highlighting the incorrect belief might increase successful correction. Methods: This study implemented an experimental design to test two science communication interventions within the context of vaccination. Participants $(n=234)$ were recruited through ProlificAcademic and were randomised receive one of two variants of a pro-vaccination message (one with myth repetition and one without) or a control message about bird feeding. Willingness to vaccinate was measured post-intervention. Results: There were no differences in willingness to vaccinate between individuals who received a pro-vaccination message and those that did not ( $\mathrm{p}>.05)$. There was no difference in vaccine-related beliefs between the two intervention conditions $(\mathrm{p}>.05)$. Trust in science was not a moderator of the intervention effect. Conclusion: Prior research has indicated that pro-vaccination messages have the potential lead to a backfire effect. This effect was not observed in this study and repetition of incorrect beliefs about vaccination in order to refute them did not result in backfire. However, the messages were also not effective in influencing individuals' beliefs about vaccination. Understanding of the factors that underlie intention to vaccinate is required in order to aid in the development of effective persuasive messages within this domain.

CORRESPONDING AUTHOR: Emily Kothe, Deakin University, ekothe@deakin.edu.au

\section{P119}

ASSOCIATION BETWEEN HOUSEHOLD INCOME AND HEALTHY FOOD ACCESS WITH COOKING AND EATING BEHAVIOURS

Julia Wolfson ${ }^{1}$, Rebecca Rampling ${ }^{2}$, Anne Palmer ${ }^{2}$

${ }^{1}$ University of Michigan School of Public Health- USA

${ }^{2}$ Center for a Livable Future, Johns Hopkins Bloomberg School of Public Health- USA

Introduction: Lack of access to healthy food is a significant barrier to healthy eating, particularly in low-income neighborhoods. More frequent cooking at home is associated with better diet quality. The relationship between food access, household income and cooking behavior is currently unknown. Methods: We designed and fielded a nationally representative online survey of adults in the United States aged 18 years $(n=1,112)$ in April 2015. We asked respondents to rate the frequency with which they face barriers obtaining healthy food (distance to store, transportation, physical disabilities, store hours, time, selection of items, quality of items, price) on a five point Likert scale from $1=$ never to $5=$ always. We used descriptive statistics to show barriers to obtaining healthy food stratified by household income (low: $<\$ 25,000$, mid: $\$ 25,000-\$ 59,000$, high: $\geq \$ 60,000$ ). We then used multivariable poisson regression to examine cooking and eating behavior by household income and frequency of encountering barriers to obtaining healthy food. Results: Overall, adults who more frequently encountered barriers to healthy food access were younger, Hispanic or Non-Hispanic Black, less educated, and lower-income. Time to shop and the price, selection and quality of items available were common barriers to obtaining healthy foods among all income groups, but were particularly prevalent among lower-income households. While there were few differences in frequency of cooking meals by income level, lower income households used packaged/boxed products more often (low vs. high income: 1.66 vs. 1.16 times/week, $\mathrm{p}=0.01$; mid vs. high income: 1.46 vs. 1.16 times/week, $\mathrm{p}=0.02$ ). Similarly, those who at least sometimes encountered barriers in accessing healthy foods cooked using packaged/boxed products and frozen products more often than those who never (packaged/boxed products: 1.60 vs. 1.27 times/week, $\mathrm{p}=0.04$; frozen products: 2.51 vs. 2.10 times/week, $\mathrm{p}=0.02$ ) or rarely (packaged/boxed products: 1.60 times/week vs. 1.23 times/week, $\mathrm{p}=0.01$; frozen products: 2.51 times/week vs. 2.12 times/week, $\mathrm{p}=0.01$ ) encountered barriers. Conclusions: Household income and barriers to healthy food access are related to cooking and eating behaviors important for diet quality and healthy eating. Targeted interventions to address time available to shop, and the price, selection and quality of healthy foods, are necessary, particularly among low-income populations.

CORRESPONDING AUTHOR: Julia Wolfson, University of Michigan School of Public Health, jwolfson@umich.edu

\section{P120}

THE EFFECT OF "PRACTA-ACTIVATING THE ELDERLY IN MEDICAL PRACTICE" E-LEARNING INTERVENTION FOR PRIMARY CARE DOCTORS EVALUATED THROUGH OLDER PATIENTS' EXPERIENCES

Marta Rzadkiewicz ${ }^{1}$, Joanna Chylińska ${ }^{1}$, Mariusz Jaworski ${ }^{1}$, Dorota Włodarczyk ${ }^{1}$

${ }^{1}$ Medical University of Warsaw- Poland

Introduction: The patients' experiences related to medical visit predict the satisfaction with care, attitude toward the treatment, and recovery. Experiences of older adults in primary care are scarcely researched, and the effects of internet-based educational interventions for doctors evaluated via patients experience are even less studied. Purpose: We investigate whether doctors' participation in different types of educational intervention might improve older patients' experience of the visit. Methods: Primary care doctors were randomly assigned to: e-learning $(n=31)$, pdf-based $(n=69)$ or no intervention $(n=65)$ condition, and the assessment concerned the experience of their older patients $(n=1527,56 \%$ female, age range 50-98 years; $\mathrm{M}=69.5 ; \mathrm{SD}=9.18$ ). The PRACTA Patient Expectations Scale - post-visit (PES-post) encompassing 6 dimensions illness explanation, treatment explanation, emotional support, health promotion, quality of life, rapport - was used. Generalized Linear Model was applied to compare the experiences of two waves of patients (before and after the intervention) between the conditions. Patient's socio-demographic, health and visit related variables were also included. Results: We found the PRACTA intervention effective for patients on most of dimensions (Wald's $\chi^{2}$ from 8.67 to 61.29 ; all $\mathrm{p}<0,02$ ). Intervention by patients' age group interaction showed particular e-learning's effects on the PRACTA PES-post dimensions of emotional support and health promotion for the older age groups (Wald's $\chi^{2}=10,97 ; p=0.027$ and $\chi^{2}=19.72 ; p=0.001$ respectively). Patient's financial status, aim of the visit, hospital use and prior attendance dominated in predicting their experience, although the detailed composition varied between the PRACTA PES-post dimensions. Conclusion: This pioneering study showed the effectiveness of internet-based intervention focused on doctors' skills evaluated via patients' experience. The e-learning education might increase older patients perception of doctors' performance, thus enhancing satisfaction with the visit and active attitude towards treatment and health.

CORRESPONDING AUTHOR: Marta Rzadkiewicz, Medical University of Warsaw, mrzadkiewicz@wum.edu.pl

\section{P121}

PSYCHOLOGICAL PREDICTORS OF PATIENT CONCERN AND ITS EFFECTS IN MEDICINE BRAND CHANGES

Kate MacKrill ${ }^{1}$, Keith Petrie ${ }^{1}$

1 University of Auckland- New Zealand

Patient-centred medicine is the idea that a patient's preferences and values should be taken into consideration when providing medical care or during 
the decision-making process. However, in economically motivated medicine brand changes, it is easy to lose sight of the patient and this can lead to frustration, worry and poor health outcomes for the patient. The purpose of this study was to investigate the situational and individual factors that increase patient concern about medicine brand switches and to examine the consequences of greater concern on patient outcomes. The study was conducted in New Zealand during an antidepressant brand switch in 2017. Participants were 319 patients taking either the branded or generic version of venlafaxine prior to a compulsory switch to a new generic version of the medication. Participants were invited to complete an anonymous online questionnaire about perceptions and experiences of the brand change. Questionnaire measures included demographic information, sources of switch information, medicine perceptions, perceived sensitivity to medicines and number of side effects. The results showed that patients who had a greater perceived sensitivity to medicines, lower trust in pharmaceutical agencies and were informed of the brand switch by someone other than a General Practitioner had a greater degree of switch concern, $\mathrm{F}(11,278)=4.48, \mathrm{p}<.001$. In turn, greater concern was associated with patients rating the new generic medicine as being less effective, $\mathrm{F}(12,269)=7.61, \mathrm{p}<.001$, and reporting a greater number of side effects from the new generic, $F(12,277)=2.11, p=.016$. Aspects of how the medicine brand change was managed and characteristics of the patient sample can foster greater concern about medicine switches. Greater concern has a flow on effect of not only influencing patients' self-rated efficacy of the new generic but also the number of side effects they experience. Fortunately, patient concern is something that can be modified. Ensuring doctors discuss medicine switches with their patients could lower concern and interventions that target medicine beliefs or increase patients' trust in pharmaceutical agencies could also be beneficial to lessen the negative effects of brand switches.

CORRESPONDING AUTHOR: Kate MacKrill, University of Auckland,kmac681@aucklanduni.ac.nz

\section{P122}

EXPERIENCES OF TRANSGENDER PRISONERS AND THEIR KNOWLEDGE, ATTITUDES AND PRACTICES REGARDING SEXUAL BEHAVIOURS AND HIV/STIS: A SYSTEMATIC REVIEW Amy Mullens ${ }^{1}$, Annette Bromdal ${ }^{2}$, Tania Phillips ${ }^{1}$, Jeff Gow ${ }^{3}$

${ }^{1}$ University of Southern Queensland, School of Psychology and Counselling, Institute for Resilient Regions - Australia

${ }^{2}$ School of Linguistics, Adult and Specialist Education- Australia

${ }^{3}$ University of Southern Queensland, School of Commerce- Australia

Introduction Despite transgender people recently gaining legal recognition within the prison systems around the world, research suggests that they are at higher risk of experiencing sexual violence compared to other prisoners and fear for their safety. Research also suggests that transgender inmates experience harassment, and physical/sexual assault by prison staff who lack transgender-specific health knowledge. There exist no systematic reviews exploring (1) What transgender prisoners' lived experiences are in different corrective sites and their knowledge, attitudes and practices regarding sexual behaviours and sexual health. Similarly, there is little understanding and no systematic reviews examining (2) What prison officers' knowledge and attitudes are towards transgender prisoners, and their sexual practices and health within sites housing transgender prisoners. This paper explores this lack of understanding for both cohorts through a recent systematic review. Methods The review followed the PRISMA guidelines and searches were conducted in four databases for the period of January to August 2017. Studies were assessed against predetermined inclusion and exclusion criteria. Included studies were peer-reviewed, written in English with online full-text availability and reported data on transgender offender outcomes relevant to one of two research questions. Results Thirteen studies met review for criteria. Transgender prisoners reported a range of challenges within correctional environments, based on reported lived experiences (e.g., sexual assault, discrimination, stigma, harassment, mental health issues) and information regarding sexual health and HIV/STI (including lack of access to genderrelated, sexual $/$ mental health services). Concordance was generally found from findings among prison staff. Conclusions The lived experience of transgender prisoners is almost uniformly more difficult than other prisoners. This 'otherness' is used as a weapon against them by fellow prisoners through violence and intimidation and by prison officers through neglect and ignorance. Prison officer skills and training in managing transgender prisoners is close to absent. This is an urgent need in prison systems if the current mistreatment of transgender prisoners is to be reduced.

CORRESPONDING AUTHOR: Amy Mullens, University of Southern Queensland, amy.mullens@usq.edu.au

\section{P123}

CHARACTERISTICS OF EARLY ADOPTERS OF HIV POINT OF CARE TESTING (POCT) AT LATE-NIGHT SEX ON PREMISES VENUE (SOPV) THEME PARTIES

Amy Mullens ${ }^{1}$, Kirstie Daken ${ }^{1}$, Jime Lemoire ${ }^{2}$, Charlotte Brownlow ${ }^{1}$, Jeff Gow ${ }^{3}$

${ }^{1}$ University of Southern Queensland, School of Psychology and Counselling, Institute for Resilient Regions - Australia

${ }^{2}$ Queensland Positive People, RAPID Clinic- Australia

${ }^{3}$ University of Southern Queensland, School of Commerce- Australia

Introduction \& Purpose Sexual theme parties have become popular among MSM (men who have sex with men). They are more commonly attended by sexually adventurous MSM, are associated with substance use, and may heighten HIV/STI transmission. HIV point of care testing (PoCT) during late-night SOPV theme parties represents a novel advance. PoCT that is peer-led with Rapid results, can reduce barriers to testing among subsets of MSM. This mixed-methods project aims to: (1) establish proof of concept, (2) evaluate the uptake and feasibility/acceptability of routine HIV/STI testing for MSM during theme parties, and (3) identify beliefs/perceptions regarding this health promotion (testing patterns, preferences) and venue (including substance use, sexual activity and harm reduction); from the perspectives of patrons and peer-testers. Methods Weekly HIV/STI PoCT was provided by trained peer-testers on Friday/ Saturday nights from 9pm-midnight, in a private room at the SOPV. Patrons requesting tests $(\mathrm{N}=207)$ were asked to complete an evaluation survey post-testing. Descriptive analyses were performed to identify socio-demographic characteristics of early adopters. Chi-square analyses examined associations between socio-demographic factors and testing patterns and preferences (e.g., whether patrons would have had a test elsewhere if the venue testing had not been available). Follow up phone interviews $(n=9)$ with patrons $(n=4)$ and peer-testers $(n=5)$ provided further qualitative data to explore survey response themes. Results Over half completed the (optional) post-testing survey, with 12 reactive HIV and 5 reactive STI tests; with higher detection rates than other PoCT settings. All those surveyed reported being "comfortable" with the community outreach testing; $96 \%$ reported peer-led testing would increase their frequency of testing; $20 \%$ 'would not have had a test' if the service did not exist (including 2 reactive HIV; 5 STI in this sub-group); and 34\% reported to have 'never had' an HIV test. Qualitative analyses revealed themes shared by patrons and peer-testers reflected 'the importance of the testing venue' and 'cultural context of the service'. Sub-themes reflected convenience of and barriers to the testing environment, non-judgmental context and professional knowledge of testers, trust and privacy, and a need to break down negative attitudes. Conclusions This project has demonstrated SOPV Rapid PoCT technologies foster high rates of opportunistic HIV/STI testing uptake amongst MSM in a high-risk setting and provides key insights into the perception and experiences of testing within harderto-reach and higher-risk MSM subgroups, resulting in high satisfaction, 
with demonstrated acceptability, feasibility and proof of concept.

CORRESPONDING AUTHOR: Amy Mullens, University of Southern Queensland, amy.mullens@usq.edu.au

\section{P124}

DEVELOPMENT OF A LIFESTYLE INTERVENTION TO ADDRESS BINGE EATING AND PROMOTE LIFESTYLE CHANGES AMONG WOMEN

Brooke Palmer $^{1}$, Virginia Gil-Rivas ${ }^{1}$, Alyssa Minnick ${ }^{1}$, Alyssa Vela ${ }^{1}$, Fary Cachelin ${ }^{12}$

${ }^{1}$ University of North Carolina, Charlotte- USA

${ }^{2}$ University of East London- United Kingdom

Introduction \& Purpose: Our program of research seeks to adapt empirically supported interventions for binge eating disorder (BED) and health behavior change for use with various populations. Binge eating is a common symptom of disordered eating, especially in women, that is associated with negative physical and mental health outcomes. Therefore, identifying and adapting interventions for a wider audience is critical to improve public health among women worldwide. The current aim was to create an intervention to reduce binge eating behaviors among women, while also increasing fruit and vegetable consumption and physical activity. Methods: Intervention development began by identifying crucial components of two cognitive behavioral therapy (CBT)-based interventions aimed at increasing women's self-efficacy and skills to change health behaviors (LEARN Manual for Weight Control; Brownell, 2004) and reduce being eating (Overcoming Binge Eating; Fairburn, 2008). Using Fairburn's six-step process as the basis of the intervention, components of Brownell's manual that address increasing fruit and vegetable consumption and physical activity were integrated into a 12 -week guided self-help protocol that includes eight support sessions conducted via telephone by trained research assistants. Results: Thirteen women $(M=26.4$ years old; $\mathrm{SD}=10.7$ ) with self-identified overeating completed the modified 12-week intervention. Overall, participants were very satisfied with the program; $100 \%$ reported excellent $(77 \%)$ or good quality $(23 \%)$ and $77 \%$ reported that the skills helped to manage their problems "a great deal". Preliminary results demonstrate a significant reduction in binge eating episodes. Reductions in frequency of binge eating at baseline (6.17, $\mathrm{SD}=5.57)$ and follow-up (2.5 episodes on average, $\mathrm{SD}=3.29 ; \mathrm{t}$ $=3.12(11), \mathrm{p}<.05)$ were reported. Conclusions: Results indicated that the modified intervention is both acceptable and effective for reducing binge eating behaviors. Also, women reported increases in physical activity and fruit and vegetable consumption. Guided self-help programs can be effective and increase treatment accessibility for groups frequently underserved. Importantly, community members could be easily trained to deliver the intervention and increase dissemination of the intervention in a variety of settings.

CORRESPONDING AUTHOR: Brooke Palmer, University of North Carolina Charlotte, bpalmer9@uncc.edu

\section{P125}

WEIGHT (MIS)PERCEPTIONS AMONG COLLEGE-AGED MEN OF VARIOUS RACIAL/ETHNIC BACKGROUNDS

Alyssa Minnick ${ }^{1}$, Brooke Palmer ${ }^{1}$, Alyssa Vela ${ }^{1}$, Virginia Gil-Rivas ${ }^{1}$, Fary Cachelin ${ }^{2}$

${ }^{1}$ University of North Carolina, Charlotte- USA

${ }^{2}$ University of East London- United Kingdom

Introduction: According to one study of 22 countries, approximately $25 \%$ of college-aged men are overweight or obese. Given that obesity is associated with poorer mental health and chronic health outcomes, it is important to identify factors that may influence treatment seeking. Research suggests that there may be racial/ethnic differences in treatment-seeking, possibly due to misperceptions of weight status; however, extant literature provides mixed support for this explanation. Methods: The current study examined the accuracy of college-aged men's $(\mathrm{N}=486)$ perception of the "overweight" and "obese" categories (based on BMI standards) using an ethnically-neutral figure rating scale. Results: Only $5.6 \%$ and $19.5 \%$ of college men correctly identified the minimum body size that reflected an "overweight" (BMI = 25) and an "obese" (BMI = 30) weight statuses, respectively. For both overweight and obese weight statuses, participants indicated a minimum body size that is larger than the BMI standards, suggesting that these men may not recognize obesity. White men and men from minority racial/ethnic backgrounds did not differ significantly on their accuracy of weight perceptions for an obese status, $\chi 2=0.29$, df $=1, \mathrm{p}=.59$; however, men from racial/ethnic minority backgrounds were slightly more accurate than White men, $x^{2}=3.58, \mathrm{df}=1, \mathrm{p}=.06$. Conclusions: Overall college men, of various racial/ethnic backgrounds, are not accurate in their perceptions of weight status. These inaccurate perceptions may influence their motivation to engage in lifestyle changes to reduce their risk for obesity/overweight and associated health conditions. Behavioral medicine should implement programs to promote accurate weight perceptions among young men which may increase motivation for health behavior change and treatment-seeking.

CORRESPONDING AUTHOR: Alyssa Minnick, University of North Carolina, Charlotte, aminnic1@uncc.edu

\section{P126}

DIGITAL PATHS TO CHANGING PROBLEMATIC ALCOHOL USE: EFFECTIVENESS OF UNGUIDED AND GUIDED INTERVENTIONS IN A STEPPED CARE MODEL

Anne H Berman ${ }^{1}$, Chris Sundström ${ }^{1}{ }^{2}$, Kristina Sinadinovic ${ }^{1}$, Mikael Gajecki $^{1}$, Magnus Johansson ${ }^{1}$,

Claes Andersson ${ }^{3}$

${ }^{1}$ Karolinska Institutet- Sweden

${ }^{2} \mathrm{CAMH}$

${ }^{3}$ Malmö University- Sweden

Introduction \& Purpose: Digital interventions for changing problematic alcohol use have shown small effect sizes in relation to control groups. A meta-analysis (Riper et al., 2014) found an overall effect size of 0.20 , with slightly higher effect sizes of 0.23 for interventions with a human guide, compared to 0.20 for unguided interventions. This presentation describes five different interventions, from unguided low-intensity to high-intensity guided interventions, evaluated in separate randomized controlled trials (RCT). Methods: Target groups included internet help-seekers and university students, with hazardous drinking according to the Alcohol Use Disorders Identification Test (AUDIT), excessive drinking based on national public health guidelines, or diagnosed alcohol use disorder (AUD). Study 1 evaluated eScreen.se, offering minimal screening and personal feedback, and alkoholhjalpen.se, a self-help program, with 633 internetbased participants reporting hazardous drinking. Study 2 evaluated the PartyPlanner and Promillekoll smartphone apps with 1932 university students reporting hazardous drinking. Study 3 evaluated the TeleCoach skills-based app with 186 university students who drank excessively. For studies 1-3 assessment-only controls were comparison groups. Study 4 compared the unguided eChange 10 -week program to a guided version with 80 internet-based participants having at least hazardous use. Study 5 with 166 participants compared the high-intensity ePlus 13-week program to the unguided eChange program in a 13-week version, and a small wait-list control group. Results: Studies 1-5 are compared with one another in terms of baseline characteristics and results. Although inclusion criteria varied, baseline AUDIT levels out of a maximum of 40 points for studies $1-5$ respectively were 20.82 (SD 6.93), 10.55 (3.90); 13.46 (4.69); 21.00 (4.90) and 23.70 (1.40). Within-group and between-group results are compared, showing greater effects for more intensive interventions. 
Conclusions: Effects vary by target groups, severity levels and interventions, but it is clear that digital interventions contribute to reduced problematic alcohol use.

CORRESPONDING AUTHOR: Anne H Berman, Karolinska Institutet, anne.h.berman@ki.se

\section{P127}

CULTURAL COMPETENCE IN HEALTH CARE: OPENING DOORS AND IMPROVING HEALTHCARE DELIVERY

Miguel Perez ${ }^{1}$

${ }^{1}$ Fresno State- USA

Cultural competence is designed to address patient health care needs taking into account their cultural backgrounds and practices. Culturally competence health care delivery at the individual and institutional level improves patient outcomes, patient satisfaction, and insures better adherence to standards of care. Aim: The purpose of this presentation is to explore the need for cultural competence among health care providers and to promote institutional assessments designed to identify the training needs of health care providers in terms of knowledge, participation in cultural awareness activities, perception of the work environment as culturally competent, and perception of culturally related barriers. Methodology: This presentation will utilize a case study format to explore the need and benefits of cultural competence. In addition, tools designed to measure institutional commitment to cultural competence will be discussed. Results: Cultural competence is a difficult road to navigate for individuals and organizations with little experience in this area. It is expected that the information and tools discussed in this presentation will enable the audience to assess their need and commitment for cultural competence. Conclusions: Improving health care outcomes is a primary concern for health care providers and institutions. A welcoming culturally competent health care workforce has been found to assist in meeting those goals.

CORRESPONDING AUTHOR: Miguel Perez, Fresno State, mperez@mail.fresnostate.edu

\section{P128}

VIEWS OF CLIENTS OF A COMMUNITY MENTAL HEALTH SERVICE TOWARDS PREVENTIVE CARE AND IMPROVING RISK BEHAVIOURS

Caitlin Fehily ${ }^{1}$, Kate Bartlem ${ }^{1}$, John Wiggers ${ }^{1}$, Lauren Gibson ${ }^{1}$, Ellen Browning ${ }^{1}$, Jenny Bowman ${ }^{1}$

${ }^{1}$ University of Newcastle- Australia

Introduction \& purpose: People with a mental illness have a high prevalence of health risk behaviours, including: smoking, poor nutrition, harmful alcohol consumption and physical inactivity; yet these behaviours are sub-optimally addressed in mental health service consultations. Barriers to providing preventive care to address such risk behaviours within mental health services include a perception that clients do not want to change their behaviours nor receive support to do so. This study examined the views towards risk behaviours and preventive care among clients of a mental health service. Methods: Participants $(n=377)$ were adult clients who attended one community mental health service in Australia over six months. Telephone interviews measured clients' perceived importance, confidence and interest in changing the four key risk behaviours; the acceptability of receiving preventive care (assessment, advice and referral) from the mental health service; and satisfaction with any preventive care that was received. Results: Preliminary results indicate that a considerable proportion of clients with risk behaviours reported currently thinking about changing their behaviours (ranging from $42 \%$ for alcohol to $66 \%$ for physical activity), and high importance of changing (ranging from $27 \%$ for nutrition to $61 \%$ for smoking. Proportions reporting high confidence in changing were lower, ranging from 17\% (smoking) to 55\% (alcohol). Clients reported high acceptability of preventive care provision: the percentage agreeing that the service should provide such care was $95 \%$ for assessment, $90 \%$ for advice and $92 \%$ for referral. The majority of clients who reported receiving preventive care from the mental health service stated that they were satisfied (assessment 64\%; advice 64\%; referral $84 \%$ ), and that it had helped them to improve their risk behaviours (80\%). Conclusions: People with a mental illness are interested in changing their risk behaviours and perceive preventive care provision by a mental health service to be highly acceptable. Moreover, when preventive care has been provided clients report high levels of satisfaction with this care. These findings reinforce recommendations for mental health services to routinely and systematically provide address clients' risk behaviours.

CORRESPONDING AUTHOR: Caitlin Fehily, University of Newcastle, caitlin.fehily@uon.edu.au

P129

AWARENESS AND USE OF TELEPHONE BEHAVIOUR CHANGE SERVICES AMONG CLIENTS OF A COMMUNITY MENTAL HEALTH SERVICE

Caitlin Fehily ${ }^{1}$, Kate Bartlem ${ }^{1}$, John Wiggers ${ }^{1}$, Lauren Gibson ${ }^{1}$, Natalie Hancox $^{1}$, Jenny Bowman ${ }^{1}$

${ }^{1}$ University of Newcastle- Australia

Introduction: The prevalence of health risk behaviours (including smoking, poor nutrition, harmful alcohol consumption and physical inactivity) among people with a mental illness is considerably higher than that of the general population. Evidence-based telephone services are available in Australia to support the community in making changes to smoking (the "Quitline") and alcohol, nutrition and physical activity (the "Get Healthy Information and Coaching service"); yet awareness and use of such telephone services by people with a mental illness is largely unknown. Method: Participants ( $\mathrm{n}=377)$ were adult clients who attended one community mental health service in Australia over a six month period. Telephone interviews measured participants' awareness and use of the Quitline and Get Healthy Information and Coaching telephone service. Preliminary results are reported. Results: Awareness and use of Get Healthy Information and Coaching service (16\% and 5\%, respectively) was considerably lower than that of the Quitline (89\% and 20\%, respectively). Of those who had heard of the telephone services, television was the most commonly reported means (74\% Get Healthy; 38\% Quitline). For both services, the majority of participants did not know that the service was free $(65 \%$ Get Healthy; $57 \%$ Quitline), nor what type of services it offered (73\% Get Healthy; $61 \%$ Quitline). $42 \%$ of participants did not know what behaviours Get Healthy provides support for. Conclusions: Use of the Quitline and Get Healthy Information and Coaching service among clients of a community mental health service is low, with awareness and use of the Get Healthy service being particularly low. These results suggest that the potential support for behaviour change that these services might offer people with a mental illness is not being realised. Research is needed to identify strategies which might promote their use.

CORRESPONDING AUTHOR: Caitlin Fehily, University of Newcastle, caitlin.fehily@uon.edu.au

P130

IDENTIFYING EFFECTIVE PERSUASIVE MESSAGES FOR VACCINATION HESITANCY: A PROTOCOL

Emily Kothe ${ }^{1}$, Mathew Ling ${ }^{2}$, Matthew Fuller-Tyszkiewicz ${ }^{3}$, Barbara Mullan $^{4}$

${ }^{1}$ Deakin University- Australia 
${ }^{2}$ Misinformation Lab, School of Psychology, Deakin UniversityAustralia

${ }^{3}$ School of Psychology, Deakin University- Australia

${ }^{4}$ School of Psychology and Speech Pathology, Curtin UniversityAustralia

Public misinformation about science threatens public health. This project will systematically test the impact of theory-informed messages designed to target different determinants one area in which misinformation is common, vaccination. This will provide crucial insight into how to combat misinformation regarding this vital public health issue without provoking backfire and will help to design brief interventions suitable for large-scale rollout. We will use an experimental design to systematically investigate the impact of persuasive messages that are tailored to components of Protection Motivation Theory (PMT) in a two-phase study. In phase 1, candidate messages for each PMT construct will be piloted. Participants $(n=480)$ will be randomised to receive a single candidate message and the impact of each message on the construct it targets will be assessed using a 4 (message) x 6 (PMT construct) design. In phase 2, participants $(n=1890)$ will be randomised to one of 7 conditions ( 6 intervention, 1 control). The impact of the message on protection motivation (primary outcome) and all PMT constructs will be evaluated.

CORRESPONDING AUTHOR: Emily Kothe, Deakin University, ekothe@deakin.edu.au

\section{P131}

A RANDOMIZED CONTROLLED TRIAL EVALUATING EFFICACY OF PROMOTING VOLUNTARY MEDICAL MALE CIRCUMCISION AMONG MALE SEXUALLY TRANSMITTED DISEASES PATIENTS IN CHINA

Joseph Lau', Zixin Wang ${ }^{2}$, Ligang Yang ${ }^{3}$, Hui Jiang ${ }^{2}$, Shujie Huang ${ }^{3}$, Chun $\mathrm{Hao}^{4}$

${ }^{1}$ The Chinese University of Hong Kong- Hong Kong

${ }^{2}$ Centre for Health Behaviours Research, JC School of Public Health and Primary Care, Faculty of Medicine, The Chinese University of Hong Kong, Hong Kong SAR

${ }^{3}$ Dermatology Hospital, Southern Medical University, Guangzhou- China ${ }^{4}$ School of Public Health, Sun Yat-sen University, Guangzhou- China

Introduction \& purpose: Male sexually transmitted diseases patients (MSTDP) form a bridge population transmitting HIV/STD from high risk population to lower risk female general populations in China. Voluntary medical male circumcision (VMMC) is an evidence-based biomedical HIV prevention but under-utilized by MSTDP. This study was to evaluate the relative efficacy of a brief theory-based and interactive intervention (a health promotion video plus brief counseling provided by clinicians) in increasing uptake of VMMC within a 6-month follow-up period, as compared to the control group. Methods: A parallel-group, non-blinded randomized controlled trial was conducted. Participants were uncircumcised heterosexual MSTDP attending the four collaborative STD clinics in three Chinese cities. Those who were known to be HIV positive were excluded. After completion the baseline face-to-face interview, 244 participants were randomized 1:1 into the intervention group $(n=108)$ and the control group $(\mathrm{n}=136)$. In addition to the health education booklets received by the control group, the intervention group watched a 10-minute video and received a brief counseling delivered by the clinicians. A total of 76 participants $(70.4 \%)$ in the intervention group and 84 participants $(61.8 \%)$ in the control group completed the telephone follow-up at Month 6. Results: Between-group differences for baseline background characteristics and potential confounders were statistically non-significant. Among those being followed up at Month 6, the intervention group reported higher prevalence of VMMC uptake within the last six months (21.1\% vs. $4.8 \%$, RR: $4.42,95 \%$ CI: $1.54,12.64$; ARR: $16.3,95 \%$ CI: 6.1 , $26.5 ; \mathrm{p}=0.002$ ), as compared to the control group. Process evaluation of the health promotion was positive. Among circumcised participants $(n=20)$, only 6 reported minor surgical complications (i.e., edema, inflammation and oozing of blood); 8 reported that they had reduced frequency of condom use with their regular female sex partners following VMMC. Conclusions: The brief intervention is effective and potential sustainable. It can be integrated into the routine service provided by the STD clinics. Such promotion should take prevention of risk compensation into account.

CORRESPONDING AUTHOR: Joseph Lau, The Chinese University of Hong Kong, johnsonstick2000@163.com

\section{P132}

LEADERSHIP DEVELOPMENT TRAINING NEEDS AMONG PSYCHOLOGISTS IN US HEALTH SETTINGS

Helen Coons ${ }^{1}$, Kathleen Brown ${ }^{2}$, Zeeshan Butt ${ }^{3}$, Wendy Ward ${ }^{4}$, Jennifer Vasterling ${ }^{5}$

${ }^{1}$ Health Psychology Solutions- USA

${ }^{2}$ Independent Consultant

${ }^{3}$ Northwestern University Feinberg School of Medicine- USA

${ }^{4}$ UAMS College of Medicine- USA

${ }^{5}$ VA Boston Healthcare System- USA

Leadership Development Training Needs Among Psychologists in US Health Settings Helen L. Coons, Ph.D., ABPP,1 Kathleen S. Brown, Ph.D.,2 Zeeshan Butt, Ph.D., ABPP,3 Wendy L. Ward, Ph.D.,4 ABPP, and Jennifer J. Vasterling, Ph.D.5 1University of Colorado School of Medicine, Aurora, CO, USA, 2Independent Consultant, Fort Myers, FL, USA, 3Northwestern University Feinberg School of Medicine, Chicago, IL USA, 4UAMS College of Medicine, Little Rock, AR USA, and 5VA Boston Healthcare System Introduction and purpose: This presentation will showcase findings from a 2017 survey of leadership development training needs among psychologists in US academic and community medical centers, hospitals and health settings. The last four decades have witnessed a marked increase in both the number of psychologists working in pediatric and adult health settings, and those advancing to leadership roles. We have limited information, however, on leadership training needs among psychologists across the career trajectory in behavioral medicine and health care. Methods: Participants completed an online survey about their interest in leadership roles, barriers to advancing to leadership positions, interest in leadership development training as well as the topics, structure and timing for leadership training programs. They rated their interest in leadership issues across five categories: communication, personnel management, administrative management, professionalism, educational leadership. Sample recruitment was conducted via the list serves of health-related Divisions of the American Psychological Association. Approximately 566 respondents initiated the survey and 311 completed the questions. Roughly 55\% worked in US academic health settings, $14 \%$ in community hospitals or health systems, $10 \%$ at VA facilities, and $4 \%$ at rehabilitation facilities. Results: Nearly $70 \%$ of the participants were interested in leadership advancement in their health setting, $22 \%$ were not sure and $8 \%$ were not interested in leadership roles. Over $55 \%$ were unsure of the pathways to leadership advancement, while $46 \%$ felt that their psychology degree was a limiting factor to leadership positions in a medical environment. Over $85 \%$ of the sample were interested in leadership development training. The presentation will highlight their preferences for the leadership training content, structure, mechanisms and timing during the day. Conclusions: Psychologists in US health care settings who participated in this on-line survey readily expressed interest in advancement to leadership roles in their health organizations, communicated a range of barriers to those positions and are extremely interested in leadership training.

CORRESPONDING AUTHOR: Helen Coons, Health Psychology Solutions, hcoons@comcast.net 
P133

FIRST CONSENSUS OF CHILEAN BARIATRIC PSYCHOLOGISTS ABOUT MENTAL HEALTH MANAGEMENT PRE AND POSTBARIATRIC SURGERY

María José Leiva Velasco ${ }^{1}$, Paula Díaz ${ }^{2}$, María Cecilia Barros ${ }^{3}$, Denisse Montt $^{4} 5$, Viviana Assadi ${ }^{3}$, Macarena Cruz ${ }^{6}$, Susana Cortés ${ }^{4}$, Luciana Marín $^{4}{ }^{6}$, Claudia Badilla ${ }^{7}$

${ }^{1}$ Clínica Las Condes- Chile

${ }^{2}$ Clínica Santa María- Chile

${ }^{3}$ Pontificia Universidad Católica de Chile- Chile

${ }^{4}$ Hospital Clínico Universidad de Chile- Chile

${ }^{5}$ Clínica Universidad de los Andes- Chile

${ }^{6}$ Clínica Alemana de Santiago- Chile

${ }^{7}$ Clínica Ciudad del Mar- Chile

Introduction \& Purpose: Bariatric surgery is the most effective and lasting alternative to weight reduction and improved general health. However, long-term weight maintenance after surgery may be challenging for some patients. Thus, multidisciplinary teams should be involved in the evaluation, treatment, and post-operative support. With the purpose of unifying criteria about mental health aspects, a scientific-based consensus was elaborated.

Methods: all members of the NPCO - Chilean Bariatric Psychologists, part of Chilean Bariatric and Metabolic Society were invited to participate, representing teams from 8 different regions throughout Chile. A questionnaire including the most important topics, such as preoperative contraindications, psychological risk factors of weight regains, postoperative support, was designed and sent to 50 national experts. When no agreement among them was achieved, the Delphi methodology was applied. Scores of $86 \%$ or greater were used for inclusion consensus. The recommendations here presented were widely discussed in several scientific meetings with active participation of all members of the group and were highly based on scientific evidence covered by the literature.

Conclusions: The main conclusions reached are that clinical findings are critical for the preoperative diagnosis and treatment adherence. The purpose of the psychological assessment has shifted from identifying contraindications for bariatric surgery and evolved towards assessing psychological and behavioral readiness. The achievement of pre-surgery preparatory behavioral changes are recommended to optimize their postoperative outcomes. Acquisition of a healthy lifestyle is the most important therapeutic tool for long-term weight maintenance. Thus, therapeutic postoperative support is fundamental. The psychologist undertaking the assessment needs specific experience and knowledge of bariatric surgery, as well as the preoperative and postoperative issues that may arise. There are specific recommendations for adolescents as this group presents special needs according to their development stage.

CORRESPONDING AUTHOR: María José Leiva Velasco, Clínica Las Condes, mjleve@gmail.com

P134

DEVELOPMENT OF TEACHING MATERIALS TO IMPROVE THE KNOWLEDGE AND SKILLS THAT MEDICAL STUDENTS SHOULD POSSESS TO PREVENT SUICIDE

Akizumi Tsutsumi $^{1}$, Hiroki Chiba ${ }^{1}$, Rika Moriya ${ }^{1}$, Akiomi Inoue ${ }^{1}$, Akihito Shimazu ${ }^{2}$

${ }^{1}$ Kitasato University School of Medicine- Japan

${ }^{2}$ Kitasato University- Japan

Introduction \& purpose Participatory training incorporating elements of behavioral science is considered an effective means of improving the skills of medical professionals to address with cases they may encounter, such as dealing with those who have attempted suicide. We tried to develop practical training that incorporates role-play. Methods In current medical education, it does not suffice to merely "know" something; medical professionals must also learn to "utilize" knowledge. Active learning-based practical training and exercises are therefore recommended. This active learning involves devising actual treatment strategies for treatment scenarios in a small group, with the objective of solving problems in role-play and hypothetical scenarios. We have thus created scenarios to make trigger video materials for active learning, where students will learn methods of interviewing patients with strong psychological burdens (familiarization, listening, support) to "empathize with the mental state of a psychologically burdened person," and dealing with people who have suicidal idealation through role-play. Results Scenario where the basics of listening closely are learned includes role-playing: a speaker is asked to freely talk about "events that were enjoyable, or pleasant, or even stressful," while a listener adopts two patterns of listening: "good listening" and "bad listening." After both the speaker and listener have reflected on how they felt about the "good listening" and the "bad listening," an interactive discussion is held between the facilitator and commentators. Scenario where psychological crisis intervention is learned includes a conversation between a woman (speaker) and her son (listener): the woman is in shock following a notification of cancer. The listener acts out two patterns of listening (also conscious of non-verbal cues): "normal (immediate problem-solving and self-centered) listening" and "crisis intervention-oriented (accepting, empathetic, supportive and mutually considerate) listening." After both the speaker and the listener have reflected on how they felt about the "normal listening" and "crisis intervention-oriented listening," an interactive discussion is held between the facilitator and commentators. Discussion While the present scenarios are basic ones with ordinary people in mind, will we investigate scenarios, such as how to handle patients with thoughts of suicide in medical settings and how they should be referred to specialist medical institutions when they visit clinics.

CORRESPONDING AUTHOR: Akizumi Tsutsumi, Kitasato University School of Medicine, akizumi@kitasato-u.ac.jp

\section{P135}

FOOD INTAKE IN OBESE PEOPLE: A RESPONSE TO STRESS OR DEFICIT IN EXECUTIVE FUNCTIONS

Mariela Gonzalez Tovar ${ }^{1}$

${ }^{1}$ Pontificia Universidad Católica de Chile- Chile

Introduction: Failures in adherence to diet programs are explained by the influence of psychological factors. From this perspective, the perspective of stress suggests that its chronicity could generate an imbalance in endocrine parameters, increasing food intake in people under a program of dietary control, which would lead to the development of obesity. It is worth noting the fact that not all people show this reactivity to stress, given that their influence on food behavior would depend, in turn, on a set of individual variables, which are fundamental to know in order to understand the relationship between stress and food intake. The present study tried to examine the mediating role of executive functions in the relationship between stress and food intake in university students. Method: An experimental design was used, this design had random assignment to two groups: an experimental group and a control group, to which a modified version of a stress induction protocol was applied, in order to manipulate the variable of perceived stress and compare both groups. The sample was non-probabilistic, intentional and consisted of 82 students from the Pontifical Catholic University of Chile, aged between 18 and 30 years $(M=21, S D=2,762)$, who decided to participate in the study under the informed consent. Results: both groups were equivalent prior to experimentation; Participants in both groups did not have high levels of stress nor cognitive and executive deficits, before being subjected to experimentation. The stress variable perceived during the experiment showed statistically significant differences between the experimental group and the control group in both activities. As for the comparative analysis of the raw scores in the variable executive functions. Only 
significant differences were obtained between the experimental group and the control group, in the gross scores of the Cognitive Flexibility variable. Finally, in the variable food intake, the experimental group had a higher intake of unhealthy foods compared to the control group. On the other hand, the stress perceived in the experimental situation designed, was associated with the intake of unhealthy foods, being a good predictor of it. Likewise, it was associated with a lower number of perseverative errors. None of the executive functions were significant predictors of unhealthy food intake. Conclusions: To understand the relationship between stress, executive functions and food intake, in university students, it is necessary to first know if there is a case of acute or chronic stress, as it is necessary to make an analogy between executive functioning and flexible coping.

CORRESPONDING AUTHOR: Mariela Gonzalez Tovar, Pontificia Universidad Católica de Chile, mgonzalez4@uc.cl

\section{P136}

GINI COEFFICIENT AS A NOVEL EVALUATOR OF SPECTRAL HEART RATE VARIABILITY INEQUALITY IN MENTAL STRESS Miguel Enrique Sánchez-Hechavarría ${ }^{1}$, Ramón Carrazana-Escalona ${ }^{1}$, Sergio de Jesús Cortina-Reyna ${ }^{1}$, Adán Andreu-Heredia ${ }^{1}$, Carlos AcostaBatista $^{2}$

${ }^{1}$ Universidad de Ciencias Médicas de Santiago de Cuba- Cuba

${ }^{2}$ Hospital Universitario "Calixto García". La Habana- Cuba

Gini coefficient is a parameter that normally is used in the economy to measure the inequality in the income inside a country, but can be used to measure any kind of unequal distribution. In the present work is shown an innovative proposal of application of the Gini Coefficient to Spectral Heart Rate Variability (HRV) like a psychophysiological indicator of mental stress. In a study crossover, 13 healthy subjects (19 $\pm 1,5$ years), intervals RR were obtained by electrocardiogram in the polygraph (AD Instruments Powerlab 8), during 5 minutes of rest and 5 minutes of response to arithmetic mental stress. The limits for the spectral HRV bands were delimited from 0.15 to $0.40 \mathrm{~Hz}$ for the high frequency band (HF), from 0.04 to $0.15 \mathrm{~Hz}$ for the low frequency band (LF), from 0.04 to 0.085 $\mathrm{Hz}$ for the first low frequency sub-band (LF1) and from 0.085 to $0.15 \mathrm{~Hz}$ for the second low frequency sub-band (LF2). Spectral Gini coefficient $(\mathrm{SpG})$ were proposed to measure the inequality in the spectral powers of the RR intervals in each spectral HRV bands employed. When comparing the rest with the mental stress was observed a decrease of HF ( $\mathrm{p}=0.046)$, a sensible increase of the heart rate $(p=0.004), L F(p=0.033)$ and LF2 $(p=0.019)$. In $\mathrm{SpG}$ were found a significant increase of Gini-LF $(p=0.009)$ and Gini-LF2 $(p=0.033)$. Which suggest that spectral inequality of the intervals RR analyzed from the Gini coefficient could be an adequate indicator of mental stress, with big potentialities in the objective assessment of the consequences of psychosomatic affections and anxiety disorders.

CORRESPONDING AUTHOR: Miguel Enrique Sánchez-Hechavarría, Universidad de Ciencias Médicas de Santiago de Cuba, miguel.sanchez881119@gmail.com

\section{P137}

TREATMENT INITIATION AMONG PEOPLE LIVING WITH HIV ( PLWH) AFTER THE LAUNCH OF EXPANDED ANTIRETROVIRAL TREATMENT (ART) STRATEGY IN CHINAFINDINGS FROM A LONGITUDINAL STUDY

Jing $\mathrm{Gu}^{1}$, Tinglong Yang ${ }^{1}$, Linghua $\mathrm{Li}^{2}$, Huifang $\mathrm{Xu}^{3}$, Chun $\mathrm{Hao}^{1}$

${ }^{1}$ Sun Yat-sen University- China

${ }^{2}$ No. Eight Hospital- China

${ }^{3}$ Guangzhou CDC- China

Introduction. China expanded her antiretroviral treatment (ART) strategy to all people living with HIV (PLWH) in 2016 right after WHO's new
ART guideline released in 2015. However, there is very limited knowledge of PLWH's actual ART up-take behavior and how ART initiation as affected by different factors. In this study, based on the health belief model (HBM), we investigated the ART initiation behavior and related factors among PLWH who were ART naïve in China. Methods. By using a prospective cohort study design, we recruited 494 PLWH who had not initiated ART during July 2016 to June 2017 from 6 communities in Guangzhou, China. The baseline information (socio-demographic variables, HIV-related characteristics, perceptions toward ART according to HBM, mental health status and social support) was collected by trained community doctors. Treatment up-take information was collected from the ART record system. Univariate and multivariate logistic regression models were applied in data analyses. Results. Of all participants, $93.8 \%$ was male and $71.1 \%$ was non-local residents; $72.4 \%$ was infected through homosexual behaviors; and $12.1 \%$ had the latest CD4 cell counts $>500$. After 6 months' follow up, 74.5\%(368/494)initiated ART. Univariate analyses showed that ART initiation was not associated with most socio-demographic and HIV-related variables but CD4 level $(\mathrm{OR}=0.48,95 \% \mathrm{CI}=0.26-0.86)$. Perceived severity $(\mathrm{OR}=1.10)$, susceptibility $(\mathrm{OR}=1.30)$, benefits $(\mathrm{OR}=1.07)$, barriers $(\mathrm{OR}=0.95)$, self-efficacy $(\mathrm{OR}=1.09)$ were all significantly associated with ART initiation $(\mathrm{p}<0.05)$. Among studied psycho-social factors, depression was significant $(\mathrm{OR}=2.20)$. In the multivariate analyses, PLWH who had higher scores in perceived benefits $(\mathrm{OR}=1.08,95 \% \mathrm{CI}=1.02-1.14)$, depression $(\mathrm{OR}=1.06,95 \% \mathrm{CI}=1.02-1.11)$ and lower scores in perceived barriers $(\mathrm{OR}=0.93,95 \% \mathrm{CI}=0.88-0.98)$ at baseline were more likely to initiate ART during the 6 months follow up period. Discussion. A significant proportion of PLWH initiated ART since the launch of expanded treatment strategy. To achieve the second $90 \%$ of the 90-90-90 strategy in HIV control, there is still space for ART promotion. Measures should be taken to improve PLWHs' confidence to overcome barriers in treatment and to enhance their beneficial perceptions. Such measure should also take into accounts of PLWHs mental health status.

CORRESPONDING AUTHOR: Jing Gu, Sun Yat-sen University, gujing0828@hotmail.com

\section{P138}

PHYSICAL ACTIVITY AMONG PARENTS OF VERY YOUNG CHILDREN: THE ROLE OF BELIEFS AND SOCIAL-COGNITIVE FACTORS

Katherine White $^{1}$, Eloise Cowie ${ }^{1}$, Kyra Hamilton ${ }^{2}$

${ }^{1}$ Queensland University of Technology- Australia

${ }^{2}$ Griffith University- Australia

Introduction and purpose: Despite the unequivocal benefits of regular physical activity, many parents engage in lower levels of physical activity (PA) following the birth of a child, a period in which PA can assist in buffering against postnatal depression. Drawing on the theory of planned behaviour (TPB) and health action process approach (HAPA), an integrative model was developed to examine variables predicting PA in parents of very young children (child under the age of 1 year). In addition, key beliefs related to PA intentions and behaviour were investigated. Methods: A prospective correlational design with two waves of data collection, spaced 1 week apart, was adopted. Initially, formative qualitative research among the target population $(\mathrm{N}=20$ parents) identified relevant underlying beliefs to inform survey content. For the main study, parents of a child under the age of 1 year $(\mathrm{N}=297)$ completed an online or paper-based questionnaire assessing TPB constructs (intention, attitude, subjective norm, perceived behavioural control, belief-based items), and family social support and planning from the HAPA. One week later, parents self-reported their PA behaviour. Data were analysed using latent variable structural equation modelling. Results: Findings revealed the proposed model was a good fit to the data, accounting for $62 \%$ and $27 \%$ of the variance in PA intention and behaviour, respectively. 
Attitude, subjective norm, and perceived behavioural control predicted intention. Family social support did not predict either planning or intention. Physical activity was predicted by planning only, with an indirect effect from intention to behaviour via planning. The effect of a number of key beliefs, focusing on time, fatigue, and motivation, were identified also. Conclusions: This research among an at-risk cohort for inactivity and negative mental health provides further understanding of the factors that influence the PA behaviour of parents of very young children, enabling theory-based targets for intervention. The findings support using integrative theoretical approaches for a comprehensive understanding of complex health behaviours, especially the addition of a volitional phase to motivational theories when there are substantial barriers to behavioural enactment.

CORRESPONDING AUTHOR: Katherine White, Queensland University of Technology, km.white@qut.edu.au

\section{P139}

HOW US PRIMARY CARE PRACTICES ARE USING THE MEDICARE INTENSIVE BEHAVIORAL THERAPY FOR OBESITY PAYMENT CODE

Jodi Holtrop ${ }^{1}$, Zhehui Luo ${ }^{2}$, Hanyue $\mathrm{Li}^{2}$, Mark Gritz ${ }^{1}$, Rowena Dolor ${ }^{3}$, Laurie Fitzpatrick ${ }^{2}$, Lauri Connelly ${ }^{1}$, Phoutdavone Phimphasone-Brady ${ }^{1}$

${ }^{1}$ University of Colorado- USA

${ }^{2}$ Michigan State University- USA

${ }^{3}$ Duke University- USA

Introduction and Purpose: In November 2011, the Centers for Medicare and Medicaid Services approved the use of a new Healthcare Common Procedure Coding System code in primary care for providing Intensive Behavioral Therapy (IBT) services for obese patients. Despite the widespread prevalence of obesity in the Medicare population, use of this benefit has been substantially lower than expected suggesting significant underutilization of this service (estimated at less than 1\%). The study purpose is to develop an understanding of the most pressing issues related to the use and non-use of the Medicare IBT for obesity in primary care and the strategies used in practices that have successfully implemented services in accordance with this benefit. Methods: The initial phase involves identifying practices that have used the IBT benefit based on primary care providers submitting claims for $>10$ Medicare beneficiaries as reported in the Medicare Provider Utilization and Payment Data: Physician and Other Supplier Public Use Files for calendar years 2012-2015. The subjects are from 14 US states in four regions. Written surveys and telephone interviews were completed with providers and staff in the identified practices. Descriptive statistics and qualitative thematic analysis were used to analyze the results. Results: Thematic analysis revealed common patterns to use of the IBT benefit: at least one provider with a passion for obesity management, willingness to try use of the code even in the face of barriers (including low levels of reimbursement), and financial flexibility to augment the IBT program. Several models of care delivery emerged (i.e. individual visits vs group classes), differing programmatic approaches (i.e. ketogenic diet vs healthy lifestyle approach), and delivery by different staff members (i.e. physicians, nurse practitioners, registered dietitians). Other approaches to obesity management included providing services outside of the bounds of the IBT benefit (e.g. becoming a coach for a weight loss company or program). Conclusions: IBT for obesity is not offered commonly, as it poses many barriers to use. However, some practices are finding creative ways to attend to obesity management which might be replicable for others if these successes can be shared.

CORRESPONDING AUTHOR: Jodi Holtrop, University of Colorado, jodi.holtrop@ucdenver.edu
P140

PSYCHOMETRIC PROPERTIES OF THE ANXIETY SENSITIVITY INDEX (ASI) IN CHILEAN PREGNANT WOMEN

Laura Rodriguez $^{1}$, Tomás Arriaza ${ }^{1}$, Gonzálo Gutierrez ${ }^{1}$, Lydia Gómez ${ }^{1}$

${ }^{1}$ Pontificia Universidad Católica de Chile- Chile

Psychometric properties of the Anxiety Sensitivity Index (ASI) in Chilean Pregnant Women Rodríguez, L.1, Arriaza, T.1, Gutiérrez, G.1, \& Gómez-Pérez, L.1 1Pontificia Universidad Católica de Chile Background: Billions of women give birth every year in the world. Between $10 \%$ and $18 \%$ of them may experience postpartum chronic pain. Anxiety sensitivity (AS) seems to be involved in the transition from acute to chronic pain. It refers to the fear of physical sensations related to anxiety due to the belief that these sensations can have potentially harmful somatic, psychological, or social consequences. AS has shown to be positively associated with labor pain; however, studies about the influence of AS in postpartum pain in the long term remain to be conducted. The Anxiety Sensitivity Index (ASI) has been widely used to assess AS because of its brevity. However, its psychometric properties among pregnant women have not been examined. Furthermore, it has not been validated for its use in Chile. Objectives: To examine the psychometric properties of the ASI in a sample of Chilean pregnant women, and whether AS predict pain disability one year postpartum. Method: Women $(\mathrm{N}=$ 111) -attended in two medical centers (Centros Médico Lira and Jan Joaquín, Red Salud UC Christus) in Santiago, Chile - completed the ASI when they were between weeks 32 and 37 of gestation. One year postpartum they completed the Brief Pain Inventory. Results: According to the results of confirmatory factor analyses, a two-factor model presented the best fit $\left(\mathrm{RMSEA}=.061 ; \mathrm{CFI}=.976 ; \mathrm{TLI}=.97 ; \chi^{2} / \mathrm{g} .1 .=1.418\right)$. Both factors, Fear of physical consequences and fear of psychological consequences presented adequate internal consistence. Cronbach alpha were .81 and .70 , respectively. The results of a regression analyses showed that Fear of psychological consequences significantly predicted pain interference one year postpartum $(\mathrm{F}=7.911 ; \mathrm{p}=.001 ; \mathrm{R} 2=.217)$. The ASI factors added predictive capacity to the model in relation to the sociodemographic variables $(\Delta R 2=.172 ; p=0,004)$, which indicated incremental validity. Conclusions: The ASI presented adequate psychometric properties in a sample of Chilean pregnant women and it was able to predict pain interference one year postpartum. Future studies about the utility of this variable to predict perinatal health are needed.

CORRESPONDING AUTHOR: Laura Rodriguez, Pontificia Universidad Católica de Chile, lcrodriguez@uc.cl

\section{P141}

WHAT CHARACTERIZES INDIVIDUALS WHO PREFER BRANDED OVER GENERIC MEDICINE?

Maria Kleinstaeuber $^{1}$, Kate MacKrill ${ }^{1}$, Keith J. Petrie ${ }^{1}$

${ }^{1}$ University of Auckland- New Zealand

Introduction \& purpose: Although generic medicines provide a safe and an economical treatment for many kinds of illnesses, negative attitudes towards generics are common. In order to understand these negative views better the current study examines individuals preferring branded drugs in comparison to individuals preferring generic drugs or having no preference with regard to sociodemographics, perceived sensitivity to medicines and other clinical variables. Methods: A representative general population sample $(\mathrm{N}=1000)$ was recruited using random digit dialing. In a telephone interview participants were asked about their preference of innovator versus generic drugs, perceived sensitivity to medicines, recently perceived symptoms, number of GP visits, health related information seeking and currently prescribed medicines. Results: Independent sample t-tests showed that the individuals preferring branded medication had lower levels of formal education, higher perceived sensitivity to medicines, more somatic symptoms, higher symptom severity, and more GP 
visits. There were no differences on whether they were currently prescribed with drugs or their medicine-related information seeking behavior. Conclusions: Clinicians should be aware that preference for branded medicine is higher in patients with lower formal education, high perceived sensitivity to medicine, a higher level of symptom burden, and with a higher level of health care utilization.

CORRESPONDING AUTHOR: Maria Kleinstaeuber, University of Auckland, m.kleinstauber@auckland.ac.nz

\section{P142}

IMPACT OF LOWER STRENGTH ALCOHOL LABELLING ON CONSUMPTION: A RANDOMISED CONTROLLED TRIAL

Milica Vasiljevic ${ }^{1}$, Dominique-Laurent Couturier ${ }^{2}$, Daniel Frings ${ }^{3}$, Antony C. Moss ${ }^{3}$, Ian P. Albery ${ }^{3}$, Theresa M. Marteau ${ }^{2}$

${ }^{1}$ University of Cambridge- United Kingdom

${ }^{2}$ Behaviour and Health Research Unit, University of Cambridge- United Kingdom

${ }^{3}$ Centre for Addictive Behaviours Research, Division of Psychology, School of Applied Sciences, London South Bank University- United Kingdom

Introduction and Purpose: Labels indicating low/light versions of tobacco and foods are perceived as less harmful which may encourage people to consume more. There is an absence of evidence concerning the impact on consumption of labelling alcohol products as lower in strength. The current study tests the hypothesis that labelling wine and beer as lower in alcohol increases their consumption. Methods: Weekly wine and beer drinkers $(n=264)$ sampled from a representative panel of the general population of England were randomised to one of three groups to taste test drinks in a bar-laboratory varying only in the label displayed; Group 1: verbal descriptor Super Low combined with $4 \%$ ABV for wine/1\%ABV for beer; Group 2: verbal descriptor Low combined with $8 \% \mathrm{ABV}$ for wine/3\%ABV for beer; Group 3: No verbal descriptors of strength (Regular). Primary outcome was total volume $(\mathrm{ml})$ of drink consumed. Results: The results supported the study hypothesis: the total amount of drink consumed increased as the label on the drink denoted successively lower alcohol strength, BLin $=.71, p=.015,[95 \% \mathrm{CI}=0.13 / 1.30]$. Group contrasts showed significant differences between those offered drinks labelled as Super Low $(M=213.77)$ compared to Regular $(M=176.85)$, $\mathrm{B}=1.43, \mathrm{p}=.019,[95 \% \mathrm{CI}=0.24 / 2.61]$. There was no significant difference in amount consumed between those offered drinks labelled as Low compared to Regular. Conclusions: These results suggest that labelling drinks as lower in strength increases the amount consumed. Further studies are warranted to test for replication in non-laboratory settings and to estimate whether any effects are at a level with the potential to harm health. Trial Registration: ISRCTN15530806

CORRESPONDING AUTHOR: Milica Vasiljevic, University of Cambridge, milica.vasiljevic@medschl.cam.ac.uk

\section{P143}

RANDOMIZED CLINICAL TRIAL OF TELEPHONEADMINISTERED INTERPERSONAL PSYCHOTHERAPY FOR DEPRESSED HIV-INFECTED RURAL PERSONS: LONG-TERM INTERVENTION OUTCOMES

Timothy Heckman ${ }^{1}$, Bernadette Heckman ${ }^{1}$, John Markowitz ${ }^{2}$, Henok Woldu $^{1}$, Timothy Anderson ${ }^{3}$, Ye Shen ${ }^{1}$, Travis Lovejoy ${ }^{4}$, Mark Sutton ${ }^{1}$, William Yarber ${ }^{5}$

${ }^{1}$ University of Georgia- USA

${ }^{2}$ Columbia University College of Physicians and Surgeons- USA

${ }^{3}$ Ohio University- USA

${ }^{4}$ Oregon Health and Science University- USA

${ }^{5}$ Indiana University- USA
Introduction and Purpose: Many of the 52,000-plus persons living with HIV in rural areas of the U.S. are in urgent need of depression treatment. This randomized clinical trial tested if telephone-administered Interpersonal Psychotherapy (tele-IPT) was efficacious in reducing depressive symptoms in 147 depressed HIV-infected rural persons over an 8-month follow-up period. Methods: Rural persons living with HIV in 28 U.S. states with comorbid depression diagnoses received either standard care $(\mathrm{SC} ; \mathrm{n}=69)$ or 9 weekly sessions of tele-IPT plus SC (tele-IPT; $\mathrm{n}=78$ ). Patients completed self-administered measures of depression (Beck Depression Inventory; BDI) at pre- and post-intervention and 4and 8-month follow-up. Patients also completed 41 weekly depression assessments (the 10-item Brief Self-Assessing Depression Scale; SADS) using interactive voice response (IVR) systems across 9 weeks of treatment and 32 follow-up weeks. Linear mixed effects models using completer-only and intent-to-treat approaches assessed intervention efficacy. Results: Intent-to-treat analyses found significant reductions in depressive symptoms in tele-IPT patients compared to SC controls at postintervention $(\mathrm{d}=.57 ; \mathrm{p}<.01), 4$-month follow-up $(\mathrm{d}=.46 ; \mathrm{p}=.05)$, and 8 month follow-up $(\mathrm{d}=.52 ; \mathrm{p}<.05)$. Tele-IPT patients also used crisis hotlines less frequently than SC controls at post-intervention and 4month follow-up ( $\mathrm{ps}<.05)$. Longitudinal analyses of weekly IVR depression data found significant depressive symptom relief in tele-IPT patients in Weeks 1 through 11 and Weeks 18 through 23 compared to SC controls but comparable depression scores at all other weeks. Conclusion: When assessed through four BDI self-administrations, tele-IPT provided shortand long-terms reductions in depression. Analyses of 41 weekly IVRbased depression assessments found short-term differences in depression between tele-IPT and SC controls that dissipated over more distal followup assessments. When conducting psychotherapy outcome analyses, the choice of assessment instrument and assessment methodology (e.g., every 4 months versus daily assessments) can influence outcome findings and subsequent clinical implications.

CORRESPONDING AUTHOR: Timothy Heckman, University of Georgia, heckman@uga.edu

\section{P144}

REDISTRIBUTION OF HEALTH BURDEN VIA SOCIAL INNOVATION: A SIMULATION STUDY OF DIFFERENT SOCIAL NETWORK TOPOLOGIES

\section{Simon K. Poon ${ }^{1}$, Mark Latt ${ }^{2}$, Peter Kim ${ }^{3}$}

${ }^{1}$ School of Information Technologies, Faculty of Engineering and IT, The University of Sydney- Australia

${ }^{2}$ Sydney Medical School, The University of Sydney- Australia ${ }^{3}$ School of Mathematics and Statistics, Faculty of Science, The University of Sydney- Australia

Introduction \& Purpose: The guardian angel concept [1] is one in which systems or individuals are responsible for monitoring and providing support to assigned individuals. In this study, an agent based model was developed to simulate to explore how individuals are affected through their levels of intrinsic positivity. A number network topologies were modelled to provide insights into the collective improvement in positivity and motivation for all participants in the network. Methods: A simulation model developed based on the concept of "emotional contagion" introduced by [2], Guardian Angel is a social innovation modelled on assisting communities in keeping motivated to reach their individual health goals. The simulation consists of two primary agents: guardians and children. We examined several network topologies using NetLogo software. Optimal performance was attained by maximizing group level motivation and positivity while exhibiting the lowest variation between nodes. Results: From a utilitarian perspective, this multi-goal objective function represents the greatest benefit for majority of users. By looking at the different network topologies in a simulated social health context, it is promising that all may provide benefit to the user. All simulated trial 
groups experienced a mean gain in the outcomes of positivity and motivation, however this does not reciprocate to all individuals benefiting from the intervention. Mean values of health outcomes do not accurately reflect disparities or distribution of resources throughout the users in each system. Conclusion: Our results highlight the need for developing grouping protocols when employing such interventions to ensure those who are inherently less motivated are connected with others in such a way that they can benefit from others motivation and positivity. Of the network models simulated, the ring topology offers the most consistent benefit to everyone as indicated by the lowest standard deviation. The standard deviation is still relatively large however and other common social network topologies should be explored in the future. Technical solution can be made through the use of mobile technologies which connect individuals to each other in a network to provide support in achieving a goal. References: 1. P. Szolovits, J. Doyle, W. J. Long, I. Kohane, and S. G. Pauker, Guardian Angel : Health Information Systems, (1994) 2. N. Christakis and J. Fowler, Connected: The Surprising Power of Our Social Networks and How They Shape Our Lives-How Your Friends' Friends' Friends Affect Everything You Feel, Think, and Do, Black Bay Books, New York, 2011.

CORRESPONDING AUTHOR: Simon K. Poon, School of Information Technologies, Faculty of Engineering and IT, The University of Sydney, simon.poon@sydney.edu.au

\section{P145}

UPTAKE OF HCV TREATMENT AND BARRIERS AMONG DRUG USERS IN METHADONE MAINTENANCE TREATMENT CLINICS IN GUANGDONG PROVINCE, CHINA

Yin Liu ${ }^{1}$, Xia Zou ${ }^{1}$, Cheng Gong ${ }^{1}$, Wen Chen ${ }^{1}$, Li Ling $^{1}$

${ }^{1}$ Sun Yat-sen University- China

Abstract Introduction \& Purpose: Despite the fact that methadone maintenance treatment (MMT) could reduce hepatitis $\mathrm{C}$ virus $(\mathrm{HCV})$ incidence, prevalence of $\mathrm{HCV}$ infection among drug users in MMT clinics was still high. Treatment for HCV was the most effective strategy to curb HCV epidemic. Here, we demonstrated the current treatment status of $\mathrm{HCV}$ among drug users in MMT clinics, and explored barriers and related factors with HCV treatment in Guangdong province, China. Methods: Crosssectional survey was conducted in 17 MMT clinics in 9 cities from June 2017 to October 2017 in Guangdong Province, China. All eligible HCV antibody positive patients were included. Data were obtained including socio-demographic characteristics, drug-use and alcohol-use related behaviors, sexual behaviors, clinical data about HIV and HCV infection, counseling about $\mathrm{HCV}$ and $\mathrm{HBV}$ infection status, status and reasons for $\mathrm{HCV}$ treatment. Results: $678 \mathrm{HCV}$ antibody positive patients with an average age of $43.3 \pm 6.65$ were enrolled with a history of abusing drugs for an average of $20.1 \pm 6.06$ years. Only $366(54.0 \%)$ reported they were infected or ever infected with HCV, of these, $42.1 \%$ (154/366) were being or ever receiving treatment but with an average delay duration of $18.8 \pm 33.5$ months later after they knew their infection status, and 15.0\% (55/366) did not complete treatment duration. The most common causes for treatment naive including unaffordable medical costs $(42.0 \%, 89 / 212)$, quite mild symptoms and thought there was no need to treat $(34.9 \%, 74 / 212)$, could not find HCV treatment sites $(20.3 \%, 43 / 212)$, and did not believe in effect of HCV treatment $(12.3 \%, 26 / 212)$. Multiple logistic regression revealed that having not stable residence (AOR: 0.510, 95\%CI: 0.265-0.983), female (AOR: 0.405, 95\%CI: 0.191-0.860), ever injecting drugs (AOR: 0.386, 95\%CI: $0.212-0.705)$ were less likely intended to receive treatment. Conclusions: Uptake of HCV treatment was relatively poor, education for $\mathrm{HCV}, \mathrm{HCV}$ care including effective referral and on-site treatment in MMT clinics, and new treatment such as direct antiviral agents (DAA) should be implemented in MMT program in China.

CORRESPONDING AUTHOR: Yin Liu, Sun Yat-sen University, liuyin2016@126.com
P146

SMOKERS LACK UNDERSTANDING OF THE CAUSES OF HARM FROM CIGARETTE SMOKING

Ron Borland ${ }^{1}$, Bill King ${ }^{1}$, Coral Gartner ${ }^{2}$, David Hammond ${ }^{3}$, Stephan Lewandowsky ${ }^{4}$, Richard O'Connor ${ }^{5}$, Hua Yong ${ }^{1}$

${ }^{1}$ Cancer Council Victoria- Australia

${ }^{2}$ University of Queensland, School of Public Health- Australia

${ }^{3}$ University of Waterloo, School of Public Health and Health SystemsCanada

${ }^{4}$ University of Western Australia, School of Psychology- Australia

${ }^{5}$ Roswell Park Cancer Institute, Department of Health Behavior- USA

Aims: To investigate smokers' understanding of the mortality/disease risks of cigarette smoking in relation to the causes or mechanisms of harm from smoking. Methods: An on-line survey administered in Australia to 1054 participants in three groups: current smokers, e-cigarette users (vapers) and recent quitters aged 18 to 25 , non-nicotine users aged 18 to 25 , and current smokers, e-cigarette users and recent quitters aged over 25. Results: Most participants reported that cancer and heart disease are major risks of smoking. A quarter accurately quantified mortality risk. Very few $(0.2 \%)$ correctly estimated the relative contributions of four component causes of harm. Even when confining risk comparisons to just two component causes - combustion products and nicotine- only $30 \%$ responded that combustion products are a more important cause. Knowledge of component causes was only weakly associated with knowledge of health risks. Conclusions: Most smokers do not understand the risks of smoking well enough to be able to think systematically about their smoking or about using non-combusted nicotine products. This is radically at odds with the popular notion that smokers make informed choices. Helping smokers gain a practical understanding of the risks they are running and of possible partial as well as complete solutions should be a priority, as it may both improve cessation outcomes and better choice of harm reduction options.

CORRESPONDING AUTHOR: Ron Borland, Cancer Council Victoria, Ron.Borland@cancervic.org.au

\section{P147}

MINORITY OLDER WOMEN LIVING WITH HIV (MOHW): A CALL FOR ATTENTION IN THE 21ST CENTURY

Rosina Cianelli ${ }^{1}$, Natalia Villegas ${ }^{1}$, Lilian Ferrer ${ }^{2}$, Nilda PeragalloMontano $^{3}$

${ }^{1}$ School of nursing and health studies, University of Miami- USA

${ }^{2}$ Escuela de Enfermería, Pontificia Universidad Católica de Chile- Chile

${ }^{3}$ School of Nursing, University of North Carolina Chapel Hill- USA

Introduction: Among adults living with HIV in the US, those 50 years and older are expected to increase from $35 \%$ in 2010 to $71 \%$ in 2020 . Older women have an increased vulnerability to HIV and AIDS due to agerelated biological changes, including declining levels of estrogen and weakening of the immune system. MOWH are disproportionately affected by HIV, African Americans and Hispanics account for 88\% (61\% and $17 \%$, respectively) of women living with HIV in the US; nevertheless, almost no study has focused on them. This study examined the characteristics and needs of MOWH living with HIV. Methods: mixed study methods conducted in a public hospital in Miami, with a convenience sample of 113 women answered a structured computerized survey in Qualtrics and 29 participated in an in depth interviews. Results: The average age of participants was 56.4 years $(\mathrm{SD}=5.1), 82 \%$ acquired HIV from heterosexual contact on average $18.2(\mathrm{SD}=8.7)$ years ago. All MOWH reported to have a comorbidity (e.g., hypertension, diabetes, hypercholesterolemia), $69 \%$ had ongoing pain, $73 \%$ have some amount of fatigue and $20 \%$ reported a fall within the last 6 months. Fifty one $(51 \%)$ reported emotional problems, $86 \%$ of being "high on drugs", and $43 \%$ stated they smoke. The majority of the OMWH were afraid to 
disclose their serostatus with family or the community because of fear of discrimination. Conclusions: MOWH have chronic pain, fatigue, mental health issues that challenge them to have a healthy lifestyle. This adds up with stigma and issues with disclosing their HIV status with family and the community. This study supports awareness in the community about MOWH and the problems that they confront to better develop the interventions they need to modify unhealthy behaviors.

CORRESPONDING AUTHOR: Rosina Cianelli, School of nursing and health studies, University of Miami, Rcianelli@miami.edu

\section{P148}

REDUCING DISPARITIES IN HIV PREVENTION THROUGH TELENOVELAS FOR YOUNG LATINA SEASONAL FARMWORKERS IN SOUTH FLORIDA

Natalia Villegas ${ }^{1}$, Anne Norris ${ }^{1}$, Rosina Cianelli ${ }^{1}$

${ }^{1}$ School of Nursing and Health Studies, University of Miami- USA

Introduction: Florida ranks first in HIV rates and there are considerable disparities among subgroups who have not received sufficient research attention. One of these subgroups is Young Latina seasonal farmworkers who work primarily in agriculture and confront several barriers that increase their HIV risk, such as isolation, immediate survival problems, being less likely to discuss HIV with others, violence and partners' risky behaviors. The design of a culturally effective HIV intervention for behavioral change tailored to reduce these women is critical. Objective: To develop a telenovela (soap opera) for HIV prevention among young Latina seasonal farmworkers. Study design: This study capitalizes upon the unique cultural appeal of telenovela, its potential for HIV prevention, and a community-based approach that incorporates these women in the telenovela development process. During phase 1, focus groups $(n=40)$ will be conducted to determine the acceptability of content and delivery features of the telenovela. During phase $2(n=50)$, telenovela stories will be developed using interactive performance focus groups (IPFG). Analysis: Focus groups in phase 1 will be transcribed and content analysis conducted by two researchers. In phase 2 , feedback from the participants and an interdisciplinary team will determine which stories are more appealing and engaging for HIV prevention. Video recordings and field notes from the IPFG will also be analyzed by the interdisciplinary team. Expected results: The telenovela will have 4 episodes of 10 minutes each which will be delivered weekly, and will be available using an online platform that will provide access from different devices. Five actors will represent different characters and the stories will be centered on the issues that Young Latina seasonal farm workers confront in their daily life. We will address during phase 1 the inclusion of the following themes: HIV risk behaviors, intimate partner violence, substance use, and prevention strategies. This CFAR study aligns with the NIH priority of reducing health disparities in the incidence of new HIV infections with a very innovative culturally sensitive approach.

CORRESPONDING AUTHOR: Natalia Villegas, School of nursing and health studies university of Miami, Navillegasr@miami.edu

P149

THE ROLE OF EMOTIONAL AND COGNITIVE INTELLIGENCE IN PREDICTING ATTITUDES TOWARDS VACCINATION

Peter Leeson ${ }^{1}$

${ }^{1}$ University of Wollongong- Australia

Introduction \& Purpose There is ongoing concern amongst a significant subset of the population about the safety of vaccination. This despite numerous studies pointing to the minimal risks associated with vaccination as well the detrimental impact on public health amongst undervaccinated populations. Given the mis-information disseminated about vaccination, it might be expected that those with more knowledge, that is people greater health literacy, will have a more positive attitudes towards vaccination. Squiers, Peinado, Berkman, Boudewyns, Squiers, Peinado, \& Cormack (2012) have claimed that health related knowledge is an important component of health literacy. But what influences health literacy? One important factor may be intelligence. Given the often emotive nature of the discussion that surrounds vaccination it may also be beneficial to look at the impact of emotional intelligence on attitudes towards vaccination. The first phase of this study explored the relationship between cognitive and emotional intelligence. While, the second phase examined the relationship between cognitive intelligence, emotional intelligence and health literacy and how these variables may predict attitudes towards vaccination. Methods Measures Phase 1254 participants were presented with the following measures: Barchard, Hensley, Anderson, and Walker's (2013) Metaphors test which measures emotional intelligence and Frederick's (2005) Cognitive Reflection Test (CRT) measuring cognitive intelligence. Phase 2 In the second phase 240 participants completed the above measures along with the following: Osborne, Batterham, Elsworth, Hawkins, and Buchbinder's (2013) Health literacy questionnaire (HLQ) and Pitts, Dyson, Rosenthal, and Garland's (2007) Vaccination in General Scale measuring attitudes towards vaccination. Results In phase 1, correlational analysis revealed that there was no significant relationship between cognitive and emotional intelligence $(\mathrm{r}=$ $-.05, \mathrm{~ns})$. While in Phase 2 hierarchical multiple regressions indicated that the two constructs differentially predicted attitudes to vaccination. Conclusions The findings of the first phase of the study illustrated that emotional intelligence and cognitive intelligence are non-overlapping constructs which may therefore deferentially predict attitudes to vaccination. Phase 2 regression analyses provided new information about the role of emotional versus cognitive intelligence in predicting responses to emotionally valenced issues such as attitudes towards vaccination. The divergence between cognitive and emotional factors may help to explain the persistence of misinformation and negative attitudes towards vaccination.

CORRESPONDING AUTHOR: Peter Leeson, University of Wollongong,pleeson@uow.edu.au

\section{P150}

THEORY-BASED PREDICTORS OF PATIENT ENGAGEMENT AND COMMUNICATION WITH HEALTHCARE PROFESSIONALS AROUND PROMPT URINARY CATHETER REMOVAL

Rashmi Bhardwaj-Gosling ${ }^{12}$, Susan Hrisos ${ }^{1}$, Sebastian Potthoff ${ }^{3}$, Clare Abley $^{12}$, Christopher Harding ${ }^{12}$, Falko Sniehotta ${ }^{1}$, Justin Presseau ${ }^{4}$

${ }^{1}$ Newcastle University- United Kingdom

${ }^{2}$ Newcastle upon Tyne Hospitals- United Kingdom

${ }^{3}$ Northumbria University, Newcastle, United Kingdom

${ }^{4}$ Ottawa Hospital Research Institute \& University of Ottawa- Canada

Introduction \& Purpose Combating catheter-associated urinary tract infections (CAUTIs) is a priority in the worldwide drive to reduce hospitalacquired infections. The longer a patient is catheterised, the risk of bacterial colonisation increases (day by day), associated with increased risk of CAUTIs. Early post-operative urinary catheter removal is an effective way of reducing CAUTIs, but current median-duration of UK postoperative catheterisation (3-days: Pickard et al 2012) remains higher than the recommended one day standard by CDC guidelines. A theory-based approach to understanding predictors of patient-engagement and communication behaviours may influence the guidance implementation to facilitate prompt catheter removal and reduce the incidence of CAUTIs. Methods A prospective correlational study involving hospitalised patients from 14 medical/surgical wards in the North-East England, UK. Adult patients $(n=140)$ requiring short-term catheterisation $(\leq 14$ days) completed a predictive questionnaire at baseline (post-insertion) and follow-up (post-removal). A Theoretical Domain Framework (TDF) questionnaire was used to first explore the breadth of potential patient behaviour determinants 
involved in engaging with $\mathrm{HCP}$ to advocate prompt catheter removal, and secondly to inform theory-selection. We then mapped TDF domains to Social Cognitive Theory (SCT), social support and habit constructs, to identify predictors of patient intention and behaviour. Results With $95 \%$ response rate at follow-up, SCT constructs self-efficacy (MEAN=3.77, $\mathrm{SD}=1.42$ ) outcome expectations ( $\mathrm{MEAN}=4.44, \mathrm{SD}=1.45)$ \& social support (MEAN=3.91, $\mathrm{SD}=2.01$ ) accounted for a medium amount of variance in patients' intention (MEAN=4.21, SD=2.38, R2adj =0.23) and with addition of habit $(\mathrm{MEAN}=4.09, \mathrm{SD}=1.93)$ accounted for small amount of variance in prediction of patients' communication behaviour (MEAN=1.01, SD=1.43, R2adj =0.03) to engage with staff. Conclusion Findings show that patients had the intention to engage with HCPs to advocate timely catheter removal. But, low variance in the behaviour model show that patients do not translate their intentions into actions. Future intervention targeting self-efficacy could encourage patients to prompt HCPs for early catheter removal leading to fewer CAUTIs.

CORRESPONDING AUTHOR: Rashmi Bhardwaj-Gosling, Newcastle University \& Newcastle upon Tyne Hospitals, rashmi.bhardwaj1@ncl.ac.uk

\section{P151}

ETHNOGRAPHIC STUDY FOR THE PROMOTION OF SEXUAL AND REPRODUCTIVE HEALTH AND THE PREVENTION OF ADOLESCENT PREGNANCY IN CHILE

Alexandra Obach ${ }^{1}$, Báltica Cabieses ${ }^{1}$, Claudia Pérez ${ }^{1}$, Michelle Sadler $^{2}$, Mariana Gerias ${ }^{3}$

${ }^{1}$ Faculty of Medicine, Clínica Alemana Universidad del Desarrollo - Chile

${ }^{2}$ Faculty of Liberal Arts, Universidad Adolfo Ibáñez- Chile

${ }^{3}$ Faculty of Educaction, Universidad del Desarrollo- Chile

Introduction: The study describes a pioneering program of intersectoral collaboration between health and education for the promotion of adolescent sexual and reproductive health and prevention of adolescent pregnancy in a commune in the city of Santiago, Chile, where health professionals work permanently in schools as part of the educational curriculum. It seeks to identify learnings and difficulties in the implementation of such program. Methods: Qualitative, ethnographic study. The research techniques of participant and non-participant observation were applied in three secondary schools, as well as semi-structured interviews $(\mathrm{N}=32)$ and discussion groups $(\mathrm{N}=8)$ with the various social actors involved in the program. Results: Three axes stand out around the perception of teachers and health professionals: (i) teachers identify cultural barriers in terms of accepting health professionals within the school territory; (ii) health professionals maintain difficulties when inserting in the educational context since they must adapt to established curricula; (iii) teachers and health professionals recognize that the intersectoral work in the educational territory is strategic and key to the well-being of adolescents. Conclusions: The development of intersectoral work for the promotion of sexual and reproductive health in adolescents entails a series of challenges for teachers and health professionals, since it involves changes in each other's sectoral cultures, as well as adapting schools for the introduction of health professionals. Emphasis is placed on the importance of promoting Health in All Policies (HiAP) as an approach with the potential to facilitate the the challanges involved in intersectoral work.

CORRESPONDING AUTHOR: Alexandra Obach, Universidad del Desarrollo, aobach@udd.cl

\section{P152}

DESIGN AND VALIDATION OF A SCALE TO EVALUATE SELFEFFICACY IN HEALTHY EATING AND EXERCISE FOR PRIMARY HEALTH CARE USERS IN CHILE

Daniela Nicoletti ${ }^{1}$, Ricardo $\mathrm{Cerda}^{2}$, Valetina Muñoz ${ }^{2}$, Macarena Peña y Lillo $^{3}$, Dergica Sanhueza ${ }^{1}$
${ }^{1}$ Universidad de Chile- Chile

${ }^{2}$ Facultad de Medicina, Universidad de Chile- Chile

${ }^{3}$ Universidad Diego Portales- Chile

The purpose of this summary is to present the validity and confidence analysis results of a Likert-type self-report scale, to measure self-efficacy in over-weight and obese users at the primary health care level.

CORRESPONDING AUTHOR: Daniela Nicoletti, Universidad de Chile,dnicolettirojas@gmail.com

\section{P153}

EALTH CARE PROVIDERS'PERCEIVED COMFORT PERFORMING A RAPID HIV TESTS, SOMETHING NECESSARY TO UNDERSTAND

\section{Lisette Irarrazabal $^{1}$, Rosina Cianelli ${ }^{2}$, Lilian Ferrer $^{1}$}

${ }^{1}$ Escuela de Enfermería, Pontificia Universidad Católica de Chile- Chile

${ }^{2}$ School of Nursing and Health Studies, University of Miami- USA

Introduction: The oral rapid HIV test (ORT) has been shown worldwide as an effective tool to increase the uptake of HIV testing and also the number of people who receive their test results. ORT has never been used in Chile for general screening. Should the technology become government approved for clinical use, a recent study in 2014 found that perceived comfort in performing a rapid HIV test was the strongest predictor of readiness to adopt ORT among Chilean healthcare professionals. Methodology: Crosssectional design, 150 healthcare professionals completed a selfadministered questionnaire that included demographic characteristics, perceived readiness and comfort in performing ORT, and HIV-related stigmatization. Participants also were asked how comfortable they would feel performing pre-counseling, oral and finger-prick rapid testing, and giving a positive or negative test result with corresponding post-test counseling. Results: A mean score of 16.21 (range 0-20), which indicates a high level of comfort with ORT. Participants felt least comfortable with giving a positive test result and providing appropriate post-test counseling to these patients. Stigmatizing attitudes were negatively related to perceived comfort performing ORT. The study's findings point to the need to address HIVrelated stigmatization among healthcare professionals and also to offer ORT training that increases providers' comfort in delivering a positive ORT result. By increasing their perceived comfort performing ORT, health care workers' readiness to adopt ORT also is likely to increase along with the greater probability of implementation success. Readiness is associated with providers behaviors, therefore it has an impact on the access to patients.

CORRESPONDING AUTHOR: Lisette Irarrazabal, Escuela de enfermería, Pontificia Universidad Católica de Chile, lisette.irarrazabal@gmail.com

\section{P154}

THE DIRECT MEDICAL ECONOMIC BURDEN OF HEALTH CARE-ASSOCIATED INFECTIONS IN PUBLIC TERTIARY HOSPITALS IN HUBEI PROVINCE, CHINA

Jing Sun ${ }^{1}, \mathrm{Hao} \mathrm{Li}^{2}$, Xinliang $\mathrm{Liu}^{2}$, Dan $\mathrm{Cui}^{2}$, Quan Wang ${ }^{2}$, Zongfu $\mathrm{Mao}^{2}$, Liang Fang ${ }^{3}$, Furong Zhang ${ }^{3}$, Ping Yang ${ }^{4}$

${ }^{1}$ Chinese Academy of Medical Sciences and Peking Union Medical College- China

${ }^{2}$ School of Health Sciences/Global Health Institute, Wuhan UniversityChina

${ }^{3}$ Department of Infection Management/Department of Logistics, The Third People's Hospital of Hubei Province- China

${ }^{4}$ Department of Infection Management, Tianmen First People's HospitalChina

Introduction and Purpose: Antimicrobial resistance (AMR) has increasingly become a global threat to human health. Healthcare associated infections (HAIs) is one important factor associated with AMR along with overuse of 
antibiotics. This study estimated the attributable direct medical economic burden of health care-associated infections (HAIs) in China, aimed to inform hospital managers and policy makers about the potential reduction of direct medical economic burden from better control of HAIs, as well as some policy implications for future actions. Methods: Data were extracted from hospitals' information systems. Inpatient cases with HAIs and non-HAIs were grouped by the propensity score matching (PSM) method. Attributable hospitalization expenditures and length of hospital stay were measured to estimate the direct medical economic burden of HAIs. Attributable hospitalization expenditures and length of hospital stay STATA 12.0 was used to conduct descriptive analysis, bivariate $\chi^{2}$ test, paired $Z$ test, PSM $(r=0.25 \sigma$, nearest neighbor 1:1 matching), and logistic regress analysis. The statistically significant level was set at 0.05 . Results: The annual HAIs prevalence rate (based on PPS) is $2.69 \%$, which is much higher than the annual HAIs rate (based on voluntary reporting) of $0.82 \%$. The HAIs group had statistically significant higher expenditures and longer hospitalization stay than the non-HAIs group during 2013 to $2015(\mathrm{P}<0.001)$. The annual average HAI attributable total expenditure, medicines expenditure, out-of-pocket expenditure, and number of hospitalization days per inpatient were (2015 US\$) 6173.02, 2257.98, and 1958.25 and 25 days during 2013 to 2015 . The direct medical cost savings was estimated at more than 2015 US\$12 billion per year in Chinese tertiary hospitals across the country. The results show a dramatic increase in 2014 and a slight decline in 2015 . The differences of all measurements are statistically significant $(\mathrm{P}<0.001)$. Conclusions: The significant attributable direct medical economic burden of HAIs calls for more effective HAI surveillance and better control with appropriate incentives. There is ample room for improvement in China and the direct medical economic burden of HAIs can be decreased through better prevention, control, and management.

CORRESPONDING AUTHOR: Jing Sun, Chinese Academy of Medical Sciences and Peking Union Medical College, sunjing@sph.pumc.edu.cn

\section{P155}

PREDICTORS OF ADHERENCE TO ANTIRETROVIRAL THERAPY: A STUDY WITH BRAZILIAN PEOPLE LIVING WITH HIV / AIDS

Eliane Seidl ${ }^{1}$

${ }^{1}$ University of Brasilia- Brazil

Introduction \& Purpose. The identification of levels of adherence to antiretroviral therapy (ART) and the understanding of variables associated with adherence behavior has been of interest to researchers and health professionals working on HIV / AIDS. The aim of this study was to investigate variables associated with adherence behavior to antiretroviral treatment, considering sociodemographic aspects (eg sex, age, educational level), clinical (eg side effects, history of ARV interruption on its own, time to treatment with antiretrovirals - ARVs), beliefs about ARV and the treatment (eg perception of difficulties in using ARVs, self-assessment of adherence behavior) and psychological (social support, self-efficacy expectancy) of people living with HIV / AIDS (PLWHA). Methods. This is a cross-sectional study with convenience sample. One hundred and fifty-six PLWHA participated, $60.4 \%(n=94)$ males, aged $18-74$ years $(M=40.5, S D=9.7)$. The instruments were interviews prepared for the study and validated scales for the Brazilian population. Adherence was measured by self-reported number of ART pills/capsules missed during the previous week and/or previous month, evaluated as satisfactory when less than $5 \%$. Data were analyzed using descriptive and inferential statistical techniques (significance level $\mathrm{p} \leq .05)$. Results. The majority of participants $(\mathrm{n}=104 ; 66,6 \%)$ reported adherence of bigger or equal $95 \%$. Preliminary bivariate statistical analyzes identified the antecedents variables with significant association $(\mathrm{p} \leq .05)$ with the criterion variable (adherence behavior), tested using logistic regression. Analysis showed that a history of ART interruption ( $\mathrm{p} \leq .05$; odds ratio=.31), self efficacy expectancy $(\mathrm{p} \leq .05$; odds ratio $=3.45)$, and self-assessment of adherence behavior $(\mathrm{p} \leq .05$; odds ratio $=2,40)$ were significant predictors and entered the final model. The percentage of variance explained in the final model was $55 \%$ according to the Nagelkerke indicator. Conclusions.
The prevalence of people with less than $95 \%$ adherence was consistent with other studies conducted in Brazil. Upgrading of care with interdisciplinary teams is needed to develop an appropriate approach to the medical and psychosocial difficulties of ART adherence by PLWHA.

CORRESPONDING AUTHOR: Eliane Seidl, University of Brasilia, eliane.seidl@gmail.com

\section{P156}

DEPRESSION AND ANXIETY SYMPTOMS IN HIV: COMPARISON BETWEEN NEWLY DIAGNOSED AND PATIENTS WHO REENGAGED IN CARE IN MEXICO CITY

Nancy Patricia Caballero-Suárez ${ }^{1}$, Ivonne Nalliely Pérez Sánchez ${ }^{2}$, Ester Gutierrez Velilla ${ }^{1}$, María Candela Iglesias ${ }^{1}$, Evelyn Rodriguez Estrada ${ }^{1}$, Gustavo Reyes Teran ${ }^{1}$

${ }^{1}$ Departamento de Investigación en Enfermedades Infecciosas del Instituto Nacional de Enfermedades Respiratorias- Mexico

${ }^{2}$ Consejo Nacional de Ciencia y Tecnología, Instituto Nacional de Enfermedades Respiratorias- Mexico

Introduction Time living with HIV and being on antiretroviral treatment have been described to influence depression and anxiety prevalence in people living with HIV (PLWH). Our aim was to compare depression and anxiety prevalence among newly HIV diagnosed and those who reengaged to care after had been in loss to clinical follow-up. Methods A cross-sectional study by convenience sample was conducted in 90 PLWH who were recruited from the Center for Research in Infectious Diseases in Mexico City from February 2016 to December 2017. Participants who were $>18$ years old, attended a mental health consultation, and who were either newly diagnosed (diagnosed $<6$ months) or lost to clinical followup (failing to attend medical appointments for $\geq 6$ months and/or stopping ART for $\geq 4$ months) were included. Spanish validated versions of Beck's Inventories were used to measure depression and anxiety. All participant provided written consent. Data were analyzed with Chi-square and U Mann-Whitney tests using SPSS-20 software. Results We included 61 newly diagnosed (NDx) and 29 re-engaged in care patients (ReCare). There were no differences between groups in sex, age, education, viral load and CD4 T cells counts. Median time since diagnosis was 1 month (IQR 1-2) in NDx and 101 months (IQR 57.5-169.5) in ReCare patients. Depression was higher in individuals reengaged in care than in those newly diagnosed $(79.3 \%$ vs. $54.1 \%, \mathrm{p}=.017)$ while anxiety prevalence was similar among both groups ( $48.3 \%$ vs. $41 \%)$. Severe depression level was obtained by $17.2 \%(n=5)$ of ReCare and $1.6 \%(n=1)$ of NDx; while severe anxiety level was obtained by $13.8 \%(n=4)$ ReCare versus $3.3 \%$ $(\mathrm{n}=2)$ of NDx. Conclusion Depression and anxiety are highly prevalent in both HIV+ groups analyzed. Patients who re-engage in care had a higher severe mental health issue than those newly diagnosed. Our results highlight the importance of assessment of mental health issues, especially in re-engaged patients to HIV-care. Future studies with a large sample and longitudinal design are necessary to obtained generalizable results.

CORRESPONDING AUTHOR: Nancy Patricia Caballero-Suárez, Departamento de Investigación en Enfermedades Infecciosas del Instituto Nacional de Enfermedades Respiratorias, nancy.caballero@cieni.org.mx

\section{P157}

COMPARING SUBSTANCE USE IN NEWLY DIAGNOSED AND IN LOSS OF FOLLOW-UP USERS AT AN HIV-CLINIC IN MEXICO CITY: A DESCRIPTIVE STUDY

Ester Gutiérrez Velilla ${ }^{1}$, Nancy Patricia Caballero-Suarez ${ }^{1}$, Ivonne Nalliely Pérez Sánchez ${ }^{1}$, Evelyn Rodríguez Estrada ${ }^{1}$, Maria Candela Iglesias $^{1}$, Gustavo Reyes-Terán ${ }^{1}$

${ }^{1}$ Departamento de Investigación en Enfermedades Infecciosas (CIENI) del Instituto Nacional de Enfermedades Respiratorias (INER)- Mexico 
Substance use in people living with HIV (PLWH) has been linked to mental health problems and worse adherence to antiretroviral treatment (ART), which leads treatment failure and possible HIV-transmission. Given the relationship between adherence and substance use, the aim was to know frequency of substance use in PLWH and to compare between newly diagnosed users and those in loss of follow-up who returned to medical care. Cross-sectional study was performed. Participants were users who went to mental health consultation at an HIV-clinic, between February 2016 to December 2017, for recent diagnosis ( $\leq 6$ months) or for returning to medical care after loss of follow-up. They were divided into those two groups. Substance use was assessed with Alcohol, Smoking and Substance Involvement Screening Test (ASSIST). SPSS software version 20 was used to calculate descriptive and nonparametric statistics to compare groups. Ninety-five users participated: 63 newly diagnosed and 32 in re-engagement. Eighty-two percent were men, $14 \%$ women and $4 \%$ transgender women, mean age was 36.6 years $(\mathrm{SD}=9.9)$. There were 56 users with moderate-high risk for tobacco, 8 for alcohol, 4 for marijuana and 6 for cocaine; 48 of patients at tobaccorisk, 4 of alcohol, 4 of marijuana and 3 of cocaine were from the newly diagnosed group. Those at risk were mostly men under 34 , except in case of marijuana, prevailing those from 35 to 50 years. When comparing ASSIST score between two groups, re-engagement patients have higher median in use of tobacco (median=57.8), alcohol (median=62.4), marijuana $($ median $=51.0)$ and cocaine $($ median $=51.8)$, although difference is only significant in use of alcohol $(\mathrm{p}<.01)$ and tobacco $(\mathrm{p}<.01)$. Substance use is present both in newly diagnosed and in re-engagement patients, which suggests need for drugs related interventions in all stages of infection. Alcohol and tobacco use occurs to a greater extent in people who have been in loss of follow-up, perhaps in part explaining it, since use of alcohol and other drugs has been related to poor adherence. Evaluating and intervening substance use since patients receives HIV diagnosis, could prevent this being a risk factor that could lead to mental health problems, to lack of adherence and finally to loss of follow-up.

CORRESPONDING AUTHOR: Ester Gutiérrez Velilla, Departamento de Investigación en Enfermedades Infecciosas (CIENI) del Instituto Nacional de Enfermedades Respiratorias (INER), estergutierrezvelilla@hotmail.com

\section{P158}

THE EFFECTS OF EDUCATIONAL GROUP PROGRAMMES DELIVERED BY HEALTH PROFESIONAL AND EXPERT PATIENTS-A SYSTEMATIC REVIEW

Mariela L. Lara-Cabrera ${ }^{1}$

${ }^{1}$ Department of Mental Health, Trondheim, Norway, Faculty of Medicine and Health Sciences, Norwegian University of Science and Technology- Norway

Background An activated patient role entails having the necessary skills, knowledge, and confidence to manage their own health. Educational group programmes co-led by expert patients are being increasingly used and closely associated with increased patient activation, however, no attempts to date have been made to systematically review this. Aim To systematic review the effects of educational group programmes co-led by expert patients in mental health settings. Methods PubMed, PsycINFO, British Nursing Index, and CINAHL were searched for RCTs published from 2004 through July 2017 that included educational group interventions co-led by expert patients. Preliminary results Four RCTs were found and the preliminary results are encouraging. Educational group interventions provided in cooperation with expert patients are a promising and innovative method to activate and engage patients in mental health services. Preliminary conclusion This is the first review on. the effects of educational group programmes co-led by expert patients in mental health settings The evidence is encouraging but limited. PROSPERO registration: CRD42015023482

CORRESPONDING AUTHOR: Mariela Loreto Lara-Cabrera, Norwegian University of Science, Tronheim, mariela.lara@ntnu.no
P159

ASSOCIATION BETWEEN DIAGNOSIS AND HOSPITALIZATION IN HIV+ INDIVIDUALS WHO DEVELOPED ANXIETY AND DEPRESSION

Rosa Guadalupe Cruz Maycott ${ }^{1}$, Lydia Perrusquía-Ortiz ${ }^{1}$, Ivonne PérezSánchez ${ }^{1}$, Nancy Caballero-Suárez ${ }^{1}$, Evelyn Rodríguez-Estrada ${ }^{1}$, Gustavo Reyes-Terán ${ }^{1}$

${ }^{1}$ Departamento de Investigación en Enfermedades Infecciosas (CIENI) del Instituto Nacional de Enfermedades Respiratorias (INER)- Mexico

Introduction and Purpuse: In Mexico City, many people living with HIV (PLWH) receive their diagnosis during hospitalization. Anxious and depressive symptoms are common among inpatients, unfortunately they don't receive adequate treatment. The objective of this study was to describe the association between anxiety and depression symptoms and the time of HIV diagnosis and hospitalization status (first- time or hospital readmission for HIV-related diseases)

Methods: All inpatients PLWH was enrolled since January 2016 to December 2017 in this cross-sectional study at the Instituto Nacional de Enfermedades Respiratorias. Participants fulfilled the Hospital Anxiety and Depression Scale and a brief interview about social demographic variables; time since HIV diagnoses and hospitalization status were obtained of their medical records. CHI2 test, partial CHI2 test and OR analysis was perform to descriptive analysis. Data conducted in SPSS 20.0.

Results: Three hundred thirty-seven PLWH participated in this study; many of them were male $(88.7 \%, \mathrm{n}=299)$ mean age was 35.7 years $(\mathrm{SD}=9.9)$ $14.8 \%(n=50)$ had depression symptoms and $23.1(n=78)$ had anxiety symptoms. Those PLWH who have more than six months with HIV diagnosed were twice prone to develop depression than those patients who have less than six month with HIV diagnoses (OR=2.06, IC [1.09,3.09]; Chi2 $=5,11 \mathrm{gl}=1, \mathrm{p}=0.018, \mathrm{PLWH}>6$ months with HIV diagnosis $62.5 \%$ vs $\mathrm{PLWH}<6$ months of HIV diagnosis $=37.5 \%$ ). We didn't identify the association between anxiety symptoms and HIV time of diagnosis. Meanwhile, hospitalization status PLWH who were in their first hospitalization were three times prone to develop depression than those who were in readmission $(\mathrm{OR}=2.93$, IC $[1.03,8.35]$; $\mathrm{Chi} 2=1 \mathrm{gl}, \mathrm{p}=0.047$, first hospitalization $88 \%$ vs readmitted $12 \%$ ) We didn't identify the association between anxiety and hospitalization status.

Conclusions: Through the Hospital Anxiety and Depression Scale we could identify the severity of depressive and anxiety symptoms. However, we didn't find the association between anxiety and depressive symptoms with the time of diagnosis and the status of hospitalization, but we collected relevant information to perform a specific psychological intervention to decrease the symptoms according to the individual's needs.

CORRESPONDING AUTHOR: Rosa Guadalupe Cruz Maycott, Departamento de Investigación en Enfermedades Infecciosas del Instituto Nacional de Enfermedades Respiratorias, rosa.cruz@cieni.org.mx

\section{P160}

COMMUNICATION IN ONCOLOGY: WHAT SHOULD THE PHYSICIAN TELL THE PATIENT?

Elisa Kern de Castro ${ }^{1}$, Bruna Madrid da Rosa ${ }^{1}$

${ }^{1}$ Universidade do Vale do Rio dos Sinos- Brazil

Introduction \& Purpose: In general, cancer patients want to know some information about their diagnosis and their potential for cure. However, the information provided by doctors is often partial, contrasting with patients' desire to know details about their illness. This difficulty in communication may generate much suffering and psychological stress for cancer patients. This study aimed to evaluate the physicians and cancer patient's perceptions about the communication regarding symptoms, causes, timeline, consequences, peronal and treatment control, illness coherence and emotional representation of the patients about their diagnosis. Method: The study is a cross-sectional design. Participants were 68 
adult patients with cancer and 58 physicians who had been working with cancer patients that answered questionnaires in an online platform. The measures were:1) Sociodemographic and clinical questionnaire; 2) Questionnaire about the perceptions of patient and doctors about information provided on diagnosis Results: The results revealed significant differences regarding the perceptions of physicians and patients about the doctor's communication. Physicians mentioned that patients are often informed about symptoms, causes, consequences, timeline and disease control, but the patient's responses are discordant $(p<0,05)$. Conclusion: It was concluded that physician's views about their communication are more positives than patients' perceptions about the same phenomena.

CORRESPONDING AUTHOR: Elisa Kern de Castro, Universidade do Vale do Rio dos Sinos, elisa.kerndecastro@gmail.com

\section{P161}

THE EFFECTS OF AN EDUCATIONAL PROGRAMME ADDED TO MENTAL HEALTH TREATMENT TO IMPROVE PATIENT ACTIVATION AND ATTENDANCE

Mariela L. Lara-Cabrera ${ }^{1}$

${ }^{1}$ Department of Mental Health, Trondheim, Norway, Faculty of Medicine and Health Sciences, Norwegian University of Science and Technology- Norway

Introduction: Educational group interventions can be a promising method to improve patient activation (1). Our RCT-study showed that an educational group intervention added to mental health treatment significantly improved patient activation (PAM-13) at 1 and 4 months (2). However, no RCTs have investigated the long term effects. Objective: To investigate the effects of an educational group intervention co-led by expert patients after 12 months. The intervention was developed and carried out in cooperation with user representatives (expert patients and peer educators), and aimed at preparing patients to actively participate in own treatment. Primary aims: To evaluate the effects on attendance and patient activation (PAM-13). Methods: Patients from two Community Mental Health Centres were randomized to a control group (control, $n=26$ ) receiving treatment as usual, or an intervention group (intervention, $n=26$ ), receiving a four-hour group educational group intervention (information about treatment, peer-support and encouragement to adopt an active role) followed by treatment as usual. Results: ITT analysis showed that there were significant group differences, in favor of the intervention group, across 1,4 and 12 months $(p=0.04)$. Results: Preliminary results showed that the intervention group had better attendance rates than controls at $4-(p=0.035)$ and 12 months $(p=0.01)$ follow-up. Conclusion: Our results provide preliminary support of the effects of pretreatment education in mental health settings. Clinical implications: Educational group interventions provided in cooperation with user representatives is a promising and innovative method to activate and engage patients in their mental health treatment. References 1: Hibbard, J. H., \& Greene, J. (2013). What the evidence shows about patient activation: better health outcomes and care experiences; fewer data on costs. Health Aff (Millwood), 32(2), 207-214. doi:10.1377/hlthaff.2012.1061 2: LaraCabrera, M. L., Salvesen, O., Nesset, M. B., De Las Cuevas, C., Iversen, V. C., \& Grawe, R. W. (2015). The effect of a brief educational programme added to mental health treatment to improve patient activation: A randomized controlled trial in community mental health centres. Patient Educ Couns. doi:10.1016/j.pec.2015.11.028

CORRESPONDING AUTHOR: Mariela Loreto Lara-Cabrera, Norwegian University of Science, Tronheim, mariela.lara@ntnu.no

\section{P162}

INFLUENCE OF BODY WEIGHT DISSATISFACTION ON EATING BEHAVIOR IN A SAMPLE OF CHILEAN ADULTS

Camila Oda-Montecinos ${ }^{1}$, Carmina Saldaña ${ }^{2}$

${ }^{1}$ Universidad de O'Higgins- Chile

${ }^{2}$ Universitat de Barcelona- Spain
Introduction \& Purpose: The aim of this research was to estimate whether the presence of body weight dissatisfaction acts as a risk factor for some disordered eating behaviors in a sample of Chilean adults of both genders. Methods: 654 participants (436 women, 218 men; age range 18-64 years) were evaluated with a battery of self-administered questionnaires. Results: Significant differences were observed in the clinical scales that evaluated disordered eating in function of the BMI of the participants; in those scales females also showed higher scores than males. Comparing participants with and without body weight dissatisfaction, the first one showed more unhealthy eating behaviors. Conclusions: The results of this study show how body weight dissatisfaction can interact and influence on eating behaviors

CORRESPONDING AUTHOR: Camila Oda-Montecinos, Universidad de O'Higgins, camilaoda@gmail.com

\section{P163}

VALIDITY OF SCREENING TEST FOR HEALTH STATE OF SUSCEPTIBILITY PHASE ON THE PRIMARY PREVENTION IN PREVENTIVE MEDICINE

Hideo Yamazaki ${ }^{1}$

${ }^{1}$ Tokoha University- Japan

Introduction: Non-communicable diseases such as circulatory diseases and metabolic syndrome are called life-style related diseases (LSRDs) in Japan. LSRDs are a global epidemic among many developed countries and not only serious causes of death but also risk factors of broken health. Furthermore, they have been steadily increasing. In general, one of the most effective ways to prevent LSRDs will be taken an early detection and early treatment in the secondary prevention. The purpose of the present study was to examine the validity of a screening test of the semihealth state for the primary prevention on preventive medicine. The semihealth state in susceptibility phase on the natural history of disease was evaluated among the community-dwelling elderly in Japan. Methods: A self-rated questionnaire consisted of 54 items included the semihealth status questions was administered to 2,113 the elderly in Japan between 2015 and 2016. Results: A principal component analysis was applied to the valid data from 1,974 samples in order to extract the multidimensional indices which were evaluated some characteristics of a spatial structure of the semihealth state. By this analysis, four principal components were extracted as. Especially, the first principal component was extracted as an index which meant a quantitative aspect of the semihealth state. Every eigenvector of its component had a positive sign. Therefore, this component was used as the semihealth index. Furthermore, a distribution of the elderly with the semihealth state was determined by using this index. The prevalence, sensitivity, specificity, positive predictive value, and negative predictive value should be calculated. Conclusions: It is likely that the screening test is able to recognize a good distinction regarding the semihealth state among the communitydwelling elderly.

CORRESPONDING AUTHOR: Hideo Yamazaki, Tokoha University, whnhx524@ybb.ne.jp

\section{P164}

PERSONALITY AND EATING BEHAVIOR OF MEXICAN UNIVERSITY STUDENTS OF HEALTH SCIENCES: EXPLORATORY STUDY

Carlos Amadeo Garcia-Ayala ${ }^{1}$

${ }^{1}$ Universidad Vasco de Quiroga- Mexico

Introduction and Purpose. Recent studies point out that obesity rather than a food addiction is a behavioral disorder of impulse control. However, our country lacks systematic studies on how personality influences eating 
behavior (EB). Purpose. Based on the Eysenck's Personality Model, determine how the three dimensions of the personality relate to the EB of students of nutrition and medicine of the UVAQ. METHOD. Observational, transversal, descriptive, correlational and analytical study.

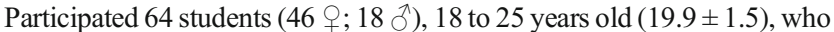
answered valid and reliable instruments: EPQR-A (personality) and CCA (eating behavior). RESULTS. The average EPQR-A scores, obtained in each personality dimension, were significantly different from each other $(\mathrm{p}<0.05)$. The CCA average score was $26.6 \pm 4.4$. A low but significant negative correlation was observed between psychoticism and EB (-0277, $\mathrm{p}<0.05)$. From 31 items that make up the CCA, $21(67.7 \%)$ were answered with "healthy" responses. However, in 10 items $(32.3 \%)$ the responses emitted were "unhealthy." The differences between "healthy" and "unhealthy" responses were statistically significant $(\mathrm{p}<0.05)$. The individual analysis of each CCA item showed that the "healthy" EB is related to low scores of neuroticism and psychoticism. In contrast, the "unhealthy" EB is related to high extraversion scores. CONCLUSION. Although exploratory, the results of the present study show that EB is influenced by the three dimensions of the personality of Eysenck's Personality Model.

CORRESPONDING AUTHOR: Carlos Amadeo Garcia-Ayala, Universidad Vasco de Quiroga, cgarcia@uvaq.edu.mx

\section{P165}

A SOUTH AFRICAN COMMUNITY'S SUGGESTIONS FOR INCREASING CONDOM USE ADHERENCE IN RESPONSE TO "CONDOM FATIGUE"

Cho Hee Shrader ${ }^{1}$, Kenisha Peters ${ }^{2}$, Roger Rochat ${ }^{3}$, Mariano Kanamori ${ }^{1}$, Pablo Montero ${ }^{1}$, Aaron Siegler ${ }^{3}$

${ }^{1}$ University of Miami- USA

${ }^{2}$ The Global Business Group on Health- USA

${ }^{3}$ Emory University- USA

Introduction South Africa has one of the highest incidences of HIV in the world. Approximately 7.1 million people currently live with HIV and there are 460,000 new infections every year (2017). In 2012, the South African Ministry of Health (MOH) identified a decrease in condom use adherence (condom use at last sex), attributing "condom fatigue" as the reason for this decrease. The MOH then piloted a colored and scented/ flavored condom distribution intervention in one university. This study seeks to understand how condom option programs could best be scaled up for the general population. This study addresses the community's perceptions of 1) whether the diagnosis of "condom fatigue" aligns with people's lived experiences, 2) free condom programs and how such programs could be improved, and 3) most desired traits in free condoms. Methods In 2014, we conducted seven focus groups ( $\mathrm{n}=40 \mathrm{men}$ ) and 20 in-depth interviews ( $n=9$ men, $n=11$ women). Participants were clients and community health workers aged $\geq 18$, from four health clinics in urban informal settlements in Cape Town, South Africa. Analysis used grounded theory principles, and transcripts were coded, managed, and analyzed in MAXQDA11. Results Participants largely did not attribute 'condom fatigue' as a main or even minor reason for lower condom use adherence. Instead, participants discussed: condoms being boring and of poor quality and alcohol use. When participants discussed using free government condoms, topics explored included perceptions that the male using the condom has lower socio-economic status and fatalistic attitudes due to perceived frequent condom breakage and inadequate lubrication. A socially marketed condom was considered ideal due to its black color and large size. Participants suggested health programs distribute condoms with: different sizes, colors, flavors, and textures; good smell before/after sex; and culturally adapted and constant novel packaging. Suggestions for program innovations included attaching condoms to alcoholic beverages bottles when sold and offering all brands of condoms at no-cost. Conclusions The $\mathrm{MOH}$ campaign to combat decreased condom use should pre-test alternative names and/or supportive slogans, and explore offering new features and/or packaging. Condoms are an extremely costeffective HIV and STD prevention strategy, and novel promotion strategies must be explored such as a continuing rotation of free/low-cost condom branding and packaging options.

CORRESPONDING AUTHOR: Cho Hee Shrader, University of Miami, cxs939@miami.edu

P166

PARENTING PRACTICES AND BODY MASS INDEX OF CHILDREN IN A MEXICAN POPULATION

Nayeli Grijalva ${ }^{1}$, Laura Ramírez ${ }^{1}$

${ }^{1}$ Universidad de Guadalajara- Mexico

Introduction \& purpose: Childhood obesity is one of the most serious public health problems of the 21 st century (WHO, 2017). Mexico is among the first places of childhood obesity (UNICEF, 2016). Family factors are associated with childhood obesity, these have been related to the increase of cases (Budd \& Hayman, 2008). Ruel and Menon (2002) found that at early ages there is a significant and positive correlation between parenting practices and nutritional status. Stang and Loth (2011) define five parenting practices associated with measures of the child's body weight such as the BMI. Despite this, most of the prevention programs have focused on working with children, obtaining unfavorable long-term results (Rodríguez and Arguello, 2014). Therefore, the objective of the study was to evaluate the influence of parenting practices on the body mass index of children and to identify differences between parenting practices of children with normal nutritional status and parents of children with overweight and obesity Method: The study was a cross-sectional, carried out in the facilities of a Primary School in the city of Guadalajara, México. A total of 110 primary school students and their parents were included, selected for convenience. Schoolchildren were mostly girls with $59.1 \%$, the average age was 6.75 years, the nutritional status was normal with $70 \%$, the rest of them were overweight or obese. Regarding the parents, most of the mothers responded to the instrument with $82.7 \%$, the average age was 33.49 . Instruments: The CFQ was used, adapted for Mexican population by Navarro \& Reyes (2016) with an internal consistency of $\alpha=.858$. For the estimation of the BMI and nutritional status of the children, the SECA 214 stadiometer and the SECA 813 scale were used for the anthropometric measures of height and weight, respectively. An informed consent letter was sent to the mothers, all those who agreed to participate answered sociodemographic and the CFQ. Their children were measured and BMI was calculated. The software SPSS v.23 were used for analysis. Results: The BMI of the children shows negative associations between perceived responsibility $(\mathrm{rho}=$ $-.199, \mathrm{p}=.042)$ and pressure to eat $(\mathrm{r}=-.211, \mathrm{p}=.031)$. The correlations between subscales by the nutritional status of the children shows that mothers of children in normal nutritional status and mothers of overweigth or obese children conincidence only in responsibility perceived with monitoring and restriction. Indicating, differences between the way in which the mothers exercise their parenting feeding practices. Discussion: The correlations found between the subscales of the CFQ and the body mass index of the children, coinciding with that found in several studies all over the world (Navarro \& Reyes, 2016; Burton, Wilder, Beech \& Bruce, 2017; Lui et al., 2014; Camci, Bas \& Buyukkaragoz, 2014). Regarding on the correlation between subscales of the CFQ and between groups of mothers of children with different nutritional status, no studies were found, important fact, since knowing how these practices interact with each other, gives us a guideline for the realization of contents for intervention programs directed to specific populations, as the case of mothers of overweight or obese children. It is considered appropriate to replicate the study in other institutions with similar characteristics for comparison and generalization.

CORRESPONDING AUTHOR: Nayeli Grijalva, Universidad de Guadalajara,naye100@gmail.com 
P167

PSYCHOSOCIAL AND ENVIRONMENTAL FACTORS OF PHYSICAL ACTIVITY AMONG CHINESE ADOLESCENTS IN BEIJING: TOWARDS SOLUTIONS TO RISING OBESITY EPIDEMIC Juan Zhang ${ }^{1}$, Wei Liao ${ }^{1}$, Shuya Cai ${ }^{1}$, Lei Ba ${ }^{2}$, Yang Liu ${ }^{1}$

${ }^{1}$ Peking Union Medical College \& Chinese Academy of Medical Sciences- China

${ }^{2}$ Chao Yang District Centers for Disease Control and Prevention- China

Background: Insufficient participation in physical activity have been observed among Chinese adolescents. The roles of social and environmental factors in shaping physical activity among Chinese adolescent is under-investigated. The purpose of the present study was to apply health behavior theories to understand the psychosocial and environmental influences of physical activity participation among Chinese adolescents in Beijing. Methods: Two middle schools from Chaoyang District of Beijing, were invited into the study. All students in grade 7 from 10 classes were invited to participate. In total, 543 students completed questionnaires during class time in October and November of 2017. Missing information left a sample of 513 students were included for data analysis. Self-administered questionnaire collected physical activity as well as psychosocial and environmental factors. The target behavior was moderate-to-vigorous physical activity (any activity that is energetic but not exhausting to any activity at a higher intensity that causes your heart to beat rapidly, and make you huff and puff) on a regular basis. RESULTS: Participants were 513 seventh-grade students $(55.94 \%$ boys and $44.06 \%$ girls), ranging in age from 11 to $15(\mathrm{M}=13.24, \mathrm{SD}=0.56)$, with $97.5 \%$ aged between 13 and 14 years. The schools offered physical education (PE) class 3 classes/week and 45 minutes/class, and $78.3 \%$ reported that they participated in moderate-to-vigorous physical activity during the PE class. In addition to PE class, $58.43 \%$ of students participated in organized sports 1-2 times/week and $16.08 \%$ reported no organized reports (boys: $12.92 \%$ vs. girls: $18.10 \%$, $\mathrm{P}<0.001$ ). However, approximately $20.08 \%$ reached the physical activity recommendation of 60 minutes daily and sex differences were observed $(\mathrm{P}<0.001,27.11 \%$ boys and $12.16 \%$ girls). The psychosocial and environmental factors-attitude towards outcome, subjective norm, perceived behavior control, self-efficacy, parental modeling, family belief, parental support- had consistent associations with the physical activity intention and participating in sufficient physical activity $(\mathrm{P}<0.001)$. The most frequently mentioned pros of participating in sufficient physical activity were both physical health and nonhealth consequences (e.g., stay in good shape $(74.60 \%)$, weight management (73.63\%), make new friends (71.99\%), and make me good at PE (70.51\%), and cons were "sweating" (62.60\%), "painful" (44.55\%), and "tired" (34.19\%). Mum (66.45\%) and dad (66.24\%) were most important referents for students to participate in sufficient physical activity, while about $18 \%$ agreed that mum/dad do not go with them for physical activity and 20\% agreed that "mum/dad thinks education/study/academic performance is more important than physical activity". Only one quarter agreed that they are confident to participate in physical activity when having assignments or exams. CONCLUSION: Results support context-specific psychosocial and environmental factors of physical activity in China. Targeting environmental factors, in particular increasing family support that may promote opportunities for physical activity as well as adolescent decision-making in China's contexts may help promote physical activity, potentially leading to increased overall physical activity and decreased obesity epidemic in China.

CORRESPONDING AUTHOR: Juan Zhang, Peking Union Medical College \& Chinese Academy of Medical Sciences, zhangjuan@sph.pumc.edu.cn

\section{P169}

DYING DIGNITY AND SELF-TRANSCENDENCE AMONG ADVANCED CANCER PATIENTS: THE MEDIATING ROLE OF SPIRITUAL COPING

Zhanling Xue $^{1}$

${ }^{1}$ Shanxi Medical Univerisity- China
Objectives To explore the mediating role of spiritual coping between dying dignity and self-transcendence among advanced cancer patients. Methods 152 advanced cancer patients were assessed by spiritual coping scale, Selftranscendence meaning of life scale and dignity scale. Results Advanced cancer patients' spiritual coping, dying dignity and self-transcendence were significantly positive correlated with each other. Advanced cancer patients' dying dignity has a direct impact on self-transcendence and spiritual $\operatorname{coping}(\beta=0.046, \mathrm{p}<0.05, \beta=0.013, \mathrm{p}<0.01)$;spiritual coping served as a partial mediator between dying dignity and self-transcendence in advanced cancer patients $(Z=44.6, p<0.01)$. Conclusions Spiritual coping played a partial mediating role on the relationship between dying dignity and self-transcendence among the advanced cancer patients.

CORRESPONDING AUTHOR: Zhanling Xue, Shanxi Medical Univerisity, 18203218526@163.com

\section{P170}

RISK AND PROTECTOR FACTORS OF STRESS IN UNDERGRADUATE MEDICAL STUDENTS

Mariantonia Lemos ${ }^{1}$, Marcela Henao ${ }^{2}$, Diana Carolina Lopez-Medina ${ }^{2}$, Ana Cristina Niño-Caldas ${ }^{2}$, Manuela Moreno-Prez ${ }^{2}$, Alejandro ArangoMachado ${ }^{1}$

${ }^{1}$ Universidad EAFIT- Colombia

${ }^{2}$ Universidad Cooperativa de Colombia- Colombia

Introduction: Chronic stress has been associated with psychosomatic disease and mental disorders. Research has shown high stress levels in medicine students but few studies have identified the main factors related to it in this population. This study evaluated stress in undergraduate students of a medicine school in Colombia to identify risks and protector factors for clinical symptomatology. Methods: Cross sectional study with a random sample of 217 undergraduate students of a medical school in Medellin (54.8\% female, $94 \%$ between 18 - 25 years) assessed during mid-term period. They completed the Perceived Stress Scale (PSS-14), General Anxiety Disorder Scale (GAD 7), Patient Health Questionnaire (PHQ 9), Medical Outcomes Study Social Support Survey (MOS-SSS) and a Coping Skills questionnaire. Additionally, we measured physiological variables related with stress. Results: $64 \%$ of the students reached clinical levels of stress, $56 \%$ of depression and $48 \%$ of anxiety. $50.7 \%$ of the students reported doing some extra-curricular activity. A cluster analysis identified two groups of students, with and without clinical symptoms $(58.9 \%$ and $41.1 \%$ respectively). A binomial stepwise logistic regression was done in order to verify which of the academic and demographic variables was involved (block 1), and which of the psychological (coping skills and social support) (block 2). Both blocks provide a statistically significant improvement over the previous model. The final Nagelkerke R2 indicated that the model accounted for the $45.3 \%$ of the total variance. The results indicated that being women $(\mathrm{OR}=2.77)$ and not doing extracurricular activity $(\mathrm{OR}=2.34)$ were risk factors for being in the group with clinical symptoms; while being in the basic cycle $(\mathrm{OR}=0.41)$ was protector. Regarding psychological factors, using aggressive reaction $(\mathrm{OR}=1.13)$ and expressing difficulties coping with stressors were risk factors $(\mathrm{OR}=1.17)$, while using problem solving $(\mathrm{OR}=0.93)$ was a protector for being in the group with clinical symptoms. Conclusions: The use of extra-curricular activities and problem-solving coping skills over emotion-focused coping skills could be promoted to moderate the consequences of stress in medical students.

CORRESPONDING AUTHOR: Mariantonia Lemos, Universidad EAFIT,mlemosh@eafit.edu.co 


\section{P171}

THE ROLE OF SMOKING CESSATION IN CLINICAL ONCOLOGY: AUSTRALIAN ONCOLOGISTS' EXPERIENCES, PREFERENCES AND PRACTICES

Christine Paul ${ }^{1}$, Fiona Day ${ }^{1}$, Freddy Sitas $^{2}$, Tina $\mathrm{Chen}^{3}$, Marianne Weber $^{2}$, Emma Sherwood ${ }^{1}$

${ }^{1}$ University of Newcastle- Australia

${ }^{2}$ University of Sydney- Australia

${ }^{3}$ Cancer Institute NSW- Australia

Introduction: Despite evidence of improved outcomes for smoking cesation following a cancer diagnosis, smoking care in oncology is relatively unstudied. This study explored the perceptions and practices of medical and radiation oncologists.

Methods: Membersof the relevant professional societieis were invited by postal mail and email to complete an anonymous survey. Results and Conclusion: Response rate $=42 \%$. More than $90 \%$ of respondents reported they assessed smoking status most of the time at the initial appointment, les often and follow-up. Less than $20 \%$ discussed cessation medications, referred for support or provided written materials for most smokers. For advanced disease, an individualised approach was favoured. While most supported cessation as part of cancer care, only $11 \%$ felt adequately trained. More than $40 \%$ reported oncologists should have advanced skills in smoking care. A lack of time and patient resistance were perceived as barriers to smoking care. A preference for GP involvement was apparent. There is a need to provide training and implement care models which involve oncologists in a targeted manner.

CORRESPONDING AUTHOR: Christine Paul, University of Newcastle, chris.paul@newcastle.edu.au

\section{$\mathrm{P} 172$}

THE REASONED ACTION APPROACH TO UNDERSTANDING HEALTH PROTECTION AND HEALTH RISK BEHAVIORS

Mark Conner ${ }^{1}$

${ }^{1}$ University of Leeds, United Kingdom

Introduction: The Reasoned Action Approach (RAA) developed out of the Theory of Planned Behavior but has not yet been widely applied to understanding health behaviors. This research used the RAA in a prospective design to test predictions of intention and action for protection and risk behaviors.

Methods: Measures of RAA components plus past behavior were taken in relation to 8 protection and 6 risk health behaviors in 385 adults. Selfreported behavior was assessed one month later.

Results: Multi-level modelling showed instrumental attitude, experiential attitude, descriptive norms, capacity and past behavior were significant positive predictors of intentions for protection or risk behaviors. Injunctive norms were only significant predictors of intention in protection behaviors. Autonomy was a significant positive predictor of intentions in protection behaviors and a negative predictor in risk behaviors. Multi-level modelling showed that intention, capacity, and past behavior were significant positive predictors of action for both protection and risk behaviors. Experiential attitude and descriptive norm were additional significant positive predictors of risk behaviors.

Conclusions: The RAA has utility in predicting both protection and risk health behaviors although the power of predictors may vary across these types of health behavior.

CORRESPONDING AUTHOR: Mark Conner, University of Leeds, m.t.conner@leeds.ac.uk
P173

PSYCHOSOCIAL RESEARCHER \& EDUCATOR

Lina Mayorga $^{1}$, Gloria Juarez ${ }^{1}$, Martin Pérez ${ }^{2}$

${ }^{1}$ Oncology Research Education Consultants- USA

${ }^{2}$ CityofHope National Medical Center- USA

The Influence Of Culture \& Language in Providing relevant and appropriate cancer risk counseling and education for underserved Latinas"

Background: Research demonstrates that Latinas are highly likely to have cancer risk associated with genetic predisposition to breast cancer and ovarian cancer. In addition underserved, Latinas have a compelling need for access to genetic cancer risk assessment (GCRA) and cancer screening and prevention measures, along with culturally appropriate education. Objectives: The primary purpose of this project was to evaluate the effectiveness of a culturally relevant GCRA educational intervention for Latinas.

Methods: Four focus groups were conducted consisting of Latinas that have undergone GCRA. Participants completed a demographic questionnaire that included items assessing perceived cancer risk and patient satisfaction. The focus groups entailed a facilitated discussion of the key study variables and other culturally relevant issues that may impact the GCRA intervention. Descriptive statistics and thematic analysis were used and analyzed.

Results: Findings show that there is a perceived sense of lack of information, education and uncertainty about what to expect appeared to play a key role in distress. Most women initially had negative expectations (expecting the worse or bad outcome), but ultimately felt hopeful that they could learn more about the GCRA process and what it meant for them. Information was cited as the primary contributor to positive psychosocial outcomes specifically increased locus of control and self-efficacy. Cultural themes identified were destiny, religious and spiritual coping mechanisms, how cultural attitudes and beliefs influence lack of information, community awareness, and public health issues.

Conclusions: Findings indicate that the pre-GCRA window may be most distressing for this population indicating that this may be the most appropriate time for psychological intervention. Along with gaps in the unavailability of culturally appropriate education, information and tools for the Latino community.

CORRESPONDING AUTHOR: Lina Mayorga, Oncology Research Education Consultants, Lina.Mayorga@yahoo.com

\section{P174}

PREDICTORS IN HOME ENVIRONMENT OF PRESCHOOL CHILDREN'S SEDENTARY TIME

Eva Roos ${ }^{1}$, Reetta Lehto ${ }^{1}$, Nina Sajaniemi ${ }^{2}$, Maijaliisa Erkkola ${ }^{2}$, Carola Ray $^{1}$, Elviira Lehto ${ }^{1}$

${ }^{1}$ Folkhälsan Research Center- Finland

${ }^{2}$ University of Helsinki- Finland

Introduction \& Purpose: Childhood obesity is a major public health concern, especially in low socioeconomic status (SES) groups. Energy balanced-related behaviors (EBRBs), including sedentary behavior, are important predictors of obesity. To be able to improve children's EBRBs and to narrows SES differences (in what), it is important to find modifiable predictors of EBRBs especially among children with low parental SES. The aim of this study is to examine whether modifiable factors in home environment are associated with objectively measured sedentary time (SED) of 3-6-year-old children and whether the associations vary depending on parental education. Methods: This research is part of the cross-sectional DAGIS study (www.dagis.fi). The parents $(n=809)$ reported (fall 2015- spring 2016) their educational level and several home physical and social environment factors related to the child's physical activity (PA) and screen time (ST). The children wore an ActiGraph accelerometer during seven days (valid data for $\mathrm{n}=784$ ). Association 
between predictors and children's SED was tested with regression analyses. Moderating effect of parental education for the associations was tested by interaction analyses. Results: Children's ST, parental opinion about suitable amounts of ST per day, and spending more time using screens when their child was present were positively associated with children's SED. Spending time with the child in nature, forest or own yard, being physically active when their child was present, finding it important to limit children's ST and finding PA to be important were negatively associated with children's SED Self-efficacy to get the child physically active and parental opinion about suitable amount of PA for children and being pleased with own child's ST was negatively associated with children's SED mainly among the low parental education group. Conclusions: Several modifiable factors in home environment are associated with objectively measured SED in 3-6 year old children. Interventions to balance EBRBs should focus on changing these predictors. Intervention aiming to narrow SES differences should, in addition, focus on predictors associated with SED mainly among children with low parental SES.

CORRESPONDING AUTHOR: Eva Roos, Folkhälsan Research Center, eva.roos@folkhalsan.fi

\section{P175}

TARGETING SLEEP PROBLEMS TO ADDRESS MENTAL HEALTH ISSUES IN POST-DISASTER COMMUNITIES: MENTAL HEALTH CONSEQUENCES IN THE AFTERMATH OF TYPHOON HAIYAN Charlie Labarda ${ }^{1}$, Christian Chan ${ }^{1}$

${ }^{1}$ The University of Hong Kong

We examined sleep disturbances, posttraumatic stress (PTS) and general psychological distress (GPD) among survivors of Super Typhoon Haiyan, which made landfall in the Philippines 2013. The study was conducted to ascertain the relationship between sleep disturbances among survivors and mental health consequences in post-disaster community setting. Sleep disturbances were hypothesized to be associated with PTS and GPD in two separate samples of respondents using Kessler-6 (K6), Insomnia Severity Index (ISI), Pittsburgh Sleep Quality Index (PSQI) and PTSD Checklist (PCL) measures. Sample $1(\mathrm{n}=223)$ was surveyed for insomnia 18 months after the storm; Sample $2(n=138)$ was surveyed 30 months after the storm.

Results from structural equation modeling indicated that insomnia was associated with both PTS and GPD (Sample 1). Poor sleep quality was associated with PTS but not with GPD (Sample 2). These findings underscore the longer-term relationship between sleep disturbances and overall sleep quality to PTS in the context of a natural disaster. In many countries, the stigma of mental health problems hinders effective population-based interventions. Public health programs addressing sleep disturbances might be able to bypass resistance to psychosocial interventions, since sleep-related problems are $c^{\circ} \mathrm{mmon}$ for many conditions.

CORRESPONDING AUTHOR: Charlie Labarda, The University of Hong Kong, clabarda@hku.hk

\section{P176}

DEGREE OF IMPACT OF PSYCHOLOGICAL ASSESSMENT DURING ALTITUDE TRAINING WITH ELITE ATHLETES Leonardo Eliecer Tarqui Silva ${ }^{1}$

${ }^{1}$ Universidad Estatal De Milagro, Facultad De Ciencias De La Educacion- Ecuador

The main purpose of this work in sport psychology aims to identify psychological effects caused by training 21 days in height with elite athletes who practice the disciplines of swimming, mountain biking, judo, boxing and triathlon; alongside the degree of impact of psychological intervention with a program of mental preparation during this period was subjectively evaluated. The research was done during sport training camps in height condition in the city of Cuenca, with a total mixed sample of 45 subjects ( 20 men and 25 woman) for different countries during the years 2007-2012, who were applying the Perceived Stress, Scale Borg, POMS (test of moods states), and Ways of Coping Check List (adapted sport) to obtain the results of in terms of stress and burnout projected between the twenty eight day of acclimatization. The results indicate a positive psychological development of scale stress and adaptation on day 20 assessment (retest), concluding that the mental training program directly affects athletes, improving mental processes of acclimatization Keywords: psychological effects, altitude trainning, stress, exhaustion, acclimatization.

CORRESPONDING AUTHOR: Leonardo Eliecer Tarqui Silva, Universidad Estatal De Milagro, 1tarquis@unemi.edu.ec

P177

PHYSICAL AND SPORTS ACTIVITY IN ADOLESCENTS SPA CONSUMERS

Leonardo Eliecer Tarqui Silva ${ }^{1}$

${ }^{1}$ Universidad Estatal De Milagro, Facultad De Ciencias De La Educacion- Ecuador

The present study shows the therapeutic approach based on physical and sports activities applied as reinforcer, channeler and behavioral modifier in conjunction with cognitive, socio-humanistic, spiritual, occupational, experiential and family therapies performed in adolescents using psychoactive substances (SPA) between Ages 14 to 17 years in the Therapeutic Community for Adolescent Males Hogar Crecer (CTHC), of the city of Cuenca - Ecuador; resulting in a reduction and channeling of aggressiveness levels, improvements in the area of socialization, self-esteem, academic performance and an adequate process of detoxification and direct incidence in the initial critical stage of abstinence. The results of the research are obtained through the application of clinical and sports psychological reagents; together provide the reports and diagnoses of weekly evolution of clinical staff during the 6 months of treatment in hospitalization modality administered in initial and subsequent periods of the psych educational process.

CORRESPONDING AUTHOR: Leonardo Eliecer Tarqui Silva, Universidad Estatal De Milagro, 1tarquis@unemi.edu.ec

P178

THE NEUROIMMUNE MECHANISM OF KP PATHWAY ACTIVATION IN RESERPINE INDUCED DEPRESSIVE-LIKE RAT MODEL

Ning $\mathrm{Wu}^{1}$, Jingjie Zhao ${ }^{2}$, Xuesong $\mathrm{Gao}^{2}$, Anna Wang ${ }^{2}$, Yongzhi Wang ${ }^{2}$, $\mathrm{Li} \mathrm{Li}^{2}$

${ }^{1}$ Southeastern Oklahoma State University

${ }^{2}$ Beijing Friendship Hospital, Capital Medical University- China

Previous studies found that Reserpine-induced depression may be useful to establish animal model. Reserpine can inhibit vesicular transporter and increase the activity of IDO that can reduce the bioavailability of internal tryptophan by decomposing tryptophan into kynurenine, which directly affects the synthesis of 5-HT in central nervous system. 24 SD male rats were divided into control and model groups. Reserpine and saline were intraperitoneal injected daily $(0.3 \mathrm{mg} / \mathrm{kg})$. After 14 days, the animals were subjected to general assessment, open field test (OFT), and forcedswimming test (FST). RT-PCR was applied to detect brain IL-1b, caspase1, and IDO genes' expression levels. The serum IL-1b and caspase1 were determined by Elisa. The brain 5-HT amount was measured by HPLC. The model group demonstrated significant horripilation, back like standing, reduced activities in cage, and irritability than that in control group. The body weight-gain rate and OFT pattern and distance showed significant 
reduction in model group $(\mathrm{P}<0.05)$. The FST time of model group was significantly increased $(\mathrm{P}<0.05)$. The amounts of IL- $1 \mathrm{~b}$ and caspase 1 in the serum were higher in model group $(\mathrm{P}<0.05)$, while the gene expression levels of IL-1b and caspase1 in brain were increased $(\mathrm{P}<0.05)$. The IDO gene expression level in model rat brain was increased significantly than that in control group $(\mathrm{P}<0.05)$. while the level of 5-HT in model group was decreased significantly than that in control group $(\mathrm{P}<0.05)$. Reserpine induced depressive-like rat model demonstrated significant depressive behavioral characteristics. Reserpine can induce the increasing of inflammatory factors in experimental animals' peripheral blood and brain tissue, activate IDO which can enhance the activity of KP pathway, and eventually reduce 5-HT production and lead to the development of depression.

CORRESPONDING AUTHOR: Ning Wu, Southeastern Oklahoma State University,nwu@se.edu

\section{P179}

PROTECTIVE EFFECTS OF CURCUMIN ON BEHAVIORAL AND IMMUNOLOGICAL FLUCTUATIONS INDUCED BY INTERMITTENT UNPREDICTABLE STRESS REFLECTING COMBAT-RELATED STRESS

Dongsoo Kim ${ }^{1}$, Chang Yul Kim², Yeon Kyung $\mathrm{Kim}^{2}$, Hyojin Koo ${ }^{1}$, Kicheol Cheon ${ }^{3}$

${ }^{1}$ Korean Air Force Academy- Republic of Korea

${ }^{2}$ Catholic University of Daegu- Republic of Korea

${ }^{3}$ Korean Agency for Defense Development- Republic of Korea

Introduction: Many of veterans have suffered from combat-related PTSD. This study conducted to investigate whether curcumin could prevent symptoms of intermittent unpredictable stress (IUS) simulating combat exposure stress. Curcumin is a kind of alkaloid that mainly contained in Indian turmeric, and is a food additive. Curcumin have been known as an antioxidant and anti-inflammatory substance. Curcumin can restore behavioral changes due to chronic stress, inhibit the hyperactivity of the HPA axis, and restore BDNF production. Recent studies also suggest that curcumin is efficacious for forgetting fear memory. However, curcumin have not been recognized as a drug because pharmacological efficacy of curcumin has not been clearly defined.

Methods: 8 week-old Balb/cByJ mice were administered orally with curcumin $(20 \mathrm{mg} / \mathrm{kg})$ solved in $200 \mathrm{ml}$ olive oil starting from a week before they were exposed to IUS until the end of the experiment. IUS, which consists of sleep deprivation, electric foot shock, and sound stimulus, was set to 3 bouts ( $24 \mathrm{~h} /$ bout) exposure in a 2 -week period.

Results: IUS caused hyperactivity and decrease of explorative activity, which were inhibited relatively by curcumin. Curcumin have no effect on attenuation of memory induced by IUS exposure. However, curcumin down-regulated elevated levels of serum corticosterone $(\mathrm{p}<0.04)$ and pro-inflammatory cytokines. IFN $\gamma(\mathrm{p}<0.001), \mathrm{TNF} \alpha(\mathrm{p}<0.02)$, and IL-6 $(\mathrm{p}<0.01)$ due to IUS exposure.

Conclusions: This study suggests that neurobehavioral and immunological adaptation to stress may not be directly associated. Curcumin may have little preventive effect on hyperactivity in locomotion and impaired memory, implying the anti-stress effect of curcumin may be restricted to peripheral stress responses.

CORRESPONDING AUTHOR: Dongsoo Kim, Korean Air Force Academy, dongsookim04@gmail.com

\section{P180}

OBSESSIVE RELATIONAL INTRUSION: COPING, RESILIENCE AND SYMPTOMATOLOGY IN MEXICO

Rozzana Sánchez-Aragón ${ }^{1}$, Daniela J. Cruz-Vargas ${ }^{1}$, Alejandra E. PérezPérez ${ }^{1}$, Berenice Jiménez-Rodríguez ${ }^{1}$

${ }^{1}$ National Autonomous University of Mexico- Mexico
When between two persons, one of the parties seeks a more intimate relationship than the other party wants, and that search persists for a certain period of time, becomes an obsessive relational intrusion (ORI) (Cupach \& Spitzberg, 2004). Given this, the victim of ORI experiences severe effects on their physical and mental health, tied with an imbalance in their social, work and financial conditions (MacKenzie et al., 2009). Given this, the person deploys certain coping tactics (Sánchez Aragón, 2014) mainly oriented to his/her self-protection. In more recent years Spitzberg (2011) has pointed out that the situation of stalking stimulates the development of mental, emotional, anxiety, social, spiritual and selfdestructive traumatic symptoms. This scenario requires that the individual improve his/her ability to face shocking experiences but to extract a benefit from them (Melillo \& Suarez, 2003). From the above, the objectives of this research were: 1) Characterize the nature of the ORI, 2) Describe the main ORI tactics, \& 3) Know the relationship of the tactics of the ORI with Coping, Symptomatology and Resilience in Mexicans. For this, we worked with a sample of 506 women and 215 men victims of harassment. The findings indicate that the situation was perceived as stalking, mainly in real space, without physical violence, threatening to harm their safety or that of their family. We also observed the notoriety of hyper-intimacy, contact during interaction, persecution by other persons and surveillance in intensity and frequency; as well as the mediated contact, invasion and aggression-violence in their intensity. Finally, when there is more frequency and intensity in the ORI tactics, more withdrawal, protect, negotiate, seek support, evade and block reactions, more psychological and sexual symptoms, and more resilience.

CORRESPONDING AUTHOR: Rozzana Sánchez-Aragón, National Autonomous University of Mexico, rozzara@unam.mx

\section{$\mathrm{P} 181$}

NEGOTIATING A GOOD NIGHT'S SLEEP DURING HOSPITAL ADMISSION

Mette Terp Hoybye ${ }^{1}$, Malene Frosch Langvad ${ }^{2}$, Lene Bastrup Jørgensen $^{3}$, Karen Schmøkel ${ }^{2}$

${ }^{1}$ Aarhus University- Denmark

${ }^{2}$ Elective Surgery Center, Silkeborg Regional Hospital- Denmark

${ }^{3}$ Department of Clinical Medicine, Aarhus University- Denmark

Introduction \& Purpose Sleep is an (in)activity attracting much attention during hospital admission as it holds key importance to the healing and rehabilitation of patients. Studies have shown however, that it is often difficult to get sufficient sleep during hospitalization. By taking an anthropological approach to nighttime and sleep in hospitals, we explored sleep as a collaboration and negotiation between different actors in the clinic, paying attention to how both environmental and social contexts affect the conditions for sleep. The study explored the environment of sleep during nighttime and the culture of sleep in patients admitted to hospital for elective orthopedic surgery. Methods Ethnographic methods of participant observation and informal conversation were employed around the clock over one month with attention to both patients, hospital staff and the environment of the ward. Following this semi-structured interviews were carried out with 10 patients after discharge from hospital. An anthropological analysis was employed moving between intentions and views expressed by the patients and staff and what was observed during the ethnographic fieldwork. Results The experience of sleep during hospital admission should be understood in relation to patient expectations of sleep. Sleep can be understood as taking place in a negotiated, social context and as a collaboration with other actors - human and nonhuman - during hospital admission. Strategies of the staff to improve sleep may not always be acceptable to patients. Conclusion The study illustrates that sleep is not only a naturally occurring, physical phenomena, but documents how obtaining and sustaining sleep is dependent on a collaboration and negotiation with other actors than the individual patients. The ideal of good care in staff may be contradictory to the patients' 
potential for choice. Clinical interventions addressing sleep should assess individual expectations of sleep and seek to navigate the dilemmas of choice and care in hospital settings.

CORRESPONDING AUTHOR: Mette Terp Hoybye, Aarhus University, hoybye@clin.au.dk

\section{P182}

STUDY ON THE DRIVING FACTORS AND FORMING MECHANISM OF COMMUNITY DOCTOR 'S FIRST DIAGNOSIS WILLINGNESS

Minzhuo Huang ${ }^{1}$, Yuxuan $\mathrm{Gu}^{1}$, Hengjin Dong ${ }^{1}$

${ }^{1}$ Zhejiang University- China

Introduction \& Purpose Primary Diagnosis at Grassroots Level system is the core basis of the grading system, and community doctors are the backbone of primary diagnosis at the grassroots level. This article is aimed to explore the driving factors and forming mechanisms of community doctor's first diagnosis willingness, and to provide reference for promoting the system of grading medical treatment. Methods Based on the KAP theory and Expectancy theory, this paper constructs a formation mechanism model of community doctor's first diagnosis willingness. And on the basis of the questionnaire survey of 430 community doctors in 4 main urban areas and 12 streets in Hangzhou city, multiple regression analysis and structural equation model are used to explore the driving factors and formation mechanism of their grassroots first willingness. Job satisfaction has an indirect effect on the community doctor's first diagnosis willingness. Results Valid data of 403. The empirical results show that community doctor's first diagnosis willingness is between the general and the satisfaction, there is still much room for improvement. Their positive expectations of grassroots first have a direct impact on their grassroots first willingness. Their capacity is the core variables that affect their willingness and their cognition of it is an important prerequisite to influence their willingness. Policy guidance is also an important factor that can't be ignored. Job satisfaction has an indirect effect on the community doctor's first diagnosis willingness. Conclusions For better policy promotion, the following points are highlighted:1. Improve the community doctor's first diagnosis cognitive level;2. Improve the first diagnosis ability for community doctors by enhancing the general practitioner training system and equipment and facilities;3. Improve supporting policies, such as health insurance policy, pharmaceutical policy and medical equipment policy;4. Establish community doctor's first diagnosis incentive mechanism;5. Introduce supervisors such as government, hospitals and ordinary citizens to achieve coordinated governance and supervision of multiple subjects.

CORRESPONDING AUTHOR: Minzhuo Huang, Zhejiang University, huangminzhuo@hotmail.com

\section{P183}

TEACHING GENERIC COMPETENCIES OF THE CANMEDS MODEL IN RESIDENCY PROGRAMS AT THE PONTIFICIA UNIVERSIDAD CATÓLICA (PUC) DE CHILE

José Pinedo Palacios ${ }^{1}$, Catalina Riquelme ${ }^{1}$, Claudia Parra ${ }^{1}$, Matías González ${ }^{1}$, María Trinidad Hoyl ${ }^{1}$

${ }^{1}$ Dirección de Postgrado, Escuela de Medicina PUC Chile- Chile

Introduction and Purpose The PUC School of Medicine, has had the initiative to implement the competencies of the CanMEDS Roles in the residency training. This model seeks that physicians not only demonstrate clinical competencies, but also generic competencies, in order to respond to society's current expectations about health care. Through a biopsychosocial and a patient-centered approach, this model allows to incorporate to the residency programs curriculum, core thematics of behavioral medicine, such as empathy, the consideration of the context and the culture, respect and confidentiality, effective communication, among others. In this context, the purpose of this proyect was to develop courses to train residents at the PUC Chile in generic competencies of the CanMEDS Roles, through a b-learning methodology. Method To create the courses in b-learning methodology, a standardized and continuous process was carried out, which included the following phases: design, production, implementation, evaluation, and redesign. The courses lasted ten weeks each and included seven online lectures and three classroom workshops. Results Nine courses were developed, addressing six Roles of the CanMEDS model. Five of these courses included in their programs the following aspects of behavioral medicine: health promotion and prevention, health inequities, physician-patient communication, ethical dimension of medical practice and teamwork. Overall, 191 residents have participated in these five courses, of which $80.1 \%(n=153)$ responded to the evaluation survey. A $90.2 \%(n=138)$, indicated that the course carried out allowed them to reflect and become aware of the course Role and $88.9 \%(n=136)$ mentioned that they would strongly recommend the course to other colleagues. Conclusions Through the implementation of courses based on the CanMEDS competency model, core aspects of behavioral medicine have been incorporated in the residency programs curriculum. The teaching of these topics contributes to the training of residents that will be better prepared to provide high quality, safe, and patient-centered health care.

CORRESPONDING AUTHOR: José Pinedo Palacios, Dirección de Postgrado, Escuela de Medicina PUC Chile, jrpinedo@uc.cl

P184

A ROAD-MAP TO BETTER UNDERSTAND, PREDICT AND PREVENT PERSISTENT POST-SURGICAL PAIN

Madelon Peters ${ }^{1}$

${ }^{1}$ Maastricht University- Netherlands

Introduction \& purpose Persistent pain is a highly unwanted but relatively prevalent outcome of surgery. Prevalence figures of persistent postsurgical pain (PPSP), usually defined as pain that persists for 6 months or longer and located at the site of surgery, range from 5 to up to $60 \%$ depending on the type of surgery. Numerous studies have identified factors that are associated with persistent postsurgical pain, the most prominent ones being presurgical pain, intense pain in the early post-operative phase and several psychological factors. The purpose of this presentation is to review the predictive role of psychological factors. Four questions will be addressed: (1) which specific psychological factors are involved; (2) how substantial is their effect; (3) what are the mechanisms by which they lead to pain persistence; and (4) can we prevent PPSP by psychological interventions. Methods First, a concise overview of previous predictor studies is given with an emphasis on orthopedic and breast surgery, which are among the procedures with the highest prevalence of PPSP. Next, new data from breast surgery patients will be presented. 281 patients filled in psychological questionnaires pre-operatively; pain reports were obtained 1 and 12 months after surgery. Finally, published and ongoing prevention studies will be reviewed. Results The most robust psychological predictors are pain catastrophizing, anxiety and expected pain with effects sizes of around $r=0.30$. In our breast surgery cohort, 125 patients $(44.5 \%)$ experienced pain at 1 -year follow-up; this was predicted by pain catastrophizing $(\beta=.14, \mathrm{p}=0.017)$ and expected pain $(\beta=.18, \mathrm{p}$ $=0.005$ ). Only few studies examined whether psychological intervention can reduce PPSP; however, preliminary results are promising. Conclusion Numerous studies have demonstrated that presurgical psychological states are related to PPSP. Future studies should elucidate the underlying mechanisms and importantly, examine whether peri-operative psychological interventions can mitigate the risk of PPSP. Randomized clinical trial such as those presented by the next speakers are of great clinical and scientific relevance. They have direct patient benefit and can establish 
whether psychological factors are causally related to PPSP.

CORRESPONDING AUTHOR: Madelon Peters, Maastricht University, madelon.peters@maastrichtuniversity.nl

P185

MEETING THE RECOMMENDATIONS OF MOVEMENTRELATED BEHAVIORS AND ITS ASSOCIATION WITH MENTAL HEALTH: A CROSS-SECTIONAL STUDY WITH BRAZILIAN STUDENTS

Alexsandra da Silva Bandeira ${ }^{1}$, Margarethe Thaisi Garro Knebel ${ }^{1}$, Valter Cordeiro Barbosa Filho ${ }^{2}$, Kelly Samara Silva ${ }^{1}$

${ }^{1}$ Universidade Federal de Santa Catarina- Brazil

${ }^{2}$ Instituto Federal do Ceará- Brazil

Introduction and purpose: Recent evidence has shown that movementrelated behaviors (i.e., physical activity, sedentary behavior and sleeping), although independent, may interact with and impact on the physical, social and mental health of adolescents. Thus, this study aimed to examine the proportion of adolescents who meet the recommendations of physical activity (PA), screen time (ST) and sleep, and the association among combinations of these behaviors with mental health indicators (quality of life and self-rated stress). Methods: A cross-sectional survey was carried out in August 2014. The sample was composed of 1,182 adolescents ( $51.5 \%$ boys) aged from 11 to 17 years from public schools in Fortaleza, northeastern Brazil. A standardized questionnaire was applied including questions about PA (weekly volume), TV and computer/ video game time (combined in ST) and sleep (duration on weekdays). We used the following recommendations: PA (420 min/week); ST ( 8 hours/ day). The mental health indicators (positive/negative) were quality of life and self-rated stress. Sex and age were control variables. Binary logistic regression models were performed (odds ratio: OR; $95 \%$ confidence interval: $95 \% \mathrm{CI}$ ), considering $\mathrm{p}<0.05$. Results: The proportion of adolescents who met the individual recommendations was $41.9 \%$ (PA), $13.8 \%$ (ST) and $38.5 \%$ (sleep). Regarding combined behaviors, we found that $16.9 \%$ (95\% CI: 14.9-19.1) of the adolescents met the recommendations of PA/sleep. While $5.2 \%$ (95\% CI: 4.1-6.6) and 4.8\% (95\% CI: 3.7-6.2) complied with the recommendations of ST/sleep and PA/ST, respectively. Only $1.9 \%$ (95\% CI: 1.2-2.8) of students attended the three recommendations. Adolescents who did not meet the recommendations of PA/sleep and PA/ST were less likely to have a positive perception of quality of life (PA/sleep: OR: $0.63 ; 95 \%$ CI: 0.42-0.94) and self-rated stress (PA/sleep: OR: 0.58; 95\% CI: 0.37-0.91; PA/ST: OR: 0.25 ; 95\% CI: 0.09-0.72). Other combinations were not associated with mental health indicators. Conclusions: Few students met all three behavioral recommendations. In addition, failure to meet PA/sleep and PA/ST recommendations were associated with a poor perception of quality of life and stress.

CORRESPONDING AUTHOR: Alexsandra da Silva Bandeira, Universidade Federal de Santa Catarina, alebandeiraufc@ gmail.com

\section{P186}

DOES ORGANIZATIONAL READINESS FOR CHANGE PREDICT HEALTH PROMOTION INTERVENTION OUTCOMES? A LONGITUDINAL STUDY AMONG HEALTHCARE PROVIDERS IN CHINA

Shan Qiao ${ }^{1}$, Wendi $\mathrm{Da}^{1}$, Xiaoming $\mathrm{Li}^{1}$, Yuejiao Zhou ${ }^{2}$, Zhiyong Shen ${ }^{2}$

${ }^{1}$ University of South Carolina- USA

${ }^{2}$ Guangxi CDC- China

Introduction \& Purpose: Existing literature suggests that organizational readiness for change (ORC) is one of critical facilitators in adapting, implementing and maintaining new projects or practices in clinical settings. However, most of the studies are based on cross-sectional data. The current study aims to explore if baseline organizational readiness for change predicts intervention outcomes based on longitudinal data collected from an RCT trial on HIV parental disclosure intervention in China. Methods: 479 healthcare providers were recruited from 42 HIV clinics across 12 cities/counties in Guangxi China for a multi-level intervention with aims to assist HIV-positive parents in making appropriate decisions about disclosing their HIV status to their HIV-negative children and provide consulting and support in post-disclosure adaption. All the participants took baseline survey before intervention assignment in 2013, after the intervention follow-up data was collected each 6 month till 2016 (five follow-ups). A series of mixed effect models on intervention outcomes (knowledge on HIV parental disclosure and self-efficacy in assisting HIV-positive parents in disclosure) were used in order to control for within-cluster and within-subject correlation over time. Collinearity of baseline background variables were checked using VIF. Results: Among the 319 participants who responded to self-efficacy, knowledge, and ORC questions at baseline, ORC was positively associated with selfefficacy in assisting parents to deal with parental HIV disclosure over time $(\beta=.096,95 \% \mathrm{CI}=[0.037,0.155], \mathrm{p}<.001)$ controlling for intervention status, demographic and working characteristics. The ORC level was also positively associated with knowledge of parental HIV disclosure, yet the association was not significant after controlling other covariates $(\beta=.029,95 \% \mathrm{CI}=[-0.009,0.065], \mathrm{p}=.14)$. Being a female healthcare providers $(\beta=.64,95 \% \mathrm{CI}=[0.14,1.13], \mathrm{p}=.03)$ and having an education level of college and above $(\beta=.61,95 \% \mathrm{CI}=[0.08,1.13], \mathrm{p}=.025)$ were positively associated with knowledge of parental HIV disclosure. Conclusions: Our findings suggest that baseline ORC level could predict some intervention outcomes over time controlling for intervention status and other demographic and working factors. It is important to integrate improving ORC level into the strategies of implementing interventions in clinical settings to improve the intervention efficacy.

CORRESPONDING AUTHOR: Shan Qiao, University of South Carolina, shanqiao@mailbox.sc.edu

\section{P188}

A RANDOMISED CONTROLLED TRIAL OF A BRIEF PSYCHOLOGICAL INTERVENTION TO REDUCE REPETITION OF SELF-HARM IN PATIENTS ADMITTED TO HOSPITAL FOLLOWING A SUICIDE ATTEMPT

Rory $\mathrm{O}^{\prime}$ Connor ${ }^{1}$

${ }^{1}$ University of Glasgow- United Kingdom ${ }^{\circ}$

Introduction: Few interventions for self-harm have been developed specifically for administration in acute settings. We investigated whether a volitional helpsheet (VHS), a brief psychological intervention, could reduce repeat self-harm in the 6 months following a suicide attempt. Methods: We conducted a randomized controlled trial in which we investigated the utility of a new brief implementation-intentions-based intervention to reduce risk of repeat self-harm in the next 6 months among those who have attempted suicide. In this study, there were three primary outcomes: (i) the number of participants who re-presented to hospital with self-harm over six months; (ii) the number of re-presentations and; (iii) cost effectiveness of the VHS. Follow-up data collection was via data linkage and patient medical records. Results: 518 patients who had attempted suicide were randomized to intervention $(\mathrm{n}=259)$ or control groups $(\mathrm{n}=259)$. We obtained complete follow-up data on $98 \cdot 8 \%$ (512/ 518 ). We conducted intention to treat (ITT) and per protocol analyses (PP) and explored whether past history of self-harm affected the efficacy of the intervention. 11 patients did not complete the VHS. Overall, the intervention did not affect the number of people who presented with selfharm or on the number of re-presentations; however, in the PP sub-group analyses, among those who had been previously hospitalised with selfharm, there was a $69 \%$ reduction in the frequency of re-presentation to the Emergency Department and a 55\% reduction in the total number of re- 
presentations. In the ITT analyses, this reduction did not reach statistical significance. There was also evidence, among those with a past self-harm history, that the VHS may be cost effective. Conclusions: The presentation ends with some general conclusions about the implications of employing brief psychological interventions as adjuncts to usual care with individuals who have attempted suicide.

CORRESPONDING AUTHOR: Rory O'Connor, University of Glasgow, rory.oconnor@glasgow.ac.uk

\section{P189}

\section{HEALTH COUNSELOR}

Ricardo Werner-Sebastiani ${ }^{1}$, Pedro Oliveira ${ }^{1}$

${ }^{1}$ Nêmeton Centro de Estudos e Pesquisas em Psicologia e Saúde- Brazil

Health Counselor is a receptive and remote service provided by multidisciplinary group of healthcare professionals that operates about 10 years, in Brazil as response to a set of requirements imposed mainly by the of labour's population urban lifestyle. During this period, such model had covered more than 5 million active members represented by 2 main groups: members linked to Corporations and the growing population of senior citizens Group (the elderly), performing an average of 100 inbound per day. Its original version provides services exclusively through a Contact Center. Today, the model is in transition to provide multimedia contact services, including through Social Networks. Its characterized by providing guidance on health in Psico, Medical, Nutricional and Pharmaco areas. The largest access occurs within the Psychobehavioral Orientation and Medical Advice. The working environment with its continuous and growing pressures causes emotional stress and somato-psychic imbalances that impact on productivity and personal performances. On the other hand, the costs generated by the search for care for these conditions impacts on employment performance with absenteeism, falling of productivity and performance. Such portrait reflexes in the family environment and private life either. Get a health care appointment may take 7, 10 days. Attendance to this appointment can consume half a day, some times, a fully day. Our records (that align with records of similar services performed in other countries) indicate that about $85 \%$ of primary health care demand are set exclusively by a professional advice. Provides customer service support structure claim initially classify the demand in degrees, as: 1st (light), 2nd (moderate) or 3rd (severe). These algorithms are guidelined, backed by renowned specialties for evidence recognized by organs and acreditated entities and defines the type of guidance or proposed reffer. The model of the Health Counselor has been modulated with different structures of benefit coverage, wether under medical, psychological or even nutritional. It's progressively contributed in the area of Pharmaceutical Assistance for questions about drug interactions; adverse events and side effects, among others. The Health Counselor has increasingly been asked for clarification of questions consequent to consultations and surveys conducted through internet search tools.

CORRESPONDING AUTHOR: Ricardo Werner-Sebastiani, Nêmeton Centro de Estudos e Pesquisas em Psicologia e Saúde, ricardo@nemeton.com.br

\section{P190}

CAN WE USE EXPECTATION EFFECTS TO IMPROVE OUTCOMES IN ORTHOPEDIC SURGERY? A META ANALYSIS AND INTERVENTION OUTLINE

Johannes Laferton $^{1}$, David Ebert ${ }^{1}$, Karolin Neubauer ${ }^{2}$, Lara Oeltjen ${ }^{3}$, Thomas Munder ${ }^{3}$

${ }^{1}$ Friedrich-Alexander-Universität Erlangen-Nürnberg- Germany

${ }^{2}$ Smart Helios

${ }^{3}$ Psychologische Hochschule Berlin- Germany
Introduction \& Purpose: Total hip and total knee arthroplasty (THA/ TKA) is one of the most frequently performed major surgery procedure. Although effective, patients do not always benefit from surgery the way doctors would predict from a medical point of view. Several prospective studies have linked positive preoperative expectations to improved outcome in orthopedic surgery patients, while other studies did not find evidence for such an association. Expectations might be a target for preoperative psychological interventions aiming to improve surgery outcome. Therefore, this study aims to meta-analytically synthesize the evidence on the relationship between pre-operative expectations on postoperative outcome in THA and TKA patients. Methods: We searched EMBASE, PsychINFO, and CINAHL for studies reporting a prospective association of preoperative expectations (according to the integrative model of patients' expectations) with surgery outcome (primary outcome: pain) in THA or TKA until August 2017. (PROPSOERO ID: CRD42017079746). Results: We identified 3803 references (after deduplication) of which 201 references remained after the abstract screening. 68 studies were ultimately included after full-text evaluation of inclusion and exclusion criteria. 50 out of the 68 included studies report statistically significant associations indicating that positive preoperative expectations lead to improved surgery outcome. Data extraction, quality assessment and pooling of the effect sizes will be completed until July 2018. Pooled effect sizes will be presented on a) the overall effect of preoperative expectations on THA/TKA outcome, b) differential effects between THA and TKA, c) differential effects of different expectation constructs (i.e. probability vs. value expectations, behavioral expectations vs. treatment expectations). Conclusions: Given a robust and sufficient association between preoperative expectations and outcome in THA/ TKA surgery, psychological expectation interventions might be used to enhance outcome in this surgery population. Based on a successful psychological intervention in cardiac surgery $(\mathrm{d}=0.82$ improved disability vs. standard care), a possible intervention module for orthopedic surgery will be discussed.

CORRESPONDING AUTHOR: Johannes Laferton, FriedrichAlexander-Universität Erlangen-Nürnberg, j.laferton@psychologischehochschule.de

\section{P192}

WHAT VARIABLES MUST USO IN ORDER TO INFLUENCE MOTIVATION AND BURNOUT? IMPACTS OF LEADERSHIP, COHESION AND ROLE CLARITY ON MOTIVATION AND BURNOUT IN TWO CHILEAN HOSPITALS

Andrés Pucheu ${ }^{1}$

${ }^{1}$ Universidad de los Andes- Chile

Introduction \& Purpose: (stating the main purposes and research question) Fomenting the motivation to work autonomously and collaboratively is a key factor in the management of people in the Health Industry; at the same time it is necessary to reduce the appearance of burnout and its effects on performance. The strategies used to encourage the motivation to make efforts to surpass the formal minimums, which in the literature is conceptualized as Organizational Citizen Behavior (CCO), and to control burnout include the strengthening of the leadership of the direct heads, facilitating the cohesion and improving the capacities of the workers, either through the structuring of tasks or the increase of the same capabilities. In this context, the purpose of this work was to compare the direct and indirect effects of these three variables over burnout and CCO, considering at the same time the mediation of burnout in the influence over CCO.

Methods: Scales of measurement of the variables studied were applied in two hospitals, with a total of 667 valid answered surveys (N1 $=292$ and $\mathrm{N} 2=375$ ), exploratory and confirmatory factorial analyzes were carried out, as well as a model of structural equations to test direct and indirect impacts 
Results: With variations in the magnitudes, similar relationships are observed in both samples. Cohesion seems to be the variable that is most strongly related to motivation and, as with leadership, it also affects burnout. At the same time, it is relevant that leadership did not influenced $\mathrm{CCO}$ in one sample, showing a relatively small influence in the other. Conclusions: The intervention through complementary strategies and the focus on cohesion could have the greatest impacts on motivation and burnout. It is possible that only one strategy could not have enough strength to be relevant.

CORRESPONDING AUTHOR: Andrés Pucheu, Universidad de los Andes, apucheu@uandes.cl

\section{P193}

COMPARING MULTIDIMENSIONAL POVERTY BETWEEN INTERNATIONAL MIGRANTS AND CHILEANS: A REPEATED ANALYSIS OF CASEN POPULATION SURVEY 2009-2015

Marcela Oyarte ${ }^{1}$, Baltica Cabieses ${ }^{1}$, Nassim Ajraz ${ }^{1}$, Piedad Galvez ${ }^{1}$, Ana María McIntyre ${ }^{1}$, Claudia Pérez ${ }^{1}$

${ }^{1}$ Programa de Estudios Sociales en Salud, Instituto de Ciencias e Innovación en Medicina ICIM, Facultad de Medicina Clínica Alemana Universidad del Desarrollo

Introduction \& Purpose International migration to Chile has exponentially grown in the past 10 years. The socieconomic heterogeneity of immigrants is large, with an expanding group of immigrants experiencing social vulnerability. Data on mutidimensional poverty amongst immigrants in Chile is outdated. The purpose of this study was to compare multidimensional poverty between international migrants and Chileans for the period 20092015. Methods Exploratory secondary analysis of nationally representative, anonymous Chilean survey CASEN 2009, 2011, 2013 and 2015 (participants belonging to about 70000 households each time). We divided the sample into self-reported immigrants (about 3500 per year) and Chileanborn (about 210 000). We did not include in the analysis those who preferred not to report their migration status (missing values about 2000 each time). We estimated and compared self-reported multidimensional poverty (4 dimensions, 12 indicators in total: access to healthcare, educational level, household living conditions, employment) between immigrants and Chileans. Results The gap of multidimensional poverty between immigrants and Chileans has widened over time (2009: 19,3\% immigrants, 19.5\% Chileans; 2015: 17,2\% immigrants, $14.5 \%$ Chileans). The factors most affecting this gap for immigrants are: no healthcare provision entitlement, overcrowding, lac of social and labour protection and poor quality of care. However, immigrants report higher levels of technical/professional education compared to the Chileans (about 2 years more of formal education than the locals). Conclusions Based on a repeated population survey analysis, international migrants are experiencing more and growing levels of multidimensional poverty than Chileans. This evidence demands urgent attention for multisector collaboration, in order to achieve "heath for all" individuals living in Chile, regardless of their migration status and socioeconomic condition.

CORRESPONDING AUTHOR: Marcela Oyarte, Programa de estudios sociales en Salud, Facultad de Medicina, Universidad del desarrollo, mdoyarte@uc.cl

\section{P194}

REASONS FOR ANXIETY ABOUT HEALTH EFFECTS CAUSED BY RADIATION AMONG COMMUNITY RESIDENTS IN FUKUSHIMA AFTER THE GREAT EAST JAPAN EARTHQUAKE: A QUALITATIVE STUDY USING TEXT MINING

Kazuhiro Watanabe ${ }^{1}$, Norito Kawakami ${ }^{2}$, Maiko Fukasawa ${ }^{2}$, Seiji Yasumura $^{3}$, Hirooki Yabe ${ }^{3}$, Michio Murakami $^{3}$, Yuriko Suzuki ${ }^{4}$, Tsuyoshi Akiyama ${ }^{5}$, Mami Kayama ${ }^{6}$
${ }^{1}$ The University of Tokyo- Japan

${ }^{2}$ Department of Mental Health, Graduate School of Medicine, The University of Tokyo- Japan

${ }^{3}$ Fukushima Medical University- Japan

${ }^{4}$ National Institute of Neurology and Psychiatry- Japan

${ }^{5}$ NTT East Medical Center- Japan

${ }^{6}$ St. Lukes International University- Japan

Introduction: Community residents in Fukushima prefecture suffered tremendous damage from the Great East Japan Earthquake. They have also suffered from anxiety about adverse effects of radiation due to the Fukushima Daiichi nuclear power plant accident. It is almost 7 years from the earthquake, expected that their feelings may change compared to those at the time. The aim of this study was to explore reasons for change of anxiety about health effects caused by radiation. Methods: A total of 2,246 adult community residents in Fukushima prefecture were recruited through 2017 and 2018, from an existing cohort. The participants were asked to complete a self-reported questionnaire. Change of anxiety about health effects caused by radiation was measured by one item rated on a 5point Likert scale ( $1=$ decreased to $5=$ increased). Reasons for the change were also collected in free writing. The text mining method was adopted to analyze reasons for change of anxiety, conducting morphological analysis and extracting words (nouns and adjectives) from their free writing. After the extraction, clusters of the words were created by conducting principal component analysis and cluster analysis. Finally, correspondence analysis was conducted to investigate the relationships between the change of anxiety and the clusters of the words. The study protocol was approved by the ethical committee of the Department of Medicine, The University of Tokyo. Results: A total of 1,420 participants (63.2\%) completed the questionnaire. Of $735(51.8 \%)$ reported decrease of anxiety, $560(39.4 \%)$ reported no change, and the other $56(3.9 \%)$ reported increase. We analyzed 477 participants who reported the change of anxiety and its reasons without missing. From their writing, 134 words were distilled. As a result of correspondence analysis, some of the words that related the participants who reported decrease of the anxiety were "decrease," "radiation," "ordinary days," "safety," "media," "fading with time," "check," "foods," "evidence," and "association." Words that related increase of the anxiety were such as "data," "eye," "forward," "young," "cause," "stress," "dysfunction," and "nation." Conclusions: Decrease of the anxiety among the community residents in Fukushima may be because they confirmed the decrease of the amount of radiation, safety of the foods, and no associations between radiation and health. On the other hand, some of the residents may still feel increased anxiety due to invisibleness of radiation, adverse effects on their descendants, and actual changes on their own health.

CORRESPONDING AUTHOR: Kazuhiro Watanabe, The University of Tokyo, kzwatanabe-tky@umin.ac.jp

\section{P195}

IMPLEMENTATION OF BEHAVIORAL MEDICINE IN A PHYSIOTHERAPY UNDERGRADUATE CURRICULUM STUDENT EVALUATIONS

Maria Sandborgh ${ }^{1}$, Maria Elvén ${ }^{1}$, Petra von Heideken Wågert ${ }^{1}$, Åsa Snöljung ${ }^{1}$, Anne Söderlund ${ }^{1}$

${ }^{1}$ Mälardalen University- Sweden

Introduction \& Purpose In 2004, Mälardalen University, Sweden, introduced a new undergraduate entry-level physiotherapy program with integrated behavioral medicine contents. Theory and evidence based behavior change techniques and clinical reasoning focused on behavior change are integral parts of the program. A competence to analyze biopsychosocial determinants of patients' behaviors in functional behavioral analyses is fundamental. Final-year students evaluate the curriculum specific goal: The student should have acquired a behavioral medicine 
perspective on the interplay between humans' everyday behaviors and biomedical factors in health problems. The aim is to describe how final year physiotherapist students rate fulfilment of the curriculum goal, and how willing, able and ready students are to implement core behavioral medicine components in their clinical work. Methods In a multiple cohort study, students from the final years 2008 to 2013 and 2015 to $2016, \mathrm{n}=$ 265 , responded to a program evaluation questionnaire in which they rated the curriculum goal fulfilment on a 5-graded Likert scale where 5 contributes to high goal fulfillment In addition, one cohort of final year students from 2016, $n=31$, rated the importance of, self-efficacy for, and perceived competence to implement seven core behavior change components in physiotherapist clinical practice. Response format was an 11graded numerical rating scale with endpoints 0 , not at all, and 10 , to a high extent. Results Response rate was $70 \%(n=186)$ Medians for students' perceived curriculum goal fulfillment varied between 4 and 5 . In the 2016 cohort the importance of implementing core behavior change components the median ratings varied between 6 and 10, self-efficacy for implementing between 6 and 8 , and perceived competence to implement between 6 and 9. Lowest importance, self-efficacy and competence ratings concerned functional behavioral analyses. Students' highest ratings were for addressing patients' physical and psychological skills in treatment. Conclusion Students state they have acquired a behavioral medicine perspective in physiotherapy. Further, the results imply that students' attitudes and self-efficacy for behavioral analyses in a health care context need more attention in education.

CORRESPONDING AUTHOR: Maria Sandborgh, Mälardalen University, maria.sandborgh@mdh.se

\section{P196}

DISEASE EXPERIENCES OF WOMEN WITH SJÖGREN'S SYNDROME: A CONCEPT MAPPING STUDY

Rinie Geenen ${ }^{1}$, Marianne Y. Visser-Noordegraaf ${ }^{2}$, Valerie Hiensch ${ }^{1}$

${ }^{1}$ Utrecht University, Utrecht- Netherlands

${ }^{2}$ National Association Sjögren Patiënts, Maarssen- Netherlands

Introduction and purpose Sjögren's syndrome is characterized by infiltration of the exocrine glands, resulting in dryness of eyes, mouth, and other parts of the body which is a burden to everyday functioning and wellbeing. The aim of this study was to categorize the full spectrum of life experiences of women with Sjögren's syndrome in an integrated, hierarchical model, and to examine the degree to which these sorted life experiences are experienced. Methods Using a concept mapping technique, 52 women with Sjögren's syndrome from the Netherlands structured and interpreted life experienced that were derived from a previous interview study (Rojas-Alcayaga et al. Musculoskeletal Care 2016;14:233-242). In a current card-sorting task, research participants individually sorted 75 experiences in piles using similarity of content as a criterion. Hierarchical cluster analysis was used to obtain an integrated, hierarchical model of these sortings. The 52 participants indicated on a 4-point Likert scale whether they had had these 75 experiences themselves: agree, agree a little, disagree a little, disagree. Results Hierarchical cluster analysis showed 14 clusters of life experiences in 6 overarching categories: 1: Primary symptoms, 2: Social functioning (relationships, role function barriers), 3: Self-management (self-care, acceptance, self-management, medical treatment), 4: Emotion processing (psychological vulnerability, crying problems, anxiety), 5: Ignorance (lack of understanding, psychologizing, not recognizing the disease, diagnosis), and 6: Experiences with physicians. Patients generally agreed to have experiences of 'primary symptoms', 'role function barriers', and 'diagnosis' and they commonly disagreed having 'psychologizing' experiences, while they differed in the degree to which they had individually experienced the other experiences. Conclusion Life experiences of women with Sjögren's syndrome were summarized in an integrated, hierarchical model. The overview with life experiences from the patient's view can be used to improve screening in clinical consults, develop a questionnaire, give direction to future research, and enhance education and self-management support. To enhance generalizability, the results of this study form the Netherlands will be integrated with results from Chile.

CORRESPONDING AUTHOR: Rinie Geenen, Utrecht University, r.geenen@uu.nl

\section{P197}

PLANNING PHYSICAL ACTIVITY FOR ADULTS WITH MENTAL HEALTH ISSUES: WHAT CONTEXTS DO PEOPLE WANT?

Nicola Burton ${ }^{1}$

${ }^{1}$ Griffith University- Australia

Introduction \& Purpose. Physical activity provides psychological and physical benefits, and could help reduce the significant morbidity and mortality associated with poor mental health. Activity opportunities that are consistent with people's interests may promote participation. This presentation summarises three projects on activity context preferences among adults with poor mental health. Methods: The Physical Activity Context Preferences Questionnaire assesses how, where and with whom activity is preferred. Responses are provided on a 5 point scale of agreement/disagreement. Study 1 focussed on mid-aged adults in the general population with elevated psychological distress $(\mathrm{N}=909)$; data were analysed using multi-level multinomial logistic regression (adjusted odds ratios and 95\% confidence intervals reported). Study 2 focussed on noninstitutionalised adults who self-identified as recovering from mental illness $(\mathrm{N}=142)$; data were analysed using logistic regression. Study 3 focussed on inpatients of a psychiatric hospital $(\mathrm{N}=101)$; data were analysed using Pearson's chi squared test. Results: In the population study, participants with elevated distress were significantly more likely than those without to prefer activities with supervision $(1.64 ; 1.32-2.03)$, scheduled sessions $(1.32 ; 1.08-1.62)$ and others of the same gender $(1.41 ; 1.12-$ 1.78). In the outpatient study, more than two thirds of participants preferred activities done alone, with scheduled sessions, close to home, and with people of the same level of ability; there were no significant differences by distress $(p>0.02)$. In the inpatient study, more than two thirds of participants preferred activities done alone, with scheduled sessions, and with a set routine; there were no significant differences by distress ( $p>0.003$ ). Study 2 and 3 participants wanted activities to be led by an exercise specialist (e.g., trainer, physiotherapist, exercise physiologist) and not medical staff. Conclusions: Adults across the continuum of poor mental health prefer scheduled activities and may be deterred by groups, especially with people of the opposite gender and different levels of ability. More work is needed to develop and evaluate appealing physical activity opportunities to support people with poor mental health.

CORRESPONDING AUTHOR: Nicola Burton, Griffith University, n.burton@griffith.edu.au

\section{P198}

BRAIN STRUCTURAL CORRELATES OF INTRUSIVE REEXPERIENCING IN SOCIAL ANXIETY DISORDER

Marie Kristin Neudert ${ }^{1}$, Raphaela Zehtner ${ }^{1}$, Rudolf Stark ${ }^{1}$, Andrea Hermann $^{1}$

${ }^{1}$ Department of Psychotherapy and Systems Neuroscience and Bender Institute of Neuroimaging, Justus Liebig University Giessen- Germany

Similar to intrusive memories in post-traumatic stress disorder (PTSD), first studies show that patients with social anxiety disorder (SAD) may also develop intrusive memories in response to stressful social events (e.g., bullying). Intrusive memories probably resulting from deficient fear and memory processes can contribute to the emergence and maintenance of social anxiety. Neuroimaging studies focusing on fear-processing and 
memory-related processes show functional abnormalities in brain regions such as insula, amygdala, hippocampus and prefrontal brain regions in both PTSD and SAD. Beside functional abnormalities, structural changes in these brain regions could also influence fear and memory effects and could be the cause or consequence of intrusive memories. However, less is known about structural abnormalities in these brain regions and intrusive re-experiencing in SAD. Therefore, the aim of this magnetic resonance imaging study was to investigate the association between intrusive re-experiencing and gray matter volume in fear-processing and memoryrelated brain regions in 36 patients with SAD. A modified version of the Posttraumatic Diagnostic Scale was used to measure intrusive reexperiencing in response to the most distressing social event. The volume of gray matter was assessed by means of voxel-based morphometry. First results show that intrusive re-experiencing was associated with greater volumes of the amygdala, insula, and hippocampus. Volume differences in these brain regions could be associated with altered fear processing and memory processes and might therefore prevent a successful emotional processing of a stressful social event.

CORRESPONDING AUTHOR: Marie Kristin Neudert, Justus Liebig University, marie.k.neudert@psychol.uni-giessen.de

\section{P199}

NEURAL CORRELATES OF EMOTION REGULATION PREDICT COGNITIVE BEHAVIORAL THERAPY RESPONSE IN SOCIAL ANXIETY DISORDER

Raphaela Zehtner ${ }^{1}$, Marie K. Neudert ${ }^{1}$, Rudolf Stark ${ }^{1}$, Andrea Hermann ${ }^{1}$ ${ }^{1}$ Department of Psychotherapy and Systems Neuroscience and Bender Institute of Neuroimaging, Justus Liebig University Giessen- Germany

An intense fear of being negatively evaluated by others is a key symptom of social anxiety disorder (SAD). These social fears and anxieties are addressed in cognitive behavioral therapy (CBT), the first line treatment for $\mathrm{SAD}$, via cognitive emotion regulation. Nevertheless, many patients remain symptomatic following treatment emphasizing the need of reliable predictors of therapy response. Recent studies suggest that neuromarkers outperform predictions based upon behavioral or demographic data. Several studies have shown that individual baseline differences in neural response patterns during cognitive emotion regulation including the medial prefrontal cortex (mPFC) and the anterior cingulate cortex (ACC) may factor into symptom reduction following CBT. Thus, brain activation during cognitive emotion regulation seem to be a promising account to target therapy success. Before a manualized CBT, 36 patients suffering from $\mathrm{SAD}$ underwent a functional magnetic resonance imaging session while performing an emotion regulation task. Patients had to either look at or reduce their negative feelings towards aversive pictures and rated their negative feelings after each picture presentation. On a behavioral level, neither demographic nor the habitual use of different emotion regulation strategies or online ratings foretold a reduction of social anxiety symptoms following treatment. On a neural level, however, results showed, that a higher activity in brain regions relevant for emotion regulation (e.g., amygdala) and cognitive control during looking at aversive vs. neutral pictures predicted a higher reduction of social anxiety symptoms when controlled for initial symptom severity. Furthermore, a decreased activation in the $\mathrm{mPFC}$ and the ACC during emotion regulation (vs. looking at aversive pictures) was associated with a greater therapy response. These results confirm assumptions of a predictive value of neuromarkers. Further research is necessary to establish reliable neuromarkers to predict therapy outcome.

CORRESPONDING AUTHOR: Raphaela Zehtner, Justus Liebig University, raphaela.zehtner@psychol.uni-giessen.de
P201

DO NOT LOOK AWAY! SPONTANEOUS FRONTAL EEG THETA/ BETA RATIO AS A MARKER FOR COGNITIVE CONTROL OVER ATTENTION TO MILD AND HIGH THREAT

Angelos Angelidis ${ }^{1}$, Muriel Hagenaars ${ }^{1}$, Dana van Son $^{2}$, Willem van der Does $^{1}$, Peter Putman ${ }^{1}$

${ }^{1}$ Leiden University- Netherlands

${ }^{2}$ Utrecht University- Netherlands

Background: Important theoretical models predict the joint influence of threat-appraisal and attentional control on the regulation of attention bias to threat. Attentional control (AC) is controlled by a bottom-up system and a top-down prefrontal cortical attentional control system, which is reflected in spontaneous frontal EEG theta/beta ratio (TBR). Low TBR is associated with greater attentional control. Attentional bias to threat is considered an important maintaining factor in anxiety disorders and is the target for novel treatments; training anxious people to attend away from threat. Importantly, the predicted role of threat-level (appraisal) of stimuli is ignored, the role of AC has mainly been studied using only subjective measures, and not one study to date has addressed their joint influences, even though they are thought to crucially determine whether attention is biased towards or away from threat. Objectives: The aim of the present study was to provide the first evidence of relations between objectively measured AC, as assessed with frontal EEG TBR, trait anxiety and attentional bias to mildly versus highly threatening stimuli. Methods: Seventy-four healthy volunteers completed a spontaneous EEG measurement, a trait anxiety questionnaire and a dot-probe task with mild and high threatening pictures. Results: TBR moderated attentional bias to different threat-levels, $\mathrm{F}(1,63)=19.19, \mathrm{p}<.001$; individuals with higher TBR (lower AC) directed their attention towards mildly threatening stimuli and avoided highly threatening pictures. Moreover, trait anxiety interacted with this moderation between TBR and threat-level dependent attentional bias, $\mathrm{F}(1,61)=7.375, \mathrm{p}=.009$; the most resilient participants, individuals with lower TBR (higher AC) and low trait anxiety, showed attention towards highly threatening stimuli instead of likely dysfunctional avoidance, while this pattern was not present for mildly threatening stimuli. Conclusions: These data confirm that attentional control and threat-level are crucial variables in the study of threat-related attentional bias, in the (treatment) of anxiety disorders. Moreover, the present results further extend the notion that TBR is a marker of cognitive control over emotional information.

CORRESPONDING AUTHOR: Angelos Angelidis, Leiden University, a.angelidis@fsw.leidenuniv.nl

P202

DEPRESSION IN PEOPLE WITH HAEMOPHILIA: ASSOCIATED FACTORS AND IMPLICATIONS FOR INTEGRATED COMPREHENSIVE CARE

Ana Cristina Paredes ${ }^{1}$, Pedro Moreira ${ }^{1}$, Armando Almeida ${ }^{1}$, Patrícia Ribeiro Pinto ${ }^{1}$

${ }^{1}$ Life and Health Sciences Research Institute, School of Medicine, University of Minho- Portugal

Introduction \& Purpose: Haemophilia is a rare genetic bleeding disorder, caused by a deficiency in coagulation factor VIII or IX. It is associated with several clinical and psychosocial challenges, such as spontaneous bleeds, demanding home-treatment regimens, acute and chronic pain, and impaired quality-of-life, which have been related to increased depression levels. In turn, high distress may have a negative impact on health-related outcomes. The aim of this study was to investigate which potentially modifiable factors were associated with the presence of depressive symptoms among adults with haemophilia. Methods: This was a crosssectional observational study examining sociodemographic, clinical and psychosocial variables among 102 patients with haemophilia A or B in 
Portugal (median age=43). Assessment measures included a socio-demographic, a clinical and a pain questionnaire, the PROMIS® depression short form, the Illness Perception Questionnaire, the A36Hemophilia-Qol and the Haemophilia Activities List. Groups with (PROMIS $\geq 8$ ) or without (PROMIS $<8$ ) significant depressive symptoms were compared, and multivariate hierarchical logistic regression models were used to analyse factors associated with the presence of depression. Results: Participants with significant depression symptoms reported more bleeds $(\chi 2(1)=7.411, p=0.006)$ and more urgent hospital visits $(\chi 2(1)=6.307$, $\mathrm{p}=0.012)$, more affected joints $(\mathrm{U}=528.500, \mathrm{p}<0.001)$ and pain $(\chi 2(1)=10.169, p=0.001)$; as well as worst levels of functionality $(\mathrm{U}=439.500, \mathrm{p}<0.001)$ and quality-of-life $(\mathrm{U}=341.500, \mathrm{p}<0.001)$. After controlling for demographic and clinical variables in multivariate hierarchical logistic regression analyses, professional status $(\mathrm{OR}=3.333$, $\mathrm{p}=0.029)$, pain interference $(\mathrm{OR}=1.347, \mathrm{p}=0.037)$, physical activity $(\mathrm{OR}=0.302, \mathrm{p}=0.024)$ and perception of haemophilia consequences $(\mathrm{OR}=1.600, \mathrm{p}=0.012)$ emerged as factors associated with depression. Conclusions: Current results highlighted a set of variables that were associated with depression in haemophilia and are amenable to change. These findings provide evidence for the development of preventive interventions, targeting specific risk factors and aiming to promote well-being and quality-of-life, in the scope of integrated comprehensive haemophilia care.

CORRESPONDING AUTHOR: Ana Cristina Paredes, Life and Health Sciences Research Institute, University of Minho, anacristinamparedes@gmail.com

\section{P203}

EFFECTS OF A MULTI-LEVEL ORAL HEALTH PROMOTION INTERVENTION INTEGRATING PRIMARY HEALTHCARE IN BRAZIL: FROM THEORY TO PRACTICE

Andreia Morales Cascaes ${ }^{1}$

${ }^{1}$ Universidade Federal de Pelotas- Brazil

In Brazil, around five million young children have dental caries, and $80 \%$ of the decayed teeth remain untreated, suggesting the low access and utilization of dental services by this segment of the population. Despite oral health promotion being a national priority in Brazil, in practice, it is operationally difficult to plan and execute it, especially for children, concurrent to the accumulated demand for dental treatments in adults. Most health professionals' practices are still based on a biomedical and curative model, becoming a hindrance to working with an integrated health promotion perspective. Changing individual behaviors is complex and arduous work influenced by many determinants, including factors beyond the individual. The effects of oral health promotion interventions informed by theory, integrated to healthcare systems and using multi-level approaches are only partially known and further evidence is needed. Few studies were conducted in low/middleincome countries, such as Brazil. This work aims to present current evidence on the effects of multi-level interventions on the reduction of oral health inequalities. Furthermore, as an example, it presents the design and effects of a theory-based multi-level intervention study aimed to promote oral health among children aged zero to five years who are registered at Primary Healthcare Centers in Brazil. This cluster randomized controlled intervention study was implemented over a two-year period in the city of Pelotas, an urban area in Southern Brazil. It targets factors at the intrapersonal/interpersonal, organizational, and community levels, following the principles of the socioecological model for health promotion. In short, the study succeeded in increasing caregivers' oral health knowledge, children's oral hygiene behaviors, preventive use of dental service and preventing dental caries in children younger than 30 months at baseline. Improvements in primary healthcare provider's practices have been observed. This innovative and feasible intervention shows its potential to address public health issues and reduce oral health inequalities and can inform the work of others seeking to reach young children and their families.
CORRESPONDING AUTHOR: Andreia Morales Cascaes, Universidade Federal de Pelotas, andreiacascaes@gmail.com

\section{P204}

BIOELECTRICAL IMPEDANCE VECTOR ANALYSIS IN CHILEAN UNIVERSITY STUDENTS

Antonio Castillo Paredes ${ }^{1}$, Jose Luis Felipe Hernández ${ }^{2}$, Ximena Palma Leal $^{3}$, Fernando Rodríguez-Rodríguez ${ }^{4}$, Itziar Págola Aldazabal ${ }^{2}$

${ }^{1}$ Universidad de Las Américas- Chile

${ }^{2}$ Universidad Europea de Madrid- Spain

${ }^{3}$ Universidad Técnica Federico Santa María- Chile

${ }^{4}$ Pontificia Universidad Católica de Valparaíso- Chile

Introduction and Purpose: Bioelectrical impedance analysis (BIA) has been used in research because it is an economic, non-invasive and safe technology. BIA data reactance (Xc) and resistance (R) through bioelectrical impedance vector analysis (BIVA) and phase angle $(\mathrm{PhA})$ have been used to evaluate cellular function and where $\mathrm{R}$ and $\mathrm{Xc}$, correlated highly with net fluid balance. For that reason, the aim of the study was to analyse the association between BIVA and physical activity level in Chilean university students sample. Methods: 497 students participated (328 men; 169 women) with an average age of $19.9+1.9$ years of 14 careers of Faculty of Engineering from the Technical University Federico Santa Maria, Chile, to whom was applied questionnaire of self-assessment of physical condition and quality of life. In addition, body composition and BIVA with Inbody S10 bioimpedance, multifrequency and octopolar was evaluated, following the inclusion and evaluation protocol for this technique. All students signed an informed consent, before voluntarily accessing the study. Results: Pearson's correlation coefficient is used to search for associations between the phase angle and BMI, fat, muscle mass, METs/min/week and self reported physical activity. All of them, reported as linked in the literature. The greatest association is observed with the muscle mass, men $\left(r=0.472^{* *}, \mathrm{p}<0.01\right)$ and women $(\mathrm{r}=$ $\left.0.516^{* *}, \mathrm{p}<0.01\right)$. We confirm what has been reported in the literature regarding the association with BMI, $\left(\mathrm{r}=0.256^{* *}, \mathrm{p}<0.01\right)$ for men and $(\mathrm{r}$ $\left.=0.386^{* *}, \mathrm{p}<0.01\right)$ in women. In this case, the BMI values are at the upper limit of the healthy category $(18.5-24.9)$, men $(m=24)$ and women $(\mathrm{m}=24.1)$. No association was found between phase angle and METs/ $\mathrm{min} /$ week, the values are $(\mathrm{r}=-0.042)$ for men and $(\mathrm{r}=0.046)$ in women. For the physical activity, a negative correlation was observed in men $(\mathrm{r}=$ $\left.0.111^{*}, \mathrm{p}<0.05\right)$ and women $(\mathrm{r}=-0.136)$. The fat reported a negative association in men $\left(r=-0.154^{* *}, p<0.01\right)$ and positive in women $(r=$ $0.067)$. Conclusions: A relationship between self-reported physical activity and BIVA was not found. In particular, there is no association between phase angle and METs/min/week. The relationship between phase angle and BMI reported in the literature is confirmed. A strong association between phase angle and muscle mass is observed. Further research would be explore the consistency of hypotheses regarding the link between phase angle and BMI, segmenting the subjects according to their BMI level. This could be explain the association that exists between phase angle and muscle mass, generating a valuable predictive health tool.

CORRESPONDING AUTHOR: Antonio Castillo Paredes, Universidad de Las Américas, acastillop85@gmail.com

\section{P205}

PREVENTIVE WORKPLACE STRESS THROUGH ORGANIZATIONAL FACTORS

Akizumi Tsutsumi ${ }^{1}$

${ }^{1}$ Kitasato University School of Medicine- Japan

Discussion: Work and health - Protective and harmful social environment at the workplace Recently, more and more countries have started to assess 
psychosocial factors at work. In some countries, the same questionnaire such as Copenhagen Psychosocial Questionnaire (COPSOQ) has been adopted. Monitoring the risk factors and/or protective factors are useful to initiate preventive action. As for protective social environment at the workplace, organizational factors such as workplace social capital has drawn attention. Bullying and offensive behaviors are major harmful social environment, on the other hand. Another recent trend is recognition of the importance of management behavior as a key factor for workers' health. It is reported that some leadership styles may become the base of bullying. Providing managers with necessary skills and information on mental health and developing appropriate management behaviors have a favorable effect on workers' mental health. In UK and some other countries, management competencies have been developed to prevent and reduce stress at work. Sick leave absence is a major cause of lost productivity and a relevant health outcome in occupational health practice. The cost of sick leave absence and disability benefits is a substantial challenge for society. Long-term sick leave absence has a very detrimental impact on the mental health and well-being of workers. Therefore, its prevention should be an important part of occupational health policy, not only to reduce costs related to sick leave absence but also to improve worker health and prevent work disability. As the discussant of this symposium, I would like to provoke discussions about: 1) similarity and differences of prevalence of adverse psychosocial environments at the workplace (including bullying and offensive behavior) and their impact on workers' health (perceived stress and sick leave absenteeism) across countries, and 2) possible preventive action through organizational factors such as workplace social capital incorporating developing managerial competency.

CORRESPONDING AUTHOR: Akizumi Tsutsumi, Kitasato University School of Medicine, akizumi@kitasato-u.ac.jp

\section{P206}

HUNGARIAN MEDICAL STUDENT'S ATTITUDE TOWARD AND KNOWLEDGE ABOUT LGBT INDIVIDUALS

Zsuzsanna Szél $^{1}$, Zsófia Török ${ }^{2}$, Zsuzsa Györffy ${ }^{1}$

${ }^{1}$ Semmelweis University, Faculty of Medicine, Institute of Behavioural Sciences- Hungary

${ }^{2}$ Semmelweis University, Faculty of Medicine, Department of Public Health- Hungary

Introduction \& Purpose The aim of our study was to investigate how knowledge of Hungarian medical students about sexual and gender minorities (SGM) is related to their attitudes towards LGBT individuals and their personal background characteristics. LGBT people often experience discrimination in the healthcare system, meanwhile they have higher risk for several mental and physical health problems. Previous studies suggest that medical professionals' attitude toward SGMs negatively correlates with their knowledge about them. Methods Our sample was 1st, 3rd and 4th year medical students of the 4 Hungarian medical universities. Participants received a paper-based, anonym, self-administered questionnaire. Our survey contained questions on general demographics and personal background characteristics, the 3-item version of G. Herek's Attitudes Toward Lesbians and Gay Men Scale (ATLG-R3), 12 items of Modern Homonegativity Scale (MHS), 14 items of modified Sex Education and Knowledge about Homosexuality Questionnaire (SEKHQ) and 14 items of a special attitude questionnaire for health professionals that we developed based on literature review about LGBT health issues (PATSM). Results Of the 568 respondents, about onethird self-identified as male; the mean age was 22 years. The average score for the SEKHQ was 8.04 (SD=3.05, range: 0-14). In our analysis, SEKHQ score was significantly $(\mathrm{p} \leq 0.05)$ inversely associated with all attitude scores (ATLG-R3, MHS-G, PATSM), conservative political views, religiosity and religious behaviour; and there was a significant $(\mathrm{p} \leq 0.05)$ positive association with age, upper grade level, female gender, non-heterosexual orientation, the presentence of LGBT acquaintances, and liberal or rather liberal political views. Conclusions Several studies agree that lack of knowledge about
SGMs associated with negative attitudes. Medical students' and physicians' adequate knowledge about LGBT people may lead to better understanding of the health issues of SGM individuals, make them more comfortable in providing care for LGBT persons, therefore reduce health inequities. Our results suggest that it is may be necessary to amend education of LGBT issues in Hungarian medical universities, to improve the knowledge and form the attitude of medical students.

CORRESPONDING AUTHOR: Zsuzsanna Szél, Institute of Behavioural Sciences, Semmelweis University, Budapest, szel.zsuzsanna@phd.semmelweis-univ.hu

\section{P207}

EFFECTIVENESS OF MOTIVATIONAL INTERVIEWING IN PREVENTING EARLY CHILDHOOD CARIES IN BRAZILIAN PRIMARY HEALTHCARE: A COMMUNITY-BASED RANDOMIZED CLUSTER TRIAL

Daniel Demétrio Faustino-Silva ${ }^{1}$, Beatriz Carriconde Colvara ${ }^{2}$, Elisabeth Meyer $^{3}$, Fernando Neves Hugo ${ }^{2}$, Juliana Balbinot Hilgert ${ }^{2}$, Roger Keller Celeste $^{2}$

${ }^{1}$ Professional Masters Graduate Program of Assessment and Production of Technologies for the Brazilian Unified Health System, Grupo Hospitalar Conceição (GHC)- Brazil

${ }^{2}$ Graduate Dentistry Program of the Universidade Federal do Rio Grande do Sul (UFRGS), Porto Alegre- Brazil

${ }^{3}$ Graduate Program in Health Sciences of the Cardiology Institute (IC/ FUC), Porto Alegre- Brazil.

Introduction \& purpose: Motivational Interviewing (MI) is a strategy aimed at changing behavior based on the premise that the reasons to do so are individual and subjective. In this approach, patients are responsible for making the choices among the different alternatives, and encouraged to make their own decisions. Thus, the aim of this study was to assess the effectiveness of MI, compared to conventional oral health education (CE), in preventing Early Childhood Caries (ECC) in a public healthcare service. Methods: Community-based randomized cluster trials with parallel groups. Twelve healthcare centers (HCs) belonging to the Community Health Service of Grupo Hospitalar Conceição (CHS/GHC) in Porto Alegre, Rio Grande do Sul state (RS), Brazil, were randomized and the population consisted of 649 children born in 2013, in the area covered by the CHS/ GHC. In six HCs randomized into the test group, professionals from Oral Health Teams (OHT) were trained in MI, while six other OHT received no specific training (control group). The study was double-blind, whereby the mothers/children and external examiners were blind to the intervention. The data were collected by calibrated examiners using questionnaires and a clinical examination based on ICDAS criteria, presented in modified dmfs format. Results: Of the 649 children born in the area, 469 received the intervention ( 244 in the control group, 245 in the test group) and 320 were examined by the study (145 in the control group, 175 in the test group), with final follow-up of $68 \%$ of the sample. Mean dmfs was 1.34 (CI95\% 0.97 1.71), 1.91 (CI95\% 1.18-2.64) in the CE group and 0.86 (CI95\% 0.56-1.16) in the MI group. There was a statistically significant difference between the groups $(\mathrm{p}=0.01)$, even when a caries rate per 100 surface-year was estimated $(p=0.02)$. The difference remained significant $(p<0.01)$ in multilevel analysis, with IRR $=0.40$ (CI95\% 0.21-0.79). Conclusion: An approach based on the principles of MI was more effective in reducing the number of surfaces affected by ECC when compared to CE. Trial registration: Brazilian Clinical Trials Registry - ReBEC (RBR-8fvwxq) and ClinicalTrial.gov (NCT02578966). Funding: Grupo Hospitlar Conceição (GHC), National Council for Scientific and Technological Development (CNPq), and Research Support Foundation for the State of Rio Grande do Sul (FAPERGS), Brazil.

CORRESPONDING AUTHOR: Daniel Demétrio Faustino-Silva, Grupo Hospitalar Conceição (GHC), ddemetrio@gmail.com 
P208

NON-LINEAR DYNAMIC OF THE CARDIOVASCULAR AUTONOMIC REGULATION DURING THE VERY DEEP HYPNOSIS

Miguel Enrique Sánchez-Hechavarría ${ }^{1}$, Ileana Cutiño-Clavel ${ }^{1}$, Elizabeth Salvador-Figueroa $^{1}$, Elizabeth De la Paz-Reyes ${ }^{1}$, Michel Torres-Leyva ${ }^{1}$, Ramón Carrazana-Escalona ${ }^{1}$, Cesar Brook-Lageire ${ }^{2}$

${ }^{1}$ Universidad de Ciencias Médicas de Santiago de Cuba- Cuba

${ }^{2}$ Hospital Genral "Dr. Juan Bruno Zayas" de Santiago de Cuba- Cuba

In the cardiovascular autonomic response under hypnotic state has described that existing a deviation in the interaction of the sympatheticvagal balance toward a parasympathetic modulation, however in this state not has elucidated the nonlinear changes in the regulatory systems of the heart rate from complexity and chaos theory. Purpose: Determining the non-linear variations of dynamics of the cardiovascular autonomic regulation during the very deep hypnosis. Methodology: In a study crossover, 15 healthy subjects of 20 years of age, the intervals RR were obtained for electrocardiogram in the equipment Medicid 4, during 10 minutes vigil at rest (closed eyes) and 10 minutes of very deep hypnosis. Were calculated the heart rate and as measures non-linear of the Heart Rate Variability the Shannon's and Sample's entropies. Results: When comparing the very deep hypnosis and the vigil at rest found a tendency not significant to the decrease of the heart rate $(\mathrm{p}=0.512)$ and to the increment of the Shannon Entropy $(p=0.098)$, reporting however significant increments in the Sample Entropy ( $p=0.013$ ). Conclusions: In spite of the fact that the heart rate in the psychophysiological state of very deep hypnosis regarding the vigil at rest does not modify, the increase of chaos becomes evident (entropies), reflecting the complex nonlinear character of the dynamics of the cardiovascular autonomic regulation in the very deep hypnosis.

CORRESPONDING AUTHOR: Miguel Enrique Sánchez-Hechavarría, Universidad de Ciencias Médicas de Santiago de Cuba, miguel.sanchez881119@gmail.com

\section{P209}

DESCRIPTIVE FINDINGS OF CULTURAL COMPETENCE LEVEL IN HEALTH WORKERS IN CHILE

Victor Pedrero ${ }^{1}$, Margarita Bernales ${ }^{1}$, Miguel Perez ${ }^{2}$, Paulina Fernandez ${ }^{3}$

${ }^{1}$ Universidad del Desarrollo- Chile

${ }^{2}$ Fresno State University- USA

${ }^{3}$ Pontificia Universidad Católica de Chile- Chile

Background Cultural competence (CC) is a necessary attribute to reduce or eliminate gaps in healthcare between disadvantage groups and the rest of population. CC is comprised of three specific areas: sensitivity - awareness of own values and beliefs and effect they have on health care, knowledge -understanding the way of seeing the world of patients in the context in which it develops, and skill -ability to adapt health care strategies according to patients' values and beliefs. Currently there are no published tools that allow quantifying $\mathrm{CC}$ levels in health workers in Chile, which makes it difficult to detect needs in this area. A recent project aims to develop a measurement instrument for these purposes. Aim To describe the level of CC in a group of primary care health workers in Chile. Methods A valid and reliable instrument to measure $\mathrm{CC}$ recently developed by the authors was used. The instrument consists of 17 Likert type response questions, grouped into three dimensions of CC. The sensitivity dimension is measured by 8 items (maximum score 40 points); knowledge by 6 items (maximum score 30 points) and skills by 3 items (maximum score 15 points). This was applied to a sample of 91 health workers belonging to an area of Santiago. Descriptive data analysis was carried out to analyze the data. Results Scores obtained were lower for sensitivity dimension (mean 24.9 points, $\mathrm{sd}=2.9$ ), than for knowledge (mean 21.6, sd=3) and skills (mean 12.7, sd=1,6). When analyzing the questions individually, it is observed that $80 \%$ of the participants consider that it is not particularly stressful to take care to patients with different beliefs and values; $22 \%$ agree or totally agree that patients with the same beliefs and values act in the same way, and $25 \%$ do not consider that their beliefs and values influence the therapeutic recommendations delivered. $40.7 \%$ do not contrast their beliefs about patients during care, but $94 \%$ declare to be capable of developing therapeutic plans together with the patient. Conclusions One central aspect of CC is the ability to be aware of preconceptions about patients. Result from this study show that although health workers state that they do not have problems in the care of diverse populations and that also feel capable of developing therapeutic plans they tend to stereotype patients and also do not consider the importance of their beliefs during care. These findings suggest the importance of developing $\mathrm{CC}$ training strategies in the national context and of complementing these results with larger samples.

CORRESPONDING AUTHOR: Victor Pedrero, Universidad del Desarrollo,vpedrero@udd.cl

\section{$\mathrm{P} 210$}

GROUP BEHAVIORAL ACTIVATION FOR PATIENTS WITH FIBROMYALGIA AND MAYOR DEPRESSION: PRELIMINARY DATA OF A PILOT STUDY

Lydia Gómez Pérez ${ }^{1}$, Paula Padilla ${ }^{2}$, Laura Rodríguez ${ }^{2}$, Isabella Soares Barreto $^{2}$, Alvaro Vergés ${ }^{2}$, Josefina Durán ${ }^{3}$, Matías González Tugas ${ }^{3}$

${ }^{1}$ Pontificia Universidad Católica de Chile- Chile

${ }^{2}$ Pontificia Universidad Católica de Chile, Escuela de Psicología, Facultad de Ciencias Sociales- Chile

${ }^{3}$ Pontificia Universidad Católica de Chile. Escuela de Medicina- Chile

Background: Chronic pain and major depression frequently co-occur. Patients with both conditions have a worse prognosis and higher disability. Behavioral activation (BA) may be a useful intervention for these patients, as it targets mechanisms of action common to both conditions. Applying this intervention in groups could be especially beneficial. Objectives: (1) To modify the brief behavioral activation therapy protocol (Lejuez, Hopko, Acierno, Daughters, \& Pagoto, 2011) so that it can be applied in groups of Spanish speaking women with fibromyalgia (FM) and major depression; (2) to examine the effects of the intervention as well as patient satisfaction. Method: A small group of Chilean women with FM and major depression $(\mathrm{N}=7)$ underwent a ten session group BA intervention at the Pontificia Universidad Católica de Chile. Depressive symptoms and FM related outcomes were assessed before, during, and after the treatment. Results: Depression remitted in $42.9 \%(n=3)$ of participants; however, $57.1 \%(\mathrm{n}=4)$ did not recover from depression. Of those who did not recover, one participant experienced clinically significant reductions in depressive symptoms (decreasing from severe to moderate); two remained the same, and one worsened along the treatment. Mean depressive symptoms were 19.5 ( $\mathrm{SD}=5.7), 16.5(\mathrm{SD}=6.3)$, and 14.7 ( $\mathrm{SD}=7.5)$, at baseline, after six weeks of treatment, and at posttreatment, respectively. Regarding pain outcomes, the percentage of patients who experienced clinically significant decreases was $42.9 \%(\mathrm{n}=$ 3) for pain intensity, $62.5 \%(n=5)$ for fibromyalgia impact, $42.9 \%(n=3)$ for general physical health symptoms, and $57.1 \%(\mathrm{n}=4)$ for pain catastrophizing. Behavioral activation increased in $62.5 \%(\mathrm{n}=5)$ of participants. Regarding satisfaction, $71.4 \%(\mathrm{n}=5)$ considered that the intervention "helped them a lot" and $28.6 \%(n=2)$ that "it helped them something". Discussion: Group BA seems to be effective to reduce depressive symptoms and improve pain-related outcomes in some individuals. Nonetheless, it may be not recommendable for all patients. Randomized clinical trials investigating the efficacy of BA in these patients are needed. In addition, studies to identify patient characteristics associated to response to therapy should be performed.

CORRESPONDING AUTHOR: Lydia Gómez Pérez, Pontificia Universidad Católica de Chile, lydiagp2@gmail.com 


\section{P211}

INTEGRATED APPROACHES FOR TOTAL WORKER HEALTH® IN CHILE - COLLABORATIVE OUTREACH AND RESEARCH WITH MUTUAL DE SEGURIDAD, CCHC

Jack Dennerlein $^{1}$, Ivan Silva ${ }^{2}$, Glorian Sorensen ${ }^{1}$

${ }^{1}$ Harvard T.H. Chan School of Public Health, Center for Work, Health, and Wellbeing- USA

${ }^{2}$ Safety Culture Center, Mutual de Seguridad de La Cámara Chlena de lad Construcción (CChC)- Chile

Introduction: All workplaces operate within a social and political framework that can define accountability and define the added value for a worksite and business incentives to for worker health and safety, which vary internationally. For example, Chilean Law number 16.744 providing social insurance against risk of industrial accidents and occupational diseases. Mutual de Seguridad, CChC is a private mutual insurance companies providing this insurance in Chile. Mutual's mission is to protect the workers of its member companies against work risks by promoting safe work environments as well as providing medical and economic benefits in case of work-related accidents. Methods and Results: Mutual has initiated efforts of disseminating integrating worker health and safety approaches for their clients especially for small and medium sized enterprises (SME). Their companies want to implement integrated programs but such programs need to translate them to the Chilean context. Mutual's programs address these challenges. Their program builds from various international resources for integrated approaches including, Healthy workplaces: a WHO global model for action, and the Harvard T.H. Chan School of Public Health's Center for Work, Health and Wellbeing's SafeWell guidelines. It frames the program to address four dimensions, Physical and Environmental Risks, Psychosocial Risk Factors, Personal Health Factors, Family and Community. Based on a pilot study in four SMEs, Mutual has adapted their program such that it now includes a scalable certification for companies to attain, an audiovisual proposal and a web support platform to monitor and support implementation. In collaboration with the Center, we have implemented a pilot study to test the efficacy of an integrated intervention for drivers within a transportation company in Chile. The trail will utilize a participatory approach to set priorities and goals utilizing the principles laid out in Mutual's program. Conclusions: Mutual and many of the social security insurance companies in Chile recognizes the impact of these integrated approaches. Through collaborations with international research centers, Mutual is impacting small and medium sized companies through their outreach and research adapted to their local policies and cultural aspects.

CORRESPONDING AUTHOR: Jack Dennerlein, Harvard T.H. Chan School of Public Health Center for Work Health and Wellbing, j.dennerlein@northeastern.edu

\section{$\mathrm{P} 212$}

EFFECT OF EXTENDED SCOPE PHYSIOTHERAPISTS ASSESSMENTS IN ORTHOPAEDIC DIAGNOSTIC SETTING: A SYSTEMATIC REVIEW

Jeanette Trøstrup ${ }^{1}$, Carsten Juhl ${ }^{2}$, Lone Ramer Mikkelsen ${ }^{1}$

${ }^{1}$ Elective Surgery Centre, Silkeborg Regional Hospital, SilkeborgDenmark

${ }^{2}$ University of Southern Denmark, Department of Sports Science and Clinical Biomechanics- Denmark

Introduction \& Purpose: The purpose was to evaluate the effectiveness of musculoskeletal assessments performed by Extended Scope Physiotherapists (ESP) compared to assessments performed by orthopaedic surgeons OS on patient satisfaction, diagnostic agreement and costs. The reason for this purpose was that musculoskeletal complaints are common with $20 \%$ of patients in primary care being referred to OS consultation. A high proportion of these referrals is not relevant for surgery and can potentially be managed entirely by ESPs; which are physiotherapist with advanced clinical competencies. Methods: A systematic review was performed searching MEDLINE, Cochrane Central Register of Controlled Trials, EMBASE, CINAHL, PEDro and reference lists of included studies as well as previously published systematic reviews. Inclusion criteria were studies evaluating adults with a musculoskeletal complaint referred to orthopaedic outpatient assessment and studies evaluating patient assessment conducted by ESP. Studies performed in primary care or emergency setting were excluded. Methodological quality (SIGN 50 checklists) was assessed independently by two reviewers. Results: 3100 studies were identified of which 23 were included. A descriptive synthesis was adopted as meta-analysis was not possible. Patient satisfaction $(n=12)$ ranged from $77-100 \%$ being satisfied with similar satisfaction in studies of low, acceptable and high methodological quality. Diagnostic agreement $(n=9)$ ranged from $65-100 \%$ between ESP and OS assessments and was highest in studies with low methodological quality. Cost analysis $(n=4)$ showed $27-49 \%$ cost savings for assessments performed by ESP compared to OS ( $n=3)$, and that ESP-led clinics were more expensive but also more beneficial compared to OS-led clinics $(n=1)$. Cost savings was found in studies of mixed methodological quality where one study of high methodological quality found that ESP-clinics were more expensive than OS-led clinics. Conclusion: ESP care may be as beneficial as or even better than assessment performed by OS in terms of diagnostic accuracy, economic costs and patient satisfaction. Results should be managed cautiously as only a few of the included studies had high methodological quality and more high quality studies are needed.

CORRESPONDING AUTHOR: Jeanette Trøstrup, Elective Surgery Centre, Silkeborg Regional Hospital, jeatro@ rm.dk

\section{P213}

PSYCHOMETRIC PROPERTIES OF THE FIBROMYALGIA SURVEY QUESTIONNAIRE (FSQ) IN CHILEAN WOMEN WITH FIBROMYALGIA: PRELIMINARY RESULTS

Carla Aguirre Cárdenas ${ }^{1}$, Maria Cecilia Oñederra ${ }^{1}$, Catalina Esparza Benavente $^{1}$, Josefina Durán Santa Cruz ${ }^{1}$, Alvaro Vergés ${ }^{1}$, Matías González Tuga ${ }^{1}$, Lydia Gómez-Pérez ${ }^{1}$

${ }^{1}$ Pontificia Universidad Católica de Chile- Chile

Background: In 2010, the American College of Rheumatology updated the diagnostic criteria for fibromyalgia (FM). The Fibromyalgia Survey Questionnaire (FSQ) was developed to assess these criteria. It comprises two subscales: The Symptom Severity Scale (SSS) and the Widespread Pain Index (WPI). A total FSQ score can be also computed. FM can be diagnosed when the following criteria are met: (1) WPI $\geq 7$ and SSS $\geq 5$ or $\mathrm{WPI}=3-6$ and $\mathrm{SSS} \geq 9$; (2) presenting the symptoms for at least 3 months; and (3) absence of a disorder other than FM that could explain the symptoms. Objective: To examine the psychometric properties of the Chilean version of the FSQ. Methods: Women with an a priori diagnosis of FM $(\mathrm{N}=114)$ or rheumatoid arthritis $(\mathrm{AR}, \mathrm{N}=100)$ attended in the rheumatology unit of a medical center in Santiago, Chile (Centro Médico San Joaquín, Red Salud UC Christus) completed a battery of questionnaires assessing several FM outcomes when they waited to be seen by their doctor (T1), as well as two weeks later (T2). The group with AR was considered the control group. Results: The FSQ presented a good internal consistency at T1 $(\alpha=.83 ; \omega=.83)$ and T2 $(\alpha=.73 ; \omega=.74)$. Test-retest reliability was adequate (ICC $=.72, \mathrm{p}<.001)$. Regarding convergent validity, FSQ scores were positively correlated with FM impact ( $r=.60, p$ $<.001)$, somatic symptoms $(\mathrm{r}=.66, \mathrm{p}<.001)$, depressive symptoms $(\mathrm{r}$ $=.50, \mathrm{p}<.001)$, pain intensity $(\mathrm{r}=.46, \mathrm{p}<.001)$, pain disability $(\mathrm{r}=.49$, $\mathrm{p}<.001)$, pain catastrophizing $(\mathrm{r}=.35, \mathrm{p}<.001)$, and hypervigilance $(\mathrm{r}$ $=.33, \mathrm{p}<.001)$, and negatively associated with quality of life $(\mathrm{r}=-.50$, $\mathrm{p}<.001)$. Considering the FM diagnosis performed by a rheumatologist's as the gold standard, an adequate level of sensitivity was observed $(88 \%)$; however, specificity was very low (45\%). The FM group showed greater 
levels of SSS $(\mathrm{U}=2865.5, \mathrm{Z}=-6.31, \mathrm{p}<.001, \mathrm{r}=.43)$, WPI $(\mathrm{U}=2273, \mathrm{Z}=$ $-7.60, \mathrm{p}<.001, \mathrm{r}=.52)$, and total FSQ scores $(\mathrm{U}=2110, \mathrm{Z}=-7.95, \mathrm{p}<.001$, $\mathrm{r}=.54$ ) than the AR group. Conclusion: The Chilean version of the FSQ presents adequate reliability, validity, and sensibility. It can be used to assist medical professionals in FM diagnosis as well as to determine FM severity. However, its level of specificity seems to be low.

CORRESPONDING AUTHOR: Carla Aguirre Cárdenas, Pontificia Universidad Católica de Chile, cpaguirre@uc.cl

\section{P214}

PSYCHOMETRIC PROPERTIES OF THE CHILEAN VERSION OF THE PAIN CATASTROPHIZING SCALE IN A SAMPLE OF CHILEAN WOMEN WITH FIBROMYALGIA

Lydia Gómez Pérez ${ }^{1}$, Ana Carolina de Obaldía ${ }^{2}$, Josefina Durán ${ }^{3}$, Carmen Ramíerez-Maestre ${ }^{4}$, Matías Gonzalez Tuga ${ }^{3}$, Alvaro Vergés ${ }^{2}$

${ }^{1}$ Pontificia Universidad Católica de Chile- Chile

${ }^{2}$ Pontificia Universidad Catñolica de Chile. Escuela de Psicología. Facultad de Ciencias Sociales- Chile

${ }^{3}$ Pontificia Universidad Católica de Chile. Escuela de Medicina- Chile

${ }^{4}$ Universidad de Málaga. Facultad de Psicología. Departamento de personalidad, evaluación y tratamiento psicológico- Spain

Catastrophizing is a fundamental variable in understanding the experience of pain in rheumatologic disorders and other chronic pain conditions. It has shown to be strongly associated with increased pain, emotional stress, depression, anxiety, and higher levels of disability in patients with chronic pain in general, and particularly in patients with fibromyalgia (Lerman \& Haythornthwaite, 2017). The Pain Catastrophizing Scale (PCS, Sullivan, 1995) is one of the scales most commonly used to assess pain catastrophizing. Despite this scale is useful for both clinicians and researchers, a culturally validated version of this questionnaire is not currently available in Chile. Objective: To culturally adapt and validate the PCS in Chilean patients with fibromyalgia. Methods: 76 women with fibromyalgia attended in a clinic (Centro Médico San Joaquín, Red Salud UC Christus) in Santiago (Chile) participated in this cross-sectional study. They completed a battery of questionnaires comprising the PCS as well as other scales assessing several pain-related outcomes while they were waiting to be seen by their rheumatologist. Two weeks after, they were asked to complete the PCS again by phone. The factor structure, internal consistency, test-retest reliability, and criterial validity of the PCS were evaluated. Results: the PCS presented a three-factor structure similar to the one found in previous studies. The fix indexes for this model were $\chi^{2} /$ $(\mathrm{gl})=1.78, \mathrm{CFI}=.98, \mathrm{TLI}=.98$. The factors were rumination $(\mathrm{RU})$, magnification (MA), and helplessness (HE). The PCS presented adequate internal consistency (Cronbach alphas PCS $=.96 ; \mathrm{RU}=.86 ; \mathrm{MA}=.80$, $\mathrm{HE}=.87$ ), and a good test-retest reliability (PCS intra-class correlation coefficients $=.84 ; \mathrm{RU}=.78 ; \mathrm{MA}=.81, \mathrm{HE}=.82$ ). The PCS scores showed a high correlation with pain interference and hypervigilance, and a moderate correlation with the fibromyalgia impact, pain intensity, and depressive symptoms. Conclusions: The Chilean version of the PCS presents adequate psychometric properties.

CORRESPONDING AUTHOR: Lydia Gómez Pérez, Pontificia Universidad Católica de Chile, lydiagp2@gmail.com

\section{P215}

CHRONIC PAIN IN RURAL PEOPLE LIVING WITH HIV: THE ROLE OF PAIN CATASTROPHIZING ON PAIN SEVERITY

Bernadette Heckman ${ }^{1}$, Tiffany Grimes ${ }^{1}$, Nicole Weaver ${ }^{1}$, Jason Blizzard ${ }^{1}$ ${ }^{1}$ University of Georgia- USA

Introduction and Purpose. Most people living with HIV (PLWH) report co-morbid pain conditions, such as gastrointestinal, neurological, and rheumatological pain. Chronic pain in PLHV is associated with greater rates of mood and substance abuse disorders and increased sleep disorders and fatigue. Pain catastrophizing is a common and powerful pain-related thought experienced by many pain patients and independently predicts pain chronicity and poor treatment outcomes. Pain catastrophizing is the magnification of the threat of the pain stimulus, feeling helpless in the context of pain, and a perceived inability to inhibit pain-related thoughts prior to, during, and/or after a painful event. Catastrophizers have difficulty cognitively disengaging from pain stimuli and demonstrate amplified activation in neural regions responsible for the processing and regulation of affective components of pain (e.g., anterior cingulate cortex). A cross sectional pilot survey was conducted to determine the role of pain catastrophizing and other predictors of chronic pain among rural PLWH. Methods. Using a cross-sectional study design, 31 patients were recruited from two AIDS-related non-governmental organizations (NGOs) in the southeastern region of the United States. All participating NGOs provided a variety of medical, psychological, and life-care services to PLWH in 10 largely rural counties. Results. Of the 31 patients, most were male (55\%), African American (76\%), single (35\%), self-identified as heterosexual (76\%), and exposed to HIV through heterosexual intercourse $(51 \%)$. Pain catastrophizing was correlated with the following variables: painrelated helplessness, $\mathrm{r}=.81, \mathrm{p}<.001$; pain-related resourcefulness, $\mathrm{r}=.60$, $\mathrm{p}<.001$; pain-related coping, $\mathrm{r}=.35, \mathrm{p}>.07$; West Haven Yale Total Scores, $\mathrm{r}=.70, \mathrm{p}<.002$; pain interference, $\mathrm{r}=.65, \mathrm{p}<.001$; pain severity, $\mathrm{r}=.73, \mathrm{p}<.001$; and BDI values, $\mathrm{r}=.49, \mathrm{p}<.01$. A forced entry multiple regression analysis was conducted to identify correlates of pain severity. While the set of predictors explained a significant amount of variability in pain severity scores, $\mathrm{R} 2=0.53, \mathrm{~F}(5,23)=5.2, \mathrm{p}=.002$, the only significant predictor of pain severity in the presence of the other predictor variables was pain catastrophizing, beta $=.64, \mathrm{t}=2.7, \mathrm{p}=.011$. Discussion. Pain catastrophizing was significantly related to pain severity suggesting that individual living with HIV and who experience chronic pain tend to magnify threat value of the pain stimulus, feel helpless in managing pain, and tend to attend to pain experience more readily. Additional research is needed to identify interventions that target catastrophic thinking and reduce pain experience among PLWH in non-urban areas.

CORRESPONDING AUTHOR: Bernadette Heckman, University of Georgia, bheckman@uga.edu

P216 AVOIDANCE POSTTRAUMATIC STRESS SYMPTOMS, FEELING OF GUILT, AND EMOTIONAL REGULATION AMONG INDIVIDUALS EXPOSED TO DIFFERENT TYPES OF TRAUMATIC EXPERIENCES

Lydia Gómez Pérez ${ }^{1}$, Elena R Serrano-Ibáñez ${ }^{2}$, Gema Teresa RuízPárraga $^{2}$, Carmen Ramírez-Maestre ${ }^{2}$, Rosa Esteve ${ }^{2}$, Alicia E LópezMartínez ${ }^{2}$

${ }^{1}$ Pontificia Universidad Católica de Chile- Chile

${ }^{2}$ Universidad de Málaga. Facultad de Psicología. Departamento de Personalidad, Evaluación, y tratamiento psicológico. Instituto de Investigaciones Biomédicas (IBIMA)- Spain

Background: Trauma exposure is associated with poorer mental health. However, not all types of trauma seem to impact health in the same way. Each traumatic experience seems to demand differential coping strategies and provoke distinct reactions to people, which may lead to differential symptom expression. Objectives: To examine differences among four groups: (1) a High Betrayal Trauma Group (HBTG, $n=66$ ), (2) a Low Betrayal Trauma Group (LBTG, $n=21$ ), (3) a Non-Interpersonal Trauma Group (NITG, $n=65$ ), and (4) a group of individuals who experienced losing a loved one (Loss-G, $n=17$ ). Method: A total of 781 undergraduate University students from the University of Málaga (Spain) completed a set of on-line questionnaires. Only those individuals that were exposed to a single type of experiential events according to the Stressful 
Live Event Screening Questionnaire Revised (Green et al., 2006) were selected for this research $(\mathrm{N}=168)$ and classified according to the type of trauma they experienced. Results: The results of Kruskal Wallis analyses showed that the HBTG presented higher re-victimization than the other three groups $(\chi 2(3)=50.71, p<.001)$; higher PTSD symptoms than the LBTG and the NITG $(\chi 2(3)=10.43, p<.05)$, higher dysphoric symptoms $(\chi 2(3)=22.41, \mathrm{p}<.001)$ and experiential avoidance $(\chi 2(3)=8.70, \mathrm{p}<.05)$ than the NITG and the Loss-G; higher feelings of guilt than the NITG $(\chi 2(3)=9.04, p<.05)$, and higher emotional regulation difficulties than the Loss-group $(\chi 2(3)=9.24, p<.05)$. Finally, the LBTG presented higher emotional regulation difficulty scores than the Loss-G (predicting $16 \%$ of the variance). Results of regression analyses showed that the level of revictimization explained the differences found in PTSD symptoms between the HBTG and the LBTG, but not any of the differences found between the HBTG and both the NITG and the LBTG. Conclusion: This is the first study in which the effect of a single type of trauma controlling for the presence of other types of trauma is examined. High betrayal trauma seems to have worse health consequences than non-betrayal trauma, which are not explained by the higher levels of revictimization experienced by these individuals. Limitations and further implications are discussed.

CORRESPONDING AUTHOR: Lydia Gómez Pérez, Pontificia Universidad Católica de Chile, lydiagp2@gmail.com

\section{P217 \\ PERCEPTIONS OF BARRIERS AND ENABLERS OF PHYSICAL ACTIVITY AMONG EMIRATI WOMEN \\ Ciarán Doyle $^{1}$, Asad Khan ${ }^{2}$, Nicola Burton ${ }^{1}$ \\ ${ }^{1}$ Griffith University- Australia \\ ${ }^{2}$ University of Queensland- Australia}

Abstract Purpose: Opportunities for physical activity need to be gender and culturally appropriate but little is known about activity attitudes among females in the United Arab Emirates. The aim of this study was to assess female Emirati university students' perceived barriers to and enablers of physical activity. Methods: Five focus group discussions were conducted with female Emirati university students $(n=25)$ aged between 18-29 years. The study used a semi structured interview. Transcripts of the discussions were analysed and emergent themes identified. Results: Participants had an average age of 20.4 (SD 2.6) years and an average BMI of 26.08 (SD 7.07), and 44\% reported difficulty managing their income some of the time. One of the most common themes identified was lack of time due to fulfilling multiple roles associated with work, family and study. Another common theme was family (husband, parent, siblings) discouragement and low support. Family concerns included safety when leaving the house and gender inappropriateness of some activities. Participants who could not drive described family declining to provide transport assistance. Another barrier theme was fatigue which was attributed to eating large portion meals mainly of meat and rice. A final major barrier theme was hot weather. The major enabler was availability of facilities close to home, that were low cost and female only. Participants wanted to do activities with friends. Another enabler was organised physical activity, scheduled into the university timetable, and for credit. An interesting theme emerged regarding social media. Time spent on social media limited discretionary time for physical activity; but social media also provided positive role models, awareness of opportunities, and social support for physical activity. Conclusion: Physical activity opportunities for young adult female Emirati students need to address family negative perceptions and be compatible with work and study commitments. Physical activity facilities need to be in the local area, female only, and provide group-based options.

CORRESPONDING AUTHOR: Ciarán Doyle, Griffith University, ciarandoyle@hotmail.com
P218

PHYSICAL ACTIVITY CONTEXT AND TYPE PREFERENCES AMONG EMIRATI UNIVERSITY STUDENTS

Ciarán Doyle $^{1}$, Asad Khan ${ }^{2}$, Nicola Burton ${ }^{1}$

${ }^{1}$ Griffith University- Australia

${ }^{2}$ University of Queensland- Australia

Abstract Purpose: Physical activity interventions may be successful if they match the preferences of the target population. However, little is known about the physical activity interests of people from non-Western cultures. The aim of the study was to assess Emirati university students' preferences for physical activity context and type. Methods: This was a cross sectional study in which a convenience sample of Emirati university students $(n=628)$ completed a self-administered online survey assessing preferences for physical activity context and type. Responses were collapsed into agreement and non- agreement. Frequency data were derived to identify common responses for males and females. Results: The majority of the sample was female $(69.1 \%$ ), with an average age of 20.6 (SD 2.9) years and an average BMI of 24.6 (SD 5.9). Both males and females preferred activities that include a fun element $(84 \%, 88.5 \%$ respectively). The leading types of activity were walking $(71.4 \%)$ for females and swimming $(56.5 \%)$ for males. Female students were also interested in swimming $(61.7 \%)$ and jogging $(43.1 \%)$ and activities done at a fixed time/scheduled session (74.7\%), and that involve little or no cost $(73.7 \%)$. Male students were also interested in walking (56\%) and jogging $(50.8 \%)$ and activities where they can choose the intensity $(74.2 \%)$, and that are done alone $(71.6 \%)$. Conclusion: These results can inform physical activity planning for university students in the UAE. Walking, swimming and jogging opportunities may be popular. Opportunities need to prioritise having fun over other types of outcomes (e.g., health). Male students may not be attracted to group-based options, but female students may be interested in organised sessions e.g., activity classes. Activity costs may be an issue for female students. Future research could explore the uptake and maintenance of activity options among university students, based on activity attributes.

CORRESPONDING AUTHOR: Ciarán Doyle, Griffith University, ciarandoyle@hotmail.com

\section{P219}

MENTAL HEALTH OF INMIGRANTS IN SANTIAGO DE CHILE: THE USE OF THE CULTURALLY FOCUSED INTERVIEW (CFI) IN ATTENDERS OF PUBLIC HEALTH SERVICES

Ramón Florenzano ${ }^{1}$, Mauricio Apablaza ${ }^{2}$, Ismael Hernandez ${ }^{1}$, Carolina Carstens $^{1}$, Matilde Bortolaso ${ }^{3}$, Arturo Roizblatt ${ }^{1}$

${ }^{1}$ Facultad de Medicina Universidad de Chile- Chile

${ }^{2}$ Facultad de Gobierno Universidad del Desarrollo- Chile

${ }^{3}$ Facultad de Psicologia Universidad del Desarrollo- Chile

Introduction \& Purpose: Migration and human displacements have increased globally and in Hispanic America in the last decade. Research about inmigrant mental health presents contradictory results, from the initial studies of Odegaard that stated they presented more severe psychotic breaks than non migrants in the USA, to data that explore the effects of boundary crossing and discrimination in the distress of migrants and their progeny. In Northern Chile previous research by Urzua et al has found that level of stress is related to geographical distance from the home country, as well as the discrimination from local populations. Lewis Fernandez et hal have developed the "Cultural Formulation Interview (CFI)" included in the 5th Edition of DSM. This scale has been adapted for Hispanic America by Alarcon and Vega-Distenmeir in Peru. The research team has been trained in the use of this interview, and this paper presents results of its application among migrants attending Santiago de Chile mental health services. The study hypothesis is that a brief version of EFC can be applied to ascertain the amount and type of psychiatric 
problems presented by migrants seeking attention, and can improve the quality of their diagnosis, treatment and prevention. Methods:The study uses a convenience sampling of cases attending Santiago de Chile public mental health outfits, in a lapse of 12 months, and applies the Chilean EFC version. Together with a quantitative questionnaire that is responded by patients and the clinician at the intake into the system. The usefulness and feasibility of its use can be then determined. Results The non-Chilean patients were younger than the Chilean ones. There were no other differences neither in socio-economic nor in clinical diagnosis in the intergroup comparison. Discussion: The methodological difficulties to analyze data refer to determine their place of birth, due to the confidentiality of that date in the current Chilean statistical system. There were no great differences in the clinical presentation or psychiatric diagnoses comparing patients born in or outside Chile. The length of the interview was an issue, and ways to shorten it is future research open.

CORRESPONDING AUTHOR: Ramón Florenzano, Facultad de Medicina Universidad de Chile, rflorenzano@gmail.com

\section{P220}

NEGATIVE COGNITION AND CORTISOL IN MAJOR DEPRESSIVE DISORDER

Susan Thomas ${ }^{1}$, Theresa Larkin ${ }^{1}$

${ }^{1}$ University of Wollongong-Australia

Introduction and purpose: Negative thinking is pervasive in major depressive disorder (MDD), and systematic cognitive distortions have been linked to vulnerability to depression as well as the onset, maintenance and rekindling of depressive episodes. Research with healthy participants indicates that inducing negative cognitions can trigger rises in cortisol, indicating that negative cognitions can act as stressors which activate the hypothalamus-pituitary-adrenal (HPA) axis. People with MDD often show elevated baseline cortisol levels, however, little is known of relationships between negative cognitions and cortisol in individuals with major depressive disorder (MDD). We investigated relationships between negative cognitions, plasma cortisol levels and depressive symptom severity in participants with MDD and healthy controls. Methods: Sixty participants with MDD (35 female) who were not receiving treatment and 60 healthy controls ( 35 female) completed the Automatic Thoughts Questionnaire which measures frequency and strength of belief in negative cognitions, and the Depression, Anxiety and Stress Scales (DASS), a measure of symptom severity. Clinical participants were interviewed using the Mini International Neuropsychiatric Interview for DSM-5 to confirm that they met diagnostic criteria for MDD. Morning plasma samples were analysed for levels of cortisol using a standard ELISA method. Results: Between-group ANOVAs indicated that participants with MDD compared to controls had higher DASS Depression scores, higher cortisol levels and higher overall ATQ scores as well as higher scores in each of the four ATQ subscales (Personal Maladjustment and Desire for Change (PMDC), Negative Self-Concepts and Negative Expectations (NSNE), Low Self-Esteem (LSE), and Helplessness (H), p <.001 in all cases). Cortisol levels were positively correlated with DASS Depression, $r$ $(118)=0.53, \mathrm{p}<.01$, overall ATQ score, $\mathrm{r}(118)=0.44, \mathrm{p}<.01$, and each subscale of the ATQ (r $(118)=0.34$ to $.41, \mathrm{p}<.01)$. Hierarchical multiple linear regressions performed on centred variables and product terms indicated that negative cognition and cortisol both significantly accounted for unique variability in depression symptom severity, $(\beta=$ $-0.79, p<0.001$ and $\beta=0.21, p<0.001$, respectively) and also that cortisol and negative thinking interacted to further predict variability in depressive symptom severity, $(\beta=-0.10, p=0.03)$. Conclusions: This is one of the first studies to directly examine associations between cortisol and negative cognitions which are a hallmark of depression. The results suggest that neuroendocrine activity is related to negative thinking in depression. In particular, distorted cognitive appraisals may be related to engagement of the HPA axis, cortisol levels and depressive symptoms.
These results provide new information about pathways between biological and psychological aspects of depression which helps to move towards more integrated models of depression.

CORRESPONDING AUTHOR: Susan Thomas, University of Wollongong, sthomas@uow.edu.au

\section{P222}

IS INFANT TUMMY TIME AN IMPORTANT INDICATOR OF PHYSICAL ACTIVITY AND RELATED HEALTH OUTCOMES IN EARLY CHILDHOOD?

Kylie Hesketh ${ }^{1}$, Katherine Downing ${ }^{1}$, Karen Campbell ${ }^{1}$, Lisa Barnett ${ }^{1}$, David Crawford ${ }^{1}$, Jo Salmon ${ }^{1}$

${ }^{1}$ Insititue for Physical Activity \& Nutrition, Deakin University- Australia

Introduction \& Purpose: Tummy time (laying prone while awake) is the first form of physical activity promoted for infants. It is associated with favourable motor development and potentially reduced risk of plagiocephaly. However it is unclear whether tummy time is predictive of physical activity levels or physical activity related health and developmental outcomes. This study aims to: (1) investigate associations between infant tummy time and physical activity in early childhood, and (2) investigate associations between tummy time and health and developmental outcomes. Methods: 523 parents participating in the Melbourne InFANT Program reported their infant's total weekly tummy time at $3 \mathrm{mo}$, and child physical and psychosocial health related quality of life (HRQoL; Pediatric Quality of Life Inventory) at $5 \mathrm{y}$. At $19 \mathrm{mo}, 3.5 \mathrm{y}$ and $5 \mathrm{y}$, physical activity was assessed by accelerometry, body mass index z-scores (zBMI) were calculated from measured height and weight, and waist circumference was measured. Object control and locomotor skills (Test of Gross Motor Development-2) were assessed at 5y. Linear regression models adjusted for clustered recruitment, intervention group and child age. Results: Tummy time at 3 mo was not associated with objectively assessed physical activity at any of the later timepoints $(18 \mathrm{mo}, 3.5 \mathrm{y}$ or $5 \mathrm{y})$. Approximately one third of infants met the public health guidelines of $30 \mathrm{~min}$ daily tummy time. Meeting tummy time guidelines at $3 \mathrm{mo}$ predicted higher physical HRQoL, locomotor skills and zBMI at 5y of age; there was no association for psychosocial HRQoL, object control skills or waist circumference. Conclusion: Tummy time did not predict physical activity levels to age $5 y$. Very early infant behaviour may not be reflective of later behaviour. However tummy time remains an important behavioural target, warranting further investigation, due to its association with important health and developmental outcomes.

CORRESPONDING AUTHOR: Kylie Hesketh, Institute for Physical Activity \& Nutrition, Deakin University, kylieh@deakin.edu.au

\section{P223}

'LOSE THE TUBE': BARRIERS/ENABLERS TO HEALTHCARE PROFESSIONAL BEHAVIOURS TO PROMPT URINARY CATHETERS REMOVAL ON IN-PATIENT WARDS: A SURVEY USING THEORETICAL DOMAINS FRAMEWORK \& SOCIAL COGNITIVE THEORY

Rashmi Bhardwaj-Gosling ${ }^{12}$, Sebastian Potthoff ${ }^{3}$, Susan Hrisos ${ }^{1}$, Christopher Harding $^{1}{ }^{2}$, Clare Abley ${ }^{1}{ }^{2}$, Falko Sniehotta ${ }^{1}$, Justin Presseau $^{4}$

${ }^{1}$ Newcastle University- United Kingdom

${ }^{2}$ Newcastle upon Tyne Hospitals- United Kingdom

${ }^{3}$ Northumbria University, Newcastle- United Kingdom

${ }^{4}$ Ottawa Hospital Research Institute \& University of Ottawa- Canada

Abstract main body Introduction \& Purpose Combating catheterassociated urinary tract infections (CAUTIs) is a priority in the worldwide drive to reduce hospital acquired infections. Minimising catheter duration 
is an effective method of reducing CAUTIs but current median duration of postoperative catheters (3-days) is higher than the recommended one day standard (CDC guidelines). A theory-based approach to understanding factors that influence the implementation of catheter removal guidance may help develop effective interventions to reduce CAUTI. This study aimed to identify theory-based predictors of guidelinerecommended healthcare-professional (HCP) behaviour (conducting daily catheter removal assessments) to prompt catheter removal. Methods Prospective correlational design involving HCPs from 11 medical and 13 surgical in-patient wards in North-East England, UK. HCPs $(n=141)$ from nursing and medical backgrounds involved in catheter care completed a Theoretical Domains Framework (TDF) based questionnaire at baseline and self-reported their catheter-removal behaviour at two-week followup. Following mapping of TDF domains to constructs of Social Cognitive Theory (SCT), social support and habit, we then assessed predictors of HCP intention and behaviour. Results With $96 \%$ response rate at followup, SCT constructs self-efficacy (MEAN=4.37, $\mathrm{SD}=1.41$ ), outcome expectations (MEAN=5.85, $\mathrm{SD}=1.16)$ ] and social support $(\mathrm{MEAN}=3.64$, $\mathrm{SD}=1.46$ ) accounted for large amount of variance in HCPs intention $(\mathrm{MEAN}=5.10, \mathrm{SD}=1.54, \mathrm{R} 2 \mathrm{adj}=0.55)$ and with addition of habit ( $\mathrm{MEAN}=4.16, \mathrm{SD}=1.83$ ) accounted for medium amount of variance in prediction of HCP behaviour (MEAN=1.31, $\mathrm{SD}=1.06, \mathrm{R} 2 \mathrm{adj}=0.20)$ to conducting daily catheter removal assessments. Conclusion HCPs are moderately motivated to perform catheter removal assessments. However, variance in behaviour shows room for improvement. Future interventions targeting intention, social support and habit may prove successful to modify clinical behaviours for prompt catheter removal to reduce CAUTIs and variance in HCP behaviour. This has potential to influence catheter removal guidelines and current clinical practice.

CORRESPONDING AUTHOR: Rashmi Bhardwaj-Gosling, Newcastle University \& Newcastle upon Tyne Hospitals, rashmi.bhardwaj1@ncl.ac.uk

\section{P224}

SELF-PERCEIVED DISCRIMINATION AMONG INTERNATIONAL MIGRANTS TO CHILE COMPARED TO LOCALS: AN URGENT PUBLIC HEALTH MATTER

Baltica Cabieses ${ }^{1}$, Sofia Astorga ${ }^{1}$, Marcela Oyarte ${ }^{1}$, Macarena Chepo ${ }^{1}$, Nassim Ajraz ${ }^{1}$, Piedad Galvez ${ }^{1}$

${ }^{1}$ Universidad del Desarrollo- Chile

Introduction: International migration is a global social determinant of health. One of the social and community factors that determines the health of migrants is discrimination, which increases social exclusion and significantly affects access to healthcare and the health status of this population. The purpose of this study was to evaluate self-perceived discrimination among the international migrant population compared to natives. Methodology: A repeated analysis of the 2015 National Socioeconomic Characterization Survey (CASEN in Spanish), an anonymous nationally representative survey. Immigrants were anyone who self-reported that their mother resided in a country other than Chile at the time of birth (immigrant versus local). Self-perceived discrimination was measured by any member of the household that reported being treated unfairly or discriminated in the last 12 months. Some specific types of discrimination were also measured (being a migrant (yes), skin color (dark), physical disability (yes) socioeconomic status (poor) and sex (female)). We compared self-perceived discrimination rates between immigrants and locals. Results: Of the households with at least one immigrant member, $28.4 \%$ would have suffered discrimination or unfair treatment, a percentage that almost doubles the presence of this indicator in the Chilean population $(14.7 \%, \mathrm{p}<0.05)$. By specific type of discrimination, in the case of immigrant population, discrimination due to being foreigner is the one that was reported most frequently (24.3\%), followed by skin color $(4.6 \%)$ and sex $(3,5 \%)$, which showed significant differences with the Chilean population $(0.6 \%$ and $2.1 \%$ respectively, $\mathrm{p}<0.05)$. We also found higher rates of discrimination due to physical disability (3.5\%) and socioeconomic level (3.7\%) in migrants versus locals, but without a statistical significance. Only discrimination by socioeconomic level was higher in Chileans $(4.1 \%)$ than in international immigrants. Conclusions: Immigrants report higher self-perceived discrimination than the Chileans, including as a cause of it simply being a foreigner. Initiatives that address the issue of intercultural health care are urgently needed to advance in social inclusion, human rights and public health.

CORRESPONDING AUTHOR: Baltica Cabieses, Universidad del Desarrollo, bcabieses@udd.cl

\section{P226}

IDENTIFICATION OF THE VASOPRESSIN SYSTEM AS A PATHWAY TO ENHANCE PLACEBO EFFECTS

Luana Colloca $^{1}$

${ }^{1}$ University of Maryland School- USA

Placebo analgesia produces pain relief in individuals by virtue of expectations and anticipations of a benefit. Placebo analgesia can also occur when placebos are used following the administration of active and effective painkillers. Pharmacological studies indicate that placebos might mimic the action of active treatments and promote the endogenous release of opioids in both humans and animals. Expectations of analgesia facilitate the activation of the systems for pain control along with the release of endogenous mediators crucially involved in placebo-induced benefits. Indeed,we have identified the vasopressinergic system as one of the promising systems contributing to placebo modulation. Here I present recent behavioral and neurobiological advances on the placebo effect. Based on a well-established proposed conceptual framework, the placebo effect is presented as the product of expectancy mechanisms in which conditioned verbal, observational, and instructional cues are centrally integrated to change behaviors and outcomes. It is becoming clear that every analgesic treatment is significantly modulated by placebo effects in clinical settings. Learned placebo analgesic effects can be elicited in patients suffering from pain disorders even when pain appears to be refractory to conventional pharmacological interventions. Since placebo effects act as reinforcers of clinical outcomes, gaining deeper understanding of predictors and moderators of the top-down mechanisms of pain modulation has enormous implications for personalizing and optimizing pain management.

CORRESPONDING AUTHOR: Luana Colloca, University of Maryland School, colloca@umaryland.edu

\section{P227}

CHILDHOOD MALTREATMENT AND ADULT MENTAL DISORDERS - FREQUENCY OF DIFFERENT MALTREATMENT FORMS AND THE CONNECTION WITH COURSE AND SEVERITY OF SYMPTOMS

Nele Struck ${ }^{1}$, Axel Krug ${ }^{1}$, Tilo Kircher ${ }^{1}$, Dilara Yüksel ${ }^{1}$, Igor Nenadić ${ }^{1}$, Udo Dannlowski ${ }^{2}$, Eva-Lotta Brakemeier ${ }^{1}$

${ }^{1}$ Philipps-Universität Marburg- Germany

${ }^{2}$ Universität Münster- Germany

Introduction: Child abuse and neglect are associated with a heightened risk for mental disorders. The present study examines whether associations between certain forms of maltreatment and specific mental disorders exist. Besides, the diagnosis of persistent depressive disorder (PDD; newly listed in DSM-5) is also considered. Childhood maltreatment is expected to play a particular role in the development of PDD. Furthermore, the influence of childhood maltreatment on onset, chronicity and severity of symptoms is examined. Methods: Within a multicenter research project patients with the diagnosis of schizophrenia (SZ; 
$\mathrm{N}=107$ ), bipolar disorder (BD, N=103) and depression (MDD, N=604) as well as healthy control persons $(\mathrm{HC}, \mathrm{N}=716)$ were examined. Of the 604 patients with depression, $\mathrm{N}=54$ could be classified as patients with PDD. The clinical symptoms were assessed with a wide test battery (e.g. SKID I, BDI-II, HAMD-21, SANS, SAPS, YMRS, HAMA). The five different forms of childhood maltreatment were assessed with the Childhood Trauma Questionnaire (CTQ-SF). Results: The three patient groups reported all five forms of maltreatment significantly more often than the healthy control group. There were no significant differences between SZ, $\mathrm{BP}$ and MDD, neither in the CTQ sum nor in the five subscales of the CTQ. However, the subgroup of patients with PDD reported significantly more emotional and physical abuse as well as emotional and physical neglect than the other patient groups. Childhood maltreatment was predictive for current symptoms of depression and anxiety but not for psychotic or manic symptoms. In the MDD and BP groups childhood maltreatment was predictive for an earlier onset while there was no association in SZ. In the MDD group childhood maltreatment was associated with a higher number of clinical stays while there was no association in BD and SZ. Conclusion: These findings underline the role of childhood maltreatment in the development and the course of mental disorders, in particular in PDD.

CORRESPONDING AUTHOR: Nele Struck, Philipps-Universität Marburg, struckne@staff.uni-marburg.de

\section{P228}

FIBROUS DYSPLASIA OF THE TEMPORAL PRESENTING WITH HEADACHE: A CASE REPORT

Yuhong Man ${ }^{1}$, Gang Yao ${ }^{1}$, Xijing Mao ${ }^{1}$, Tingmin $\mathrm{Yu}^{1}$

${ }^{1}$ Department of Neurology, 2nd Hospital of Jilin University- China

Purpose: Fibrous dysplasia of bone(FDB) is an benign self-limiting disease, which pathological feature is the replacement of medullary bone by histologically benign fibro-osseous tissue. FDB usually occurs throughout the skeleton with a predilection for skull, but the temporal bone is rarely affected. Here we report a case to develop the diagnosis and treatment of FDB. Methods: We collected all the clinical features and associated test of a 29 year-old male who presented with headache. He rejected any preventive therapy and manifested the similar pain quality, intensity, frequency and during a follow-up 2 years. Results: The patient pain paroxysms was starting in both temporal scalp 2 years ago. Pain frequency was 3-4 times per month and last for 30 minutes per time. He had no history of fever, dizzy, nausea, vomit and discharge of vision an hearing. He was smoking for 10 years with 10 cigarettes per day. Physical examination and neurological test were normal. Brain CT expressed the left temporal bone thicken throughout the lesion which is characterized by uniformly dense bone. He rejected all the treatment and expressed all the features until now. Conclusions: The pathogenesis of FDB may be implicated with the proliferation and inappropriate differentiation of the osteoprogenitor cells, as well as the activating mutation of the $\alpha$ subunit of $G$ protein gene. Trauma and endocrinology abnormalities are involved in some reports. It is often expressed with progressive conductive hearing loss, occlusion of external auditory canal and intamescentia of temporal bone. High-resolution CT is the main diagnostic method. Surgery is the most important therapy which should be taken by clinical symptom and image examination. This description may expand the clinical manifestation of FDB.

CORRESPONDING AUTHOR: Yuhong Man, Department of Neurology, 2nd Hospital of Jilin University, myh1996@163.com

\section{P229}

OF PREMATURE DEMISE - A FOLLOW-UP STUDY OF YOUNG MEN EXPOSED TO VIOLENCE IN SWEDEN
Ingemar Kåreholt ${ }^{1}$, My Lilja $^{2}$, Ebba Hedström ${ }^{3}$, Sven Trygged ${ }^{2}$

${ }^{1}$ Institute of Gerontology, School of Health and Welfare, Jönköping University, Jönköping- Sweden

${ }^{2}$ University of Gävle, Faculty of Health and Occupational Studies, GävleSweden

${ }^{3}$ The Swedish National Board of Health and Welfare, StockholmSweden

Introduction \& Purpose The discourse of violence has largely focused on female victims of male violence, and partly ignored male victims. More men than women die of homicide and are exposed to non-fatal violence. Being physically assaulted is a traumatic experience with serious and long-lasting consequences. It is primarily young men aged 16-24 who suffer from violence. It can be difficult for these men to be in the role of victims, and this can be reinforced by norms that say masculinity stands for power and strength while the role of crime victims is rather an expression of weakness. The purpose of the study are to analyze mortality patterns among men aged 18-24 who have been exposed to violence leading to hospital care. Methods Register data including all men in Sweden 18-24 years that received at least one night of hospital care after injury from another person in 1992-2005. For each hospitalized man, nine age-matched men were randomly selected from the population each year. Data is available from the year before hospitalization/inclusion and 10 years after (or to 2006 when follow-up ends). Results Analyses are based on Cox regressions, controlled for age, days of inpatient care from violence, days of hospital care for other reasons, degree of urbanization, country of birth, presence of preschool children, gainfully employed/ not, financial assistance/not, education, and income. Compared to men from the population, exposed men had $>3$ times higher risk $(\mathrm{HR}=3.34)$ for all-cause mortality, $>4$ times higher risk $(\mathrm{HR}=4.16)$ to die from suicide, and about 20 times higher risk $(H R=19.63)$ to die from violence. The difference between exposed and non-exposed men in all-cause mortality, decrease significantly over the 10-year follow-up from HR 4.48 to 2.66 , in mortality from violence from HR 45.23 to 2.06 . Regarding mortality from violence, the difference between exposed and non-exposed men was not significant after 10 years. The risk difference to die from suicide remained stable over the follow-up period. Conclusions Paying attention to the long-term consequences from violence, there are also opportunities to change the situation and allow for alternative development for men who have been abused. This is especially important when it comes to the risk for suicide.

CORRESPONDING AUTHOR: Ingemar Kåreholt, Institute of Gerontology, School of Health and Welfare, Jönköping University, Jönköping, ingemar.kareholt@ju.se

\section{$\mathrm{P} 230$}

MINIMIZING NOCEBO EFFECTS ON ITCH BY CONDITIONING

Andrea Evers ${ }^{1}$, Danielle Bartels ${ }^{1}$, Antoinette van Laarhoven ${ }^{1}$

${ }^{1}$ Leiden University- Netherlands

Nocebo effects are known to contribute to the experience of physical symptoms such as pain and itch. However, little is known about how to reduce nocebo effects in itch or other physical symptoms. We tested whether nocebo effects can be minimized by positive expectation induction with respect to electrical and histaminic itch stimuli. After inducing negative expectations about electrical itch stimuli were induced by conditioning, participants were randomized to either the experimental group or one of the control groups. In the experimental group, positive expectations were induced by conditioning, while in the control groups either the negative expectation induction was continued or an extinction procedure was applied. Afterwards, a histamine application test was conducted. Scratching behavior was videotaped during the whole session. Results demonstrated that positive expectation induction resulted in a significantly smaller nocebo effect in comparison with both control groups and even 
changed into a placebo effect. Comparable effects were also found for histamine application, while there were no effects on behavioral outcomes of scratching. This study for the first time demonstrate that nocebo effects for physical symptoms, such as itch, can be minimized and even reversed by conditioning procedures. The results of the current study indicate that learning via counterconditioning represents a promising strategy for diminishing nocebo responses.

CORRESPONDING AUTHOR: Andrea Evers, Leiden University, a.evers@fsw.leidenuniv.nl

\section{P231}

COMMITMENT TO THE RELATIONSHIP A RESOURCE THAT MEDIATES THE RELATIONSHIP BETWEEN INTERNALIZED HOMOPHOBIA AND SAME-SEX RELATIONSHIP SATISFACTION Fabiola Gómez ${ }^{1}$, Jaime Barrientos ${ }^{2}$

${ }^{1}$ Pontificia Universidad Católica de Chile- Chile

${ }^{2}$ Universidad de Santiago de Chile- Chile

According to Minority Stress Model, some gay men and lesbian could have self-devaluation by internalizing the negative attitudes toward homosexuality (called internalized homophobia). This process has been mentioned as one of most important stressor derived from sexual stigma. There is evidence that relates, negatively, the internalized homophobia with same-sex relationship satisfaction, but to our knowledge, current literature lacks research that identifies the processes underlying this relationship, and no studies have examined possible differences by age. According to that, the purpose of this study was to investigate commitment to the relationship as a mediator in the relationship between internalized homophobia and relationship satisfaction among gay men and lesbians. Additionally, we explore possible differences by age. A type of snowball chain sampling was used, and to test the main hypothesis of this study, a structural equation model analysis with latent constructs was performed. The final sample consisted of 259 gay men and lesbians who were currently involved in a romantic relationship. The age of participants ranged from 18 to 66 years $($ Mage $=27.8, \mathrm{SD}=7.8$ ). For the analysis, age was dichotomized into 2 age groups: 18-29 years old (emergent adults, $69.5 \%$ ) and 30 or older (adults, $30.5 \%$ ). When controlling for the effects of all the other variables, the direct path coefficient from internalized homophobia to relationship satisfaction was not significant (B $=-0.07, \mathrm{SE}=0.04, \mathrm{p}=.10,95 \% \mathrm{CI}[-0.144,0.000])$, but we found $\mathrm{a}$ significant indirect effect (BIndirect $=-0.10, \mathrm{SE}=0.04, \mathrm{p}=.02,95 \% \mathrm{CI}$ $[-0.172,-0.031])$. The multi-group analysis allows to put out that the mediational model is only met in the emergent adult group. For Adult group, only commitment to the relationship was, significantly, related to relationship satisfaction. These results provide evidence on the importance of commitment to the relationship as a resource that intervenes positively in the relationship between internalized homophobia and same-sex relationship satisfaction, especially, among emergent adult people. This study discusses whether the impact of sexual stigma on the same-sex relationships in adulthood affects emergent adults in the same way.

CORRESPONDING AUTHOR: Fabiola Gómez, Pontificia Universidad Católica de Chile, fagomez3@uc.cl

\section{P232}

STRESS RESPONSE BEFORE AND AFTER A LUNG CANCER DIAGNOSIS

Hrönn Harðardóttir ${ }^{1}$, Unnur Valdimarsdóttir ${ }^{1}$, Heiðdís Valdimarsdóttir ${ }^{2}$

${ }^{1}$ University of Iceland- Iceland

${ }^{2}$ University of Reykjavík- Iceland

Background:A diagnostic work-up leading to a diagnosis of lung cancer is a severely stressful experience as illustrated in the dramatic rise in mental disorders, suicide and cardiovascular deaths in this patient population. Data are scarce on the underlying psychobiological stress-sequel during this critical time window. The aim of this study was to assess the association between physical state, psychological symptoms and urinary catecholamines as well as to describe the pre- to post diagnosis change in these parameters. Methods: The LUCASS study (Lung CAncer, Stress and Survival study) is a prospective cohort study targeting all individuals referred to a 24-36 hour diagnostic work-up at Landspitali National University Hospital in Iceland, due to radiologic- or clinical suspicion of lung cancer. The study protocol is integrated with clinical assessments at two time points, i.e. during the diagnostic work-up and at follow-up visit 1-3 weeks after diagnosis but before treatment. At both time points, participants complete measure of psychological distress (HADS) and provided urine samples for catecholamine assessments. Symptoms of posttraumatic stress is measured after diagnosis (Impact of event scale revised (IES-R). Preliminary results: Of 155 recruited participants, 92(59.4\%) completed questionnaires and overnight urine collection at both assessments. Mean age was 69 years, women $43.5 \%$. A lung cancer was diagnosed by $70(76 \%)$ patients. The HADS-total score among patients diagnosed with lung cancer was 9.2(+5.9) pre- and 10.8(+6.4) postdiagnosis; with $45.9 \%$ presenting with clinical significant symptom burden (HADS score $>11$ ). In contrast, patients diagnosed with benign causes of their lung pathology had an average of $8.5(+6.6)$ pre- and $7.6(+5.6)$ post diagnosis. Post diagnosis $88.5 \%$ of patients diagnosed with lung cancer reported symptom burden suggestive of PTSD ( $>33$ on IES). Pre- and post- diagnosis urinary-catecholamines are currently being analyzed and will be presented at the congress. Conclusion: These preliminary results confirm the heavy psychological burden associated with the diagnostic work-up and diagnosis of lung cancer.

CORRESPONDING AUTHOR: Hrönn Harðardóttir, University of Iceland, hronnhardardottir@simnet.is

\section{P233}

ASSESSMENT OF THE EFFECT OF CHRONIC URBAN NOISE ON LIFE HABITS AND CORTISOL IN PRIMARY CHILDREN

Everardo Camacho ${ }^{1}$, Claudia Vega-Michel ${ }^{1}$, Sugei González ${ }^{1}$, Jordan García $^{1}$, Bárbara Doddoli ${ }^{1}$, Juan Carlos Conrigue ${ }^{1}$, Demian Ortega ${ }^{1}$ ${ }^{1}$ ITESO- Mexico

The objective of this research was to evaluate the effect of chronic exposure to noise in children of primary. (a) Participants: 56 children from sixth grade of both genders. 28 children who have studied from first year in a primary in a noisy area (more than $80 \mathrm{db}$ on average during class hours) and 28 children from a primary in a not noisy area (levels of noise equal or less than $65 \mathrm{Db}$ on average during class hours). (b) Instruments: participants applied the questionnaire of child life consisting of 58 questions evaluating schedule of sleep, power, work, recreation, use of electronic equipment, time transfer and collaboration at home habits. In the analysis of salivary cortisol test enzyme-immuno-assay (ELISA - enzimelinked immunoabsorbent essay), detecting complex Antigen - antibody with pigmentation of peroxidase is used. Using a commercial team Salimetrics 1-3002-5. A sound level meter brand Steren model HER400 was used for the measurement of noise levels. (c) Design: a design comparison groups, was used by measuring cortisol levels and establishing differences between the experimental group and control the variable cortisol and lifestyle variables. (d) Procedure: the measurements of the noise levels were made to select the primaries that met the criteria previously set noise levels. Requested access to schools with principals specifying the purposes of the investigation and was managed the letter of consent by the parents of the children. We selected children based on the criterion of seniority in the primary (having completed all grades of primary school). The questionnaire of child life habits and surrendered the tubes for the collection of samples of saliva, instructing children the procedure for collecting samples and at what hours. Samples per child 
were collected upon waking, within half an hour of waking up, two hours have woken up and before going to sleep. (e) Analysis of data. The data were analyzed by experimental group and control to identify differences in cortisol and life habits using the T test. (f) The significant differences encountered are reported and discussed regarding the practical implications of the urban design of the children's educational centers.

CORRESPONDING AUTHOR: Everardo Camacho, ITESO, ecamacho@iteso.mx

\section{P234}

DIRECT AND INDIRECT EFFECTS OF PERCEIVED STIGMA ON POSTTRAUMATIC GROWTH IN A SAMPLE OF GAY MEN AND LESBIANS IN CHILE

Jaime Barrientos ${ }^{1}$

${ }^{1}$ Escuela de Psicologia, Universidad de Santiago de Chile- Chile

This study examined the direct and indirect effects of perceived stigma on posttraumatic growth in a sample of gay men and lesbian women in Chile, with coping strategies (positive reappraisal, social support seeking, and active coping strategies) as intermediate variables. Data from 467 gay men $(57.4 \%)$ and lesbian women $(42.6 \%)$ were analyzed. A respondent-driven sampling (RDS) method was used. The hierarchical regression analysis indicated the important predictive role of active coping, $\beta=.23$, and positive reappraisal, $\beta=.45$, in posttraumatic growth, $\mathrm{R} 2=.31, \mathrm{p}<.001, \mathrm{f} 2=0.16$. Results revealed that, in the presence of positive reappraisal coping as an intermediate variable, partial indirect effects are detected between perceived stigma and posttraumatic growth. Seeking instrumental social support did not produce indirect effects between perceived stigma and posttraumatic growth, whereas active coping produced partial indirect effects. These findings suggest that the positive reappraisal of a traumatic experience is essential for reporting personal growth. Implications of these more complex relations for counseling interventions and further research are discussed

CORRESPONDING AUTHOR: Jaime Barrientos, Universidad de Santiago de Chile, jaime.barrientos@usach.cl

\section{$\mathrm{P} 235$}

GLUCOSE LEVELS AND ITS RELATION TO EMPATHY AND RECOGNITION OF FACIAL EXPRESSION

Roberto La Marca ${ }^{1}$, Niclà Lozza ${ }^{1}$, Pearl La Marca-Ghaemmaghami ${ }^{1}$, Silja Sollberger ${ }^{1}$, Wolfgang Langhans ${ }^{2}$, Myrtha Arnold ${ }^{2}$, Ulrike Ehlert ${ }^{1}$ ${ }^{1}$ Clinical Psychology and Psychotherapy, Department of Psychology, University of Zurich, Zurich- Switzerland

${ }^{2}$ Institute of Food, Nutrition and Health, Department of Health Sciences and Technology, Swiss Federal Institute of Technology (ETH) Zurich, Zurich- Switzerland

Introduction \& Purpose: The brain and its capacity to conduct resource-intensive tasks rely heavily on the availability of glucose. Low glucose availability has been associated with unsocial behavior, while acute glucose intake was shown to improve self-reported prosocial behavior. The aim of the present study is to explore associations between glucose levels at baseline and in response to an oral glucose tolerance test (OGTT) and empathy and facial emotion recognition. Methods: This study is part of a larger project. Normaland overweight, but otherwise healthy young men $(\mathrm{N}=147)$ participated in a $75 \mathrm{~g}$ OGTT in the afternoon, after seven hours of fasting. Blood samples were collected, via a venous catheter, immediately before, and then again 1 and 2 hours after glucose intake. The area under the curve with respect to increase (AUCi) for glucose was calculated as the overall response to the OGTT. Prior to the OGTT, participants completed a test assessing facial emotion recognition (Reading the Mind in the Eyes Test, RMET; BaronCohen et al., 2001) and responded to a questionnaire on empathy (FEP; Maes et al., 1995). Results: The OGTT resulted in a significant increase of plasma glucose levels $(\mathrm{p}<.001)$. While baseline glucose levels were unrelated to FEP and RMET scores, the AUCi for glucose was positively correlated with empathy $(p<.01)$ and negatively with the percentage of correctly recognized faces depicting negative emotions $(p<.05)$. The AUCi was unrelated to the performance in recognizing positive or neutral facial expression and the total RMET score. Conclusions: In a sample of healthy men, a larger glucose response to the OGTT was associated with greater empathy and reduced accuracy of processing negative facial expression. Therefore, an overall higher glucose availability after glucose intake may provide a resource to engage empathetically with the social environment. This ability might be supported by a reduced recognition of negative emotions and, thus, decreased negative emotional contagion. Funding: The project was funded by the Swiss National Science Foundation (SNSF), grant no. 105314 149529. The first author received a further grant from the Forschungskredit of the University of Zurich, grant no. FK-15-075.

CORRESPONDING AUTHOR: Roberto La Marca, University of Zurich,r.lamarca@psychologie.uzh.ch

\section{P236}

SOCIAL CAPITAL AND DEPRESSION IN THE BRAZILIAN LONGITUDINAL STUDY OF ADULT HEALTH (ELSA-BRASIL)

Ester Souto $^{1}$, Enirtes Caetano ${ }^{1}$, Arlinda B Moreno ${ }^{1}$, Rosane H Griep ${ }^{1}$

${ }^{1}$ Fundação Oswaldo Cruz. Rio de Janeiro, RJ- Brazil

Introduction: Recent studies emphasize the multidimensionality of the social capital and characterize it as the presence of resources that individuals can access through a social network. Its association with depression has also been explored, as it is believed that having a greater number of resources available through the social networks can act as a protective effect against depression. This study investigates the gender differences in the association between the social capital and the incidence and maintenance of depressive episodes. Methods: Cross sectional and longitudinal investigation (4 years of follow-up) of 14,390 adults, aged 35-74 years from the Brazilian Longitudinal Study of Adult Health (ELSA-Brasil). It is a multicenter cohort study involving public servants at six teaching and research institutions in six towns in Brazil. The social capital was assessed using the Resource Generator, composed of two distinct dimensions: social support and prestige/education. The depressive episodes were assessed using the Clinical Interview Schedule-Revised (CIS-R) questionnaire. The statistical analysis was stratified by gender and used the multinomial regression model with adjustments for the possible confusion factors (age, marital status and education). Results: Among men, only the social support dimension has proven to be associated both with the incidence $(\mathrm{RRR}=1.66$, IC 95\%: $1.01-2.72)$ and with the maintenance (RRR $=1.56$, IC 95\%: $0.58-4.24)$ of depressive episodes. Among women, both dimensions of social capital were associated with the maintenance of depressive episodes in four years (social support $-\mathrm{RRR}=$ 2.66, IC 95\%: 1.61 - 4.41; prestige/education - RRR = 1.38, IC 95\%: 0.86 - 2.24). Among women, the social capital was not associated with the incidence of depressive episodes. Conclusions: For women, lower social capital in both dimensions was positively associated with the maintenance of depressive episodes. Among men, the social support was identified as a risk for the incidence and the maintenance of depressive episodes. The results highlight different patterns of association for different genders, confirming the gender difference found in the literature for both social capital and mental health.

CORRESPONDING AUTHOR: Ester Paiva Souto, Fiocruz, esterpaiva@hotmail.com 
$\mathrm{P} 238$

DIMENSIONAL AND CORRELATIONAL VALIDITY OF THE BRAZILIAN VERSION OF THE EFFORT-REWARD IMBALANCE IN DOMESTIC AND FAMILY WORK SCALE

Ilmeire Vasconcellos ${ }^{1}$

${ }^{1}$ Escola Nacional de Saúde Pública. Fundação Oswaldo Cruz- Brazil

Abstract Objective: To present the results of the dimensional and correlational and validity of the Brazilian version of the scale "Effort-Reward Balance in Domestic and Family Work" (Domestic ERI). Methods: In a cross-sectional study, we applied a three-dimensional scale (effort, reward, and over commitment) among 468 female nursing workers from a public hospital in Rio de Janeiro; all of them were married and had children. Data were collected from October 2012 to May 2013. Dimensional validity analysis was performed using exploratory factorial analysis. The final factorial structure was confirmed by observing adjustment indexes (IFC, TLI and RMSEA). Correlational analysis was performed using sociodemographic characteristics, working conditions (professional and domestic) and health conditions (physical and mental) by Spearman correlation coefficient and significance level of 5\% was adopted. Results: The mean age of the sample group was 44 (SD = 10.3 ) years, $62.4 \%$ with higher education level or more and $42.1 \%$ selfdeclared themselves as white. The best selected model in the dimensional analysis was a single scale with 22 items and 5 dimensions: Excess of commitment with 5 items (loads ranging from 0.767 to 0.927 ); effort loaded with 7 items (loads ranging from 0.691 to 0.920 ); recognition and affection of children with 3 items (loads varying from 0.652 to 0.932); partner recognition with 3 items (loads varying from 0.829 to 0.930 ); and intrinsic value and social value with 4 items (loads ranging from 0.342 to 0.745 ). The adjustment indexes were satisfactory (RMSEA $=0.036, \mathrm{CFI}=0.98$ and TLI $=0.99$ ). Correlations of effort, over commitment and psychosocial stress in domestic work were significant and positive with the variables domestic work hours, domestic overload and common mental disorders, and negative with age, per capita income and demands on professional work. Conclusions: The observed correlations were within the expected second similar studies and the dimensional validity presented adequate results. This indicates that the Domestic ERI scale showed acceptable psychometric properties in Brazilian reality.

CORRESPONDING AUTHOR: Ilmeire Vasconcellos, Fundação Oswaldo Cruz, ilmeiredevasconcellos@gmail.com

\section{P239}

\section{THE EXPERIENCE OF MOTHERHOOD DURING CANCER} TREATMENT

Elisa Kern de Castro ${ }^{1}$, Ana Luisa Kenne Dornel ${ }^{1}$, Miguel Alves de Souza ${ }^{1}$ ${ }^{1}$ Unisinos- Brazil

Introduction \& Purpose: Cancer may affect negatively the lives of women who are mothers. This factor causes a series of difficulties to reconcile the responsibilities of treatment with young children's care. Furthermore, it is common that mothers start living in fear of dying and leaving their children helpless. Therefore, cancer may cause physical, behavioral and emotional changes that could end up affecting negatively women's lives, interfering with the quality of relationship between mother and child. Therefore, the aim of this study was to investigate the experience of motherhood on women with cancer diagnosis and mothers with at least one child under 10 years of age. Method: The study is a qualitative exploratory design. Ten mothers on treatment at different stages of disease and with different types of cancer were interviewed. The measures were: 1) sociodemographic and clinic questionnaire; 2) semi-structured interview. The interviews were audio-recorded and transcribed for content analysis. The data was analyzed in three stages: a) initial reading without judgement ("naive"); b) structural analysis and categorization of content; and c) critical interpretation and discussion. Two independent judges evaluated the emerging categories.
The Kappa index was 0,738. Results: Five thematic categories were identified: 1) Be a mother is ... being afraid of death / cancer recurrence and leaving their child orphan; 2) Be a mother is ... changing the values / meaning of life with the illness; 3 ) Be a mother is ... changing the family routine / child routine; 4) Be a mother is ... having conflict / defeat / ambivalent feelings; 5) Be a mother is ... having difficulties to attend their children. Conclusion: Observation showed that motherhood and cancer diagnosis experiences overlap and intensify the suffering of these mothers. At the same time, both experiences place those women in situations that lead them to re-signify their lives and their priorities, which, in turn, enhances mother-child relationship in a positive way.

CORRESPONDING AUTHOR: Elisa Kern de Castro, Universidade do Vale do Rio dos Sinos, elisa.kerndecastro@gmail.com

\section{P240}

RELATION OF PERCEIVED BARRIERS AND PHYSICAL ACTIVITY LEVEL IN BRAZILIAN OLDER ADULTS

Daniela Lopes dos Santos ${ }^{1}$, Nicanor Dornelles ${ }^{1}$, Temistocles Barros ${ }^{1}$, Barbara Silva ${ }^{1}$, Patricia Soares ${ }^{1}$

${ }^{1}$ Federal University of Santa Maria- Brazil

INTRODUCTION \& PURPOSE: Despite the well-known benefits of physical activity, there is still a great amount of the population leading a sedentary lifestyle. The causes may be explained by the barriers perceived to physical activity, so the determination of these barriers may be an important tool for the increase of a population's physical activity level (PAL). The purpose of this study was to analyze the association between perceived barriers and PAL in older adults. METHODS: This was a descriptive cross-sectional study conducted within the elderly population living in three neighborhoods of the urban area of a medium-sized city of southern Brazil. The PAL was evaluated with the IPAQ (transport and leisure domains) and the perceived barriers with the instrument proposed by Reichert et al. (2007) validated for the study population. The chi-square test was used to analyze the associations between perceived barriers and PAL. RESULTS: $52.7 \%$ of the studied population was classified as physically active, $37.18 \%$ as insufficiently active and $10.25 \%$ as physically inactive. The barrier perceived the most among the insufficiently active elderly was having an injury or illness that interferes in physical activity $(58.62 \%)$ and among the physically inactive, feeling lazy and tired (62.5\%). Physically inactive and insufficiently active older adults reported a greater number of barriers in greater frequencies and similar perceived barriers (having an injury or illness that interferes in physical activity, feeling lazy and tired, and lack of companionship). In the analysis of the difference in the perception of barriers between the groups stratified by PAL, a statistically significant difference $(\mathrm{p}<0.05)$ was found in the barriers "feeling old for practicing physical activity" ( $\mathrm{p}=0.034)$ and "having an injury or illness that interferes in physical activity" ( $p=0.004)$. CONCLUSIONS: The findings of this study showed the impact that the perception of barriers exerts on PAL, showing that the higher the number of perceived barriers, the lower tends to the PAL of the older adults. Health professionals working with this population should be aware of intervention possibilities that may reduce or minimize the negative effects of perceived barriers on PAL.

CORRESPONDING AUTHOR: Daniela Lopes dos Santos, Federal University of Santa Maria, lopesdossantosdaniela@gmail.com

\section{P241}

A QUALITATIVE EXAMINATION AND THEORETICAL MODEL OF ANXIETY IN ADULTS WITH EPILEPSY

Amelia Scott, Louise Sharpe ${ }^{1}$

${ }^{1}$ The University of Sydney- Australia

Objective. There is an elevated prevalence of anxiety disorders among people with epilepsy, and the co-morbidity of anxiety in epilepsy is 
associated with adverse medical and psychosocial outcomes. Despite its importance, little is known about what psychosocial or epilepsy factors may be associated with the development of anxiety. The aim of this qualitative study was to determine what factors may explain why some people with epilepsy develop anxiety disorders and others do not. Methods. Adults with epilepsy were recruited from an outpatient epilepsy service. Semi-structured interviews were conducted with 26 participants, 15 of whom reported clinically significant levels of anxiety. Grounded theory analysis was used to develop a theoretical model of anxiety development in the context of epilepsy. Results. Qualitative analyses revealed a number of processes that appeared to account for the development of anxiety in the context of epilepsy. These including inflated estimates regarding epilepsy-specific risks, and excessive attempts to avoid these risks. Such excessive avoidance often resulted in greater interference with participants' role functioning, thus risking ongoing quality of life. A number of pre-morbid and contextual factors also appeared implicated in the development of anxiety. Conclusion. This investigation provides a comprehensive account for the development of anxiety in epilepsy which bears consistency with existing theories of anxiety development and maintenance. Importantly, this model provides a foundation for appropriate treatment strategies and future research to address anxiety in people with epilepsy.

CORRESPONDING AUTHOR: Amelia Scott, The University of Sydney, ameliajanescott@gmail.com

\section{$\mathrm{P} 242$}

IN TUNE WITH THE BODY: CARDIAC VAGAL TONE PREDICTS CALORIC INTAKE IN HIGH, BUT NOT IN LOW STATUS INDIVIDUALS

Mario Weick, ${ }^{1}$ Milica Vasiljevic ${ }^{2}$

${ }^{1}$ University of Kent- United Kingdom

${ }^{2}$ Behaviour and Health Research Unit, University of Cambridge- United Kingdom

Introduction and Purpose: The aim of the present study was to examine the link between (subjective and objective) status and cardiac vagal tone - an established marker of implicit self-regulation. We examined two distinct routes through which status may impact the association between implicit self-regulation and eating behaviour. First, drawing on previous studies on externalising behaviours, we reasoned that elevated status may be associated with greater cardiac vagal tone withdrawal during active coping, which may provide an indication of better self-regulation. Second, drawing on the assumption that low status individuals are more strongly guided by external cues than high status individuals, we also examined the correspondence between cardiac vagal tone and caloric intake as a second route through which variations in status may impact implicit selfregulation. Methods: Ninety-six participants completed the study (80 females; MAge $=20.28$, SDAge $=4.58$ ). Participants had a diverse socio-economic background (on both objective and subjective indices of deprivation). Participants were invited to take part in a chocolate taste test in the laboratory. Prior to the taste test, indices of cardiac vagal tone were derived from resting periods and during two challenging tasks. Results: We found no support for the mediational route whereby higher status bolsters the responsiveness of parasympathetic influence on the heart during active coping (i.e., vagal tone withdrawal). However, we found evidence that resting vagal tone modulated the caloric intake of high [BSubjectiveStatus $=-.45, \mathrm{SE}=.17$, $\mathrm{t}(90)=-2.64, \mathrm{p}=.010, \mathrm{sr}=-.26$, BObjectiveStatus $=-.37, \mathrm{SE}=.15$, $\mathrm{t}(80)=-2.55, \mathrm{p}=.013, \mathrm{sr}=-.27]$, but not of low status participants [ps $=.323$ and .076], resulting in a significant interaction between resting cardiac vagal tone and status [psubjective $=.015$; pobjective $=.005]$. Conclusions: Cardia vagal tone - a marker of implicit selfregulation - predicts caloric intake in high, but not in low status individuals. This suggests that relative to low status individuals, high status individuals' eating behaviour may be more strongly guided by internal states. Furthermore, unlike low status individuals, high status individuals' self-regulatory efforts may benefit from the involvement of implicit processes.

CORRESPONDING AUTHOR: Mario Weick, University of Kent, m.weick@kent.ac.uk

P243

CROSS-NATIONAL COMPARISON OF EDUCATION-BASED INEQUALITIES IN UNTREATED CARIES AMONG ADULTS IN AUSTRALIA, CANADA, CHILE, NEW ZEALAND AND THE UNITED STATES

Iris Espinoza ${ }^{1}$, Gloria Mejia ${ }^{2}$, Jay Kaufman ${ }^{3}$, Murray Thomson ${ }^{4}$, Hawazin Elani ${ }^{5}$, Sam Haper ${ }^{3}$, Lisa Jamieson ${ }^{6}$, Ichiro Kawachi ${ }^{5}$, Ju Xiangqun ${ }^{6}$

${ }^{1}$ Centro de Epidemiología y Vigilancia de las Enfermedades Orales, Universidad de Chile- Chile

${ }^{2}$ University of South Australia- Australia

${ }^{3}$ Department of Epidemiology, Biostatistics, \& Occupational Health. McGill University- Canada

${ }^{4}$ University of Otago- New Zealand

${ }^{5}$ Harvard University- USA

${ }^{6}$ The University of Adelaide- Australia

PURPOSE: To conduct cross-national comparison of education-based inequalities in untreated caries among adults in Australia, Canada, Chile, New Zealand and the United States. METHODS: Nationally representative data from Australia's National Survey of Adult Oral Health; Canadian Health Measures Survey; Chile's First National Health Survey Ministry of Health; US National Health and Nutrition Examination Survey; and the New Zealand Oral Health Survey were used. We examined the prevalence of untreated caries in 25-year-old and older dentate populations. We used education as a measure of socioeconomic position and measured absolute inequality with the Slope Index of Inequality (SII). We used random-effects meta-analysis to summarize inequality estimates. RESULTS: Adjusted prevalences of untreated caries (\% decayed) by education level were analyzed and the $95 \%$ confidence intervals presented in brackets. Chile showed the highest prevalence of caries in all education levels across countries: $85.5 \%(79.5 ; 85.6)$ in primary-educated people; $70.3 \%(65.9 ; 74.8)$ in secondary; $66.3 \%(40.8 ; 62.7)$ in postsecondary and $44.8 \%(34.2 ; 55.4)$ in university. This was followed by New Zealand with $50.4 \%(41.2 ; 59.5)$ in primary, $45.4 \%(39.2 ; 51.6)$ in secondary, $39.9 \%(35.0 ; 44.8)$ in post-secondary and $32.6 \%(25.9 ; 39.2)$ in university. Australia showed $36.0 \%(31.4 ; 40.6)$ in primary, $30.9 \%$ $(24.8 ; 37.1)$ in secondary, $26.3 \%(22.8,29.8)$ in post-secondary and $16.2 \%(13.5 ; 18.8)$ in university. Canada had $32.7 \%(25.2 ; 39.9)$ in primary, $25.7 \%(20.1 ; 31.3)$ in secondary, $18.3 \%(15.2 ; 21-3)$ in post-secondary and $10.4 \%(7.4 ; 13.3)$ in university. Finally, the US had $29.0 \%(24.3 ; 33.7)$ in primary, $19.2 \%(14.0 ; 29,4)$ in secondary, $16.5 \%(12.9 ; 20.1)$ in postsecondary and $8.7 \%(5.6 ; 11.8)$ in university. The SIIs were 38.3 $(28.0 ; 48,6)$ in Chile, Canada $29.6(20.0,39.3)$, Australia $23.0(16.6 ; 29.4)$, USA $22.6(16.6 ; 28.6)$ and the lowest was found in New Zealand at 19.4 (8.2;30.6). CONCLUSIONS: There was a consistent social gradient in the prevalence of untreated dental caries by education level across the countries studied. Chile, a country with a lower level of development than the other countries included in this analysis, showed the highest prevalence of untreated caries and the widest absolute inequality. However, New Zealand showed that a high prevalence is not invariably associated with wide inequalities.

CORRESPONDING AUTHOR: Iris Espinoza, Centro de Epidemiología y Vigilancia de las Enfermedades Orales, Universidad de Chile, iespinoz@u.uchile.cl 
P244

SOCIAL DISPARITIES IN FERMENTABLE CARBOHYDRATE INTAKE PROFILE IN CHILDHOOD IN METROPOLITAN AREA, CHILE

Maria Jose Monsalves ${ }^{1}$, Iris Espinoza ${ }^{2}$, Josefina Aubert ${ }^{3}$, Patricia Moya ${ }^{3}$, Oscar Arteaga ${ }^{4}$, Doris Duran ${ }^{5}$, Macarena Valdes ${ }^{5}$, Shrikant Bangdiwala ${ }^{6}$

${ }^{1}$ Universidad San Sebastian- Chile

${ }^{2}$ Centro de Epidemiologia y Vigilancia de las Enfermedades Orales, Universidad de Chile- Chile

${ }^{3}$ Facultad de Odontología, Universidad Finis Terrae- Chile

${ }^{4}$ Facultad de Medicina, Escuela de Salud Pública, Universidad de Chile, Santiago- Chile

${ }^{5}$ Facultad de Medicina, Universidad San Sebastián, Santiago- Chile

${ }^{6}$ McMaster University- Canadá

Objective: To characterize the profile of fermentable carbohydrate intake in children 1-6 years old in the Metropolitan Region (MR), Chile. Methods: A cross-sectional multilevel study (school/communes) was conducted in a sub-sample of the FONIS-SA13I20130 project with a questionnaire for caregivers of children 1-6 years old in 13 communes of the MR in Chile during 2014-2015. Intake frequency of 5 food-groups was assessed: biscuits/cakes/cream cakes; sugary juice/beverages; jam/ honey; sweets/candy; and tea/coffee/milk with sugar. Six categories for intake-frequency were considered never; several times a month, once a week, several times a week, at least once a day every day, several times in a day. Social characterization was obtained with educational level of the caregiver and family income. Statistical analysis included uni-bivariate analysis of the proportions (Chi-squared). Coefficients/odds of food intake-frequency for each food-group by education and income in the high intake-frequency category (several times a day) were obtained with ordinal logistic multilevel regression. Results: Sample-size was 1989. Three food-groups had the highest intake-frequency of at least once a day every day: sugary juice/beverages with a $48.92 \%$; tea/coffee/milk with sugar with $38.67 \%$, and biscuits/cakes/cream cakes with $27.77 \%$. In relation to social characterization, educational level was associated with intake frequency of sugary juice/beverages; tea/coffee/milk with sugar; and Sweets/candy $(\mathrm{p}<0.05) .88 \%$ higher odds of having a high intake frequency of tea/coffee/milk with sugar, $35 \%$ for sugary juice/ beverages, $34 \%$ for jam/honey was found for caregivers with primary education. $71 \%$ lower odds of never consuming tea/coffee/milk with sugar, $46 \%$ for sugary juice/beverages and for sweets/candy between the highest family income in relation to the lowest. Conclusions Sugary juice/beverages; tea/coffee/milk with sugar, and biscuits/cakes/cream had the highest frequencies of intake, and the higher differences between socioeconomic groups. Primary educational level and low family income present the higher frequency of intake for food-groups related to caries, evidencing their disadvantaged position to have better oral-health.

CORRESPONDING AUTHOR: Maria Jose Monsalves, Universidad San Sebastian, iespinoza@odontologia.uchile.cl

\section{$\mathrm{P} 245$}

A THERAPEUTIC MODEL TO STIMULATE NEUROADAPTIVE SELF-REGULATION

Eduardo Muñoz ${ }^{1}$, Nicolás Zalaquett

${ }^{1}$ Neuroespejo- Chile

Chronic neuropathic pain, loss of motor, sensor and linguistic function in stroke and the traumatic experience often associated constitute health issues that demand measures beside pharmacological and physical treatment. The proposed model is based on the premise of a fundamental ability of the brain for neuroplastic reorganization of ill adapted networks that chronify pain, sickness and trauma. It uses a valuation of psychological and psychosocial chronifying factors, a detection and amplification of potential resources and, through a detailed articulation of personal preferences, enables the patients subjectivity to begin, in a personalized and creative manner, a transition of state, and hopefully the emergence of new healthy neural networks. The model's method is implemented using a protocolized circuit which includes the use of a designed multisensorial peripersonal installation that allows the patient to interact with their self image, using a digital mirror, reflecting new possibilities for the brain. The method capitalizes on the activity of mirror neurons which previously have allowed for an explanation of the effectiveness of mirror therapy in ghost pain, regional complex pain and other pathologies that compromise the central and peripheral nervous system. Their activity in the contralateral hemisphere increase the neural activity in areas involved with attention and cognitive control, increase the motor activity, produce analgesia and neuroplastically rearrange the somatosensory and motor cortex. The use of a peripersonal space is to amplify the activation of mirror neurons and of the central nervous system to the stimuli. The method, highly personalized, executed in at most eight sessions, has been used to treat ghost pain, stroke, severe traumatic memories, phobias, self-image distortion and other pathologies that compromise the central nervous system. The main results are reported case by case, using the EVA pain scale, a new instrument named three mirrors and subjective reports. The preliminary results indicate that it can enable adaptive neuroplastic events where ill formed networks are reorganized, pain experience is reduced, some function recovery is obtained, self image can be modified and in general negative networks can be reorganized.

CORRESPONDING AUTHOR: Eduardo Muñoz, Neuroespejo, emunozh@uc.cl

\section{P246}

A PILOT STUDY OF THE BENEFITS OF MUSIC TO IMPROVE ATTENTION IN TWO PATIENTS WITH MORQUIO-A AND MAROTEAUX-LAMY SYNDROMES

D. Carolina Cárdenas-Poveda ${ }^{1}$, Nolly N. Castañeda-Ibáñez ${ }^{1}$, Mayerli A. Prado-Rivera ${ }^{1}$, Alejandra Rizo-Arévalo ${ }^{1}$, Jessica Vásquez-Salazar ${ }^{1}$

${ }^{1}$ Corporación Universitaria Minuto de Dios - UNIMINUTO- Colombia

Morquio-A and Maretoaux-Lamy syndromes (mucopolysaccharidosis MPS IV-A and MPS VI) are a rare type of multisystemic lysosomal storage disorders caused by an inherited deficiency in different enzymes, involved in lysosomal degradation of glycosaminoglycans. The exact incidence of these syndromes is unknown, although it is estimated to occur in 1 in 200,000 to 300,000 individuals for Morquio and in 1 in 250,000 to 600,000 newborns for Maroteaux-Lamy. Prevalence of MPS varies according to the country. In Colombia, the most frequent variant is the Morquio-A. There are several studies focused on genetic status or clinical manifestation related to these disorders due to their classification as inborn errors of metabolism disorders, but specific research on psychological or neurocognitive conditions are very scarce, even when behavioral aberrations and cognitive deficits are observed. Morquio-A and Maroteaux-Lamy syndromes usually has not been associated with neurological involvement nor with intellectual impairment measured by scales of intelligence, with some studies reporting normal average IQ for patients with Morquio-A and inconsistent results for Maroteaux-Lamy patients. Nonetheless, the presence of cognitive and behavioral impairment that might impact long term functional outcomes are investigated, with some studies reporting alterations in visual and sustained attention in children. Despite advances in medical treatments, no interventions have been reported to improve the cognitive functioning of these patients. Music therapy, which within its objectives has the use of various techniques to have cognitive and intellectual benefits, could be an alternative to stimulate attention. Therefore, the aim of this study was to describe the attentional functioning in two patients diagnosed with MPS IV-A and MPS VI evaluated before and after a stimulation program using music therapy elements. Participants were one female with acquired visual loss, 23 years old, diagnosed with Maroteaux-Lamy syndrome and one male 
diagnosed with Morquio-A syndrome, 16 years old with hearing loss. Both were borne in Colombia and treated with enzyme replacement therapy. A neuropsychological test-retest were conducted to evaluated sustained, verbal and visual attention, before and after 12 sessions of a stimulation program using music therapy elements included melodic and percussion instruments. Sessions were conducted for 45 minutes each, 3 times per week. For the Maroteaux-Lamy patient, sustained attention was evaluated with digit detection from the Brief Neuropsychological Evaluation (NEUROPSI) and verbal attention with digit span from WAIS-III. For the Morquio A patient, the digit progression and cancellation test from the Neuropsychological Assessment of Children (ENI) were applied to evaluate visual and auditory attention. The results show a normal-low performance for the age and schooling of both patients in the pretest. The posttest shows an improvement in sustained attention for the Maroteaux-Lamy syndrome patient and a maintenance of audio verbal and auditory attention in Morquio-A syndrome patient. These results suggest that it is worth exploring this type of stimulation protocols for these patients, especially taking in to account their sensorial difficulties and low school level. Even so it is necessary to increased population and intervention programs in the future to determine the relationship between stimulation programs, the maintenance of the improvement and the disease progression in these syndromes.

CORRESPONDING AUTHOR: D. Carolina Cárdenas-Poveda, Corporación Universitaria Minuto de Dios, dccardenasp@unal.edu.co

\section{P247}

PSYCHOMETRIC PROPERTIES OF THE CHILEAN VERSION OF THE REVISED FIBROMYALGIA IMPACT QUESTIONNAIRE (FIQR) IN A SAMPLE OF CHILEAN WOMEN WITH FIBROMYALGIA: PRELIMINARY RESULTS

Alejandra Escobar ${ }^{1}$, Camila Román-Butrón ${ }^{1}$, Alvaro Vergés ${ }^{1}$, Lydia Gómez ${ }^{1}$

${ }^{1}$ Pontificia Universidad Católica de Chile- Chile

Background: The Fibromyalgia Impact Questionnaire Revised (FIQ-R) is a widely used instrument to assess the impact of fibromyalgia (FM) on the daily lives of those who suffer from it. It comprises three subscales: function, overall impact, and symptom severity. It has been translated and validated in a large number of countries; however, its Chilean version remains to be validated. Objectives: To examine the psychometric properties of the Chilean version of the FIQ-R. Method: Women with fibromyalgia $(\mathrm{N}=94)$ attended in a clinic (Centro Médico San Joaquín, Red UC Christus in Santiago, Chile) completed a battery of questionnaires while they were waiting to be attended by their rheumatologists. The FIQ$\mathrm{R}$ was filled again two weeks later, during a telephone interview. Results: According to the results of confirmatory factor analyses, a bifactorial model with a general factor and three specific correlated subfactors (function, overall impact, and symptom severity) presented the best fit, $\mathrm{X} 2 / \mathrm{df}=$ 1.233, $\mathrm{p}=0,022$; RMSEA $=.05$ (CI 90\%: .02-.07); CFI $=.97$; and $\mathrm{TLI}=.96$. Factorial loadings were between .47 and .97 . Cronbach's alpha and Omega were .91 and .96 for the total FIQ-R score, .89 and .92 for function, 79 and .87 for overall impact, and .88 and .90 for symptoms severity. Regarding test-test reliability, ICC were $.90, .85, .56$, and .88 , for the FIQ-R total score and its subscale, respectively. Pearson correlation analyses showed that the FIQ-R total score presented high correlations with pain intensity $(\mathrm{r}=.61, \mathrm{p}<.001)$, somatic symptoms $(\mathrm{r}=.60, \mathrm{p}<.001)$, pain interference $(\mathrm{r}=.76 \mathrm{p}<.001)$, and depressive symptoms $(\mathrm{r}=.59$, $\mathrm{p}<.001)$, and moderate correlations with quality of life $(\mathrm{r}=.39, \mathrm{p}<.001)$ indicating convergent validity. Conclusions: The Chilean version of the FIQ-R is valid and reliable and can be used to assess fibromyalgia impact in Chilean women with fibromyalgia.

CORRESPONDING AUTHOR: Alejandra Escobar, Pontificia Universidad Católica de Chile, alescobarz87@gmail.com
P248

PSYCHOMETRICS PROPERTIES OF THE CHILEAN VERSION OF THE PAIN VIGILANCE AND AWARENESS QUESTIONNAIRE (PVAQ) IN WOMEN WITH FIBROMYALGIA

Camila Román-Butrón ${ }^{1}$, Alejandra Escobar ${ }^{1}$, Tomás Arriaza ${ }^{1}$, Álvaro Vergés ${ }^{1}$, Matías González ${ }^{2}$, Josefina Durán ${ }^{2}$, Lydia Gómez-Pérez ${ }^{1}$

${ }^{1}$ Pontificia Universidad Católica de Chile. Escuela de Psicología. Facultad de Ciencias Sociales- Chile

${ }^{2}$ Pontificia Universidad Católica de Chile. Escuela de Medicina- Chile

Background: Pain hypervigilance is usually increased among patients with chronic pain and it has been associated with poor pain related outcomes and higher disability. The Pain Vigilance and Awareness Questionnaire (PVAQ) has been widely employed to assess pain hypervigilance in patients with several pain disorders, including fibromyalgia. However, this scale has never been validated for its use in Chile. Objective: To examine the psychometric properties of the Chilean version of the PAVQ. Method: Women with fibromyalgia $(\mathrm{N}=119)$ attended in a clinic in Santiago (Centro Médico San Joaquín, Red UC Christus) completed the PVAQ while they were waiting to be seen by their rheumatologists and two weeks later during a telephone interview. Results: The fit of several structural models previously reported in the literature were compared using confirmatory analyses. Namely, a one factor model, a two-factor model (Esteve et al., 2013; Wing et al., 2011; Roelofs et al, 2002) and a three-factor model (McWilliams \& Asmundson, 2001) were compared. In addition, because the total PVAQ scores are usually calculated, we also tested several bifactorial models including a general factor representing the total score, and specific subfactors. The two-factor and three-factor unidimensional models did no present a good fit. A bifactorial model comprising a general factor (GF) and three specific correlated subfactors presented the best fit $(\chi 2 / \mathrm{gl}=1.63$; RMSEA $=$ 0.073; CFI 0.98 and TLI=0.97). The three specific factors were similar to those previously reported in the literature: Passive awareness (PA), Active vigilance (AV), and Intrusion (IN). The resulting questionnaire comprised 15 items, as item 16 was deleted because it presented low weights in all the factors. The general factor as well as three subscales showed a good internal consistency (GF $\alpha=.88$, PA $\alpha=.90, \mathrm{AV} \alpha=.82$ and IN $\alpha=.51)$. Test-retest reliability was excellent $(\mathrm{GF}$ ICC $=.94, \mathrm{CI}$ .91-.95; PA ICC $=.84$, CI .75-.89; AV ICC = .67, CI 48- .79; and IN ICC $=$ .84, CI .76-89). Conclusions: The Chilean version of the PAVQ present a structure similar to those found in previous studies as well as adequate internal consistence and test-retest reliability.

CORRESPONDING AUTHOR: Camila Román, Pontificia Universidad Católica de Chile, cbroman@uc.cl

\section{P250}

HIGHER-ORDER COGNITIVE FLEXIBILITY: INFLUENCES OF CATECHOLAMINES, TVNS AND STRESS

Klodiana-Daphne Tona ${ }^{1}$, Hans Revers ${ }^{2}$, Bart Verkuil ${ }^{3}$

${ }^{1}$ Clinical Psychology Unit, Leiden University- Netherlands

${ }^{2}$ Cognitive Psychology Unit, Institute of Psychology and Leiden Institute for Brain and Cognition, Leiden University- Netherlands

${ }^{3}$ Clinical Psychology Unit, Institute of Psychology and Leiden Institute for Brain and Cognition, Leiden University- Netherlands

INTRODUCTION Cognitive flexibility is an important function for successfully "juggling" between different responsibilities in important domains in our daily life. Previous literature shows that the catecholamines norepinephrine and dopamine are involved in cognitive flexibility in general, but their involvement in higher-order flexibility (indexed by taskswitching) in humans is unknown. This study investigates the involvement of catecholamines in higher-order cognitive flexibility by presenting two studies in which we manipulated catecholamine levels pharmacologically (Study 1), via transcutaneous vagus nerve stimulation (tVNS; 
Study 2a) and stress manipulation (Study 2b). METHODS Task: participants repeatedly switched between two cognitive tasks. On each trial, a cue indicated the task to be performed. The cue-stimulus interval was varied to manipulate the time to prepare for the switch. Study 1: 28 participants performed the task once after administration of atomoxetine (selective NE transporter blocker) and once after placebo administration (7 days apart). Study 2: 72 male participants were asked to perform the task during tVNS and during sham stimulation (7 days apart). To investigate the effect of tVNS intensity, 24 participants where tested at intensity of $0.5 \mathrm{~mA}$ and 24 participants at intensity of $1 \mathrm{~mA}$. To investigate the effect of stress, 24 participants were tested after stress was induced by means of the socially evaluated cold pressor task. Psychophysiological measurements: alpha-amylase, cortisol and mood were assessed throughout the experimental sessions. RESULTS Subjects showed typical switch costs, which decreased with the time available for preparation. Atomoxetine, tVNS, and stress-induction increased levels of salivary aamylase and cortisol but did not affect switch costs or the preparation effect. These findings suggest that catecholamines are not involved in higher-order cognitive flexibility.

CORRESPONDING AUTHOR: Klodiana-Daphne Tona, Clinical Psychology Unit, Leiden University, klodato@gmail.com

\section{$\mathrm{P} 251$}

CHARACTERISTICS OF INVOLUNTARY REPETITIVE RECALLS (FLASHBACKS) ON TRAUMATIC EVENT OF SEXUAL HARASSMENT OF WOMEN IN THE WAR

Slavica Kozina ${ }^{1}$

${ }^{1}$ Department of Psychological Medicine School of Medicine University of Split- Croatia

Objective of research was to evaluate quality and intensity of involuntary, intrusive autobiographical memories, and flashbacks in testimony of women sexual harassment in the war. Method: We analyzed testimonies of 58 women-victims of rape and sexual harassment. We applied noninterventionist strategy, encouraging free memory float to recall thoughts and emotions about their experiences. Testimony contains descriptions of observed phenomena, without any researcher intention to judge, which could affect validity of testimony. We analyzed quality and intensity of involuntary autobiographical memories, intrusive memories and flashbacks in testimony by Kvavilashvili (2014). We included rape circumstances if: a) women were raped once by one person, multiple times by multiple persons or by one person; b) the person was isolated or not; c) there were witnesses of the rape; and d) the rapist was known or unknown to the victim. Results: Womenvictims of rape and sexual harassment that were raped once by multiple persons have significantly more often: flashbacks $(9 / 20,45 \%$, $\mathrm{P}=0.011$ ), experiencing repetition of intrusive autobiographical memories of traumatic event $(6 / 20,30 \%, \mathrm{P}=0.041)$, spontaneous retrieval of flashbacks $(9 / 20,45 \%, \mathrm{P}=0.011)$, highest frequency of flashbacks repetition $(6 / 20,30 \%, \mathrm{P}=0.056)$, often evaluate events emotionally negative $(9 / 20,45 \%, \mathrm{P}=0.011)$, vivid memories $(9 / 20,45 \%, \mathrm{P}=0.011)$, actively avoiding memories $(9 / 20,45 \%, \mathrm{P}=0.011)$, more often reexperiencing event $(9 / 20,45 \%, \mathrm{P}=0.011)$. Frequency and significance of disruption of current activity by flashbacks in these victims is statistically borderline significant ( $\mathrm{P}=0.050)$. When there were witnesses of the rape event are present, flashbacks frequency is higher $(11 / 32,34.4 \%, P=0.048)$. Conclusions: Incidence and intensity of involuntary intrusive recalls are especially highlighted in victims who were raped once by multiple persons and in those who were raped in presence of a witness. Unlike that, knowing the rapist and isolation during harassment does not affect incidence and quality of flashbacks. Clinicians need to be aware of and attempt to avoid assigning degrees of traumatization (PTSD or not) for women-victims with different expressions of traumatic experiences of the rape.
CORRESPONDING AUTHOR: Slavica Kozina, Department of Psychological Medicine School of Medicine University of Split, slavica.kozina@mefst.hr

\section{$\mathrm{P} 252$}

THE BRIDGE AND COMMUNICATION OF COMMUNITY MENTAL HEALTH SERVICES FOR GERIATRIC

Qian Yang ${ }^{1}$, Wenqi Weng ${ }^{2}$, Shulin Chen ${ }^{2}$

${ }^{1}$ The Department of Public Health of Zhejiang university

${ }^{2}$ The Department of Psychology and Behavioral Sciences- Chin

The mental health Gap which proposed by WHO pointed out that although every community and age group are troubled with mental health problems across all income countries, there is a GAP that most of the people affected $-75 \%$ in many low-income countries - do not have access to the treatment they need. China has entered the aging society gradually, and its aged population will reach 400 million by 2035 . As a typical New Normal society, it is a key issue for China to build a bridge between the mental health needs and services of age group in community. Take the $\mathrm{COACH}$ model funded by $\mathrm{NIH}$ as a case, we conducted two focus group interviews which contained 8 villages leaders and 8 community directors in Zhejiang Province. We also tested main leaders of 20 rural research points and 21 urban communities study sites by survey of model continue and dissemination after the project coordinator (i.e. the researcher group) has left. The results summarized the experience and barriers of building the bridge in Chinese rural and urban. And it has pointed out that the bridge can be built and retain successfully when the project coordinator has left only if the area has enough motivation, project support, human resource, information and leaders' empowerment.

CORRESPONDING AUTHOR: Qian Yang, The Department of Public Health of Zhejiang university, chianyoung@zju.edu.cn

\section{P253}

FAMILY SOCIAL COHESION AND HEALTH RELATED QUALITY OF LIFE IN ADOLESCENTS: A LONGITUDINAL ANALYSIS

Ester Villalonga $^{1}$, Tracy Richmond ${ }^{2}$, Ichiro Kawachi ${ }^{3}$

${ }^{1}$ Pharmaceutical Health Services Research Department. University of Maryland- USA

${ }^{2}$ Boston Children's Hospital- USA

${ }^{3}$ Harvard T.H. Chan School of Public Health- US

Introduction: Though studies have demonstrated a relationship between social capital and Health Related Quality of Life (HRQoL) in adults, studies in children, particularly longitudinal studies, are lacking. Our objective was to examine the association of one indicator of social capital, family social cohesion, and changes in HRQoL across 5 years of early to mid-adolescence. Methodology: We used data from the Healthy Passages study, a prospective cohort of 5250 5th grade students followed over 3 waves of data collection into grade 7 and then 10 . We used principal component analysis to create a family cohesion score based on a validated 10-item family cohesion scale (e.g., family members ask each other for help, family togetherness is very important). Longitudinal mixed-effects linear regression models were constructed to study the relationship between family social cohesion and overall HRQoL as well as physical and psychosocial health subdomains of HRQoL adjusting for age in 5th grade, wave, gender, race/ethnicity, highest parental education and household income category. We estimated the association of predictors with the three outcomes over time. A random intercept for individual was included to account for multiple measurements of the outcomes across waves. Results: Overall HRQoL improved from Wave 1 to 2 and worsened from Wave 2 to 3 . Family social cohesion was positively associated with overall changes in HRQoL $(0.70$ (SE $0.15, \mathrm{p}=0.01)$ ); similar patterns were observed in the subdomains for physical health $(0.55$ (SE $0.17 \mathrm{p}=0.01))$ 
and psychological health $(0.70$ (SE $0.18 \mathrm{p}=0.01)$ ). Demographic factors were also associated with changes in HRQoL: being male and highest parental education and household income were both positively associated with changes in overall HRQoL. African-Americans had greater improvement in HRQoL from Wave 1 to 3 than Whites (0.14 (SE 0.47, $\mathrm{p}=0.03$ )), whereas Hispanics had lower improvement in HRQoL than Whites $(-0.96$ (SE $0.49, \mathrm{p}=0.03)$ ). Discussion: Family cohesion has a protective association on changes in HRQoL from early to mid-adolescence. We contribute to the literature that supports interventions on family cohesion.

CORRESPONDING AUTHOR: Ester Villalonga, Pharmaceutical Health Services Research Department. University of Maryland, ester.villalonga@rx.umaryland.edu

P254

DIET: PERCEPTIONS AND BEHAVIORS, AND THE ASSOCIATION WITH WELL-BEING

Sandra Winter ${ }^{1}$, Haley Hedlin ${ }^{1}$, Vidhya Balasubramanian ${ }^{1}$, Catherine Heaney ${ }^{1}$

${ }^{1}$ Stanford University- USA

Introduction: There is evidence that the perceptions people have about their health behaviors are associated with their health outcomes, even after taking into account the actual health behaviors and other determinants such as health status and sociodemographic variables. This study examined the association of self-reported diet with well-being, and the extent to which this association was mediated by participants' perceptions of the influence of their diet on their well-being. Method: Participants $(n=597$, mean age $=45.1 \pm 17.3$, women $=73 \%$, years of education $=16.0$ \pm 3.9 , marital status-married $=45 \%$, never married $=32 \%$, other $=23 \%$ ) completed the Stanford WELL for Life on-line survey that included questions about 10 domains of well-being: social connectedness, lifestyle behaviors (including diet, physical activity, sleep, and tobacco and alcohol use), physical and emotional health, stress/resilience, purpose and meaning, sense of self, finances, spirituality/religiosity, and creativity. For each domain, a score from 0-10 was created. Within the lifestyle domain, a sub-domain score from 0-10 was created for each lifestyle behavior. An overall well-being score was created by summing the 10 domain scores. Participants were also asked if their current health behaviors influenced their well-being. Linear regression analyses were conducted, controlling for age, gender, education and marital status. Results: A $10 \%$ improvement in the diet domain score was associated with a $2.4 \%$ increase in the overall well-being score $(95 \%$ CI 1.78, 2.97). When the perceived influence of diet was added to the model, this association was attenuated but still statistically significant - the increase in well-being decreased from $2.4 \%$ to $1.8 \%$, with most of the explanation due to people who perceived their diets as harmful to their well-being. Conclusion: On average, participants in this study who reported healthier diet behaviors also reported higher levels of well-being. This association was partially mediated by participants' reports of the influence of diet on their wellbeing. However, a sizable portion of each of the associations was independent of participants' awareness. Future Directions: The methodology adopted in this study is being replicated in China, Taiwan and Singapore, providing future opportunities for cross cultural comparisons of the associations between actual and perceived diet and well-being.

CORRESPONDING AUTHOR: Sandra Winter, Stanford University, sjwinter@stanford.edu

\section{P255}

HEALTH HABITS QUESTIONNAIRE IN UNIVERSITY STUDENTS: PSYCHOMETRIC EVIDENCE

Monica Cassaretto ${ }^{1}$, Patty Vilela ${ }^{1}$, Juan Carlos Saravia ${ }^{1}$

${ }^{1}$ Pontificia Universidad Católica del Perú - Perú
One of the most used instruments in research with university students is the Lifestyles Questionnaire for Young University Students Part I (CEVJU I), an instrument developed in Colombia by Arrivillaga, Salazar \& Gómez (2002). This instrument has 69 items and has been used in Peru on several occasions but has shown difficulties in terms of its psychometric properties. Because of this, Chau and Saravia (2016) suggested that the instrument have a better functioning with changes in phrasing and response format, and the reduction of the number of items to a final number of 30 . For this reason, a new instrument is proposed, renamed as Health Habits Questionnaire in University Students (CHSU). This questionnaire has its main focus on lifestyles behavioral aspects evaluation, without the assessment of the motivational and cognitive aspects that could involve the concept of lifestyle. An analysis of the reliability and validity evidence of the CHSU in a Peruvian students sample is proposed. The study participants were 980 students from 5 universities in Peru. With ages between 16 and 30 years old, $62 \%$ of them were women, $60 \%$ of the students came from private universities. The Questionnaire was applied to the whole sample, which is a 30-item instrument, with a response format of 4 options from Never to Always. It assesses 6 areas, which represents key health practices and habits: physical exercise and sport; rest organization and leisure time; self-care and medical care; eating habits; drug consumption control; \& sleep organization. A factor analysis of the main components was performed in order to elucidate the structure of the scale, this analysis shows the existence of 6 factors that coincide with the suggested structure (Test $\mathrm{KMO}=0.80$, Bartlett's sphericity test $\left.\chi^{2}(435)=6611.05 ; \mathrm{p}<0.00\right)$ and explain $49.12 \%$ of the questionnaire's total explained variance. All items exhibit adequate levels of factorial loading. In terms of reliability, Cronbach's alpha indexes of 0.61 to 0.79 for the 6 areas were found, all of the items show item-test correlations higher than 0.30 . The CHSU is presented as an instrument with enough evidence of validity and reliability to be used in research with university population.

CORRESPONDING AUTHOR: Monica Cassaretto, Pontificia Universidad Católica del Perú, mcassar@pucp.edu.pe

\section{P256}

AN EVIDENCE-BASED APPROACH TO IMPLEMENTING THE PATIENT REPORTED OUTCOMES MEASUREMENT INFORMATION SYSTEM IN A BOLIVIAN PUBLIC CANCER CENTER

Steven J Schuetz ${ }^{1}$, Rina S. Fox ${ }^{1}$, Claudia B. Soliz P. ${ }^{2}$, Maribel Marmol C. ${ }^{3}$, Edgar F. Apaza O. ${ }^{2}$, Mayra V. Alaca M. ${ }^{2}$, Frank J. Penedo ${ }^{1}$, David Cella ${ }^{1}$, Richard C. Gershon ${ }^{1}$, PROMIS®-Bolivia Collaborative ${ }^{2}$

${ }^{1}$ Department of Medical Social Sciences, Northwestern University Feinberg School of Medicine-USA

${ }^{2}$ PROMIS®-Bolivia Organization-Bolivia

${ }^{3}$ Instituto Chuquisaqueño de Oncología-Bolivia

Introduction \& Purpose: Evaluating patient-reported symptom experiences enhances health outcomes by promoting individualized care, improving patient-provider communication, and facilitating shared decision-making. The Patient-Reported Outcomes Measurement Information System (PROMIS $®$ ) is a comprehensive set of tools to measure self-reported physical, mental, and social health in people ages 5-90. While PROMIS $®$ is available in over 40 languages and has been widely used in high-income countries, it has not been implemented in Latin America despite the availability of high-quality Spanish-language translations. The purpose of the present study was to implement PROMIS ${ }^{\circledR}$ symptom screening in a multicultural, low-resource public cancer center in Bolivia, the Instituto Chuquisaqueño de Oncología (ICO). Methods: An implementation strategy was developed by incorporating the Expert Recommendations for Implementing Change into the EPIS phases of implementation (Exploration, Preparation, Implementation, Sustainment). A multidisciplinary Implementation Coalition was 
developed, consisting of ICO leadership and medical providers, patient advocates, and international PROMIS ${ }^{\circledR}$ and implementation science experts. This coalition developed an initial implementation protocol based on the International Society for Quality of Life Research standards, with local contextualization based on an analysis of implementation barriers and facilitators using the Consolidated Framework for Implementation Research (CFIR) domains. Results: Pre-implementation site assessment demonstrated that ICO would be an appropriate center for the initial PROMIS ${ }^{\circledR}$ pilot study. The protocol was iteratively refined before and during implementation by the Implementation Coalition. Adjunctive data collection tools were developed and integrated into ICO's medical records to enhance assessment of patient language preferences, health literacy skills, and pertinent sociodemographic information.

Conclusions: Multiple frameworks exist to facilitate evidence-based and sustainable implementation of patient-reported outcome assessment using PROMIS ${ }^{\circledR}$ in diverse settings. Future studies can use these frameworks to successfully assess patient-reported outcomes in low-resource health systems on national and international levels.

CORRESPONDING AUTHOR: Steven J Schuetz, Department of Surgery, Department of Medical Social Sciences, Northwestern University Feinberg School of Medicine, s-schuetz@ northwestern.edu

\section{INVITED SYMPOSIUM}

\section{S01}

GLOBAL APPROACHES TO INCREASING THE UPTAKE OF THE HPV VACCINE: EXAMPLES FROM FOUR COUNTRIES

Sheinfeld Gorin ${ }^{1}$

${ }^{1}$ NYPAC, NCI- USA

High risk types of HPV are necessary though not sufficient causes of the vast majority of cervical cancers. Cervical cancer is the fourth most common cancer of women worldwide. In addition, HPV is responsible for more than $90 \%$ of anal cancers, about $70 \%$ of vaginal and vulvar cancers, and more than $60 \%$ of penile cancers. About $70 \%$ of cancers of the oropharynx may be linked to HPV. While vaccines against HPV are on the national schedule in 82 countries, full coverage has been slow and uneven. According to a recent pooled analysis, globally, 47 million women have received a full course of HPV vaccine, with country-specific coverage rates ranging from less than $5 \%$ to more than $80 \%$. The most vulnerable populations, which would benefit most from vaccination, may still remain unprotected. Australia, Chile, and the US have national immunization programs; in Japan, vaccination uptake in adolescent girls plummeted after government withdrawal of proactive recommendations for the vaccine due to unconfirmed reports of adverse events in the media. Australia has among the highest HPV vaccine coverage in the world; in 2016, the 3-dose, school-based vaccination program immunized $78.6 \%$ of eligible girls and $72.9 \%$ of eligible boys. In the US, with a 2-dose, provider-based program for adolescents aged 13-17, uptake is $60 \%$. Through the newer Chilean national program (2-dose, grade 4, schoolbased), in 2017 , country-wide coverage ranged from $70.0 \%$ to $91.3 \%$. Only Australia has an HPV vaccine surveillance registry that can systematically track HPV vaccinations and assess their impact on HPV infections, cervical lesions and cancers. Each of these three countries has a different HPV vaccination policy, delivery system, and vaccination protocol. Aims. The aims of this symposium are to: (1) describe the predictors of HPV vaccination and, where collected, rates of HPV vaccination (initiation and completion), with particular attention to variations by socioeconomic and racial/ethnic subgroups. (2) examine the multi-level (policy, media, community, medical practice, family and child) supports and barriers to national programs; (3) discuss multi-level approaches to increasing HPV vaccine uptake to reduce cervical, anogenital and oropharyngeal cancers worldwide.
S01.1

TRANSLATING KNOWLEDGE ON HPV VACCINE TO THE REAL LIFE SITUATION IN CHILE: FIRST 4-YEARS RESULTS

Catterina Ferreccio ${ }^{1}$

${ }^{1}$ Pontificia Universidad Católica de Chile- Chile

Introduction and Purpose: To secure a successful vaccination Program, the vaccination strategy has to be practical, acceptable for providers and the public. Achieving adequate coverage with the standard regimen of 3 doses over six months is difficult and costly. In 2012 we proposed an alternative strategy for a HPV vaccination program to the Ministry of Health $(\mathrm{MoH})$ of Chile. We will present its rationale, 2014-18 coverage, and the MoH's communication approach to improve coverage and to respond to anti-vaccines groups. Method. Based on the scientific evidence for HPV vaccines immunogenicity and the efficacy of different number of doses, dosing intervals, and expected health benefits, we proposed a practical, cost-saving and acceptable, yet efficacious, HPV vaccination schedule. Results. We gathered evidence that: 2 or 3 doses in young women were efficacious; larger dose intervals improved immunogenicity; there were higher cancer prevention benefits for girls: $92.4 \%$ than boys: $7.6 \%$ (based on Chile's incidence rates from 2003-07). At younger ages, there is higher immunogenicity; school vaccination programs reached higher coverage than private; bi-valent or quadrivalent vaccines were similarly efficacious. Concordantly, we proposed a school vaccine program, for girls 9-12 years, 2-dose every 12 months; with a catch-up for girls in 13-16 years. Early in the process, anti-vaccines and anti-sexually-transmitted vaccine's groups commenced social-network propaganda; these were effectively neutralized by the $\mathrm{MoH}$ ("Juntos por ella" campaign) and the scientific community. As a result, $30 \%$ of refusals reverted; anti-vaccine groups had little impact in coverage; mainly in counties they controlled, where they required parents' signed consent. Coverage (2-D) has increased yearly reaching $87 \%$ in 2017 (countrywide range $70.0 \%$ to $91.3 \%$ ). Chile has the lowest reported rate of events associated to the VPH vaccination. Conclusions. The lower cost of a 2dose regimen, the logistic benefits of a yearly schedule and a rapid and efficacious response from the $\mathrm{MoH}$ and the national scientific community greatly contributed to achieving high coverage and acceptability of the Program.

CORRESPONDING AUTHOR: Catterina Ferreccio, Pontificia Universidad Católica de Chile, cferrec@med.puc.cl

S01.2

WHO AND WHAT ARE THE ENEMIES OF THE JAPANESE HPV VACCINATION PROGRAMME?

Sharon JB Hanley ${ }^{1}$

${ }^{1}$ Department of Women's Health Medicine, Hokkaido Graduate School of Medicine- Japan

Introduction and Purpose. The bivalent HPV vaccine was licenced in Japan in October 2009 and the quadrivalent vaccine in July 2011. From December 2010, special funding was provided for girls aged 12-16 years whereby the national government paid $50 \%$ of the vaccine cost, if local governments also paid $50 \%$. Due to unexpectedly high demand after public funding was introduced, vaccine stock decreased drastically nationwide and many girls who wanted the vaccine could not initiate vaccination. During this period, the media fiercely criticized the government for not stocking enough of the cancer preventing vaccine. From February 2013, after it became clear the government was to include the HPV vaccine in the national immunization programme (NIP), unconfirmed reports of adverse events after immunization (AEFI) began to appear in the media and a 'Victims' Support Group was established. The HPV vaccine was introduced into the NIP in April 2013 and just two months later, on June 14th, 2013, proactive recommendations for the HPV vaccines were suspended due to the unconfirmed media reports of AEFI. 
Very quickly uptake dropped from $>70 \%$ to $<1 \% 1$, and four and a half years later, this suspension remains, despite no evidence to suggest the vaccine is responsible for the reported symptoms. This presentation will elucidate the complex social and scientific reasons why proactive recommendations for the HPV vaccine have not recommenced in Japan. Methods. Data from recent epidemiological and government studies as well as media activities will be presented. Results and Conclusions. Poor risk communication and risk management, a public health infrastructure that makes it difficult to convince the public that the vaccine is both effective and safe, and an anti-vaccine movement more organized than the government and supported by the media, has made it difficult for the Japanese government to restart proactive recommendations the HPV vaccine.

CORRESPONDING AUTHOR: Sharon JB Hanley, Department of Women's Health Medicine, Hokkaido Graduate School of Medicine, sjbh1810@mta.biglobe.ne.jp

\section{S01.3}

HPV VACCINATION IN AUSTRALIA: IS HPV ELIMINATION POSSIBLE?

Julia Brotherton ${ }^{1}$

${ }^{1}$ Registries and Research, Victorian Cytology Service Registries ${ }^{-}$ Australia.

Introduction and Purpose: Australia is in its 12th year of HPV vaccination. Prior to 2018, the program used 3 dose quadrivalent HPV vaccine, with male vaccination commencing in 2013. In 2018, the program is using two dose nonavalent vaccine in the routine school based program. This presentation will assess whether Australia's coverage, barriers to higher coverage, and program impacts so far, are consistent with eventually achieving elimination of targeted HPV infection and disease in Australia. Methods: The National HPV Vaccination Program Register enables monitoring of vaccination coverage and supports measures of vaccine impact by making vaccination history available when studying outcome data. Results: This presentation will review the uptake of dose one of nonavalent HPV vaccine in Australia (due to commence delivery from February 2018) and compare it with previous coverage trends for males and females and by age for quadrivalent HPV vaccine, including for disadvantaged groups. We will review recent research updating progress in HPV and disease reduction in Australia, and exploring remaining barriers to higher uptake, in the context of emerging models that suggest that the eventual elimination of vaccine targeted HPV is possible. Conclusion: School-based vaccine delivery provides high and equitable coverage. Australia's multi-cohort catch up program has brought forward vaccine benefits considerably in the population and we may be one of the first countries to eventually achieve elimination of vaccine targeted types and cervical cancer.

CORRESPONDING AUTHOR: Julia Brotherton, Registries and Research, Victorian Cytology Service Registries, jbrother@vcs.org.au

\section{S01.4.}

HPV VACCINATION IN AN OPPORTUNISTIC SYSTEM: LESSONS FROM THE UNITED STATES

Rebecca Perkins ${ }^{1}$, Terresa Eun ${ }^{1}$, Anny Fenton ${ }^{1}$, Emma Trucks ${ }^{1}$, Emily Jansen $^{2}$, Nina Batista ${ }^{2}$, Anna Hassan ${ }^{2}$, Sandy Preiss ${ }^{2}$

${ }^{1}$ Boston University School of Medicine/Boston Medical Center- USA

${ }^{2}$ Boston University of Medicine/Boston Medical Center- USA

Introduction \& Purpose. In the US, HPV vaccination is part of the recommended national vaccination program, and is generally available for low or no cost, but provision is largely dependent on interactions between clinicians and patients. Vaccination is opportunistic, and provided primarily in medical settings. Vaccination is not typically provided in schools, and is mandatory for school entry without exemptions in only 1 of the 50+ US states and territories. Early research indicated that provider recommendation was the most important predictor of HPV vaccine utilization. We therefore conducted provider-focused education and training programs in 14 healthcare systems in diverse geographic locations to determine their impact on HPV vaccination rates. Methods. Providers and office staff participated in a structured program with four key components: (1) data collection and feedback, (2) education on HPV-related cancers, HPV vaccine effectiveness and safety, and recommended communication techniques, (3) quality improvement training and/or support, and (4) whole-staff involvement in choosing and implementing a clinicwide action plan for improving HPV vaccination rates. Programs ran over 6-9 months. Physicians received mandatory credits for maintaining specialty licensure for participating in the program. Results. Individualized data collection allowed identification of clinic-specific gaps in vaccine provision. Several sites noted that their HPV vaccine series initiation rates were more than $20 \%$ higher than their series completion rates. Further investigation revealed that this was due in large part to the highly mobile nature of patients seen in publicly-funded healthcare settings in the US. These sites chose to begin vaccination at ages 9-10 instead of ages 11-12. Implementation of vaccination at younger ages was highly successful, with $60-80 \%$ of eligible patients initiating the vaccine series. As dosing is recommended annually, we anticipate seeing increases in completion rates among 10-12 year olds in mid-2018. Additional strategies implemented included reminder/recall systems, involvement of clinic leadership, and creation of clinic scorecards with HPV vaccination rates. Conclusions. In the absence of organized national programs, intensive efforts with healthcare systems can achieve improvements in HPV vaccination rates.

CORRESPONDING AUTHOR: Rebecca Perkins, Boston University School of Medicine/Boston Medical Center, rbperkin@bu.edu

\section{S02.}

DEVELOPING AND IMPLEMENTING PSYCHOSOCIAL ONCOLOGY IN DIVERSE PATIENTS

Betina Yanez ${ }^{1}$

${ }^{1}$ Northwestern University Feinberg School of Medicine- USA

Psychosocial research in oncology is crucial for the development and implementation of effective interventions that improve health-related quality of life (HRQoL) among diverse cancer patients and survivors. As an example, greater attention to the unique needs among the rapidly increasing Hispanic/Latino population in the U.S. is critical in order to develop culturally-sensitive interventions and community-based approaches that improve outcomes among this underserved population. Innovative methods for delivering interventions through technology communications, such as Smartphones and web-based platforms, are becoming increasingly common but more research is needed to establish their feasibility among underserved patients and scalability outside of the research context. In this symposium, the speakers will present findings on four studies in topics that range from cancer prevention and screening to survivorship and span four common cancer disease sites: breast, colorectal, prostate, and cervical. The first speaker will present data from the U.S. Hispanic Community Health Study/Study of Latinos (HCHS/SOL) Study $(\mathrm{N}=5,313)$ examining sociocultural and individual determinants of adherence to breast, colorectal, cervical, and prostate cancer screening recommendations. The second speaker will present findings from an e-health study $(\mathrm{N}=111)$ to reduce symptom burden and improve HRQoL among U.S. Hispanic/Latina breast cancer survivors. The third speaker will present findings from a study that assessed patient eligibility and interest in enrolling in an evidence-based randomized clinical trial for colorectal cancer survivors in the Netherlands. The final speaker will provide findings from a study that evaluated the implementation of an evidence-based 
website to support re-entry adjustment after completing breast cancer treatment $(\mathrm{N}=254)$. Finally, the discussant will reflect on some of the strengths of psychosocial interventions in oncology as well as lessons learned from these studies and future directions.

S02.1

DISTRESS SCREENING IN COLORECTAL CANCER SURVIVORS FOR A RANDOMIZED CONTROLLED TRIAL TO TEST THE EFFECTIVENESS OF BLENDED COGNITIVE BEHAVIOR THERAPY TO REDUCE DISTRESS

Judith Prins ${ }^{1}$, Sarah Döking ${ }^{1}$, Belinda Thewes ${ }^{2}$, Annemarie Braamse ${ }^{3}$, Hans de Wilt ${ }^{4}$, Joost Dekker ${ }^{5}$

${ }^{1}$ Radboud University Medical Centre- Netherlands

${ }^{2}$ The University of Sydney- Australia

${ }^{3}$ University of Amsterdam- Amsterdam

${ }^{4}$ Radboud University Medical Centre- Netherlands

${ }^{5}$ Free University Of Amsterdam- Amsterdam

Introduction \& Purpose: Approximately one third of colorectal cancer survivors (CRCS) experience high levels of psychological distress, even 5 years after medical treatment. Distress in CRCS is related to anxiety, dealing with physical consequences after treatment, fear of cancer recurrence (FCR) and depressive symptoms. Research on psychological interventions for CRCS is scarce. To meet the need of an evidence based intervention, the CORRECT blended therapy was developed and will be tested in a randomized controlled trial (RCT). Eligible CRCS can be included in the RCT if they have high distress and a need for help. The aim of this study is to investigate the eligibility of CRCS, patient recruitment and patient inclusion for this trial. Methods: Currently, the CORRECT blended therapy and care as usual are studied in a two-arm multicenter randomized controlled trial (RCT), registered in the Netherlands Trial Register (NTR6025). From 2016 to 2019 CRCS will be recruited up to 5 years after surgery retrospectively via hospital registries and prospectively during medical follow-up. The Distress Thermometer (DT) is used for distress screening. A sample of 160 CRCS with a DT score of 5 or higher needs to be recruited. Results: In January 2018, after one year, 405 patients in two academic hospitals and three local hospitals fulfilled the medical inclusion criteria and received written study information of their physicians. 215 CRCS (53\%) responded, 65 CRCS (16\%) showed interest to participate and together with 11 prospectively recruited patients, $75 \mathrm{CRCS}$ filled in the DT. Of the total 416 CRCS only 46 screened patients $(10,8 \%)$ scored high on distress. 18 patients (39\%) had a need for help and were included. Thus, $24 \%$ of the screened patients and $4 \%$ of the eligible CRCS were included in the RCT. Conclusions: This intervention study has a low inclusion rate of $4 \%$ of eligible CRCS. The percentage of CRCS experiencing high distress was lower than expected based on findings in the literature. In our sample of CRCS up to 5 year after surgery only $10,8 \%$ had high distress. More than half of the highly distressed patients had no need for help or already received psychological help.

CORRESPONDING AUTHOR: Judith Prins, Radboud University Medical Centre, judith.prins@radboudumc.nl

\section{S02.2}

DATA-MINING TECHNIQUES TO EVALUATE USER ENGAGEMENT OF A SELF-HELP WEBSITE FOR EARLY BREAST CANCER SURVIVORS IN REAL-WORLD IMPLEMENTATION

Judith Prins ${ }^{1}$, Sanne van den Berg ${ }^{2}$, Kaz Roomer ${ }^{3}$, Maurtis Henneke ${ }^{4}$, Marie Postma ${ }^{3}$

${ }^{1}$ Radboud University Medical Centre- Netherlands

${ }^{2}$ Karify- Netherlands

${ }^{3}$ Tilburg University- Netherlands

${ }^{4}$ Ippz.- Netherlands
Introduction Implementation of evidence-based online interventions into real-world settings comes with inevitable changes and barriers. Log-data analysis enables continuous evaluation outside research context. This study aims to evaluate user engagement and patterns of use of a selfhelp website supporting re-entry adjustment after breast cancer. Methods An evidence-based self-guided low-intensity cognitive behavioral intervention was found to reduce distress in early breast cancer survivors1. Next step was the implementation of this intervention. Baseline user characteristics were collected for a consecutive sample of real-world users, who paid $€ 20$ (\$23) and registered between October 2014 and November 2015. Data of the real-world users were compared to those of the RCT patients1. Technical data of online behavior included: log-data (timestamps for responses and views), response data (whether or not a user filled in something), quantitative (self-tests, multiple choice format) and qualitative data (text/open question format). Using datamining and text-mining techniques, exploratory data analyses were performed. Results 254 of 390 registered users were included in the final dataset. Mean age was 50.8 (range 26-89) and similar to the RCT patients. Mean time since breast cancer treatment was 10 months (range 0-120 months) and larger than in the RCT patients (range 2-4 months). All women were found to be highly distressed on the Hospital Anxiety and Depression Scale (HADS-T>11) compared to 32\% in the RCT patients. Patients with high and low user engagement did not differ on distress, demographic or clinical variables. $29 \%$ of paid users completed all four program phases, compared to $44 \%$ in the RCT. Discussion The results showed that in real world implementation users and usage differed from the original RCT. 1Van den Berg SW, Gielissen MFM, van der Graaf WTA, Custers J, Ottevanger PB, Prins JB. BREATH an internet-based self-help program for emotional adjustment after primary breast cancer: results of a multicenter randomized controlled trial. Journal of Clinical Oncology 2015, Sep 1;33(25):2763-71.

CORRESPONDING AUTHOR: Judith Prins, Radboud University Medical Centre, judith.prins@radboudumc.nl

\section{S02.3}

CANCER PREVENTION SELF-EFFICACY AND ADHERENCE TO NATIONAL CANCER SCREENING GUIDELINES: RESULTS FROM THE HISPANIC COMMUNITY HEALTH STUDY/STUDY OF LATINOS (HCHS/SOL)

Patricia Moreno ${ }^{12}$, Betina Yanez ${ }^{1}$, Steven J. Schuetz ${ }^{1}$, Katy Wortman ${ }^{1}$, Linda C. Gallo ${ }^{3}$, Catherine Benedict ${ }^{4}$, Carrie E. Brintz ${ }^{5}$, Jianwen $\mathrm{Cai}^{5}$

${ }^{1}$ Northwestern University. - USA

${ }^{2}$ Feinberg School Of Medicine- USA

${ }^{3}$ San Diego State University/University Of California, San Diego- USA

${ }^{4}$ Hofstra Northwell School Of Medicine- USA

${ }^{5}$ University Of North Carolina At Chapel Hill- USA

Introduction: Sociocultural factors, such as health insurance status, income, education, and acculturation, have been shown to predict cancer screening among U.S. Hispanics/Latinos. However, these factors can be difficult to modify. Therefore, more research is needed to identify individual-level modifiable factors that can be targeted to improve screening behaviors and subsequent cancer outcomes in this population. Purpose: The primary aim of this study was to examine cancer prevention self-efficacy (i.e., confidence in one's ability to lower his/her chances of developing cancer) as a determinant of adherence to national screening guidelines for colorectal, breast, prostate, and cervical cancer among Hispanics/Latinos, while adjusting for well-established determinants of cancer screening. Methods: Participants were from the multi-site Hispanic Community Health Study/Study of Latinos (HCHS/SOL) Sociocultural Ancillary Study $(\mathrm{N}=5,313)$. The National Cancer Institute (NCI) Health Interview National Trends Survey was used to assess cancer prevention self-efficacy and receipt of cancer screening. Adherence was defined as receipt of screening within recommended time 
frames according to guidelines from the United States Preventive Services Task Force and the American Cancer Society that were in effect during the study period. Results: Adjusting for age, health insurance status, income, education, acculturation, Hispanic/Latino background, and study center, greater cancer prevention self-efficacy was marginally associated with greater adherence to screening for colorectal (OR 1.13, $95 \%$ CI [.99 - 1.30], $\mathrm{p}=.07)$, breast (OR 1.16, 95\% CI [.99 - 1.36], $\mathrm{p}=$ .07 ) and prostate cancer (OR 1.18, 95\% CI [.97 - 1.43], $\mathrm{p}=.10$ ), but not cervical cancer $(p>.10)$. Conclusions: Findings suggest that cancer prevention self-efficacy may be a viable target for interventions aimed at improving screening adherence for the three most common cancers among Hispanics/Latinos. Nevertheless, the effects of cancer prevention self-efficacy were small and marginal, underlining that sociocultural factors like health insurance status, income, and acculturation are more robust determinants of cancer screening adherence among Hispanics/ Latinos.

CORRESPONDING AUTHOR: Patricia Moreno, Northwestern University Feinberg School of Medicine, patricia.moreno@northwestern.edu

\section{S02.4}

THE MY GUIDE INTERVENTION FOR HISPANIC BREAST CANCER SURVIVORS

Betina Yanez ${ }^{1}$, Diana Buitrago ${ }^{1}$, Joanna Buscemi ${ }^{2}$, Judy Guitleman ${ }^{3}$, Alejandra Perez-Tamayo ${ }^{4}$, Frank Penedo ${ }^{1}$

${ }^{1}$ Northwestern University Feinberg School of Medicine- USA

2 DePaul University- USA

${ }^{3}$ Latina Association for Breast Cancer- USA

${ }^{4}$ University of Illinois at Chicago- USA

Breast cancer is the most commonly diagnosed type of cancer among Hispanic women living in the U.S. Relative to non-Hispanic white women, Hispanic women report poorer quality of life (QOL) after treatment. This study describes the development and preliminary testing My Guide, a smartphone-based intervention to improve QOL and reduce symptom burden among Hispanic breast cancer survivors. METHODS: My Guide was developed over several phases. First, we conducted semi-structured field interviews with English or Spanish-speaking Hispanic cancer survivors $(n=9)$ and coded the interviews for thematic content. Next, our study team conducted preliminary usability testing on participants and community stakeholders $(n=9)$ to further refine the design of My Guide. Next, we enrolled Hispanic breast cancer survivors $(n=25)$ in a 4-week field trial. In the final phase of My Guide, Hispanic breast cancer survivors $(n=80)$ are currently being enrolled in an 8-week randomized controlled trial. RESULTS: The prevalent themes in the semi-field interviews were fear of recurrence, patient-provider communication and social support, managing the side effects of cancer treatment, psychological well-being, and lack of breast cancer knowledge. In the field trial, the study recruitment rate was $75.8 \%$ and the study retention rate was $91.6 \%$. The mean number of hours spent using the application was 9.25 hours. Mean score on the satisfaction survey was 65.91 (range 42-70), in which higher scores reflect greater satisfaction. Participants' scores on the Breast Cancer Knowledge Questionnaire significantly improved from study baseline $(\mathrm{M}=9.5, \mathrm{SD}=$ $2.92)$ to the post-intervention assessment $(\mathrm{M}=11.14, \mathrm{SD}=2.66), \mathrm{d}=.59$. Participants' QOL scores improved throughout the 4-week field trial, but these improvements were not statistically significant. In the ongoing randomized trial of My Guide, the study enrollment rate is $93 \%$ and the retention rate is $100 \%$. CONCLUSIONS: Study findings underscore the relevant needs and concerns for Hispanic breast cancer survivors and suggest that the My Guide Smartphone application may be a feasible intervention. Considerations for developing a culturally adapted intervention as well as working with community stakeholders will be discussed.

CORRESPONDING AUTHOR: Betina Yanez, Northwestern University Feinberg School of Medicine, betina.yanez@northwestern.edu

\section{INVITED SYMPOSIUM}

$\mathrm{S} 03$

THE IMPACT OF EARLY ENVIRONMENTS ON MATERNAL AND CHILD OBESITY AND OVERWEIGHT

Barbara Mullan ${ }^{1}$

${ }^{1}$ Curtin University- Australia

Overweight and obesity are a global problem and their impact on physical and mental health is enormous. Fifty percent of women enter pregnancy overweight/obese and more than $50 \%$ of women gain excessive weight during pregnancy. Further over 41 million children worldwide are overweight/obese and these rates are increasing. The impact of early environments on maternal and child obesity and overweight and on understanding predictors of weight gain; what interventions should target and who the best people to administer such interventions should be is investigated in this symposium. We draw on research from four research groups, across two continents to provide the most current, topical research on the impact of early environments on obesity and overweight. Using a variety of methodological approaches including content analysis, predictive studies and systematic review we provide a coherent narrative on the importance of research to better inform both research and clinical practice. Midwives are an import resource for expectant mothers and weigh management is often difficult and can lead to negative maternal and child health outcomes. Using a combination of content analysis and cross sectional data Emily Kothe explores the importance of social cognitive factors in understanding the provision of midwife support to expectant mothers around weight management. Marita Hennessy explores the effectiveness of health professional-delivered interventions which aim to reduce the risk of overweight and obesity in children. She finds that there is inconsistency in reporting and in the effectiveness of interventions. Using both cross sectional and longitudinal data Barbara Mullan looks at the importance of psychosocial predictors of intervention in parents of young children. Her research highlights some of the key determinants of health feeding behaviours and suggests targets for future research. Briony Hill addresses the question of why the success of lifestyle interventions to prevent excessive gestational weight gain is suboptimal and variable and suggest key recommendations for future research. In combination this research highlights the importance of early environments for tackling the global obesity epidemic.

\section{S03.1}

CHILDHOOD OBESITY PREVENTION: THE EFFECTIVENESS AND ACTIVE INGREDIENTS OF INTERVENTIONS DELIVERED BY

Marita Hennessy ${ }^{1}$, Caroline Heary ${ }^{2}$, Rachel Laws ${ }^{3}{ }^{4}$, Luke Van Rhoon ${ }^{5}$, Elaine Toomey ${ }^{5}$, Molly Byrne ${ }^{5}$

${ }^{1}$ National University Of Ireland Galway- Ireland

${ }^{2}$ School Of Psychology, Nui Galway- Ireland

${ }^{3}$ Institute For Physical Activity And Nutrition.-Australia

${ }^{4}$ Deakin University- Australia

${ }^{5}$ Health Behaviour Change Research Group, School Of Psychology, Nui Galway- Ireland

Introduction \& Purpose: In 2014, over 41 million children worldwide were overweight/obese. Several factors identifiable during the first 1,000 days are associated with the development of overweight/obesity; increasingly these are intervention targets. Parents of young children have multiple routine contacts with health professionals during this period providing opportunities to integrate obesity prevention practices into routine services. This review aims to synthesise the evidence for the effectiveness of health professional-delivered interventions which aim to reduce the risk of overweight and obesity in children under the age of two and explore what behaviour change theories and techniques are associated with intervention outcomes. Methods: Systematic review of randomized and quasi-randomized controlled trials. Eligible studies were 
identified through systematic searches of 11 electronic databases, three trial registry databases, and cross-checking reference lists of full-texts retrieved. Intervention details were extracted according to the TIDieR reporting guidelines. Intervention procedures were coded using the Behaviour Change Technique Taxonomy (v1), intervention functions using the Behaviour Change Wheel, and theories employed using the Theory Coding Scheme. The methodological quality of studies was being assessed using the Cochrane Collaboration Tool for Assessing the Risk of Bias and generalisability of studies assessed using an external validity checklist. All procedures were conducted independently by two reviewers. A narrative synthesis was conducted. Results: Of the 16,044 records identified, 8,043 abstracts screened and 110 full-texts reviewed, 153 papers, representing 35 trials, were included. Interventions demonstrated inconsistent effects on child weight and related outcomes. Several methodological limitations were observed within and across trials, particularly in relation to intervention reporting which limits efforts to establish the active ingredients of interventions. Conclusions: While interventions identified had limited effects on child weight outcomes, they hold promise in terms of impacting on weight-related behaviours. The findings have implications for the conduct, reporting, and translation/ scalability of interventions.

CORRESPONDING AUTHOR: Marita Hennessy, National University of Ireland Galway, m.hennessy11@nuigalway.ie.

\section{S03.2}

LIFESTYLE INTERVENTION TO PREVENT OBESITY DURING PREGNANCY: A VIEW TO THE FUTURE

Briony Hill $^{1}$, Lisa Moran $^{12}$, Helena Teede ${ }^{12}$, Helen Skouteris ${ }^{1}$

${ }^{1}$ Monash Centre For Health Research And Implementation.- AUSTRALIA

${ }^{2}$ Monash University, Australia

Introduction: Fifty percent of women enter pregnancy overweight or obese and more than $50 \%$ of women gain excessive weight during pregnancy. Maternal obesity and excessive gestational weight gain (GWG) are associated with a range of negative maternal and offspring outcomes, including infant macrosomia and higher weight status in infancy through to adulthood. However, isolated lifestyle interventions to address weight concerns in pregnancy appear to have only modest benefit and individual responses to intervention vary. This presentation aims to address the question of why the success of lifestyle interventions to prevent excessive GWG is suboptimal and variable and suggest key recommendations for future research. Method: A narrative review of the literature. Results: There are inherent barriers to lifestyle change within pregnancy as a life stage that may contribute to tempered impact of interventions, including the short window available for habit formation; the choice for women not to prioritise their weight during pregnancy; competing demands including physiological, financial, relationship, and social situations; and lack of self-efficacy among healthcare professionals on this topic. In order to address these problems, we propose that just like all successful public health approaches seeking to change behaviour, individual lifestyle interventions must be provided in the context of a supportive environment that enables, incentivises and rewards healthy changes. Conclusions: Future research should focus on a systems approach that integrates the needs of individuals with the context within which they exist, removing potential barriers to individual lifestyle change at organisation and policy levels. We also need to truly understand the needs of individuals to design interventions appropriately tailored to these needs. This approach should also be applied to the preconception and postpartum periods for comprehensive prevention approaches. Additionally, relevant policy needs to reflect the changing evidence-based climate. Interventions in the clinical setting need to be integrally linked to multipronged obesity prevention efforts in the community, so that healthy weight goals are reinforced throughout the system.

CORRESPONDING AUTHOR: Briony Hill, Monash Centre for Health Research and Implementation, briony.hill@monash.edu.
S03.3

CURRICULUM BARRIERS FOR PROVIDING ANTENATAL WEIGHT MANAGEMENT ADVICE

Emily Kothe ${ }^{1}$, Carlye Weiner ${ }^{12}$, Michelle Lamb ${ }^{12}$, Skye McPhie ${ }^{12}$, Helen Skouteris $^{34}$, Other Cate Nagle ${ }^{56}$, Caryl Nowson ${ }^{6} 7$, Briony Hill ${ }^{34}$ '

${ }^{1}$ Deakin University- Australia

${ }^{2}$ School Of Psychology-Australia

${ }^{3}$ Monash Centre For Health Research And Implementation- Australia

${ }_{5}^{4}$ Monash University- Australia

${ }^{5}$ School Of Nursing And Midwifery

${ }^{6}$ Deakin University- Australia

${ }^{7}$ School Of Exercise \& Nuturition Sciences- Australia

Introduction \& Purpose: Excessive gestational weight gain and gestational overweight and obesity are independent predictors of post-partum weight retention, gestational diabetes, pregnancy complications and increased risk of negative child health outcomes. Provider counselling is a modifiable predictor of women's weight management during pregnancy. However, only half of patients report that they received this advice and many midwives report reluctance to provide weight management advice to women. This project focuses on curriculum barriers within pre-clinical midwifery training to address this gap in clinical practice. Methods: The project consists of two phases (1) a content analysis of Australian midwifery curricula and (2) a survey of the predictors of student midwives intention to provide weight management advice once they enter practice. Results: Phase 1: Review of 252 units of study across 20 Australian universities identified only one subject that made explicit reference to the role of midwives in providing weight management advice. While general health promotion was a prominent focus, there was a little evidence that skills in the provision of weight management advice or the value of providing such advice is explicitly taught to student midwives. Phase 2: 187 student midwives completed the phase 2 questionnaire. In general the student midwives were positive about providing weight management advice during pregnancy. Theory of planned behaviour constructs (attitude, subjective norm, and perceived behavioural control) accounted for $39 \%$ of intention to provide antenatal advice. Subjective norm and attitude were independent predictors of intention $(\mathrm{p}$ 's $<.01)$. Conclusions: Intentions to provide weight management advice are highest amongst those who hold positive attitudes towards provision of such advice and feel that others would approve of them providing such advice. However, at present current midwifery curricula includes little content to help students form such beliefs. Incorporating explicit content about the importance of antenatal weight management to child and maternal health and the role of midwives in supporting women manage weight during pregnancy into the midwifery curricula may be an important step in improving midwives clinical practice.

CORRESPONDING AUTHOR.: Emily Kothe, Deakin University, ekothe@deakin.edu.au.

S03.4

USING THEORY TO EXAMINE THE FEEDING PRACTICES OF PARENTS OF YOUNG CHILDREN

Barbara Mullan ${ }^{1}$, Megan McGee ${ }^{1}$, Enrique Mergelsberg ${ }^{1}$, Peter McEvoy ${ }^{1}$, Ben Gardner ${ }^{2}$, Kyra Hamilton ${ }^{3}$, Emily Kothe ${ }^{4}$

${ }^{1}$ Curtin University- Australia.

${ }^{2}$ Kings College London- United Kingdom.

${ }^{3}$ Griffith University- Australia.

${ }^{4}$ Deakin University- Australia.

Background Obesity is a global problem, with rates of child obesity increasing around the world. In Australia, approximately $25 \%$ of children are overweight or obese. Since obesogenic behaviours are very hard to change in adulthood, understanding eating behaviours in early childhood is essential. The aim of the current research is to understand the 
contributing factors of healthy feeding intention and behaviour by using an extended theory of planned behaviour. Methods Questionnaires related to the theory of planned behaviour, habit, and past behaviour were reported at baseline by 444 parents. Of these, 236 completed a longitudinal healthy eating questionnaire one week later. Results Using the maximum-likelihood method of structural equation modelling it was shown that the theory of planned was a good fit to the data at time 1 and at follow-up. At both times, the goodness of fit increased when we integrated habit strength, such that habit strength was predicted by attitude, subjective norm, perceived behavioural control and intention and habit strength predicted behaviour. Furthermore, the interaction between habit strength and intention was significant. At followup this indicated that habit strength moderated the intentionbehaviour relationship. For parents with low intentions, habit strength was associated with healthier feeding behaviours. No such effect was observed amongst parents with high intentions. Discussion These results suggest the extended theory of planned behaviour is an appropriate framework to use when predicting dietary adherence in the parents of toddlers and provides practical opportunities and targets for future interventions. The results highlight the importance for researchers and policy makers to focus not just on rational decision making like intentions but on automatic processes like habit.

CORRESPONDING AUTHOR: Barbara Mullan, Curtin University, barbara.mullan@curtin.edu.au.

S04.

MENTAL HEALTH OF INTERNATIONAL MIGRANTS IN LATIN AMERICA: DISENTANGLING UNDERLYING MECHANISMS FOR ACTION IN PUBLIC HEALTH

Baltica Cabieses ${ }^{1}$

${ }^{1}$ Universidad del Desarrollo- Chile

Introduction \& Purpose International migration represents a global phenomenon. There is a vast amount of international evidence discussing the relationship between migration status and por mental health. Most studies support a link between experiencing social vulnerability during migration and mood disorders, while findings on psychosis are conflicting. Little research on the matter has been conducted in Latin America and Chile. The purpose of this simposium is to update current evidence on the mental health of international migrants, its plausible underlying mechanisms and actions in public health. Methods We will organise the simposium in four consecutive presentations and final time for discussion. Presenters come from Costa Rica and Chile. The focus will be mental health of international migrants in the region, existing evidence, ongoing solutions, and possibilities for public health action. Results The first presentation (K Carpio, IOM Costa Rica) will describe the international migration in Latin America and existing specific evidence and solutions for the mental health of immigrants, for health promotion and prevention, as well as diagnosis and recovery. The second presentation (B Cabieses, UDD Chile) will describe general characteristics of immigrants to Chile, their access to healthcare and their mental health services use and needs, as well as ongoing solutions from primary care for immigrants to Chile. The third presentation (D Sirlopu, UDD Chile) will expand on the process of social integration of international migrant families, their underlying acculturation processes and its consequencues on mental health. The final presentation (M Chepo, UDD Chile) will describe the living conditions of immigrant families with children and how families in socioeconomic vulnerability report less access to healthcare and poorer health outcomes. She will also describe contextual factors affecting the mental health of immigrants. Conclusions This unique symposium will update assistants on current evidence, theory and solutions to protect the mental health of international migrants. Critical issues like our understanding on intercultural health, quality of data, and need of monitoring will be discussed.
S04.1

DIGGING INTO MIGRATION PROCESS: ACCULTURATION, ATTACHMENT STYLES, AND SOCIO-CULTURAL

David Sirlopú $^{1}$

${ }^{1}$ Universidad Del Desarrollo- Chile

Introduction \& Purpose (stating the main purposes and research question) In recent years, the migratory flows around the world have been expanded significantly. This situation has also caused that social scientist should cross their disciplinary boundaries. An example of this is the simultaneous use of concepts from anthropology, individual psychology and social psychology. Following this model, the objective of this study is to connect three central construals in the field of migration but that they have been studied separately such as acculturative orientations, attachment styles, and socio-cultural adaptation. All these concepts were used in order to understand the situation of a group of Peruvian immigrants (adolescents and adults) who are settled in Chile. Methods Four-hundred adult immigrants ( 230 women and 170 men) between 18 and 58 years of age $(\mathrm{M}=33.4, \mathrm{SD}=9.1$ years $)$ and 298 Peruvian schoolchildren $(152$ women and 146 men) between 13 and 18 years old $(\mathrm{M}=15.5, \mathrm{SD}=1.25$ years) were interviewed in Santiago. Descriptive and multivariate statistical analyzes were used to determine the association between the variables of interest. Results It was found differences in acculturative orientations and attachment styles between adolescent and adult immigrants. Moreover, attachment styles in both samples showed differentiated effects on contact with Chileans, perception of discrimination, and homesickness. Conclusions Some individual variables such as secure attachment promote the linking of Peruvian immigrants with Chilean society, while others such as fearful attachment lead Peruvian immigrants to be more reluctant to contact and engage with Chilean culture.

CORRESPONDING AUTHOR: David Sirlopú, Universidad del Desarrollo,dsirlopu@udd.cl.

\section{S04.2}

MIGRATION FLOWS AND MENTAL HEALTH: DEVELOPING EVIDENCE-BASED POLICIES AND ACTIONS IN LATIN AMERICA

Macarena Chepo ${ }^{1}$.

${ }^{1}$ Organización Internacional para las Migraciones.- Costa Rica.

Migration is now considered the megatrend of our time (1). In terms of migration realities, the American sub-regions (South America, Central America, North America, and the Caribbean) are known to be one of the world's main migration corridors in the world, currently holding a mix of intra-regional and regional migration flows that make migration management efforts very complex $(2,3)$. The topic has become one of the main issues of high level international dialogues at United Nations, including: the 71st UN general Assembly High-level Plenary Meeting on Large Movements of Refugees and Migrants in 2016, and the agreement to develop a Global Compact for Safe, Orderly and Regular Migration by 2019 (1). This presentation aims to render the relationship between migration processes and mental health consequences visible. Understanding that mental health is not only the absence or presence of mental disorders in migrant populations, but as defined by WHO, "a state of well-being in which every individual realizes his or her own potential, can cope with the normal stresses of life, can work productively and fruitfully, and is able to make a contribution to her or his community" (18). In such understanding, mental health actions and policies entail not only the response to mental disorders among migrant populations, but all actions regarding mental health promotion, prevention and treatment, throughout the migration process. The presentation also aims to provide evidence-based recommendations for the development of public health and mental health policies and actions to protect the right to health of migrant populations in Latin America. 
CORRESPONDING AUTHOR: Karen Carpio-Barrantes, Organización Internacional para las Migraciones, kcarpio@iom.int.

\section{S04.3}

THE LIVING CONDITIONS OF IMMIGRANT FAMILIES WITH CHILDREN IN CHILE AND THE GAPS IN ACCESS TO HEALTHCARE SERVICES BETWEEN MIGRANT AND LOCAL CHILDREN IN CHILE

Macarena Chepo ${ }^{1}$, Baltica Cabieses ${ }^{2} 3$.

${ }^{1}$ Universidad Del Desarrollo- Chile

${ }^{2}$ Programa De Estudios Sociales En Salud.- Chile

${ }^{3}$ Facultad De Medicina Clínica Alemana Universidad Del Desarrollo.-Chile

Background: International migration represents a global phenomenon that has reached relevance in Chile. Studies in the adult population suggest that there are gaps in access to healthcare services between immigrants and locals. Equity in health is a fundamental value of health systems. This concept includes equity in access, with potential access (or healthcare coverage) being its main indicator. Equity gaps in access to health services in immigrant versus Chilean children in Chile have not been measured yet. The objective of this work is describe the socioeconomic vulnerability of foreign children in Chile and its relationship with access to health services, between 2009 and 2015. It also deals with the implications of these findings on mental health of this age group Methods: Secondary analysis of Socioeconomic Characterization Surveys (CASENs) 2009, 2011, 2013 and 2015, for population under 18 years. The variables reviewed were migration status (immigrant/local), multidimensional poverty, poverty by income, age, access to health (healthcare provision: yes/no), sex and reporting any health problem in the last 3 months. All variables were parent-reported. Crude and adjusted gaps in access to healthcare between immigrant and local children were measured through weighted descriptive and multivariate logistic regressions in Stata 14. Results: The population of immigrant children that reports having no healthcare provision was between 3 and 14 times larger than the population of Chilean children in the same condition (2009: $2.25 \% \mathrm{v} / \mathrm{s}$ 8.4\%; 2011: $1.23 \% \mathrm{v} / \mathrm{s} 20.11 \%, 2013: 1.25 \% \mathrm{v} / \mathrm{s} 11.95 \%$ and $2015: 1.57 \% \mathrm{v} / \mathrm{s} 22.27 \%$ ). Crude and adjusted analysis showed that the possibility of not having a healthcare provision was at least $10 \%$ higher in immigrant versus local children ( $\mathrm{p}<0.01$ in years 2011, 2013 and 2015). Multidimensional poverty was the factor that was associated most strongly with having no healthcare provision for all the years of the survey analyzed (OR between 2.90 [95\% CI 2.08-4.07] and 3.96 [95\% CI 2.90-5.36]). Conclusions: Despite descriptions of descriptions in Chile to favor access to the health system, there is still a gap between the international and native immigrant population, where those children in multidimensional poverty would present a greater disadvantage. According to the available literature, this situation can exert effects on mental health directly or indirectly to both children and their families, generating scenarios of problems in parenting, domestic hostility, dissatisfaction marriage, conflict and aggression within families, among others.

CORRESPONDING AUTHOR: Macarena Chepo, Universidad del Desarrollo, macarena.chepo@gmail.com.

\section{INVITED SYMPOSIUM}

\section{S05}

SYSTEMATIC LIGHT EXPOSURE TO TREAT CANCER- AND CANCER TREATMENT-RELATED SYMPTOMS

Lisa $\mathrm{Wu}^{1}$.

${ }^{1}$ Northwestern University Feinberg School of Medicine- USA.

Cancer and its treatments are associated with a variety of co-occurring symptoms, including fatigue, sleep disturbances, depressive symptoms, and cognitive impairment. These problems may be present before the initiation of treatment and may continue for years after treatment has ended. Emerging evidence suggests that disruption of circadian rhythms (CRs) may underlie these cancer-related symptoms. This symposium will present recent research from the US and Europe on the use of systematic light exposure (sLE) to control/diminish cancer-related symptoms. It is hypothesized that sLE entrains CRs and thereby reduces or prevents fatigue, sleep disturbances, depressive symptoms, and cognitive impairment. Figueiro will present the rationale for light's effects on circadian rhythms. Amidi will present background research about CR disruption associated with cancer/cancer treatment and then present findings from studies that have used sLE to treat or prevent cancer-related fatigue. $\mathrm{Wu}$ will present findings from a pilot trial that examined sLE to treat cancerrelated cognitive impairment. Fox will present results from another pilot trial that examined sLE to treat sleep disturbance in cancer survivors. Valdimarsdottir will present results from a recent pilot trial that examined the effects of sLE using ambient light on depressive symptoms in cancer patients. Finally, Redd will discuss future directions for this research. This new area of supportive care research holds promise for reducing suffering among cancer patients/survivors. Indeed, sLE, no matter the delivery method, is a low burden intervention that is easy to deliver and inexpensive.

\section{S05.2}

LIGHT AS A STIMULUS FOR THE CIRCADIAN SYSTEM: IMPACT ON HEALTH AND WELL-BEING

Mariana Figueiro ${ }^{1}$

${ }^{1}$ Lighting Research Center at Rensselaer Polytechnic Institute- USA.

Introduction and Purpose: The circadian system keeps people synchronized with the 24-hour day by regulating internal biological cycles that repeat themselves on a daily basis, such as digestion, the release of hormones, the control of core body temperature, and when people feel alert or sleepy. The patterns of light and dark received by our eyes are the major synchronizer of the circadian system's master clock to our local position on Earth. Without the cues provided by these light-dark patterns, the numerous physiological and behavioral systems that help to ensure our well-being, if not survival, become asynchronous with each other and the environment. Research has associated such asynchrony with a number of mental and physical health problems, particularly including elevated risk for metabolic diseases such as obesity, diabetes, and cancer. Lighting characteristics affecting our circadian systems are, however, different than those affecting vision. The circadian system has a higher threshold for activation and is sensitive to short-wavelength light. When and how long light is received is also important for the circadian system. Methods: This presentation will discuss lighting as it affects the circadian system. It will present a new metric, circadian stimulus (CS) used to characterize circadian-effective light and how it was used to develop a lighting intervention designed to promote entrainment. CS has been used in various applications to promote entrainment to support good sleep and mood. Results: A series of field studies where the CS lighting intervention was used to promote entrainment will be presented. High CS during the day and low CS in the evening improved sleep and reduced depression and agitation in Alzheimer's disease patients, improved sleep and mood in office workers, increase alertness in office workers and Submariners, and improved sleep, fatigue, and mood in cancer patients. Conclusions: Light that is designed to maximally affect the circadian system can be used to improve sleep, mood and behavior in various populations. This presentation will set the stage for the subsequent talks in this symposium by providing a general overview of the importance of appropriately specifying and measuring light for health and well-being.

CORRESPONDING AUTHOR: Mariana Figueiro, Lighting Research Center at Rensselaer Polytechnic Institute, figuem@ @ri.edu 
S05.5

REDUCING CANCER-RELATED FATIGUE WITH SYSTEMATIC LIGHT EXPOSURE

Ali Amidi ${ }^{1}$, Lisa $\mathrm{Wu}^{2}{ }^{3}$, Birgitte Offersen ${ }^{145}$, Sonia Ancoli-Israel ${ }^{6} 7$, Heiðdís Valdimarsdóttir ${ }^{6} 8$, William Redd ${ }^{10}$, Robert Zachariae ${ }^{111}$.

${ }^{1}$ Aarhus University Hospital- Dinamarca

${ }^{2}$ Department of Medical Social Sciences- USA

${ }^{3}$ Northwestern University Feinberg School of Medicine, Chicago, ILUSA.

${ }^{4}$ Department of Oncology- USA

${ }^{5}$ Denmark- Dinamarca

${ }^{6}$ Department of Psychiatry- USA

${ }^{7}$ University of California, San Diego, CA- USA.

${ }^{8}$ Reykjavik University, Reykjavik, Iceland

${ }^{9}$ Department of Population Health Science and Policy- USA

${ }^{10}$ Icahn School of Medicine at Mount Sinai, New York, NY- USA.

11 Department of Oncology and Department of Psychology and Behavioural Sciences.

Introduction: Emerging evidence indicates that circadian rhythms may be disrupted following cancer and its treatment, and that circadian rhythm disruption may be an underlying pathophysiological mechanism of cancer- and cancer treatment-related symptoms such as cancer-related fatigue (CRF) and other co-occuring symptoms. Given the detrimental effect of CRF on cancer survivors' quality of life, there's a pressing demand for an effective, low cost and low burden intervention to treat CRF. Systematic exposure to bright white light (BWL) is a novel intervention with the potential to treat CRF. The effect of BWL on CRF is hypothesized to be through "nonvisual" pathways affecting a unique nonimage forming photoreceptor system with the potential to normalize disrupted circadian rhythms. Methods: In several ongoing and completed research protocols, BWL is administered using a small light box to be placed on a table about $45 \mathrm{~cm}$ from the patient's head and within $45^{\circ}$ of the visual field for a duration of 30 minutes every morning for 4 weeks. The light box uses premium white light emitting diodes emitting light at approximately $1500 \mathrm{lux} / 10.000 \mathrm{~K}$. An identical-appearing device emitting dim light in the red frequency range is used as a comparison condition as photoreceptors are relatively insensitive to this light color frequency. Results: The effects of BWL on fatigue prevention and reduction have been investigated in several studies with cancer patients. In a mixed group of fatigued cancer survivors, it has been shown that exposure to BWL is associated with a statistically significant decrease in fatigue symptoms compared with a control condition. In a group of breast cancer patients undergoing chemotherapy, it has been shown that BWL exposure is associated with fatigue prevention during treatment. Currently, several ongoing randomized controlled trials are being conducted with the aim of replicating these initial preliminary findings. Furthermore, in order to test the underlying assumption regarding normalization of circadian rhythms, several studies are also investigating different markers of the circadian timing system, such as circadian activity rhythms and cortisol rhythms. Conclusion: Preliminary research supports systematic light exposure with BWL as a potential treatment for CRF. Systematic light exposure, thus, holds significant promise as a low-cost and low-burden intervention for ameliorating fatigue in cancer populations. Further studies are needed to replicate findings and to investigate hypothesized circadian mechanisms.

CORRESPONDING AUTHOR: Ali Amidi, Aarhus University Hospital, sayamidi@gmail.com

\section{S05.1}

Systematic light exposure and cognition in hematopoietic stem cell transplant survivors: A pilot study.

Lisa $\mathrm{Wu}^{1}$, Ali Amidi ${ }^{2}$, Heiddis Valdimarsdottir ${ }^{3}$, Sonia Ancoli-Israel ${ }^{4}$, Gary Winkel ${ }^{5}$, Ariella Hoffman-Peterson ${ }^{5}$, William Redd ${ }^{5}$.

${ }^{1}$ Northwestern University Feinberg School of Medicine- USA
${ }^{2}$ Aarhus University Hospital \& Aarhus University- USA

${ }^{3}$ Reykjavik University- USA

${ }^{4}$ University of California, San Diego- USA

${ }^{5}$ Icahn School of Medicine at Mount Sinai- USA

Introduction and Purpose: Hematopoietic cell transplant (HCT) survivors may experience a range of co-occurring cancer-related symptoms including cognitive impairment (CRCI). Systematic light exposure using bright white light (BWL) has shown promise as a treatment for cancer-related symptoms (e.g, fatigue). This study investigated the preliminary efficacy of BWL to treat CRCI and assessed treatment satisfaction. METHODS: Fifty-six HCT survivors 1-5 years post-transplant (mean age 60) screened for mild cognitive impairment using computerized neuropsychological tests (i.e., $\geq 1$ SD below the mean on $\geq 1$ subtest) were randomized to BWL or an established comparison dim red light (DRL) condition. Participants were instructed to use the light box for 30 minutes/morning for 28 days. Standardized measures of objective (primary outcome) and self-reported cognitive functioning (secondary outcome) were administered at baseline, the end of the intervention, and 3-weeks post-intervention. Preliminary efficacy was examined using linear mixed models. Treatment satisfaction was assessed using an established 4 point Likertscale question. RESULTS: There were statistically significant improvements over time in both groups in objective $(\mathrm{p}<.0001)$ and self-reported cognition ( $\mathrm{p}=.034)$. Linear mixed models indicated no significant time by group effects for objective $(\mathrm{p}=.20)$ nor self-reported cognition $(\mathrm{p}=.41)$. Seventy-two percent of participants were satisfied with the intervention to some extent (scores between 2 and 4) with no significant difference between groups ( $\mathrm{p}=.87$ ). CONCLUSION: Although improvements to objective and self-reported cognition were evident in both groups, there was no specific hypothesized effect of BWL over DRL. It is unclear whether changes over time were due to placebo effects, real therapeutic effects of both light conditions, changes unique to each group and unrelated to the intervention, or in the case of objective cognitive functioning, whether both groups were simply displaying practice effects. Due to the small sample size, follow-up research is warranted potentially with a usual care group.

CORRESPONDING AUTHOR: Lisa Wu, Northwestern University Feinberg School of Medicine, lisa.wu1@ northwestern.edu

\section{S05.6}

PRELIMINARY FINDINGS FROM A BRIGHT LIGHT INTERVENTION FOR SLEEP DISTURBANCE AND SYMPTOM BURDEN IN OVARIAN AND ENDOMETRIAL CANCER SURVIVORS Rina Fox ${ }^{1}$, Kathryn Reid ${ }^{1}$, Katrin Bovbjerg ${ }^{1}$, Cody Boland ${ }^{1}$, Lisa Wu ${ }^{1}$, Shohreh Shahabi ${ }^{1}$, Jason Ong ${ }^{1}$, Phyllis Zee ${ }^{1}$, Frank Penedo ${ }^{1}$

${ }^{1}$ Northwestern University Feinberg School of Medicine- USA

Introduction \& Purpose Research evaluating the extent to which psychosocial and behavioral treatments can improve sleep quality and impact health outcomes among gynecologic cancer survivors is limited. A novel, promising, non-pharmacological intervention that targets sleep by promoting circadian regulation is bright light. This pilot study assessed the preliminary efficacy of systematic bright light exposure on symptom burden and self-reported and objectively-measured sleep among women with a history of ovarian or endometrial cancer. Methods Participants were women diagnosed with ovarian $(n=4)$ or endometrial $(n=14)$ cancer who had completed primary treatment, and reported sleep disturbance (i.e., Pittsburgh Sleep Quality Index (PSQI) global score $\geq 5$ ). Women were randomized to receive 45 minutes of bright white light (BWL; $\mathrm{n}=9$ ) or dim red light (DRL; $\mathrm{n}=9$ ) each morning for 4 weeks using ReTimer ${ }^{\circledR}$ glasses. Participants wore a wrist actigraph for 1 week prior to and throughout the duration of the intervention, and completed assessments of psychosocial functioning and subjective sleep quality pre- and post-intervention. High acceptability and feasibility of this protocol has previously been shown. Results Unadjusted mixed model ANOVAs 
demonstrated significant group by time interactions for improved subjective sleep quality $(\mathrm{F}(1,14)=5.00, \mathrm{p}=.04, \mathrm{np} 2=.26)$ and reduced depression $(\mathrm{F}(1,16)=6.25, \mathrm{p}=.02, \mathrm{np} 2=.28)$. Compared to participants in the DRL group, those in the BWL group reported significant improvements in PSQI sleep quality (Cohen's d=1.50 vs. .30) and PROMIS Depression scores (Cohen's $d=1.00$ vs. .10) from pre- to post-intervention. Results also demonstrated a significant group by time interaction for actigraphymeasured wake after sleep onset (WASO; $\mathrm{F}(1,15)=4.87$, $\mathrm{p}=.04$, пp2=.25). Participants in the BWL group demonstrated decreased WASO (Cohen's $\mathrm{d}=0.18$ ), while those in the DRL group demonstrated increased WASO (Cohen's $\mathrm{d}=-.47$ ) from pre-intervention to the fourth week of the intervention. Conclusions BWL may be an effective strategy to diminish sleep disturbance and improve symptom burden among women with a history of ovarian or endometrial cancer. Future studies should evaluate these associations in larger samples, and explore the underlying mechanisms thereof.

CORRESPONDING AUTHOR: Rina Fox, Northwestern University Feinberg School of Medicine, rina.s.fox@gmail.com.

\section{S 05.4}

PROGRAMMED ENVIRONMENTAL ILLUMINATION DURING AUTOLOGOUS STEM CELL TRANSPLANTATION HOSPITALIZATION FOR THE TREATMENT OF MULTIPLE MYELOMA REDUCES SEVERITY OF DEPRESSIVE SYMPTOMS Heiddis valdimarsdottir ${ }^{1}$,William Holden ${ }^{1}{ }^{2}$, Jason Chen $^{2}$, Ariella Hoffman-Peterson ${ }^{2}$, Julia Granski ${ }^{2}$, Nina Prescott ${ }^{2}$, Alejandro Vega ${ }^{2}$, Natalie Stern ${ }^{2}$.

${ }^{1}$ Reykjavik Univeristy-Iceland.

${ }^{2}$ Icahn School Of Medicine At Mount Sinai- USA

Purpose: A significant number of multiple myeloma (MM) patients undergoing an Autologous Stem Cell Transplant (ASCT) report clinical symptoms of depression. Depression during hospitalization predicts depressive symptoms and mortality following ASCT. We report initial results of a randomized clinical trial testing the effects of Programmed Environmental Illumination (PEI) using circadian stimulating bright white light (BWL) from 7-10AM daily on depressive symptoms during ASCT hospitalization. Methods: Patients $(n=190)$ scheduled to receive an ASCT were assessed for eligibility. Those meeting study eligibility criteria $(n=42)$ completed the Center for Epidemiological Studies Depression Scale (CES-D) during a baseline assessment. They were then randomly assigned to PEI, using BWL or circadian ineffective, dim white light (DWL). BWL patients received 1,300 lux at the eyes while DWL patients received 90 lux at the eyes. All patients completed CES-D assessments at days 2 and 7 post-transplant, on the third day of engraftment and at one and three months post-discharge. Results: Linear mixed-model analyses revealed that there was no difference between the groups in CES-D total score at baseline $(p=0.7948)$. The interaction between time of assessment and light condition was significant $[\mathrm{F}(5,146)=2.63 ; \mathrm{p}=$ 0.0263; Effect Size $($ eta $2=0.08)]$. Compared to patients in the BWL condition, patients exposed to DWL reported greater levels of depressive symptoms 7 days post-transplant and on the third day of engraftment after controlling for potential demographic and medical covariates. Follow up assessments indicated that CES-D total scores declined for both groups post-discharge resulting in no significant group difference. On the third day of engraftment $72.22 \%$ of the participants in the DWL condition meet the criteria for clinically significant depressive symptoms compared to $41.18 \%$ in the BWL condition. Conclusion: PEI using BWL during ASCT hospitalization for the treatment of MM reduces the severity of depressive symptoms during hospitalization. Future studies should examine the potential mechanisms whereby PEI reduces depressive symptoms.

CORRESPONDING AUTHOR: Heiddis valdimarsdottir, Reykjavik Univeristy, heiddisb@ru.is.
S05.3

FUTURE DIRECTIONS IN LIGHT THERAPY RESEARCH

William Redd ${ }^{1}$

${ }^{1}$ Icahn School of Medicine at Mount Sinai- USA

A growing body of empirical evidence supports the use of light therapy (systematic light exposure (sLE) and programmed environmental illumination $(\mathrm{PEI})$ ) to reduce negative sequela of cancer and it's treatment. However, research on this area is still in its infancy. Future research directions include the study of: 1) how differing exposure to natural light (daylight) is associated with negative sequela cancer and its treatment (e.g., are there associations between how much light a person receives in hospital rooms and the severity of cancer-related fatigue, depression and sleep problems); 2) the impact of exposure to circadian stimulating light (i.e., healthy light) from engineered lighting fixtures in hospital and home environments (e. g., Can PEI prevent/reduce negative side effects?); 3) biological mechanisms underlying the impact of systematic light exposure (E.g., What biological processes underlie the beneficial effects of sLE and PEI?); 4) combining light interventions with other treatments for cancer-related fatigue and depression (e. g., Are the positive effects of light interventions enhanced by integration with CognitiveBehavior Therapy?); 5) the impact of sLE and PEI on disease processes (What is the impact of sLE on metabolic syndrome during adjuvent chemotherapy for breast cancer?) and 6) SLE and PEI used with other disease groups (e. g., What is the effect of sLE and PEI on fatigue, depression and cognitive impairment among patients with neurodegenerative disorders, including Parkinson's Disease, Alzheimer's and MS?). Research discussed in this symposium is based on the broad impact of circadian rhythms on biological and behavioral processes and evidence showing that light exposure is a primary means of entraining rhythms and thereby reducing symptoms of circadian rhythm disruption.

CORRESPONDING AUTHOR: William Redd, Icahn School of Medicine at Mount Sinai, william.redd@mssm.edu.

S06.

HOW STRESS AFFECTS WOUND HEALING: HORMONES, CYTOKINES, GENE EXPRESSION, AND IMMUNE CELLS John Weinman ${ }^{1}$

${ }^{1}$ Kings College London- United Kingdom

Research has shown that psychological stress can slow wound healing, and that psychological interventions can reduce stress and improve healing. It is generally understood that stress causes changes to stress hormones and these in turn affect immune response and healing, yet the detailed mechanisms are not fully understood. This symposium aims to further investigate the biological mechanisms that link psychological factors to stress hormones, immune function, and healing in order to improve our understanding of pathways in psychoneuroimmunology and guide clinical interventions. The symposium will be chaired by Professsor John Weinman, who will briefly introduce the context of stress and wound healing and highlight gaps in our knowledge. Associate Professor Christopher Engeland will then present data from two studies. The first study examines whether stress from University examinations affects healing and alters inflammatory mediators in mucosal wounds. The second study investigates whether administered corticosteroids affect the healing of mucosal wounds as well as inflammation in the tissue. Longer-term stress slowed mucosal healing, likely via increased inflammation in mucosal tissues, whereas the administration of corticosteroids (stress hormones) reduced tissue inflammation and did not slow mucosal healing. Associate Professor Elizabeth Broadbent, will present findings from an immunohistological analysis of cells in healing skin from punch biopsy wounds in the upper arm. This study compares the effects of an emotional disclosure writing intervention versus a control writing condition on the numbers and activation of immune cells in the skin. The 
results suggest that Langerhans cells play a key role. $\mathrm{PhD}$ candidate Astrid Meesters will present data on the relationship between cytokines/growth factors and healing in a study of a mindfulness intervention. Results indicated that the effect of mindfulness on skin permeability early in the healing process was mediated through its decreasing effects on pro-inflammatory cytokines (i.e. IL-6 and IL-1 $\beta$ ). To close, Professor Weinman will present a discussion of what these results mean for our understanding of psychoneuroimmunology, implications for patients in clinical applications, and areas for future research.

\section{S06.1}

THE RELATIONSHIP BETWEEN PRO-INFLAMMATORY CYTOKINES, GROWTH FACTORS AND WOUND HEALING AFTER MINDFULNESS MEDITATION

Astrid Meesters ${ }^{1}$, Yvo In den Bosch-Meevissen ${ }^{1}$, Madelon Peters ${ }^{1}$

${ }^{1}$ Maastricht University- United Kingdom

Introduction \& Purpose It was previously demonstrated in our lab that mindfulness-based stress reduction (MBSR) can increase the speed of wound healing in healthy volunteers, but only in those who actually benefitted from the intervention in terms of increased mindfulness from pre- to post-intervention. The purpose of the present study was twofold. First, we aimed to study whether participants showing high levels of worry benefitted most from the intervention. In particular, it was hypothesized that MBSR would aid highly ruminating participants, as these are the individuals for whom wound healing might be slowed down. Second, as cytokines and growth factors (GF) play a vital role in the healing process, we examined whether the effect of MBSR on wound healing was mediated through its effect on the level of pro-inflammatory cytokines and GF in wound exudate. Methods Participants were randomly assigned to a wait-list control or an 8-week MBSR group. They completed psychological questionnaires before and after the waiting/intervention period. Standardized skin blisters were induced on the volar surface of the forearm by means of suction, which allowed us to measure both cytokines and GF in the wound exudate 3, 6 and 22 hours after injury. Healing was monitored by assessing trans-epidermal water loss (TEWL) and wound size at days 3, 4, 5, 6, 7 and day 10 after wounding. Results Rumination at pre-intervention was not found to moderate the relationship between the intervention and mindfulness levels, TEWL, wound size, cytokines, or GF. Results indicated that the effect of mindfulness on TEWL at day 3 was mediated through its effect on IL- 6 and IL- $1 \beta$ at 22 hours after injury. For wound size, no mediators were found, but wound size at day $3,4,5,6$, 7 , and day 10 was correlated with IL- 6 at 3 hours and IL- 6 at 6 hours after wounding for the whole sample $(\mathrm{r}[.32 ; .68]$, p's $<.03)$, indicating that an increase in this inflammatory marker may be unfavorable for healing. Further, wound size at day 3, 4, 5, and 6 was associated with IL-8 at 3 hours and IL- 8 at 6 hours after wounding in the MBSR group ( $\mathrm{r}[.53 ; .85]$, $\mathrm{p}$ 's $<.02)$. Conclusion These preliminary findings require further research to elucidate the potentially clinically relevant effect of mindfulness on wound healing.

CORRESPONDING AUTHOR: Astrid Meesters, Maastricht University, astrid.meesters@maastrichtuniversity.nl.

\section{S06.2}

EXPLORING THE CELLULAR MECHANISMS BEHIND THE BENEFICIAL EFFECTS OF EXPRESSIVE WRITING ON HEALING Elizabeth Broadbent ${ }^{1}$, Paul Jarrett ${ }^{2}$, Kavita Vedhara ${ }^{3}$, John Tarlton ${ }^{4}$, Christine Whiting ${ }^{4}$, Hayley Robinson ${ }^{1}$,

${ }^{1}$ The University Of Auckland- New Zealand

${ }^{2}$ Counties Manukau District Health Board - New Zealand

${ }^{3}$ The University Of Nottingham- United Kingdom.

${ }^{4}$ University Of Bristol- United Kingdom.
Introduction \& Purpose: Written emotional disclosure (expressive writing) has been shown to improve the healing of small punch biopsy wounds, yet how this occurs is not understood. This study aimed to analyse the biological mechanisms behind these effects by conducting an immunohistochemistry analysis of the healing tissue in expressive writing and control groups. Methods: 122 participants were randomly allocated to four groups in a 2 (timing) by 2 (writing type) design. Groups performed either expressive writing or control (neutral) writing either during the 7 days before or the 7 days after a $4 \mathrm{~mm}$ punch biopsy wound. A $5 \mathrm{~mm}$ punch biopsy was taken 14 days after the original $4 \mathrm{~mm}$ wound over the same site. Forty-eight of the $5 \mathrm{~mm}$ biopsy tissue samples were randomly selected from the original sample of 122 participants (12 participant samples from each of the four groups) for immunohistochemistry analysis. The skin tissue samples from the $5 \mathrm{~mm}$ biopsy were tested for number of macrophages, neutrophils, T cells, Langerhans cells (rating of activation and cell count), immune cell activation and matrix metallaoproteinases 2 and 9. Results: There was a significant difference between groups in scoring of epidermal Langerhans cells in the healing tissue $(\mathrm{p}=.050)$. Participants who performed expressive writing before wounding had significantly higher scoring than whose who performed neutral writing after wounding $(2.21 \mathrm{vs} 1.29, \mathrm{p}=.024)$. Participants who performed expressive writing after wounding also had significantly higher scoring than those who performed neutral writing after wounding ( 1.83 vs $1.29, \mathrm{p}=.016)$. Differences between groups in macrophages, neutrophils, T cells, immune cell activation and matrix metallaoproteinases were not statistically significant. Conclusions: Expressive writing either prior to after wounding increases Langerhans cells in the healing skin compared to neutral writing. Langerhans cells are involved in the first-line defense of the epidermal barrier and are important in the inflammatory phase of acute wound healing. This supports previous results showing that expressive writing can speed epithelialization, and provides evidence of one biological mechanism.

CORRESPONDING AUTHOR: Elizabeth Broadbent, The University of Auckland, e.broadbent@auckland.ac.nz

\section{S06.3}

STRESS, INFLAMMATION, AND MUCOSAL TISSUE REPAIR Christopher Engeland ${ }^{1}$,Phillip Marucha ${ }^{2}$,Praveen Gajendrareddy ${ }^{3}$

${ }^{1}$ Penn State University- USA

${ }^{2}$ Oregon Health and Science University- USA

${ }^{3}$ University of Illinois at Chicago- USA

Psychological stress has been reported to slow mucosal healing, although the underlying mechanism is unclear. One possibility is via excessive inflammation, which may be particularly detrimental in mucosal tissues. Relevant to this, corticosteroids are potent anti-inflammatory hormones used in oral surgery but no study has objectively assessed the effects of corticosteroids on mucosal healing. Using a within-subjects design, the present two studies examined the effects of 1) stress and 2) corticosteroid administration on healing rates and inflammation in oral mucosal wounds. Healing rates were determined using a well-established mucosal wound model. Three small wounds were placed by a periodontist on the hard oral palate under local anesthesia. In Study 1, 65 dental students received these wounds at two time points: during academic examinations (stress) and during summer vacation (non-stress). In Study 2, 63 healthy adults received either methylprednisolone (125 mg iv) or placebo just prior to wound placement. This protocol was repeated 4 weeks later on the other side of the mouth using the opposite treatment. Healing rates were determined using daily wound videographs. Gene expression for inflammatory mediators was determined directly in tissue obtained at $6 \mathrm{~h}$ and $24 \mathrm{~h}$ post-wounding using real-time PCR. In Study 1, higher tissue inflammation was seen during the stress period. Specifically, IL-1 $\beta$, IL-6, IL-8, MIP-1a, MCP-1, ICAM and e-selectin were more highly expressed at $6 \mathrm{~h}$ post-wounding during the stress condition $(\mathrm{P}<.05$ or better). At $24 \mathrm{~h}$ post-wounding expression of IL-6 and MIP-1a remained elevated 
compared to the non-stress condition $(\mathrm{P}<.10, \mathrm{P}<.05)$. Wound closure was delayed during examinations compared to vacation $(\mathrm{P}<.05)$. In Study 2, corticosteroid therapy resulted in reduced tissue inflammation at each time point, indicating the drug had strong physiological effects. However, no significant differences in healing rates were observed between placebo and corticosteroid treatments, regardless of sex or phase of the menstrual cycle (follicular vs. luteal). To sum, the slower mucosal healing observed in stressed individuals likely stemmed, at least in part, from the exaggerated inflammatory response observed in healing tissues. Corticosteroid administration did not alter mucosal healing rates despite obvious reductions in tissue inflammation, suggesting that clinicians should not be hesitant to administer corticosteroids in oral surgery for fear of impaired healing in healthy adults.

CORRESPONDING AUTHOR: Christopher Engeland, Penn State University, cge2@psu.edu

\section{S06.4}

DISCUSSION OF SYMPOSIUM ON HOW STRESS AFFECTS WOUND HEALING

Prof. John Weinman ${ }^{1}$

${ }^{1}$ kings college London- United Kingdom

Discussion of Symposium on "How stress affects wound healing: Hormones, cytokines, gene expression and immune cells". John Weinman, Institute of Pharmaceutical Sciences, Faculty of Life Sciences \& Medicine, Kings College London, London, UK Abstract. Taken together, these three papers provide a really good snapshot of the state of the art in stress/wound healing research. That stress has deleterious effects on wound healing in humans is now firmly established, and current research is focused on understanding the mechanisms and temporal parameters involved as well as the potential for intervention development. All three papers explore underlying mechanisms as well as the effects of contrasting interventions. While both psychological interventions facilitated wound healing in the epidermis, the mediating mechanisms and temporal effects of these showed interesting differences. In contrast, the other study investigated the effects of corticosteroids, which are well known anti-inflammatory agents, and showed no effects on the healing speed of wounds in mucosal tissue, even though stress was clearly associated with higher gene expression of a range of inflammatory mediators. In the discussion of these papers, it will be important to (i) explore the nature and measurement of the stressors involved in these wound healing studies; (ii) contrast the different types of intervention which have been found to facilitate wound healing in terms of their comparative effects on wound repair mechanisms in the skin and mucosa. The discussion will not only aim to integrate the findings but also to generate questions for the speakers and encourage the audience to produce further questions and discussion points.

CORRESPONDING AUTHOR: Prof. John Weinman, kings college London- United Kingdom, john.weinman@kcl.ac.uk

\section{S07}

INTEGRATING CULTURE, PSYCHOLOGY, AND BEHAVIOR: RESEARCH ON TREATMENT ADHERENCE, CONTINUITY OF CARE, AND WILLINGNESS TO SEEK PSYCHOTHERAPY IN THE USA, MEXICO, AND CHILE

Hector Betancourt ${ }^{12}$

${ }^{1}$ Loma Linda University- USA

${ }^{2}$ UFRO- Chile

The aim of the symposium is to examine the role of cultural, psychological, and behavioral factors influencing health behavior and outcome in three healthcare domains and countries. All studies are conceptually grounded in Betancourt's Integrative Model of Culture, Psychology, and Health Behavior, (Betancourt et al., 1010, 2011; Flynn et al., 2011; 2015). Mixed-methods were implemented across studies, and structural equation modeling (SEM) was used to test the structure of relations among socio-structural, cultural, and psychological factors as determinants of the corresponding behavioral and/or biological health phenomenon: The focus of research was adherence to diabetes treatment in the USA, consequences of unfair treatment among primary care patients in Mexico, and willingness to seek psychotherapy among college students in Chile. In the first presentation, Diabetes Patients' cultural beliefs about the unfair treatment of people with diabetes predicted diabetes distress, which in turn predicted $\mathrm{HbAlc}$. The analysis of indirect effects showed that cultural beliefs exerted an indirect effect on $\mathrm{HbA} 1 \mathrm{c}$ through diabetes distress. In the second presentation, research with primary care patients in Mexico shows that cultural beliefs about health professionals influence perceptions of unfair treatment, which in turn results in negative emotions and behaviors relevant to continuity of care, such as avoidance or delay in making future healthcare appointments. In the third presentation, results suggest that expectations about psychotherapy as a process, a factor previously found to influence adherence to psychotherapy, does in fact influence seeking psychological help and is at least in part a function of cultural believes about psychotherapy. As a whole, the studies reported in this symposium highlight not only the role of cultural and psychological factors relevant to health research and care, but also the need for theoretical models that can guide research on the multivariate nature of the structure of relations among the determinants of health behavior and outcome. The results and conclusions of all presentations will be discussed from the perspective of the integrative model and the implications for healthcare research, policy, and intervention

\section{S07.1}

CULTURAL AND PSYCHOLOGICAL FACTORS RELATED TO SEEKING PSYCHOLOGICAL HELP IN CHILEAN YOUNG ADULTS

Natalia Salinas ${ }^{1}$

${ }^{1}$ Universidad de La Frontera- Chile

Introduction \& Purpose., College students, as young-adults, are at a particularly high risk for mental health problems. Still, they are less likely than other populations to seek psychological help. Previous research (Salinas, 2015), guided by Betancourt's integrative model of culture, psychology, and behavior (Betancourt et al., 2010, 2011; Flynn et al., 2011;2015) revealed that cultural beliefs and expectations about psychotherapy influence adherence to psychotherapy among adult women in Chile. The aim of the research reported here was to examine the structure of relations among socio-structural factors, cultural beliefs about mental health services, and psychological processes associated with seeking psychotherapy among college students. Based on Betancourt's Integrative Model, it was hypothesized that higher levels of negative cultural beliefs about psychotherapy would be associated with less favorable expectations about psychotherapy, which would be associated with lower willingness to seek psychotherapy. Methods. Participants were 391 students recruited from universities in Temuco, Chile. The instrument consisted of scales previously developed (Salinas et al., 2017), employing the mixedmethods cultural research approach (Betancourt et al., 2010), designed to measure cultural beliefs about psychotherapy, expectations about psychotherapy, and related psychological and behavioral phenomena. In addition, participants indicated their willingness to seek psychological help. Results. As expected, male participants reported higher scores on negative cultural beliefs about psychotherapy female participants $(\mathrm{t}=4.68, \mathrm{p}<$ .05). Consistent with the integrative model, negative cultural beliefs about psychotherapy were associated with less favorable expectations about psychotherapy, but only for female participants (female $\mathrm{r}=-.22, \mathrm{p}<$ .05 ; male $r=-.15, p>.05$ ). For both male and female students, more favorable expectations about psychotherapy were in turn associated with 
greater willingness to seek psychotherapy (female $\mathrm{r}=.32, \mathrm{p}<.05$; male $\mathrm{r}$ $=.27, \mathrm{p}>.05)$. Conclusions: Results demonstrate the need to consider the structure of relations among cultural, psychological, and behavioral in order to understand seeking psychological help in the young adult population.

CORRESPONDING AUTHOR: Natalia Salinas, Universidad de La Frontera, natalia.salinas@ufrontera.cl

\section{S07.2}

EXTENDING BETANCOURT'S INTEGRATIVE MODEL OF CULTURE, PSYCHOLOGICAL FACTORS, AND HEALTH BEHAVIOR TO BIOLOGICAL OUTCOMES

Patricia Flynn ${ }^{1}$, Hector Betancourt ${ }^{12}$

${ }^{1}$ Loma Linda University- USA

${ }^{2}$ UFRO- Chile.

Introduction. Type 2 diabetes (T2D) is a growing global epidemic that disproportionately affects ethnic and socioeconomically disadvantaged minorities. Prior research employing Betancourt's Integrative Model of Culture, Psychological Factors, and Health Behaviors (Betancourt \& Flynn, 2009), revealed that cultural beliefs indirectly predict diabetes self-care behaviors through psychological factors (Ung, 2015). The aim of the present study was to examine the impact of cultural and psychological variables on a biological outcome of diabetes control (HbAlc). It was hypothesized that cultural beliefs about the unfair treatment of people with diabetes would impact $\mathrm{HbAlc}$ directly and/or indirectly through diabetes distress among culturally diverse individuals with T2D in the US. Methods. Participants were recruited from diabetes treatment centers in California and included 149 culturally diverse individuals with T2D. The cultural scale, previously developed through the mixed-methods cultural research approach to instrument development (Betancourt et al., 2011), included three items reflecting cultural beliefs about the unfair treatment of people with diabetes $(\alpha=.87)$. Psychological distress was measured with two items from the Diabetes Distress Scale $(\alpha=.80$; Polonsky et al., 2005). HbA1c was self-reported. Results. Structural equation modeling indicated that the model including the hypothesized relations fit the data well $(\mathrm{CFI}=.97 ; \chi 2[19]=27.03, \mathrm{p}>.05$; $\mathrm{SRMR}=.05$ : RMSEA $=.05,90 \%$ CI $[.00, .09])$. As expected, cultural beliefs about the unfair treatment of people with diabetes predicted diabetes distress $(\beta=$ $.21, \mathrm{p}<.05)$, which, in turn, predicted $\mathrm{HbA1c}(\beta=.28, \mathrm{p}<.05)$. Consistent with the integrative model, cultural beliefs exerted an indirect effect on $\mathrm{HbA} 1 \mathrm{c}$ through diabetes distress $($ Bindirect $=.06, \mathrm{p}<.05$ ). Conclusions: Findings provide preliminary support for the extension of the integrative model to include biological outcomes. Results also underscore the importance of examining the indirect effect of culture on biological outcomes through psychological processes. Had only the direct effect of culture on $\mathrm{HbAlc}$ been examined, findings would incorrectly conclude that culture does not play an important role in treatment adherence and outcome.

CORRESPONDING AUTHOR: Patricia Flynn, Loma Linda UniversityUSA, UFRO- Chile, pflynn@1lu.edu.

\section{S07.3}

CULTURAL BELIEFS ABOUT PHYSICIANS AND PERCEIVED MISTREATMENT IMPACT PATIENTS' EMOTIONS AND CONTINUITY OF HEALTHCARE IN MEXICO

María José Baeza Rivera ${ }^{1}$, Rolando Diaz Loving ${ }^{2}$

${ }^{1}$ Universidad Católica de Temuco- Chile

${ }^{2}$ Universidad Nacional Autónoma de México- México.

Introduction \& Purpose. Healthcare mistreatment and perceived discrimination have important consequences for patients and healthcare in general, particularly for minority and economically disadvantaged populations (PNUD, 2017). According to previous research, caregivers' cultural beliefs about health professionals and perceived discrimination influence behaviors related to continuity of children's healthcare (BaezaRivera, 2015). The aim of this study was to examine the role of sociostructural factors, socially-shared (cultural) beliefs about physicians, and psychological processes associated with interpersonal mistreatment as potential determinants of health behavior and outcome. Based on Betancourt's Integrative Model of Culture, Psychology, and Behavior (Betancourt et al., 2010, 2011; Flynn et al., 2011, 2015), it was hypothesized that higher levels of negative cultural beliefs about physician would be associated with high interpersonal mistreatment, which would be negatively impact behaviors relevant to future healthcare. Methods. Participants were 323 male (35.3\%) and female (64.7\%) adults, from Mexico City and Tabasco, Mexico. They responded to an instrument that included a series of scales to measure cultural beliefs about physicians, $(=0.88)$, perceived interpersonal mistreatment $(=$ $0.89)$, emotions and affective states $(=0.94)$ and healthcare consequences $(=0.77)$. Structural equation modeling was used to examine the structure of relations among the variables and test the hypotheses of the study. Results. Consistent with the model guiding the research, negative beliefs about physicians influence perception of mistreatment ( $\beta=0.36, p<0.001$ ), which in turn had influenced avoidance of future care, indirectly, through emotions associated with interpersonal mistreatment ( $\beta=0.62, p<0.001 ; \beta=0.32, p<0.001$, respectively). In addition, negative beliefs impacted avoidance directly $(\beta=0.20, p$ $<0.001)$. Conclusions: A causal model including all the hypotheses of the study provided an excellent fit of the data, confirming the proposed structure of relations among the various factors investigated as determinants of health behavior in Mexico.

CORRESPONDING AUTHOR: María José Baeza Rivera, Universidad Católica de Temuco, mariajosebaezarivera@gmail.com.

\section{INVITED SYMPOSIUM}

S08

NOVEL NONPHARMACOLOGICAL APPROACHES TO PAIN MANAGEMENT

Ana-Maria Vranceanu ${ }^{1}$

${ }^{1}$ Harvard Medical School/Massachusetts General Hospital- USA

Pain affects millions of men and women around the world. Although extensive evidence shows that one of the best ways to achieve positive outcomes for pain is through non-pharmacological treatments that promote patients' self-management skills, uptake of such treatments is limited. With the recent opioid epidemic there is perhaps a new impetus. Now more than ever we need novel interventions that bypass barriers to non-pharmaceutical treatments, such as stigma, length, and access, for people with acute and chronic pain across a range of settings. This symposium presents findings from three, novel, brief interventions that show good feasibility, acceptability, and preliminary effects on pain outcomes. In the first presentation, Dr. Vranceanu will provide an overview of The Toolkit for Optimal Recovery after injury, a 4-session skills training program delivered via live video to patients with acute orthopedic injuries at risk for chronic pain and disability. In the second presentation, Prof. McCracken will provide an overview of a brief online treatment designed to promote psychological flexibility for people with chronic pain. In the third presentation, Dr. Addington will provide an overview of a promising brief intervention targeting positive affect developed for adults with HIV that is being adapted for the needs of patients with chronic pain. Dr. Pedro Perez will summarize these findings, place them into the larger context of the pain research, and provide directions for future research. 
S08.1

THE TOOLKIT FOR OPTIMAL RECOVERY; MEETING THE RECOVERY NEEDS OF PATIENTS WITH ORTHOPEDIC INJURIES ANA-MARIA VRANCEANU, EMILY ZALE, CHRIS FUNES, ANN LIN, \& DAVID RING

Ana-Maria Vranceanu ${ }^{12}$, Emily Zale ${ }^{3}$, Chris Funes ${ }^{3}, \mathrm{Ann} \mathrm{Lin}^{3}$, Marylin Hang $^{3}$, David Ring ${ }^{3}$

${ }^{1}$ Massachusetts General Hospital

${ }^{2}$ Harvard Medical School

${ }^{3} \mathrm{MGH} / \mathrm{HMS}$.

Orthopedic injuries are the leading cause of hospital admissions. Catastrophic thinking about pain and pain anxiety are risk factors for disability after orthopedic injury. We iteratively developed the "Toolkit for Optimal Recovery", a 4-session program for patients with orthopedic injuries at risk for poor recovery. The Toolkit bypasses barriers to psychosocial care such as stigma (by being depicted as a coaching mind body intervention) and access (by being delivered through live video). The Toolkit teaches mind body skills (relaxation response, acceptance, problem solving, daptive thinking, activity pacing). We aimed to determine the feasibility and acceptability of the Toolkit through a pilot RCT against usual care. We also tested whether participation in the Toolkit would be associated with more improvement in physical function, pain, mood and coping than participation in usual care, and whether these improvements would maintain 3 months later. Patients were recruited from the Orthopedic Trauma Center of a major-medical center. A research assistant screened the medical record for eligible patients, and performed recruitment, screening, consenting, randomization, and video set-up. Data was collected electronically. The Toolkit was delivered by trained clinical psychologists. Out of 101 participants screened, $50(50 \%)$ were depicted as at risk (scores above median split on catastrophic thinking about pain or pain anxiety), met inclusionary/exclusionary criteria and were randomized (25/ arm). Of these, 45 completed the intervention (90\%), and $38(84.5 \%)$ completed the 3 -months follow-up, which is ongoing. Patients (M age $=51)$ were women in majority $(62 \%)$, white $(80 \%)$, employed $(82 \%)$, without a work-related injury $(91 \%)$ and married $(52 \%)$. Satisfaction with the intervention was high ( $M=5, \mathrm{SD}=0,1-5$ scale). Patients in the Toolkit improved significantly more from baseline to post intervention on pain $(\mathrm{p}=.08 ; \mathrm{ES}=.20)$, and physical function $(\mathrm{p}=.004 ; \mathrm{ES}=.31)$ and in all secondary outcome variables $(\mathrm{p}=.001-.02 ; \mathrm{ES}=.20-.50)$, when compared to those in usual care. Results maintained 3 months later. Orthopedic patients are receptive to mind body skills building when delivered via secure live video. Results support a fully powered efficacy RCT.

CORRESPONDING AUTHOR: Ana-Maria Vranceanu, Massachusetts General Hospital, avranceanu@mgh.harvard.edu

\section{S08.2}

FEASIBILITY AND PRELIMINARY CLINICAL OUTCOMES FROM BRIEF ONLINE TREATMENT DESIGNED TO PROMOTE PSYCHOLOGICAL FLEXIBILITY FOR PEOPLE WITH CHRONIC PAIN Lance McCracken ${ }^{1}$, Joseph Chilcot ${ }^{1}$, Whitney Scott ${ }^{1}$

${ }^{1}$ King's College London- United Kingdom.

Cognitive Behavioral Therapy (CBT) for chronic pain is now well established, widely delivered, and known to help. At the same time developments in CBT continue, including a focus on specific therapeutic processes, such as psychological flexibility, and on efficient modes of delivery, such as online. The study reported here combines these two elements, in the form of randomized, two-arm, feasibility trial of Acceptance and Commitment Therapy (ACT) for general chronic pain. As far as we know this is the first trial of its type developed and conducted in the UK. In the current study, participants with chronic pain $(N=63)$ were recruited from a central London specialty service and allocated to either online ACT plus treatment as usual (TAU) or TAU alone.
Participants completed self-report measures at baseline and 3- and 9months following randomization. While the primary purpose of the trial was to determine feasibility, outcome and process measures also were administered, including pain-related interference, depression, work and social adjustment, medication use, healthcare visits, and psychological flexibility. Recruitment rate was 63 out of 139 (45\%) during 10 months, and retention rates were $76 \%$ and $78 \%$ for the 3- and 9-month assessments, respectively. Sixty-one percent of participants in the ACT online arm completed treatment. $56.5 \%$ in the ACT arm rated themselves as "much improved" or "very much improved," compared to $20 \%$ in the control arm. Effect sizes at 3-months ranged from small to large favoring ACT online participants for functioning, mood, medication and healthcare use, and psychological flexibility processes. Small to medium effects were maintained for functioning, healthcare use, and committed action at 9-months. Additional analyses of process variables will be presented and discussed, as will adjustments made in the treatment following with feasibility study.

CORRESPONDING AUTHOR: Lance McCracken, King's College London, Lance.McCracken@kcl.ac.uk.

\section{S08.3}

TARGETING POSITIVE AFFECT TO IMPROVE EMOTION REGULATION AND PAIN MANAGEMENT

Elizabeth Addington ${ }^{1}$, Elaine Cheung ${ }^{1}$, Judith Moskowitz ${ }^{1}$

${ }^{1}$ Northwestern University Feinberg School of Medicine- USA.

Introduction: Pain often is associated with emotional distress, which can exacerbate both the severity and chronicity of pain. Positive affect, which uniquely contributes to beneficial psychological and physical health outcomes, has the potential to disrupt this harmful spiral. The IRISS and LARKSPUR studies therefore examine whether a brief positive affect skills intervention improves pain management. Methods: We developed a theory- and evidence-based intervention that teaches eight skills for increasing positive affect: noticing and capitalizing on positive events, gratitude, mindfulness, positive reappraisal, personal strengths, goal attainment, and acts of kindness. The intervention has been delivered in face-to-face and internet-based formats to improve psychological and physical health outcomes among dementia caregivers and adults with chronic illness. In intention-to-treat analysis of a randomized controlled trial of the positive affect skills intervention versus supportive interviews for people newly diagnosed with HIV $(\mathrm{N}=159)$, longitudinal growth models tested for group-based differences in pain-related outcomes. Results: Analyses examining headache-related bother revealed a significant quadratic effect. Participants receiving the positive affect skills intervention reported significantly less headache-related bother than controls at post-intervention $(\mathrm{d}=-.27)$ and at 10 -month follow-up $(\mathrm{d}=-.34)$. In addition, rates of opioid use at the 10- and 15-month follow-up assessments were lower among participants in the intervention $(14.6 \%, 15.5 \%)$ versus the control group $(25.8 \%, 29.7 \%)(10$-month $\mathrm{d}=-.36 ; 15$-month $\mathrm{d}=-.43)$. Conclusions: These findings suggest that positive affect skills may improve pain management in adults with chronic illness. We therefore are using stakeholder-engaged methods to adapt the intervention for online delivery to adults seeking medical care for chronic musculoskeletal pain.

CORRESPONDING AUTHOR: Elizabeth Addington, Northwestern University Feinberg School of Medicine, elizabeth.addington@northwestern.edu

\section{INVITED SYMPOSIUM}

S09

THE ROLE OF OXYTOCIN IN HEALTH AND BEHAVIOR

Philip McCabe ${ }^{1}$

${ }^{1}$ University of Miami- USA 
The neuropeptide, oxytocin, has been widely studied in terms of reproductive physiology and social behavior. More recently, there has been increasing interest in the role oxytocin may play in both physical and mental health. Although much of this research has focused on CNSpituitary oxytocin and plasma or cerebrospinal fluid levels of the peptide, it is now clear that a variety of bodily tissues also express and secrete oxytocin. Both central and peripheral sources of oxytocin have been proposed to regulate basic physiological processes and also potentially mitigate pathophysiological events. Because of the likely importance of this peptide in mental and physical health, this symposium will explore the role of oxytocin in social behavior and mental health, in ovarian cancer, in glycemic control and diabetes, and in atherosclerosis and inflammatory processes.

\section{S09.1}

THE ROLE OF OXYTOCIN IN ATHEROSCLEROSIS AND INFLAMMATION

Philip McCabe ${ }^{1}$, Armando Mendez ${ }^{2}$, Angela Szeto ${ }^{1}$, Ni Sun-Suslow ${ }^{1}$, Jesica Westwright ${ }^{1}$

${ }^{1}$ University of Miami- USA

${ }^{2}$ University of Miami Miller School of Medicine- USA

Introduction: In addition to its established role in reproduction and social behavior, the neuropeptide oxytocin (OXT), working via its receptor (OXTR), has anti-inflammatory properties in a variety of tissues. We hypothesized that OXT may play an important role in inflammatory diseases, such as atherosclerosis. Atherosclerosis can be triggered by vascular injury/dysfunction or hyperlipidemia, which leads to plaque formation due to local inflammation and and oxidative stress. Methods: Cultured cells were incubated with physiological doses of OXT, and cellular inflammation and oxidative stress were measured. In animal models of disease, OXT was chronically infused and aortic atherosclerosis and inflammatory markers were assessed. Results: We demonstrated that OXT decreases inflammation and oxidative stress in cultured human vascular endothelial and smooth muscle cells, monocytes, and macrophages. A subsequent study using cultured macrophages demonstrated that expression of the OXTR is dramatically up-regulated in response to inflammatory signals. It was concluded that the OXTR is an acute-phase protein and that its increased expression is regulated by the nuclear transcription factor, NF-kB. When activated by OXT, the OXTR functions to attenuate cellular inflammatory responses in macrophages. We also demonstratred that chronic OXT infusions in an animal model of hyperlipidemia and atherosclerosis, the ApoE -/- knockout mouse, attenuated aortic atherosclerosis in a site-specific manner and inhibited the ex vivo secretion of the inflammatory cytokine, IL-6, from visceral adipose tissue. Similarly, in vivo infusion of OXT in the genetically hyperlipidemic WHHL rabbit significantly reduced atherosclerosis and was associated with decreased systemic inflammation, as reflected by circulating $\mathrm{C}$-reactive protein. Conclusions: Taken together, these findings suggest that peripheral OXT may work directly on vascular tissue, as well as visceral fat, to reduce inflammation and the pathophysiological sequelae leading to atherosclerosis. The fact that OT influences both macrophages and adipose tissue, which are not specific to the cardiovascular system, suggests that peripheral OXT may have widespread effects on inflammation and disease in other tissues.

CORRESPONDING AUTHOR: Philip McCabe, University of Miami, pmccabe@miami.edu.

\section{S09.2}

OXYTOCIN, COUPLE INTERACTION AND CYTOKINERESPONSES TO ACUTE DERMAL WOUND-APPLICATION

Beate Ditzen ${ }^{1}$, Friederike Winter ${ }^{1}$, Corina Aguilar-Raab ${ }^{1}$, Severin Laeuchli $^{2}$, Guy Bodenmann ${ }^{2}$, Markus Heinrichs ${ }^{3}$, Ulrike Ehlert ${ }^{2}$.
${ }^{1}$ University Hospital Heidelberg- Germany

${ }^{2}$ University Hospital Zurich- Suiza

${ }^{3}$ University of Freiburg- Germany

Please sort this abstract with Phil McCabe's planned symposium, entitled: "The Role of Oxytocin in Health and Behavior". Introduction: Romantic couple behavior has repeatedly been shown to affect individual mental and physical health and one mediating mechanism might be improved immune responses in those with positive interaction behavior between partners. On a neuroendocrine level, oxytocin (OXT) has been suggested to modulate immune responses. We, thus, hypothesized that couple behavior would interact with intranasal OXT administration and alter cytokine responses to suction blister wound application. Methods: At the Dermatological Clinic, University Hospital, Zurich, Switzerland, 80 healthy adult couples ( $\mathrm{N}=160$ individuals) received up to four standard suction blister wounds. Unstimulated cytokines (IL1beta, IL6, TNFalpha) were assessed from wound liquid at $1 \mathrm{~min}, 90 \mathrm{~min}$ and $24 \mathrm{hrs}$ after wounding. Immediately after wound application in a 4-group randomized design, couples were assigned to either instructed positive couple interaction (10min couple appraisal task) or no specific interaction and intranasal OXT or placebo administration. During the interaction, couple behavior was videotaped and coded for friendly and dominant behavior based on the Circumplex model. Results: Overall, local cytokine responses to wounding were very low and of the three cytokines only TNF-alpha at $90 \mathrm{~min}$ and $24 \mathrm{hrs}$ after wounding provided sufficient data for analysis. These data suggest no main effect of group assignment on TNF-alpha levels. However, friendly behavior during couple interaction predicted lower TNF-alpha levels at $24 \mathrm{hrs}$ post wounding and intranasal OXT and friendly behavior interacted to further reduce TNF-alpha in both, men and women. Conclusions: These findings suggest an interacting role of couple behavior and OXT on pro-inflammatory cytokine responses to a standard wound application procedure. The data, thereby, point towards OXT as a possible underlying neuroendocrine mechanism which might mediate the beneficial effect of close social relationships on health.

CORRESPONDING AUTHOR: Beate Ditzen, University Hospital Heidelberg, beate.ditzen@med.uni-heidelberg.de

\section{S09.3}

THE ROLE OF OXYTOCIN IN THE PANCREAS AND GLYCEMIC CONTROL

Armando J Mendez ${ }^{12}$, Phillip M McCabe ${ }^{2}$, Angela Szeto ${ }^{2}$, Raisa Ahmend $^{2}$, Natalie Hickerson ${ }^{2}$, Jesica S Westwright ${ }^{2}$,

${ }^{1}$ Miller School of Medicine- USA

${ }^{2}$ University of Miami- USA

Introduction. Oxytocin (OXT) is produced in the hypothalamus and is secreted into the bloodstream by the posterior pituitary gland and is associated with reproduction and lactation. Additionally, OXT has been associated with social affiliative behaviors. Recently, there has been increased interest in the role of the OXT in glycemic control, metabolism, and in the treatment of obesity and type-2 diabetes (T2D). In the current study we show that OXT injection affects glycemia in mice, confirm the presence of OXT and its receptor (OXTR) in the mouse and human islet, identify the presence of OXT within vesicles and evaluate pathways that stimulate OXT secretion from pancreatic cells. Methods: We evaluated the effect of exogenous OXT administration in obese mice after intraperitoneal injections and measured blood glucose and insulin. To evaluate OXT expression in tissues, isolated islets and the MIN6 mouse beta-cell line, mRNA was measured by qPCR and protein expression by immunohistochemistry and immunoassay. Insulin secretion in the presence or absence of OXT and or glucose was evaluated in isolated islets and beta cell lines. Results: We demonstrate that OXT injection into obese, hyperglycemic mice reduced blood glucose and was associated with an 
increase in serum insulin levels. Evaluation of isolated mouse and human islets demonstrated the presence of mRNA for OXT and OXTR. Immunofluorescence of mouse pancreatic tissue revealed the presence of OXTR within both alpha and beta cells of the islet, while OXT appeared confined predominately to the beta cell population based on colocalization with insulin. Using cultured cell lines we also evaluated conditions that may affect OXT secretion from islet cells and discuss mechanisms that maybe involved Conclusions: We demonstrated that OXT is present in and can be secreted from islet cells, and these data suggest that the exocrine pancreas may contribute to peripheral levels of OXT. Administration of OXT to hyperglycemic mice reduced blood glucose levels confirming the insulinogenic properties of the peptide in vivo. Together these data suggest that the OXT/OXTR pathway may function to influence pancreatic beta cell function and glycemic control.

CORRESPONDING AUTHOR: Armando J. mendez, Miller School of Medicine- University of Miami, amendez2@med.miami.edu.

\section{S09.4}

OXYTOCIN: A NOVEL BIOMARKER RELATED TO INFLAMMATION AND QUALITY OF LIFE IN OVARIAN CANCER Michaela Cuneo ${ }^{1}$, Susan Lutgendorf ${ }^{1}$, Angela Szeto ${ }^{2}$, Lauren Davis ${ }^{3}$, Phil McCabe ${ }^{2}$, Anil Sood ${ }^{4}$, Armando Mendez ${ }^{2}$

${ }^{1}$ University of Iowa- USA

${ }^{2}$ University of Miami- USA

${ }^{3}$ University of Michigan- USA

${ }^{4}$ MD Anderson Cancer Center- Spain

Research on the central and peripheral hormone oxytocin (OT) has primarily focused on neurohypophyseal OT and its role in human social behavior, bonding and the stress response. Less research has examined relationships of peripheral OT with psychosocial factors. OT is synthesized and produced locally by multiple peripheral organs, including the ovary. In vitro and in vivo studies suggest a protective role for OT in ovarian cancer via anti-inflammatory and anti-tumor effects. However, no studies have examined relationships between peripheral OT, inflammation and psychosocial variables that may be influenced by inflammation (e.g. quality of life) in a cancer population. This study examines endogenous OT from the tumor microenvironment (ascites fluid) and its potential relationship with quality of life (QOL) outcomes in ovarian cancer. OT was measured in the ascites fluid of 108 ovarian cancer patients and relationships between OT, markers of inflammation (IL-6) and QOL were examined. High levels of naturally occurring OT (> 200 times plasma levels of healthy, non-pregnant women) were observed in the ascites fluid of ovarian cancer patients (mean $=191.2 \mathrm{pg} / \mathrm{mL}$; range $=1.7$ $2,383 \mathrm{pg} / \mathrm{mL}$ ). Ascites OT was inversely related to both ascites IL-6 $(\beta=-.27, p=.007)$ and plasma IL-6 $(\beta=-.20, p=.055)$, suggesting antiinflammatory activity. To test the hypothesis that OT inhibits inflammatory activity in ovarian cancer, in-vitro studies were performed and revealed 1) basal OT secretion from four ovarian cancer cell lines, and 2) a direct inhibitory effect of exogenous OT on IL-6 secretion (IL-6 levels reduced by $40 \% ; \mathrm{p}=.03$ ). Given known relationships between inflammation and poorer QOL in cancer patients, we examined whether ascites OT was associated with QOL via its relationship with IL-6. Path analyses indicated that higher levels of ascites OT were associated with higher patient total QOL (FACT-G; $\beta=.20, p=.02$ ), higher ovarian cancer specific QOL (FACT-O [subscale]; $\beta=.29, \mathrm{p}<.01$ ) and less patient-rated physical disability (Performance Status; $\beta=-.21, p=.02$ ), controlling for age, stage, and correlations among the outcome variables. In bootstrapped mediation analyses, the effect of ascites OT on overall QOL $(\beta=.07,95 \%$ $\mathrm{CI}=.01, .12)$ and patient-rated disability $(\beta=-.06,95 \% \mathrm{CI}=-.11,-.01)$ was found to be fully mediated by IL- 6 . The effect of ascites OT on higher ovarian cancer specific QOL was only partially mediated by IL-6 ( $\beta=.06$, $95 \% \mathrm{CI}=.002, .12)$. This research identifies OT as a novel biomarker in the ovarian tumor microenvironment with both direct anti-inflammatory effects and relevance for patient QOL. Ongoing research is investigating the source of OT in the ovarian tumor microenvironment and examining potential top-down processes that may contribute to individual differences in OT in the tumor microenvironment.

CORRESPONDING AUTHOR: Michaela Cuneo, University of Iowa, michaela-cuneo@uiowa.edu

\section{INVITED SYMPOSIUM}

$\mathrm{S} 10$

BEHAVIORAL AND PSYCHOSOCIAL FACTORS IN CARDIOVASCULAR DISEASE

Kenneth Freedland ${ }^{1}$.

${ }^{1}$ Washington University School of Medicine

Cardiovascular disease is the leading cause of death in middle- to highincome countries and one of the top three causes of death in low-income countries. International research in behavioral medicine has produced robust evidence that behavioral and psychosocial factors play important roles in the development and outcomes of cardiovascular disease. Work is also in progress on preventive strategies as well as interventions to help patients cope with cardiovascular disease and to reduce their risk of adverse outcomes. This symposium will highlight the findings of recent studies in several different areas of cardiovascular behavioral medicine. The first presentation examines sex differences in inflammatory and vascular mechanisms underlying mental stress-induced myocardial ischemia. The second focuses on the effects of low perceived social support on the physical and mental components of health-related quality of life following cardiac hospitalization. The third presents the findings of a recent trial of an innovative stress management training intervention for patients undergoing cardiac rehabilitation, including both psychosocial and cardiovascular outcomes. Finally, the fourth paper presents recent research on the effects of posttraumatic stress disorder (PTSD) symptoms on endothelial function and ambulatory blood pressure in previously deployed military veterans. These presentations demonstrate the diversity of contemporary research in cardiovascular behavioral medicine and highlight significant new findings about several forms of cardiovascular disease.

\section{S10.1}

LOW PERCEIVED SOCIAL SUPPORT AND QUALITY OF LIFE IN COLOMBIAN PATIENTS WITH HEART DISEASE.

Mariantonia Lemos ${ }^{1}$, Paula Rios ${ }^{2}$

${ }^{1}$ Universidad EAFIT- Colombia

${ }^{2}$ Universidad Catolica de Oriente- Colombia

Purpose: Social Support (SS) has been linked to a worse prognosis of Health-Related Quality of Life (HRQoL) in cardiac patients. Latin American countries differ from other countries because families are bigger and social relationships are an important part of the everydayness. This leads to general reports of high SS levels in cardiac patients and general population. We sought to determine if low social support is related with HRQoL prognosis in Colombian cardiac patients following six months after discharge. Method: Cardiac patients $(n=66)$ were enrolled in this study during an admission to an urban hospital and followed up for six months after discharge. Medical Outcomes Study - Social Support Survey was administered to assess perceived SS, and the Medical Outcomes Study Short Form SF 36 was used to assess HRQoL. A twoway mixed design ANOVA with two levels of SS (low vs. high) as a between factor and three time points (discharge, three months, six months) as a within-subject factor. Results: Fifty-seven percent of the participants were men; mean age, $62.9+11.3$. The effect of time was significant for the physical and mental components of HRQoL, F (1.646, $102.076)=7.915, \mathrm{p}<0.01$ and $\mathrm{F}(1.890,117.207)=4.085, \mathrm{p}<0.05$ 
respectively; also SS was found to be statistically significant for both components of HRQoL, $\mathrm{F}(1,62)=7.995, \mathrm{p}<0.01$ for the physical component and $\mathrm{F}(1,62)=4.535, \mathrm{p}<0.05$ for the mental component. The Time $\mathrm{x}$ Social Support interaction was not significant. Conclusions: This study suggests that physical quality of life tends to improve in cardiac patients after discharge, but the trajectories are different for those who have high versus low perceived SS. The mental component tends to improve in patients with high perceived SS but not in those with less SS.

CORRESPONDING AUTHOR: Mariantonia Lemos, Universidad EAFIT, mlemosh@eafit.edu.co.

\section{S10.2}

PTSD AND CARDIOVASCULAR DISEASE RISK IN DEPLOYED POST-9/11 US MILITARY VETERANS

Matthew Burg ${ }^{1}$, Casey Cavanagh ${ }^{2}$, Lindsey Rosman ${ }^{2}$, Joseph Schwartz ${ }^{3}$, James Dziura $^{1}$, Cynthia Brandt ${ }^{2}$, Sally Haskell ${ }^{2}$

${ }^{1}$ Yale University School of Medicine- USA

${ }^{2}$ VA Connecticut Healthcare System- USA

${ }^{3}$ SUNY Stoney Brook- USA

Purpose: Meta-analyses demonstrate that PTSD increases risk for incident cardiovascular disease (CVD) and cardiac mortality. Early markers are needed to better risk stratify deployed military veterans while also determining the dose-response of risk associated with PTSD symptom severity. We present findings concerning markers of vascular health endothelial function and 24-hr ambulatory blood pressure (ABP) - in relation to PTSD symptom severity among deployed veterans. Method: Participants in this ongoing study are veterans who were previously deployed for post-9/11 military operations. The psychosocial battery includes PTSD diagnosis, PCL-M (PTSD symptom severity), prior trauma exposure, and current stress. Participants complete 1) assessment of endothelial function by reactive hyperemia and in response to psychological stressor; 2) 24-hr ABP monitoring (readings every 30-min) with electronic diary of ecological experiences completed at each daytime ABP assessment; 3) 7-day sleep quality actigraphy; and 4) 7-day electronic diary sampling of PTSD relevant symptoms and experiences (3 times per day). Results: Preliminary findings indicate that PTSD symptom severity is inversely related to endothelial function: as severity increases, function declines. In addition, PTSD symptom severity is directly and highly correlated with ecologically assessed PTSD relevant experiences over 7-day monitoring, and inversely associated with sleep quality. veterans with PTSD diagnosis demonstrate higher day and night time average ABP and greater ABP reactivity to ecological stressful events during the day, compared to veterans without diagnosis and low symptom severity. Conclusions: Both PTSD diagnosis and subsyndromal PTSD symptom severity are associated with markers of vascular health, and risk factors ABP, sleep quality - for incident cardiovascular disease. It is essential to establish risk surveillance healthcare models to identify CVD risk in military veterans.

CORRESPONDING AUTHOR: Matthew Burg, Yale University School of Medicine, matthew.burg@yale.edu

\section{S10.3}

INFLAMMATORY AND VASCULAR RESPONSES TO STRESS AND MENTAL STRESS INDUCED ISCHEMIA IN WOMEN AND MEN WITH STABLE CORONARY ARTERY DISEASE

Viola Vaccarino ${ }^{1}$, Samaah Sullivan ${ }^{1}$, Muhammad Hammadah ${ }^{2}$, Ayman Alkhoder $^{2}$, Bradley Pearce ${ }^{1}$, Amit Shah ${ }^{1}$, J Douglas Bremner ${ }^{2}$, Paolo Raggi $^{3}$, Arshed Quyyumi ${ }^{2}$.

${ }^{1}$ Emory Univ / Rollins School of Public Health- USA

${ }^{2}$ Emory University School of Medicine- USA

${ }^{3}$ Mazankowski Alberta Heart Institute- USA
Purpose: Mental stress-induced myocardial ischemia (MSIMI) is associated with adverse outcomes in patients with coronary artery disease (CAD), yet the mechanisms are unclear and may differ by sex. We measured inflammatory and vascular responses to laboratory mental stress and their association with MSIMI in women and men with stable CAD. Method: 819 CAD patients underwent mental stress (speech task) with myocardial perfusion imaging. Inflammatory markers, measured at rest and 90 min after stress, included interleukin-6 (IL-6), high-sensitivity Creactive protein (CRP), monocyte chemoattractant protein-1 (MCP-1), and matrix metallopeptidase-9 (MMP-9). We measured digital vasoconstriction during stress with peripheral arterial tonometry (PAT), and endothelium dependent flow-mediated dilation of the brachial artery (FMD) and of the digital vessels [reactive hyperemia index (RHI)] at rest and 30 min after stress. We assessed hemodynamic workload by rate-pressure product (RPP) and used mixed models to adjust for clinical risk factors and to examine sex and age differences. Results: The mean age was 60y; $31 \%$ were women. Mental stress increased IL-6, MCP-1, and MMP-9 $(p<0.001)$ but not CRP. Younger women had higher IL- 6 at baseline and in response to stress compared with men of same age $(\mathrm{p}<0.05$ for interaction). Younger women also showed lower resting RHI but higher RPP and PAT ratio during stress. While inflammatory response to stress did not differ by MSIMI status in either men or women, baseline levels of inflammation (IL-6 and MMP-9) predicted MSIMI in younger women only. A lower PAT ratio during stress (i.e., more peripheral vasoconstriction) was related to MSIMI in women only ( $\mathrm{p}=0.003)$, with a larger effect in younger women. A higher RPP response to stress was related to MSIMI in men only $(\mathrm{p}=0.04)$. FMD and RHI were not associated with MSIMI in men or women. Conclusions: In men, MSIMI is due to supplydemand mismatch secondary to stress-induced hemodynamic workload. In women, it is driven by microvascular vasoconstriction and baseline inflammation. Younger women are especially vulnerable to MSIMI through these mechanisms.

CORRESPONDING AUTHOR: Viola Vaccarino, Emory Univ / Rollins School of Public Health, viola.vaccarino@emory.edu.

\section{$\mathrm{S} 10.4$}

THE VALUE OF STRESS MANAGEMENT TRAINING IN EXERCISE-BASED CARDIAC REHABILITATION

James A. Blumenthal ${ }^{1}$

${ }^{1}$ Duke University School of Medicine

Cardiac rehabilitation (CR) is the standard of care for patients with coronary heart disease (CHD). Despite epidemiologic evidence that high stress is associated with worse health outcomes, stress management training (SMT) is not included routinely in CR. We sought to determine whether CR enhanced by SMT can reduce stress and improve CHD biomarkers and medical outcomes compared with standard CR. Methods: One hundred fifty-one outpatients referred for CR at Duke University or the University of North Carolina at Chapel Hill were randomized to standard CR or CR enhanced by SMT. Prior to randomization, and after 12 weeks, patients completed a psychometric stress battery and an assessment of CHD biomarkers and were followed for a median of 2.6 years to assess medical outcomes. Seventy-five randomly selected patients who declined to engage in CR formed a no-CR control group. The primary outcome was a composite measure of self-reported stress. Secondary outcomes included CHD biomarkers and major adverse cardiovascular events (MACE) consisting of all-cause mortality, non-fatal myocardial infarction, coronary revascularization, and stroke/transient ischemic attack. Results: Patients randomized to CR+SMT exhibited greater reductions in stress levels compared with those randomized to $\mathrm{CR}$ alone $(\mathrm{P}=0.022)$ and reported less distress $(\mathrm{P}=0.049)$, anxiety $(\mathrm{P}$ $=.025)$, and perceived stress $(\mathrm{P}=.063)$. Both $\mathrm{CR}$ groups achieved significant, and comparable, improvements in CHD biomarkers. Participants in $\mathrm{CR}$ and $\mathrm{CR}+\mathrm{SMT}$ were at significantly lower risk of MACE compared 
with no-CR patients ( $17 \%$ vs. $37 \%, \mathrm{HR}=0.40[0.23,0.67], \mathrm{P}<.001)$ and participants in $\mathrm{CR}+\mathrm{SMT}$ also had lower rates of MACE compared with participants in CR ( $11 \%$ vs. $24 \%, \mathrm{HR}=0.43[0.19,0.99], \mathrm{P}=.049)$. Conclusions: CR enhanced by SMT provides incremental benefit when combined with standard CR and should be incorporated routinely into $\mathrm{CR}$ programs.

CORRESPONDING AUTHOR: James A. Blumenthal, Duke University School of Medicine, james.blumenthal@duke.edu.

S11

DEVELOPING AND EVALUATING SELF-DIRECTED BEHAVIOUR CHANGE INTERVENTIONS

Charles Abraham ${ }^{1}$

${ }^{1}$ University of Exeter Medical School- UK

Interventions to promote behaviour patterns associated with health and illness prevention are central to public health practice. Behaviour change usually requires more than education and interventions requiring multiple face-to-face contact can be expensive, and prohibitively so, when resources are limited. Consequently, many self-directed interventions have been developed. By "self-directed", we mean those requiring no professional contact or no contact after introductory sessions. Such interventions require users to self-regulate and generate change without professional assistance. They can be implemented with existing infrastructure and staffing and embedded in users' everyday lives. In this symposium, four speakers will consider evidence from systematic reviews of selfdirected interventions, including applications to weight loss (Prof. Charles Abraham, University of Exeter Medical School, UK) and the reduction of alcohol use (Ms. Renee O'Donnell, Deakin University, Melbourne). We will also present analyses of the development and evaluation of inhibition training to reduce smoking (Assoc' Prof. Petra Staiger, Deakin University, Melbourne) and two leaflets designed to promote screening for diabetic retinopathy (Dr. Amelia Lake, The Australian Centre for Behavioural Research in Diabetes, Melbourne). We will discuss the use of different delivery modes such as leaflets, manuals and smartphone applications. We will also consider appropriate development of intervention materials, optimal user targeting and rigorous evaluation methods. We will present evidence that self-directed intervention can be effective in generating behaviour change and can augment the effectiveness of face-to-face interventions. Finally, we will provide guidance for the development of self-directed interventions with the potential to have public health impact.

\section{S11.1}

LESSONS FROM THE APPLICATION OF SELF-DIRECTED INTERVENTIONS TO PROMOTE WEIGHT LOSS

Charles Abraham ${ }^{1}$, Jason Tang ${ }^{2}$

${ }^{1}$ University of Exeter Medical School- United Kingdom

${ }^{2}$ University of Dundee- United Kingdom

Many self-directed weight-loss interventions (SDWLIs) have been developed employing a variety of delivery modes (e.g., websites and smartphone applications). A systematic review and meta-analysis was conducted to ascertain whether such interventions are effective. MEDLINE, Embase, PsycINFO, CINAHL, and the Cochrane Library were searched for randomised controlled trials evaluating self-directed interventions in relation to weight-loss outcomes in adults. Standardised mean differences (SMD) and 95\% confidence intervals (CI) were calculated using a random effects model. Twenty-seven trials incorporating 36 comparisons met the specified inclusion criteria. Participants using selfdirected interventions lost significantly more weight $(\mathrm{MD}=-1.56 \mathrm{~kg}, \mathrm{CI}$ $-2.25,-0.86$ ranging from 0.6 to $5.3 \mathrm{~kg}$ ) than those in minimal intervention or no-treatment groups at 3 months follow-up. The majority of interventions were internet based (18) and this sub-group were effective at 3 months $(\mathrm{MD}=-1.74 \mathrm{~kg}, \mathrm{CI}-2.65,-0.82$ ranging from 0.6 to $4.8 \mathrm{~kg}$ ) $(\mathrm{SMD}=-0.48,95 \% \mathrm{CI}-0.72,-0.24, \mathrm{I} 2=82 \% ; \mathrm{p}<.0001 ; 16$ evaluations) and 6 months follow-up $(\mathrm{MD}=-2.71 \mathrm{~kg}, \mathrm{CI}-4.03,-1.39$ ranging from 2.2 to $5.3 \mathrm{~kg})(\mathrm{SMD}=-0.59,95 \% \mathrm{CI}-0.99,-0.19, \mathrm{I} 2=76 \% ; \mathrm{p}=$ $.004 ; 4$ evaluations). Thus motivated users can use such interventions to lose modest but noticeable weight over three to six month periods. Beyond six months, self-directed interventions may need to be supplemented by those requiring professional contact if clinically meaningful weight loss is to be achieved. Since such interventions are likely to be cost-effective, when effective, they can provide useful adjuncts to existing community and clinical services. Lessons for the design, promotion and application of self-directed interventions across a range of health-related behaviours are drawn from this example.

CORRESPONDING AUTHOR: Charles Abraham, University of Exeter Medical School, c.abraham@exeter.ac.uk

\section{S11.2}

THE USE OF LEAFLETS TO PROMOTE SELF-DIRECTED HEALTH BEHAVIOR CHANGE: TARGETING TWO POPULATIONS AT RISK OF VISION LOSS FROM DIABETIC RETINOPATHY

Amelia Lake ${ }^{1}$, Jessica Browne ${ }^{1}$, Gwyn Rees ${ }^{2}$, Charles Abraham ${ }^{3}$, Jane Speight ${ }^{1}$,

${ }^{1}$ The Australian Centre for Behavioural Research in Diabetes- Australia.

${ }^{2}$ Centre for Eye Research- Australia

${ }^{3}$ University of Exeter Medical School- Australia

Introduction and purpose: All people living with diabetes are at risk of diabetic retinopathy (DR), a common microvascular complication of diabetes which damages the blood vessels in the back of the eye. Retinal screening is key to early detection of DR and prevention of vision loss. Despite this, not all people with diabetes screen for DR and retinal screening uptake is low among some populations. The purpose of this presentation is to provide key outcomes and learnings from a program of research focussed on developing and evaluating two retinal screening promotion leaflets, tailored to priority populations with type 2 diabetes (T2D) identified as being at increased risk of both DR and low retinal screening uptake. Methods: Using a mixed-methods approach, we conducted a comprehensive needs assessment (literature review, interviews and online survey). Cohort-specific determinants were mapped to evidence-based persuasive messaging and incorporated into a print-based leaflet for each priority population. A pragmatic randomized controlled trial was planned and customized to the characteristics of each group. Results: The literature review demonstrated that although the majority of previous individual-level retinal screening promotion interventions focussed on increasing knowledge and awareness of DR and retinal screening guidelines, social cognitive factors, such as attitudes and beliefs also play an important role. The mixed methods study highlighted salient motivational factors impacting retinal screening behavior specific to each priority population, providing a pathway for the development of tailored, psychoeducational messaging. The interventions were developed and trialled within real-world conditions, and distributed to over 27,000 adults utilising existing infrastructure. Future directions involve incorporation of the leaflet into 'starter packs' provided to all eligible newly-diagnosed Australians with T2D registered on a nationwide diabetes database. Conclusions: This study illustrates the importance of taking a flexible approach to health behavior change intervention development for specific priority populations. Such findings are relevant in order to ensure we maximize engagement and relevance of health behavior interventions to the target audience.

CORRESPONDING AUTHOR: Amelia Lake, The Australian Centre for Behavioural Research in Diabetes, alake@ deakin.edu.au 
S11.3

DEVELOPMENT AND PILOT EVALUATION OF ONLINE RESPONSE INHIBITION TRAINING TO REDUCE SMOKING

Petra Staiger ${ }^{1}$, Karen Guo ${ }^{1}$, Melissa Hayden ${ }^{1}$, Laura Hughes ${ }^{1}$, Jason Bos $^{1}$, Natalia Lawrence ${ }^{2}$

${ }^{1}$ Deakin University- Australia.

${ }^{2}$ University of Exeter- United Kingdom

Introduction and Purpose: A recent meta-analysis reports that response inhibition training (utilising the Go/no-go task) can reduce unhealthy snacking and over consumption of alcohol. Recently, overweight individuals who participated in an online version of a response inhibition training task (4 training sessions) reported significant weight-loss 6 months post completion compared to controls. Online self-directed delivery of response inhibition training is highly cost effective and hence could be applied to other problem behaviours such as smoking. This paper reports on the findings of three studies which guided the translation of the food response inhibition training task to target smoking cessation. Methods: The three studies consisted of the following methods. An initial needs assessment (Pilot study 1) was conducted with smokers $(\mathrm{N}=7)$ in order to ensure the ecological validity of the smoking pictures utilised in the training task. Pilot Study 2 investigated the feasibility and acceptability of completing the online training for a four week period $(\mathrm{N}=16,44 \%$ male, aged $\mathrm{M}=35.5$ years). Pilot study 3 focussed on evaluation data regarding craving and smoking outcomes following two weeks of daily training $(\mathrm{N}$ $=13,69 \%$ male, aged $M=44$ years). Results: Key findings are reported. Participants in Pilot 1 preferred smoking stimuli that included salient smoke cues and less distractor imagery. Pilot study 2 found that participants found the training acceptable online but preferred to complete it on a daily basis for a shorter time period (ie two weeks). Pilot study 3 found that $54.5 \%$ of the participants reported decreased cravings for cigarettes at post-intervention, and $27 \%$ reported a clinically relevant reduction in cigarette smoking at two weeks follow-up. Conclusions: Given the limited success of most smoking interventions these outcomes are promising. This is particularly the case given the low-cost nature of this self-directed intervention. A randomised controlled trial is almost complete and is briefly described. If effective in reducing smoking, our development methodology could be adopted as a prototype to adapt this training to a range of additional problem health behaviours.

CORRESPONDING AUTHOR: Petra Staiger, Deakin University, petra.staiger@deakin.edu.au

\section{S11.4}

HOW EFFECTIVE ARE SMARTPHONE APPLICATIONS FOR PROVIDING ALCOHOL AND OTHER DRUG INTERVENTIONS? Renee O'Donnell ${ }^{12}{ }^{3}$, Paul Liknaitzky ${ }^{12} 3$, Lilani Arulkadacham ${ }^{24} 5$, Petra Staiger ${ }^{123}$

${ }^{1}$ Deakin University- Australia

${ }^{2}$ School of Psychology- Australia

${ }^{3}$ Geelong- Australia

${ }^{4}$ Monash University- Australia

${ }^{5}$ Melbourne- Australia

Risky drinking and drug use remain a significant global public health concern. Given the prolific use of smartphone devices, recent studies have evaluated whether smartphone applications (apps) are an effective mode of delivery for self-directed interventions targeting the reduction of Alcohol and Other Drug (AOD) use. Findings have been promising. For example, in one large trial, individuals with diagnosed alcohol dependence reported substantially fewer risky drinking days (1.13 per month) compared to participants in the control group (2.60 per month), 12 months later. This paper reports on a systematic review which aims to synthesise this literature and describe: (a) types of interventions that have been delivered using a smartphone, and (b) the effectiveness of these interventions. Method: A total of 9 studies were identified that examined the effectiveness of an AOD intervention delivered using an app. Results: The large majority of apps were designed to reduce alcohol use (6/9) with a smaller subset designed to reduce cannabis use (3/9). Certain intervention components were in common across six of the nine apps: selfmonitoring, psychoeducational information, and AOD refusal strategies. In terms of effectiveness, a significant reduction in quantity and frequency of alcohol use (five out of six studies) and marijuana consumption (two out of three studies) was found. It is important to note that there was very little consistency across studies with varying design, theoretical underpinning and client characteristics. Conclusion: These findings provide preliminary evidence that smartphone apps have the potential to assist people to reduce their alcohol and/or cannabis use. Importantly, as apps have the potential to reach individuals who may not otherwise receive help (e.g., due to geographical and financial barriers, privacy concerns, time constraints, etc.), the implications of these results are significant for the translation of behaviour change interventions more broadly.

CORRESPONDING AUTHOR: Renee O'Donnell, Deakin University, Renee.odonnell@deakin.edu.au

$\mathrm{S} 12$

ADJUSTING TO BREAST CANCER SURVIVORSHIP: CHALLENGES OF ONGOING TREATMENT AND SYMPTOMS Zoe Moon $^{1}$

${ }^{1}$ King's College London- United Kingdom

Whilst advances in breast cancer treatment have led to improved prognosis and survival rates, some women still face a number of challenges with regards to psychosocial and functional wellbeing post treatment. Ongoing symptoms such as pain and fatigue can persist for years once treatment has been completed. Some women also experience psychological distress including anxiety, depression and fear of recurrence. In addition to this, after primary treatment, women with oestrogen receptor positive breast cancer are offered endocrine therapy to reduce the likelihood of the cancer returning. This is associated with increased survival rates, but also with a number of side effects which reduce quality of life, and adherence to the treatment can be low. This symposium will bring together advances in research around understanding these psychosocial issues, and developing ways to support women with these issues. It will showcase results from four quantitative studies highlighting key cognitive and behavioural factors which influence fatigue, medication adherence and depression. The first study, by Hughes and colleagues, found that increased symptom focusing, all-or-nothing behaviour, avoidance behaviour and health anxiety prior to chemotherapy were associated with presence of ongoing fatigue post chemotherapy. In the second presentation, Cahir and colleagues examined 1,606 women prescribed endocrine therapy and identified a number of factors associated with non-adherence, including beliefs about capabilities and beliefs about consequences. Next, Moon and colleagues followed 345 women through their first year of tamoxifen prescription and found that women who had negative medication beliefs and lower perceived behavioural control were more likely to be nonadherent. Finally, Stanton and colleagues studied 460 women over a one year period and found that coping through cancer-related avoidance was associated with increased likelihood of depression related outcomes. The results from these studies enhance our understanding of what factors might be driving these psychosocial issues. By identifying modifiable cognitive and behavioural factors associated with fatigue, depression and adherence, interventions aiming to modify these factors can be developed.

\section{S12.1}

PSYCHOSOCIAL PREDICTORS OF NON-ADHERENCE TO TAMOXIFEN IN BREAST CANCER SURVIVORS: A LONGITUDINAL ANALYSIS

Zoe Moon $^{1}$, Rona Moss-Morris ${ }^{1}$, Myra Hunter ${ }^{1}$, Lyndsay Hughes ${ }^{1}$

King's College London- United Kingdom. 
Introduction and Purpose: Previous research has shown that up to 50\% of breast cancer survivors prescribed tamoxifen do not take it as recommended, which is associated with increased risk of recurrence and mortality. This study aimed to assess changes in adherence rates over time, and to identify clinical, demographic and psychosocial factors associated with changes in non-adherence. Methods: 345 breast cancer survivors who were in their first year of tamoxifen prescription were sent four questionnaires over a 12 month period. Questionnaires assessed demographic and clinical factors, side effects, illness perceptions, medication beliefs, social support, distress and adherence. Adherence was assessed using the Medication Adherence Rating Scale. Latent Growth Modelling was used to identify predictors of tamoxifen non-adherence. Results: Reported rates of non-adherence increased over time $(37-48 \%)$. Unintentional non-adherence (35-43\%) was reported more frequently than intentional non-adherence $(7-10 \%)$ at baseline and 12 months. Several predictors of non-adherence were identified, the most consistent were: being from minority ethnic groups; having lower necessity/concern differentials; and lower perceived behavioural control over medication taking (all $\mathrm{p}<.05)$. Unintentional non-adherence was associated more strongly with clinical and demographic factors, whereas intentional non-adherence was associated more with psychosocial factors. Conclusions: Reported rates of non-adherence to tamoxifen rise over time, with unintentional non-adherence being reported more frequently than intentional. These results identified demographic and clinical variables which can be used to identify women at higher risk of non-adherence. The modifiable psychosocial variables can be used as the basis for psychological interventions to improve adherence in this population. Interventions should focus on both intentional and unintentional nonadherence separately as they have different determinants.

CORRESPONDING AUTHOR: Zoe Moon, King's College London, zoe.moon@kcl.ac.uk

\section{S12.2}

DEVELOPING A MODEL OF THEORY BASED BEHAVIOURAL INFLUENCES ON ENDOCRINE THERAPY MEDICATION TAKING BEHAVIOUR IN WOMEN WITH BREAST CANCER

Caitriona Cahir ${ }^{1}$, Stephan U Dombrowski ${ }^{2}$, Linda Sharp ${ }^{3}$, Kathleen Bennett $^{1}$

${ }^{1}$ Royal College of Surgeons- Ireland

${ }^{2}$ University of Stirling- United Kingdom

${ }^{3}$ University of Newcastle- Australia

Introduction \& Purpose: Taking endocrine therapy for 5-10 years is recommended to prevent breast cancer recurrence. Despite the proven clinical efficacy of this treatment many women do not take their therapy as prescribed. The aim of this study was to develop a model of the theorybased behavioural determinants of endocrine therapy medication taking behaviour (MTB). Methods: Women with a diagnosis of stage I-III breast cancer prescribed endocrine therapy were identified from the National Cancer Registry Ireland $(\mathrm{N}=2,423)$ and invited to complete a postal survey based on the Theoretical Domains Framework (TDF), an integrative framework consisting of 14 domains of behaviour change, developed to inform intervention design and the Functional Assessment of Cancer Therapy and endocrine subscale (FACT-ES). MTB was measured by participant self-report with early treatment discontinuation classified as non-persistence and failure to take the correct dosage at the prescribed frequency classified as non-adherence. The theory-based model of MTB was tested using structural equation modelling. Results: 1,606 women completed the questionnaire (response rate $=66 \%) ; 1,211(75 \%)$ women were adherent; $180(11 \%)$ women were non-adherent and; $215(14 \%)$ women were non-persistent. The final model included 7 TDF domains and produced a reasonable fit $(\chi 2(321)=964, p<0.001$; RMSEA $=0.03$; $\mathrm{CFI}=0.96$ and $\mathrm{WRMR}=1.35$ ). Significant direct pathways on MTB were determined for 5 domains: Beliefs about Capabilities, Beliefs about
Consequences, Goals, Behaviour Regulation and Memory, Attention, Decision Processes and Environment (all $\mathrm{p}<0.01)$. The domains Beliefs about Consequences, Goals and Behaviour Regulation also had an indirect effect on MTB $(\mathrm{p}<0.01)$. These behavioural determinants accounted for $62 \%$ of the variance in MTB. Contrary to expectation, endocrine therapy-related adverse effects had neither a direct nor a mediating effect on MTB. Conclusions: The results suggests that an intervention to improve endocrine therapy MTB should target these domains using effective behaviour change techniques in order to prevent breast cancer recurrence and mortality in women with breast cancer.

CORRESPONDING AUTHOR: Caitriona Cahir, Royal College of Surgeons in Ireland, Caitrionacahir@rcsi.com

\section{S12.3}

CANCER-RELATED FATIGUE AND FUNCTIONAL IMPAIRMENT - TOWARDS AN UNDERSTANDING OF COGNITIVE AND BEHAVIOURAL FACTORS

Alicia Hughes ${ }^{1}$, Katharine Rimes ${ }^{1}$, Trudie Chalder ${ }^{1}$, Sahil Suleman

${ }^{1}$ King's College London- United Kingdom

Introduction \& Purpose: Fatigue is a highly prevalent and debilitating symptom experienced by women with breast cancer, both during and after chemotherapy. This study investigated the cognitive, behavioural, interpersonal and affective responses associated with and predictive of fatigue and social adjustment. Methods: 151 women diagnosed with breast cancer, currently undergoing or about to undergo a course of chemotherapy, completed a range of measures. Correlational and multiple regression analyses explored associations between fatigue severity, social adjustment and a range of psychological, behavioural, demographic and clinical variables. Fifty-two patients who completed measures prior to chemotherapy were followed-up prospectively, to examine the relationship between psychosocial variables and fatigue and social adjustment, as measured after three cycles of chemotherapy. Results: Fatigue and social adjustment were associated with a range of cognitive, behavioural and affective variables. In cross-sectional analyses key cognitive and behavioural correlates included increased symptom focusing, all-or-nothing behaviour, avoidance behaviour and health anxiety (all $\mathrm{p}<.001$ ). In a multiple regression analyses for fatigue, demographic/clinical predictors accounted for $10 \%$ of the variance, with the psychological predictors (sleep, symptom focusing and all-or-nothing behaviour) accounting for an additional $19 \%$ $[\mathrm{R} 2=.29 ; \mathrm{F}(4,106)=10.61, \mathrm{p}<.001]$. For social adjustment, clinical predictors accounted for $15 \%$ of the variance, with the psychological predictors (depression and avoidance behaviour) accounting for an additional $41 \%$. After 3 cycles of chemotherapy, fatigue (but not social adjustment) significantly increased $[\mathrm{t}(51)=4.48, \mathrm{p}<.001]$. Prospective analysis showed increased fatigue was predicted by prior beliefs about cancerrelated embarrassment avoidance, expectation of future fatigue and sleep $(\mathrm{R} 2=.42 ; \mathrm{F}(3,44)=7.18 ; \mathrm{p}=.001)$. Conclusions: Psychological and behavioural factors contribute to cancer-related fatigue and associated impairments. Such factors are potentially amenable to change within the context of cognitive behavioural therapy.

CORRESPONDING AUTHOR: Alicia Hughes, King's College London, alicia.hughes@kcl.ac.uk

\section{S12.4}

BEYOND THE SNAPSHOT: SUSTAINED COPING AS A PREDICTOR OF DEPRESSION IN WOMEN WITH BREAST CANCER

Annette Stanton ${ }^{1}$,Karen Weihs ${ }^{2}$, Joshua Wiley ${ }^{3}$

${ }^{1}$ University of California, Los Angeles

${ }^{2}$ University of Arizona Cancer Center

${ }^{3}$ Monash University 
Introduction and Purpose Most studies that address the relationships of coping processes with psychological outcomes during stressful experiences are cross-sectional in design, measure coping processes at only one assessment point, or predict only the linear slope of depressive symptoms. The authors investigated engagement in cancer-related approachand avoidance-oriented coping processes across time as predictors of depressive symptoms, symptom trajectory classes (consistently high, recovery, consistently low), and major depressive episodes over 12 months in the breast cancer context. Method Women $(\mathrm{N}=460)$ within four months of a breast cancer diagnosis completed reliable and valid assessments of cancer-related coping strategies, depressive symptoms, and major depressive episodes at seven points across one year. Results Beyond sociodemographic and medical variables, coping through cancer-related avoidance an average of two months after diagnosis was associated with likelihood of a consistently high depressive symptom trajectory and occurrence of a major depressive episode during the year. Less decline in avoidant coping over time also predicted poor outcomes. In contrast, high initial engagement in approach-oriented coping, as well as increases in coping specifically through emotional expression and acceptance, were associated with lower depressive symptoms across assessments and higher likelihood of being in the recovery or low as compared with the consistently high depressive symptom trajectory class over one year. Conclusions Greater engagement in cancer-related avoidant coping was associated with greater risk on all three indicators of depression, and greater approach-oriented coping was related to more favorable outcomes (except the occurrence of major depressive episodes). Sustained or increasing coping with the experience of breast cancer through emotional expression or acceptance predicted recovery from initially high depressive symptoms. Approach- and avoidance-oriented coping are promising malleable targets for preventive and ameliorative approaches to reduce depression.

CORRESPONDING AUTHOR: Annette Stanton, University of California, Los Angeles, astanton@ucla.edu

\section{$\mathrm{S} 13$}

HOW DIGITAL TECHNOLOGIES ARE DISRUPTING HEALTH CARE DELIVERY IN CHINA

Brian Oldenburg

University of Melbourne, School of Population and Global HealthAustralia

The health of China's population has improved remarkably in just one generation. Although there have been many significant reforms in China's health policy and health care delivery over the last 15 years, with a population of almost 1.5 billion people, there are still many challenges. NCDs like cancers, diabetes and cardiovascular diseases and the associated behavioral and clinical risk factors have increased rapidly and now account for more than $80 \%$ of China's disease burden. There are also increasing socioeconomic health inequalities, particularly between the population living in more highly urbanized eastern China, compared to those who still live in more rural and western China. There have been recent global rapid advances in new information and communication technologies and rapid uptake of internet-enabled smart devices and wearables and their application to health. There is rapidly accumulating evidence about how such "digital health innovations" (DHI's) can help prevent and manage chronic conditions like diabetes, heart diseases and cancers. This symposium will highlight three exciting new research programs being undertaking in China that are investigating the potential benefits of DHI's. Dr. Bennett will detail findings from trials designed to test the feasibility of a fully automated texting intervention for weight loss among adults with overweight and obesity in urban China. Enying Gong will detail the development and implementation of a trial in rural China on the secondary prevention of stroke incorporating both providerfacing and patients-facing digital health technologies. Dr Oldenburg will detail findings from a research collaboration with Shanghai CDC and other universities in China to evaluate the quality, functionality and use of diabetes- related apps in China. Each of the presenters will identify the various challenges and the potential for DHI's to disrupt current approaches to prevention and health care delivery in China. Key lessons learnt and emergent themes will then be discussed by Dr Zhong and the audience will be invited to discuss other digital health programs and relevant developments from China and other countries.

S13.1

THE QUALITY AND FUNCTIONING OF MOBILE APPLICATIONS FOR DIABETES SELF-MANAGEMENT: A SYSTEMATIC REVIEW OF MOBILE APPLICATIONS IN CHINA

Brian Oldenburg ${ }^{1}$, Enying Gong ${ }^{2}$, Zongmuyu Zhang

${ }^{1}$ The University of Melbourne- Australia

${ }^{2}$ Duke Kunshan University- China

${ }^{3}$ The University of Melbourne- Australia

INTRODUCTION: China has the largest national burden of diabetes mellitus in the world with more than 110 million people with diabetes (PWD). However, many people with diabetes are still unidentified and among those identified, the control rate is less than half. With the very rapid uptake of digital technologies throughout China, there has also been a similarly rapid increase of mobile applications for PWD. However, very little research has reviewed the quality and functioning of Apps available to assist PWD in China. The objectives of our study are: (1) to provide an overview of the available Chinese language mobile Apps for PWD; (2) to evaluate the quality of these applications; and (3) to investigate the key functions and features of these applications in promoting diabetes management and control. METHODS: We undertook a systematic review and assessment of publicly available Chinese Apps for diabetes management in the Apple Store and three other major App stores in China (Tencent, 360 Mobile Assistant, Baidu). A list of relevant free Apps was compiled after two reviewers independently screened the "candidate" apps. The Apps were further evaluated by trained reviewers using the Mobile App Rating Scale, IMS Functionality Score and a designed checklist of functions on diabetes management. Twenty percent of the applications were independently evaluated by more than one reviewer. RESULTS: So far, we have identified more than 120 Apps related to diabetes self-management from the Tencent App store. Twenty percent of these Apps have very few downloads $(<50)$ or are not active anymore. Twenty of the apps $(17 \%)$ account for $90 \%$ of all downloads. Key App functions include information, recording of clinical, behavioral and other data, setting notifications for medication taking and appointment scheduling. Less than $10 \%$ of Apps incorporate specific strategies for behavior change. App quality is very variable and few have any formal evaluation. CONCLUSIONS: There are many applications available for PWD in China, however, the overall quality is poor, most are not widely used and have never been properly evaluated. Specific attention to behavior change strategies and selfmanagement of diabetes is lacking. The implications for the future use and scalability of Apps and other available technology platforms for chronic disease self-management in China will be discussed.

CORRESPONDING AUTHOR: Brian Oldenburg, The University of Melbourne, boldenburg@unimelb.edu.au

\section{S13.2}

IMPLEMENTATION OF DIGITAL HEALTH SYSTEMS FOR IMPROVING THE SECONDARY PREVENTION OF STROKE IN RURAL CHINA: LESSONS LEARNED FROM THE SINEMA TRIAL Enying Gong ${ }^{1}$, Wanbing $\mathrm{Gu}^{1}$, Lijing $\mathrm{Yan}^{1}$, Li-Qun $\mathrm{Xu}^{2}, \mathrm{Na} \mathrm{Wu}^{2}$

${ }^{1}$ Duke Kunshan University.

${ }^{2}$ Center of Excellence for mHealth and Smart Healthcare, China Mobile Research Institute 
Introduction: Stroke is the leading cause of deaths in rural China where the largest number of economically disadvantaged population resides and the healthcare system is fragmented and inadequate to provide highquality care. Digital health innovations have the potential to empower both healthcare providers and patients. The aim of our study is to test the feasibility and effectiveness of a system-integrated technology-enabled model of care (SINEMA) for secondary prevention of stroke designed for rural China. Methods: We developed and are evaluating the SINEMA model in a one-year cluster-randomized controlled trial in Nanhe County from July 2017-June 2018. Fifty villages with about 25 stroke patients per village were randomized in a 1:1 ratio to intervention: control arm. The primary outcome of systolic blood pressure will be evaluated via baseline and follow up surveys among all 1,299 patients. Village doctors in the intervention arm were provided with an androidphone with the SINEMA application designed for this study to enhance their capacity through training, monthly patient followups and performance-based payments. Voice messages containing information on reminders and health education were delivered to patients in the intervention arm daily to promote their medication adherence and physical activities. Semi-structure interviews were conducted quarterly during the trial among village doctors and patients to collect data regarding their engagement in these digital health innovations and the impact on behavior changes. Interim Results: We report interim results from two quarterly evaluations. Among the 25 village doctors in the intervention arm, $96 \%$ regarded the SINEMA app as the first health-related apps ever used. Features that village doctors benefited from most included recording patients' data for future review, performance feedback and quality monitoring. Challenges were also identified: integration with existing electronic system, perceived better flow for traditional paper-based practices, and high work load from existing public health services. About $50 \%$ of the patients could receive voice messages every day. Patients receiving the messages considered it as good reminders and benefited from the health education information. However, sharing a phone with family members and difficulties in understanding due to low education level are key obstacles in receiving the messages. Final results will be available in November. Conclusion: Digital health technologies are feasible and have the potential to empower both healthcare providers and patients in promoting secondary prevention of stroke, but challenges exist in implementing and diffusing such innovations.

CORRESPONDING AUTHOR : Enying Gong, Duke Kunshan University, enying.gong@dukekunshan.edu.cn

\section{S13.3}

TEXTING INTERVENTIONS FOR WEIGHT LOSS IN CHINA

Gary Bennett ${ }^{1}$

${ }^{1}$ Duke University

Introduction: Overweight and obesity prevalence have increased dramatically in China during the past quarter-century. Only the United States has a larger absolute number of individuals with obesity. With nearly 1.5 billion mobile phones and extremely high use of messaging technologies (e.g., text messaging, WeChat), digital health approaches have promise for extending the reach of evidence-based obesity treatments to China's large and diverse population. Methods: We present findings from multiple investigations, including a randomized controlled trial evaluating 6month changes in weight among individuals randomized to either a texting intervention or a control arm. The intervention used a culturallyadapted version of interactive obesity treatment approach (iOTA) and comprised tailored behavior change goals, daily self-monitoring with automated feedback delivered via text messaging, and 5 coaching calls. We recruited 123 Chinese adults (40\% male), ages 30-50, BMI> 24, who used a mobile phone. Results: Intervention participants had significantly greater change in weight $(0.24 \pm 0.28 \mathrm{vs}-1.60 \pm 0.28 \mathrm{~kg}, \mathrm{p}<.0001)$ and waist circumference $(-0.13 \pm 0.43$ vs $-2.69 \pm 0.43 \mathrm{~cm}, \mathrm{p}<.0001)$. We also observed significantly larger changes in systolic and diastolic blood pressure among intervention vs control participants. We observed significant associations between intervention engagement (defined as daily use of text messaging-based self-monitoring features) and both anthropometric and cardiometabolic outcomes. Conclusion: Digital strategies have great potential for delivering evidence-based obesity treatments in China. Intervention engagement appears to be the most robust predictor of successful intervention outcomes. Texting offers advantages over other digital approaches including low development and service costs, use proximity, and potential for rich tailoring. These advantages are magnified in China, where messaging technologies enjoy high penetration into individuals' daily activities.

CORRESPONDING AUTHOR: Gary Bennett, Duke University, gary.bennett@duke.edu

\section{S14}

PAIN, LIFESTYLE AND MENTAL HEALTH IN ADOLESCENTS

Steven Kamper ${ }^{1}$

${ }^{1}$ University of Sydney- Australia

Behavioural health risks such as physical inactivity and substance use, musculoskeletal pain, and mental health issues are important health concerns for adolescents. They have in common; high prevalence, substantial individual and societal health burden, and implications for chronic illhealth in adulthood. This symposium reports three studies exploring the health impacts of pain, behavioural health risks and mental health factors in adolescents. Along with recognition of the burden of musculoskeletal pain in adolescents has come the search for cause. School backpacks have been implicated to the extent that articles are common in the popular media; professional associations and clinicians commonly endorse certain brands; and various bodies mandate specific backpack guidelines. The first study presents findings of a systematic review including 69 studies $(n=72,627)$ to synthesise the evidence that underpins these views. While smoking and alcohol consumption, and depression and anxiety are targets of public health campaigns in adolescents, the same is not true for pain. The question of whether pain should be considered in the adolescent public health context has not been addressed. A strong relationship between pain and other well-acknowledged health risks would provide an argument in favour of such consideration. The second study uses two observational datasets with a combined sample $>6000$ adolescents to investigate the relationship between pain, and substance use and mental health measures. There has been a recent upsurge of interest in the role resilience plays in behavioural health risk. There appears good theoretical basis for thinking boosting resilience could reduce risk behaviours such as substance use, and decrease the risk of mental health problems. Emerging evidence also suggests that increasing resilience may impact on pain outcomes. The third study will present the results of a large cluster RCT $(n>3000)$ of school-based resilience intervention delivered to adolescents in Australia. The symposium will finish with facilitated discussion aimed at exploring future directions for design and implementation of clinical and population health programs for adolescents, in light of current evidence.

\section{S14.1}

\section{DO SCHOOLBAGS CAUSE BACK PAIN IN CHILDREN AND} ADOLESCENTS? A SYSTEMATIC REVIEW

Tie Yamato ${ }^{1}$, Chris Maher ${ }^{2}$, Adrian Traeger ${ }^{2}$, Christopher Williams ${ }^{3}$, Steven Kamper $^{2}$

${ }^{1}$ Universidade Cidade de Sao Paulo- Brazil

${ }^{2}$ University of Sydney- Australia

${ }^{3}$ University of Newcastle

Background: The media and many professional associations commonly cite schoolbag use as the cause back pain in children. However, the 
evidence on this matter has not been systematically reviewed in a rigorous manner. Objective: To investigate whether characteristics of schoolbag use (e.g. weight, duration of use, bag design, method of carrying bag, perceived weight) are risk factors for back pain in children and adolescents. Data sources: Electronic searches of MEDLINE, EMBASE and CINAHL databases up to November 2017. Eligibility criteria for selecting studies: Prospective cohort studies, cross-sectional and randomised controlled trials conducted with children or adolescents. The primary outcome was report of back pain. We weighted evidence from longitudinal studies above that from cross-sectional. The risk of bias of the longitudinal studies was assessed by a modified version of the Quality in Prognosis Studies tool. Results: We included 69 studies $(n=72,627)$, of which five were prospective longitudinal and 64 cross-sectional or retrospective. We found evidence from five prospective studies that schoolbag characteristics such as weight, design, and carriage method do not increase the risk of developing back pain in children and adolescents. There is some evidence that the perception of heaviness is associated with back pain. The included studies were at moderate to high risk of bias. Evidence from cross-sectional studies aligned with that from longitudinal studies (i.e. there was no consistent pattern of association between schoolbag use or type and back pain). We were unable to pool results due to clinical and methodological heterogeneity. Conclusion: The available evidence shows no consistent relationship between schoolbag characteristics and back pain. These findings call into question the various guidelines and statements that endorse specific weight limits and bag types for children and adolescents.

CORRESPONDING AUTHOR: Tie Yamato, Universidade Cidade de Sao Paulo, tiparma@gmail.com

\section{S14.2}

ADVERSE HEALTH RISKS IN ADOLESCENTS WITH BACK PAIN Christopher Williams ${ }^{1}$, Steven Kamper ${ }^{2}$, Zoe Michaleff ${ }^{3}$, Paul Campbell ${ }^{3}$, John Wiggers ${ }^{1}$, Rebecca Hodder ${ }^{1}$,Kate Dunn ${ }^{3}$

${ }^{1}$ University of Newcastle- Australia.

${ }^{2}$ University of Sydney- Australia

${ }^{3}$ Keele University- Australia

Background Adverse health risk indicators such as substance use and psychological distress are public health priorities for adolescents. They are drivers of long term adverse health and social outcomes. For this reason they are common targets of public health campaigns delivered in schools. Prevalence of back pain rises steeply in adolescence and has significant consequences for many, including: distress, missing school, interference with physical activity, and use of health care services and medication. There is a question as to whether pain forms part of a cluster of behaviours and factors indicative of poor adolescent health. The aim was to assess whether adolescents that experience back pain more frequently are also more likely to report health risk behaviours and poor mental health. Methods Two cross-sectional samples of 14-15 year-olds. One from across Australia $(n=3,896)$, and one from the Newcastle area $(\mathrm{n}=1,831)$. Participants were asked how frequently they experienced back pain in the past 6 months, and about smoking, alcohol consumption, missed school, and depression and anxiety. Test-for-trend analyses were conducted to determine whether experiencing back pain more often was associated with adverse health behaviours and feelings of anxiety and depression. Results In total, $3.2 \%$ and $9.3 \%$ of participants had drunk alcohol in the two samples, and $4.4 \%$ and $12.6 \%$ had smoked. Alcohol consumption and smoking in both samples increased steadily with more frequent back pain. Of those who reported pain every day; $12.2 \%$ and $21.3 \%$ had drunk alcohol, and $12.7 \%$ and $20.8 \%$ had smoked. The same trend for increased prevalence of feelings of anxiety and depression with increasing back pain frequency was evident, although absolute differences between strata were small. Discussion There was the same, consistent relationship between frequency of back pain and adverse health risk indicators in two independently-collected samples of adolescents. These data indicate that frequent back pain is part of a picture of poor health and adverse health risk. These findings have implications for identifying a population at-risk of poor health, and indicate a need for more integrated services for adolescents with back pain.

CORRESPONDING AUTHOR: Christopher Williams, University of Newcastle, christopher.m.williams@newcastle.edu.au

\section{S14.3}

EFFECTIVENESS OF A UNIVERSAL SCHOOL-BASED 'RESILIENCE' INTERVENTION IN REDUCING PAIN IN ADOLESCENTS: A CLUSTER-RANDOMISED CONTROLLED TRIAL Rebecca Hodder ${ }^{1}{ }^{2}$, Hopin Lee ${ }^{2}{ }^{3}$, Steven Kamper ${ }^{4}$, John Wiggers ${ }^{1}{ }^{2}$, Christopher Williams ${ }^{12}$

${ }^{1}$ Hunter New England Population Health- Australia

${ }^{2}$ The University of Newcastle- Australia

${ }^{3}$ University of Oxford- Australia

${ }^{4}$ The University of Sydney- Australia

Introduction and purpose: Musculoskeletal pain is the leading cause of disability worldwide. Pain during adolescence is common, with persistent pain during adolescence associated with an increased risk of chronic pain in adulthood. Emerging evidence suggests the potential of resilience interventions in improving pain outcomes in chronic pain populations, however there are no studies that investigate this in children. The aim was to investigate the effectiveness of a school-based 'resilience' intervention in reducing pain frequency, problematic pain and pain intensity, and increasing pain self-efficacy and resilience in secondary school students. Methods: A cluster-randomised controlled study that examined the effectiveness of a school-based resilience intervention in Grade 9 students (aged 14-15 years) in 32 Australian secondary schools (20 intervention; 12 control). A three-year intervention involving school staff implementation of programs and resources that addressed individual and environmental resilience protective factors was implemented. Outcome data was collected online and included the primary outcome of pain frequency, and secondary outcomes of problematic pain, pain intensity, pain selfefficacy and resilience protective factors (individual and environmental). Results: Data from 1831 students (intervention $=1201$; control $=630$ ) were analysed. No significant differences were found between intervention and control for primary: pain frequency: OR 0.98 (95\%CI: 0.73 to 1.31 , $\mathrm{p}=0.88$ ) or secondary outcomes: problematic pain prevalence: OR 0.93 ( 0.74 to $1.18, \mathrm{p}=0.54)$; pain intensity: $\mathrm{MD}-0.09(-0.34$ to $0.16, \mathrm{p}=0.47)$; pain self-efficacy: MD $0.61(-1.38$ to $2.61, \mathrm{p}=0.53)$; individual resilience: MD -0.02 (-0.11 to $0.07, \mathrm{p}=0.65)$; environmental resilience: MD 0.04 (0.07 to $0.14 \mathrm{p}=0.48$ ). Post hoc mediation analyses showed resilience was not a mediator of pain frequency. Conclusions: The school-based resilience intervention was not effective in reducing pain frequency, problematic pain, pain intensity, or improving the pain self-efficacy or resilience in secondary school students. The results suggest resilience, as defined and measured in this study, may not be a suitable intervention target for reducing pain frequency in general adolescent populations.

CORRESPONDING AUTHOR: Rebecca Hodder, Hunter New England Population Health/The University of Newcastle, rebecca.hodder@hnehealth.nsw.gov.au

\section{S 15}

ADOLESCENT HEALTH-RISK BEHAVIORS IN A SOCIAL CONTEXT: INTERNATIONAL PERSPECTIVES

Annette La Greca ${ }^{1}$

${ }^{1}$ University of Miami- USA

Adolescence is a key development period for the initiation and maintenance of health-risk behaviors that have strong implications for adult 
morbidity and mortality. Behaviors established in adolescence, such as drug and alcohol use, sedentary behavior, and sleep problems, can persist into adulthood and are linked to a variety of health-related problems, including cardiovascular disease, obesity, and injuries. Further, these health-risk behaviors often occur in the context of peers. As adolescents assume greater independence from family members, peer relations become a key source of support for adolescents' health and well-being. Yet, peer relations can also represent a source of stress and contribute to the occurrence of risky health behaviors. In contrast, parents who remain engaged in adolescents' lives, as through monitoring of their activities, typically have better health-risk profiles. The dual purpose of this symposium is to (a) provide an international perspective on adolescent health risk behaviors and (b) examine some of the social (i.e., peer and parental) factors that contribute to or mititgate adolescents' health risk. The first presentation, by Cumsille and Martinez, examines the interplay of alcohol and marijuana use among Chilean adolescents and the role of parental monitoring in reducing such substance use. The second presentation, by La Greca and colleagues, examines the potentially contrasting roles of peer-related cybervictimization and peer support in predicting U.S. adolescents' sedentary behaviors. The third presentation, by Brodar and colleagues, examines the role of peer processes, such as peer pressure and conflict with friends, on U.S. adolescents' sleep problems during the transition from primary to secondary school. The final presentation, by Sivertsen and colleagues, examines associations between bullying, sleep, and academic outcomes among Norweigian adolescents. Overall, across the presentations and diverse international samples, findings illustrate the roles of peers and parents in promoting or mitigating adolescents' health risks. Health intervention and promotion programs that address both peer and family influences have the best chance of making a lasting impact on adolescents' lives and health.

\section{S15.1}

CYBER VICTIMIZATION: A KEY RISK FACTOR FOR ADOLESCENT GIRLS' SEDENTARY BEHAVIOR?

Annette La Greca ${ }^{1}$, Naomi Tarlow ${ }^{1}$, Kaitlyn Brodar ${ }^{1}$, BreAnne Danzi ${ }^{1}$ ${ }^{1}$ University of Miami- USA

Introduction \& Purpose. Cyber victimization (CV), or being the target of peers' aggression via technology, is a critical problem affecting adolescents' well-being. CV is associated with psychological risk among adolescents (e.g., symptoms of depression and anxiety), and may affect girls more than boys. Yet, little is known about the health impact of CV; this gap is surprising since adolescents are frequent users of technology, especially for texting and social networking. Thus, we examined the impact of CV on adolescents' sedentary behavior (a risk factor for obesity) (Aim 1), and also whether peer support moderated this association (Aim 2). We focused on adolescents from predominantly Latino/Hispanic backgrounds, as rates of obesity in the US are high among such youth. Method. Adolescents ( $\mathrm{N}=523, \mathrm{M}$ age $=14.23$ years, 58\% girls) completed standardized measures of $\mathrm{CV}$ and peer support; sedentary behavior was assessed by items from the Youth Risk Behavior Survey (e.g., TV viewing, non-school related screen time). Regressions examined associations between PV, PS, and sedentary behavior. Results. Analyses showed that girls reported more peer support than boys $(\mathrm{t}=-3.12, \mathrm{p}=.002)$; no gender differences were found for CV or sedentary behavior ( $\mathrm{ps}>.510$ ). Thirty-six percent of adolescents reported watching two or more hours of TV per school day, and $57 \%$ spent three or more hours on video games or the computer. For Aim 1: Greater CV was associated with more sedentary behavior in girls $(b=.90, \mathrm{SE}=.35, \mathrm{t}=2.59, \mathrm{p}=.010)$ but not in boys $(\mathrm{b}=.05$, $\mathrm{SE}=.31, \mathrm{t}=.17, \mathrm{p}=.867)$. For Aim 2: Peer support was unrelated to sedentary behavior $(\mathrm{p}=.456)$, and did not moderate the impact of $\mathrm{CV}$ on sedentary behavior $(\mathrm{p}=.508)$ for boys and girls. Conclusions. For girls, the stress of $\mathrm{CV}$ experiences may lead to greater sedentary behavior, which in turn puts girls at risk for health problems. Even the availability of supportive friends did not mitigate the stressful impact of CV for girls.
Perhaps girls experiencing CV withdraw from social activities and spend more time in solitary and sedentary tasks. Alternatively, sedentary girls, who also may be more overweight, might be $\mathrm{CV}$ targets. Further research should examine CV's impact on girls' health over longer time periods and with more comprehensive health measures.

CORRESPONDING AUTHOR: Annette La Greca, University of Miami, alagreca@miami.edu

\section{S15.2}

PARALLEL GROWTH IN ALCOHOL AND MARIHUANA USE IN CHILEAN ADOLESCENTS

Patricio Cumsille ${ }^{1}$, Maria Loreto Martínez ${ }^{1}$

${ }^{1}$ Pontificia Universidad Católica de Chile- Chile

Introduction and Purpose: Alcohol and marihuana use has increased in Chilean adolescents over the last 2 decades. The increase in Marihuana use has been particularly steep over the last decade. Our aim was to examine the parallel growth in alcohol and marihuana use over a 4-year period, and examine the predictive power of the initial level of use in each substance over the growth in the use of the other. We also examined the influence of parental monitoring over the growth in both substances. Methods: A multi-cohort sample of high school Chilean adolescents $(\mathrm{N}=1660, \mathrm{M}$ age $=14.96$ years, $52.8 \%$ girls) was followed annualy for 4 years. The students reported their alcohol and marihuana use (Likert scale with anchors $1=$ never, $7=$ more than once a week) over the entire period, and the level of parental monitoring in the second wave of measurement. A parallel latent growth curve analysis was used to model the initial level and change in alcohol and marihuana use over the entire period. Initial level of alcohol and marihuana use were modelled as predictors of change in rates of marihuana and alcohol use and parental monitoring at wave 2. Parental monitoring was also used as a predictor of changes in the trajectories of alcohol and marihuana use. Results: As expected, the initial level of alcohol use was higher than of marihuana ( $\mathrm{M}=3.089$ versus $\mathrm{M}=1.468)$ and both alcohol and marihuana use increased over the 4-year period ( $\beta=.404, p<.001, \beta=.235, p<.001$, respectively). Initial level of alcohol and marihuana $(\mathrm{r}=.67, \mathrm{p}<.001)$ and rate of change in alcohol and marihuana $(r=.47, p<.001)$ were related. Initial level of alcohol use was directly related to the increase in marihuana $(\beta=.083, \mathrm{p}<.001)$ but initial level of marihuana use was inversely related to the rate of change in alcohol use $(\beta=-.157, p<.001)$. Initial level of alcohol and marihuana use did not predict subsequent parental monitoring; monitoring was inversely related to the changes in alcohol use $(\beta=-.157, p=.015)$, and marginally related to changes in marihuana use $(\beta=-.039, \mathrm{p}=.058)$. Conclusions: Changes in alcohol and marihuana use in adolescence are related and level of use in both substances are related to rates of change in the other substance. Consistent with the gateway hypothesis, high initial level of alcohol use predicts a steeper increase in marihuana use, but the opposite is not true.

CORRESPONDING AUTHOR : Patricio Cumsille, Pontificia Universidad Católica de Chile, pcumsill@uc.cl

\section{S15.3}

THE LINK BETWEEN ACADEMIC OUTCOMES, BULLYING, AND SLEEP PROBLEMS IN ADOLESCENCE

Borge Sivertsen ${ }^{1}$, Kyrre Breivik ${ }^{2}$, Mari Hysing ${ }^{2}$

${ }^{1}$ Norwegian Institute of Public Health- Norway

${ }^{2}$ Uni Research Health- Norway

Introduction and Purpose: Previous research on adolescents has linked bullying to sleep problems, but the nature of the association remains unclear. Also, the association between bullying and sleep, and academic outcomes has received little attention. Thus, the aim of the current study was twofold: 1) to determine whether involvement in bullying as a victim 
or bully, was associated with a higher risk of sleep problems among high school students in Norway; and 2) to examine the separate and joint contribution of bullying and sleep problems to school absence and academic grades. Methods: A large population based study in Hordaland county in Norway conducted in 2012, the ung@hordaland study, surveyed 10,220 adolescents aged 16-18 years (54\% girls) about bullying and sleep. Bullying was assessed by the Revised Olweus Bully/Victim Questionnaire, while self-reported sleep measures provided information on sleep duration and DSM-5 insomnia. Academic outcomes were obtained from official administrative registries. Results: In all, $2.2 \%$ of the adolescents $(n=206)$ reported being victims of bullying, while being a bully was reported by $1.5 \%(\mathrm{n}=142)$. Compared to the sleep duration of adolescents not involved in bullying (6hrs 26 mins), both victims and bullies had significantly shorter sleep duration ( $5 \mathrm{hrs} 45 \mathrm{mins}$ and $5 \mathrm{hrs}$ 33 mins, respectively; $\mathrm{p}<.001)$. Both victims $(36.5 \%)$ and bullies $(27.2 \%)$ also had a significantly higher prevalence of insomnia $(\mathrm{P}<$ $.001)$, compared to their peers (18.2\%). Both insomnia and bullying were significantly associated with lower grade point average (GPA) and more school absence. Adolescents reporting both insomnia and bullying had significantly higher risk of poor academic outcomes $(\mathrm{OR}=3.09 ; 95 \% \mathrm{CI}$ : $1.82-5.25)$, compared to reporting either insomnia $(\mathrm{OR}=1.51 ; 95 \% \mathrm{CI}$ : $1.32-5.25)$ or bullying alone $(\mathrm{OR}=2.36 ; 95 \% \mathrm{CI}: 1.64-3.42)$. Conclusions: We found that both bullying and sleep problems were both prevalent and strongly associated in adolescence. The significant effect of bullying and sleep problems on poor academic outcomes emphasizes the importance of assessing and addressing these risk factors during this important period of transition.

CORRESPONDING AUTHOR: Borge Sivertsen, Norwegian Institute of Public Health, borge.sivertsen@fhi.no

\section{S 15.4}

PEER PROBLEMS CONTRIBUTE TO SLEEP LOSS IN ADOLESCENTS TRANSITIONING TO HIGH SCHOOL

Kaitlyn Brodar ${ }^{1}$, Annette M. La Greca ${ }^{1}$, Naomi Tarlow ${ }^{1}$, BreAnne A. Danzi $^{1}$

${ }^{1}$ University of Miami- USA

Introduction and Purpose: The transition to secondary/high school is a critical time for adolescents to develop and maintain peer relationships. Poor quality peer relationships may have a negative impact on health and well-being. Specifically, little is known about how negative peer relationships affect adolescents' sleep. Our aim was to examine two common peer-related stressors (peer pressure and conflict with a close friend) as factors that could contribute to sleep difficulties; this may occur as many adolescents communicate with friends via text message or social media around bedtime, which could intrude on sleep. We also examined whether adolescents who tend to ruminate have more sleep problems, as they may struggle to stop thinking about relationship problems at night. Methods: Adolescents entering the 9 th grade $(\mathrm{N}=521, \mathrm{M}$ age $=14.23$ years, $56.8 \%$ girls, $89.5 \%$ Hispanic/Latino/a) reported on peer pressure, conflict with friends, rumination tendencies, and sleep, using items from the Adolescent Stress Questionnaire, the Network of Relationships Inventory, the Perseverative Thinking Questionnaire, and the Youth Risk Behavior Survey, respectively. Multiple regression analyses, controlling for sex, tested whether peer relationship problems significantly predicted sleep loss, and if rumination moderated this relationship. Results: Greater peer problems and more rumination were associated with more difficulty sleeping $(\mathrm{R} 2=.19, \mathrm{~F}(4,518)=30.77, \mathrm{p}<.001)$. Peer pressure $(\beta=.19, p<.001)$, friendship conflict $(\beta=.08, p<.05)$, and rumination $(\beta=.32, p<.001)$ each uniquely predicted variance in sleep problems. Neither gender nor rumination moderated the relationship between peer stress and sleep problems. Conclusions: Both peer relationship problems and rumination tendencies contribute to adolescents' sleep loss during the transition to high school. Adequate sleep is critical for adolescent health and successful academic functioning. Sleep interventions for adolescents might incorporate strategies for reducing rumination and enhancing peer support. Future research should assess the bi-directional relationship between these variables over time and incorporate additional measures of sleep disturbance, such as actigraphy.

CORRESPONDING AUTHOR: Kaitlyn Brodar, University of Miami, kbrodar@miami.edu

S16

\section{BEATING THE HIV EPIDEMIC}

Deborah Jones ${ }^{1}$

${ }^{1}$ University of Miami Miller School of Medicine- USA

HIV is a manageable disease with an essentially normal lifespan provided those affected remain in care. However, $25 \%$ of HIV-infected individuals are non-adherent to treatment, intermittently retained in care, or lost to follow up, the consequences of which are premature morbidity and mortality and increased likelihood of HIV transmission to others. Patients are also vulnerable to the comorbidities of HIV, e.g., cardiovascular disease, substance use, depression, accentuating the need to identify, engage, treat and retain HIV-infected patients. This symposium addresses challenging patients in Argentina, the USA and South Africa. Applying the ecological model, panel presentations examine individual, interpersonal, normative, and environmental factors confronting HIV affected individuals and explore approaches to overcoming barriers to care, stimulating engagement, increasing retention and optimizing health outcomes. Are large scale programs designed to get patients into care effective? Can providers work together with struggling HIV-infected patients to motivate their engagement in care? How can providers track and treat HIV-infected women in rural communities to optimize neonatal and maternal health? This panel will address challenges to early enrollment in care (nationwide private clinic system, Argentina), interventions to motivate engagement and retention (public and private clinic and hospital based clinical trial, Argentina), the impact of substance use on retention in interventions (intervention in urban USA) and factors that reduce retention and care during pregnancy (community clinic-based clinical trial, South Africa). Presenters will discuss strategies to improve engagement and retention outcomes. Presenters: O Sued, Argentina "Predictors of engagement and retention among challenging patients in Argentina: an opportunity for intervention" D Cecchini, Argentina "Late presentation and virologic suppression in HIV-infected population of an ambulatory HIV care center in Buenos Aires, Argentina" M Kumar, USA "Retention of HIV-infected and cocaine using individuals at risk of cardiovascular disease" D Jones, USA "Challenges to engagement and retention of HIVinfected perinatal women in rural South Africa" SM Weiss, USA "Beating the HIV Epidemic."

\section{S16.1}

RETENTION OF HIV-INFECTED AND COCAINE USING INDIVIDUALS AT RISK OF CARDIOVASCULAR DISEASE

Violeta Rodriguez ${ }^{1}$, Suat Babayigit ${ }^{1}$, Arnetta Phillips ${ }^{1}$, Stephen Weiss ${ }^{1}$, Mahendra Kumar ${ }^{1}$

${ }^{1}$ University of Miami- USA

Introduction \& Purpose. Engagement and retention in care can reduce the risk of cardiovascular disease (CVD), and HIV infection and cocaine use have been associated with CVD risk. This study examined drop out and an intervention to reduce CVD risk in men and women with subclinical atherosclerosis. Methods. Participants were HIV-infected and uninfected, using and not using cocaine (HIV-C- $\mathrm{n}=28$; HIV $+\mathrm{C}-\mathrm{n}=6$; HIV $+\mathrm{C}+\mathrm{n}=$ 5 ; HIV- $\mathrm{C}+=20$ ), assessed by carotid ultrasound to quantify carotid intima-media thickness (IMT) and plaques as precursors to subclinical atherosclerosis. Participants with plaques or IMT $>=2.0 \mathrm{~mm}$ attended a 
standard of care plus motivational interviewing visit addressing CVD, IMT, blood pressure and BMI results, followed by a 12 month health behavior assessment. Results. Those dropping out were compared with those not discontinuing. Greater depression, worse eating habits, and higher total cholesterol were associated with attending a follow-up visit ( $\left.\mathrm{p} \_\mathrm{s}<0.01\right)$. In multivariable logistic regression, higher cholesterol $(\mathrm{AOR}=1.04[1.01,1.06])$ and being HIV+ C- status $(\mathrm{AOR}=9.09$ $[1.02,81.21]$, ref $=$ HIV- C-) were associated with attending a followup visit after the SOC+ visit, controlling for depression and eating habits. HIV-infected individuals were less likely to drop out of care, while individuals with lower cholesterol were more likely to be HIV-uninfected and drop out of care. Conclusions. Despite risk of CVD, some individuals may minimize risk, while HIV-infected individuals may be more motivated and actively engaged in care, more habituated to routine HIV clinic visits and testing, and have more concerns about managing their health status. Among HIV-infected individuals, results are promising regarding engagement and follow up and have important implications for interventions for those identified as being at increased CVD risk. Motivational interviewing be a useful adjunct to behavioral interventions regarding CVD risk among HIV-infected individuals. NIH grant R01DA034589

CORRESPONDING AUTHOR: Violeta Rodriguez, University of Miami,vjrodriguez@med.miami.edu

\section{$\mathrm{S} 16.2$}

PREDICTORS OF ENGAGEMENT AND RETENTION AMONG CHALLENGING PATIENTS IN ARGENTINA: AN OPPORTUNITY FOR INTERVENTION

Omar Sued ${ }^{1}$, Suat Babayigit ${ }^{2}$, Lissa Mandell ${ }^{2}$, Tae Lee ${ }^{2}$, Violeta Rodriguez $^{2}$, Stephen Weiss ${ }^{2}$, Diego Cecchini ${ }^{3}$, Isabel Cassetti ${ }^{3}$

${ }^{1}$ Fundación Huésped

${ }^{2}$ University of Miami Miller School of Medicine- USA

${ }^{3}$ Helios Salud- Argentina

Introduction \& Purpose. Argentina offers no cost treatment for HIV, however, not all patients are engaged or retained in care. COPA2 seeks to optimize health; clinics participating were randomized to experimental (provider motivational interviewing) or control (standard of care) conditions. This preliminary review examined predictors of engagement and retention among patients previously lost to care. Methods. Participants were from 6 urban Argentine clinics. Engagement was defined as office visit attendance and pharmacy pickups; retention was 6-month follow-up; active patients attended follow-up; missing patients did not. Results. Participants $(\mathrm{N}=198)$ were $79 \%$ active, $17 \%$ missing, and $4 \%$ deceased. Participants' age was $\mathrm{M}=39+11.22 ; 56 \%$ were male. Comparing retention categories, active participants reported greater perceived stigma $(\mathrm{M}=6.12, \mathrm{p}=0.011)$. Participants deceased following enrollment reported more treatment motivation ( $\mathrm{p}=0.022)$, but had higher viral load $(\mathrm{M}=5.16$ vs. Mact=4.39, Mmiss=4.78, $\mathrm{p}=0.006$ ), more missed appointments $(\mathrm{F}=4.29, \mathrm{p}=0.015)$, and were more likely to abuse drugs $(\mathrm{p}=0.013)$. Missing participants had more missed pharmacy pick-ups (AOR= $4.419, \mathrm{p}<0.001)$. Public clinic attendees were more adherent $(\mathrm{AOR}=.348, \mathrm{p}=0.045)$ and adherence was lower among those with emotional problems $(\mathrm{OR}=.171, \mathrm{p}<0.001)$. Experimental participants $(73 \%)$ were more adherent by pharmacy pick-up than controls $(27 \%)$ $(\mathrm{OR}=1.549, \mathrm{p}=0.031)$. Conclusions. Results highlight the need for repeated efforts to re-engage lost patients, given the consequences of loss to care and the impact of drug use on potential re-engagement despite motivation to improve. Integration of motivational interviewing and mental health assessment and treatment in HIV primary care could be important contributors to active engagement in care. NIH grant R01MH110242.

CORRESPONDING AUTHOR : Omar Sued, Fundación Huésped' omar.sued@huesped.org.ar
S16.3

LATE PRESENTATION AND VIROLOGIC SUPPRESSION IN HIVINFECTED POPULATION OF AN AMBULATORY HIV CARE CENTER IN BUENOS AIRES, ARGENTINA

Diego Cecchini ${ }^{2}$, Edgardo Bottaro ${ }^{2}$, Suat Babayigit ${ }^{1}$, Paula Rodriguez Lantorno $^{2}$, Isabel Cassetti ${ }^{2}$

${ }^{1}$ University Of Miami Miller School Of Medicine- USA

${ }^{2}$ Helios Salud- Argentina

Introduction:In Argentina, a high percentage of HIV-infected individuals do not enter the healthcare system until late in the course of infection. In this population, it is mandatory to achieve virologic suppression in order to reduce morbidity and prevent further transmission of HIV. This study aimed to evaluate trends in late presentation and virologic suppression among patients supported by Argentina's leading HIV healthcare clinic in Buenos Aires city metropolitan area within the national Health Maintenance Organization (HMO)-like system. Method:Electronic medical records for patient attendance and pharmacy records were collected from the healthcare clinic. An adherence team provided regular follow up of patients identified as medication noncompliant, based on pharmacy records, and requiring intervention. Retrospective analysis of admissions of newly diagnosed patients and their virologic outcomes at 12 months were examined using electronic medical records (InfHos); period 20132016.The following definitions applied:1) Late presentation (LP):presenting for care with a CD4 count $<350$ cell/uL; 2) Advanced HIV disease $(\mathrm{ADH})$ : presenting for care with a CD4 count $<200 \mathrm{cell} /$ $\mathrm{uL}$.Data were processed using R.Results:There was an increasing trend in admissions from 314 in 2013 to 572 in 2016 ( $p=0.04$ ). Examining LP and ADH among those admitted, the proportion of neither LP nor ADH changed over time ( $p=0.07$ and $p=0.12$, respectively). An increase in 12 month virologic suppression was observed among newly admitted patients: from $82.4 \%$ in 2013 to $91.6 \%$ in 2016 ( $\mathrm{p}=0.03$ ). Among LP and $\mathrm{ADH}$, virologic suppression remained above at levels $>85 \%$ for the whole period, were variable between years $(p=0.01$ and $p=0.03$, respectively).Conclusion: Virological suppression improved among the whole population. Despite increasing admissions, no change in the proportion of LP was observed, indicating an urgent need of campaigns promoting HIV diagnosis. Of note, high levels of virologic suppression were observed in these patients during the whole period. Reduction of LPs should be considered a high priority within the national AIDS policy and implementation of adherence teams within the different healthcare subsections could contribute to improved virological outcomes.

CORRESPONDING AUTHOR: Diego Cecchini, Helios Salud, dcecchini@heliossalud.com.ar

\section{S16.4}

CHALLENGES TO ENGAGEMENT AND RETENTION OF HIVINFECTED PERINATAL WOMEN IN RURAL SOUTH AFRICA Deborah Jones ${ }^{1}$, Violeta Rodriguez ${ }^{2}$, Stephen Weiss ${ }^{1}$, Karl Peltze ${ }^{1}$

${ }^{1}$ University Of Miami Miller School Of Medicine- USA

${ }^{2}$ University of Georgia- USA

Introduction.The World Health Organization recommends that optimal perinatal care consists of a minimum of eight perinatal care visits. Despite the critical role of perinatal care and adherence within the South African prevention of MTCT (PMTCT) guidelines, women living with HIV (WLHIV) in rural South Africa may access care only once during pregnancy, thereby representing the only opportunity for perinatal and PMTCT psychoeducation, which can also reduce the risk of vertical transmission of HIV. This study aimed to identify predictors of perinatal retention among pregnant WLHIV in rural South Africa. It was theorized that results could inform programmatic decisions designed to optimize perinatal care among HIV-infected women and their neonates. Methods. Participants $(\mathrm{N}=1161)$ were WLHIV recruited from antenatal care clinics 
in rural SA. Women were 2-30 weeks pregnant at entry $(17.52+5.77)$; baseline demographics, depression, intimate partner violence, male involvement, adherence, and knowledge were assessed. Women and neonatal mortality were assessed at 12 months postpartum. Retention was defined as $>1$ visit during this period. Results. Women were aged $\mathrm{M}=28 \pm$ 6 years; $23.0 \%$ [95\% CI 20.7, 25.2]) were lost post-baseline. Maternal mortality rates were $0.2[0.0,0.5]$, and neonatal mortality, $9.3[7.7,10.9]$. One-third had completed $>=12$ years of education; half had monthly income of $\sim \mathrm{US} \$ 49$.In logistic regression, decreased depression $(\mathrm{AOR}=$ $0.69, \mathrm{p}=0.045]$ and neonatal mortality $(\mathrm{AOR}=0.01, \mathrm{p}<0.001]$, and increased education $(\mathrm{AOR}=1.68, \mathrm{p}=0.014]$, medication adherence $(\mathrm{AOR}=1.47, \mathrm{p}=0.045]$, and baseline PMTCT knowledge predicted retention in PMTCT care (all ps $<0.05$ ), controlling for HIV-related stigma and infant HIV status. Conclusions. Findings support provision of psychoeducational and mental health services in perinatal rural South African clinics to optimize retention. Rates of infant mortality should be examined and may reflect home deliveries, mixed breastfeeding, birth complications, maternal nutrition and limited access to clean water. Future research should explore brief interventions to treat depression in the perinatal setting, which may enhance engagement and retention in care among pregnant women. NIH grant R01HD058481

CORRESPONDING AUTHOR: Deborah Jones Weiss, University Of Miami Miller School Of Medicine, DJONES@MED.MIAMI.EDU

\section{S25 \\ SOCIAL INEQUALITY IN HEALTH - THE SCANDINAVIAN PARADOX \\ Espen Dahl ${ }^{1}$ \\ ${ }^{1}$ Oslo Metropolitan University - Norway}

Introduction Scandinavian countries are known for their ability to reduce income inequality without compromising economic growth. It has been a tradition in Scandinavia to not only support marginalized citizens but also take shared responsibility for preventing the negative effects of a socioeconomic development that generates wealth and health for some and poverty and poor health for others. Welfare policies have focused on equity through several decades but only more recently on health equity. It has turned out to be a challenge to translate small inequalities in wealth into small inequalities in health - the question is why? Mortality in different socioeconomic groups are the most comparable measure of inequality across counties. In Scandinavia, the mortality rates decline with higher education for both men and women. Socio-economic determinants in childhood influence on health literacy, access to education and healthcare and the risk of getting a chronic disease later in life. In adulthood, the effect of social position on health is mediated through differences in employment, working and living conditions, income, social support and health behaviors. In addition, unequal consequences of disease exist in terms of the opportunities of getting treatment, rehabilitation and further in terms of survival, disability and quality of life. Lack of integration and inequities in health care might contribute. Perspectives The presentations in this symposium will give examples of the scope of social inequality in health in Denmark, Sweden, Norway and Finland as well as reflect on possible mechanisms. Based on the presentations we will discuss what can be learned from the Nordic experiences of tackling health inequalities by addressing several different policy areas locally.

S25.1

MULTIPLE ADVERSE CHILDHOOD EXPERIENCES, ADULT SOCIOECONOMIC POSITION AND RISK OF WORK DISABILITY Jussi Vahtera $^{1}$

${ }^{1}$ Section of Behavioral Medicine, Finnish Society of Social MedicineDenmark
Background: Work disability, with musculoskeletal and mental disorders being the most common causes, represents a major economic burden to the society. Early life adversities and later life socioeconomic characteristics are associated with increased rates of work disability. Objectives: To estimate the extent to which the association between multiple adverse childhood experiences (ACEs) and cause-specific work disability is mediated by adulthood education, occupation and neighbourhood disadvantage. Methods: Data were from 40181 employed adults from two cohorts: the Finnish Public Sector (2008-2011) and the Health and Social Support (1998-2013) studies. ACEs were reported at baseline. Information for work disability was obtained from national registers. Results: Age and sex-adjusted associations between ACE and work disability due to musculoskeletal disorders $(\mathrm{HR}=1.34,95 \% \mathrm{CI} 1.14-3.26)$ and other physical diseases ( $\mathrm{HR}=1.57,95 \% \mathrm{CI} 1.30-1.90)$ were partially attributable to socioeconomic factors in adulthood. In contrast, the association between multiple ACEs and disability due to mental disorders $(\mathrm{HR}=2.57$, 95\% CI 2.02-3.26) was largely independent of those adulthood factors. Conclusions: People with multiple ACEs have less favourable socioeconomic profile contributing to their increased risk of work disability due to musculoskeletal and other physical diseases. The excess work disability due to mental disorders was independent of adulthood socioeconomic factors.

CORRESPONDING AUTHOR: Jussi Vahtera, Section of Behavioral Medicine, Finnish Society of Social Medicine, jussi.vahtera@utu.fi

\section{$\mathrm{S} 25.2$}

DOES SOCIOECONOMIC POSITION INFLUENCE THE RISK OF CARDIOVASCULAR EVENTS FOLLOWING PROSTATE CANCER?

Ida Rask Moustsen ${ }^{1}$, Anne Sofie Friberg ${ }^{1}$, Signe Benzon Larsen ${ }^{2} 3$, Susanne Oksbjerg Dalton ${ }^{1}$.

${ }^{1}$ Danish Cancer Society Research Center- Denmark

${ }^{2}$ Department of Oncology- Denmark

${ }^{3}$ Copenhagen University Hospital Rigshospitalet- Denmark

Introduction and purpose: Men with low socioeconomic position (SEP) experience higher mortality after prostate cancer compared to men with higher SEP. This may be due to difference in stage at diagnosis, access to optimal treatment or preexisting comorbidity by socioeconomic group. Currently, we do not know if SEP is associated with risk of late effects such as cardiovascular events after prostate cancer, however it could explain more of the observed inequality in survival of this growing population of cancer survivors. In this study, we investigate whether SEP as measured by education influences the development of severe cardiovascular disease (CVD) following prostate cancer and whether potential socioeconomic differences in the risk for CVD may be explained by pre-cancer lifestyle, comorbidity and treatment. Method: This is a cohort study of 1546 prostate cancer patients diagnosed among the 25436 men in the Danish prospective Diet, Cancer and Health study. Participants completed questionnaires and anthropometry at study enrollment. We retrieved information on education, income, and CVD-related hospital contacts from National Danish Registries, excluding men with CVD prior to prostate cancer diagnosis. Data on stage, primary treatment and anthropometry was collected from medical records. Acute myocardial infarction (AMI), stroke and heart failure (HF) were the outcomes of interest. We calculated hazard ratios for the combined CVD outcomes according to SEP using Cox proportional hazards models. Results: 182 of the 1546 men with prostate cancer experienced AMI, stroke or HF during followup. Preliminary unadjusted analyses showed a more than twofold risk of CVD in men with 12 years of education. Adjusting for smoking and BMI reduced the HRs to statistically significant 1.96 and 1.92 for medium or short compared to long education. Conclusion: Our study indicates that men with short education have a twofold increased risk of severe CVD following prostate cancer compared to men with higher education. The 
association is partly mediated by smoking and BMI. Further analyses will determine whether physical activity, preexisting hypertension, hypercholesterolemia and diabetes, and treatment further mediate the association.

CORRESPONDING AUTHOR: Ida Rask Moustsen, Danish Cancer Society Research Center, idamou@cancer.dk

\section{S25.3}

MECHANISMS IN SOCIAL INEQUALITY IN HEALTH

Ingelise Andersen ${ }^{1}$, Helene Nordahl ${ }^{2}$, Theis Lange ${ }^{1}$, Jimmi Mathiesen ${ }^{1}$ 3 , Finn Diderichsen ${ }^{13}$

${ }^{1}$ University of Copenhagen, Department of Public Health- Denmark.

${ }^{2}$ AstraZeneca, Nordic Baltic MC, Medical \& Regulatory Nordic Baltic, Södertälje, Sweden- Denmark

${ }^{3}$ Section of Social Medicine- Denmark

Introduction \& Purpose Social gradients in health are evident across countries. To intervene on such socioeconomic differences in health, it is important to understand the mechanisms that work to create and uphold them. The mechanisms of differential exposure and differential vulnerability have been proposed as pathways that can link an individual's social position to disease risk. Differential exposure corresponds to the effect of socioeconomic position on a given health outcome being mediated through a given risk factor. Differential vulnerability corresponds to an interaction between the risk factor and socioeconomic position on the outcome. The purpose here is to give examples of those analyses addressing those mechanisms illustrated by the educational inequality in all-cause mortality. We want 1) to quantify how much of the educational inequality was mediated by smoking. 2) To separate the effects of differential exposure and differential vulnerability to smoking simultaneously Methods By means of methods using the counterfactual approach we use marginal structural models and a three-way effect decomposition and estimate the mediated proportion of smoking and clarified the role of the interaction between smoking and educational exposure. Results In terms of Differential exposure: we saw that $22 \%$ is attributed to an indirect effect through smoking. Also $9 \%$ of the additional cases among low educated vs high is explained by different levels of smoking between the two educational groups. In terms of Differential vulnerability we saw that $13 \%$ is attributed to mediated interaction between education and smoking. This means that $13 \%$ is attributed to differential vulnerability in the two eductional groups. The mediated effect through smoking on all cause mortality was $(22 \%+13 \%=35 \%)$ Conclusions Differential exposure and vulnerability should be addressed simultaneously, as these mechanisms are not mutually exclusive and may operate at the same time. (Epidemiology 2014;25: 389-396)

CORRESPONDING AUTHOR: Ingelise Andersen, University of Copenhagen, Department of Public Health, inan@sund.ku.dk

\section{$\mathrm{S} 25.4$}

SMOKING AND ALCOHOL USE CONTRIBUTE TO INCOME DIFFERENCES IN LIFE EXPECTANCY - EVIDENCE USING DANISH, FINNISH, NORWEGIAN AND SWEDISH REGISTER DATA

Olof Östergren ${ }^{1}$, Pekka Martikainen ${ }^{2}$, Lasse H. Tarkianien ${ }^{2}$, Jon Ivar Elstad $^{3}$, Henrik Brønnum-Hansen ${ }^{4}$

${ }^{1}$ Department of Public Health Sciences, Stockholm University - Sweden

${ }^{2}$ Population Research Unit, University of Helsinki - Finland

${ }^{3}$ NOVA, Oslo Metropolitan University - Norway

${ }^{4}$ Department of Public Health, University of Copenhagen - Denmark

Despite being comparatively egalitarian welfare states, the Nordic countries have not been successful in reducing health inequalities. Previous studies have suggested that smoking and alcohol contribute to this pattern. Few studies have focused on variation in smoking and alcohol-related mortality within the Nordic countries. We assess the contribution of smoking and alcohol to differences in life expectancy between countries and differences in life expectancy by income quintile within countries.

We collected data from registers in Denmark, Finland, Norway and Sweden. The data covered men and women aged 25-79 during 19952007 with register-based information on income. Alcohol-related mortality was estimated on the basis of underlying and contributory causes of death on individual death certificates, and smoking-related mortality on the basis of an indirect method that used lung cancer mortality as an indicator for the population-level impact of smoking on mortality.

The results show that about $40 \%$ to $70 \%$ of the between country differences in life expectancy in the Nordic countries can be attributed to smoking and alcohol consumption. The contribution of alcohol was smaller among women due to lower levels of alcohol-related mortality. Alcohol and smoking-related mortality also made substantial contributions to income differences in life expectancy within countries. The magnitude of the contributions ranged from about $20 \%$ to $55 \%$.

The findings suggest that significant social differences in smoking and alcohol use are part of the explanation as to why the Nordic welfare state have not been able to reduce inequalities in health. There are substantial differences in the patterns of smoking- and alcohol-related mortality by gender and between the Nordic countries.

CORRESPONDING AUTHOR: Olof Östergren, Department of Public Health Sciences, Stockholm University, olof.ostergren@su.se

\section{S25.5}

TOWARDS AN EXPLANATION OF THE SCANDINAVIAN PARADOX? THE CASE OF NORWAY

Espen Dahl

${ }^{1}$ Oslo Metropolitan University- Norway

Introduction The presentation aims at explaining part of the "Scandinavian Paradox" by an in-depth examination of the nature and development of social and behavioral determinants of health in Norway over the past 30 years. Methods Statistical information on social inequalities in morbidity and mortality over the past 30 years was compiled. This was supplemented with relevant findings from comparative research carried out in European countries. Information on (changes in) the social distribution of a number of social and behavioral determinants of health was also collected and assessed. These include childhood living conditions, economic resources, labour market conditions, physical and psychosocial working conditions, use of welfare benefits and services, and health behaviours. Results The analysis indicates that albeit mortality levels have fallen markedly and life expectancy has increased over the past decades, social inequalities in mortality and life expectancy have widened. Educational inequalities in mortality are at about the same level as in other rich European welfare states, also in less egalitarian ones that have less comprehensive and generous welfare systems. The assessment of the empirical evidence indicates that inequality in many of the social determinants of health persists, e.g. inequalities in drop-out from high school and in working conditions, and in several instances even increase, e.g. income, wealth and relative child poverty. Research also shows that educational inequalities in smoking have been particularly large in Norway as compared with other countries. The smoking epidemic seems to follow the pattern of «diffusion of innovations». Now, towards the end of the epidemic, the educational inequalities in smoking are about to decline. Conclusions In Norway there is an «infrastructure» of inequalities in material and immaterial goods, resources and exposures. This persisting infrastructure of inequality, in combination with inequalities in smoking behaviors, help explain the international position and the increasing trend in inequality in mortality in Norway.

CORRESPONDING AUTHOR: Espen Dahl, Oslo Metropolitan University, espen.dahl@hioa.no 


\section{INVITED SYMPOSIUM}

S17

THE EFFECTS OF STRESS FACTORS AND INTERVENTIONS IN CANCER: BIOBEHAVIORAL MECHANISMS

Susan Lutgendorf ${ }^{1}$

${ }^{1}$ University of Iowa - USA

There is emerging evidence for the effects of stress factors and stress modulating interventions on health outcomes across the cancer continuum. Basic research on stress processes provides leads for investigating biobehavioral processes that may underlie associations reported to date. This symposium will include 4 presentations focusing on basic and clinical human research across multiple cancers, examining the effects of stress processes and stress modulating interventions on ongoing and longer-term health status, and the role of putative biobehavioral mechanisms in explaining these effects. The first paper presents a series of longitudinal studies examining the association of insomnia with immune status and infectious disease symptoms across the period of surgical and adjuvant cancer treatment in women with breast and gynecological cancers. The 2nd paper presents basic and clinical investigations demonstrating the effects of social isolation/support and beta-adrenergic signaling on the epithelial-mesenchymal transition [EMT], a critical step underlying tumor metastasis, in both tumor and exosomes (circulating vesicles derived from tumor cells) in women with ovarian cancer. The 3rd paper presents a series of results from randomized controlled trials in breast cancer patients providing evidence that post-surgical group-based cognitive behavioral stress management interventions can modulate stress factors and improve longterm clinical health outcomes and modulate neuroendocrine, anti-viral, inflammatory, and pro-metastatic signaling in circulating leukocytes that may underlie these psychological and health effects. The 4th paper presents the results of placebo-controlled RCTs in breast and colorectal cancer patients showing that pharmacologic blockage of 2 stress-associated pathways (SNS and COX2 signaling) during the peri-surgical period is associated with decreased EMT, down-regulated beta adrenergic signaling and pro-inflammatory gene expression, enhanced anti-viral cytokine production and reductions in tumor proliferation markers. Dr. Erica Sloan, discussant, will address development of theory-driven and empiricallysupported models to understand and intervene on stress factors and their underlying biobehavioral processes.

\section{S17.1}

TITLE: DO POST-SURGICAL STRESS MANAGEMENT INTERVENTIONS IMPROVE ADAPTATION, IMMUNE CELL SIGNALING AND HEALTH OUTCOMES IN BREAST CANCER PATIENTS?

Michael Antoni ${ }^{1}$

${ }^{1}$ University of Miami - USA

Breast cancer (BCa) patients who do not adapt well reveal poorer longterm outcomes. Cognitive behavioral stress management (CBSM) intervention showed concomitant 12-month improvements in adaptation, downregulated leukocyte pro-inflammatory and pro-metastatic gene expression, implicating decreased $\mathrm{NFkB} / \mathrm{Rel}$ and GATA family transcription factors and increased glucocorticoid receptor expression as mediators. Women in CBSM also showed greater 11-yr median overall and disease-free survival (DFS) than controls, and greater DFS was related to greater reductions in leukocyte pro-inflammatory signaling in the year after CBSM. METHODS and RESULTS. Because women attending 5/ 10 CBSM sessions showed comparable effects to those attending all sessions, and Relaxation Training (RT) and Cognitive Behavioral Therapy (CBT) skills accounted for many of its effects, we conducted a dismantling trial in $183 \mathrm{BCa}$ patients. We found 5 -wk post-surgical interventions (CBT, RT) improved adaptation during treatment vs a 5-wk Health Education (HE) control. We determined NFkB DNA binding in leukocyte cell nuclei by electrophoresis and found those in RT or CBT showed decreased NFkB DNA binding over 12 -months vs HE $(\mathrm{p}<.05)$. Those in RT or CBT showed reduced (vs HE) ligands (e.g., s100A8/A9) for the Receptor for Advanced Glycation End Products (RAGE) $(\mathrm{p}<.05)$, which is implicated in NFkB activation and MAPK-mediated processes promoting tumor cell survival and metastasis. CONCLUSION. Providing post-surgical $\mathrm{BCa}$ patients with stress management can improve adaptation and immune signaling during primary treatment, and may improve long-term health outcomes.

CORRESPONDING AUTHOR: Michael Antoni, University of Miami, mantoni@miami.edu

\section{S17.2}

TITLE: BIOBEHAVIORAL EFFECTS ON EPITHELIAL MESENCHYMAL TRANSITION (EMT) IN OVARIAN CANCER Susan Lutgendorf ${ }^{1}$, Premal Thaker ${ }^{2}$, Michael Goodheart ${ }^{1}$, Sarah Strack ${ }^{1}$, Jesusa Arevalo ${ }^{3}$, Anil Sood ${ }^{4}$, Steve Cole ${ }^{3}$

${ }^{1}$ University of Iowa - USA

${ }^{2}$ Washington University Medical School - USA

${ }^{3}$ University of California Los Angeles - USA

${ }^{4}$ UT MD Anderson Cancer Center - USA

Epithelial mesenchymal transition (EMT) is a process by which transformed epithelial cells undergo changes to a more embryonic (mesenchymal) phenotype, thereby enhancing metastasis. We examined effects of beta-adrenergic signaling and/or biobehavioral exposures on EMT polarization in primary ovarian tumor cells and in exosomes, cellderived vesicles involved in intercellular signaling which can promote metastasis. Methods: Genome-wide transcriptome profiling of advanced stage ovarian tumors from 98 patients was performed, and EMT profiles of those above vs. below a median split on tumor norepinephrine (NE; median: 1.05 $\mathrm{pg} / \mathrm{mg}$ tumor) were compared, adjusting for age, BMI, grade, and stage. In vitro stimulation of ovarian cancer cells with $\mathrm{NE}$ was used to assess effects of exposure to NE on EMT mediators. We also examined exosomal profiles from 40 ovarian cancer patients with low vs. high social support for EMT polarization and gene expression related to inflammation and betaadrenergic signaling. Following exosomal isolation and RNA extraction, microarray analysis of the transcriptome was performed. Results: High-NE tumors showed significant polarization to a more mesenchymal phenotype, as well as decreased expression of a variety of anti-metastatic genes. In 2 ovarian cancer cell lines, exposure to stress-concentrations of NE significantly increased transcription of SNAI1, SNAI2, and IL6, all of which mediate EMT. In exosomes, there was a significant up-regulation of 67 mesenchymal-characteristic gene transcripts and down-regulation of 63 epithelial-characteristic transcripts in patients with low levels of social support, demonstrating decreased EMT polarization in this group $(\mathrm{p}=0.0002)$. Promoter-based bioinformatics also indicated increased levels of CREB/ $\operatorname{ATF}(p=.0019 ; p=.0049)$ and NFKB $(p=0.0109)$ family transcription factors in exosomes from patients with low social support. Conclusions. Induction of EMT gene expression programs by NE and beta-adrenergic signaling provides one plausible pathway through which psychosocial risk factors may promote ovarian cancer progression. Results also highlight the possibility of leveraging exosomes as a non-invasive assessment of biobehavioral factors to help direct personalized treatment approaches.

CORRESPONDING AUTHOR: Susan Lutgendorf, University of Iowa, susan-lutgendorf@uiowa.edu

\section{S17.3}

TITLE: IS INSOMNIA ASSOCIATED WITH IMMUNE ALTERATIONS AND INFECTIOUS RISK IN CANCER PATIENTS? Josée Savard $^{1}$, Sophie Ruel ${ }^{1}$, Hans Ivers ${ }^{1}$.

${ }^{1}$ Université Laval - Canada 
Insomnia affects 30 to $60 \%$ of cancer patients and is especially prevalent in women receiving chemotherapy for breast or gynecological cancer. Experimental studies in healthy individuals have consistently found relationships between sleep deprivation and immune alterations. Evidence from highly-controlled experiments also shows associations of sleep impairments (e.g., lower sleep efficiency) with an increased risk for the common cold. The overarching goal of the naturalistic longitudinal studies that will be presented was to assess whether insomnia is associated with immune alterations and an increased infectious risk in cancer patients. Methods. In Study 1, 962 patients with mixed cancer sites were assessed at 6 time points over an 18-month period following surgery with a curative intent. In Study 2, 52 women about to initiate chemotherapy for breast or gynecological cancer completed measures at 8 time points: prechemotherapy, at immunosuppression and recuperation phases of cycles 1 and 2, at post-treatment, and at 3- and 6-month follow-ups. In both studies, patients were administered a clinical interview assessing the presence of an insomnia syndrome and of infections. In addition, blood was collected in Study 2 for immune measures. Results. Results of Study 1 indicated that patients with an insomnia syndrome at one time point were at a significantly higher risk of reporting at least one infectious episode at the subsequent time point $(\mathrm{OR}=1.31, \mathrm{p}=.04)$, as compared to good sleepers. Study 2 showed that patients with an insomnia syndrome at baseline had significantly lower counts of white blood cells and neutrophils at post-treatment (T6) as compared to good sleepers. Although between-groups differences were not significant, there was a similar trend at the recuperation phase of Cycle 2 (T5). In addition, patients with an insomnia syndrome had a significantly higher risk of reporting infectious symptoms at T5 as compared to those with insomnia symptoms. Conclusions. Together these studies provide some evidence that insomnia accentuates the immunosuppression secondary to chemotherapy, limits the immune recuperation between cycles and at post-treatment, and increases the risk for infections during cancer treatment.

CORRESPONDING AUTHOR: Josée Savard, Université Laval, josee.savard@psy.ulaval.ca.

S17.4

TITLE: BLOCKADE OF STRESS AND INFLAMMATORY RESPONSES IN COLORECTAL AND BREAST CANCER PATIENTS DURING THE PERIOPERATIVE PERIOD IMPROVES PRO-METASTATIC INDICES IN THE EXCISED TUMOR AND IN REPEATED BLOOD SAMPLE

Shamgar Ben-Eliyahu ${ }^{1}$

${ }^{1}$ Tel Aviv University - Israel.

Excess release of catecholamines and prostaglandins was shown to mediate pro-metastatic processes of stress and surgery, specifically during the perioperative period. Here, in two randomized placebo controlled biomarker clinical trials in colorectal $(\mathrm{CRC}, \mathrm{n}=34)$ and in breast cancer patients $(B C, n=38)$, we tested the combined perioperative use of the $\beta$ blocker, propranolol, and the COX2-inhibitor, etodolac, scheduled for 20 perioperative days, starting 5 days before surgery. Tumor samples were subjected to histological analyses, whole genome mRNA profiling, and transcriptional control pathways analyses. Repeated blood samples in BC patients were assessed for cytokine levels and immune indices. Drugs were well tolerated. In both studies, whole genome mRNA profiling of excised tumors showed decreased epithelial-to-mesenchymal transition (EMT); down-regulation of the transcriptional activity of CREB, NFkB, GATA family, and STAT3; reduced presence of tumor-associated monocytes; and increased presence of NK cells in CRC and B cells in BC tissue. In blood samples of BC patients, treatment reduced serum IL-6 and CRP levels even before surgery, improved markers of NK cytotoxicity, and enhanced induced production of IFN $\gamma$ and IL-12, without affecting anti-inflammatory soluble factors (cortisol and IL-10). The tumor proliferation marker Ki67 was tested in $\mathrm{BC}$ patients, and was significantly reduced by drug treatment. In CRC patients, three-year follow-up showed large but statistically insignificant improvement in disease free survival (DFS) in the treatment group (1/15 vs 5/19), further suggesting the safety of the paradigm. Overall, these findings clearly show the efficacy of this combined drug regimen, and suggest its metastatic-reducing impact, which should be tested in larger clinical trials. Such a stressinflammatory-reducing approach may enable us to exploit the critical perioperative period to reduce cancer recurrence, improving long-term survival rates.

CORRESPONDING AUTHOR: Shamgar Ben-Eliyahu, Tel Aviv University, shamgar@post.tau.ac.il

\section{S18}

TITLE: BEHAVIORAL INTERVENTIONS FOR PREVENTION AND MANAGEMENT OF TYPE 2 DIABETES AND COMPLICATIONS: TRANSLATION AND CROSS-LEARNING ACROSS SETTING AND DISEASE SPECTRUM

Pilvikki Absetz ${ }^{1}$

${ }^{1}$ University of Eastern Finland - Finland

Type 2 diabetes (T2DM) and its complications are increasingly burdening health care and individuals in high as well as low and middle-income countries. While they can be prevented and managed with behavioral interventions that target lifestyle and self-care behaviors, translation across settings and cross-learning along the spectrum of disease is rare. In this symposium, we will describe three studies implemented in different countries, including South Africa, Sweden, Uganda, Australia and Singapore with interventions that target lifestyle and self-care behaviors at different stages on the spectrum of disease from increased risk to diagnosed disease to complications and multi-morbidity. All the interventions apply principles from behavior change theories and methods and aim to provide several or all of the following functions of support: assistance in daily lifestyle and management, social and emotional support, linkage to clinical care, and ongoing availability of support. In the first study, Dr Absetz describes development and implementation of a health care and community support program for participants with a high risk or a recent diagnosis of T2DM in semi-rural communities in Uganda, an urban township in South Africa, and urban, vulnerable area in Sweden. In the second study, Dr Oldenburg reports on engagement as well as the outcomes of a virtual coaching program among patients with type 2 diabetes in Australia. In the third study, Dr Griva presents an evaluation of a brief, clinic-integrated intervention to improve outcomes among patients with coexisting end stage renal disease and diabetes mellitus (DM-ESRD) in Singapore. In this symposium, we will focus on the feasibility of these interventions, identify potential for cross-learning and discuss the potential of the interventions for translation and scale-up.

\section{S18.1}

TITLE: RANDOMIZED CONTROLLED EVALUATION OF AN MHEALTH PROGRAM FOR PEOPLE WITH TYPE 2 DIABETES: MY DIABETES COACH

Brian Oldenburg ${ }^{1}$, Shaira Baptista ${ }^{1}$, Dominique Bird ${ }^{2}$, Suman Shetty ${ }^{1}$, Jillian Zemanek ${ }^{1}$

${ }^{1 .}$ University of Melbourne - Australia

${ }^{2}$ University of Queensland - Australia

Type 2 diabetes (T2D) involves complex self-management which most healthcare systems are not able to support. My Diabetes Coach is an mHealth program using an interactive relational agent, 'Laura', supporting blood glucose monitoring and providing personalized feedback and advice. The aim of this presentation is to describe the baseline characteristics of study participants and 6-month data on program use. METHODS: MDC is a real world RCT undertaken in Australia. 
Participants were recruited through the National Diabetes Services Scheme, consumer diabetes organisations and Facebook. Participants completed clinical tests, demographics, clinical and self-report measures were collected at baseline, 6- and 12-months. There were also extensive analytics for evaluating program use. RESULTS: 187 participants were randomized to a waitlist control $(n=94)$ or intervention $(n=93)$ condition. Participants were aged $56-10$ years, $45 \%(n=83)$ were women. At baseline, they had an HbA1c (average blood glucose) of 7.32-1.58\%. Almost half of the study participants were employed full-time (47\%); most were between 50 and 69 years old (69\%); and the majority had completed at least 12 years of education (53\%). Of the 93 participants randomly assigned to the intervention group, $56 \%$ used a smartphone, $14 \%$ used a tablet, and $30 \%$ used both to access the MDC app. At 6 months, $n=79$ (85\%) were still using the MDC app to complete chats with Laura, selfmonitor their glucose levels, access the program website, and/or participate in the MDC online community discussions. Of these, $50 \%$ were weekly chats, $15 \%$ fortnightly, $18 \%$ monthly and $17 \%$ were less than once a month. Over the 6 months, participants had responded to a total of 57,265 statements and questions asked by Laura; and an average of 622 statements per participant. DISCUSSION: We have recruited a diverse sample of people with diabetes from around Australia. Smartphones were preferred for program access. Program uptake and use was excellent when compared with other similar technology interventions. Program delivery resulted in on-going, weekly use for most MDC participants. The level and frequency of program use suggests a high degree of program engagement. The implications and potential of this kind of mhealth program for wider implementation and scale-up are discussed.

CORRESPONDING AUTHOR: Brian Oldenburg, University of Melbourne, boldenburg@unimelb.edu.au

\section{S18.2}

TITLE: IMPROVING OUTCOMES IN MULTIMORBID PATIENTS WITH DIABETES AND ON DIALYSIS - THE CDIRECT: A BRIEF INTERVENTION TO SUPPORT SELF-CARE AND ADJUSTMENT Konstadina Griva ${ }^{1}$, Mooppil Nandakumar ${ }^{2}$, Moothathamb Rajeswari ${ }^{2}$, Deenie Choong ${ }^{3}$, Eric YH Khoo ${ }^{4}$, Newman P. Stanton ${ }^{5}$

${ }^{1}$ Lee Kong Chian School of Medicine, Nanyang Technological University - Singapore

2. National Kidney Foundation Singapore - Singapore

${ }^{3}$ National University of Singapore - Singapore

${ }^{4}$ National University Hospital - Singapore

${ }^{5}$ City University of London - London

The study assessed the feasibility and acceptability of a nurse-led intervention based on motivational interviewing (MI) and self-management and its potential efficacy to improve glycaemic control, as well as psychosocial and self-care outcomes of patients with coexisting End stage renal disease and diabetes mellitus (DM-ESRD) compared with usual care. METHODS: In this feasibility single-blinded randomised trial [ISRCTN10546597], a total of $\mathrm{N}=44$ patients with DM-ESRD and glycated hemoglobin (HbA1c) $>8 \%$ were $\mathrm{r}$ randomly assigned to intervention $(n=20)$ and treatment-as-usual $(n=24)$ arms. Patients were recruited across five dialysis centers in Singapore that cater for disadvantaged patients. The intervention [CDIRECT] consisted of 3 weekly chair-side sessions delivered by diabetes specialist nurses. Facilitators were trained in motivational interviewing and self-management as tools to support behavioral change. We collected data on rates of recruitment, randomization, and retention. As secondary outcomes, clinical endpoints (HBA1c,) and measures of emotional distress, adherence and self-management skills were obtained at baseline and at 12 weeks post baseline. A qualitative evaluation using interviews was conducted at the end of the trial. RESULTS: Recruitment, retention and acceptability of delivery of CDIRECT yielded no major problems. The HbAlc levels at 12 weeks decreased in both groups $(\mathrm{p}=.02)$ but significantly more CDIRECT participants had levels $<8$ at follow up $(\mathrm{p}=.008)$. Significant improvements in role limitations due to physical were noted for CDIRECT whereas levels remained stable in usual care. No statistically significant differences between groups were observed for other clinical markers and other patient-reported outcomes. QOL and depression outcomes were significantly worse in Chinese patients compared to non-Chinese (ps <.01). CONCLUSIONS: In this feasibility study, a brief intervention delivered on bedside as part of routine dialysis care showed some benefit in glycaemic control and on QOL domain compared with usual care, although no effect was observed in other clinical or secondary outcomes. Further research is needed to design and assess interventions to promote diabetes self-management in socially vulnerable patients.

CORRESPONDING AUTHOR: Konstadina Griva, Lee Kong Chian School of Medicine, Nanyang Technological University, konstadina.griva@ntu.edu.sg

\section{S18.3}

TITLE: SMART2D - A SELF-MANAGEMENT APPROACH LINKING HEALTH CARE AND COMMUNITY IN PREVENTION AND MANAGEMENT OF TYPE 2 DIABETES

Pilvikki Absetz ${ }^{1}$, Josefien Van Olmen ${ }^{2}$, Helle Alvesson ${ }^{3}$, Peter Delobelle $^{4}$, Francis Kasujja ${ }^{5}$, David Guwatudde ${ }^{5}$, Thandi Puoane ${ }^{6}$, Meena Daivadanam ${ }^{7}$

${ }^{1}$ University of Eastern Finland \& Collaborative Care Systems Finland Finland

${ }^{2}$ Institute of Tropical Medicine - Belgium

${ }^{3}$ Karolinska Institutet - Sweden

${ }^{4}$ University of Western Cape - South Africa

${ }^{5}$ Makerere University - Uganda

${ }^{6}$ University of Western Cape - South Africa

${ }^{7}$ University of Uppsala \& Karolinska Institutet - Sweden

Type 2 diabetes (T2DM) and its complications are increasing rapidly in low- and middle-income countries (LMIC), as well as among vulnerable populations such as immigrants in high-income countries (HIC). Health systems are poorly equipped to tackle the problem, due to scarce resources and emphasis on communicable diseases in the LMIC, and cultural gaps and special needs among the immigrants that are poorly understood in the HIC. The SMART2D is a European Commission funded project that implements and evaluates the feasibility of a lifestyle and self-management intervention for participants newly diagnosed with prediabetes or type 2 diabetes in semi-rural communities in Uganda, an urban township in South Africa, and urban vulnerable communities in Sweden. Our aim was to develop an intervention that would share common key functions without compromising a good cultural fit and feasibility. The intervention program design was conducted in three steps facilitated by a coordinating team (PA and JVO): 1) Mapping of patient/person-at-risk processes and identification of needs and potential for intervention in health care and community. This step was based on comprehensive situational analyses conducted at each site with scoping reviews and qualitative and quantitative data collection within health systems and communities; workshops and teleconferences with the local teams; and stakeholder collaboration. 2) Designing generic intervention strategies and tools targeting the needs and utilizing the potential identified in the mapping step. This step was conducted in joint workshops and teleconferences with all teams, and as desk research. 3) Translating the generic strategies and tools into local context. This step was conducted by the local teams. The resulting intervention includes a health facility program with two generic strategies - organization of care process and strengthening patient role in self-management - and a communitybased program with three generic strategies - community mobilization, strengthening support from social and physical environment, and linkage between facility and community. In this presentation, we will describe the process and outputs of the SMART2D intervention program design and 
translation

CORRESPONDING AUTHOR: Pilvikki Absetz, University of Eastern Finland, pilvikki.absetz@gmail.com

S19

TITLE: IMPULSIVITY, SUBSTANCE USE, AND SUBSTANCE USE DISORDERS

Alvaro Vergés ${ }^{1}$

${ }^{1}$ Pontificia Universidad Católica de Chile - Chile

Substance use and substance use disorders (SUDs) are associated with a number of negative consequences, including health, behavioral, social, and economic outcomes, thus becoming a significant, increasing public health problem worldwide. One major risk factor for substance use and SUDs that has received attention in recent years is impulsivity, a multidimensional construct that can be broadly defined as the tendency to engage in behavior prematurely or without appropriate planning. Recently, different specific facets of impulsivity have been identified and linked with substance use and SUDs. In particular, specific dimensions of impulsivity have been shown to predict substance use initiation, substance use intensity and substance-related problems among current users, SUD onset, and SUD treatment outcomes. In this symposium, recent work examining how impulsivity relates to the course, treatment outcomes, and neurobiology of substance use and SUDs will be presented. Dr. Kenneth J. Sher will present data regarding the longitudinal association of impulsivity and substance use across young and middle adulthood. Dr. Andrew K. Littlefield will present the results of a study showing evidence of change in impulsivity during residential treatment. Dr. Mario J. Hitschfeld will present evidence of how impulsivity relates to substance use recurrence after inpatient detoxification in adults. Finally, Dr. Alvaro Vergés will present data comparing two models of impulsivity in predicting substance use initiation among adolescents.

\section{S19.1}

TITLE: IMPULSIVITY FACETS AND SUBSTANCE USE INITIATION

Alvaro Vergés ${ }^{1}$, Andrew Littlefield ${ }^{2}$

${ }^{1}$ Pontificia Universidad Católica de Chile - Chile.

${ }^{2}$ Texas Tech University - USA

Introduction \& Purpose: Several types of impulsivity have been linked to various substance use outcomes. The UPPS-P framework has received major focus within the field of substance use research. However, this framework is not without limitation. An alternative framework is the Two-Factor Model of impulsivity, which posits that rash impulsivity and reward drive are the central, if not sufficient, domains of impulsivity. Unfortunately, the extant literature is quite limited in terms of work that have directly compared the UPPS-P framework to the Two-Factor Model of impulsivity, particularly in prospective designs focused on the initiation of common, problematic forms of substance use among adolescents (i.e., alcohol and marijuana use). Methods: The UPPS-P measures were compared to dedicated measures of the Two-Factor Model of impulsivity in a sample of Chilean adolescents who were lifetime abstainers of alcohol or marijuana use at baseline $(\mathrm{N}=541)$ to predict the initiation of use for these substances at a one and two-year follow-up. Results: The TwoFactor Model had superior predictive utility compared to the UPPS-P measures, and only rash impulsivity $(\mathrm{OR}=1.39[\mathrm{CI}=1.00,1.93])$ and reward drive $(\mathrm{OR}=1.37[\mathrm{CI}=1.05,1.80])$ were significant predictors at the one-year follow-up in a multivariate model that simultaneously considered UPPS-P and Two-Factor Model assessments. Conclusions: The Two-Factor Model should be considered to index risk of substance use initiation to guide prevention efforts and highlight the importance of direct comparisons of alternative measurement and theoretical frameworks of impulsivity within the field of substance use research.

CORRESPONDING AUTHOR: Alvaro Vergés, Pontificia Universidad Católica de Chile, ajverges@uc.cl

S19.2

TITLE: ASSESSMENT OF INTERACTION BETWEEN INHIBITORY CONTROL AND CRAVING AS PREDICTOR OF SUCCESS AFTER ADDICTION TREATMENT COMPLETION

Mario Hitschfeld ${ }^{1}$, Melissa Martinez ${ }^{2}$, Patricia Flores ${ }^{3}$, Daniela Alvo ${ }^{4}$, Paulina Puebla ${ }^{4}$

${ }^{1}$ Universidad Diego Portales - Chile

${ }^{2}$ Hospital Clínico Universidad de Chile - Chile

${ }^{3}$ P. Universidad Católica de Chile - Chile

${ }^{4}$ Hospital Sótero Del Río - Chile

Inhibitory control, as part of neuropsychological functioning, has been linked to poor outcomes in Substance Use Disorder (SUD) patients. Craving has often been linked to the preoccupation/anticipation stage of addiction, which consists in executive function deficits leading to excessive salience to substance-paired cues and subsequent relapse. There are studies that evaluate the interaction of executive function impairment with self-efficacy, social network, and previous treatments; but no with craving. This study aimed to determine the interaction existing between executive function, specifically inhibitory control, and craving at treatment admission, and its association with relapse during 1-year post-treatment follow-up among SUD patients. Methods: This was a prospective cohort study design among all adults with an SUD undergoing detoxification at Psychiatry Inpatient Unit of the Sotero Del Rio Hospital in Santiago, Chile. The main predictor was inhibitory control which was measured by Stop Signal Reaction Time (SSRT). The effect modifier was craving which was measured by the Penn Alcohol Craving Scale (PACS). The main outcome was relapse, whose assessment took place every 1 month after discharge until 1-year follow-up completion. Results: The study included 31 patients who were admitted for detoxification; 20 $(65 \%)$ were males and mean \pm SD age was $39 \pm 8$ years. The primary substance of abuse was cocaine for $24(77 \%)$ subjects. The mean SSRT at initial evaluation was $0.23 \pm 0.1 \mathrm{seg}$. The mean PACS score at initial evaluation was $15.2 \pm 3.7$. After adjustment for covariates, to have either a higher SSRT and PACT score was associated with higher relapse rate at 1-year follow-up (For SSRT: HR = 1.19, 95\% CI: $1.01-1.38, \mathrm{p}=0.03$; for PACS: $\mathrm{HR}=1.82,95 \% \mathrm{CI}: 1.32-2.31, \mathrm{p}=0.01$ ). There was no interaction between SSRT and PACS score in their association with relapse at 1year follow-up. Conclusions: Patients with impaired inhibitory control and elevated craving at detoxification beginning have higher risk of relapse after 1-year post-treatment follow-up. There is no interaction between inhibitory control and craving, which means the effect of inhibitory control on relapse is not heterogeneous across strata of the variable craving.

CORRESPONDING AUTHOR: Mario Hitschfeld, Universidad Diego Portales, mjhitschfeld@gmail.com

\section{S19.3}

TITLE: IMPULSIVITY AND SUBSTANCE USE DISORDER: A DEVELOPMENTAL PERSPECTIVE

Kenneth Sher ${ }^{1}$

${ }^{1}$ University of Missouri - USA

Traits related to impulsivity are likely the strongest dispositional correlates of alcohol use disorders (AUDs) and other substance use disorders (SUDs). While the link has long been recognized, the extent to which impulsivity represents a risk factor or a consequence has been the subject of much debate. Objectives: In this presentation, we review findings from 
longitudinal studies from my group and others demonstrating that various measures tapping facets of impulsivity predict the later development of AUDs (and other SUDs) and may change as a function of recent substance use. Methods: Review of longitudinal studies Results: Perhaps most critically, changes in impulsivity associated with normal aging covary with alcohol-related symptomatology and implicate personality development as a major factor in determining the course of AUDs and highlight the importance of personality change in "maturing out" of substance use problems and "natural recovery." Additionally, these personality changes are associated with drinking motivations which may represent a proximal manifestation of deeper personality change. We also consider methodological challenges in distinguishing personality from alcohol-specific pathology (i.e., some symptoms/diagnostic criteria appear to represent occasion-specific manifestations of impulsive behavior). We also review recent research documenting durable personality changes following relatively brief courses of psychological treatment. Conclusion: Overall, the implications of understanding the impulsivity-SUD relation are wide-ranging in terms of assessing risk for future problems, monitoring treatment progress, and identifying new treatment targets.

CORRESPONDING AUTHOR: Kenneth Sher, University of Missouri, waudbyc@missouri.edu

S19.4

TITLE: STABILITY AND CHANGE IN MULTI-METHOD MEASURES OF IMPULSIVITY ACROSS RESIDENTIAL ADDICTIONS TREATMENT

Andrew Littlefield ${ }^{1}$

${ }^{1}$ Texas Tech University - USA

Although the relations between constructs related to impulsivity and substance use disorders (SUDs) are well established, recent research suggests that changes in impulsivity may be an important mechanism in the recovery process. However, this evidence is primarily based on studies that have examined the relation between changes in impulsivity and substance involvement across the span of several years. Thus, it is unclear if these changes are linked across shorter time intervals. Importantly, impulsivity is a multifaceted construct that can be assessed using both self-report and behavioral methods. However, the extent to which changes in different types of impulsivity, assessed using both self-report and behavioral paradigms, serve as treatment mechanisms for SUDs remains unclear. Using prospective data from 43 participants (mean age $=35.06 ; 60 \%$ female) enrolled in residential substance abuse treatment, the degree to which seven facets of impulsivity (i.e., questionnaire-assessed delay discounting, lack of perseverance, lack of planning, negative urgency, positive urgency, sensation seeking, and behaviorally-assessed inhibitory control) changed across approximately 4 weeks of treatment was examined. Dependent group t-tests suggested significant reductions in negative urgency and lack of planning, and significant improvements in inhibitory control. Findings indicate that specific impulsivity facets show change across relatively short time frames and suggest nonspecific effects of residential treatment on levels of impulsivity. These results inform the functional relation between facets of impulsivity and substance involvement. Further, these findings have implications for assessment and treatment approaches for SUDs; the current results are consistent with emerging work suggesting that personality features that are associated with SUDs can be modified through various treatment approaches and that changes in impulsivity may serve as a mechanism of change for established treatment approaches for addictive behaviors. Existing, theory-driven interventions for changing personality features, such as those related to impulsigenic traits, as well as approaches that target coping skills related to personality characteristics, will also be discussed. Methodological considerations regarding the aforementioned body of research will also be noted.
CORRESPONDING AUTHOR: Andrew Littlefield, Texas Tech University, andrew.littlefield@ttu.edu

S20

TITLE: COGNITIVE AND EMOTIONAL FACTORS IN MEDICALLY UNEXPLAINED PHYSICAL SYMPTOMS

Henriëtte van der Horst ${ }^{1}$

${ }^{1}$ VU University Medical Center - Amsterdam

Medically unexplained symptoms (MUS) are associated with high levels of symptom-related burden and disability in everyday functioning. Several studies have demonstrated difficulties in emotional processing and expression in MUS patients. Recently published systematic reviews have demonstrated that cognitive behavior therapy (CBT) is one of the best evidenced psychological treatments for patients with chronic MUS, but effect sizes are moderate or even small. A potential option to increase efficacy would be to enrich CBT with strategies for improving processing and regulation of emotions. In this symposium we present cutting-edge research on cognitive and emotional aspects in the course of MUS. The contributors will present new results from three large longitudinal studies, performed in The Netherlands, Germany and the UK, respectively. The aim of this symposium is three-fold: 1) to present results of a cohort study of MUS patients focusing on the longitudinal relationship between a variety of cognitive and emotional aspects and the course of MUS; 2) to present the findings of an RCT assessing the effectiveness of an Cognitive Behavioral Therapy intervention enriched with emotion regulation training in MUS patients; 3) to present results of a three-armed $\mathrm{RCT}$, looking into the effectiveness of therapist delivered telephone CBT versus a web-based CBT programme and versus usual care in patients with irritable bowel syndrome. The discussant will provide an integrative view on cognitive and emotional factors in MUS, within the context of a model on the adaptation to chronic stressors.

\section{S20.1}

TITLE: PREDICTING THE 2-YEAR COURSE OF PERSISTENT PHYSICAL SYMPTOMS

Nikki Claassen ${ }^{1}$, van Desse ${ }^{1}$, Johannes C. van der Wouden ${ }^{1}$, Jos W.R. Twisk $^{1}$, Joost Dekker ${ }^{1}$, Henriette E. van der Horst ${ }^{1}$

${ }^{1}$ VU University Medical Center - Amsterdam

Objective To identify patients at risk for persistent physical symptoms (PPS more knowledge about predictors of the course of PPS in different clinical settings is needed. Therefore, we developed prediction models for the course of PPS in terms of symptom severity and related functional status during a 2-year follow-up period. Methods We used data of the PROSPECTS cohort study, consisting of 325 PPS patients from several health care settings (general practice, mental health care, rehabilitation centres, pain clinics). Symptom severity (PHQ-15), physical functioning (RAND-36 PCS) and mental functioning (RAND-36 MCS) were assessed at baseline and 6,12 and 24 months afterwards. We applied mixed model analyses to develop prediction models for all three abovementioned outcomes, using all follow-up measurements. Our choice of potential predictors was based on empirical and theoretical literature and these were measured at baseline. By incorporating questionnaires about illness anxiety, hypervigilance, negative illness perceptions, and illness cognitions we covered several potential predictors, including emotions and cognitions. Results Predictors for an adverse course of symptom severity, physical functioning and mental functioning were: physical comorbidity, higher severity and longer duration of PPS, anxiety, catastrophizing cognitions, embarrassment, fear avoidance cognitions, avoidance behaviour and neuroticism. Predictors of a favourable course were: limited alcohol use, higher education, higher levels of physical and mental functioning at baseline, symptom focusing, damage cognitions and extraversion. Explained interpersonal variance of the multivariate 
models varied between $70.5 \%$ and $76.0 \%$. Sensitivity analyses revealed that performance of the models was comparable in primary and secondary/tertiary care patients. Conclusion We identified several relevant demographic, medical, psychological and behavioural predictors for adverse and favourable courses of PPS. External validation is needed prior to clinical implementation.

CORRESPONDING AUTHOR: Nikki Claassen, VU University Medical Center, n.vandessel@vumc.nl

S20.2

TITLE: A RANDOMIZED CONTROLLED TRIAL (ACTIB) OF CLINICAL AND COST EFFECTIVENESS OF THERAPIST DELIVERED COGNITIVE BEHAVIOURAL THERAPY AND WEBBASED

Rona Moss-Morris ${ }^{1}$

${ }^{1}$ King's College London - United Kingdom

Irritable bowel syndrome (IBS) affects $10-22 \%$ of the population. Current treatment includes lifestyle advice and drug therapies, but many people suffer ongoing symptoms. Objectives: To determine the clinical and cost-effectiveness of therapist telephone delivered CBT (TCBT) and web based CBT self-management (WCBT) compared to treatment as usual (TAU) in IBS. Design: 3-arm randomised controlled trial. Ethical approval awarded 11th June 2013 (13/SC/0206) Setting: UK primary and secondary care Participants: Adults with refractory IBS recruited from 74 general practices and 3 gastroenterology centres over 23 months (May 2014 to March 2016). Randomisation: Conducted by an independent clinical trials unit using random block sizes stratified by treatment centre. Blinding: Randomization by concealed allocation, blinding of outcome assessors and the trial statistician. Interventions: TCBT: IBS self-help manual with six 60-minute telephone therapy sessions over nine weeks + two 60-minute booster sessions ( 8 hours therapist time). WCBT: Eight session tailored IBS self-management website (Regul8) plus three 30minute telephone therapy sessions over 9 weeks + two 30-minute boosters ( 2.5 hours therapist time). Therapists and treatment fidelity: 10 CBT therapists, 1-day training in trial interventions, monthly supervision, post-trial ratings of fidelity. Primary outcomes: Irritable bowel syndrome symptom severity score and Work and Social Adjustment Scale at 12 months. Affect measures: Positive Affect, HADS, Beliefs about Emotions Scale Data sources: Participants completed measures online at baseline (pre-randomization), 3, 6 and 12 months after randomization. Data analysis: Data analysed under intention-to-treat assumptions using longitudinal linear mixed modelling, including outcome measures at all time points and adjusting for the stratification variable (treatment centre) and baseline measures. Results: 558 (36.6\%) of 1525 patients screened for eligibility were recruited. 186 randomised to TCBT, 185 to WCBT and 186 to TAU alone. Data have been analysed but because of embargo on releasing trial results pre-publication from journals, the trial team has asked these to be withheld until published or presented at conference. Registration ISRCTN44427879 Funding NIHR HTA (11/69/02)

CORRESPONDING AUTHOR: Rona Moss-Morris, King's College London, rona.moss-morris@kcl.ac.uk

\section{S20.3}

TITLE: DOES COMPLEMENTING COGNITIVE BEHAVIOR THERAPY WITH EMOTION REGULATION TRAINING FOR PATIENTS WITH MEDICALLY UNEXPLAINED PHYSICAL SYMPTOMS ENHANCE THE OUTCOME? FINDINGS OF A MULTICENTER RCT

Maria Kleinstaeuber ${ }^{1}$, Johannes Schwabe ${ }^{2}$, Winfried Rief ${ }^{2}$

${ }^{1}$ University of Auckland - New Zealand

${ }^{2}$ Philipps-University Marburg - Germany
Medically unexplained symptoms are likely to have a persistent course and to be associated with disability and high health care costs. Cognitive behaviour therapy (CBT) is recommended as first-choice treatment in various national treatment guidelines. Although CBT has a strong evidence of efficacy, effect sizes are only moderate. Previous research demonstrated deviations in emotional processing in MUS patients from healthy subjects. Therefore the central aim of the current study was to find out if the therapy outcome can be improved when $\mathrm{CBT}$ is complemented with emotion regulation training. Methods: For a multicenter RCT 255 patients were recruited and randomly assigned to 20 sessions of either conventional CBT $(\mathrm{N}=128)$ or CBT with emotion regulation training (ENCERT; $N=127$ ). Eligible participants had to be diagnosed with at least 3 persisting, distressing and disabling MUS. The primary outcome (somatic symptom severity) and secondary outcomes (symptom disability, depressive symptoms, health anxiety, general psychopathology, symptom coping, quality of life, emotion regulation skills) were assessed at pre-treatment, session 8, end of therapy, and a 6-month followup. Results: Hierarchical linear mixed-effect models with baseline adjustment and study centre as random effect revealed for primary and secondary outcomes highly significant effects in the ITT-samples of both treatment groups. Significant time*group interactions indicated a stronger beneficial effect of ENCERT for the outcomes health anxiety, emotion regulation skills, general psychopathology, and symptom coping. Results of moderator and mediator analyses allude to higher effects of ENCERT in subgroups with comorbid mental disorders. Conclusions: Current findings are based on a high-quality randomized controlled study design and a large, thoroughly screened sample. The results are therefore of high validity and reliability and demonstrate that CBT as well as CBT complemented with emotion regulation training have strong effects on primary as well as secondary outcomes in MUS patients. The results allow conclusions about ENCERT to be more beneficial than conventional CBT for patients in specific subgroups of the sample. ENCERT Research Group: A. Martin, B. Löwe, P. Henningsen \& C. Lahmann, A. Schröder \& J. Heider, R. Stark, J. Bailer, W. Rief, J. Rau, J. Schwabe, M. Kleinstäuber.

CORRESPONDING AUTHOR: Maria Kleinstaeuber, University of Auckland, m.kleinstauber@ auckland.ac.nz

\section{INVITED SYMPOSIUM}

\section{S21}

TITLE: RESEARCH IN RESILIENCE AND HEALTH: THE ROLE OF PROTECTIVE FACTORS IN DISEASE AND ILLNESS CONDITIONS

Eduardo Remor ${ }^{1}$

${ }^{1}$ Universidade Federal do Rio Grande do Sul - Brasil

The concept of resilience has stimulated extensive research, focusing on factors that promote health and well-being, as a basis for health promotion. Resilience describes a phenomenon under which an individual does not or only temporarily, experiences mental health problems despite being subjected to psychological or physical stressors of acute or chronic duration. This symposium will present communications focusing on resilience and health. Specifically, will describe (a) the development and evaluation of interventions that aimed to foster psychological resilience and prevent stress-related desease conditions; (b) research that adressed resilience as predictor of quality-of-life in chronic illness. On the other hand, (c) we will bring to discussion how research on resilience has been conducted within Latin American region througout an updated review on research focusing in chronic illness.

\section{S21.1}

TITLE: INTERVENTION PROGRAM TO PROMOTE EMPOWERMENT AND RESILIENCE IN HEALTHY INDIVIDUALS Eduardo Remor ${ }^{1}$, Gabriela Mondelo ${ }^{1}$

${ }^{1}$ Universidade Federal do Rio Grande do Sul - Brasil 
The "Programa +Recursos" (original title in Spanish; Remor \& AmorósGómez, 2013) is a manualized psychological intervention that foster psychological resources to deal with adversities. The intervention is delivered in group format by two trained facilitators, consists of 10 sessions (up to 2 hours each), addressing psychological resources such as assertive communication, empathy, optimism, positive coping, forgiveness, gratitude and the capacity for emotional self-regulation. Purpose: This presentation will describes the program content, summarizes and discuss results observed across all samples assessed to date. Implications of the intervention usefulness to resilience improvement in healthy individuals and preventive potential of the intervention will be addressed as well. Method: Non-random samples and quasi-experimental designs were applied in order to evaluate evidence for intervention effectiveness. Results: Early studies. The development of the program started in 2003, and underwent evaluation of results in samples of Spanish $(n=61)$ and Latin-American (El Salvador, $n=24$ ) participants. Results observed indicated enhancement effects on mood, dispositional optimism, coping with stress, satisfaction with life. In addition, decreasing effects were shown in depressive mood, stress, anxiety and subjective health complaints (All ps $<0.05$ ). Currently, we are developing the adaptation of the program to Brazil. The manual was translated to Portuguese. A pilot intervention in Brazilian population $(n=13)$ was completed with attrition rate of $38 \%$. Those that completed $(n=8)$ provided a positive feedback in terms of satisfaction ( $87 \%$ very satisfied), learned skills and comprehension of content $(100 \%$ very satisfied). Analysis of the results indicated benefits in empathy, optimism, gratitude, forgiveness, stress, assertiveness, mood, satisfaction with life, resilience, and perceived health, however results were not statistically significant. Replications of the intervention is currently in progress, in order to improve the sample size and evaluate feasibility and effects in the selected outcomes. Conclusions: The program is able to promote personal resources in healthy individuals and have potential as preventive psychological intervention.

CORRESPONDING AUTHOR: Eduardo Remor, Universidade Federal do Rio Grande do Sul, remor.eduardo@ gmail.com

\section{S21.2}

TITLE: PEOPLE LIVING WITH HIV/AIDS: RESEARCH ON RESILIENCE, RELIGIOUS COPING AND QUALITY OF LIFE

Hérica Landi de Brito $^{1}$, Eliane Maria Fleury Seidl ${ }^{1}$

${ }^{1 .}$ Centro Universitário Alves Faria - Brasil

Studies have shown that coping strategies, especially in their religious and /or spiritual dimensions, and resilience are factors that allow people with HIV/AIDS to adapt to seropositivity and have a better quality of life (QoL). This study aimed to investigate predictors of QoL, considering sociodemographic and clinical variables, religious coping and resilience in people living with HIV/AIDS (PLWHA). Methods. Two hundred HIV positive patients $(105$ men) with a mean age of 39 years $(\mathrm{SD}=10.9)$ participated in the study, constituting a convenience sample, followed at an outpatient clinic of a hospital specialized in HIV / AIDS. The instruments applied were: questionnaire on sociodemographic, clinical and religious aspects; the Ways of Religious Coping Scale; the Resilience Scale and the WHOQoL-HIV Bref. Data were analyzed using descriptive and inferential statistical techniques (significance level $\mathrm{p} \leq .05$ ). Results. The positive religious coping (PRC) average was greater than the negative religious coping $(\mathrm{NRC}): 3.5(\mathrm{SD}=0.52)$ and $1.7(\mathrm{SD}=0.68)$ respectively, which means that participants used more PRC than NRC, which is an indicator of more adaptive ways to deal with stressors related to seropositivity. Participants showed good average levels of resilience $(\mathrm{M}=3.06 ; \mathrm{SD}=0.41)$ and thus they are able to cope and overcome the difficulties arising from seropositivity and learn from them. Correlation analysis indicated that resilience was associated significantly and positively to the PRC ( $r s=0.37 ; p=.01$ ); with the NRC this association was negative ( $r s=-0.10 ; p=.04)$. Patients with HIV/AIDS obtained good mean scores of QoL, classified as intermediate, and the environment dimension had the lowest average. The predictors of QoL were: resilience $(\beta=0.29$; $\mathrm{p} \leq .001)$, level of education $(\beta=0.20 ; \mathrm{p} \leq .01)$ and NRC $(\beta=-0.22$; $\mathrm{p} \leq .001$ ). Thus, $25 \%$ (24\% adjusted) of the variance of QoL can be predicted based on these three antecedent variables in this sample of PLWHA. Conclusions. The results revealed the important role that resilience and religious coping can play in overcoming adversities related to HIV / AIDS and highlights its influence on the quality of life of PLWHA.

CORRESPONDING AUTHOR: Hérica Landi de Brito, Centro Universitário Alves Faria, herica.landi@unialfa.com.br

S21.3

TITLE: LATIN AMERICAN RESEARCH ON RESILIENCE IN CHRONIC ILLNESS: A REVIEW

Stefano Vinaccia Alpi ${ }^{1}$, Japcy Margarita Quiceno ${ }^{2}$

${ }^{1}$ Universidad Del SINU; Fundacion Universitaria Sanitas - Colombia

${ }^{2}$ Universidad de Medellin - Colombia

Over the last decades have had a high increase about resilience studies in different sort of samples like children, young people and adults with or without organic pathologies, and the vast majority studies emphasize in that resilience is a protection factor on mental and physical health. Purpose: Therefore, the aims of this work is describe the main studies about resilience which have been developed in Mexico, Costa Rica Venezuela, Colombia, Peru, Chile, Argentina, Brazil, Uruguay. In addition, psychometric instruments which have been used to measurement it are mentioned, as well as, physical pathologies that have been studied until now. On the other hand, this work also emphasizes in resilience programs with chronic illness, as resilience intervention programs are the last frontier in the area, if we may say it so. Method: Literature review from the year 2000 to the year 2018 for the collection of articles the bases were used of PubMed and SciELO data Results: having resilient characteristics would help in some way harmonize exposure to stress situation caused by the disease. Conclusions: Research about resilience in health settings in Latin America is flourishing, however the production of literature is uneven across countries

CORRESPONDING AUTHOR: Stefano Vinaccia Alpi, ${ }^{1}$ Universidad Del SINU; Fundacion Universitaria Sanitas, vinalpi47@hotmail.com

S22

TAILORED DHEALTH INTERVENTIONS: INNOVATIONS AND EFFECTS AND COST-EFFECTIVENESS

Hein de Vries ${ }^{1}$

${ }^{1}$ Department of Health Promotion- Netherlands

dHealth interventions aimed at improving lifestyles have become popular and involve eHealth and mHealth interventions. In this symposium we will provide examples of new innovative approaches, outline the theoretical and communication strategies used. We will discuss the effects of different applications and innovations, such as the effects of interactive and intensive text messaging program for pregnant smokers (Abroms); the (cost) effectiveness of video, text and game based (lifestyle) tailoring (De Vries); automated text messages plus personalized texts from mentors (Westmaas); and effects of video, cartoon tailoring for obesity prevention, t2Diabetis and physical activity (Cheung) and smoking cessation for smartphones (Herbec).

\section{S22.1}

COMPUTER TAILORED DIGITAL HEALTH INTERVENTIONS, ALSO FOR LSES GROUPS? INNOVATIONS AND COSTEFFECTIVENESS

Hein de Vries ${ }^{1}$

${ }^{1}$ Maastricht University- Netherlands 
Computer tailored digital Health Interventions, also for LSES groups? Innovations and cost-effectiveness Background: Traditionally computer tailored methods use text based messages. Yet, this method may be less effective for people with a lower socio-economic background (LSES). How to best offer health communication using dHealth is not immediately clear. We conducted three RCTs to experimentally test effects of simultaneous or sequential tailored lifestyle information, to assess potential differences between text and video tailoring, and to analyze the effects of adding gamification to tailoring. Method: We will present the results of three RCTs testing the effects and cost-effectiveness of innovative tailored dHealth interventions. Study 1 will discuss the effects and costeffectiveness of a lifestyle program on 5000 Dutch adults and will compare sequential and simultaneous lifestyle tailoring. Study 2 will discuss the differential results of text and video tailoring on 2100 Dutch adult smokers for smoking cessation. Study 3 will discuss the effects of using gamification in computer tailored programs to prevent binge drinking on 2600 adolescents. Results: Study 1 showed that computer tailoring resulted in significant effects on increasing lifestyles in Dutch Adults. The program was effective after 12 and 24 months, and also cost-effective. Both sequential and simultaneous tailored interventions were effective, but effects differed between 12 and 24 months. Study 2 showed that video tailoring was effective for LSES adults, and also for HSES adults. The video tailored program was also more cost effective. Study 3 showed that the binge drinking prevention was most effective for the 15 and 16 year olds, and that a dose-response relationship was found suggesting that a greater effectiveness when all modules were adhered to. Conclusions: Our results confirm results found in our previous studies as well as international studies on the effects of computer tailoring. Several challenges are still to be addressed, such as how to cope with high drop-out rates, which multiple imputation technique to be used, how to transfer eHealth to mHealth, and how to implement effective programs nationally.

CORRESPONDING AUTHOR: Hein de Vries, Maastricht UniversityNetherlands, hein.devries@maastrichtuniversity.nl

\section{S22.2}

PREFERENCES AND NEEDS OF SMOKERS AND RECENT EXSMOKERS REGARDING SMOKING CESSATION APPS - A SYNTHESIS OF FINDINGS FROM THREE INTERVIEW STUDIES Aleksandra Herbec ${ }^{1}$, Olga Perski ${ }^{1}$, Ildiko Tombor ${ }^{1}$, Lion Shahab ${ }^{1}$, Jamie Brown $^{1}$, Robert West ${ }^{1}$

${ }^{1}$ University College London- United Kingdom.

Introduction \& Purpose: Smartphones apps could deliver cessation support to smokers, but we need to know more about what smokers would find beneficial, acceptable, and desirable in such programmes. Such perceptions are likely to be important in driving engagement. In line with user-centred approaches to the design of digital programmes, the study aimed to identify core needs, preferences and expectations of potential users of stop smoking apps. Methods: As part of three projects to develop and evaluate smoking cessation apps, we conducted 50 qualitative semi-structured interviews with adult UKbased smokers and recent ex-smokers. The interviews explored participants' experiences, preferences, views on apps or app prototypes, and their smoking cessation needs. We were particularly interested in issues around craving management, use of nicotine replacement therapy (NRT), and assessment of carbon monoxide (CO) levels with personal, smartphone-enabled monitors. The Interview transcripts were analysed using inductive thematic analysis, and relevant data and findings across the projects were synthesised under a unifying coding framework. Results: The analyses identified several core themes relevant to app development across all projects: (1) Factual content (e.g. information, advice); (2) Core functionality (e.g. monitoring, feedback, craving management); (3) Functionality related to the specific focus of apps (e.g. video demonstrations of CO testing or NRT use); (4) Supportive features (e.g. reminders, tailoring, diary); (5) Auxiliary support (e.g. peer, integration with traditional programmes); (6) Information architecture and user journeys (e.g. location and timing of advice); and (7) Underlying needs and expectations (e.g. information, motivation, engagement, usability, relevance). Some expectations of potential users may conflict with research, clinical and technical priorities. Conclusions: Smokers interested in using apps to support them to quit are able to articulate a wide range of needs and preferences relating to cessation apps. Sustaining smokers' motivation to quit and addressing momentary cravings in an engaging manner emerged as key functions of cessation apps. These findings will help inform app design and tailoring of content to fit users' needs.

CORRESPONDING AUTHOR: Aleksandra Herbec, University College London, aaherbec@gmail.com.

\section{S22.3}

PEER MENTORING AND AUTOMATED TEXT MESSAGES FOR SMOKING CESSATION: A RANDOMIZED PILOT TRIAL

Johann Westmaas $^{1}$, Justin White ${ }^{2}$, Severine Toussaert ${ }^{3}$, Johannes Thrul ${ }^{4}$, Lorien Abroms ${ }^{5}$.

${ }^{1}$ American Cancer Society- USA.

${ }^{2}$ University of California, San Francisco- USA

${ }^{3}$ Oxford University- USA

${ }^{4}$ Johns Hopkins Bloomberg School Public Health- USA

${ }^{5}$ George Washington University

Background: Computer programs that deliver texts with information and support for quitting to smokers' smartphones have shown promise, but they lack the socially supportive component inherent in peer-to-peer or mentoring programs shown to promote behavior change. Peer mentoring providing emotional and informational support for smoking cessation delivered by text messaging has not been tested as a potentially more effective intervention compared to computer-generated text messaging for cessation. Methods: In February and March 2017, 200 smokers who were planning to quit were recruited online and randomly assigned to receive smoking cessation support for $8-10$ weeks via (i) automated text messages similar to NCI's SmokefreeTXT program (control group) ( $\mathrm{n}=99$ ), or (ii) automated text messages plus personalized supportive texts sent from trained mentors who were former smokers $(n=101)$. Mentors' texts were available in an online library which allow customization and personalization by mentors. We evaluated the efficacy, acceptability, engagement, and user experience of the intervention. The analyses were performed on an intent-to-treat basis. Results: After three months, selfreported 7-day point prevalence abstinence was $21.8 \%$ among those receiving mentor support versus $12.1 \%$ among those in the control group. Adjusting for baseline characteristics, the mentor intervention increased self-reported abstinence by 12.1 percentage points $(\mathrm{p}=0.03)$ and increased biochemically verified abstinence by 6.2 percentage points above the control group mean of $2.6 \%(\mathrm{p}=0.05)$. The intervention was viewed as acceptable: $78 \%$ of the mentored group felt someone cared if they quit versus $51 \%$ in the control $(\mathrm{p}<0.01) ; 78 \%$ felt like they knew how to quit versus $56 \%$ in the control group $(\mathrm{p}=0.03)$; and $78 \%$ liked participating versus $74 \%$ in the control $(\mathrm{p}=0.59)$. Opt-out rates were similar across treatment arms (15\% versus $18 \%$ ). Users were able to differentiate between the automated and personal messages from a mentor: about $90 \%$ in each group correctly identified whether they had received personal messages. Nearly two-thirds of those in the mentor support group preferred the personal messages to the automated messages, and $81 \%$ were satisfied with their mentor. Implications: Text-based peer mentoring from former smokers holds potential as an acceptable, effective, and engaging smoking cessation intervention and may be an attractive, convenient, option for smokers who desire ongoing peer support in their attempts to quit. Future research should examine whether this modality is an effective tool for health coaches to assist employees in their quit smoking attempts.

CORRESPONDING AUTHOR: Johann Westmaas, American Cancer Society, lee.westmaas@cancer.org 
S22.4

INNOVATIONS IN DHEALTH: TEXT OR VIDEOS; EHEALTH OR MHEALTH?

Kei Long Cheung ${ }^{1}$, Hein de Vries ${ }^{1}$

${ }^{1}$ Maastricht University- Netherlands

Introduction \& Purpose: Computer tailored interventions have shown to be effective for a wide range of behaviors, including physical activity and eating behavior. Yet, reaching the population is still a big challenge, certainly when another Internet site is just one click away. We will discuss the effects of using different methods of providing dHealth communication. Methods: Study 1 used a RCT to test the effects on Dutch adults of an obesity prevention intervention with three conditions: 1 . Text tailoring, 2. Video tailoring, and 3. control group. Study 2 consisted of a RCT with three conditions: 1. eHealth, 2. mHealth, and 3. Control group to assess the effects on physical activity. Results: Study 1 showed that both the video tailored conditions were more effective than the control group after 6 months on energy intake. The video condition was most effective and resulted in significant changes concerning energy intake after 6 and 12 months. Video tailoring was also evaluated more positively than text tailoring. Study 2 found that receiving tailored feedback resulted in more physical activity after 6 months than in the control group. Compared to the mHealth condition, the eHealth program was most effective, had lower drop-out rates, and was appreciated better. Conclusions: The delivery of tailored messages is important. Text messages are less attractive than video messages and also less effective. Future research needs to identify the optimal balance between using text and other communication methods. mHealth has the advantage of being easily accessible but may also result in more drop-out. How to best use mHealth - with or without eHealth - is important to assess in future studies.

CORRESPONDING AUTHOR: Kei Long Cheung, Maastricht University, kl.cheung@maastrichtuniversity.nl

\section{$\mathrm{S} 22.5$}

EFFECTIVE CO-CREATION OF D-HEALTH INTERVENTIONS FOR HEALTH PROMOTION AND CARE

Silvia Matrai ${ }^{1}$, Hein de Vries ${ }^{2}$, Fleur Braddick ${ }^{3}$, Antoni Gual ${ }^{3}$, Kei Long Cheung $^{2}$

${ }^{1}$ Hospital Clínic de Barcelona- Spain

${ }^{2}$ Maastricht University- Netherlands

${ }^{3}$ Hospital Clínic de Barcelona- Spain

Introduction \& Purpose: d-Health is considered as having great potential for personalized healthcare. Yet the high turnover and short lifecycle of most d-Health solutions is evidence of the limited uptake and scarce adherence by their intended users. To ensure a sustained use of $d-$ Health, the whole range of targeted users should be mapped and involved from the concept stage of product development, so that the solutions delivered meet their priorities, motivations, needs and expectations, and draw on their specific knowledge and skills. This process is referred to as co-creation. Yet, various stakeholders appear to have varying motivations related to the use of d-Health, speak different jargons, and often lack specifics on areas outside of their sector. There is thus a need to create a knowledge and resource base for stakeholders to understand why and how to use co-creation methods and processes in conceptualizing, designing, deploying and evaluating d-Health. Methods: We assessed needs for knowledge and resources for co-creation among a range of stakeholders including d-Health businesses, academia, patients, champions and opinion leaders, healthcare professionals, providers, payers, and policy makers. An online stakeholder survey and two interactive multistakeholder workshops were deployed to identify gaps in attitudes, knowledge and skills related to co-creation of d-Health, and to establish a basis for guidance and a modular training programme on designing processes for effective co-creation of dHealth. Results: Three main thematic pillars were identified: the integration of the motivational approach into d-Health development; innovative collaborative business models in d-Health; and models, methods and challenges of co-creation in d-Health. For each pillar, a number of specific topics were established, related to practical knowledge and skills to co-creating d-Health in real world settings. The pillars and topics form the skeleton of a guidance document and the modular training programme that is being developed and will be pilot tested twice by October 2018 in order to fine tune the market-ready version. Conclusions: Practical guidance and continuing training in co-creating are expected to enable stakeholders to boost the efficacy of d-Health interventions.

CORRESPONDING AUTHOR: Silvia Matrai, Hospital Clínic de Barcelona, smatrai@clinic.cat

\section{S22.6}

A RANDOMIZED TRIAL OF A TEXT-MESSAGING PROGRAM TO PROMOTE SMOKING CESSATION IN PREGNANT SMOKERS

Lorien Abroms ${ }^{1}$, Leavitt ${ }^{1}$, PR. Johnson ${ }^{2}$, Cleary ${ }^{1}$, T. Brandon ${ }^{3}$, J. Schindler-Ruwisch ${ }^{1}$, J. Bushar ${ }^{4}$,

${ }^{1}$ Milken Institute School of Public Health at the George Washington University- USA

${ }^{2}$ Voxiva, Inc- USA

${ }^{3}$ offitt Cancer Center- USA

${ }^{4}$ Zero to Three- USA

While automated text messaging programs have been found to be successful for adult smoking cessation, their efficacy for pregnant smokers is unknown. The Quit4baby study explores whether an interactive and intensive text messaging program can promote smoking cessation in pregnant smokers. Women were recruited from Text4baby, an existing text messaging program aimed at pregnant women. From July 2015 to February 1016, over 35,000 Text4baby subscribers were sent a recruitment text message. Interested and eligible women were enrolled $(n=497)$ and randomized to receive Text4Baby + Quit4Baby (intervention) or Text4Baby (control). Participants were surveyed at 1-month, 3-month and 6-months post-enrollment, and saliva samples were collected at 3months for biochemical verification of smoking status. Using an Intention-to-Treat analysis, where missing are assumed to be smoking, $28.80 \%$ of the intervention group and $15.79 \%$ of control group reported not smoking in the past 7 days at 1 -month $(\mathrm{p}<.01)$ and $35.20 \%$ of the intervention group and $22.67 \%$ of the control group reported not smoking in the past 7 days at 3 -month $(\mathrm{p}<.01)$. Biochemical verification of smoking status at 3-months indicated no significant differences among groups, though quit rates favored the intervention group. Results will also be presented for the 6-month follow-up. Current results provide mixed support for the efficacy of the Quit4baby program. Future study is warranted.

CORRESPONDING AUTHOR: Lorien Abroms, Milken Institute School of Public Health at the George Washington University, lorien@gwu.edu

S23.

COUPLE-BASED RESEARCH IN CANCER: NOVEL APPROACHES TO ASSESSMENT AND INTERVENTION Shelby Langer ${ }^{1}$

${ }^{1}$ Arizona State University-USA

There is a burgeoning literature describing the impact of cancer diagnosis and treatment on couples' relationships, as well as couple-based interventions designed to improve the adjustment of both patients and partners. However, there are still notable gaps in our knowledge of couple functioning in the context of cancer, due in part to the methodological 
limitations of studies to date. Most descriptive studies have relied on cross-sectional designs which limit our ability to discern temporal patterns and retrospective self-report measures which require participants to both remember and summarize their behavior. Most couple-based interventions have been broad in scope and have focused primarily on patients with early stage cancer; many have also suffered from difficulties with recruitment. Thus, novel methodological and interventional approaches are needed both to advance the science and to understand how to best meet the needs of couples facing cancer. This symposium will highlight three examples of innovative approaches for assessing couple functioning and conducting couple-based interventions in the context of cancer. The first speaker, Dr. Shelby Langer, will present data from a smartphonebased ecological momentary assessment study that was designed to examine temporal associations between how patients and partners communicate, their mood, and their relationship satisfaction. The second speaker, Dr. Laura Porter, will describe a novel caregiver-assisted intervention for pain management among patients with advanced cancer, focusing on the ways in which a dyadic intervention can be employed to improve symptom management, caregiver burden, and relationship functioning. The third speaker, Dr. Tanja Zimmermann, will describe a dyadic coping intervention for breast cancer survivors and their partners that was targeted to couples screened for individual and/or dyadic distress. The discussion, led by Dr. Tracey Revenson, will focus on implications of these studies for improving our understanding of couple functioning in the context of cancer and designing interventions that meet the needs of these couples.

\section{S23.1}

USING SMARTPHONE TECHNOLOGY TO EXAMINE INTRAAND INTER-PERSONAL ASSOCIATIONS OF COMMUNICATIVE BEHAVIOR WITH MOOD AND RELATIONSHIP SATISFACTION IN PATIENTS WITH CANCER AND THEIR SPOUSAL CAREGIVERS

Shelby Langer ${ }^{1}$, Mike Todd ${ }^{1}$, Joan Romano ${ }^{2}$, Karen Syrjala ${ }^{3}$, Jonathan Bricker $^{3}$, Niall Bolger ${ }^{4}$, John Burns ${ }^{5}$, Neeta Ghosh ${ }^{3}$

${ }^{1}$ Arizona State University- USA

${ }^{2}$ University of Washington- USA

${ }^{3}$ Fred Hutchinson Cancer Research Center- USA

${ }^{4}$ Columbia University- USA

${ }^{5}$ Rush University- USA

The extent to which persons with cancer and their spouses express their feelings and respond to one another is of paramount importance to the adjustment of both dyad members. In this study, patients with stage 2-4 breast or colorectal cancer and their spousal caregivers $(\mathrm{N}=74$ couples, $\mathrm{M}=51.7$ years old, $3 \%$ Hispanic, $87 \%$ Caucasian, $62 \%$ female patients, and $38 \%$ female caregivers) independently completed twice-daily assessments of communication, negative affect (NA), and relationship satisfaction via smartphone application in the early afternoon and evening for 14 sequential days ( $89 \%$ response rate). We examined intra- and interpersonal associations (i.e., actor and partner effects) between afternoon communication (the extent to which participants expressed their feelings, held back from expressing their feelings, supported their partner, and criticized their partner) and evening NA and relationship satisfaction using linear mixed models employing an Actor Partner Interdependence Model. Two actor, or within-person, effects emerged for NA. Patients' holding back was positively related to their own evening NA $(b=0.06 \mathrm{p}=$ .043) and caregivers' support of the patient was negatively related to caregivers' own NA ( $b=-0.09, p=.013)$. A partner effect also emerged, such that caregivers' disclosure was positively related to patients' NA (b $=0.07, p=.008)$. With relationship satisfaction as the outcome, actor effects for supporting one's partner were found for both dyad members. Being supportive was positively related to one's own evening relationship satisfaction $(b=0.17, p=.022$ for patients; $b=0.21, p=.011$ for caregivers). An actor effect was also found for caregiver's criticism of his/her patient partner such that relatively high criticism was related to lower relationship satisfaction for the caregiver $(b=-0.20, p=.036)$. With respect to partner effects, patients' reports of supporting their partners and criticizing their partners were, respectively, positively $(b=0.10, p=$ $.045)$ and negatively $(b=-0.13, p=.035)$ related to caregivers' relationship satisfaction. Likewise, caregivers' criticism of patients was negatively related to patients' relationship satisfaction $(b=-0.12, p=.049)$. Findings from this smartphone-enabled ecological momentary assessment study (to our knowledge, the first in cancer) underscore the importance of patient and caregiver disclosure and responsiveness in explaining later adjustment both within and across dyad members.

CORRESPONDING AUTHOR: Shelby Langer, Arizona State University, shelby.langer@asu.edu

\section{S23.2}

CAREGIVER-GUIDED PAIN MANAGEMENT FOR PATIENTS WITH ADVANCED CANCER

Laura Porter ${ }^{1}$, Francis Keefe ${ }^{1}$

${ }^{1}$ Duke- USA

Purpose: Pain a major concern of patients with advanced cancer and their family caregivers. Behavioral pain management strategies have proven effective but have rarely been tested in patients with advanced disease or included caregivers. We developed a caregiver-guided pain management training (CGPMT) intervention for patients with advanced cancer and pain and are currently testing its efficacy in a multi-site randomized clinical trial. Outcomes include caregiver self-efficacy for helping the patient manage pain, caregiver adjustment, patient self-efficacy, patient pain, and patient psychological distress. The aim of this presentation is to provide a description of the intervention and its implementation. Methods: Patient eligibility criteria include age $\geq 18$, Stage IV cancer, at least one month life expectancy, worst pain rating $\geq 4$ and a family caregiver also willing to participate. Dyads are randomized to either the CGPMT intervention or to an enhanced standard care condition which provides them with pain-focused educational materials. Patients and caregivers complete assessments at baseline and post intervention, and caregivers complete follow-ups 3 and 6 months following patient's death. To date, 155 dyads have enrolled. Patients are $60 \%$ male, mean age $=64.5$ years $(\mathrm{SD}=11.2), 79 \%$ Caucasian. The most frequent cancer sites are lung (20\%), GI (19\%), and prostate (13\%). Caregivers are $27 \%$ male, mean age $=60$ years $(\mathrm{SD}=13.4), 74 \%$ Caucasian; $69 \%$ are the spouse/partner of the patient. Results: To meet the needs of patients with serious illness and their caregivers, the CGPMOT intervention is brief ( 3 sessions) and delivered via videoconference using tablet computers which are provided to participants. Sessions are conducted jointly with the patient and caregiver. The intervention includes training patients and caregivers in (a) individual pain coping skills such as relaxation and pleasant imagery, which can also be used by the caregiver for management of stress/distress, and (b) communication skills to enhance the dyad's ability to communicate effectively about pain and its impact and to set goals together for increasing pleasurable and meaningful activities. Patients and caregivers learn to jointly practice and apply pain coping skills as a way of decreasing their distress and burden and enhancing a sense of meaning and closeness during this stressful and demanding time. The presentation will include a case example describing implementation of the protocol with a patient-partner dyad. Conclusion: A caregiver-guided intervention that combines training in pain coping skills and relational skills is a novel approach to pain management in the context of advanced cancer that may be of significant benefit to patients and their partners/family caregivers.

CORRESPONDING AUTHOR: Laura Porter, Duke, laura.porter@duke.edu 
S23.3

COUPLES COPING WITH CANCER: A COUPLES-BASED SKILLS INTERVENTION FOR BREAST CANCER PATIENTS AND THEIR PARTNERS - RESULTS OF TWO RCTS

Tanja Zimmermann ${ }^{1}$

${ }^{1}$ Hannover Medical School- Germany

A cancer diagnosis is a stressful event that poses formidable and enduring challenges to the women and to their intimate partners. A conjoint process of mutual support has been suggested to be an effective coping strategy for patients who are in committed relationships. The primary aim of this study was to gather data on the potential benefits of a brief psychological intervention ("Side by Side") designed to assist women and their partners to cope with breast cancer in two different studies. Two randomizedcontrolled studies were conducted at two different time points. Study I: $\mathrm{N}=72$ couples were randomly assigned to either the couples' component ("Side by Side"), or an information component directly after the diagnosis whereas in study II $\mathrm{N}=46$ couples were randomly assigned to "Side by Side" or relaxation after completing the medical treatment. Moreover in this second study couples were screened in advance for individual and/or dyadic distress before they receive the intervention. Results of study I suggest that women receiving Side by Side showed larger reductions in cancer-related distress and fear of progression post-intervention compared to women in control group. Furthermore, couples in the Side by Side intervention showed less avoidance in dealing with the cancer, more posttraumatic growth, and better communication skills and dyadic coping relative to the control group. Study II showed a reduction of cancerrelated distress in both groups but only in the Side by Side group improvements of relationship satisfaction occured. Short-term changes in cancer-related functioning may be improved by enhancing couples' dyadic coping skills. The findings highlighted the importance of helping both, the woman and her partner, to effectively cope with cancer. Different implications of couple-based intervention were discussed.

CORRESPONDING AUTHOR: Tanja Zimmermann, Hannover Medical School, zimmermann.tanja@mh-hannover.de

\section{S24}

INTERDISCIPLINARY RESEARCH ON COGNITIVE IMPAIRMENT AND WELLBEING AMONG OLDER PEOPLE EPIDEMIOLOGICAL, BIOMEDICAL AND SOCIAL PERSPECTIVES

Shulin Chen ${ }^{1}$

${ }^{1}$ Zhejiang University- China

WHO points out that cognitive impairment among older people is a global health priority. Health care work and research needs to incorporate interdisciplinary perspectives. A collection of such research is presented in this symposium to illustrate importance of employing various approaches to understand problems and impact of cognitive impairment. Three of the four presentations are based on empirical data and one involves a review of the most update literature. The first presentation is based on a large scale longitudinal study collected data from 1998 to 2014, and reported trends of increase in cognitive impairment followed by a decrease; multidimensional factors were identified to inform health workers, demonstrating the importance of epidemiological studies. The second presentation reminds us of the importance of biomedical research in developing effective prevention for dementia which is urgently warranted, and looks at the potential impact of vascular risk factors which are modifiable. The third presentation reminds us of importance of psychosocial factors, social support in particular, and issues of geographic disparity which may be related to variations levels of psychosocial factors. The last presentation reminds us importance of working at the primary care setting, and that cognitive function is an important factor of quality of life, among other factors, users of primary care services. The symposium hence reflects the interdisciplinary nature of behavioral medicine that integrates epidemiological, biomedical and social sciences approaches. It also introduces important areas of research on cognitive impairment among older people that have been conducted in China.

S24.1

THE REGIONAL DISPARITIES OF SOCIAL SUPPORT AND COGNITIVE FUNCTIONING AMONG ELDERS IN RURAL CHINA Jiang Xue ${ }^{1}$, Shulin Chen ${ }^{1}$, Jiaming Liang ${ }^{1}$, Peiyuan Qiu ${ }^{2}$

${ }^{1}$ Zhejiang University- China

${ }^{2}$ Sichuan University

Objectives: Social support has generally been found to have positive effects on cognitive function of rural elders. The effect is likely to vary between regions because rural regions are socio-economically and environmentally diverse. Previous studies reported various prevalence of cognitive impairment in different regions of China. This study explored the region-specific effect of social support on cognitive function among rural elders in East and West China. Methods: Elders aged 60 years or more were randomly selected from Jiande County (East China) and Fushun County (West China) respectively. The assessment package included: General Characteristics Questionnaire, DSSI (Duke Social Support Index), and MMSE (Mini-Mental State Examination). The analysis methods included descriptive statistics and logistic regression. Results: The final sample included 218 participants from Jiande County and 318 participants from Fushun County. The prevalence of cognitive impairment is $26.15 \%$ in Jiande and $33.65 \%$ in Fushun. Participants in Jiande had higher education level and better financial status. They also reported higher social support than participants in Fushun. The logistic regression implied that social support is negatively associated with cognitive impairment. Perceived social support shows moderating effect on reducing the regional disparities of cognitive impairment in China. Conclusions: This study reported the high prevalence of cognitive impairment in rural areas of East China, which is more significant in rural West China. The model presented in the study shows that the regional difference of cognitive impairment is related to the disparities of social support in two regions. This model provides good support for the future scale-up preventive strategies for cognitive impairment, which is applicable to the East and West China.

CORRESPONDING AUTHOR: Jiang Xue, Zhejiang University, jiang_xue@urmc.rochester.edu

\section{$\mathrm{S} 24.2$}

TRENDS IN THE PREVALENCE OF COGNITIVE IMPAIRMENT IN CHINESE OLDER ADULTS: BASED ON THE CLHLS COHORTS FROM 1998 TO 2014

Weihong Kuang ${ }^{1}, \mathrm{M} \mathrm{Gao}^{1}, \mathrm{~L} \mathrm{Tian}^{1}$, Y Wan ${ }^{2}$, Peiyuan Qiu ${ }^{2}$

${ }^{1}$ Mental Health Center, Sichuan University, Chengdu, China

${ }^{2}$ Public Health School, Sichuan University, Chengdu, China

Objective: This study aims to examine trends in cognitive impairment (CI) prevalence of people aged 65+ years old in China over time. Method: Data were drawn from the seven waves (1998, 2000, 2002, 2005, 2008-2009, 2011-2012, 2014) of the Chinese Longitudinal Healthy Longevity Survey (CLHLS), a national mixed longitudinal cohort. Our study sample consisted of older adults aged $65 \sim 105$. We excluded those who reported blindness, deafness or depression. The Chinese version of the Mini-Mental State Examination (CMMSE) was used to measure CI. A CMMSE score of 18 points or lower was used as the standard for diagnosing CI. Crude and age-standardized prevalence of CI by gender were calculated. Risk factor adjusted trends in the prevalence over time were examined using multilevel regression models. Results: The sample size were 7882 in 1998, 9604 in 2000, 14076 in 
2002, 13482 in 2005, 13432 in 2008, 8266 in 2011, and 6079 in 2014. The total age-standardized prevalence of CI was $11.00 \%$ in $1998,10.47 \%$ in $2000,11.83 \%$ in $2002,11.67 \%$ in $2005,11.84 \%$ in $2008,9.14 \%$ in 2011 and $8.88 \%$ in 2014. Older age, female, less education, rural residence, not married, lack of physical and cognitive activities, suffering from stroke, vision and hearing impairment and ADL disability were risk factors for CI. Discussion: The result showed an increasing trend in the prevalence of CI first and a decreasing trend after 2008. In China, rapid ageing may lead to increased prevalence; while more years of education, higher economic status, and efforts of prevention of chronic diseases may lead to decreased prevalence. More time points after 2014 are needed to determine whether the prevalence of $\mathrm{CI}$ in China is decreasing.

CORRESPONDING AUTHOR: Peiyuan Qiu, ${ }^{2}$ Public Health School, Sichuan University, qiupeiyuan0218@163.com

\section{S24.3}

VASCULAR BURDEN AND CLINICAL EXPRESSION OF DEMENTIA SYNDROME: AN OPPORTUNITY FOR INTERVENTION?

Cheng Xuan Qiu ${ }^{1}$

${ }^{1}$ Aging Research Center, Karolinska Institutet-Stockholm University, Stockholm, Sweden

Introduction \& Purpose: Dementia has been recognized by WHO as a global public health priority. There is currently no cure or a diseasemodifying therapy for dementia. Identifying the interventions to reduce risk of dementia has therefore become critical. We sought to summarize multidisciplinary research that examined the relationship between vascular burden and risk of dementia, and thus providing evidence for possible interventions against dementia by targeting vascular pathways. Methods: We search the literature of population-based epidemiological, neuropathological, and neuroimaging studies investigating the presence and load of cardiovascular risk factors and vascular diseases in association with the risk of dementia. Results: Vascular lesions in the brain are increasingly common and accumulated with advancing age. In the last two decades, evidence from numerous epidemiological studies has repeatedly shown that vascular risk factors (e.g., smoking, hypertension and diabetes) and related vascular diseases (e.g., atrial fibrillation, heart failure, and cerebrovascular lesions) were associated with an increased risk of dementia. Further, neuropathological and neuroimaging studies confirmed that clinical and silent cerebrovascular lesions (e.g., brain infarcts, white matter lesions, cerebral microbleeds and lacunes) alone or in combination with brain degenerative pathologies (e.g., loss of neurons and amyloid- $\beta$ ) could promote or accelerate the onset and clinical expression of the dementia syndrome. Conclusions: A greater load of cerebrovascular burden is associated with clinical expression and progression of dementia. This implies that interventions targeting vascular risk factors to reduce the burden of vascular pathologies in the brain should be effective in delaying the onset of dementia syndrome, thus reducing its societal burden.

CORRESPONDING AUTHOR: Dr. Cheng Xuan Qiu, Aging Research Center, Karolinska Institutet-Stockholm University, chengxuan.qiu@ki.se

\section{S24.4}

\section{FACTORS ASSOCIATED WITH HEALTH-RELATED QUALITY OF} LIFE IN CHINESE OLDER ADULTS IN PRIMARY CARE

Baoliang Zhong, ${ }^{1}$ Yeates Conwell ${ }^{2}$

${ }^{1}$ Wuhan Mental Health Center, Tongji Medical College of Huazhong University of Science \& Technology, Wuhan China

${ }^{2}$ Department of Psychiatry, University of Rochester Medical Center, Rochester, NY, USA

Background: The ongoing healthcare system reform in China has made great efforts to strengthen primary care health services for both urban and rural older adults (OAs). Health-related quality of life (QOL) is as important in older age as at any other time of life, particularly for OAs with physical illnesses, however, the QOL of OAs treated in primary care is understudied in China. This study investigated the level and correlates of QOL in OAs treated in Chinese primary care. Methods: A total of 744 OA patients $(65+$ years) were consecutively recruited from 13 primary care clinics in Wuhan, China, and interviewed with a standardized questionnaire, concerning socio-demographic characteristics, chronic conditions, felling of burden to family, depression, loneliness, cognitive function, and sensory impairments. QOL was measured with the six-item QOL scale. Multiple linear regression was used to identify correlates of QOL. Results: The average QOL score of primary care OA patients was (20.7 \pm 2.5 ). Factors significantly and independently associated with poor QOL included being manual workers before older adulthood $(\beta=-0.550$, $\mathrm{P}=0.011)$, burdensome to family $(\beta=-0.563, \mathrm{P}=0.039)$, depressive symptoms $(\beta=-1.261, P<0.001)$, no physical exercise habit $(\beta=-0.161$, $\mathrm{P}=0.002)$, loneliness $(\beta=-0.843, \mathrm{P}=0.001)$, and poor cognitive function $(\beta=-0.058, P=0.007)$. Conclusions: OAs of Chinese primary care report a moderate level of QOL. To improve QOL of Chinese primary care OAs, mental health problems such as depression and cognitive decline, social problems such as loneliness and burdensome, and unhealthy lifestyle need to be addressed.

CORRESPONDING AUTHOR: Baoliang Zhong, Wuhan Mental Health Center, Tongji Medical College of Huazhong University of Science \& Technology, haizhilan@gmail.com

O01.1

MODERATORS OF ADVERSE EFFECTS OF SOCIAL CONSTRAINTS ON DISTRESS IN SURVIVORS TREATED WITH STEM CELL TRANSPLANT: THE ROLE OF RELATIONSHIP SATISFACTION AND NEGATIVE NETWORK ORIENTATION

Christine Rini ${ }^{1}$, Jane Austin ${ }^{2}$, Lisa $\mathrm{Wu}^{3}$, Heiddis Valdimarsdottir ${ }^{4}$, Scott Rowley ${ }^{1}$, William Redd ${ }^{5}$

${ }^{1}$ Hackensack University Medical Center- USA

${ }^{2}$ William Paterson University- USA

${ }^{3}$ Northwestern University- USA

${ }^{4}$ Reykjavik University- Iceland

${ }^{5}$ Icahn School of Medicine at Mount Sinai- USA

Background and aims: Research shows elevated distress in cancer survivors with higher social constraints on disclosure of thoughts and feelings about their treatment. The present study examined 2 factors that may moderate adverse effects of social constraints on cancer-related distress: the quality of survivors' relationship with the confidante and survivors' dispositional tendency to avoid seeking or accepting support (negative network orientation). Focusing on social constraints with a partner - the most important source of support for partnered adults - we hypothesized that adverse effects of social constraints would be reduced for survivors with negative network orientation because constraints are consistent with their negative orientation, but that adverse effects would be increased for survivors with a more satisfied relationship with their partner because constraints are inconsistent with how they normally interact with their partner. Methods: Participants were 250 married or partnered cancer survivors who had undergone stem cell transplant. Data were from the prerandomization baseline assessment of a randomized controlled trial that included the Social Constraints Scale, Network Orientation Scale, and Impact of Event Scale. Results: Controlling for potential confounds, higher partner social constraints was positively associated with distress $(\mathrm{p}<.001)$. A more negative network orientation was marginally associated with higher distress $(p=.07)$, as was higher relationship satisfaction $(\mathrm{p}=.06)$. There was no interaction between partner social constraints and negative network orientation ( $\mathrm{p}=.41$ ), suggesting that adverse effects of constraints are independent of a survivor's orientation toward accessing support resources. An interaction between partner social constraints and 
relationship satisfaction $(\mathrm{p}=.001)$ showed that the association between constraints and distress became stronger as relationship satisfaction increased. Conclusion: These findings have implications for couplefocused interventions after cancer. We recommend screening for and intervention to reduce partner social constraints in cancer survivors who are partnered, with particular attention to survivors with partner social constraints despite a satisfied partner relationship.

CORRESPONDING AUTHOR: Christine Rini, Hackensack University Medical Center, christine.rini@hackensackmeridian.org

\section{O01.2}

THE EFFECT OF SOCIAL CONSTRAINTS ON FEAR OF CANCER RECURRENCE AND PSYCHOLOGICAL ADJUSTMENT AMONG PARENTS OF ADOLESCENTS AND YOUNG ADULTS WITH CANCER

Tracey Revenson ${ }^{1}$, Emilia Mikrut ${ }^{1}$, Aliza Panjwani ${ }^{1}$

${ }^{1}$ Hunter College \& The Graduate Center - USA

Introduction and Aims: Parents of adolescents and young adults (AYAs) diagnosed with cancer often require social support themselves, experience psychological distress, and worry their child's cancer will recur. Because social constraints can inhibit disclosure, fear of cancer recurrence (FCR) may become magnified. We examined the: 1) direct effects of social support and social constraints on psychological distress; 2) buffering effect of social support on social constraints and distress; and 3) indirect effect of FCR on social constraints and distress. Methods: Data were collected in an online survey of 71 parents (93\% mothers) of AYAs (aged 15-39) with cancer. Psychological distress was assessed using validated measures of depressive symptoms (PHQ-9), anxiety (GAD-7), and post-traumatic stress (IES-R). FCR was assessed with an adaptation of Vickberg (2003). Support and constraints were measured with the Contextual Illness-Specific Support Scale (CISS) and Lepore's Social Constraints Scale (SCS). Results: $32 \%$ of parents reported moderate-severe depressive symptoms, 30\% moderatesevere post-traumatic stress (PTS), and 14\% moderate-severe anxiety; $60 \%$ of parents reported moderate-high FCR. Social support predicted depressive symptoms $(\mathrm{p}=.02)$, but not anxiety or PTS. Social constraints predicted all three distress outcomes ( $\mathrm{p}$ 's $<.001)$. Social support did not moderate the relationship between social constraints and any distress outcome. The total indirect effect of social constraints on FCR and on all distress outcomes was significant, suggesting an indirect relationship between social constraints and distress mediated by FCR (total indirect effect for PTS, $\beta=.32$, CI $[0.11$, $0.58]$; depressive symptoms, $\beta=.07$, CI [0.03, 0.14], anxiety, $\beta=.05$, CI $[0.02,0.12])$. This indirect effect accounted for $25 \%, 16 \%$ and $16 \%$ of the variance in PTS, depressive symptoms, and anxiety, respectively. Conclusion: Among parents of AYAs with cancer, the negative aspects of social relationships on adjustment may outweigh the positive. The data suggest a possible pathway (FCR) through which social constraints affect distress. Future caregiver interventions may benefit from including skills-based work on improving family communication to minimize social constraints.

CORRESPONDING AUTHOR: Tracey Revenson, Hunter College \& The Graduate Center, Tracey.Revenson@hunter.cuny.edu

\section{O01.3}

PREVALENCE AND PREDICTORS OF RELATIONSHIP DISTRESS AMONG HEMATOPOIETIC CELL TRANSPLANT SURVIVORS: A LARGE CROSS-SECTIONAL STUDY

Shelby Langer ${ }^{1}$, Karen Syrjala ${ }^{2}$, Jean $\mathrm{Yi}^{2}$, Stephanie Lee ${ }^{2}$

${ }^{1}$ Arizona State University- USA

${ }^{2}$ Fred Hutchinson Cancer Research Center- USA

Introduction: Hematopoietic cell transplantation (HCT) is a rigorous treatment for hematologic malignancies and other diseases, with established medical sequelae. Less is known regarding impacts on relationships, in particular, the extent to which patients report long-term marital adjustment or lack thereof, especially given caregiving demands placed on significant others. We sought to determine the prevalence and predictors of relationship distress in a large sample of HCT survivors. METHODS: In this cross-sectional survey, HCT survivors completed items designed to assess demographic and medical characteristics, the Cancer Treatment Distress scale, and the Revised Dyadic Adjustment Scale (RDAS). Single-sample t-tests afforded comparison of our RDAS scores to pooled means across (1) community samples and (2) couple therapy samples, as reported in Anderson et al. (2014). Logistic regression analyses were conducted to identify predictors of relationship distress $(<48$ on the RDAS). Predictors were: age at transplant, years post-transplant, sex, race, presence of graft-versus-host disease, and cancer treatment distress. RESULTS: 2036 survivors (45\%) responded to the survey. Our analysis sample included married and partnered/ cohabiting survivors who completed at least $50 \%$ of the RDAS items $(\mathrm{n}=1317)$. Demographic and clinical characteristics were: $M(S D)$ age $=58.06$ (11.48), $44 \%$ female, $3 \%$ Hispanic, 90\% Caucasian, $\mathrm{M}(\mathrm{SD})$ years post-transplant $=11.77$ (9.56), and 69\% allogeneic transplant. The average RDAS total score (possible range $0-69$ ) was 53.21 ( $\mathrm{SD}=7.95$ ). 19\% of survivors scored in the relationship-distressed range. Total scores did not differ from community samples $(\mathrm{t}=1.21, \mathrm{p}=.228)$ and were higher than couple therapy samples $(t=57.47, p<.001)$. Results of regression analyses revealed predictors of relationship distress: more years post-transplant $(\mathrm{OR}=1.02$, $\mathrm{p}=.015)$, non-White race $(\mathrm{OR}=1.88, \mathrm{p}=.003)$, and higher cancer treatment distress $(\mathrm{OR}=1.81, \mathrm{p}<.001)$. CONCLUSION: While, on average, our HCT survivors scored in the relationship-adjusted range and did not differ from community samples, $19 \%$ reported relationship distress. Minorities and those with persisting transplant-related distress are important targets for screening and intervention.

CORRESPONDING AUTHOR: Shelby Langer, Arizona State University, shelby.langer@asu.edu

\section{O01.4}

POST-SURGICAL PAIN INTENSITY PREDICTS INTRUSIVE THOUGHTS AND DEPRESSED AFFECT FOLLOWING TREATMENT FOR NON-METASTATIC BREAST CANCER

Hannah Fisher ${ }^{1}$, Chloe Taub ${ }^{1}$, Suzanne Lechner ${ }^{1}$, Charles Carver ${ }^{1}$, Michael Antoni ${ }^{1}$

${ }^{1}$ University of Miami- USA

: Introduction \& Purpose: Many women experience pain following surgery for non-metastatic breast cancer. Post-surgical pain is linked to poorer long-term mental health, yet the longitudinal mechanism of this relationship is unclear. Intrusive thoughts regarding the breast cancer experience may play a role in the association between pain and longterm emotional well-being. This study aimed to test a mediation model elucidating the longitudinal relationship between post-surgical pain and depressed affect 1 year later via intrusive thoughts following primary treatment. Method: Stage 0-III breast cancer patients $(\mathrm{N}=240)$ were recruited 4 to 10 weeks post-surgery and completed a baseline (T1) questionnaire measuring pain, intrusive thoughts, and depressed affect. Women were reassessed 6- (T2) and 12-months (T3) later. Structural equation modeling in Mplus was used to test the proposed mediation model. Age, stage, receipt of chemotherapy and/or radiation, condition, and proceeding values of the mediator and outcome variables served as covariates. Results: The specified model was consistent with the data $(\chi 2$ $(14)=17.50, p=.23$; RMSEA $=.03$ (90\% CI $[.00, .07])$; CFI=.98; $\mathrm{SRMR}=0.03)$. Significant direct effects emerged from $\mathrm{T} 1$ pain intensity to T2 intrusive thoughts $(\beta=.15,95 \%$ CI $[.03, .30], \mathrm{p}<.05)$, and to T3 depressed affect $(\beta=.14,95 \%$ CI $[.01, .27], p<.05)$. Intrusive thoughts at $\mathrm{T} 2$ did not predict T3 depressed affect, and there was no evidence of mediation. Conclusions: Greater pain in the weeks following surgery 
may serve as a reminder of one's breast cancer diagnosis and treatment, contributing to more frequent intrusive thoughts up to 6-months later. Although greater post-surgical pain was associated with more depressed affect 1 year following surgery, this relationship was not mediated by more frequent intrusive thoughts. Avoidance or withdrawal from social and/or pleasant activities should be explored as alternative mediators. Clarifying the relationship between pain experiences earlier on the in breast cancer trajectory and subsequent emotional functioning may inform more precise pain management.

CORRESPONDING AUTHOR: Hannah Fisher, University of Miami, hannah.fisher@miami.edu

\section{O01.5}

INTRUSIVE THOUGHTS, SOCIAL CONSTRAINTS AND THE EFFICACY OF GENETIC COUNSELING FOR BREAST CANCER SUSCEPTIBILITY

Heiddis B. Valdimarsdottir ${ }^{1}$, Kamilla R. Jóhannsdóttir ${ }^{1}$, Ariella HoffmanPeterson $^{2}$, Mary Kate Ladd ${ }^{3}$, Christine Rini ${ }^{4}$, Beth N. Peshkin ${ }^{3}$, Kristi Graves $^{3}$, Marc D. Schwartz ${ }^{3}$

${ }^{1}$ Reykjavik Univeristy- Iceland

${ }^{2}$ Icahn School of Medicine- USA

${ }^{3}$ Georgetown University- USA

${ }^{4}$ Hackensack University Medical Center- USA

Introduction: Consistent with the cognitive processing theory of emotions, research has shown that emotional expression buffers the negative impact of intrusive thoughts on distress. However, it is not known if emotional expression also buffers the effects of intrusive thoughts on cognitive processes, such as retention of new knowledge. We examined whether genetic counseling for breast cancer susceptibility was less effective for women who experience both high levels of cancer-specific intrusive thoughts and social constraints in expressing these concerns. We hypothesized that women who report high levels of cancer-specific intrusive thoughts and who also report feeling socially constrained in expressing their concerns to family and friends, would gain less knowledge from genetic counseling than women with high intrusive thoughts but low social constraints. Patients and methods: Participants were 531 women at high risk for carrying a BRCA1/2 mutation who underwent genetic counseling. Prior to counseling, the women completed the intrusive subscale of the Impact of Events Scale, the social constraint scale, and the Breast Cancer Genetic Counseling Knowledge Questionnaire (BGKQ). One week following counseling, the women repeated the BGKQ. Results: The results from General Linear Model, entering baseline knowledge as a covariate, showed that the interaction between social constraints and intrusive thoughts was significant $(p=0.03)$. Subsequent analyses showed that women with high levels of cancer-specific intrusive thoughts and high levels of social constraint had the lowest knowledge scores after genetic counseling. Discussion: Women with high levels of cancer-specific intrusive thoughts benefit less in terms of knowledge gained from genetic counseling for BRCA testing than women with low levels of intrusive thoughts, but only if they also feel constrained in expressing their concern about breast cancer. These findings suggest that women undergoing genetic counseling may benefit from interventions aimed at assisting them with expressing their emotions and concerns about breast cancer.

CORRESPONDING AUTHOR: Heiddis B. Valdimarsdottir, Reykjavik Univeristy, heiddisb@ru.is

\section{O01.6}

LATENT GROWTH MODELING OF BRIEF PSYCHOSOCIAL INTERVENTION EFFECTS ON CANCER-RELATED INTRUSIVE THOUGHTS OVER THE FIRST 12 MONTHS OF PRIMARY TREATMENT IN NON-METASTATIC BREAST CANCER PATIENTS
Chloe Taub ${ }^{1}$, Hannah Fisher ${ }^{1}$, Amanda Ting ${ }^{1}$, Suzanne Lechner ${ }^{1}$, Charles Carver $^{1}$, Michael Antoni ${ }^{1}$

${ }^{1}$ University of Miami- USA

Introduction: Breast cancer (BCa) treatment imposes significant psychological and physical challenges with negative psychosocial effects. These challenges have been effectively targeted with stress management interventions, such as group-based cognitive-behavioral stress management (CBSM), which combines cognitive-behavioral (CB) techniques and relaxation training (RT). However, shorter interventions may be optimal during primary treatment. Methods: A randomized controlled dismantling study enrolled 183 women with stage 0 -III BCa $2-10$ weeks post-surgery to test the impact of three 5-week interventions: $\mathrm{CB}$, RT, and a health education control (HE). Latent growth modeling examined intervention effects on cancer-specific distress measured by the Impact of Event ScaleIntrusions Subscale (IES-I) over 4 points: baseline, 2 months (post-intervention), 6 months, and 12 months. Results: The model with HE as the reference showed good fit $(\mathrm{X} 2 \mathrm{p}=.054$, RMSEA $=.074, \mathrm{CFI}=.973$, SRMR $=.031)$. CB showed greater pre-post intervention reductions in IES-I vs. HE $(=-.166, \mathrm{SE}=.085, \mathrm{p}=.049)$ and the difference in rate of change over 12 months was marginally greater for CB vs HE ( $=.024$, SE $=.013, \mathrm{p}=.067)$. There were no significant differences for RT vs HE. To examine the difference between $\mathrm{CB}$ and $\mathrm{RT}$, the model was rerun with $\mathrm{CB}$ as the reference and showed good fit $(\mathrm{X} 2 \mathrm{p}=.054$, RMSEA $=.074, \mathrm{CFI}=$ $.973, \mathrm{SRMR}=.026$ ). CB showed greater pre-post intervention reductions in IES-I compared to RT $(=.213, \mathrm{SE}=.088, \mathrm{p}=.016)$ and a significantly greater rate of change vs RT over 12 months $(=-.030, \mathrm{SE}=.014, \mathrm{p}=.030)$. Analyses of mood and quality of life demonstrated greater pre-post intervention change in $\mathrm{CB}$ and RT vs HE (p's $<.05)$ but effects were not maintained at 12-months. Conclusions: Modeling of stress management intervention effects over the 12-month period of primary treatment for $\mathrm{BCa}$ demonstrates significantly greater reductions in cancer-specific intrusive thoughts with a brief 5 -week CB-based stress management intervention compared to attention-matched RT or HE. A greater understanding of the mechanisms through which psychosocial interventions are functioning will aid in the development of more concise and targeted interventions for patients with $\mathrm{BCa}$.

CORRESPONDING AUTHOR: Chloe Taub, University of Miami, cjt87@miami.edu

O02.1

QUALITY OF LIFE OF ISLAND-BASED PUERTO RICAN WITH CORONARY HEART DISEASE, STROKE AND DIABETES

Yarimar Rosa-Rodríguez ${ }^{1}$, Leonell Torres-Pagán ${ }^{2}$

${ }^{1}$ University of Puerto Rico- Puerto Rico

${ }^{2}$ Center for Puerto Rican Studies, CUNY- USA

Introduction \& Purpose: In spite of some progress in healthcare at the national level, heart disease, diabetes and cerebrovascular diseases are among the top leading causes of death in Puerto Rico. As the country face numerous public health and financial constraints, the quality of life among adults with these conditions are expected to aggravate in a near future. Therefore, the present study seeks to examine quality of life changes (mental and physical) in subjects with any of three conditions: coronary heart disease (CHD), stroke and diabetes. Methods: We perform a secondary database analysis using the Puerto Rico Behavioral Risk Factor Surveillance System from 2010 and 2015. This database comprises a representative sample of 8,947 Puerto Ricans (3,220 men; 5,727 women). Statistical analysis was performed using STATA. Prevalence risk ratios for quality of life including mental and physical health (last 30 days) by selected health outcomes were computed as the result of multivariate logistic regression analysis. Results were adjusted for demographic and socio-economic status (SES) proxies. Results: Diabetes was found on a total of 1,824 participants (21\%), while CHD on 881 participants $(9.9 \%)$ 
and stroke on $247(2.7 \%)$. The majority of the sample reported a SES below $\$ 10,000(3,485 ; 48.0 \%)$. Bivariate analysis indicated an association between quality of life and selective health outcomes. Fully adjusted models for the quality of life reported by adults with CHD (Mental Health $-\mathrm{aPR}=2.0,95 \%$ CI 1.6, 2.5; Physical Health $-\mathrm{aPR}=2.1,95 \%$ CI 1.7, 2.5), and stroke (Mental Health- aPR $=1.7,95 \%$ CI 1.1, 2.7; Physical Health- aPR $=1.6,95 \%$ CI 1.0, 2.4) showed an improvement ranging from $10 \%$ to $40 \%$ between 2010 and 2015 . On the other hand, worse quality of life for both mental $(\mathrm{aPR}=1.1,95 \%$ CI $1.0,1.3)$ and physical health $(\mathrm{aPR}=1.5,95 \% \mathrm{CI} 1.3,1.7)$ domains among adults with diabetes increased $10 \%$. Conclusions: Findings highlighted the importance of quality of life experienced by adults with diabetes as an integral part of healthcare. Implications for public policy and practice are discussed.

CORRESPONDING AUTHOR: Yarimar Rosa-Rodríguez, University of Puerto Rico, yarimar.rosa@upr.edu

\section{O02.2}

PSYCHOLOGICAL CORRELATES OF TYPE 2 DIABETES IN MIGRANTS ALONG THE UNITED STATES (U.S.)-MEXICO BORDER Jessica L. McCurley ${ }^{1}$, Jesus A. Naranjo ${ }^{2}$, Juan M. Peña ${ }^{3}$, Jose L. Burgos ${ }^{3}$, Adriana Carolina Vargas-Ojeda ${ }^{4}$, Victoria D. Ojeda ${ }^{2}$, Scott Roesch $^{5}$, Paul Mills $^{2}$

${ }^{1}$ Massachusetts General Hospital/Harvard Medical School- USA

${ }^{2}$ University of California, San Diego- USA

${ }^{3}$ University of New Mexico- USA

${ }^{4}$ Universidad Autónoma de Baja California- USA

${ }^{5}$ San Diego State University- USA

Introduction \& Purpose: Along the U.S.-Mexico border, diabetes prevalence is 2-3 times higher than national averages in either country. This region is home to a high concentration of migrants and deportees at risk for diabetes due to low socioeconomic status, housing/food insecurity, and chronic stress. Investigation of diabetes in migrants and deportees is scant; little is known about the unique risk profiles of these populations. This study aimed to assess prevalence of diabetes and prediabetes in a high-risk border population in Tijuana, Mexico, examine the relationships of depression symptoms, anxiety symptoms, and adverse childhood experiences (ACEs) with diabetes prevalence and glucose regulation, and explore indirect effects of social support in these relationships. Methods: Participants were recruited from a walk-in primary care clinic which primarily serves low-income migrants near the US-Mexico border. Diabetes was diagnosed by hemoglobin $\mathrm{A} 1 \mathrm{c}(\mathrm{HbA} 1 \mathrm{c})$ value $\geq 6.5 \%$, or by self-report of previous diagnosis by a physician, or use of oral antihypoglycemics. Psychosocial variables were measured via self-report. Sample characteristics were estimated in SPSS Statistics; regression models were conducted in MPlus. Results: Participants were 220 adults aged 19-83 years $(\mathrm{M}$ age $=47.2, \mathrm{SD}=11.9)$. The majority was male $(74.5 \%)$ and of Mexican nationality $(89.1 \%)$. Over $70 \%$ reported history of migration to the U.S.; $58.6 \%$ reported at least one deportation. Prevalence of clinically significant depression was $33.2 \%$, clinically significant anxiety was $30.5 \%$, and $49 \%$ of the sample reported $\geq 4$ ACES items. Prevalence of diabetes was $17.3 \%$; prediabetes (HbA1c: $5.7 \%$ $6.4 \%$ ) was $29.1 \%$. Controlling for age, sex, education, and income, psychological variables were not significantly associated with diabetes or $\mathrm{HbAlc}$. Indirect effects were found from depression symptoms and ACEs through social support to HbA1c. Conclusions: This is the first study to our knowledge to examine prevalence and correlates of diabetes in a large number of migrants and deportees, an underserved and hard-toreach population in the U.S.-Mexico border region. Results suggest the need for accessible diabetes prevention interventions in this context that adopt an integrated biopsychosocial approach.

CORRESPONDING AUTHOR: Jessica L. McCurley, Massachusetts General Hospital/Harvard Medical School, jess.mccurley@gmail.com
O02.3

ADVANCES AND CHALLENGES OF THE THEORETICAL MODELS OF HEALTH-RELATED QUALITY OF LIFE (HRQOL) FOR TYPE 2 DIABETES

Eliozka Nuñez ${ }^{1}$

${ }^{1}$ Ministry of Health of Chile- Chile

Introduction \& Purpose: Quality of life (QoL) according to the WHO is the individual perception of the position in life in the context of the culture and value system in which one lives and its relationship with goals, expectations, standards and interests, however not has achieved the consensus of all researchers. Thus, the Quality of Life Related to Health (HRQOL) emerges as a concept that refers to the level of well-being derived from the evaluation that the person performs in various domains of his life, considering the impact that their state of health has on them. Diabetes is one of the chronic noncommunicable diseases with greater importance in public health. The purpose of this article is to analyze the different theoretical models that exist around the HRQOL and Type 2 Diabetes. Methods: A narrative review was carried out, with a systematic search of scientific evidence in electronic databases, such as Pubmed, Medline, LILACS, Cochrane Library, EMBASE, CINAHL, Google Scholar, from January 1990 to December 2017. Key words were used: quality of life, diabetes, quality of life related to health. Results: Seventy relevant articles were selected, 22 of them being specific in the measurement of HRQoL in patients with type 2 Diabetes. Two distinctions can be identified, Quality of Life and Quality of Life Related to Health, identifying that few authors make a distinction with the term of QoL. Despite this, psychometric instruments have been incorporated for their measurement, which associate the different subjective and objective and multidimensional aspects (eg EQ-5D, SF-36 and DQOL) Conclusions: There is a discrepancy between the definitions of QoL and HRQoL, which requires a deeper analysis and a global discussion to agree on a definition of each concept. However, there is agreement that the incorporation of the HRQOL measure has been one of the greatest innovations in evaluations, since it essentially incorporates the patient's perception of their well-being, delivering a valid, reliable tool, and providing empirical evidence with scientific basis to the process of decision making in health.

CORRESPONDING AUTHOR: Eliozka Nuñez, Ministry of Health of Chile, eoriele@gmail.com

\section{O02.4}

RISK FACTORS FOR COGNITIVE IMPAIRMENTS CAUSED BY TYPE 2 DIABETES MELLITUS

Jin $\mathrm{An}^{1}$, Yanyan Ren ${ }^{1}$, Xiaoxuan $\mathrm{Li}^{1}$, Peiyuan $\mathrm{Lv}^{1}$

${ }^{1}$ Hebei General Hospital- China

Background: Type 2 diabetes mellitus (T2DM) is a chronic multi-system disease that is characterized by hyperglycemia. Due to the increasing incidence of T2DM in recent years, more researchers have shown that T2DM is closely related to cognitive impairment. It seriously lowers the quality of life of patients and caregivers and poses a large economic burden for families and countries. Therefore, researchers must explore the risk factors for cognitive impairment and control the consequences of these factors. Methods: We searched PubMed for reports published from 2000 to 2017 using keywords including "diabetes and cognitive impairment". We analyzed and summarized the risk factors and the treatment based on the most recent research progress. Result:This disease has an insidious onset and complex etiology. With the development of an auxiliary examination method for cognitive impairment, researchers are better able to provide objective evidence to support the diagnosis and differential diagnosis. However, unified standards and the penetration rate are still unavailable. The pathogenesis of T2DM has been studied for many years, including blood glucose levels, blood lipid metabolic disorder, insulin resistance, oxidative stress, chronic inflammation, the 
psychological barrier, and intestinal dysbacteriosis. Strict and effective control of the blood sugar level is required to prevent cognitive impairment caused by diabetes. In addition, appropriate exercise, improved food structure, antioxidants, improved brain metabolism, inhibition of neuronal apoptosis, increased cerebral blood flow perfusion, probiotics, hyperbaric oxygen, traditional Chinese medicine and adjuvant therapy partially improve the cognitive impairment caused by diabetes. Conclusions: Based on strong evidence, T2DM is an independent risk factor for cognitive impairment. Early intervention and treatment for cognitive impairment caused by diabetes is critical to prevent dementia or slow its progression.

CORRESPONDING AUTHOR: Jin An, Hebei General Hospital, 944786931@qq.com

\section{O02.5}

MAIN AND INTERACTIVE EFFECTS OF ILLNESS REPRESENTATIONS OF DIABETES AND HYPERTENSION ON DEPRESSION IN THE CONTEXT OF DIABETES PATIENTS COMORBID WITH HYPERTENSION

Lu MA ${ }^{1}$, Nelson Chun Yiu YEUNG ${ }^{1}$, Alice Pik Shan $\mathrm{KONG}^{2}$, Rong $\mathrm{SHI}^{3}$, Joseph Tak Fai LAU ${ }^{1}$

${ }^{1}$ The Jockey Club School of Public Health and Primary Care, The Chinese University of Hong Kong- China

${ }^{2}$ Department of Medicine \& Therapeutics, The Chinese University of Hong Kong, Hong Kong- China

${ }^{3}$ School of Public Health, Shanghai University of Traditional Chinese Medicine, Shanghai- China

Introduction \& Purpose: Studies have found that illness representationsdiabetes mellitus (IR-DM) (people's perceptions about DM) were associated with mental outcomes in DM patients. However, in DM patients co-morbid with hypertension (HT), whether IR-HT (people's perceptions about HT) is also important is still unclear. This study aimed to examine: 1) whether IRDM was associated with depression; 2) whether IR-HT could explain additional variances in depression beyond and above IR-DM; 3 ) whether IR-HT moderate associations between IR-DM and depression. Methods: 524 DM patients co-morbid with HT ( $63.5 \%$ female, Mean age $=64.9$ years $)$ were recruited from community health centers in Shanghai, China. IR-DM, IR-HT, and depression were measured in a cross-sectional survey. IR-DM and IRHT were measured using Brief Illness Perception Questionnaire, including identity, consequence, timeline, personal control, treatment control, coherence, and emotional response. Results: Hierarchical regression analyses showed that after adjusting for background variables, IR-DM significantly explained $20.2 \%$ of variance in depression: attributing symptoms to DM (identity, $\beta=0.10)$, perceptions of consequence $(\beta=0.11)$, negative emotional response $(\beta=0.32)$, perceptions of timeline $(\beta=-0.14)$, and coherence $(\beta=-$ 0.08 ) were significantly associated with depression. Beyond IR-DM, IR-HT additionally explained $7 \%$ of variance in depression: identity-HT $(\beta=0.10)$ and emotional response-HT $(\beta=0.25)$ were significantly associated with depression. The interaction between coherence-DM and coherence-HT $(\beta=-$ $0.10, \mathrm{p}<0.05)$ significantly explained $1 \%$ of variance in depression. Simple slopes test showed that negative association between coherence-DM and depression was stronger among patients who reported higher coherenceHT. Conclusions: Findings supported the main and interactive effects of IR-DM and IR-HT in explaining depression among DM patients comorbid with HT. Interventions targeting on altering perceptions of DM (reducing identity, consequence, emotional response, and increasing timeline and coherence) and perceptions of HT (reducing identity and emotional response) may reduce depression. The benefits of increasing coherence-HT on depression were more evident among patients with higher coherence-HT.

CORRESPONDING AUTHOR: Lu MA, The Jockey Club School of Public Health and Primary Care, The Chinese University of Hong Kong, 1155080347@link.cuhk.edu.hk
O02.6

INTERVENTION BASED ON THE COMMON-SENSE MODEL OF SELF-REGULATION IN IMPROVING ILLNESS REPRESENTATIONS AND SELF-CARE AMONG CHINESE TYPE 2 DIABETES MELLITUS: A RANDOMIZED CONTROLLED TRIAL Phoenix $\mathrm{Mo}^{1}$, Virginia Chan ${ }^{1}$, Joseph Lau ${ }^{1}$

${ }^{1}$ School of Public Health and Primary Care, The Chinese University of Hong Kong- China

o Introduction \& Purpose Diabetes Mellitus (DM) is a major public health concern. Multiple studies reported that up to half of the individuals with DM had unsatisfactory diabetic control. It is recommended that diabetes self-management education should address both cognitive and emotional issues, as emotional well-being is significantly associated with diabetes outcomes. The Common-sense Model of Self-Regulation (CSM), which considers both cognitive emotional illness representations (IR) in promoting health, can potentially be useful in promoting self-care among individuals with DM. The present study evaluated the efficacy of an intervention based on the CSM in improving illness representations and selfcare among Chinese individuals with Type 2 DM in Hong Kong. o Methods A randomized controlled trial was used. A total of 457 individuals with Type $2 \mathrm{DM}$ was recruited from a hospital and randomly assigned to the intervention $(\mathrm{N}=232)$ or control group $(\mathrm{N}=225)$. Participants in the control group were invited to take part in 5 weekly group sessions delivered by a psychologist. The intervention sessions covered both cognitive and emotional IR as well as positive coping strategies based on the CSM. Participants in the control group received standard care from the hospital and booklets about DM self-care. Participants were assessed at baseline, post-interventions, and 6 months after the intervention. o Results A total of 410 participants completed the follow-up assessment. More than $80 \%$ of them completed at least 3 group sessions. Results from the 2(group) $\mathrm{x} 3$ (time) ANCOVA showed that after controlling for significant background variables, significant time $(\mathrm{F}(1$, $405)=8.036, \mathrm{p}<.01)$ and time $\mathrm{x}$ group interaction effect $(\mathrm{F}(1$, $405)=4.996, \mathrm{p}<.05$ ) on cognitive IR of timeline (cyclical), and significant and time $\mathrm{x}$ group interaction effect $(\mathrm{F}(1,405)=12.674, \mathrm{p}<.001)$ on $\mathrm{DM}$ self-efficacy were observed. Significant group $(F(1,405)=9.059, p<.01)$ and time $\mathrm{x}$ group interaction effect $(\mathrm{F}(1,405)=12.843, \mathrm{p}<.001)$ was also reported in self-care activity. o Conclusions Intervention based on the CSM could improve IR, DM self-efficacy and self-care among individuals with DM and such effects can be maintained up to 6 months. CSM can potentially be a useful framework for improving DM self-care.

CORRESPONDING AUTHOR: Phoenix Mo, School of Public Health and Primary Care, the Chinese University of Hong Kong, phoenix.mo@cuhk.edu.hk

\section{O03.1}

THE DANISH STUDY OF FUNCTIONAL DISORDERS (DANFUND) - COHORT INTRODUCTION WITH MEASURES OF FUNCTIONAL SOMATIC SYNDROME PREVALENCES, AUTONOMIC REGULATION AND LIFESTYLE CHARACTERISTICS

Thomas Dantoft ${ }^{1}$, Marie Eliasen ${ }^{1}$, Andreas Schröder ${ }^{2}$, Marie Weinreich Petersen ${ }^{2}$, Lene Eplov ${ }^{3}$, Louise Brinth ${ }^{4}$, Per Fink ${ }^{2}$, Betina Thuesen ${ }^{1}$

${ }^{1}$ Centre for Clinical Research and Prevention, Capital Region- Denmark

${ }^{2}$ The Research Clinic for Functional Disorders and Psychosomatics, Aarhus University Hospital- Denmark

${ }^{3}$ Mental Health Center Copenhagen, Capital Region- Denmark

${ }^{4}$ Coordinating Research Unit, Bispebjerg Frederiksberg HospitalDenmark

Aim Functional somatic syndromes (FSS) are associated with poor health status and increased health care cost. The etiology of FSS is only partly elicited, and how to delimit FSS remains unclear. The Danish study of 
Functional Disorders (DanFunD) was initiated to outline the epidemiology of FSS. The specific aims are to test delimitations of FSS, estimate occurrence, identify risk factors, delimitate the pathogenic pathways, and explore the consequences of FSS. Method The study population comprises a random sample of 9,656 adults from the general population. The various FSS were defined according to standard criteria based on symptom reports in questionnaires complemented by telephone diagnostic assessment for FSS and bodily distress syndrome (BDS) in a subsample. Questionnaires on physical and mental health, personality traits, lifestyle, use of health care services, and social factors are combined with physical measures of cardiorespiratory, morphological and metabolic fitness, neck mobility, heart rate variability (HRV), and conditioned pain modulation. A biobank has been established and the cohort is linked to central registries data. The first re-examination (5 year) of the cohort is currently ongoing. Results FSS will be studied in a bio-psycho-social model reflected by a hypothesized causal web on which the analytical plan for DanFunD is based. Selected characteristics of the FSS population will be depicted, incl. prevalence, lifestyle and HRV. Prevalence of FSS were fibromyalgia (3.6\%), irritable bowel syndrome $(4.6 \%)$, chronic fatigue syndrome (8.6\%), whiplash associated disorder (1.7\%), multiple chemical sensitivity $(2.0 \%)$ and BDS $(16.1 \%)$. Lifestyle measures showed that FSS cases differed from non-cases on numerous parameters e.g. poor self-perceived health and higher mental distress $(\mathrm{P}<0.001)$, less education, higher BMI, less exercise capacity, and lower muscle strength $(\mathrm{P}<0.05)$. HRV measures supported a sympathetic predominance of the autonomic nervous system associated with FSS, most prominent for fibromyalgia and BDS (P $<0.05$ ). Conclusion DanFunD data show that FSS are common in the Danish population and that FSS cases differ from non-cases on mental health, socioeconomic factors, physical fitness and autonomic regulation.

CORRESPONDING AUTHOR: Thomas Dantoft, Centre for Clinical Research and Prevention, Capital Region, thomas.meinertz.dantoft@ regionh.dk

\section{O03.2}

THE EPIDEMIOLOGY OF HEALTH ANXIETY OR ILLNESS ANXIETY IN THE GENERAL DANISH POPULATION. P R E V A L E N C E A N D S O C I O D E M O G R A P H I C CHARACTERISTICS. THE DANFUND STUDY

Per Fink ${ }^{1}$, Marie Weinreich Petersen ${ }^{1}$, Tine Blom Osterland ${ }^{1}$, Tina Carstensen $^{1}$, Lene Falgaard Eplov ${ }^{2}$, Thomas Meinertz Dantoft ${ }^{3}$, Torben Jørgensen ${ }^{3}$, Lisbeth Frostholm ${ }^{1}$

${ }^{1}$ The Research Clinic for Functional Disorders and Psychosomatics, Aarhus University Hospital- Denmark

${ }^{2}$ Mental Health Services in the Capital Region- Denmark

${ }^{3}$ Centre for Clinical Research and Prevention, Capital Region- Denmark

Introduction and purpose The prevalence of Health Anxiety (HA) in the general population is not very well described. Furthermore, prevalence estimates have been based on self-report questionnaires, and to our knowledge, no general population studies are based on clinical diagnoses established by standardized diagnostic research interview. The aim of this study is to estimate the prevalence, comorbidity and sociodemographics of Health Anxiety in the general population measured by research criteria as well as by DSM-V criteria for Illness Anxiety Disorder (IA) and Somatic Symptom Disorder (SDD). Methods The DanFunD general population cohort includes 7493 individuals. A stratified subsample $(\mathrm{n}=$ 1604) including a random sample of $1 / 10$ of all individuals and all high scores on the screening questionnaire completed a diagnostic research interview, i.e. a brief version of Schedules of Clinical Assessment in Neuropsychiatry (SCAN), conducted by 3 trained family physicians per telephone. For the overall cohort, the Whiteley-7 index was used for case identification. For the interviewed sample, established research criteria for HA were used, and the DSM-V criteria for IA and SSD were also applied. Results $13.6 \%$ (95 \% CI: 12.9-14.3) (n=1306) (41.0\% males and $59.0 \%$ females) of the overall sample had a Whiteley-7 score above the clinical threshold of 23.5. For the interviewed subsample, the prevalence of HA was $4.1 \%$ (95\% CI: 2.6-5.6) among males and $7.6 \%$ (95\% CI: 5.9-9.3) among females. For IA, prevalence was $1.4 \%$ (95\% CI: 0.5 2.3) for males and $2.6 \%$ (95\% CI: $0.5-2.3$ ) for females, while prevalence of SSD was $3.8 \%$ (95 \% CI: 2.3-5.3) and $6.0 \%$ (95\% CI: 4.5-7.5), respectively. There were no obvious differences across age. Depending on the diagnostic criteria used, between $0.4 \%$ (95\% CI: 0.1-0.7) and 1.3 $\%(0.8-1.9)$ had a comorbid depression, and between $0.6 \%$ (95\% CI: $0.2-$ $1.0)$ and $1.5 \%$ (95\% CI: 0.9-2.1) had a comorbid anxiety disorder. Sociodemographic and other characteristics of the HA will be presented as well. Conclusion This study shows that illness worrying is common in the general population and in clinical cases of HA. Prevalence is higher among females than males.

CORRESPONDING AUTHOR: Per Fink, The Research Clinic for Functional Disorders and Psychosomatics, Aarhus University Hospital, maleskjo@rm.dk

\section{O03.3}

ECONOMIC EVALUATION OF A HEALTH BEHAVIOUR INTERVENTION FOR MUSCULOSKELETAL CONDITIONS

Emma Robson ${ }^{1}$, Steven Kamper ${ }^{2}$, Bruno Saragiotto ${ }^{3}$, Christopher Williams $^{1}$, Kate O'Brien ${ }^{1}$, Amanda Williams ${ }^{1}$, Rebecca Hodder ${ }^{1}$, Hopin Lee $^{4}$, Luke Wolfenden ${ }^{1}$

${ }^{1}$ Hunter New England Health Distric and University of NewcastleAustralia

${ }^{2}$ University of Sydney- Australia

${ }^{3}$ Universidade Cidade de São Paulo- Brazil

${ }^{4}$ University of Newcastle

Introduction and purpose: Musculoskeletal conditions and associated health behaviour risks are both costly and burdensome. Owing to their growing costs and burden, economic evaluations which estimate cost implications and cost-effectiveness of potential treatments facilitates decision about real world application. The purpose of this study was to perform economic evaluation of a health behaviour intervention focused on healthy lifestyle (weight loss, physical activity and diet) for musculoskeletal conditions. Methods: We analysed data from two randomised controlled trials; a trial of 160 patients with low back pain, and a trial of 120 patients with knee osteoarthritis, which compared a six-month healthy lifestyle intervention with usual care. We compared total costs between groups and performed cost-effectiveness analyses. The primary effectiveness outcome was quality-adjusted life years (QALYs). Secondary effectiveness outcomes included pain intensity, disability, weight, and body mass index (BMI). Costs included intervention costs, healthcare utilisation costs (healthcare services and medication use) and absenteeism costs from low back pain. We conducted a primary analysis from the societal perspective (including all cost measures). Results: In the back pain trial, the mean total costs were lower in the intervention group than in the control group (-\$614; $95 \% \mathrm{CI}:-3133$ to 255$)$. From the societal perspective, for all outcomes, the intervention was on average less expensive and more effective than usual care and the probability of the intervention being cost-effective compared to usual care was relatively high (i.e. 0.81) at a willingness-to-pay of $\$ 0 /$ unit of effect. For QALYs, this probability increased to 0.90 at a willingness-to-pay of $\$ 17,000 / \mathrm{QALY}$ and reached a maximum of 0.96 at $\$ 67,000 / Q A L Y$. For the knee osteoarthritis trial, there were no differences between groups for total costs. For all outcomes, the probability of the intervention being cost-effective compared with usual care was less than 0.33 at all willingness-to-pay values. Conclusions: The healthy lifestyle intervention, appeared to be costeffective compared to usual care for low back pain, however the intervention was not cost-effective for knee osteoarthritis.

CORRESPONDING AUTHOR: Emma Robson, Hunter New England Health Distric and University of Newcastle, emma.robson@hnehealth.nsw.gov.au 
O03.4

EFFECTIVENESS OF SMOKING CESSATION AND WEIGHT LOSS INTERVENTIONS FOR MUSCULOSKELETAL PAIN

Bruno Saragiotto $^{1}$, Steve Kamper ${ }^{2}$, Rebecca Hodder ${ }^{3}$, Luke Wolfenden ${ }^{3}$, Hopin Lee ${ }^{3}$, Vinicius Oliveira ${ }^{4}$, Priscila Silva ${ }^{1}$, Emma Robson $^{3}$, John Wiggers $^{3}$

${ }^{1}$ Universidade Cidade de São Paulo- Brazil

${ }^{2}$ University of Sydney- Australia

${ }^{3}$ University of Newcastle- Australia

${ }^{4}$ Universidade Federal dos Vales do Jequitinhonha e Mucuri, DiamantinaBrazil

Introduction and purpose: Several health behavioural risk factors have been shown to increase the risk of musculoskeletal conditions such as low back pain and osteoarthritis. Most compelling are links between obesity and smoking, and musculoskeletal conditions; meta-analyses show 1.3 to 3.2 times the odds of developing persistent pain in people who are overweight or smoke. While weight loss and smoking cessation is widely recommended for patients with musculoskeletal conditions who are overweight or smoke, there is no recent synthesis of evidence about effectiveness of intervention strategies. We conducted two systematic reviews evaluating effectiveness of weight loss or smoking cessation interventions in patients with musculoskeletal pain. Methods: Eight databases were searched up to August 2017. Randomised controlled trials of interventions that aimed to reduce smoking or weight of patients with musculoskeletal pain, to improve pain or disability were included. Primary outcomes were pain and disability. Secondary outcomes included weight, physical performance measures, mental health, physical activity, dietary outcomes, and quality of life. We used the Cochrane Risk of Bias tool to assess risk of bias and GRADE to evaluate the quality of evidence. Results: 18 trials were included in the reviews (16 weight loss and 2 smoking cessation), including a total of 3070 and 99 participants respectively. Meta-analysis showed there is moderate quality evidence that behavioural weight loss interventions compared to usual/minimal care for osteoarthritis improved pain (SMD $-0.54,95 \%$ CI -0.91 to-0.17) and disability (SMD $-0.28,95 \%$ CI -0.45 to -0.11 ), interventions that included exercise were more effective. Only one trial $(n=96)$ was identified for low back pain, which supported (pharmacological) weight loss. There was low to very low quality evidence that smoking cessation did not reduce pain or disability. Conclusions: Multicomponent weight loss interventions improve pain and disability in patients with musculoskeletal pain, but there is very limited evidence regarding smoking cessation interventions. Multicomponent weight loss interventions and those that included exercise were more effective than dietary weight loss alone.

CORRESPONDING AUTHOR: Bruno Saragiotto, Universidade Cidade de São Paulo, bruno.saragiotto@gmail.com

\section{O03.5}

CAUSAL MECHANISMS OF A HEALTH BEHAVIOUR INTERVENTION FOR PATIENTS WITH MUSCULOSKELETAL PAIN

Steven Kamper ${ }^{1}$, Amanda Williams ${ }^{2}$, Hopin Lee ${ }^{3}$, Kate O'Brien ${ }^{2}$, John Wiggers $^{2}$, Sze Lin Yoong ${ }^{2}$, Rebecca Hodder ${ }^{2}$, Emma Robson $^{2}$

${ }^{1}$ School of Public Health, University of Sydney- Australia

${ }^{2}$ Hunter New England Population Health, Newcastle- Australia

${ }^{3}$ Centre for Rehabilitation Research and Centre for Statistics in Medicine, Nuffield Department of Orthopaedics Rheumatology and Musculoskeletal Sciences, University of Oxford- United Kingdom

Introduction and purpose: Low back pain and knee osteoarthritis are common conditions with a high global burden. Health behavioural risk factors may adversely influence the clinical course of LBP and knee $\mathrm{OA}$ and are hypothesised treatment targets to reduce pain and disability. The purpose of this study was to test the causal mechanisms of an integrated clinical and lifestyle focused intervention for patients with LBP and knee OA who are overweight or obese. Methods: Causal mediation analyses of aggregated data $(n=280)$ from two RCTs; which included patients chronic low back pain and knee osteoarthritis. Participants were randomised via one central randomisation schedule, to receive health behaviour intervention, or usual care. The intervention involved clinical advice and education, and referral to a six-month telephone-based healthy behaviour coaching service focusing on behaviour change to support weight loss, physical activity and diet modification. We hypothesised the primary mediator was weight, and alternative mediators were diet, physical activity and pain beliefs. Outcomes were pain, disability and quality of life (QoL). Data were analysed using causal mediation analysis with sensitivity analyses for sequential ignorability. All mediation models were specified a priori in a published protocol. Results: The health behaviour intervention had no effect on pain intensity, disability or QoL in patients with low back pain or knee osteoarthritis. The intervention did not reduce weight, or the alternative mediators (diet, physical activity, pain beliefs), and these mediators were not associated with the outcomes (with one exception; poor diet was associated with lower mental quality of life). Conclusions: The health behaviour intervention delivered primarily using the telephone did not change the intended targets of weight, diet, physical activity and pain beliefs. The hypothesised mediators were not associated with the outcomes, meaning their role as treatment target might be questioned for treating musculoskeletal pain. However, targeting these mediators, in particular the lifestyle risk factors, may offer other health benefits such as improved cardiovascular disease risk, particularly for overweight or obese patients.

CORRESPONDING AUTHOR: Steven Kamper, School of Public Health, University of Sydney, steven.kamper@sydney.edu.au

\section{O03.6}

CHRONIC PAIN AND WHIPLASH - IS CAUSAL ATTRIBUTION ASSOCIATED WITH ABILITY TO WORK?

Solbjørg Makalani Myrtveit Sæther ${ }^{1}$, Heidi Johansen ${ }^{2}$, Lisbeth Frostholm ${ }^{3}$

${ }^{1}$ Department of Health Promotion, Norwegian Institute of Public Health, Bergen- Norway

${ }^{2}$ Institute for Sosial Medicine, UiT Norwegian Arctic University- Norway ${ }^{3}$ The Research Clinic for Functional Disorders, Aarhus University Hospital, Aarhus- Denmark

Background: Though most individuals who experience whiplash recover rapidly, some develop chronic whiplash; a condition characterized by pain and reduced ability to work. Aim: We aimed to investigate whether attributing pain (neck pain or any chronic pain) to whiplash is associated with full time work and receipt of disability pension. Any such association would further be investigated in relation to differences in reported pain and psychological complaints between those attributing pain to whiplash and those not. Methods: Data from the sixth wave of the Tromsø Study $(2007-08, n=12,981)$ were analyzed. Using logistic regression analyses, the odds ratios (OR) of working full time and of receiving disability pension was computed for individuals attributing their chronic neck pain to whiplash compared to individuals reporting chronic neck pain due to other causes (reference category). Analyses were adjusted for socio-demographics, pain characteristics and psychological distress. These same analyses were run comparing individuals attributing any chronic pain to whiplash compared to individuals reporting any chronic pain due to other causes. Results: In individuals reporting chronic neck pain and in individuals reporting any chronic pain, the odds of working full time was significantly lower (crude OR= 0.73 (95\%CI: 0.55 $0.97)$ and $\mathrm{OR}=0.61$ (95\% CI: $0.47-0.80)$ respectively), and the odds of receiving disability pension significantly higher (crude $\mathrm{OR}=1.58$ 
(95\%CI: 1.18-2.12) and $\mathrm{OR}=2.02(95 \% \mathrm{CI}: 1.53-2.66)$ respectively) when pain was attributed to whiplash. For both chronic neck pain and any chronic pain, the association between pain attribution and work and benefit status remained significant after adjusting for pain and psychological distress. Conclusions: Individuals reporting whiplash as a cause of their chronic pain are less likely to work full time and more likely to receive disability pension than individuals with chronic pain due to other causes. This association remains after adjusting for pain characteristics and psychological distress.

CORRESPONDING AUTHOR: Solbjørg Makalani Myrtveit Sæther, Department of Health Promotion, Norwegian Institute of Public Health, Bergen, malene.skjoeth@aarhus.rm.dk

\section{O04.1}

THE EFFECT OF TESTOSTERONE REPLACEMENT THERAPY ON COGNITIVE FUNCTIONS IN MEN - A SYSTEMATIC REVIEW AND META-ANALYSIS OF RANDOMIZED CONTROLLED TRIALS

Cecilie Dorthea Rask Clausen ${ }^{1}$, Helene Johanne Rask Dalby ${ }^{2}$, Ali Amidi $^{1}$, Claus H. Gravholt ${ }^{3}$, Robert Zachariae ${ }^{1}$

${ }^{1}$ Unit for Psychooncology \& Health Psychology, Department of Psychology, Aarhus University, and Department of Oncolody, Aarhus University Hospital- Denmark

${ }^{2}$ Department of Clinical Medicine, Aarhus University Hospital and Aarhus University, Aarhus- Denmark

${ }^{3}$ Department of Endocrinology and Internal Medicine and Department of Molecular Medicine, Aarhus University Hospital, Aarhus, Denmark

Purpose: Testosterone (T) replacement therapy (TRT) is generally assumed to improve cognitive functions in particularly aging men. The reported effects are, however, inconsistent. The present study reviewed the available research and analyzed the combined effects of TRT on overall cognitive functioning and individual cognitive domains in men and explored between-study differences as possible moderators of these effects. Methods: Searches in PubMed, PsycINFO, Web of Science, The Cochrane Library, CINAHL, and Embase yielded 3869 unique records. Titles and abstracts were examined by two independent reviewers, resulting in the inclusion of 22 papers reporting results of 24 randomized controlled trials investigating 1793 patients. Neuropsychological tests were categorized into 11 cognitive domains. The study was preregistered in PROSPERO (\# CRD42017060530). Results: The small improvement (Hedges's $\mathrm{g}=0.09$ ) found for overall cognitive function did not reach statistical significance $(K=24, p=0.12)$. A small statistically significant improvement was found for the cognitive domain of attention/working memory $(\mathrm{K}=12, \mathrm{~g}=0.20, \mathrm{p}<0.00)$. The effects for the remaining cognitive domains did not reach statistical significance $(g=-0.10-0.17$, $\mathrm{p}=0.20$ - 0.86). Small statistically significant improvements were found in studies assessing $\mathrm{T}$ at random times during the day $(\mathrm{K}=6$, $\mathrm{g}=0.32, \mathrm{p}=0.04)$, in studies administering $\mathrm{T}$ with injection $(\mathrm{K}=12$, $\mathrm{g}=0.23, \mathrm{p}=0.02$ ), and in studies failing to adjust for missing data $(\mathrm{K}=9, \mathrm{~g}=0.26, \mathrm{p}=0.02$ ). The small Fail-safe N's found (range: 3-12) indicate that the results were less robust. Conclusions: The results suggest that TRT may lead to small improvements in attention/ working memory and that the effect of TRT on overall cognitive function may be strongest in studies administering $\mathrm{T}$ with injection, assessing $\mathrm{T}$ at random times during the day, and failing to adjust for missing data. However, the effects were negligible, less than robust, and may be of insufficient magnitude to be of clinical relevance.

CORRESPONDING AUTHOR: Cecilie Dorthea Rask Clausen, 1Unit for Psychooncology \& Health Psychology, Department of Psychology, Aarhus University, and Department of Oncolody, Aarhus University Hospital, cdrc@psy.au.dk
O04.2

A RANDOMIZED TRIAL OF A NOVEL PATIENT-CENTERED PREVENTIVE INTERVENTION TO REDUCE ALCOHOL USE AMONG MEDICALLY VULNERABLE YOUTH - CONFIRMING A THEORY OF CHANGE

Elissa R Weitzman ${ }^{1}$, Lauren E Wisk, Kara Magane, Julie Lunstead, Sharon Levy

${ }^{1}$ Harvard Medical School, Boston Children's Hospital- USA

Introduction \& Purpose: Alcohol poses unique risks for youth living with a chronic medical condition (YCMC), yet many drink. We created a preventive intervention for YCMC to deliver content specific to alcohol's effects on their disease and health. We sought to confirm the logic model for motivating health protecting behavior change among these youth by testing hypothesized associations among behavior, knowledge and perceived risk. Methods: We recruited 210 adolescents with type 1 diabetes (T1D), inflammatory bowel disease (IBD) or rheumatic disease (RD) to participate in an RCT of a novel, brief intervention to reduce alcohol use. Participants self-reported: alcohol use, perceptions of alcohol-related risk and knowledge of alcohol's impacts on their condition. Post-intervention youth in the treatment arm reported perceived risk and knowledge. We used multivariable logistic regression to evaluate knowledge, perceived risk and use. Results: Subjects (63\% response) were 16.2 years old on average $(\mathrm{SD}=1.38), 56.7 \%$ female, $81.6 \%$ white non-Hispanic, $23.3 \%$ reported past year alcohol use at baseline. In all, $62.8 \%$ of YCMC thought that consuming $\geq 1$ drink per occasion was "safe for them"; this group had nearly five times the odds (AOR 4.77, 95\% CI:3.82, 5.95) of past year alcohol use as youth who reported "no amount of alcohol was safe for them." Greater knowledge was associated with lower odds of any past 3 month drinking (AOR: 0.65, 95\% CI: 0.42-0.99, $\mathrm{p}=0.045$ ) and lower odds of drinking monthly or more in that time (AOR: $0.57,95 \% \mathrm{CI}: 0.35-0.93$, $\mathrm{p}=0.025$ ). After viewing the intervention, knowledge about alcohol's effects on their condition improved, with the percentage of correct responses demonstrated for youth with T1D, RD, or IBD improving to $81.7 \%$ (from $59.6 \%$ ), $79.4 \%$ (from $67.6 \%$ ), and $87.5 \%$ (from $68.1 \%$ ), respectively. Similarly, $35.6 \%$ reported decreased risk tolerance for alcohol use. Conclusion: Results confirm strong associations among alcohol use, perceived risk of drinking and knowledge of alcohol's effects on health. Findings confirm the logic of the intervention model for YCMC and suggest the potential efficacy of a patient-centered preventive intervention for reducing alcohol use when tailored to the needs, beliefs and concerns of medically vulnerable youth.

CORRESPONDING AUTHOR: Elissa R Weitzman, Harvard Medical School, Boston Children's Hospital, elissa.weitzman@childrens.harvard.edu

\section{O04.3}

PILOTING A COGNITIVE-BEHAVIORAL INTERVENTION TO IMPROVE HBA1C, DISTRESS, DEPRESSIVE SYMPTOMS AND PSYCHOLOGICAL WELL-BEING AMONG TYPE 2 DIABETES MELLITUS PATIENTS

Joel Omar González Cantero ${ }^{1}$, Roberto Oropeza Tena ${ }^{2}$, Jorge Armando Casillas Rodríguez ${ }^{3}$

${ }^{1}$ Universidad De Guadalajara- Mexico

${ }^{2}$ Universidad Michoacana de San Nicolás de Hidalgo

${ }^{3}$ Universidad Tecnológica de Guadalajara- Mexico

Introduction \& Purpose In Mexico, only one of four people with type 2 diabetes mellitus has an acceptable glycemic control. Psychological intervention can serve as a template for programs to be developed to help people with type 2 diabetes mellitus to control their illness and improve their quality of life. However, many studies in Mexico do not (or only partially) meet with CONSORT criteria, putting into question the effects achieved in the interventions. Also, it is necessary that interventions include variables related to quality of life and attempt to improve follow-up. 
Thus, the purpose of this study was to identify the effects of a cognitivebehavioral intervention to control $\mathrm{HbAlc}$, decrease distress and depressive symptoms and increase psychological well-being in people with type 2 diabetes mellitus. Methods A quasi-experimental pretest posttest design with a follow-up of three months was used. The intervention was composed of 12 sessions of an hour and a half, provided weekly. To analyze data Hedges' g was used to identify the size effect. Results $\mathrm{HbA1c}$ had a decrease from the pre-test $8.43(\mathrm{SD}=2.42)$ to the post-test $8.14(\mathrm{SD}=$ $1.70)$ and to the follow-up $8.02(\mathrm{SD}=1.87)$; however, it was not significant. Distress was $51.75(\mathrm{SD}=6.89)$ in the pre-test, $24.00(\mathrm{SD}=23.39)$ in the post-test and $20.75(\mathrm{SD}=24.85)$ in the follow-up, which represents a very large effect in both cases, from the pre-test to post-test $(g=1.399)$ and from this one to the follow-up ( $g=1.478)$. Depressive symptoms were $45.00(\mathrm{SD}=13.68)$ in the pre-test, $40.75(\mathrm{SD}=12.60)$ in the posttest and $36.00(\mathrm{SD}=12.19)$ in the follow-up, which means a small effect to pre-test to post-test $(\mathrm{g}=.281)$ and medium effect from post-test to follow-up $(\mathrm{g}=.604)$. Psychological well-being was $147.25(\mathrm{SD}=$ $11.58)$ in the pre-test, $157.75(\mathrm{SD}=25.47)$ in the post-test and 175 (SD $=30.82)$ in the follow-up, which means a small effect from pre-test to post-test $(\mathrm{g}=-0.461)$ and a large effect from post-test to follow-up $(\mathrm{g}=$ 1.306). Conclusions The intervention is effective in decreasing distress and increasing psychological well-being. More time is needed to decrease $\mathrm{HbA1c}$ and depressive symptoms.

CORRESPONDING AUTHOR: Joel Omar González Cantero, Universidad De Guadalajara, joel.gonzalez@academicos.udg.mx

\section{O04.4}

A PILOT TRIAL OF A BRIEF ACCEPTANCE AND COMMITMENT THERAPY INTERVENTION AIMING TO PREVENT PERSISTENT POSTSURGICAL PAIN

Katherine Hadlandsmyth ${ }^{1}$, Lilian Dindo ${ }^{2}$, Sonia Sugg ${ }^{3}$, M. Bridget Zimmerman $^{4}$, Lori Stout ${ }^{5}$, Barbara Rakel ${ }^{6}$

${ }^{1}$ University of Iowa Hospitals and Clinics- USA

${ }^{2}$ Baylor College of Medicine, Menninger Department of Psychiatry and Behavioral Sciences- USA

${ }^{3}$ University of Iowa, Carver College of Medicine, Department of Surgery- USA

${ }^{4}$ University of Iowa, College of Public Health, Department of Biostatistics- USA

${ }^{5}$ University of Iowa Hospitals and Clinics, Department of AnesthesiaUSA

${ }^{6}$ University of Iowa, College of Nursing- USA

Introduction and Purpose: Psychological distress is associated with risk for developing persistent pain following breast surgery. The purpose of this study is to assess a psychological intervention in women at risk for persistent postsurgical pain. Method: This study is a pilot randomized control trial of a brief, single session Acceptance and Commitment Therapy (ACT) intervention compared to Treatment as Usual (TAU). Participants were screened preoperatively, based on "at risk" criteria including: elevated depressive symptoms (PHQ- $8 \geq 10$ ); anxious symptoms (GAD-7 $\geq 10$ ); pain catastrophizing ( $\mathrm{PCS} \geq 30$ ); pre-existing chronic pain conditions; or young age $(<50)$. Results: During the recruitment period, 264 women scheduled for first time breast surgery to treat cancer or ductal carcinoma in situ were approached, 115 expressed interest and 64 met screening criteria, of whom 62 were consented and randomized. Three were later excluded (due to medical complications or delayed surgery) and 3 withdrew, resulting in a final $\mathrm{N}=56(\mathrm{ACT}=26$; $\mathrm{TAU}=30)$. Of the 26 randomized to ACT, 2 participants did not complete the intervention as designed (per protocol $=24$ ACT participants). Preliminary analyses on the $\mathrm{N}=56$ participants did not find a significant difference between groups in the number of patients with moderate to severe pain in their breast or arm at 3 -months post-surgery (odds ratio $=1.18 ; 95 \%$ CI: $0.24-$ $5.83 ; \mathrm{p}=.84)$. Per protocol analyses $(\mathrm{n}=54)$ also did not find a significant difference between groups on pain (odds ratio $=1.69 ; 95 \%$ CI: $0.28-$ $10.13 ; \mathrm{p}=0.56$ ). Written qualitative responses from participants who received the ACT intervention indicated that the majority of these participants understood the concepts and reported continued application of exercises learned during the session. Conclusion: While qualitative responses suggest interest in the ACT intervention, understanding of the material, and subsequent application of skills, we did not find a significant impact on pain severity following breast surgery. This may have been affected by the low overall rate of persistent pain reported in this sample. This suggests the need for better identification of patients at risk for persistent postsurgical pain.

CORRESPONDING AUTHOR: Katherine Hadlandsmyth, University of Iowa Hospitals and Clinics, katherine-hadlandsmyth@uiowa.edu

\section{O04.5}

DO MINDFULNESS-BASED INTERVENTIONS IMPROVE HEALTH OUTCOMES FOR ADULTS LIVING WITH CHRONIC ILLNESS? RESULTS FROM A SYSTEMATIC REVIEW AND META-ANALYSIS

Lori Scott-Sheldon $^{1}$, Elena Salmoirago-Blotcher ${ }^{1}$, Marissa Donahue ${ }^{1}$, Melissa Feulner ${ }^{1}$, Brittany Balletto ${ }^{1}$, Julie DeCosta ${ }^{1}$, Emily Gathright ${ }^{1}$, Eugene Dunne ${ }^{1}$, Dean Cruess ${ }^{2}$

${ }^{1}$ The Miriam Hospital and Brown University- USA

${ }^{2}$ University of Connecticut- USA

Introduction/Purpose: The purpose of this systematic review and meta-analysis was to examine the efficacy of mindfulness-based interventions (MBIs) on psychological, immunological, and disease outcomes among adults living with cardiovascular disease (CVD), type II diabetes (T2DM), or human immunodeficiency virus (HIV). Methods: Comprehensive searches identified studies that (a) evaluated a MBI in adults with CVD, T2DM, or HIV, (b) used a randomized controlled trial (RCT) design, (c) assessed psychological outcomes (e.g., anxiety, depression) or physiological markers (e.g., systolic blood pressure $[\mathrm{SBP}]$ ), immunological (e.g., CD4+ counts), and disease (e.g., HbA1c) outcomes, and (d) available through December 2017. Independent raters coded study, sample, design, intervention content, and methodological quality criteria. Results: Of the 6,697 records identified through comprehensive searches, 33 RCTs met the inclusion criteria. Studies were published (or available) between 1996 and 2017 and conducted in multiple countries within North America (47\%), Asia (28\%), and Europe (25\%). The studies sampled adults with CVD $(\mathrm{N}=1406, \mathrm{M}$ age $=57,40 \%$ women $), \mathrm{T} 2 \mathrm{DM}(\mathrm{N}$ $=450, \mathrm{M}$ age $=59,57 \%$ women $)$, or $\mathrm{HIV}(\mathrm{N}=943, \mathrm{M}$ age $=43$, $22 \%$ women). Participants were recruited through clinic $(63 \%)$, community $(16 \%)$, or clinic and community $(22 \%)$ venues. The MBI used most often was Mindfulness-Based Stress Reduction (KabatZinn, 1990). Comparison conditions included passive (56\%; e.g., usual care, wait-list) or active (44\%; e.g., aerobic exercise) controls. Participants receiving a MBI improved on psychological outcomes (e.g., fewer depressive symptoms) and physiological markers (e.g., lower SBP) compared to passive controls. The findings were mixed when MBIs were compared to active controls. Too few studies assessed markers of disease progression to draw conclusions. Conclusions: MBIs can improve psychological and physiological markers in adults with chronic illness but limited evidence exists to support MBIs as an effective therapeutic approach compared to other treatments. RCTs that directly compare MBIs to other evidence-based approaches and include measures of disease progression are needed to determine the potential benefits for adults living with chronic illness.

CORRESPONDING AUTHOR: Lori Scott-Sheldon, The Miriam Hospital and Brown University, lori_scott-sheldon@brown.edu 
O04.6

MEASURING THE EFFECTIVENESS OF PSYCHOLOGICALLYORIENTED INTERVENTIONS BY USING BIOMARKERS: A META-ANALYSIS

Andres Gomez-Acosta ${ }^{1}$, Alejandra Herrera-Pinilla ${ }^{1}$, Stefania AguileraDíaz ${ }^{1}$, Miguel Puentes-Escamilla ${ }^{2}$

${ }^{1}$ Fundación Universitaria Sanitas- Colombia

${ }^{2}$ Universidad Nacional de Colombia

Introduction: Although the evidence-based psychological approaches mention some effectiveness indicators related to the reduction of emotional distress, and even considering the existence of several studies about how complaints can negatively affect people's somatic states, very few studies show if those approaches can actually modify biomarkers, and, in turn, improve somatic processes. Purpose: Analyzing psychologically-oriented interventions studies related to health (published since 2012) which have proven the effectiveness of those interventions by using biomarkers. Method: Meta-analysis of papers published in scientific databases (BVS, Embase, Medline, PsycArticles, and ProQuest), using the following descriptors: "psychological intervention" OR "psychosocial intervention" AND "biomarkers" OR "psychoneuroendocrinoimmunology". Results: a) psychologically-oriented interventions are related to attention to patients with chronic diseases (hypertension, diabetes, breast cancer, HIV, irritable bowel disease, coronary disease, Alzheimer's disease), and also are related to people involved in stressful situations; b) most of the analyzed studies have a pre-experimental methodology; c) only a few of them refer explicit size-effect measures, or enough information to make that type of calculations; $d)$ the reported biomarkers included alpha-amylase $(d=0.12)$, cholesterol $(d=10)$, cortisol $(d=0.51-1.85)$, dopamine $(d=0.34)$, epinephrine $(d=0,39)$, glucose $(d=0.20-2.62)$, neopterin $(d=2.44)$, norepinephrine $(d=0.54)$, platelets $(d=0.14)$, diastolic blood pressure $(d=0), 04$ $2.55)$, systolic blood pressure $(\mathrm{d}=0.05-4.21)$, pulse $(\mathrm{d}=0.41)$, and triglycerides $(\mathrm{d}=0.14)$. Conclusions: psychologically-oriented interventions can be useful for reducing oxidative stress and allostatic load, decelerating apoptosis processes, and also regulating anatomic-physiological functioning. Nevertheless, evidence found is not conclusive, and that could be because of the heterogeneity of diseases and techniques, the duration of those techniques, the severity of symptoms, and the lack of a more consistent monitoring.

CORRESPONDING AUTHOR: Andres Gomez-Acosta, Fundación Universitaria Sanitas, cesagomez@unisanitas.edu.co

\section{O05.1}

PREVALENCE AND SOCIOECONOMIC DETERMINANTS OF WORKPLACE BULLYING IN JAPAN AND ITS COMPOSITIONAL AND CONTEXTUAL EFFECTS ON EMPLOYEES' MENTAL HEALTH

Kanami Tsuno ${ }^{1}$, Norito Kawakami ${ }^{2}$

${ }^{1}$ Wakayama Medical University- Japan

${ }^{2}$ University of Tokyo- Japan

Introduction \& Purpose: For decades, workplace bullying has received worldwide attention. Our study aimed to reveal 1) prevalence and risk groups of workplace bullying and 2) its direct or indirect effects on employees' mental health, using the data of the Japanese national representative sample and employees in public service, respectively. Methods: First, a questionnaire survey was administered to a nationally representative community-based sample of 5,000 residents in Japan aged 20-60 years. The questionnaire included questions about employment, occupation, company size, education, household income, and subjective social status (SSS). We inquired about both the witnessing and personal experience of workplace bullying during the past 30 days. Second, a longitudinal study was conducted among Japanese workers in the public sectors $(\mathrm{N}=3,242)$. Both the baseline and follow-up questionnaires inquired about demographic and occupational characteristics, experiences of workplace bullying (NAQ-R), psychological distress (K6), and intention to leave. We conducted three-level (individualdivision - department) multilevel analyses to examine the effect of division or department-level workplace bullying on individual-level psychological distress and intention to leave. Results: The study of representative Japanese sample showed that $6 \%$ and $15 \%$ of the total sample reported experiencing or witnessing workplace bullying, respectively. After adjusting for gender and age, temporary employees (Odds Ratio [OR]: 2.45 [95\% CI = 1.03-5.85]), junior high school graduates (OR: 2.62 [1.01-6.79]), workers with lowest household income (OR: 4.13 [1.58-10.8]), and workers in the lowest SSS stratum (OR: 4.21 [1.66-10.7]) were at increased risk of experiencing workplace bullying. Workplace bullying has a significant association with depression and anxiety, physical complaints, and low work performance. The results of multilevel analyses revealed that division-level workplace bullying was associated with increased individual-level psychological distress (coefficient $=0.185, \mathrm{p}<0.01)$ as well as increased individuallevel intentions to leave the workplace (coefficient $=0.022, \mathrm{p}<0.01$ ) This association remained even after adjustment for individual experiences of workplace bullying and psychological distress or intention to leave at baseline. Conclusions: Workers in low socioeconomic status were more likely to experience workplace bullying. Also, our findings suggest that the presence of bullying in the workplace can have a detrimental effect on employees' mental health even if they are not personally victimized.

CORRESPONDING AUTHOR: Kanami Tsuno, Wakayama Medical University, tsuno@wakayama-med.ac.jp

\section{O05.2}

BENEFICIAL OR HARMFUL ? FINDINGS FROM A LITERATURE REVIEW OF THE RELATIONSHIP BETWEEN SOCIAL CAPITAL IN THE WORKPLACE AND EMPLOYEE'S HEALTH

Emiko Ando ${ }^{1}$, Kanami Tsuno ${ }^{2}$, Hisashi Eguchi ${ }^{3}$

${ }^{1}$ Osaka University- Japan

${ }^{2}$ Wakayama Medical University- Japan

${ }^{3}$ Kitasato University- Japan

Although the concept of social capital in community settings has not reached consensus, it could be briefly summarized as networks, norms, and trust that facilitate action and cooperation for mutual benefit. These features, such as the norm of reciprocity and trust in others facilitate cooperation, people's interaction for mutual benefit, are said to have potential to improve individual health outcomes, but the findings among community-dwelling people sometimes indicate its harmful effects (i.e., the dark side of social capital). In occupational settings, researchers have been also attracted to its concept and explored the health effect of workplace social capital in line with Job Demand-Control-Support Model or Job Demand-Resource Model, as a higher-level resource. Prior to a literature review, we re-conceptualize social capital in workplace settings. With search term based on this re-constructed concept, we systematically review the previous findings regarding the association with employee's health. (ex. blood pressure, depression, health behavior, etc)

CORRESPONDING AUTHOR: Emiko Ando, Osaka University, andoemiko-tky@umin.ac.jp

\section{O05.3}

\section{INTEGRATED APPROACHES IN DENMARK}

Ole Steen Mortensen ${ }^{1}$, Sanna Autrup ${ }^{1}$, HenrikGreiffenberg ${ }^{1}$, Jonas Winkel-Holm ${ }^{1}$, Lars L Andersen ${ }^{2}$, Emil Sundstrup ${ }^{2}$

${ }^{1}$ Copenhagen University Hospital Holbæk- Denmark

${ }^{2}$ National Research Center for the Working Environment- Denmark

Introduction and purpose: In Denmark, there has been focus on the prevention of work-related diseases through legislation. This legislation has 
focused on how to regulate work-related exposures to injury and illness. At the end of the 1990s, several health promotion initiatives were launched, mostly focused on healthy food in canteens and increased physical activity through gym memberships. Later in the mid 00's, the focus changed to the psychosocial working environment. Despite the preventive initiatives and an increased prevalence of health promotion activities, the rate of absence due to sickness and exclusion from the labor market was unchanged. Both preventive and health promoting initiatives had no significant effect on the incidence of musculoskeletal symptoms which occurred at the same rate as before the interventions. Methods: As a result of these disappointing results, research projects were initiated to support the development of further initiatives. The research projects targeted the most common musculoskeletal problems and investigated whether training of the most affected muscle groups increased strength and could reduce musculoskeletal symptoms. The next challenge was to find ways these interventions could be implemented and sustained in workplaces. Results: Randomized studies conducted showed significant reductions in forearm, neck and shoulder pain and a significant reduction in sickness absence due to low back pain. A combination of physical training with a cognitive based pain management strategy appeared to increase the efficiency of the interventions. Results also indicated that it was possible to do both physical and psychological initiatives during working hours; especially when that the interventions were implemented in a group setting. However, drop-out rates increased when the complexity of the intervention increased. We also showed that the health promotion activities at the workplace reached the relevant target groups. A cluster randomized study targeting low back pain including a specific focus on organizational support was implemented. The study showed that the intervention resulted in less low back pain and a lower physical work load. Conclusions: The translation of research results has occurred including strategies focused on workplace policies and organizational change; but success depends on the feasibility of the initiatives. Initiatives focused on musculoskeletal problems in workers should also include a cognitive approach to cope with pain. In addition, government support for these initiatives strengthens the chance for sustainable outcomes.

CORRESPONDING AUTHOR: Ole Steen Mortensen, Copenhagen University Hospital Holbæk, osm@ regionsjaelland.dk

O05.4

BULLYING AT THE WORKPLACE AND PERCEIVED STRESS: WHO IS AT RISK? WHO SUFFERS MORE?

Adrienne Stauder ${ }^{1}$, Barna Konkoly Thege ${ }^{2}$,

${ }^{1}$ Institute of Behavioural Sciences, Semmelweis University, BudapestHungary

${ }^{2}$ Waypoint Centre for Mental Health Care, Penetanguishene, and Dept. of Psychiatry, University of Toronto, Ontario- Canada

Introduction and goals: We examined the relationship between exposure to bullying at the workplace and self-reported stress level. Methods: Data source was a cross-sectional on-line survey in Hungary. The sample contained 13,104 actively working individuals, weighted to be representative of the working population according to sex, age groups, education, and occupational sectors. Questions on bullying, stress, burnout, and sleeping problems were from the Copenhagen Psychosocial Questionnaire (COPSOQ-II). The shortened version of the Beck Depression Inventory (BDI) and the Perceived Stress Scale (PSS4) were also included in the analysis. Results: $48.2 \%$ of the sample ( $44.3 \%$ of men and $52.7 \%$ of women) reported experiencing an offensive behavior (bullying, sexual harassment, threats of violence or physical violence). Frequent (weekly or more) exposure was $9.1 \%$ (7,7 for men and 10.7 for women). The highest prevalences were found in the health and social care, and defense sectors. Stress, burnout, sleeping troubles, and depression scores were significantly higher among the "occasionally bullied" compared to the "not bullied"; and among the "frequently bullied" compared to both previous groups. Almost all
(92\%) of those who reported bullying also reported other significant psychosocial stressors. Based on the number of stressors, $57.5 \%$ of the frequently bullied met our criteria for high psychosocial risk at the workplace. Conclusions: We found a strong association between offensive behaviors at the workplace and symptom scores. Our results suggest that bullying is typically not an isolated problem, other psychosocial risk factors are also present and contribute to the negative health outcomes.

CORRESPONDING AUTHOR: Adrienne Stauder, Institute of Behavioural Sciences, stauder.adrienne@med.semmelweis-univ.hu

O05.5

PSYCHOSOCIAL RISK IN THE WORKPLACE AND SICK LEAVE ABSENTEEISM

Juan Perez-Franco ${ }^{1}$, Macarena Candia ${ }^{1}$

${ }^{1}$ Superintendence of Social Security- Chile

Introduction. Research in Chile suggests that many variables are associated with sick leave absenteeism, although it is difficult to establish a general pattern from published results. This work looks for clear associations between psychosocial risk, other variables and sick leave absenteeism. Method. Assessment of psychosocial risk with COPSOQ 1 is mandatory in Chile under conditions of anonymity and confidentiality. Their unnamed results are preserved in Superintendencia de Seguridad Social (SUSESO). The independent variables were treated as discrete and in some cases they were dichotomized, as is the case of the psychosocial risk in which the upper tercile was used to define high risk. The statistical model was the Zero Inflated Negative Binomial, given the characteristics of the dependent variable (days of sick leave), not normal, with overdispersion and excess of zeros. The model has two parts: one measures the probability of not having one day of leave (the excess of zeros, or its inverse, having one day of sick leave), and the other part measures the probability of having more than one day of sick leave in those employees who could have a sick leave (count model). Results. We analyzed 20,575 records, 9,529 men and 11,046 women, only full time, in all occupations except agricultural workers and armed forces. High psychosocial risk is associated with having a sick leave $(\mathrm{OR}=1.57, \mathrm{p}<0.001)$, controlling for sex, salary, occupation, type of shift, severity of personal debt, general health, mental health, vitality, and reason for the workplace evaluation. The count model showed that high psychosocial risk is associated with more days of sick leave (IRR $=1.15, \mathrm{p}<0.001)$, controlling for the same previous variables. Discussion. The analysis of a broad database of Chilean workers shows that high psychosocial risk is a factor associated with sick leave absenteeism and more days of sick leave. It should be stressed that the risk was calculated controlling a series of confounding factors, which shows that high psychosocial risk by itself could be a cause of absenteeism. A longitudinal study is required to estimate if this hypothesis is true, but the current data is a valid approach to this problem.

CORRESPONDING AUTHOR: Juan Perez-Franco, Superintendence of Social Security,jperez@suseso.cl

\section{O05.6}

DEPRESSION ILLNESS AND PAID-SICK LEAVE IN COLOMBIA: EVIDENCE FROM A HEALTH INSURER IN A UNIVERSAL HEALTH INSURANCE CONTEXT

Hernan G. Rincon-Hoyos ${ }^{1}$,Sergio Prada ${ }^{2}$, Melissa Perez ${ }^{2}$, Valentina Serna ${ }^{1}$

${ }^{1}$ Fundación Valle Del Lili/ Universidad Icesi / Medicina Inteligente SasColombia

${ }^{2}$ Instituto Proesa, Universidad Icesi- Colombia

Introduction: The effect of behaviour disorders on labor market outcomes is unknown in Colombia. This paper estimated whether people suffering 
of depression comorbid with a medical illness have more paid sick-leave (PSL) days than people with the medical illness without depression. DATA AND METHODS: The study used claims data from 2016 of the main health insurer in the southwestern region in Colombia. Depression was identified using an international algorithm that detects chronic conditions from diagnosis and pharmacy use data. Outcome variables were: paid sick-leave allowed by the insurer in one year, and the amount paid. We used a multivariate regression to estimate the causal effect of depression disorders after pre-processing the data using a matching algorithm. Leave must be signed by an MD and must include a principal diagnosis. Analyses were run separately by major leave diagnosis groups as per the ICD10 classification of disease. Only paid-sick leave diagnosis in which there were at least one person with depression and one without depression were included. RESULTS: A total of 44,782 people with any PSL were included and 59,383 PSL were analysed. We found that individuals diagnosed with depression disorder tend to have more days of sick leave throughout the year than individuals with no depression illness. The diseases with statistically significant more days were infectious (1 day), endocrine, nutritional and metabolic diseases (11), ear (4), circulatory (7 days) and musculoskeletal illness (5 days). Regarding costs we found an incremental cost between US\$11 and US\$118 per year for patients with depression as compared to individuals with no depression illness. To put it in perspective US $\$ 118$ is equivalent to $91 \%$ of the annual amount paid to the insurer from the government for males in the age bracket 19-44. CONCLUSIONS: Depression comorbid with a medical illness increases number of days and consequently costs associated with paid-sick leaves. The additional cost seems high when compared to what private insurers receive annually to cover for health services. Additional research is warranted to identify cost-effective prevention activities that can lower the burden detected.

CORRESPONDING AUTHOR: Hernan G. Rincon-Hoyos, Fundación Valle Del Lili/ Universidad Icesi / Medicina Inteligente Sas, hernangrincon@gmail.com

\section{O06.1}

CARDIOVASCULAR HEALTH FACTORS AND RISK OF DEPRESSION AND COGNITIVE IMPAIRMENT

Pablo Toro ${ }^{1}$

${ }^{1}$ Pontificia Universidad Católica De Chile- Chile

Background: The American Heart Association defined in 2010 the concept of cardiovascular health factors (CVHF) changing the focus from risk factors to the promotion of health. Recent studies showed that these $\mathrm{CVHF}$ are associated to reduced overall and cancer specific mortality and a multicentric study showed that CVHF were associated to better cognitive performance. This association has not been studied in Southamerican population and, furthermore, the relation of CVHF and depressive symptoms needs to be addressed. Methods: This is a cross-sectional study based on data collected from the ongoing protocol of the Maule Cohort (MAUCO) for Chronic Diseases 2014-2024 that seeks to analyze the natural history of chronic diseases in the county of Molina $(40,000$ inhabitants) in the Maule Region, Chile. This cohort is funded to follow 10,000 subjects in the range of 38 to $74 \mathrm{y}$ for 10 years. In particular, participants were investigated by using the Mini Mental State Examination (MMSE), the Adenbrooke's Cognitive Examination (Muñoz et al., 2012) and Trail Making Test. Normative values were obtained from the population. Depressive symptoms were assessed using PHQ-9 (Spitzer R et al., 1999). Results: until now, data was collected and analysed for 6106 participants, including measures of CVHF, cognitive function and assessment of depressive symptoms. Mean age $=53,2 \pm 9,5$ years, $59,6 \%$ women. The mean number of ideal CVHF was: $2,48 \pm 1,1$; only $16 \%$ of the population had $\geq 4$ ideal CVHF, and no one had all 7 ideal factors. Diet and physical activity were the factors with less prevalence in ideal category. The mean for MMSE and ACE-R was $26.0 \pm 3.7$ and 72.4 \pm 14.3 , respectively. We found associations of CVHF to cognitive function, in particular to cognitive domains related to psychomotor speed and executive functions. Conclusions: CVHF are associated to cognitive function in the MAUCO population. The association of CVHF to depressive symptoms will be presented at the conference. This study underlines the importance of promotion of cardiovascular health as an intervention for mental health.

CORRESPONDING AUTHOR: Pablo Toro, Pontificia Universidad Católica De Chile, toropab@gmail.com

O06.2

CHRONIC NON-PSYCHIATRIC MEDICAL ILLNESS, INFLAMMATION AND DEPRESSION

Constanza Caneo ${ }^{1}$, Michael King ${ }^{2}$, Louise Marston ${ }^{2}$

${ }^{1}$ Pontificia Universidad Católica de Chile- Chile

${ }^{2}$ University College London- England

Introduction: There is a large body of evidence to support a bi-directional relationship between physical illness and depression. Observational studies have demonstrated that people with depression have higher rates of physical illness, while patients with physical illness have higher rates of depression, a risk that follows a dose-response relationship. The aim of this study was to explore and compare the impact of chronic medical conditions with and without an inflammatory profile on the incidence of depression. Methods This is a secondary analysis with an a priori hypothesis, conducted within the completed Spanish cohort of the predictD study, a cohort study with 12 months follow up to predict the incidence of depression in primary care attendees. 5,347 participants formed the predictD-Spain sample. The assessment of the risk of depression according to physical condition was conducted using multivariable logistic regression. Odds ratios and $95 \%$ confidence intervals are reported. All analyses were conducted using Stata $12 \AA$. Results The odds of depression were higher for all inflammatory illnesses before and after adjustment for confounders. Most non-inflammatory illnesses showed no increased odds of depression, except for locomotor pain disorders (OR 2.24 95\%CI $1.36-3.71$ ), but this association was not statistically significant after adjustment for confounders (OR 1.57 95\% CI 0.84 2.92). The regression model estimated that the odds of depression increased with inflammatory load. The highest odds of depression was associated with the presence of autoimmune disorders plus any other inflammatory disorder, which is our best proxy for a very high inflammatory state (OR $6.52,95 \%$ CI 2.14 - 19.83). Similarly, the odds of depression in the groups with three or more cardio-metabolic disorders was higher than the odds where there was one cardio-metabolic disorder (OR 2.38, 95\% CI $1.22-4.61$ for more than three cardio-metabolic disorders versus an OR of $1.28,95 \%$ CI $0.84-1.97$ for one cardiometabolic disorder). Conclusions: Inflammation may be part of the pathway by which chronic physical illness leads to depression. Future studies should examine the role of inflammation in the prevention and management of depression.

CORRESPONDING AUTHOR: Constanza Caneo, Pontificia Universidad Católica de Chile, cmcaneo@uc.cl

O06.3

DOES THE PREVALENCE OF MCI DECLINE? EVIDENCE FROM A PROSPECTIVE, POPULATION-BASED LONGITUDINAL STUDY WITH A FOLLOW-UP OF 25 YEARS

Johannes Schröder ${ }^{1}$

${ }^{1}$ University of Heidelberg- Germany

Background: Recent reports suggest declining prevalence of dementia. If reliable, a comparable decline should be detectable in the prevalence of 
Mild Cognitive Impairment (MCI) widely accepted as a preclinical stage of dementia. We tested this hypothesis in two birth cohorts on reaching age about 65 years some 18 years apart. Cohorts were born 1930-1932 (C 30) or 1950-1952 (C50). Both cohorts were followed up from 1993 up to 2016 in our Interdisciplinary Longitudinal Study of Adult Development and Ageing (ILSE). Methods: ILSE cohorts from either Heidelberg or Leipzig regions underwent medical and neuropsychological assessments on four occasions between 1993 and 2016. We contrasted prevalence of MCI and Mild Cognitive Disorder due to medical conditions (MCD) in the Heidelberg region between C30 at the second examination $(1998, \mathrm{n}=$ 222; mean age: ) and C50 at the fourth examination (2016, n=166; mean age: ). The effect of diagnoses of MCI/MCD on neuropsychological test performance was investigated using ANOVAs with respect to attention, memory, psychomotor speed/mental flexibility, visuo-spatial and abstract thinking. Results: $25.23 \%$ of C30 met criteria for MCI in 1998, while $9.64 \%$ of C50 were diagnosed with MCI in 2016. Moreover, $12.16 \%$ of $\mathrm{C} 30$ were diagnosed with $\mathrm{MCD}$ at the second examination wave in contrast to $7.83 \%$ of C 50 at the fourth examination wave. Chi-Square analysis was significant with $\chi 2=19.46, \mathrm{df}=2, \mathrm{p}<.0001$. Effects of diagnoses (cognitively healthy, $\mathrm{MCI}$ ) on neuropsychological test performance were identified for tests assessing abstract thinking, memory, attention, verbal fluency, visuo-spatial thinking, and mental flexibility. However interactions between cognitive diagnoses and cohort (C 30/C 50) were restricted to a test assessing abstract thinking abilities. Conclusion: Findings from our prospective study clearly confirm a decreasing prevalence of MCI in a cohort born 1950-52 when compared with a cohort born 1930-32. This effect conforms to an increased cognitive reserve as school education was significantly longer in the young than the old cohort. Additional factors (I.e. better control of hypertension or hypercholesterimia) also have to be considered as effects were confirmed after adjustment for school education. Hence, the profile of neuropsychological deficits was similar between cohorts except for abstract thinking as a domain which particularly involves cognitive reserve.

CORRESPONDING AUTHOR: Johannes Schröder, University of Heidelberg, ptoro@uc.cl

\section{O06.4}

MIND-BODY TREATMENT FOR GEOGRAPHICALLY DIVERSE ADOLESCENTS WITH NEUROFIBROMATOSIS (NF); A PILOT RANDOMIZED CONTROLLED TRIAL VIA LIVE VIDEOCONFERENCING

Ana-Maria Vranceanu ${ }^{1}$, Eric Riklinn ${ }^{2}$, Vanessa Merker ${ }^{1}$, Scott Plotkin ${ }^{1}$

${ }^{1}$ Massachusetts General Hospital/Harvard Medical School- USA

${ }^{2}$ Fordham University- USA

Adolescents with NF are at high risk for poor quality of life and psychosocial concerns. Despite advances in biomedical care that have substantially improved outcomes, there remains an enormous need for interventions to reduce the emotional toll that NF places on adolescents. Toward this end, our team has adapted a mind body program - The Relaxation Response Resiliency Program - that teaches relaxation response, stress awareness and positive psychology skills, for the specific needs of adolescents with NF (3RP-NF). The program was developed iteratively for delivery via secure live group videoconferencing to bypass barriers to care for adolescents with this rare disease. We tested the feasibility, acceptability and preliminary effect of the $3 \mathrm{RP}-\mathrm{NF}$ versus an educational control in improving quality of life (QoL), depression, anxiety, pain intensity and pain interference. Patients were recruited through a USA based international NF registry. Screening and consent occurred via live video with the adolescent and at least one parent. Data was collected electronically. The 3RP-NF and control ( 8 sessions, 45 minutes) were delivered by a trained clinical psychologist. Adolescents had a mean age of 14.36 (12-17 range), equally distributed in terms of gender ( $N=25$ females), predominantly white $(\mathrm{N}=37)$, from USA $(\mathrm{N}=45)$, with mean number years of education of 8.4 (5-12). About half reported having a learning disability $(\mathrm{N}=27)$ and living with both parents $(\mathrm{N}=33)$. Out of 61 adolescents screened, 51 entered the study, completed consent, provided baseline and were randomized ( 27 intervention, 24 control). Of these, $45(88.3 \%)$ completed the intervention and provided post-test data, and $37(74.5 \%)$ provided 6 months follow up data. Satisfaction with participation was high and similar in both groups. Participants in the 3RPNF improved significantly more than those in control on Psychological QoL (13.17 points; $\mathrm{p}=.03$ ), Physical QoL (10.20 points; $\mathrm{p}=.03$ ), pain intensity (2.44 points; $\mathrm{p}=.006)$, anxiety ( 2.32 points, $\mathrm{p}=.03$ ) and pain interference $(8.05 ; \mathrm{p}=.038)$. Improvements were clinically meaningful and maintained over time. Adolescents with NF are receptive to live video mind body skills building. Results support a fully powered efficacy RCT.

CORRESPONDING AUTHOR: Ana-Maria Vranceanu, Massachusetts General Hospital/Harvard Medical School, avranceanu@mgh.harvard.edu

\section{O06.5}

A RANDOMIZED CONTROLLED TRIAL OF THE DISASTER WORKER RESILIENCY TRAINING PROGRAM Adam Gonzalez ${ }^{1}$, Daniel Mackin ${ }^{1}$, Brittain Mahaffey ${ }^{1}$

${ }^{1}$ Stony Brook University- USA

Introduction \& Purpose. Disaster workers are at significant risk for mental health problems as a result of traumatic exposures and disaster response efforts. Depression and posttraumatic stress disorder (PTSD) symptoms are primary sequelae from disaster work. In addition, disaster-related psychiatric symptoms are associated with greater engagement in negative health behaviors. One possible way to offset the negative effects of disaster work is to build-up coping resources and enhance resilience prior to disaster exposures. The Disaster Worker Resiliency Training Program (DWRT) is a brief resilience-training workshop developed by the National Institute for Environmental Health Sciences and the Substance Abuse Mental Health Systems Administration. The primary aim of this study was to evaluate the efficacy of the DWRT program for enhancing resilience over a 3-month period in active disaster workers previously exposed to Hurricane Sandy. Methods. Disaster workers were recruited from local volunteer and professional organizations. Interested persons completed a phone screen to assess eligibility and were then randomly assigned to complete the DWRT or to a 3-month waitlist condition. We hypothesized that participants completing the DWRT would evidence greater improvements in resilience and mental health, as compared to waitlist controls, as demonstrated by self-reported improvements on validated measures of health behaviors and PTSD and depression symptoms at 3-month follow-up. Results. Participants included 167 disaster workers (54\% female; $70 \%$ White) randomized to either the DWRT $(n=78)$ or waitlist condition $(n=89)$. Participants in the DWRT versus waitlist condition reported greater improvements from pre-intervention (T1) to 3-month follow-up (T2) in healthy lifestyle behaviors $(\eta 2=.03 ; \mathrm{p}=.03)$, stress management $(\eta 2=.03, \mathrm{p}=.04)$, and spiritual growth $(\eta 2=.03, \mathrm{p}=.02)$. There was also a significant time by intervention interaction for participants reporting additional trauma exposures $(\mathrm{n}=99)$ between $\mathrm{T} 1$ and $\mathrm{T} 2$, such that participants in the waitlist versus DWRT condition reported a significant increase in PTSD $(\eta 2=.05, \mathrm{p}=.03)$ and depression $(\eta 2=.07, \mathrm{p}<.01)$ symptoms at follow-up. Conclusions. The DWRT program is an efficacious training for building resilience and preserving mental health in disaster workers. This preventative intervention may be useful to provide to disaster worker prior to response efforts. Future research is needed to understand mechanisms of action and ways to optimize dissemination.

CORRESPONDING AUTHOR: Adam Gonzalez, Stony Brook University, adam.gonzalez@stonybrook.edu 
O06.6

MINDFULNESS AND ENHANCEMENT OF SELF REGULATION: RESULTS FROM A PILOT STUDY USING FIRST AND THIRD PERSON PERSPECTIVES

Sebastian Medeiros ${ }^{1}$, Carla Crempien ${ }^{2}$, Vasquez Alejandra ${ }^{1}$, Catherine Andreu $^{2}$

${ }^{1}$ Depto. Psiquiatría de la Universidad Católica y MIDAP- Chile

${ }^{2}$ Millenium Institute for Research in Depression and Personality- Chile

Introduction: Emotional self-regulation is a central component of health. Poor emotional regulation underlies various emotional and medical conditions. Mindfulness practice- non-judgemental awareness of present moment experience - may enhance emotional regulation capacities. The goal of this study is to deepen the understanding of the potential self-regulatory mechanisms of mindfulness using mixed methodological approaches. Methods: Subjects $(n=6)$ vulnerable to stress and depression participated in Mindfulness based Cognitive Therapy (MBCT; 8 weeks). Psychological functioning (self report), micro-phenomenological subjective accounts (synchronic and diachronic), psychophysiological (HRV) and neurobehavioral (Flanker) accounts were measured pre/post intervention. Results: All participants completed the program. There was significant improvement in overall symptoms (OQ-45, $\mathrm{z}=-2,201$ ), mindfulness skills (FFMQ, Observation $z=-2,060$, No reactivity $z=-2,207$ ), Structural functions (OPD-SQ, Self-Perception $z=-2,201$ and Self-regulation $(z=-$ 2,207). Moreover, first person analysis correlated with quantitative results, highlighting body awareness and acceptance as fundamental aspects of change. A single case analysis shows concordance between self-report and present moment subjectivity and psychophysiological responses. No changes were observed in global neurobehavioral measures. Conclusions: Increased ability for emotional regulation is a likely mechanism explaining reduction in symptoms after mindfulness practice. First person methodologies contribute in understanding new forms of relating to distress - via awareness and acceptance. Future studies with larger samples should replicate these results.

CORRESPONDING AUTHOR: Sebastian Medeiros, Depto. Psiquiatría de la Universidad Católica y MIDAP, sebastianmedeiros@gmail.com

\section{O07.1}

ATTENTIONAL BIAS FOR FOOD CUES AFTER SLEEVE GASTRECTOMY

Arturo Zumaeta ${ }^{1}$, Francisco Aboitiz ${ }^{2}$

${ }^{1}$ Universidad de Chile- Chile

${ }^{2}$ Universidad Católica de Chile- Chile

Introduction: In cognitive models, pathological behaviors are the consequence of maladaptive knowledge structures that determine altered processing of information, including attentional, memory and judgment bias. Attentional Bias $(\mathrm{AB})$ refers to a predominant sensitivity for specific environmental stimuli. $\mathrm{AB}$ for specific stimuli has been associated with disorders, playing a central role in causation and maintenance of the pathology. $\mathrm{AB}$ has been described in a wide variety of mental pathologies; including anxiety disorders, depression, substance use disorders, and eating behavior disorders. $\mathrm{AB}$ for food cues in obesity is a recent area of study. According to incentive sensitization theory, the rewarding effect of high-calorie food (with high concentration of sugar and fat), can produce a pathologic modification on the dopaminergic system. This modification generates an abnormal dopaminergic response to foodrelated stimuli, transforming these stimuli in attention-grabbing cues, which induce craving and approach behavior. In this study, we explore the presence of $\mathrm{AB}$ for food cues in patients after at least 1 year from sleeve gastrectomy. Methods: 30 women participants were recruited: 15 bariatric patients (BP), and 15 control participants matched by age and body mass index. Two tasks were performed by every participant: foodmodified Stroop task with EEG recording and a visual task with eye tracking recording. ERPs were extracted from EEG signal, and the amplitude of p300 was analyzed, as an electrophysiological marker of AB. From eye tracking recording we analyzed the first fixation, to calculate a direction bias score, which represent a marker for $\mathrm{AB}$. Results: There is no significant difference between groups in socio-demographics aspects. Food-modified Stroop task with EEG recording: no significant difference were found in reaction times between groups, but intrasubject difference were found. BP show a higher p300 amplitud in food words trials, compared to control words trials. BP show increased p300 amplitud and latency in food words trials, compared to control group. Visual task with Eye Tracking recording: Bariatric group has decreased direction bias to diet stimuli compared to control group. Conclusions: $\mathrm{AB}$ for food cues can play a central role in maladaptive eating behavior, including obesity. Growing evidence support the presence of $A B$ to food in obesity. Our study shows an objective methodology to explore $\mathrm{AB}$ for food, combining a behavioral approach with electrophysiological recording. Our results show altered processing of food cues in the bariatric group, including high amplitud of P300, an electrophysiological marker of AB.

CORRESPONDING AUTHOR: Arturo Zumaeta, Universidad de Chile, drzumaeta@gmail.com

\section{O07.2}

SHORT- AND LONG-TERM PSYCHOLOGICAL EFFECTS OF BARIATRIC SURGERY

Martti T. Tuomisto ${ }^{1}$, Maaria Nikunen ${ }^{2}$, Satu-Sisko Koivula ${ }^{1}$, Jyrki Ollikainen $^{1}$, Lauri Parkkinen ${ }^{1}$, Ulla Siljamäki-Ojansuu ${ }^{2}$

${ }^{1}$ University of Tampere- Finland

${ }^{2}$ Tampere University Hospital- Finland

Introduction \& Purpose: The treatment of choice for extreme obesity is bariatric surgery. Besides body weight, it affects psychological variables to a great extent. Our aim was to monitor social and behavioural changes during the process of assessment, and treatment of obesity using bariatric surgery. Methods: Psychological assessments have so far been conducted on four occasions: first assessment (some months before the second assessment), second assessment two months before the operation, third assessment two months after and fourth assessment at one-year followup. The following questionnaires were used in this research: (1) Questionnaire of Risk Situations for Overeating, (2) Rosenberg SelfEsteem Scale, (3) Motivation Scale, (4) Symptom Check List 90 (SCL90), and (5) Penn State Worry Questionnaire (PSWQ). Results: Data from about 25 patients have been collected through all the phases of the research. We tested linear trends in the development of the factors. The mean BMI of the group developed as follows: 43.3 (SD = 4.3), 42.4 (4.5), 37.2 (4.2), and 32.9 (5.3). Mean frequency of risk situations (number of possible situations $=40)$ was reduced $(\mathrm{F}=11.1, \mathrm{p}=.003): 2.1(0.5)$, $2.0(0.5), 1.8 .(0.5)$, and $1.8(0.6)$, and mean of the actual risk $(0-4)$ was reduced $(\mathrm{F}=36.2, \mathrm{p}=00005): 2.2(0.6), 1.9(0.6), 1.6(0.5)$, and $1.5(0.5)$. Mean Rosenberg scores improved $(\mathrm{F}=77.6, \mathrm{p}=.00005)$ : $13.6(2.4), 20.6$ (5.5), 21.1 (4.7), and 21.7 (4.1). Motivation scores improved ( $F=18.5, p$ $=.00005)$ : 7.6 (2.7), 7.1 (2.7), 8.8 (2.6), and 9.4 (2.4). The following scales were collected on the last three occasions. In SCL 90, the total score development was quadratic and somewhat impaired $(\mathrm{F}=8.8, \mathrm{p}=$ $.01): 30.3$ (16.3), 31.6 (28.0), and 49.3 (46.1). The same was almost true for PSWQ ( $\mathrm{F}=3.3, \mathrm{p}=.09)$ : 37.4 (7.6), 36.2 (9.2), and $39.8(12.5)$. However, the results for both of these questionnaires were within the normal range. Conclusions: Many favourable changes occurred before and just after bariatric surgery. Most of these changes improved further at 1-year follow-up. However, not all changes were positive at 1-year follow-up and the changes were more variable than earlier. It will be important to study these changes in detail and with further follow-ups.

CORRESPONDING AUTHOR: Martti T. Tuomisto, University of Tampere, martti.tuomisto@uta.fi 
O07.3

CHANGES IN PSYCHOLOGICAL HEALTH FOLLOWING BARIATRIC SURGERY: UNDERSTANDING THE 'HEAD SPACE' OF BARIATRIC CLIENTS POST-SURGERY

Karena Burke $^{1}$, Susan Williams ${ }^{1}$, Rebecca Campbell ${ }^{1}$

${ }^{1}$ Central Queensland University- Australia

Introduction \& Purpose: Bariatric surgery is considered the most effective treatment for weight loss maintenance following obesity. However, while the treatment is undoubtedly effective physically, results regarding the psychological impact of this approach remain contradictory. This presentation will explore the results of two studies examining psychological health following bariatric surgery. Methods \& Results: Study 1 focussed on the impact of length of time since surgery on psychological health and on body image. Snowball sampling via Social Media support groups yielded responses from 299 individuals who had undergone bariatric surgery, most of whom were female $(n=241)$. Participants completed the DASS-21, the Subjective Happiness scale, the trait version of the Physical Appearance State and Trait Anxiety scale and the Body shape questionnaire. The findings from Study 1 suggested that Psychological health peaks during the period 6-12 months post-operatively, and that those who had their surgery more than 2 years prior had lower psychological health and lower body image compared to those who had had surgery more recently. Study 2 extends upon the findings from Study 1 by focussing on recruitment through bariatric surgeons and further examining changes experienced after having bariatric surgery, and explored the impacts of time, post-surgery procedures (i.e. follow up doctor visits) and general life events that may explain the worsening of psychological health over time that was found in Study 1. Conclusions: Preliminary results suggest a range a factors may impact on longer term psychological health, including the patient's relationship with their surgeon and ongoing relationship with their doctor. Both studies reinforce the need for continued monitoring of psychological health following bariatric procedures, and the importance of ensuring health professionals are aware of the psychological changes that can occur alongside the physical changes as a result of undergoing bariatric surgery.

CORRESPONDING AUTHOR: Karena Burke, Central Queensland University, k.j.burke@cqu.edu.au

\section{O07.4}

CHANGES IN BODY IMAGE IN ADOLESCENTS UNDERGOING BARIATRIC SURGERY

Claudia Cruzat-Mandich

Universidad Adolfo Ibáñez. Centro de Estudios de la Conducta Alimentaria, CECA. Escuela de Psicología- Chile

Objectives: To describe the body image of Chilean adolescents and young people and to identify the changes in their components in adolescents with obesity who undergo bariatric surgery. Methodology: Mixed study (quantitative-qualitative), designed in phases, correlational and longitudinal, using a non-experimental design. In the first phase, of validation of the MBSRQ, the population was made up of 1,438 adolescents and young people of both sexes, between 15 and 25 years old, belonging to different socioeconomic levels, enrolled in school or in the firsts years of higher education. In the application phase of the questionnaire to adolescents and young people with obesity, the population was made up of 112 young people who consult for bariatric surgery in the public and private system, and who are subsequently operated on. In the qualitative phase, the final sample was defined by theoretical saturation, remaining in 30 people. Regarding the instruments and strategies for data collection, the Multidimensional Body-Self-Relations Questionnaire (MBSRQ), the Eating Disorders Inventory (EDI-2) and the SCL-90_R were applied. Meanwhile, in the qualitative phase, semi-structured interviews were conducted with patients in the follow-up phase (6 months after surgery). With regard to ethical safeguards, all participants sign an informed consent. The identity of the participants is safeguarded, the data is confidential and only the members of the research team and the supervising agents of the study access them. Results: The psychometric properties of MBSRQ in the Chilean population are described, which allows the evaluation of relevant dimensions of body image in this population. It was possible to carry out a screening of what is happening in some group of the adolescent and normal young population in relation to the body Image, and how this affects its relation with the weight: in general women appear with greater difficulties and dissatisfaction. In addition, it was possible to evaluate a specific sample of adolescents and young people with obesity who decide for a surgery, which allows a detailed description.

CORRESPONDING AUTHOR: Claudia Cruzat-Mandich, Universidad Adolfo Ibáñez. Centro de Estudios de la Conducta Alimentaria, CECA. Escuela de Psicología, claudia.cruzat@uai.cl

\section{O07.5}

BARIATRIC SURGERY IN ADOLESCENTS. REMOTE PSYCHOLOGICAL AND NUTRITIONAL EVOLUTION IN ADOLESCENTS UNDERGOING BARIATRIC SURGERY AT 5 YEARS IN CLINICAL HOSPITAL OF THE UNIVERSITY OF CHILE Susana Cortes ${ }^{1}$

${ }^{1}$ Hospital Clínico de la Universidad de Chile- Chile

The increasing prevalence of obesity in Chile and the world impacts not only adults but also children and adolescents. Obesity is associated with serious medical co-morbidities, as well as psychological discomfort and social deterioration. Before consulting for bariatric surgery as a treatment opción, many adolescents have made numerous strategies to reduce their weight, such as diet, behavioral modification, physical exercise, and in some cases pharmacotherapy, without succeeding in such an attempt, which carries a significant impact on psychosocial well-being. This is why bariatric surgery has become a viable solution for that purpose. However, bariatric surgery remains controversial in this population, given the developmental stage in which they are located; still in maturation of their identity, so it is presumed that the evolution of these could be different from what is reported in adult patients. In this group of patients the reports in the literature on remote post-operative follow-up are scarce and with small samples of patients. The present study examines the evolution of psychosocial variables and nutritional aspects such as weight and pattern of food intake, in adolescents between 18 and 25 years of age with diagnosis of obesity and pre-operative psychological and nutritional evaluation undergoing bariatric surgery in the Clinical Hospital of the University of Chile, between the years 2012-2013, after 5 years of post-op follow-up. The study is a descriptive study. Thirty four adolescents patients, undergoing bariatric surgery are evaluated after 5 years of surgery, with a semi-structured interview, the SF 36 health scale; the Symptom Inventory of Derogatis rev SCL-90-R; the Multidimensional Body Self-Self MBSRQ and Record of the alimentary behavior. The variables measured in the pre-operative evaluation and the results in the 5-year post-op evaluation are described; plus the results obtained in the post-op evaluation instruments. The relevance of this study is that it contributes in clarifying the indications and contraindications for bariatric surgery in this age group, expanding existing knowledge. This makes possible to improve the mechanisms of selection and intervention in the treatment of future patients and intervene in a more efficient way on psychological and nutritional aspects in adolescents patients already operated.

CORRESPONDING AUTHOR: Susana Cortes, Hospital Clínico de la Universidad de Chile, susanacortesm@yahoo.es 
O07.6

FIRST CONSENSUS OF CHILEAN BARIATRIC PSYCHOLOGISTS ABOUT MENTAL HEALTH MANAGEMENT PRE AND POSTBARIATRIC SURGERY.

María José Leiva ${ }^{1}$, Paula Díaz ${ }^{2}$, María Cecilia Barros ${ }^{3}$, Denisse Montt ${ }^{4}$, Viviana Assadi ${ }^{3}$, Macarena Cruz ${ }^{5}$, Susana Cortés ${ }^{4}$, Luciana Marin ${ }^{4-5}$, Claudia Badilla ${ }^{6}$

${ }^{1}$ Clínica Las Condes- Chile

${ }^{2}$ Clínica Santa María- Chile

${ }^{3}$ Universidad Católica de Chile- Chile

${ }^{4}$ Hospital Clínico Universidad de Chile - Clínica Universidad de los Andes- Chile

${ }^{5}$ Clínica Alemana- Chile

${ }^{6}$ Clínica Ciudad del Mar Chile

INTRODUCTION \& PURPOSE: Bariatric surgery is the most effective and lasting alternative to weight reduction and improved general health. However, long-term weight maintenance after surgery may be challenging for some patients. Thus, multidisciplinary teams should be involved in the evaluation, treatment, and post-operative support. With the purpose of unifying criteria about mental health aspects, a scientific-based consensus was elaborated. METHODS: all members of the NPCO - Chilean Bariatric Psychologists, part of Chilean Bariatric and Metabolic Society were invited to participate, representing teams from 8 different regions throughout Chile. A questionnaire including the most important topics, such as preoperative contraindications, psychological risk factors of weight regains, postoperative support, was designed and sent to 50 national experts. When no agreement among them was achieved, the Delphi methodology was applied. Scores of $86 \%$ or greater were used for inclusion consensus. The recommendations here presented were widely discussed in several scientific meetings with active participation of all members of the group and were highly based on scientific evidence covered by the literature. CONCLUSIONS: The main conclusions reached are that clinical findings are critical for the preoperative diagnosis and treatment adherence. The purpose of the psychological assessment has shifted from identifying contraindications for bariatric surgery and evolved towards assessing psychological and behavioral readiness. The achievement of pre-surgery preparatory behavioral changes are recommended to optimize their postoperative outcomes. Acquisition of a healthy lifestyle is the most important therapeutic tool for long-term weight maintenance. Thus, therapeutic postoperative support is fundamental. The psychologist undertaking the assessment needs specific experience and knowledge of bariatric surgery, as well as the preoperative and postoperative issues that may arise. There are specific recommendations for adolescents as this group presents special needs according to their development stage.

CORRESPONDING AUTHOR: María José Leiva, Clínica Las Condes, mleiva@clc.cl

\section{O08.1}

TRENDS IN HEALTH AND PHYSICAL FITNESS AMONG CHINESE CHILDREN AND ADOLESCENTS DURING THE RECENT EPIDEMIC OF PHYSICAL INACTIVITY: A MULTIPLE CROSS-SECTIONAL STUDY BETWEEN 1985 AND 2014

Jun $\mathrm{Ma}^{1}$

${ }^{1}$ Peking University- China

Objective: This study aimed to assess the trends in health and physical fitness, including obesity, hypertension, myopia, cardiorespiratory endurance, and muscle strength, during the recent epidemic of physical inactivity in Chinese children and adolescents. Methods: Data on 1,303,616 participants aged 7-18 years were obtained from the Chinese National Survey on Students' Constitution and Health (CNSSCH) from 1985 to 2014 , which is the largest nationally representative sample of school-aged children in China. Overweight and obesity were classified with the International Obesity Task Force definition. Hypertension and were recognized according to the reference values of sex- age- and height-specific 95th centile. The myopia was defined using unaided distance visual acuity and the subjective refraction method. Trends in health fitness, including cardiorespiratory endurance (determined by endurance run test), muscle strength (determined by grip strength and standing long jump), and 50-meters dash, were also assessed. Results: Children and adolescents who reported they participated in physical activity for more than 1 hour per day was only $23.9 \%$ in 2014 . The prevalence of overweight and obesity increased steadily from 1985 ( $0.8 \%$ and $0.04 \%$, respectively) to 2014 (14.3\% and $4.1 \%$, respectively). The prevalence of hypertension decreased between $1985(10.1 \%)$ and $2005(4.3 \%)$, but increased in $2010(6.1 \%)$ and $2014(5.8 \%)$. The prevalence of myopia increased from $47.5 \%$ in 2005 to $57.1 \%$ in 2014. Additionally, the performance of cardiorespiratory endurance, muscle strength, and 50 meters dash declined constantly from 1995 to 2014. Conclusion: Worsening trends in health and physical fitness were observed in Chinese children and adolescents. Intervention aimed to increase physical activity could provide an opportunity to curb these trends.

CORRESPONDING AUTHOR: Jun Ma, Peking University, majunt@bjmu.edu.cn

\section{O08.2}

PARENTS' RULES FOR EATING AND SCREEN TIME BEHAVIORS: DOES FOCUS AND CONTENT OF RULES MATTER? Heather Hardin ${ }^{1}$, Sarah Jones ${ }^{1}$, Abdus Sattar ${ }^{1}$, Shirley Moore ${ }^{1}$, Matt Plow ${ }^{1}$, Elaine Borawski ${ }^{1}$

${ }^{1}$ Case Western Reserve University- USA

Introduction \& Purpose: Previous research has evaluated the influence of parents' rules on subjective reports of adolescent eating and physical activity behavior, but little research has evaluated the effect of parents' rules on objective measures of eating and physical activity. The purpose of our study was to evaluate the relationships between parents' rules for eating and screen time on adolescents' objectively measured eating and physical activity behaviors. Methods: This was a cross-sectional secondary analysis of behavioral data from 360 low-income, urban adolescents ages 11-12 years and a parent participating in a weight loss program. Parents completed the Rules for Eating and the Rules for Screen Time questionnaires. Adolescents' diet and physical activity were objectively measured through multi-pass dietary recalls using the Nutrient Data System for Research and accelerometry. Regression analyses were performed using binary logistic regression and log-linear regression. Results: Parents most often reported using eating rules that emphasized family time (Must help with clean up after meals at home), rather than eating rules that emphasized healthy food choices (No desserts except fruit). Parents' rules for eating was marginally significant $(\mathrm{p}=.07)$ for more vegetables eaten, but had no effect on calories, fat, fruit, or sugar sweetened beverages consumed. Parents most often reporting using screen time rules that emphasized academic achievement (No TV before homework), rather than rules that emphasized physical activity (No TV or computer unless exercised first). More screen time rules resulted in a marginally significant reduction in percent time in moderate to vigorous physical activity $(\mathrm{p}=.09)$, while having no effect on sedentary behavior. Conclusions: Parents' rules were not effective for influencing diet and physical activity behaviors in this sample of urban adolescents. Parents reported frequent use of rules, but the most commonly reported rules focused on family time and academics, rather than healthy eating and physical activity. Contrary to our hypothesis, more restrictive rules concerning screen time reduced percent time in moderate to vigorous physical activity. These results suggest that the content and focus of parents' rules are important in promoting adolescents' health outcomes. Future research should evaluate the effectiveness of influencing the 
content and focus of parents' rules for eating and physical activity behaviors.

CORRESPONDING AUTHOR: Heather Hardin, Case Western Reserve University, hkh10@case.edu

\section{O08.3}

RELIABILITY AND VALIDITY OF THE INTRINSIC MOTIVATION INVENTORY FOR PEDIATRIC WEIGHT MANAGEMENT

Alan Delamater ${ }^{1}$, Elizabeth Pulgaron ${ }^{1}$

${ }^{1}$ University of Miami Miller School of Medicine- USA

Introduction: Intrinsic motivation (IM) is an important construct for understanding management of various health conditions. The aim of this study was to develop a self-report measure of IM for weight management in overweight children. Our purpose was to determine the reliability and criterion-related and predictive validity of the new measure, Intrinsic Motivation Inventory for Pediatric Weight Management (IMI-PWM). We hypothesized that greater IM would be associated with better dietary intake and greater physical activity as well as predicting participation in a treatment program. Methods: Participants were recruited at a primary care pediatric visit. After determining children's overweight status ( $>85$ th \%ile for age and sex BMI), parents were invited to participate in a web-based treatment program. The sample included 65 8-12 year-old children with mean age of 9.6 years; $33.8 \%$ were male; $72 \%$ were Hispanic, and $78.9 \%$ were White. Children completed the IMI-PWM, as well as items from the CDC 2012 Youth Physical Activity (PA) and Nutrition Survey. Composite scores measured sedentary behavior, moderate-vigorous PA, healthy eating, and unhealthy eating, with parallel forms completed by parents about their children. The IMI-PWM consists of 16 items rated on a 7-point Likert scale, with higher scores indicating greater IM. Results: Internal consistency of the scale was very good $(\alpha=.87)$. As predicted, significant associations were observed between the IMI-PWM total score and PA $(\mathrm{r}=.34, \mathrm{p}<.003)$, sedentary behavior $(\mathrm{r}=-.20, \mathrm{p}<.05)$, healthy eating $(r=.23, p<.03)$, and unhealthy eating $(r=-.27, p<.02)$. Similarly, children's IM was significantly associated with parent reports of children's PA $(r=.27, \mathrm{p}<.02)$, sedentary behavior $(\mathrm{r}=-.21, \mathrm{p}<.05)$, and unhealthy eating $(\mathrm{r}=-.22, \mathrm{p}<.04)$. Children who later utilized the web program (48\%) had higher baseline IM scores than those who did not (4.5 vs. $3.9, \mathrm{p}<.01)$. Conclusions: These findings support the reliability and validity of the IMI-PWM. Greater IM for weight management was associated with healthy eating and PA, and also predicted participation in a webbased treatment program. These results suggest that interventions to increase IM for weight management may be helpful in the treatment of overweight children

CORRESPONDING AUTHOR: Alan Delamater, University of Miami Miller School of Medicine, adelamater@med.miami.edu

\section{O08.4}

SCHOOLCHILDREN IN JAPAN PERCEIVE DIABETES AS A DISEASE RELATED TO "EATING TOO MUCH SUGAR": A SCHOOL-BASED QUESTIONNAIRE SURVEY

Shinomi Takahashi ${ }^{1}$, Pilar Suguimoto ${ }^{2}$, Patou Masika Musumari ${ }^{2}$, Teeranee Techasrivichien ${ }^{2}$, Tomoyuki Kawamura ${ }^{3}$, Masako OnoKihara $^{2}$, Masahiro Kihara ${ }^{2}$

${ }^{1}$ Kyoto University- Japan

${ }^{2}$ Department of Global Health and Socio-Epidemiology, Kyoto University School of Public Health, Kyoto- Japan

${ }^{3}$ Department of Pediatrics, Osaka City University Graduate School of Medicine, Osaka- Japan

1. Introduction and aim For people living with diabetes, self-care of glycemic control is key to enjoy life without any complications, but it is not easy. Furthermore, concealment of diabetes has been reported as an important barrier for adequate self-care. Some children with diabetes are reluctant to disclose their status because they fear the negative reaction from friends and the stigma attached to the disease, but it is unclear how children without diabetes perceive the disease. Therefore, the aim of our study was to explore the perceptions of diabetes and the sources of knowledge among schoolchildren in Japan. 2. Methods We conducted a schoolbased questionnaire survey between September 2017 and February 2018. We used a convenience sample of elementary, junior high, and high schools in Japan recruited during a nationwide workshop for school teachers held in August 2017. We asked all students: (1) "Do you know the words "diabetes", "type 1 diabetes", "type 2 diabetes", and "lifestyle diseases"?"; (2) "Where/from whom have you heard/seen each of the words?"; and (3) "What do you know about diabetes?". Free descriptions were analyzed using content analysis. 3 . Results These are our preliminary results. A total of 3 elementary, 10 junior high, and 6 high schools gave a total of 2850 valid questionnaires; each contributing with 212 , 1600 , and 1038, respectively. The majority of students knew the words "diabetes" (92\%) and "lifestyle diseases" (84\%). Their sources of knowledge were mainly TV (49\% and 21\%) and school (32\% and 54\%). However, few students were familiar with the words "type 1 diabetes" (9\%) and "type 2 diabetes" (8\%). Among all students, 38\% perceived diabetes as related to "eating too much sugar"; $9 \%$ associated to a "fat person"; and 5\% to an "abnormal urine". 4. Conclusions Among schoolchildren, TV and school education are the main sources of information about diabetes, and lack of self-control ("eating too much sugar") was a salient perception about the disease. This perception may perpetuate the shame and stigma frequently attached to diabetes, in turn, reducing the willingness of children with diabetes to disclose their condition. Future research needs to examine ways to explain diabetes in schools and through mass media in Japan.

CORRESPONDING AUTHOR: Shinomi Takahashi, Kyoto University, takahashi.shinomi.45u@st.kyoto-u.ac.jp

\section{O08.5}

INFANT FEEDING AND ITS ASSOCIATION WITH BODY COMPOSITION GROWTH TRAJECTORIES IN THE FIRST 6 MONTHS OF LIFE: THE ETHIOPIAN IABC BIRTH COHORT

Gregers Andersen ${ }^{1}$, Rasmus Wibaek ${ }^{2}$, Tsinuel Girma ${ }^{2}$, Mubarek Abera ${ }^{2}$, Bitiya Admassu ${ }^{2}$, Pernille Kæstel ${ }^{3}$, Dorte Vistisen ${ }^{1}$, Jonathan Wells ${ }^{4}$, Henrik Friis $^{3}$

${ }^{1}$ Steno Diabetes Center Copenhagen- Denmark

${ }^{2}$ Jimma University Specialized Hospital- Ethiopia

${ }^{3}$ Department of Nutrition, Exercise and Sports, University of Copenhagen- Denmark

${ }^{4}$ UCL Institute of Child Health, London- United Kingdom

Infant feeding is influencing early body composition which may have long term effects on growth, development and risk of diseases, including obesity. However, current evidence is inconsistent and mainly from high income countries. We assessed associations of infant feeding with trajectories of body composition from birth to 6 months (mo) in apparently healthy Ethiopian infants. METHODS: We followed 428 term-born Ethiopian infants on a monthly basis from birth to 6 mo of age and used air displacement plethysmography to measure body composition. Infant feeding status was collected through questionnaires at each visit following birth and classified into exclusively breastfed (EBF), predominantly breastfed (PBF), or predominantly non-breastfed (NBF) at 6 mo. Fat- and lean mass trajectories were modelled using multiple linear mixed-effects models with an infant-specific random intercept and slope. Age was specified with natural cubic splines and models were adjusted for sex, birth weight, maternal BMI $(\mathrm{kg} / \mathrm{m} 2) 6$ mo postpartum and maternal education at delivery. RESULTS: EBF comprised 143 (33.4\%) infants, while 264 (61.7\%) were PBF and 21 (4.9\%) were NBF. NBF infants were either 
formula- and/or cow milk-fed. Mean \pm SD birth weight was $3.04 \pm 0.42 \mathrm{~kg}$ and did not differ between groups. EBF and PBF infants gained more weight at a higher rate between 1-4 mo of age, compared to the NBF. This difference was attributable to a greater fat mass accretion (figure 1a). In contrast, the lean mass trajectories did not differ between infant feeding groups (figure 1b). CONCLUSIONS: The higher fat mass gain associated with breastfeeding is likely to have a beneficial effect as an energy buffer during a period with high growth velocity, brain development and increased risk of infections. The long term effects on growth, development and disease risk in an apparently healthy low income country population needs to be studied further.

CORRESPONDING AUTHOR: Gregers Andersen, Steno Diabetes Center Copenhagen, gregers.stig.andersen@ regionh.dk

\section{O08.6}

DETERMINANTS OF BODY MASS INDEX VARIATIONS IN EARLY CHILDHOOD. CENTER-BASED CARE PROGRAMS TEND TO CONTROL THE BMI VARIATIONS BY REDUCING THE PROBABILITY OF BEING IN AN OUT-OF-NORMAL CATEGORY Kasim Allel ${ }^{1}$, Marigen Narea ${ }^{1}$

${ }^{1}$ Pontificia Universidad Católica de Chile- Chile

Childhood overweight and obesity is growing at an alarming rate and represents one of the main challenges of public health globally, including many developing countries. In Chile, about $34 \%$ of children under 6 years old are overweight or obese (Chilean Ministry of Health, 2014), the highest rate in Latin-America (WHO, 2016). Socioeconomic and lifestyle factors that may lead to inadequate eating behaviors in early-childhood, such as parental characteristics, and household routines, are the main drivers of childhood overweight (Reilly John, et al., 2005). However, attendance to center-based care at early ages tends to moderate overweight growth (Maher Erin, et al., 2008). We examined the effect of attendance of center-based care on the probability that a child is overweight, and the determinants of BMI variation in toddlers. We use panel data (2010 and 2012) from the Longitudinal Survey in Early Childhood (ELPI), a Chilean nationally representative dataset. The sample is children who were $<24$ months old in $2010(n=1,396)$. First, we used a difference-in-difference model to compare changes in the BMI of children participating in a center-based care program and children in maternal care. Second, we used a logit model, controlling for baseline covariates, to estimate the probability that children who were $<24$ months old in 2010 were overweight in 2012. We defined being overweight employing the bmi-for-age index using WHO standards for children with the same age and sex. Finally, we examined the association between the probability of being overweight in 2012 and the BMI gains/losses between 2010 and 2012 by maternal and center-based care. Overall, the results showed smaller BMI differences between 2010 and 2012 for children who attended center-based care compared to children in maternal (P-value $<0.05)$. Conditional on general characteristics, the variables most strongly associated with child overweight in 2012 were mother's BMI $(p<0.001)$, mother's chronic diseases, and child hours of TV watching and sleeping. The results suggest that public policies should encourage the participation in center-based care to avoid childhood overweight. Furthermore, controlling sudden increases in BMI should be promoted to fight this problem faced currently by many countries worldwide.

CORRESPONDING AUTHOR: Kasim Allel, Pontificia Universidad Catolica de Chile, kasim.allel@gmail.com

\section{O09.1}

A ROADMAP FOR INSTITUTIONALIZING COLLABORATIVE CARE FOR DEPRESSION IN LARGE INTEGRATED HEALTHCARE SYSTEMS
Karen Coleman ${ }^{1}$, Daniel Hackett ${ }^{1}$, Alisa Aunskul ${ }^{1}$,Mark Dreskin ${ }^{1}$ ${ }^{1}$ Kaiser Permanente Southern California- USA

Introduction and Purpose: Collaborative models for depression care have not been widely adopted throughout the U.S. It is possible that this limited dissemination is because there are no available, successful roadmaps for implementing these types of models in large, complex healthcare systems. This presentation is designed to provide such a roadmap through a case study of the institutionalization of a collaborative care model for depression in a large integrated healthcare system serving 4.2 million members, over 400,000 of whom suffer from depression. Methods: The model was implemented by a collaborative care team with a nurse, social worker, or mid-level provider as the treatment care manager, administrative support for patient outreach and engagement, and consulting primary care and psychiatric physicians. In addition, the intervention included systematic case review and treat-to-target guidelines, an extensive patient registry based on the electronic medical record, and extensive performance metrics. Institutionalization occurred over two years from 2015 2017, involving 13 care teams, targeting 47,922 patients newly diagnosed with mild-to-moderate depression. A "playbook" was developed with specific workflows, training materials, treatment guidelines, and documentation standards for the initiative. Outcomes were improvement (defined as at least $50 \%$ reduction in symptoms) and remission (defined as a PHQ9 less than 5) of depression symptoms. Follow-up of depression symptoms was also collected at 6 months following discharge. Results: Over a two-year period, 11,059 patients were treated in the program. The average course of treatment was 4.5 months (ranging from 3-8 months); $61 \%$ of patients experienced improvement in symptoms and $47 \%$ experienced remission of their depression at the time of discharge. These rates were maintained at the 6-month follow-up. Conclusions: Collaborative care for depression can be institutionalized in large healthcare systems and be sustained with a specific, detailed roadmap that includes workflows, training, treatment guidelines, and clear documentation standards that are linked to performance metrics. Extensive stakeholder engagement at every level is also critical for success.

CORRESPONDING AUTHOR: Karen Coleman, Kaiser Permanente Southern California, Karen.J.Coleman@kp.org

\section{O09.2}

STIGMA TOWARD MENTAL ILLNESS AMONG PRIMARY CARE PROFESSIONALS: A NATIONAL SURVEY STUDY IN CHILE

Jaime Camilo Sapag Muñoz de la Peña ${ }^{1}$, .Ruben Alvarado ${ }^{1}$, Paola Velasco ${ }^{1}$, Fernando Poblete ${ }^{1}$, Luis Villarroel ${ }^{1}$, Ana Jofre ${ }^{1}$, Cinthia Alvarez $^{1}$, Samanta Anriquez ${ }^{1}$

${ }^{1}$ Departments of Public Health and Family Medicine, School of Medicine, Pontificia Universidad Católica de Chile- Chile

Introduction \& Purpose: Stigma related to mental illness (MI) is a significant barrier to accessing good quality of care. Special efforts are being made in Chile to strengthen mental health services in primary health care (PHC). The overall objective of this study is to examine and understand in depth the phenomenon of stigma towards people with MI in PHC in Chile; and to explore whether and how specific factors may influence it. Methods: Cross sectional survey study conducted with health professionals and users from 32 PHC centres of the public health system across Chile. Socio-demographic and other key variables were assessed. In the case of professionals, the self-administered survey included previously adapted international scales: the Opening Minds Scale for Health Care Providers (OMS-HC) (Kassam et al., 2012); the Mental Illness: Clinicians Attitudes Scale (MICA) (Kassam et al., 2010); and the Modified Borgadus Social Distance Scale (Link et al., 1987). In the case of users ( $>=18$ years age/attended in PHC in the last three months for any MI), their capacity to consent was evaluated first. Their perception of sigma in PHC was assessed by a face-to-face survey which included 
instruments already validated in Chile:the Perceived DevaluationDiscrimination Scale developed by Link (1987) and the Discrimination Experience subscale of the Internalized Stigma of Mental Illness (ISMI) scale (Ritsher et al., 2003). The Marlowe-Crowne Social Desirability Scale was also considered. Results: 830 PHC professionals and 450 PHC users participated in the study. The results of the analysis will be presented:(1) Student's t-test, ANOVA test and multiple comparisons with Hochberg correction performed to assess differences of continuous variables between groups, while chi-squared tests to assess differences of proportions;(2) Wilcoxon rank-sum test and Kruskal-Wallis test to compare medians between groups;(3) Spearman's rank correlation coefficient to assess the linear relationship between stigma scores and/or numerical variables;(4) General linear models to determine independent factors associated with stigma scores, and to account for possible confounders;(5) Cohen's kappa statistics or intraclass correlation (ICC) to assess the degree of agreement or concordance between stigma scores. Conclusions: We confirmed that there is stigma toward people with MI among PHC professionals in the Chilean public health system. The phenomenon of stigma is complex, affected by different factors, and there are potential opportunities to prevent or mitigate its negative effects. This is the first National study to explore MI stigma among PHC professionals in Chile.

CORRESPONDING AUTHOR: Jaime Camilo Sapag Muñoz de la Peña, Pontificia Universidad Catolica de Chile, jsapag@med.puc.cl

\section{O09.3}

PROFESSIONALS' PERCEIVED BARRIERS TO INTERVENE WITH YOUNG OFFENDERS' FAMILIES IN DRUG TREATMENT Mónica Lobato Concha ${ }^{1}$, Robbert Sanderman ${ }^{1}$, Marcela Soto ${ }^{2}$, Decio Mettifogo $^{2}$, Mariët Hagedoorn ${ }^{1}$

${ }^{1}$ University medical Center Groningen, University of GroningenNetherlands

${ }^{2}$ Facultad de Psicología, Universidad de Chile- Chile

Introduction. To improve drug treatment programs through involving the family in an individual-centered program design, it is necessary to know the current professionals' experiences within the current program. Our aim was to explore professionals' perspectives of perceived barriers to intervene with parents or responsible adults of young offenders with substance use disorder. Methods. Semistructured in-depth interviews were conducted with professionals (14 women, 4 man) in charge to contact and/or intervene with families from one of the 18 teams participating in this research, belonging to the Chilean National Drug Treatment Program (2016-2017). Traditional content analysis was conducted. Results. Main barriers experienced with these families were related with: 1) the complex profile of families (i.e., family resignation, externalization of parental responsibility) that hinder adherence and willingness of parents to participate; 2) the families' problematic and dangerous context that discourage family involvement and difficult to obtain results; 3) a justice, social and health system that does not work integrally (i.e., different focuses to understand and intervene with these families); and 4) tensions with the program design (i.e., perception that the current model does not guide adequately the intervention with these families). Conclusions. Professionals have a negative concept of young offenders' families, the family context and the intervention itself. It makes them feel hopeless and they are pessimistic about the effectiveness of the current program. When innovating the treatment modalities intended to improve treatment outcomes, it is very important to take into account professionals' perspectives to make use of their expertise but also since their current perspective might be a barrier when suggesting a change.

CORRESPONDING AUTHOR: Mónica Lobato Concha, University medical Center Groningen, University of Groningen, lobatoc.monica@gmail.com
O09.4

FROM EFFICACY TO EFFECTIVENESS: IMPLEMENTATION OF AN HIV RISK REDUCTION FOR YOUNG GAY AND BISEXUAL MEN

Jeffrey Parsons ${ }^{1}$, Tyrel Starks ${ }^{2}$, Sitaji Gurung ${ }^{2}$, Demetria Cain ${ }^{3}$, Thomas Borkowski $^{3}$, Jonathan Marmo ${ }^{3}$

${ }^{1}$ Hunter College - CUNY- USA

${ }^{2}$ Graduate Center - CUNY- USA

${ }^{3}$ Center for HIV/AIDS Educational Studies and Training- USA

Introduction \& Purpose: Young gay and bisexual men (YGBM) remain disproportionally affected by HIV yet only one evidence-based intervention - the Young Men's Health Project (YMHP) exists to reduce condomless anal sex and substance use. YMHP is a four-session motivational interviewing intervention originally tested in a research setting, delivered by doctoral students, and for YGBM ages 18-29. It is unclear how such an evidence-based program will work in the "real world." Efforts were made to adapt YMHP for 1) delivery in HIV clinic settings and in community-based organizations by paraprofessionals; 2) remote delivery; and 3) for YGBM under the age of 18. Methods: Three phases of implementation research were conducted. First, qualitative methods were conducted at three HIV clinics and three community-based organizations serving YGBM. Second, YMHP intervention was pilot tested, focused on remote delivery, with a sample of 10 YGBM ages 13-18 recruited through advertisements on Facebook. Six of the Phase 2 participants completed a post-intervention interview and feedback was used for further revisions. Third, the final revised intervention was pilot tested with another 20 YGBM. Results: In Phase 1, feedback from 18 staff members was used to create developmentally appropriate consent materials for YGBM under the age of 18 and we successfully applied for a waiver of parental permission. Focus groups with 25 YGBM generated suggestions for overcoming barriers to remote delivery: 1) use of video chat rather than telephone to build rapport and facilitate being able to "open up" about sensitive behaviors; 2) ensuring flexible scheduling to avoid disclosure to parents, guardians, or peers; and 3) ensuring counselors were relatable and non-judgmental. The Phase 2 pilot revealed challenges with delivery of YMHP, most notably that youth had many basic "sex ed" questions about gay sexuality, various sexual behaviors, how to find partners, etc. As a result, for Phase 3, YGBM were offered an online educational program ("Queer Sex Ed") prior to YMHP. This phase has been more successful - retention across sessions has been higher, with the majority completing all four YMHP sessions. Conclusions: Adaptation generated developmental and technology-related insights. YGBM have a need for psychoeducation around sex and motivation to reduce HIV risk can be generated through conversations focused on sexual health (e.g., sexual identity issues, dating habits, relationship preferences). Providing all YGBM with access to an online sexual education program improved engagement and retention. Remote service delivery (via phone, Skype, FaceTime) presented initial challenges in establishing rapport and scheduling, which, with further adaptations, were able to be overcome.

CORRESPONDING AUTHOR: Jeffrey Parsons, Hunter College CUNY,jparsons@chestnyc.org

\section{O09.5}

IMPLEMENTATION OF THE BRECONDA DECISION SUPPORT RESOURCE FOR WOMEN WITH BREAST CANCER

Kerry Sherman ${ }^{1}$, Christopher Kilby ${ }^{1}$, Elisabeth Elder $^{2}$, Kathy Wells ${ }^{3}$

${ }^{1}$ Macquarie University- Australia

${ }^{2}$ Westmead Breast Cancer Institute- Australia

${ }^{3}$ Breast Cancer Network Australia

Introduction and Purpose Women with breast cancer face difficult decisions regarding surgery following mastectomy. We have previously 
developed and evaluated the BRECONDA (Breast Reconstruction Decision Aid) web-based intervention through two RCTs. Following demonstration of the efficacy of BRECONDA to reduce decisional conflict and increase satisfaction with information, we have undertaken nationwide implementation of this online patient resource through the Breast Cancer Network Australia, a consumer support organisation. The aim of this study was to characterise the nature of users of BRECONDA, and to determine change in decisional conflict and satisfaction with information following access to the intervention. Methods All registrants accessing BRECONDA first completed an online survey documenting their status (patient/relative/health professional), age and place of residence. Then all women considering breast reconstruction completed questions assessing decisional conflict with the 4-item SURE (1=conflicted, 4=not conflicted) and satisfaction with information using a Likerttype single item ( $1=$ not at all satisfied to $4=$ very satisfied). Two months after accessing the BRECONDA website a subset of participants completed optional repeat measures of decisional conflict and satisfaction with information. Results Since implementation (February 2017), there have been 1532 registered BRECONDA users. The majority (81\%) were women diagnosed with breast cancer considering breast reconstruction, as well as another $5 \%$ of women who had already completed breast cancer surgery/treatment; $3 \%$ relatives/friends; and $11 \%$ health professionals (including breast surgeons and breast care nurses). The majority of patient users were Australian residents (99\%), aged 51- 60 years. In total 178 users consented to provide pre- and post- measures. t-tests indicated a significant improvement in decisional conflict [Pre $1.29(\mathrm{SD}=1.29)$ vs. Post 2.99 ( $\mathrm{SD}=1.36) ; \mathrm{p}<.0005$ ] following access to BRECONDA. Specifically, of the 160 women who were conflicted prior to accessing the intervention, 84 $(52.5 \%)$ no longer experienced any conflict at follow-up. Study participants also reported significant increases in satisfaction with information [Pre $2.89(\mathrm{SD}=.77)$ vs. Post $3.32(\mathrm{SD}=.78), \mathrm{p}<.0005]$ following access to BRECONDA. Conclusions These findings demonstrate that BRECONDA is being widely used for breast cancer patients in the process of surgical decision making and is being utilised by health professionals. Further corroborating the efficacy trials, these findings provide further support for the benefits of BRECONDA in a real-world setting.

CORRESPONDING AUTHOR: Kerry Sherman, Macquarie University, kerry.sherman@mq.edu.au

\section{O09.6}

THE IMPORTANCE OF CREATING MENTAL MODELS IN SUCCESSFUL IMPLEMENTATION OF CHRONIC DISEASE MANAGEMENT

Jodi Holtrop ${ }^{1}$, Laurie Fitzpatrick ${ }^{2}$, Lauri Connelly ${ }^{1}$, Georges

Potworowski ${ }^{3}$, Lee Green ${ }^{4}$

${ }^{1}$ University of Colorado- USA

${ }^{2}$ Michigan State University- USA

${ }^{3}$ University at Albany - SUNY- USA

${ }^{4}$ University of Alberta- USA

Introduction and Purpose: Mental model is the term cognitive and organizational scientists apply to an individual's or team's understanding of how some aspect of the world around them does and should work; about the work they do, why they do it, how processes do or should work, how the context affects them, what actions produce what consequences under what conditions, and through what mechanisms. We studied 24 primary care medical practices in three organizations who had been implementing chronic disease management delivered by care managers; and qualitatively examined the concept of mental models as it applied to the use of this service. Methods: Through individual interviews and care managers shadowing, over 110 primary documents were produced. Both a grounded theory hermeneutic editing approach and a template approach using the framework of macrocognition (includes the concept of mental models) were used for analysis. Results: We found differences in mental models across practices which appeared to be associated with more or less successful chronic disease management implementation. Several aspects of mental models were important. First, the mental model needs to be accurate and 'rich.' Does each practice member know what the different care team members actually do and what actions they take to help patients? Do they have a detailed understanding of the care manager role? Second, the mental model needs to be shared among practice members. Do some practice members think the care manager helps elderly patients to find nursing home care and others think the care manager calls patients who over-use the emergency department? Last, there need to be no major conflicting beliefs to the mental model. Even if a physician has a clear understanding of the care manager role, if $\mathrm{s} /$ he believes that $\mathrm{s} / \mathrm{he}$ should not 'share care' with another practice member such as a care manager, this belief system about the role will interfere with effective implementation. Conclusions: The lack of an accurate, rich, favorable, and shared mental model can seriously impede implementation efforts. Strategies to get practice team members "on the same page" with a high degree of buyin is important to adoption, implementation and maintenance of a new intervention.

CORRESPONDING AUTHOR: Jodi Holtrop, University of Colorado, jodi.holtrop@ucdenver.edu

O10.1

"TOBACCO USE AND PREVALENCE OF HEAD AND NECK CANCERS AMONG MALAYALI TRIBES, YELAGIRI HILLS, TAMIL NADU, INDIA"

Delfin Lovelina Francis ${ }^{1}$

${ }^{1}$ Tamil Nadu Dr. M.G.R. Medical University- India

BACKGROUND: India has the second largest tribal population of the world next to the African countries. Despite remarkable world-wide progress in the field of diagnostic, curative and preventive medicine, still there are large populations of people living in isolation in natural and unpolluted surroundings far away from civilisation, maintaining their traditional values, customs, beliefs and myths. Hence the present study was conducted to assess the oral health status, treatment needs among the Malayali tribes in the Yelagiri hills, Tamil nadu. AIM: 1. To assess the prevalence of head and neck cancers among the Malayali tribes, Yelagiri Hills, Tamil Nadu, India. 2. To assess the association of tobacco use with head and neck cancers among the Malayali tribes, Yelagiri Hills, Tamil Nadu, India. METHODOLOGY: A cross-sectional descriptive study was conducted to assess the tobacco use and head and neck cancer prevalenceamong 660 Malayali tribes in the Yelagiri Hills. Inhabitants of the villages aged 18 to 75 years who were residing for more than 15 years were included. Data was collected using a survey proforma which comprised of a questionnaire and WHO Oral Health Surveys - Basic Methods Proforma(1997). The collected data was subjected to statistical analysis. RESULTS: Results showed that among 660 study population, $57.7 \%$ had no formal education, $64.5 \%$ had indigenous brushing habits. $58 \%$ had the habit of tobacco, of which $37 \%$ were males and $21 \%$ were females. The percentage of oral mucosal lesions observed were as follows: $19.09 \%$ leukoplakia, $29 \%$ ulceration and $6 \%$ malignant tumor. $37 \%$ of the study populations had other abnormal conditions like candidiasis and OSMF. Prevalence of oral mucosal lesions in the study population was due to tobacco usage and alcohol consumption and lack of awareness regarding the deleterious effects of the products used. CONCLUSION: The oral health status of Malayali tribes was poor with high prevalence of periodontal disease and dental caries. Oral cancer and cancerous lesions were at a very high percentage. Regular oral examination by dental professionals, dental health education and motivation to maintain oral hygiene should be insisted to improve the oral health status of this community.

CORRESPONDING AUTHOR: Delfin Lovelina Francis, Tamil Nadu Dr. M.G.R. Medical University, delfin_lovelina@yahoo.co.in 


\section{2}

ETHNIC DISPARITIES IN PSYCHOLOGICAL DISTRESS AND UNMET PSYCHOSOCIAL NEEDS AMONG HISPANIC AND NON-HISPANIC WHITE COLORECTAL CANCER SURVIVORS Anita Kinney ${ }^{1}$, Cindy Blair ${ }^{1}$, Rachael Ruckman ${ }^{1}$, Jean McDougall ${ }^{1}$, Ashwani Rajput ${ }^{1}$, Charles Wiggins ${ }^{1}$

${ }^{1}$ University of New Mexico- USA

Introduction and Purpose: Psychosocial concerns and distress are among the most commonly reported unmet needs among post-treatment cancer survivors. Psychological distress is associated with adverse outcomes, including decreased quality of life, functional and social role limitations, and increased pain and mortality. Yet little is known about post-treatment psychosocial sequelae and concerns among Hispanic colorectal cancer (CRC) survivors. This study's objective was to investigate psychological distress and unmet psychosocial needs in an ethnically diverse population-based sample of post-treatment CRC survivors. Methods: Two-hundred sixtythree men and women diagnosed with localized or regional CRC were ascertained by a statewide cancer registry and completed a mailed questionnaire or telephone surveys about their CRC survivorship experience, measures of psychological distress using PROMIS measures, and unmet psychosocial needs. Chi-square tests were used to estimate bivariate associations and multivariable logistic regression was used to estimate adjusted odds ratios (OR) and 95\% confidence intervals $(\mathrm{CI})$. Results: Compared to non-Hispanic whites, Hispanic CRC survivors were more likely to report higher levels of depressive symptoms $(\mathrm{p}<0.001)$, anxiety $(\mathrm{p}<0.001)$, fatigue $(p=0.05)$, poor ability to participate in social roles and activities $(p=0.03)$ and pain interference $(\mathrm{p}=0.002)$. Controlling for sociodemographic characteristics, self-reported health, and cancer-related symptoms, Hispanic CRC survivors were more likely to report they wanted help with connecting with other survivors $(\mathrm{OR}=2.03 ; \mathrm{CI}=1.50-3.91)$, coping with sadness $(\mathrm{OR}=2.41 ; \mathrm{CI}=1.25-4.64)$, sharing thoughts and feelings $(\mathrm{OR}=3.85, \mathrm{CI}$ $=1.98-7.47)$; overcoming fears $(\mathrm{OR}=2.64 ; \mathrm{CI}=1.29-5.38)$ and finding meaning in life $(\mathrm{OR}=5.24$; $\mathrm{CI}=2.24-12.30)$. Conclusion: The study's findings suggest substantial ethnic disparities. Hispanic CRC survivors may be at greater risk of developing psychosocial difficulties and have related unmet needs due to their unique challenges of coping with cancer. The need for interventions to reduce psychosocial morbidity in cancer survivors, especially Hispanics, is critical and may offset increased health care costs among distressed cancer survivors.

CORRESPONDING AUTHOR: Anita Kinney, University of New Mexico, aykinney@salud.unm.edu

$\mathrm{O} 10.3$

"MY PEOPLE ARE DESTROYED FROM LACK OF KNOWLEDGE": A SPIRITUALLY-BASED TEXT MESSAGING PILOT PROGRAM TO PROMOTE CERVICAL CANCER PREVENTION AMONG AFRICAN AMERICAN WOMEN

Daisy Le ${ }^{1}$, Cheryl Holt ${ }^{2}$

${ }^{1}$ Johns Hopkins University: Bloomberg School of Public Health- USA

${ }^{2}$ University of Maryland, College Park: School of Public Health

Introduction/Purpose: On a national level, African American women have a $34 \%$ higher incidence of cervical cancer and are twice as likely to die of the disease when compared to White women. In response to the need to improve cervical cancer prevention and Pap test screening knowledge and utilization, we developed and pilot tested a 16-day SMS text message-based intervention. The CervixCheck study was designed to develop, pilot test, and evaluate the feasibility, acceptability, and initial efficacy of a spiritually-based SMS text messaging intervention aimed at increasing cervical cancer awareness and Pap test screening intention in church-attending African American women ages 21-65. Methods/ Approach: The Theory of Planned Behavior guided the development of the CervixCheck intervention. In this article, we present findings from
Phase 3 of the study. Phase 3 utilized a non-experimental one-group pretest-posttest design to pilot test the intervention. Of the 52 participants at baseline, 46 completed the post-program survey. Results/Findings: The current study provides evidence for the early feasibility, high acceptability, and some initial efficacy of the CervixCheck intervention. There was a significant pre-post increase observed for knowledge about cervical cancer and the Pap test $(p=.001)$ and subjective norms $(p=.006)$. Findings post-intervention also revealed that $83 \%$ of participants reported being either "satisfied" or "very satisfied" with the CervixCheck intervention and $85 \%$ found the SMS text messages either "useful" or "very useful". Conclusions/Significance: A spiritually-based SMS text messaging intervention could be a culturally appropriate and cost-effective method of promoting cervical cancer early detection information to churchattending African American women.

CORRESPONDING AUTHOR: Daisy Le, Johns Hopkins University: Bloomberg School of Public Health, drdaisyle@gmail.com

\section{O10.5}

IMPLEMENTATION OF PROMIS® SYMPTOM SCREENING IN A BOLIVIAN PUBLIC CANCER CENTER: PRELIMINARY RESULTS Steven J Schuetz ${ }^{1}$, Rina S. Fox ${ }^{1}$, Claudia B. Soliz P. ${ }^{2}$, Maribel Marmol C. ${ }^{3}$, Edgar F. Apaza O. ${ }^{2}$, Mayra V. Alaca M. ${ }^{2}$, Frank J. Penedo ${ }^{1}$, David Cella ${ }^{1}$, Richard C. Gershon ${ }^{1}$, PROMIS®-Bolivia Collaborative ${ }^{2}$

${ }^{1}$ Department of Medical Social Sciences, Northwestern University Feinberg School of Medicine-USA

${ }^{2}$ PROMIS®-Bolivia Organization-Bolivia

${ }^{3}$ Instituto Chuquisaqueño de Oncología-Bolivia

Introduction \& Purpose: Evaluation of patient-reported outcomes (PROs) enhances cancer care and improves quality of life. However, despite the availability of valid and reliable tools such as the Patient-Reported Outcomes Measurement Information System (PROMIS®), assessment of PROs in low-resources settings is limited. The purpose of the present study was to assess the preliminary feasibility of PROMIS $®$ symptom screening among a diverse population of ambulatory cancer patients in Bolivia. Methods: All patients receiving cancer treatment at the Instituto Chuquisaqueño de Oncología (ICO) from June 5 - July 11, 2018 ( $N=$ 266) were considered for inclusion in the present pilot program. Participants were eligible to complete PROMIS $®$ symptom screening if they were over 18 years old and self-reported basic Spanish proficiency; participants were excluded if they had a cognitive or physical impairment that precluded participation. Symptom domains evaluated included anxiety, depression, pain interference, fatigue, and anger; all domains were assessed inperson utilizing iPad-administered Computerized Adaptive Tests (CATs). In addition, participants self-reported sociodemographic information and completed the 18-item Short Assessment of Health Literacy - Spanish (SAHLS). Results: The sample was predominantly female (83.0\%). Approximately one fifth (20.9\%) preferred to speak the local indigenous Quechua language, and over half of the sample (54.5\%) reported less than 6th-grade education. Nearly one-third of the sample (32.6\%) demonstrated inadequate health literacy (SAHL-S score < 14). Of potential participants, $85.0 \%(n=226)$ were eligible to complete PROMIS $®$ screening; reasons for ineligibility included language barriers $(n=31)$, physical impairment $(n=5)$, and age $(n=4)$. The majority $(86.7 \%, n=196)$ of those eligible participated in PROMIS $®$ screening, and nearly all patients who initiated screening completed the full evaluation $(95.4 \%, n=187)$. Conclusions: Preliminary results support the feasibility of implementing PROMIS ${ }^{\circledR}$ symptom screening in a low-resource ambulatory cancer center. Future objectives include evaluating the acceptability and appropriateness of this protocol, as well as the differential functioning of PROMIS $®$ items in this population.

CORRESPONDING AUTHOR: Steven J Schuetz, Department of Surgery, Department of Medical Social Sciences, Northwestern University Feinberg School of Medicine, s-schuetz@northwestern.edu 
O10.5

DECISION SUPPORT AND NAVIGATION TO INCREASE COLORECTAL CANCER SCREENING AMONG HISPANICLATINO PATIENTS

Ronald Myers ${ }^{1}$, Brian Stello ${ }^{2}$, Constantine Daskalakis ${ }^{1}$, Randa Sifri ${ }^{1}$

${ }^{1}$ Thomas Jefferson University- USA

${ }^{2}$ Lehigh Valley Health Network- USA

Background: Colorectal cancer (CRC) screening rates are low among Hispanic primary care patients who are 50 to 75 years of age. Effective interventions are needed to raise these rates. Methods: The research team consented and enrolled 400 Hispanic patients from five primary care practices of the Lehigh Valley Health Network (LVHN) in southeastern Pennsylvania who were eligible for, but were not up to date with CRC screening. We randomized participants either to an SI Group $(n=203)$ or a DSNI Group $(n=197)$. Both groups were sent a CRC screening kit with instructions in English and Spanish (an informational booklet on screening, a stool blood test (SBT), and colonoscopy screening instructions). The DSNI Group also received a telephone call from a bilingual patient assistant (navigator) who reviewed the booklet with each recipient, determined the individual's preferred screening test and likelihood of adherence, and helped the participant implement a personal screening plan. Follow-up survey and medical records data were used to assess 12month overall and test-specific screening adherence, screening decision stage, and knowledge and perceptions about screening. Results: CRC screening adherence was significantly higher in the DSNI Group than the SI Group $(\mathrm{OR}=4.83)$. The DSNI Group, compared to the SI Group, also displayed a higher SBT screening rate $(\mathrm{OR}=4.20)$, a higher colonoscopy screening rate $(\mathrm{OR}=8.79)$, and a higher screening decision stage $(\mathrm{OR}=4.91)$. There were no significant study group differences in participant screening knowledge or perceptions. Conclusions. The DSNI strategy had a substantially greater positive impact on CRC screening adherence than the SI approach.

CORRESPONDING AUTHOR: Ronald Myers, Thomas Jefferson University, ronald.myers@ jefferson.edu

\section{O10.6}

DIFFERENCES IN QUALITY OF LIFE BY SOCIODEMOGRAPHIC AND CLINICAL FACTORS IN PATIENTS TREATED WITH A STEM CELL TRANSPLANTATION

Liliana Mey Len Rivera Fong ${ }^{1}$, Rebeca Robles García ${ }^{2}$, Corina Benjet ${ }^{2}$, Lara Traeger ${ }^{3}$, Juan José Sánchez Sosa ${ }^{1}$, Brenda Lizeth Acosta Maldonado ${ }^{4}$, Luis Manuel Valero Saldaña ${ }^{4}$, Silvia Rivas Vera ${ }^{4}$

${ }^{1}$ Universidad Nacional Autónoma de México- Mexico

${ }^{2}$ National Institute of Psychiatry- Mexico

${ }^{3}$ Massachusetts General Hospital; Harvard Medical School- USA

${ }^{4}$ National Cancer Institute- Mexico

Hematopoietic stem cell transplantation (HSCT) has been associated with a decrease in patients' quality of life (QOL), but there is little evidence regarding which individual variables contribute to such impact. AIM: To compare QOL domains in patients treated with HSCT along such sociodemographic and clinical variables. METHODS: Comparative, cross-sectional study which included patients treated at public hospital in Mexico. Patients in different phases of an HSCT (pre-transplant, during the transplant and post-transplant) were enrolled from 2016 to 2017. The European Organisation for Research and Treatment of Cancer's Quality of Life Questionnaire (EORTC QLQ-C30) was applied to 121 patients (scores ranging from 0 to 100 with higher scores meaning better QOL). PARTICIPANTS: $49 \%$ of patients had lymphoma, $29 \%$ had leukemia, $16 \%$ had multiple myeloma, and $6 \%$ had a non-malignant disease. The mean age of patients was $40 ; 58 \%$ were males; $55 \%$ were single. RESULTS: Patients' global QOL mean was 83 , and subscales' means ranged from 76 (social functioning) to 84 (physical and emotional functioning). Males reported higher physical ( $\mathrm{x}=88 \mathrm{vs} \mathrm{x}=80 ; \mathrm{t}=2.2$; $\mathrm{p}=.03$ ) and cognitive ( $\mathrm{x}=88$ vs $\mathrm{x}=78 ; \mathrm{t}=2.4 ; \mathrm{p}=.02$ ) functioning as compared to women. Single patients showed lower emotional functioning $(\mathrm{x}=81$ vs $\mathrm{x}=87 ; \mathrm{t}=-2.1 ; \mathrm{p}=.04)$ as compared to married patients. Posttransplant outpatients showed better physical functioning compared with hospitalized patients and those that are candidates for HSCT ( $\mathrm{x}=88$ vs $\mathrm{x}=83$ vs $\mathrm{x}=80$ respectively; $\mathrm{x} 2=7.7, \mathrm{p}=.02$ ). To make comparisons by age, participants were classified into two groups considering mean age (40 years old). This analysis showed that the younger group has higher physical $(\mathrm{x}=89$ vs $\mathrm{x}=80 ; \mathrm{t}=2.9 ; \mathrm{p}<.01)$ and role functioning $(\mathrm{x}=86$ vs $\mathrm{x}=77$; $\mathrm{t}=2.0 ; \mathrm{p}<.05)$, as well as global QOL $(\mathrm{x}=86$ vs $\mathrm{x}=80 ; \mathrm{t}=2.1 ; \mathrm{p}=.04)$, but the older group reported better emotional functioning ( $\mathrm{x}=87 \mathrm{vs} \mathrm{x}=80$; $\mathrm{t}=2.9 ; \mathrm{p}<.01)$. There were no differences by the type of transplant or the education level. CONCLUSIONS: While QOL was relatively high across patients, specific QOL domains differed by sociodemographic factors and timing relative to HCT. Clinicians and researchers should be keenly aware of the patients' global context while designing or evaluating QOL interventions.

CORRESPONDING AUTHOR: Liliana Mey Len Rivera Fong, Universidad Nacional Autónoma de México, lmeylenf@hotmail.com

\section{O11.1}

LATE EVENING FOOD CONSUMPTION AND DEVELOPMENT OF PREDIABETES OR DIABETES: A PROSPECTIVE ANALYSIS FROM THE WHITEHALL II STUDY

Kristine Færch ${ }^{1}$, Jonas Salling Quist ${ }^{1}$, Adam Hulman ${ }^{2}$, Daniel Rinse Witte $^{2}$, Adam Tabak ${ }^{3}$, Eric Brunner ${ }^{4}$, Mika Kivimaki ${ }^{4}$, Marit Eika Jørgensen ${ }^{1}$, Satchidananda Panda ${ }^{5}$

${ }^{1}$ Steno Diabetes Center Copenhagen- Denmark

${ }^{2}$ Aarhus University and Danish Diabetes Academy

${ }^{3}$ Semmelweis University- Hungary

${ }^{4}$ University College London- United Kingdom

${ }^{5}$ Salk Institute- USA

INTRODUCTION \& PURPOSE: We examined whether late evening food consumption was associated with development of prediabetes or diabetes. METHODS: From the Whitehall II study, we included 3,587 individuals with normal glycaemia $(\mathrm{HbAl}<39 \mathrm{mmol} / \mathrm{mol})$ and followed them for five years. Using recursive partitioning modelling, we studied the association of time of last meal (TLM) before the examination day with the development of prediabetes or diabetes $(\mathrm{HbAl} \mathrm{c} \geq 39 \mathrm{mmol} / \mathrm{mol}$ ). Baseline HbA1c, age, sex, BMI, cholesterol, blood pressure, and TLM (continuous variable in whole hours from 16:00 to 3:59) were included as explanatory variables. RESULTS: 1,565 individuals (44\%) developed prediabetes or diabetes. Figure 1 shows the survival tree. Among women with $\mathrm{HbA} 1 \mathrm{c} \leq 32 \mathrm{mmol} / \mathrm{mol}$, those consuming their last meal after 21:00 had a $55 \%$ higher 5 -year risk of developing prediabetes or diabetes than those consuming their last meal between 16:00-20:59 (_P_<0.001). TLM was not associated with progression to prediabetes/diabetes among men or among women with $\mathrm{HbAl}>32 \mathrm{mmol} / \mathrm{mol}$. CONCLUSIONS: Among women with a good glycaemic control, eating late in the evening is associated with increased risk of developing prediabetes or diabetes.

CORRESPONDING AUTHOR: Kristine Færch, Steno Diabetes Center Copenhagen, kristine.faerch@ regionh.dk

\section{O11.2}

EVALUATION OF A LITERACY TAILORED SELFMANAGEMENT GROUP INTERVENTION FOR PEOPLE living with DIABETES CHILE

Claudia Bustamante ${ }^{1}$, Claudia Alcayaga ${ }^{1}$

${ }^{1} \mathrm{PAHO} /$ WHO Collaborating Center, Escuela de Enfermería Pontificia Universidad Católica de Chile- Chile 
Introduction: Non-communicable diseases are a public health issue. The degree in which people are able to access, understand, evaluate, and communicate information to cope with health demands is critical to maintain health throughout their lifetime. The challenge for people living with a chronic condition is to achieve self-management on a day-to-day basis and the role of professionals is to support patients' decision-making and implementation. This research describes the effect of an intervention based on health literacy for people with DM2 to increase metabolic compensation and self-efficacy. Methods: A RCCT was conducted in 4 Family Health Centers in La Florida commune. Control group (n 53) received usual care and intervention group (n 67) received a 6-month intervention (telephone counseling and group sessions). Two ethic committees approved the study. Trained nurses provided the literacy-tailored intervention: 1) telephone counselling and 2) group sessions to allow participants to strengthen self-management by improving their understanding and management of DM2, relationship between behavior and outcomes, perception of barriers, and behavioral changes that promote self-management. Contents focused on: medication use, eating habits, physical activity, self-monitoring and therapeutic goals. The program was based on "The Latinos en Control Intervention Manual" provided and authorized by Dr. Rosal from University of Massachusetts Medical School. The primary outcome was metabolic compensation evaluated through the level of glycosylated hemoglobin and the secondary endpoints are self-efficacy, health literacy, self-management practices, and anthropometric parameters. Results: Women $66.7 \%$, age average was 61.63 years (SD 7.30 years) and the average BMI was 31.26 (DS 5.90). Average attendance to all sessions was $50 \%$. The intervention group showed significant difference in $\mathrm{HbAlc}$ between the baseline and final measurements, in which the control group increases by one point, that is, it deteriorates with respect to the IG. However, no effect was observed in the perception of self-efficacy in any of the groups. Conclusions: This study was the first experience in Chile applying a health literacy approach to tailor the intervention and including specific health literacy measures. Literacy tailored self-management group intervention was more effective than the usual interventions to improve HbAlc levels, this targets behavioral change.

CORRESPONDING AUTHOR: Claudia Bustamante, PAHO/WHO Collaborating Center, Escuela de Enfermería Pontificia Universidad Católica de Chile, Cqbustam@uc.cl

O11.3

WHAT IS THE EFFECT OF A TAILORED, EVIDENCE-BASED LEAFLET ON DIABETIC RETINOPATHY SCREENING UPTAKE AMONG YOUNG ADULTS WITH TYPE 2 DIABETES? FINDINGS FROM A RANDOMIZED CONTROLLED TRIAL

Amelia Lake ${ }^{1}$, Jessica Browne ${ }^{1}$, Gwyn Rees ${ }^{2}$, Jane Speight ${ }^{1}$

${ }^{1}$ The Australian Centre for Behavioural Research in Diabetes- Australia ${ }^{2}$ Centre for Eye Research Australia

Introduction and purpose: Young adults with type 2 diabetes (T2D, 18-39 years) are a growing population, characterized by sub-optimal glycemia and considerable diabetes complication burden by mid-life. Diabetic retinopathy, a common complication of diabetes, is the leading cause of blindness and vision loss in working age adults. Retinal screening is the proven clinical pathway to early diabetic retinopathy detection, treatment, and prevention of vision loss. Despite this, many young adults with T2D do not engage in retinal screening in a timely manner after diabetes diagnosis. The purpose of this study was to evaluate the effectiveness of an evidence-based retinal screening promotion leaflet tailored to this priority population in a Randomized Controlled Trial (RCT, ACTRN1261400111067). Methods: 5,345 young Australian adults with T2D were invited to complete an online survey about 'diabetes self-management' and participate in the RCT. Participants were randomized to leaflet intervention or no-leaflet control, stratified by prior engagement with retinal screening. Primary and secondary outcomes (retinal screening uptake, change in modifiable behavioral determinants) were collected pre-intervention and four weeks postintervention. Study purpose was disclosed and control group participants invited to receive the leaflet after study completion. Results: 227 eligible young adults with T2D registered and were randomized, with 101 providing post-intervention data. Moderate and significant intervention effect was observed for secondary outcome: knowledge of diabetic retinopathy $(p=.03$, partial eta squared=.05). No further intervention effects were observed. Conclusions: Power to test intervention effect on the primary outcome was curtailed by low recruitment $(4 \%)$, high attrition $(55 \%)$ and higher than anticipated baseline retinal screening rate (72\%). This study suggests that a well-designed retinal screening leaflet tailored to young adults with T2D can increase knowledge of diabetic retinopathy, an important retinal screening facilitator. Lessons learned from conducting behavioral research with this priority population include increasing engagement for a population traditionally considered 'hard-to-reach' and non-conventional clinical trial design alternatives.

CORRESPONDING AUTHOR: Amelia Lake, The Australian Centre for Behavioural Research in Diabetes, alake@ deakin.edu.au

\section{O11.4}

DEVELOPMENT OF A COMPLEX INTERVENTION TARGETING WOMEN WITH PRIOR GESTATIONAL DIABETES MELLITUS AND THEIR FAMILIES THROUGH CO-CREATION

${ }^{1}$ Karoline Kragelund Nielsen, Anne Timm ${ }^{1}$, Line Hillersdal ${ }^{1}$, Helle Terkildsen Maindal $^{1}$

${ }^{1}$ Steno Diabetes Center Copenhagen- Denmark

Introduction \& Purpose: Gestational diabetes mellitus (GDM) is a transient condition affecting pregnant women and associated with long-term adverse health outcomes in the woman and her offspring. This association is modified and influenced by factors operating at the social, familial, individual and health system levels. We therefore sought to develop a complex health promotion intervention targeting women with prior GDM and their families following the UK Medical Research Council's Framework. The aim of this presentation is to demonstrate the process of developing a complex intervention in practice and address the knowledge gap of evidence based program development. Methods: Co-creation was a key feature of the intervention development, which consisted of three stages: 1) evidence review and stakeholder consultations, 2) co-creation of intervention materials and resources, and 3) prototyping. Results: At stage 1, we carried out systematic literature reviews of behavioral interventions seeking to prevent diabetes in women with prior GDM and of the barriers and determinants of GDM related services. We also conducted qualitative research exploring the experiences of women with prior GDM and their partners as well as the views of healthcare providers in Denmark to inform our intervention with context specific evidence. Stakeholder consultations consisted of two review meetings with national and international researchers in the area, an international symposium and focus group discussions with health visitors. Stage 2 consisted of cocreation workshops with healthcare providers as deliverers of the intervention and women with prior GDM and their families as the target group where intervention ideas and materials were generated and tested. Finally, at stage 3 the draft intervention resources and guidelines were reviewed by a group consisting of experts in the field of GDM, health promotion, epidemiology and health technology as well as healthcare providers and women with prior GDM. Conclusions: The result of this process is a theory- and evidence-based complex intervention design, which will be presented at the conference. Further, the outline of qualitative and quantitative evaluations (effectiveness) will be given to inspire and support future program developers.

CORRESPONDING AUTHOR: Karoline Kragelund Nielsen, Steno Diabetes Center Copenhagen, karoline.kragelund.nielsen@ regionh.dk 
O11.5

EXPLORING REAL-TIME CONTINUOUS GLUCOSE MONITORING SYSTEMS (RT-CGMS) AS A BEHAVIOR CHANGE TECHNOLOGY IN TYPE 2 DIABETES

Ian Zajac ${ }^{1}$, Pennie Taylor ${ }^{1}$

${ }^{1}$ Commonwealth Scientific and Industrial Research OrganisationDenmark

OBJECTIVE: Wearable health technologies might be useful for facilitating long-term behavioural changes resulting in improved health outcomes. Real-time continuous glucose monitoring systems (RT-CGMS) provide frequent, accurate monitoring of blood glucose over extended periods (i.e. weeks/months), enabling users to receive immediate biological feedback in response to diet, physical exercise and medication management. However, to date, no published studies have assessed the extent to which the availability of real time glucose feedback influences behaviour change in the context of self-management of type 2 diabetes (T2DM) and thus, whether this technology is effective for supporting long-term behavioural improvements. METHODS: This feasibility study explored the efficacy of RT-CGMS for motivating behavioural change to facilitate improved management of T2DM. Participants $(\mathrm{N}=20)$ were assigned to either a Masked condition (RT-CGMS wear without visual glucose feedback) or an Unmasked condition (glucose feedback visible). Participants wore the device for 12-weeks and behavioural measures relevant to T2DM self-management were taken at week-6 and week-12. RESULTS: After 6-weeks, participants in the unmasked condition reported significantly greater impact of RT-CGMS on their knowledge of dietary factors (e.g., carbohydrates and sugars) impacting on their blood glucose levels $(\mathrm{d}=1.24, \mathrm{p}=.01)$, with the effect becoming more pronounced after 12-weeks of wear $(\mathrm{d}=2.06, \mathrm{p}<.001)$. Additionally, at 12weeks, there was a trend $(\mathrm{p}=.08)$ towards unmasked participants reporting greater dietary change $(\mathrm{d}=0.81)$ compared to masked participants. Analysis of change in global diabetes self-management behaviour scores from baseline to 6 weeks was significant for both groups $(p<.001)$, with a significant interaction indicating a divergence in degree of change between week- 6 and week-12 favouring the unmasked condition $(\mathrm{p}=.05)$. CONCLUSIONS: This study demonstrates the potential efficacy of wearable health technology in the form of real-time continuous glucose monitoring systems resulting in significant improvements in diabetes selfmanagement behaviours, and that this is linked directly to the provision of real-time glucose feedback.

CORRESPONDING AUTHOR: Ian Zajac, Commonwealth Scientific and Industrial Research Organisation, ian.zajac@csiro.au

\section{O11.6}

"ONCE MY DOCTOR SAID IT, IT WAS GOOD ENOUGH FOR ME": A QUALITATIVE STUDY EXPLORING ATTENDANCE AT DIABETES STRUCTURED EDUCATION PROGRAMMES IN IRELAND

Molly Byrne ${ }^{1}$, Jenny McSharry ${ }^{1}$, Margaret Humphries ${ }^{2}$, Sean Dinneen ${ }^{1}$

${ }^{1}$ National University of Ireland- Ireland

${ }^{2}$ Cork University Hospital- Ireland

Purpose: Diabetes self-management education (DSME) programmes aim to facilitate people to self-manage diabetes. Attendance at DSME has been recommended internationally for people with Type 2 diabetes (T2DM), though attendance rates are consistently low. The purpose of this study was to explore barriers and facilitators to attendance at T2DM DSME programmes in Ireland. Methods: People with T2DM who had been invited to attend structured education programmes offered in Ireland and educators from these programmes took part in semi-structured telephone interviews, which were audio-taped, transcribed and analysed using inductive thematic analysis. Results: 15 people with T2DM and 14 educators were interviewed. People with T2DM described a lack of information at initial diagnosis but were overwhelmingly positive about their experience of DSME. Reasons for non-attendance included not knowing programmes were available, not appreciating the potential benefits, and not wanting to admit to the reality of diabetes. Analysis also identified the key role of healthcare professionals in promoting structured education. Conclusions: Interventions should focus on the process of referral onto programmes within routine care. We need to "sell" DSME to healthcare professionals so they can encourage attendance.

CORRESPONDING AUTHOR: Molly Byrne, National University of Ireland, molly.byrne@nuigalway.ie

\section{O12.1}

DEPRESSION, ANXIETY AND PERCEIVED STRESS SCORES COMPARISON BETWEEN PATIENTS WITH CARDIO-RESPIRATORY FAILURE WITH AND WITHOUT COGNITIVE IMPAIRMENT

Monica Rivera ${ }^{1}$, Viridiana Peláez Hernández ${ }^{1}$, Arturo Orea Tejeda ${ }^{1}$, Alejandro Figueroa Herrera ${ }^{1}$, Dulce Gonzalez Islas ${ }^{1}$, Clyo Chávez Méndez $^{1}$, Carmen Olivo Villalobos ${ }^{1}$, Giselle Dávila Said ${ }^{1}$, Luis Felipe Castillo Aguilar ${ }^{1}$

${ }^{1}$ Instituto Nacional de Enfermedades Respiratorias- Mexico

Introduction and Purpose: Heart failure (HF) patients have high levels of anxiety, depression and perceived stress, which may be linked with cognitive impairment in many cases. However, there is no information about patients with cardio-respiratory failure, who have major oxygenation tissue problems, which affects the brain and its functions, including those cognitive and emotional. The purpose of this study was compare anxiety, depression and perceived stress scores between patients with cardio-respiratory failure with and without cognitive impairment. Material and Methods: A cross-sectional study was realized. Sixty-six patients with of cardiorespiratory failure were included heart failure NYHA I-III and chronic obstructive pulmonary disease (COPD), GOLD 1-3. All participants were evaluated with the following tests: a) Hospital Anxiety and Depression Scale (HADS), with 14 items to evaluate anxious and depressive symptomatology; b) Perceived stress scale in the 10 items version, and c) Montreal Cognitive Assessment (MOCA) alternate mexican version 7.2 to determine the presence of cognitive impairment if the score is less or equal to 26 points. According to the MOCA, the participants were divided into two groups: Group $1(\mathrm{G} 1, \mathrm{n}=8)$ : without cognitive impairment, and Group 2 (G2, n=58), with cognitive impairment. The data was analyzed with SPSS v. 21 for Windows, data didn't present a normal distribution, so the U Mann Whitney test was used. Results: Cognitive impairment was found in the $87.87 \%$. There were no differences in the socio-demographic variables between both groups. Patients with cognitive impairment have major scores of anxiety (G1: $3.88 \pm 1.959, \mathrm{G} 2: 5.47 \pm 4.168, \mathrm{p}<0.05)$ and depression (G1: $4 \pm 2.777, \mathrm{G} 2: 5.97 \pm 4.176, \mathrm{p}<0.05$ ) according to the HADS; besides of a major perceived stress (G1: 18.50 $\pm 5.155, \mathrm{G} 2: 23.36 \pm 6.282$, $\mathrm{p}<0.05)$. Conclusions: Patients with cardio-respiratory distress has a high prevalence of cognitive impairment and poor quality of life (more anxiety and depression). The interdisciplinary evaluation and intervention as part of non pharmacological treatment is necessary.

CORRESPONDING AUTHOR: Monica Rivera, Instituto Nacional de Enfermedades Respiratorias, monica.rivera8@hotmail.com

\section{O12.2}

PERCEIVED NEIGHBORHOOD SOCIAL TRUST AND HYPERTENSION CONTROL IN CHILE: RESULTS FROM A NATIONAL HEALTH SURVEY, ENS 2009-2010

Alvaro Passi Solar ${ }^{1}$, Paula Margozzini ${ }^{1}$

${ }^{1}$ Pontificia Universidad Católica de Chile- Chile

Introduction: Hypertension treatment coverage in Chile is the highest in Latinamerica but control (HC) rates are still lower than expected. The aim 
of this study was to explore the association between perceived neighborhood trust (PNT) and HC in treated hypertensives. Methods: ENS2009-2010 is a national random household study including 5412 adults aged 15 and over, 1.124 treated hypertensive adults were analysed. Backward Logistic regression for HC and PNT(defined with the "lost wallet" question method) was used. Adjustment variables included gender, age, number and type of hypertensive drugs used, education, rurality, body mass index, salt intake, alcohol use and time since last blood pressure measurement. Results: $\mathrm{HC}$ crude rates were $44 \%, 50 \%$ and $33 \%$ for the total, female and male population. HC in hypertensives with high vs low PNT was $56 \%$ vs $39 \%$. The highest adjusted odds $(95 \% \mathrm{CI})$ in the model was 2.9 (1.2-6.9) for NT. Other significant variables were : gender, age, thiazide diuretic use and number of hypertensive drugs used. Conclusions: this national survey suggests a higher level of PNT is independently asociated with a better $\mathrm{HC}$ in treated hypertensives. Chronic disease care programmes and national health promotion policy should consider this important health determinant

CORRESPONDING AUTHOR: Alvaro Passi Solar, Pontificia Universidad Católica de Chile, passi.solar@gmail.com

\section{O12.3}

PREVALENCE OF LIFESTYLE AND BEHAVIORAL FACTORS AMONG MIDDLE-AGED AND ELDERLY PATIENTS WITH ISCHEMIC STROKE IN SHANDONG, CHINA

Chengxuan $\mathrm{Qiu}^{1}$, Bo Bai ${ }^{2}$

${ }^{1}$ Karolinska Institutet- Sweden

${ }^{2}$ Jining Medical University- China

Background \& Purpose: The link of stroke to lifestyle or behavioral factors has been well-established. We sought to investigate the prevalence of major lifestyle and health behavioral factors in middle-aged and elderly patients with ischemic stroke in China, while taking into account gender differences. Methods: This was a baseline cross-sectional study of stroke patients who participated in an ongoing behavioral intervention study and were hospitalized into two tertiary hospitals in Jining, Shandong. The inclusion criteria are: first-ever ischemic stroke or transient ischemic attacks; age $\geq 40$ years; and no loss of consciousness. Data were collected through interviews, clinical examinations, and laboratory tests. We followed international criteria to define lifestyle and behavior-related factors, such as smoking, alcohol use, physical inactivity, obesity (body mass index $\geq 30 \mathrm{~kg} / \mathrm{m} 2$ ), hypertension, high cholesterol, and diabetes. Data were analyzed using descriptive statistics. Results: In October 2015January 2017, a total of 2082 patients with ischemic stroke (mean age 61.6 years; $36 \%$ women; $94.4 \%$ cerebral infarcts) were recruited. The prevalence of lifestyle or behavioral factors was $41.1 \%$ (men vs. women: $61.7 \%$ vs. $6.0 \%, \mathrm{p}<0.001$ ) for current smoking, $33.9 \%$ (54.4\% vs. $2.4 \%$, $\mathrm{p}<0.001)$ for alcohol consumption, $71.5 \%$ (71.9\% vs. $70.8 \%$ ) for physical inactivity, $73.8 \%(70.5 \%$ vs. $79.6 \%, \mathrm{p}<0.05)$ for hypertension, $27.8 \%$ $(24.0 \%$ vs. $34.5 \%, \mathrm{p}<0.001)$ for diabetes, $7.9 \%$ (6.3\% vs. $10.7 \%$, $\mathrm{p}<0.01)$ for obesity, and $7.3 \%$ (5.3\% vs. $10.9 \%$, p <0.01) for high total cholesterol. Conclusions: Lifestyle and behavioral risk factors are highly prevalent among middle-aged and elderly patients with ischemic stroke in China, with a substantial gender difference. These results call for genderspecific interventions to modify unhealthy lifestyle and behavior.

CORRESPONDING AUTHOR: Chengxuan Qiu, Karolinska Institutet, chengxuan.qiu@ki.se

O12.4

CARDIAC MISCONCEPTIONS, RISK PERCEPTIONS AND SELFEFFICACY IN ADOLESCENTS : IMPLICATIONS FOR PREVENTIVE BEHAVIOUR

Maria Joao Figueiras ${ }^{1}$, David Dias Neto ${ }^{1}$

${ }^{1}$ Instituto Piaget/RECI- Portugal
Introduction: Health beliefs have been associated with the adoption of preventive behaviours. The aims of this study were to investigate the type of beliefs related to cardiovascular disease, including cardiac misconceptions, causal attributions, individual and compared risk perception, perceived severity and self-efficacy, in a sample of adolescents. Methods: This was a cross-sectional design in which 455 healthy students (both sexes) from two public secondary schools in a metropolitan area of Lisbon completed a questionnaire which included socio-demographic information, self-rated health, illness perceptions and causal attributions, cardiac misconceptions, individual and compared risk perception and self-efficacy. These variables were analyzed by gender, age group, study area, smoking habits and family history. Results: The strongest cardiac misconception is related to the need to avoid stress and excitement. Known risk factors showed higher scores for the causal attributions. It has been found that the perceived seriousness for cardiovascular disease is very high. However, the perception of individual and compared risk perception are low. There were significant interaction effects of sociodemographic variables, smoking habits and family history on cardiac misconceptions, self-efficacy and compared risk. Conclusions: This exploratory approach enables the identification of factors which are relevant for assessing risk for cardiovascular disease as well as the type and strength of cardiac misconceptions. These results can contribute in a meaningful way for the development of cardiovascular disease prevention strategies in adolescence.

CORRESPONDING AUTHOR: Maria Joao Figueiras, Instituto Piaget / RECI, figueirasmar@gmail.com

\section{O12.5}

PSYCHOLOGICAL CARE FROM THE INTEGRATIONIST MODEL WITH ACUTE MYOCARDIAL INFARCTION IN PHASE I OF CR: SEEKING QUALITY OF CARE AND SECONDARY PREVENTION Teresa Rodríguez Rodríguez ${ }^{1}$

${ }^{1}$ Sociedad de psicología de la Salud de Cuba- Cuba

Introduction: Concept of Cardiac Rehabilitation (CR) was instituted by the WHO. It consists of three phases: I or Hospitable, II or Convalescent, III or Maintenance. Psychological care is essential in this process. To evaluate the effectiveness of a psychological intervention to get the control of emotions and to begin secondary prevention in patients with Acute Myocardial Infarction in acute phase has been the purpose of the study. Scientific question: What impact will have psychological intervention developed during phase I or hospitable on the clinical stability and secondary prevention in patients who has suffered from AMI? Method: Quasi-experimental design was performed with patients with AMI in the acute phase. As an independent variable was taken intensive psychological treatment in special situations (integrationist model), psychological and physiological variables were dependent variables. Investigation was done in the Cardiology Room of Dr. Gustavo Aldereguía Lima University Hospital, Cienfuegos. Cuba. Fundamental results: Diagnostic phase: High situational anxiety $(100 \%)$, depression $(50.5 \%)$, extreme vulnerability to stress $(54.6 \%)$ and family dysfunctionality $(71.0 \%)$ were predominant. Knowledges about heart-healthy lifestyles were insufficient in all subjects. Post intervention: $95.9 \%$ of patients were aware about the need to change, $34.6 \%$ had situational anxiety (high or medium) and depression $15.5 \%$. Relaxation Techniques applied led to favorable changes in psychological and physiological parameters. The acquisition of knowledges were increased significantly and important attitudinal modifications were done. Two months after discharge, the effectiveness of the psychological treatment was confirmed by the favorable and significant changes in lifestyle of the most patients. Functional families had more participation in the compliance of rehabilitation treatment than dysfunctional ones. Conclusions: Most of the patients reveled favorable behavioral changes in the modification of toxic and nutritional habits and incorporation of emotional regulation mechanisms. These indicators 
support the durability of the actions carried out. Repercussion in physiological parameters favored the clinical stability of the patient. Participation of family in rehabilitation process was successful.

CORRESPONDING AUTHOR: Teresa Rodríguez Rodríguez, Sociedad de psicología de la Salud de Cuba, teresa.rodriguez@gal.sld.cu

\section{O12.6}

REVERSION FROM PREDIABETES TO NORMOGLYCAEMIA REDUCES THE RISK OF A CARDIOVASCULAR DISEASE EVENT Dorte Vistisen $^{1}$, Daniel R Witte ${ }^{2}$, Eric J Brunner ${ }^{3}$, Mika Kivimäki ${ }^{3}$, Leigh Perreault $^{4}$, Adam Tabák ${ }^{3}$, Marit E Jørgensen ${ }^{1}$, Kristine Færch ${ }^{1}$

${ }^{1}$ Steno Diabetes Center Copenhagen- Denmark

${ }^{2}$ Aarhus University- Denmark

${ }^{3}$ University College London- United Kingdom

${ }^{4}$ University of Colorado Anschutz Medical Campus- USA

INTRODUCTION \& PURPOSE: Reversion from prediabetes to normoglycaemia has been shown to improve a range of cardiovascular risk factors. However, whether this translates into a reduction in risk of a cardiovascular disease (CVD) event has yet to be proven. Our aim was to study the probability of reversion from prediabetes to normoglycaemia by subtypes of prediabetes and the impact of reversion on risk of future CVD. METHODS: From the prospective Whitehall II study, we studied 881 participants with prediabetes at baseline by fasting plasma glucose (FPG) only (PreDMFPG, $n=609$ ) or by HbA1c (PreDMHbA1c, $n=272$ ), using the American Diabetes Association criteria. Reversion probabilities to normoglycaemia at 5-year follow-up as well as rates for CVD (fatal- or non-fatal CHD or stroke) thereafter were calculated and compared between subgroups, using Poisson regression analysis with risk time as offset. RESULTS: Of the 881 with prediabetes, 190 (22\%) had reverted to normoglycaemia at 5 years. Only 7 (4\%) of the 190 participant reverting to normoglycaemia had an $\mathrm{HbAlc}$ in the prediabetic range (PFPG and PreDMHbA1c). Overall, reverting to normoglycemia was associated with a $46 \%$ reduction in the rate of a future CVD event $(2.1$ vs 3.9 per 1000 person-years, $\mathrm{P}=0.056$ ). We found no difference in CVD risk between participants who reverted from PreDMFPG vs PreDMHbA1c $(P=0.162)$. CONCLUSIONS: Reversion to normoglycemia is less likely from an $\mathrm{HbAlc}$ than from a FPG in the prediabetic glucose range. Nevertheless, reversion to normoglycemia is associated with a strong trend toward lower rates of CVD.

CORRESPONDING AUTHOR: Dorte Vistisen, Steno Diabetes Center Copenhagen, dorte.vistisen@ regionh.dk

\section{O13.1}

IMPLICIT NOCEBO CONDITIONING INCREASES PAIN AFFECT Anne-Kathrin Bräscher ${ }^{1}$, Michael Witthöft ${ }^{1}$

${ }^{1}$ University of Mainz- Germany

Introduction \& Purpose: It is well known that expectation and classical conditioning can both induce nocebo effects. According to current models, conscious expectation necessarily mediates conditioned nocebo effects when affecting consciously accessible processes as e.g. in pain perception. Limited evidence, however, has shown that conditioning with subliminally presented cues can lead to nocebo hyperalgesia, too. In this study, we tested whether nocebo hyperalgesia can develop after implicit conditioning using supraliminally presented cues.

Methods: In a differential conditioning design $(\mathrm{N}=48$ healthy participants), moderately and weakly painful electrical stimuli were coupled with various visual stimuli that consistently only differed in symmetry/ asymmetry of one component (CS+/CS-) and contained further distractor components. In the test phase, only the weakly painful electrical stimulus followed both CS and the conditioned effect with regards to subjective intensity and aversiveness was tested. A contingency test and a postexperimental questionnaire assessed contingency awareness. Additionally, electrodermal activity was continuously measured.

Results: Increased subjective aversiveness after CS+ but not after CS- in the test phase indicated successful conditioning $(p=.037, d=0.31)$. In the subjective intensity ratings, no significant difference between $\mathrm{CS}+$ and CS- emerged $(p=.0190 ; d=0.19)$. Further, increased skin conductance level confirmed the successful learning $(p=.009, d=0.39)$. These effects appeared independently of contingency awareness.

Conclusions: The results show that implicit conditioning of nocebo hyperalgesia is feasible. A learning effect emerged on the subjective as well as on the psychophysiological level. Conditioning with supraliminally presented cues avoids many pitfalls associated with subliminal stimulus presentation. Implicitly conditioned nocebo effects might have been underestimated so far, despite vast clinical implications, e.g. for the treatment of chronic pain and other systems affected by nocebo effects.

CORRESPONDING AUTHOR: Anne-Kathrin Bräscher, University of Mainz, abraesch@uni-mainz.de

\section{O13.2}

TESTING THE ABILITY OF TWO DIFFERENT TYPES OF MESSAGE INSTRUCTIONS TO REDUCE NOCEBO HEADACHES IN AN EXPERIMENTAL PARADIGM

Suzanne Helfer ${ }^{1}$, Fawn Caplandies ${ }^{2}$, Ben Colagiuri ${ }^{3}$, Kate Faasse ${ }^{4}$, Andrew Geers ${ }^{2}$

${ }^{1}$ Adrian College- USA

${ }^{2}$ University of Toledo- USA

${ }^{3}$ Sydney University- Australia

${ }^{4}$ University of New South Wale- Australia

Introduction and Purpose: Practitioners and clinical researchers are ethically bound to inform individuals about possible undesired treatment effects, however, the provision of this information risks elevating the likelihood of those adverse effects, a phenomenon called the nocebo effect. Developing experimental models of this phenomenon and identifying communication strategies that minimize and perhaps even prevent nocebo effects would provide opportunities to avoid this risk. Here we present the results of two laboratory experiments (Study $1 \mathrm{~N}=126$; Study $2 \mathrm{~N}=174$ ) that tested the ability of two different types of message instructions, effect type and attribute framing, to reduce nocebo headaches. Methods: Both experiments consisted of a between-subjects design in which volunteer participants were given instructions suggesting that a lab task would induce headaches, or they were not given this headache expectation information. For the participants given the headache expectation, we also manipulated effect type and attribute framing instructions. The effect type instructions compared the magnitude of nocebo effects when headaches were described as the primary effect or the side effect of a treatment. The attribute frame instructions compared the magnitude of nocebo effects when headaches were described as either $70 \%$ likely to occur versus $30 \%$ unlikely to occur. Results: Both experiments found evidence of nocebo effects, with headache instructions significantly increasing headache reports compared with a no-headache forewarning control condition. Further, primary-effect participants reported significantly more headaches than side-effect participants. There were no effects of the attribute framing manipulation in either study. The findings were consistent across measures of headache occurrence, frequency, and maximum level. Conclusions: The present experiments demonstrate that nocebo effects can be moderated by effect-type framing instructions. Importantly, these result can inform attempts at reducing nocebo effects in clinical practice.

CORRESPONDING AUTHOR: Suzanne Helfer, Adrian College, shelfer@adrian.edu 
O13.3

SIDE EFFECT INFORMATION FRAMING AND THE NOCEBO EFFECT

Kate Faasse ${ }^{1}$, Anna Huynh ${ }^{1}$, Sarah Pearson ${ }^{1}$, Ben Colagiuri ${ }^{2}$, Andrew Geers $^{3}$

${ }^{1}$ University of New South Wale- Australia

${ }^{2}$ University of Sydney- Australia

${ }^{3}$ University of Toledo- USA

Introduction and purpose: The nocebo effect is a powerful psychological phenomenon in which an inert treatment, like a sugar pill, can produce harmful unpleasant side effects. One potential strategy for reducing nocebo effects while still providing accurate treatment information is to change the way this information is framed. Methods: Participants $(\mathrm{N}=$ 112) were randomly assigned to ingest a treatment described as a benzodiazepine (actually placebo) or to a no treatment control group. Participants received side effect information that was either negatively framed (e.g. headache is a possible side effect, and 20 out of 100 people will experience headache after taking this medication) or positively framed (e.g. headache is a possible side effect, but 80 out of 100 people will remain headache-free after taking this medication). After information provision, participants' side effect expectations were assessed. Nocebo side effects were assessed 20 minutes after placebo ingestion, and again at 24-hour follow-up. Results: At 20 minutes post-ingestion, participants in the positive framing condition had significantly lower nocebo side effect scores compared to those in the negative framing condition. While positive framing significantly reduced the formation of nocebo side effects, participants' side effect expectations did not differ significantly, suggesting that differences in negative expectations cannot fully explain these results. At 24-hour follow-up, nocebo side effects in the two framing conditions did not differ. Conclusions: The current results indicate that while positive information framing can buffer the formation of nocebo side effects, this framing effect may only be temporary. Further research is needed to assess other possible mechanisms underlying this effect, and to investigate whether framing effects can be enhanced to be more durable.

CORRESPONDING AUTHOR: Kate Faasse, University of New South Wale,k.faasse@unsw.edu.au

\section{O13.4}

NOCEBO EFFECTS: THE ROLE OF LEARNING AND NEGATIVE EMOTIONAL STATES

Ben Colagiuri, Veronica Quinn ${ }^{1}$,Victoria Lim-Howe ${ }^{1}$

${ }^{1}$ University of Sydney- Australia

Introduction \& Purpose: The nocebo effect is a pervasive phenomenon that causes patients substantial harm. It is clear that expectancies induced by verbal instruction and classical conditioning are responsible for triggering the nocebo effect. However, much remains known about the psychological processes that mediate expectancies' effects on negative health outcomes. The current studies tested the extent to which negative emotional states, namely, fear and anxiety, contribute to the nocebo effect. Methods: Across two studies, healthy volunteers underwent a nocebo conditioning procedure using experimentally-induced pain and then were tested over multiple pain trials. The first experiment tested the extent to which fear contributed to nocebo hyperalgesia via both self-report and autonomic (skin conductance) measures. The second experiment extended this by implementing an anxiety manipulation whereby one group of participants was randomised to receive nocebo conditioning with unpredictable pain intensities relative to standard nocebo conditioning with predictable pain intensities. Results: In both experiments, conditioning led to a reliable nocebo effect relative to controls and this nocebo effect did not diminish over the course of the test phase. In terms of self-reported fear and anxiety, fear was significantly related to the magnitude of the nocebo effect across individuals, but anxiety was not. In terms of the autonomic measure, higher skin conductance was not associated with the magnitude of the nocebo effect, but did predict its persistence over the test phase. Conclusions: The current findings confirm that the nocebo effect is readily established following conditioning and, concerningly, that it persists over time. The results also demonstrate that while fear and autonomic arousal contribute to the nocebo effect's magnitude and persistence, respectively, that anxiety does not appear to play a role. These findings suggest that interventions that target fear could be effective for reducing the burden of nocebo effects.

CORRESPONDING AUTHOR: Ben Colagiuri, University of Sydney, ben.colagiuri@sydney.edu.au

\section{O13.5}

CAN AN EXPLANATION OF THE NOCEBO EFFECT REDUCE SYMPTOM BURDEN?

Keith Petrie $^{1}$, Dominic Burton ${ }^{1}$, Mark Lane ${ }^{2}$, Maggie Ow ${ }^{1}$, Kate MacKrill $^{1}$, Maria Kleinstäuber

${ }^{1}$ University of Auckland- New Zeland

${ }^{2}$ Auckland Hospital- New Zeland

This study investigated whether explaining the nocebo effect reduced patient symptom reporting and side effects following colonoscopy investigation. Eighty patients scheduled for colonoscopy were randomised to receive a nocebo explanation or standard preparation prior to the colonoscopy investigation. The intervention or standard preparation was administered by a specialist gastroenterology nurse prior to the colonoscopy following scripts developed for the study. Patients were assessed immediatly following the procedure at three days later by a research assistant blind to group assignment. The nocebo explanation was effective in reducing side effects at the three day follow-up in the intervention group compared to control $(\mathrm{p}<.05)$ and seemed to have particular benefit for patients with high levels of anxiety prior to the procedure in terms of reducing symptom reporting $(\mathrm{p}<.05)$ and the attribution of symptoms as side effects $(\mathrm{p}<.05)$. The study suggests a simple nocebo explanation may be a useful intervention for reducing symptom burden for patients undergoing medical investigations.

CORRESPONDING AUTHOR: Keith Petrie, University of Auckland, kj.petrie@auckland.ac.nz

\section{O13.6}

THE EFFECT OF OPERANT PAIN-RELATED AVOIDANCE LEARNING ON PLACEBO AND NOCEBO OUTCOMES Ann Meulders ${ }^{1}$

${ }^{1}$ KU Leuven- Belgium

Introduction \& Purpose: Placebo and nocebo research has mostly relied on Pavlovian conditioning procedures to induce expectations. However, operant learning procedures may more closely mimic real-life situations in which placebo/nocebo effects occur. In this study, we introduce a novel operant learning task investigating placebo/nocebo responses following operant learning. Methods: Healthy participants $(n=58)$ performed a robotic arm reaching task in two contexts; three trajectories were possible to reach the target (T1-3). During acquisition, in the experimental context, pain intensity decreased by performing the more effortful (in terms of distance and exerted force) movement trajectories ( $\mathrm{T} 1=$ high intensity pain, $\mathrm{T} 2=$ medium intensity pain; $\mathrm{T} 3=$ low intensity pain). In the control context, the opposite contingencies applied, i.e. pain intensity increased with effort. During both test phases, all movement trajectories were followed by the same medium intensity pain stimulus. In a voluntary movement test, participants were still free to choose which movements to perform. During a subsequent instructed movement test, participants 
were probed to perform specific movements. Results: Participants acquired differential movement preferences (Context x Trajectory, $\mathrm{F}(2,1368)=164.31, \mathrm{p}<.001)$, as well as differential pain-movement expectations (Context $\mathrm{x}$ Trajectory $\mathrm{F}(2,1311)=6.53, \mathrm{p}=.002)$. Changing the reinforcement scheme during voluntary movement test quickly extinguished established movement preferences across contexts, $\mathrm{F}(2,1368)=0.74, \mathrm{p}=.48$. However, differential pain expectations (Context $\mathrm{x}$ Trajectory $\mathrm{F}(2,1311)=18.30, \mathrm{p}<.001)$ and reported pain intensity (Context $x$ Trajectory, $F(2,1002)=4.40, p=.013$ ) remained significant, although within-context comparisons showed that these effects were more pronounced in the control context than in the experimental context. Differences between contexts disappeared during the instructed movement test. Conclusion: Changing the reinforcement schedule swiftly altered movement preferences. However, pain expectations and pain intensity ratings persist to some extent after disconfirmation. These results may explain difficulties in updating pain-related expectations and their lasting impact on pain.

CORRESPONDING AUTHOR: Ann Meulders, KU Leuven, ann.meulders@kuleuven.be

O14.1

ASSOCIATIONS BETWEEN ADOLESCENT TOBACCO AND ALCOHOL USE, AND TOBACCO AND ALCOHOL USE RISK AND PROTECTIVE FACTORS

Rebecca Hodder ${ }^{1-2}$, Elizabeth Campbell ${ }^{2}$, Conor Gilligan ${ }^{2}$, Hopin Lee ${ }^{2}$, Christophe Lecathelinais ${ }^{1}$, Sue Green ${ }^{1}$, Meghan MacDonald ${ }^{1}$, John Wiggers

${ }^{1}$ Hunter New England Population Health- Australia

${ }^{2}$ The University of Newcastle- Australia

Introduction and purpose: Tobacco and alcohol use contributes significantly to global rates of morbidity and mortality with initiation to such use typically occurring during adolescence. A large number of studies have sought to identify the factors that either increase (risk factors) or decrease (protective factors) the likelihood of such use. However relatively fewer studies have attempted to determine the relative association between such factors and tobacco and alcohol use, nor the consistency of associations between such factors and both tobacco and alcohol use. Such investigation may identify common risk and protective factors that can be prioritised as intervention targets for reducing both tobacco and alcohol use within a single prevention intervention. The study aimed to determine the strength and consistent direction of associations between use of tobacco and alcohol, and tobacco and alcohol risk and protective factors. Methods: A cross sectional survey of Grade 9-10 (aged 15-17 years) students from 32 Australian secondary schools was conducted (2014). A self-report survey collected data regarding tobacco use (ever/recent), alcohol use (ever/recent/“binge drinking'), risk factors (e.g. tobacco and alcohol use/permissive attitude to tobacco and alcohol by friends/sibling/ parents), and protective factors (e.g. self-efficacy; school/home/community support; peer caring relationships). Results: Risk factors were the variables with the strongest association for each tobacco and alcohol use outcome. Friends use of tobacco (OR range:3.79-4.35) and alcohol (OR range:4.27-5.32), and permissive attitude to tobacco (OR range:3.41-4.78) and alcohol (OR range:2.11-2.82) consistently had the strongest associations with each of the five measures of tobacco and alcohol use. Sibling use of tobacco and alcohol, belief health not damaged from smoking, personal income and pro-social were also important factors for some outcomes (OR range:1.64-2.47). Conclusions: The strength and consistent direction of associations of certain risk factors suggest such factors should be prioritised for interventions focused on reduction of both tobacco and alcohol use.

CORRESPONDING AUTHOR: Rebecca Hodder, Hunter New England Population Health/The University of Newcastle
O14.2

A SYSTEMATIC REVIEW OF SMOKING PREVENTION AND CESSATION IN VOCATIONAL SCHOOLS

Charlotte Demant Klinker ${ }^{1}$, Britt Svane ${ }^{2}$, Anneke Vang Hansen ${ }^{1}$, Helle Terkildsen Maindal ${ }^{3}$

${ }^{1}$ Steno Diabetec Center Copenhagen, Diabetes Prevention ResearchDenmark

${ }^{2}$ Independent

${ }^{3}$ Department of Public Health, University of Aarhus- Denmark

Introduction and purpose: The prevalence of smoking is high in youth who attend vocational schools - e.g. $37 \%$ compared to $12 \%$ among Danish high school students. Vocational schools are therefore an important setting for health promotion, and effective interventions are urgently needed to prevent the uptake of smoking and increase smoking cessation. A systematic review was conducted to investigate the effectiveness of behavioral smoking prevention and smoking cessation interventions targeting students at vocational schools. Methods: Pubmed, ERIC, PsycInfo, SCOBUS and Web of Science were searched using MeSH terms and keywords for studies published in English prior to September 2017. All intervention studies reporting effects on vocational students on prevention of smoking, reduction in daily smoking or smoking cessation were included. Results: Of 1,251 articles identified, five studies met the inclusion criteria. All studies were based on controlled designs and two were cluster randomized. Two studies targeted smoking cessation (SC), two smoking prevention (SP) and one targeted both (SCP). Two studies (SC1+SP1) demonstrated significant positive effects on smoking at long term follow-up ( $>=12$ months), two studies (SC2+SP1) had no significant effects on primary outcomes but significantly reduced daily smoking at short-term follow-up ( $<6$ months). One study (SCP) had adverse negative effects with a significant lower risk perception in non-users at 6 months follow-up. Three studies included a curriculum component with the add-on of one or more other interventions components, e.g. peer-support or policy-development. One study was based on eHealth technologies and one was structural with an emphasis on improving social cohesion among students. Conclusions: This review provides some evidence of the effectiveness of interventions in vocational schools aiming to prevent or reduce smoking among students. However, current evidence for the effectiveness of smoking interventions in vocational schools is equivocal, largely due to lack of rigorous research, differences in study design and intervention content.

CORRESPONDING AUTHOR: Charlotte Demant Klinker, Steno Diabetec Center Copenhagen, Diabetes Prevention Research, charlotte.demant.klinker@ regionh.dk

\section{O14.3}

EFFECTIVENESS OF BEHAVIOURAL AND MOOD MANAGEMENT INTERVENTIONS FOR SMOKING CESSATION IN PEOPLE WITH DEPRESSION: IMPACT OF VARIATION IN FUNCTIONS AND DELIVERY

Gemma Taylor ${ }^{1}$, Kate Bartlet ${ }^{2}$, Paul Aveyard ${ }^{3}$, Regina Van der Meer ${ }^{4}$, Bobby Stuijfzand $^{1}$, David Kessler ${ }^{1}$, Marcus Munafö ${ }^{1}$

${ }^{1}$ University of Bristol- United Kingdom

${ }^{2}$ University of Newcastle- Australia

${ }^{3}$ University of Oxford- United Kingdom

${ }^{4}$ Public Health Service of Haaglanden- Netherlands

Introduction: People with depression are twice as likely to smoke and find it harder to quit than those without depression. A Cochrane review of RCTs of smoking cessation treatment for smokers with current/past depression found that adding mood management to usual care improved quit rates (van der Meer, 2013). With the aim of informing future interventions, we examined if variation in mood management delivery or behaviour change functions impacted treatment effectiveness, and if smoking cessation treatment alters depression symptoms. Methods: 
PROSPERO ID: CRD42017070741. Studies were identified by a previous Cochrane review by searching CENTRAL, MEDLINE, EMBASE, PsycINFO, other reviews, and expert input. We included RCTs in smokers, $\geq 18$ years with current depression. Outcomes were: treatment effect for smoking cessation (risk difference, RD) and change in depression. We used meta-regression to determine: 1) the association between variation in delivery of mood management (i.e. Template for Intervention Description and Replication checklist) and treatment effect, and 2) the association between behaviour change functions (i.e. Taxonomy of Behaviour Change techniques) and treatment effect, and 3) the standardised mean difference (SMD) in change in depression between treatment arms from baseline to follow-up. Results: We included 16 RCTs of behavioural and/or medicinal treatments. There were insufficient data to determine the impact of variation in mood management delivery. We found no evidence for an association between behaviour change functions and treatment $\operatorname{effect}(\mathrm{F}(6,5),=0.78, \mathrm{P}=0.62)$. There was some evidence that smoking cessation treatment improved depression vs control, SMD -0.09(95\%CI: -0.19 to $-0.00, \mathrm{P}=0.04$ ); subgroup analyses suggested that the effect was not associated with treatment type $\left(X^{2}=8.64\right.$, $\mathrm{P}=0.19)$, or presence of mood management $\left(\mathrm{X}^{2}=5.04, \mathrm{P}=0.08\right)$. Conclusion: Many studies did not provide sufficient details about treatment content or delivery, therefore limiting our analyses. Based on details that were available we found no evidence for an association between behaviour change function and treatment effect. In studies that provided enough data to calculate SMDs, we found that smoking cessation treatment led to a small improvement in depression symptoms.

CORRESPONDING AUTHOR: Gemma Taylor, University of Bristol, gemma.taylor@bristol.ac.uk

\section{O14.4}

SUSCEPTIBILITY TO SMOKING AND DETERMINANTS AMONG MEDICAL STUDENTS: A REPRESENTATIVE NATIONWIDE STUDY IN CHINA

Tingzhong Yang ${ }^{1}$, Xiaozhao Yang ${ }^{2}$

${ }^{1}$ Zhejiang University- China

${ }^{2}$ Murray State University- China

Background: Susceptibility to smoking among medical students may relate to smoking after they becoming physicians. But no literature explored this issue. The objective of this study was to examine susceptibility to smoking and determinants among medical students. Methods: Participants were 11,954 students, who were identified through a multistage survey sampling process that included 50 universities. Subsequent analysis focused on 8,916 non-smokers among medical students. Descriptive statistics were calculated to determine the prevalence of susceptibility to smoking. Both unadjusted and adjusted logistic methods were considered in the data analyses, and utilized to examine these associations. Results: The prevalence of susceptibility to smoking was $23.0 \%$. Multiple variables logistic regression found: Exposure to SHS, cigarette advertisement and promotional activities on campus were positively associated with susceptibility to smoking. Positive attitude toward health professions (HPs) tobacco control were negatively associated with susceptibility to smoking. Those who received information about the dangers of smoking and did not agree that light cigarettes are less harmful to health were less susceptible to smoking. Caring about exposure to second-hand smoke and advising family members to stop smoking were negatively associated with susceptibility to smoking. Conclusions: To our knowledge, this is the first study to examine susceptibility to smoking and determinants among medical students in China and elsewhere. These findings underscore the importance of tobacco control training and establishing smoke free campuses for reducing susceptibility to smoking among medical students.

CORRESPONDING AUTHOR: Tingzhong Yang, Zhejiang University, Tingzhongyang@zju.edu.cn
O14.5

PROCESS EVALUATION OF PATIENT NAVIGATION COUNSELING IN AN EFFICACIOUS SMOKING CESSATION INTERVENTION WITH LOW INCOME PATIENTS

Lisa Quintiliani $^{1}$, Ve Truong ${ }^{2}$, Karen Lasser ${ }^{1}$

${ }^{1}$ Boston University- USA

${ }^{2}$ Boston Medical Center- USA

Introduction \& Purpose: Racial/socio-economic disparities are present in smoking prevalence and effectiveness of interventions for cessation. In a randomized controlled intervention trial among adult daily smokers receiving primary care at a large safety-net hospital, we found higher rates of biochemically confirmed cessation among intervention participants (patient navigation delivered over six months + financial incentives) versus control group participants. Intervention participants were eligible to receive up to 4 hours of contact with a patient navigator who used motivational interviewing (MI) to conduct smoking cessation counseling. Subgroup analysis revealed the intervention was particularly efficacious for those who received the minimum intervention dose $(\geq 1$ session in which an entire MI guide was completed), among other groups. No white smokers quit. The objective of this process evaluation was to examine relationships between receipt of counseling through patient navigation and patient socio-demographic characteristics. Methods: We analyzed data from intervention participants $(n=177)$ and grouped participants into three categories: 1) no or minimal contact with the navigator; 2) some navigation; or 3) minimum dose or higher achieved. Results: Bivariable analyses showed that non-Hispanic white participants (12.9\%), Hispanic (12.9\%), and other race/ethnicity smokers $(10.7 \%)$ received the minimum intervention dose less frequently compared to non-Hispanic black $(63.4 \%)$ participants $(\mathrm{p}<0.05)$ and that participants who received the minimum intervention dose were older (51.9 years) than participants who received no or limited contact (48.1 years) or some navigation (47.0 years) $(\mathrm{p}<0.05)$. In a logistic regression controlling for education, age, gender, income, stress, hassles, life chaos, and smoking amount, nonHispanic whites had lower odds $(0.38 ; 95 \%$ CI $0.17-0.85)$ of receiving the minimum intervention dose or higher compared to all other race/ ethnicity categories. Conclusions: White smokers were less likely to receive sufficient patient navigation; future research should explore the underlying reasons including racial/ethnic concordance between navigator and participant, comorbid mental health or substance use, and hesitation to use navigator services.

CORRESPONDING AUTHOR: Lisa Quintiliani, Boston University, lisa.quintiliani@bmc.org

\section{O14.6}

UNDERSTANDING ATTITUDES ABOUT ELECTRONIC CIGARETTES AMONG NON-USERS

Matthew Olonoff ${ }^{1}$, Christina Ciecierski ${ }^{2}$, Hardik Marfatia ${ }^{2}$, Brian Hitsman $^{1}$

${ }^{1}$ Northwestern Feinberg School of Medicine- USA

${ }^{2}$ Northeastern Illinois University- USA

Introduction: As the number of alternative tobacco products increases, so does the number of users, especially young adults. Individuals who have been exposed both e-cigarettes may have stronger positive beliefs about e-cigarettes. This in turn, could lead to future initiation of these products. The purpose of this study was to identify relevant covariates of e-cigarette attitudes. Methods: An online survey was administered at a HispanicServing university in Chicago, IL, USA in December 2017. Questions included demographics, other tobacco use, lifetime and current e-cigarette, and social proximity to e-cigarette users. Attitudes questions covered beliefs about e-cigarettes, including health, susceptibility, and quit characteristics that were scored as sums of individual item scores. Higher scores indicated more favorable attitudes of e-cigarettes and a score of 0 
represents a neutral attitude (range $=[-20,20])$. Univariate correlations were examined on the overall sample. Multivariate regression analyses were performed testing demographic, other tobacco product, and social proximity variables on attitude scores among never users. Results: Of the total sample ( $\mathrm{N}=696), 82 \%$ were never users of e-cigarettes, $11 \%$ were noncurrent-ever users, and $7 \%$ were current e-cigarette users. E-cigarette users $(n=127)$ had more positive attitudes than never users, $72 \%$ vs $36 \%$. Never users $(\mathrm{n}=569)$ were predominantly female, $74 \%$, Hispanic, $30 \%$, and $46 \%$ white. This majority viewed e-cigarettes negatively, $58 \%$, and $5 \%$ were neutral. Regression analysis on attitude scores showed that demographic and proximity to other e-cigarette users were significant $(\mathrm{F}(6,490)=8.00, \mathrm{p}<.001)$. Living with a current e-cigarette user was positively associated with e-cig attitudes while non-white status was negatively correlated with scores. Social proximity was also significant for scores of susceptibility, health, and quit characteristics. Conclusions: In this sample of diverse U.S. college students, more positive attitudes about e-cigarette use were associated with previous tobacco use and proximity to other e-cigarette users. As e-cigarettes increase in worldwide availability, understanding what contributes to attitudes of e-cigarettes may be important to predict susceptibility to e-cigarette use.

CORRESPONDING AUTHOR: Matthew Olonoff, Northwestern University Feinberg School of Medicine, matthewolonoff2023@u.northwestern.edu

\section{O15.1}

DOES EXPECTATION MANAGEMENT DIMINISH CANCER TREATMENT SIDE EFFECTS? RESULTS OF THE MULTISITE RANDOMIZED CONTROLLED PSY-BREAST TRIAL

Pia von Blanckenburg ${ }^{1}$, Yiqi Pan $^{2,3}$, Sarah Heisig ${ }^{2}$, Meike SheddenMora $^{2}$, Ute-Susan Albert ${ }^{1}$, Peyman Hadji ${ }^{4}$, Isabell Witzel ${ }^{2}$, Arthur Barsky ${ }^{5}$, Winfried Rief ${ }^{1}$

${ }^{1}$ Philipps-University of Marburg- Germany

${ }^{2}$ University Medical Center Hamburg-Eppendorf- Germany

${ }^{3}$ Schön Klinik Hamburg-Eilbek- Germany

${ }^{4}$ Krankenhaus Nordwest- Germany

${ }^{5}$ Harvard Medical School- USA

Introduction. Although patients' treatment expectations have been shown to substantially modulate the efficacy and tolerability of medical treatments, expectation-based interventions focusing on the prevention of nocebo mechanisms are lacking. This clinical trial aims to evaluate the efficacy of an expectation optimization training to reduce side effects and improve health-related quality of life in patients undergoing endocrine treatment for breast cancer. Methods. Patients with hormone-receptorpositive breast cancer and indication for adjuvant endocrine therapy (AET) were assigned 1:1:1 to a three-session expectation management intervention (EXPECT), a manualized attention control group (SUPPORT), or treatment as usual (TAU). Our primary outcome was number of side effects at 3 and 6 months after AET start. Secondary outcomes included coping with side effects and health-related quality of life (EORTC-QLQC30 and BR23). Linear mixed models for repeated measures were conducted with adjustments for baseline values, medical, and psychological variables. Results. Among 197 randomized patients (mean age: 58.0 years, $70.6 \%$ postmenopausal), $158(80.2 \%)$ and 160 $(81.2 \%)$ completed the follow-ups at 3 and 6 months. In Intention-totreat analyses, we have found that primary and secondary outcomes did not differ by group. Moderation analyses at $6 \mathrm{M}$ showed that among patients who expected moderate or severe side effects, EXPECT reported fewer side effects than SUPPORT $(-4.00,95 \%$ CI $[-7.25 ;-0.75], \mathrm{ES}=0.9)$ and TAU $(-3.80,95 \%$ CI $[-7.51 ;-0.08], \mathrm{ES}=0.8)$. The same pattern was found for unspecific side effects (SUPPORT:-2.69, 95\% CI [-4.79;$0.60], \mathrm{ES}=0.9$; TAU:-2.38, 95\% CI [-4.22,-0.54],ES=1.0). Higher scores were also found for quality-of-life functioning compared to SUPPORT $(9.33,95 \%$ CI $[1.42 ; 17.24], \mathrm{ES}=0.9)$. No significant effect was seen for coping with side effects. Conclusions. A psychological expectation optimization training showed no overall effects at reducing side effects and improving quality of life during adjuvant endocrine treatment. However, significant group differences of clinically significant magnitude were found for patients with negative baseline expectations. Expectation management showed beneficial effects for a targeted group of breast cancer patients.

CORRESPONDING AUTHOR: Pia von Blanckenburg, PhilippsUniversity of Marburg, Pia.vonBlanckenburg@staff.uni-marburg.de

\section{O15.2}

SEXUALITY AND BODY IMAGE IN WOMEN AFTER CANCER: THE EFFICACY OF THE AUSTRALIAN WOMEN'S WELLNESS AFTER CANCER PROGRAM

Janine Porter-Steele ${ }^{1}$, Charrlotte Seib ${ }^{1}$, Amanda McGuire ${ }^{1}$, Alexanda McCarthy ${ }^{2}$, Debra Anderson ${ }^{1}$

${ }^{1}$ Griffith University- Australia

${ }^{2}$ University of Auckland- New Zeland

Introduction: Cancer diagnosis and treatment can have profound psychological, psychosocial and psychosexual effects on women. Health professionals are reluctant to discuss sexuality concerns with their patients with reasons for this being cited as lack of knowledge, embarrassment, or because the patient has not overtly expressed concern. Often by the time a patient realises there is cause for concern, treatment has finished, there is less contact with the health care team, and they are less able to access support in this area. This presentation examines the efficacy of a wholeof-lifestyle intervention on sexual health and body image in women after cancer treatment. Methods: The Women's Wellness after Cancer program was a national randomised clinical trial of 351 women engaging in an ehealth-enabled lifestyle intervention that broadly aimed to improve the health and wellness of women after cancer. Moreover, this trial addresses existing gaps in supportive cancer care by embedding the topic of sexuality within a broader whole-of-lifestyle intervention. Results: In this study, many participants were sexually inactive. Of those who were sexually active, most reported sexual dysfunction $(\leq 26.55$ points $)$ on the Female Sexual Function Index (FSFI), Body image scale (BIS), and health-related quality of life (measured by the FACT-G). Notably, women in the intervention group reported statistically significant improvements in mean scores from pre-intervention to post-intervention on the FSFI subscales and overall FSFI $(\mathrm{p}<.05)$. The intervention group also reported a significant improvement in body image, functional wellbeing, and overall quality of life scores overtime $(\mathrm{p}<.05)$. Conclusion: This study provides evidence that an e-enabled, whole-of-lifestyle intervention incorporating health professional support improves sexual wellbeing and body image after a cancer diagnosis and could help improve overall health-related quality of life. The findings of this study have implications for health promotion practice and health professional education in the cancer setting.

CORRESPONDING AUTHOR: Janine Porter-Steele, Griffith University, j.porter-steele@griffith.edu.au

\section{O15.3}

PERCEIVED STRESS AND RESILIENCE IN WOMEN AFTER CANCER: EXAMINING THE IMPACT OF A LIFESTYLE INTERVENTION IN WOMEN PREVIOUSLY TREATED FOR BREAST, GYNECOLOGICAL OR BLOOD CANCER

Charrlotte Seib ${ }^{1}$, Debra Anderson ${ }^{1}$, Amanda McGuire ${ }^{1}$, Janine PorterSteele $^{1}$, Alexandra McCarthy ${ }^{2}$

${ }^{1}$ Griffith University- Australia

${ }^{2}$ University of Auckland- New Zeland

Introduction: For most women a cancer diagnosis is associated with considerable stress and anxiety. While symptoms can abate over time, some 
women report persistently elevated distress and this has been linked with a number of adverse health outcomes. This paper examines the reciprocity between perceived stress and resilience over a 24 week period and explores the potential impact of a lifestyle intervention to decrease stress and enhance resilience. Methods: Longitudinal data from 351 women previously treated for breast, gynaecological or blood cancer from the Australian Women's Wellness After Cancer Program (WWACP) were examined. Perceived stress (Perceived Stress Scale) and resilience (Connor-Davidson Resilience Scale 10) were measured across 3 time points (baseline, 12 weeks post-intervention, and 24 weeks for sustained change) and were analysed using hierarchical regression models. Results: At baseline, more than half (56\%) of women reported moderate (PSS range from14-26) or high (PSS $\geq 27$ ) perceived stress. Correlations between perceived stress and resilience at all time points suggested that while the indices were correlated, they were empirically distinguishable (Time 1: $\mathrm{r}=-.54$; Time 2: $\mathrm{r}=-.61$; Time 3: $\mathrm{r}=-.65$ ). Linear mixed-effect models estimated the relationships between perceived stress and resilience by group (intervention vs. control) over time. Results showed significant within-group $(\mathrm{p}<.05)$ and interaction (time $\mathrm{x}$ group, $\mathrm{p}<.01)$ effects for perceived stress and a significant interaction effect $(\mathrm{p}<.05)$ for resilience between baseline and 24 weeks. Conclusions: Findings suggested that while perceived stress as relatively high in this group, symptoms were amenable to intervention. This study highlights the importance of stress reduction and positive coping strategies to promote emotional wellbeing for women previously treated cancer

CORRESPONDING AUTHOR: Charrlotte Seib, Griffith University, c.seib@griffith.edu.au

\section{O15.4}

PROMOTING EXERCISE SELF-EFFICACY AND PHYSICAL ACTIVITY IN WOMEN AFTER CANCER

Amanda McGuire ${ }^{1,2}$, Charrlotte Seib $^{2}$, Janine Porter-Steele ${ }^{2}$, Debra Anderson ${ }^{2,3}$

${ }^{1}$ Griffith University- Australia

${ }^{2}$ Menzies Health Institute Queensland- Australia

${ }^{3}$ School of Nursing and Midwifery- Australia

Introduction: Regular physical activity and exercise has a range of physical and mental health benefits for cancer survivors (WCRF \& AICR, 2007). Current physical activity recommendations for cancer survivors are generic, but all suggest reducing sedentary time and increasing regular physical activity (Buffart, et al., 2014). In relation to promoting behaviour change, there is evidence that self-efficacy beliefs are strongly correlated with exercise behaviour in adults (Bauman et al., 2012). The purpose of this study was to evaluate the efficacy of an e-health enabled multiple health behaviour change intervention (the Women's Wellness after Cancer Program) to increase exercise self-efficacy and promote physical activity in women after cancer. Methods: The WWACP intervention includes easy to read, structured evidence based information about physical activity and exercise, with strategies to improve exercise self-efficacy including nurse coaching and goal setting to support positive mastery experience and behaviour change. Data were collected using self-report online and interview administered survey. Physical activity was measured using the Short IPAQ (Craig et al., 2003) and exercise self-efficacy using the Self-Efficacy for Exercise scale (Bandura, 2006). Descriptive and inferential analysis was undertaken using Statistical Package for Social Sciences version 25.0 (IBM Corp, 2017) and compared the difference between intervention and control groups post intervention (using ANCOVAs and Cohen's d). Results: A total of 351 women participated in the randomised controlled trial, with an average age of 53 years $(\mathrm{SD}=$ 8). After adjusting for pre-intervention scores there was a significant difference between intervention and control groups for exercise selfefficacy $(\mathrm{p}<0.05)$ post intervention, with a small to moderate effect size noted (Cohen's d). While there was no statistically significant difference in walking, moderate or vigorous physical activity between groups, there was a clinically significant increase in all types of physical activity in the intervention group over time. Conclusions: Outcomes of this study suggest that the WWACP intervention is effective in promoting exercise self-efficacy and physical activity in female cancer survivors. With increased survival rates and evidence of the multiple benefits of regular physical activity in increasing fitness, reducing fatigue and depression, interventions such as the WWACP have potential to contribute to improved health and wellbeing of women following cancer treatment.

CORRESPONDING AUTHOR: Amanda McGuire, Griffith University/ Menzies Health Institute Queensland, a.mcguire@ griffith.edu.au

\section{O15.5}

UTILIZING AN E-HEALTH INTERVENTION TO PROMOTE HEALTH-RELATED QUALITY OF LIFE IN WOMEN AFTER CANCER: THE AUSTRALIAN WOMEN'S WELLNESS AFTER CANCER PROGRAM

Debra Anderson ${ }^{1}$, Amanda McGuire ${ }^{1}$, Janine Porter-Steel ${ }^{1}$, Alexandra McCarthy $^{2}$, Charrlotte Seib ${ }^{1}$

${ }^{1}$ Griffith University- Australia

${ }^{2}$ University of Auckland

Introduction: Advances in medical technologies have improved many cancer survival rates (e.g., 5-year survival is $90 \%$ for breast cancer) and thus patients previously treated for cancer (or cancer survivors) represent a growing population. Despite this however, treatment-related health problems are common and are likely to significantly and negatively impact on quality of life. The project aimed to test the efficacy of 12-week ehealth multimodal lifestyle intervention to improve health-related quality of life (HRQoL) in Australian women previously treated for breast, gynaecological and blood cancers. Methods: The single-blinded multicentre randomized controlled trial recruited a total of 351 women within 24 months of completion of active cancer treatment. Women were randomly assigned to either a usual care or intervention group. The intervention, designed to enhance self-efficacy and promote positive lifestyle behaviours, comprised an interactive iBook and journal, web interface, and three virtual health coaching consultations by experienced cancer nurses. Data were collected on sociodemographic, lifestyle behaviours, and several health variables through structured online questionnaires at baseline (t0), 12 weeks (t1), and 24 weeks (t2). This paper used hierarchical models to examine within- and between- group changes $(\mathrm{t} 0-\mathrm{t} 2)$ in the main outcome variable of HRQoL (using the Short Form 36 or SF 36). Results: The average age of women in this study was 53 years $(\mathrm{SD}=8)$. Over two-thirds of the sample were married (77\%), many were Australian born (69\%), and over half had completed a university degree (59\%). Most Australian states were represented with around three-quarters of participants coming for Queensland, New South Wales, and Victoria. Linear mixed models showed significant within- and between- group increments in several SF 36 domains including Physical Functioning, Role Physical, Physical Component Scores $(\mathrm{p}<.05)$ while several other domains also showed significant improvements over time (Role Emotional, Social Function, Mental Component Score) though differences between groups were not noted. Conclusions: Despite generally good cancer survival rates, many women after cancer face a number of residual health problems which are amenable to interventions. The conclusion of active treatment is a key 'teachable moment' in which sustainable positive lifestyle change can be achieved if patients receive education and psychological support which targets key treatment related concerns and known chronic disease risk factors.

CORRESPONDING AUTHOR: Debra Anderson, Griffith University, debra.anderson@griffith.edu.au 
O15.6

INTERNET-DELIVERED COGNITIVE-BEHAVIORAL THERAPY FOR INSOMNIA IN BREAST CANCER SURVIVORS: EFFECTS ON SELF-REPORTED COGNITIVE FAILURES

Louise Stroem $^{1,2}$, Ali Amidi ${ }^{1,2}$, Malene Damholdt ${ }^{1,2}$, Jesper Dahlgaard ${ }^{3}$, Frances Thorndike $^{4}$, Lee Ritterband ${ }^{4}$, Robert Zachariae ${ }^{1,2}$

${ }^{1}$ Aarhus University Hospital- Denmark

${ }^{2}$ Aarhus University- Denmark

${ }^{3}$ VIA University College- Denmark

${ }^{4}$ University of Virginia School of Medicine- USA

Introduction: Sleep difficulties, fatigue, and cognitive impairment are common co-occurring symptoms associated with breast cancer (BC) and its treatment. Approximately $40 \%$ of breast cancer survivors report clinical levels of sleep disturbances. Although sleep disturbances and cognitive impairment are distinct symptoms, they may be reciprocally related, and thus, treatment for one symptom may impact the other. We have previously shown in a randomized controlled trial that internetdelivered and fully automated cognitive-behavioral therapy for insomnia (iCBT-I) is an effective intervention for sleep disturbances in women treated for breast cancer. Here we present the results of secondary analyses of the effect of iCBT-I on self-reported cognitive failures. Methods: A total of 255 Danish $\mathrm{BC}$ survivors (age $=53.2, \mathrm{SD}=8.8$ ) experiencing sleep disturbances (Pittsburg Sleep Quality Index (PSQI) $>5$ ) were recruited and randomly allocated to iCBT-I $(n=133)$ or waitlist control $(n=122)$. The fully automated iCBT-I program consists of 6 cores and is completed by participants within 6-9 weeks. Participants responded to questionnaires at baseline and post-intervention. Sleep was measured with the PSQI and the Insomnia Severity Index (ISI). Self-reported cognitive failures were assessed with The Cognitive Failures Questionnaire (CFQ), a 25-item questionnaire assessing daily cognitive failures. Results: Statistically significant $(p \leq 0.02)$ time $\times$ group interactions were found for all sleeprelated outcomes from pre- to post-intervention with small to large effect sizes (Cohen's $d=0.33-1.17)$. Baseline CFQ was associated with PSQI $(\mathrm{r}=0.16, \mathrm{p}=0.01)$ and ISI $(\mathrm{r}=0.29, \mathrm{p}<0.001)$. Baseline CFQ for all participants was 31.42 ( $\mathrm{SD}=14.94)$. Groups did not differ on CFQ at baseline $(\mathrm{p}=0.8)$. Within-group analyses revealed improvements on CFQ from baseline (mean=31.5, $\mathrm{SD}=14.0$ ) to follow-up (mean=30.1, $\mathrm{SD}=13.0)$ in the intervention group. Changes did not reach statistical significance $(\mathrm{p}=0.07)$. No between-group difference (time $\mathrm{x}$ group interaction) across time was found $(\mathrm{p}<0.33)$. Conclusion: No convincing evidence was found for improvements on self-reported cognitive failures following an internet-delivered CBT-I intervention in a Danish cohort of BC survivors with sleep disturbances.

CORRESPONDING AUTHOR: Louise Stroem, Aarhus University Hospital and Aarhus University, louisestroem@hotmail.com

\section{O16.1}

MANGUARD: AN ONLINE CARDIOVASCULAR DISEASE PREVENTION PROGRAM FOR MEN AT MIDLIFE

Marita McCabe ${ }^{1}$

${ }^{1}$ Institute for Health and Ageing- Australia

INTRODUCTION \& PURPOSE: Men aged 40-65 years report substantial modifiable risk factors for cardiovascular disease (CVD), such as obesity, smoking, and physical inactivity. There is a need for an accessible, cost-effective strategy targeting men that focuses on prevention. AIM: The current research implemented an online program targeting middle-aged men in the workplace to address CVD risk factors and modify behavior. METHOD: In total, 60 men (30 in the intervention group, 30 in the control group) were recruited. Men in the intervention group completed $4 \times 1.5$ hour modules online that utilized activities based on cognitive behavioral therapy (CBT) and motivational interviewing (MI) to assist men to identify and challenge their target behavior (e.g., exercise, eating patterns, smoking, alcohol intake) to adopt positive behavior change. The program focussed on goal setting, managing of lapses and relapses. Baseline, post-intervention and 3 months follow-up measures were taken of blood pressure, body mass index (BMI) and waist circumference. Measures of physical activity, dietary intake, alcohol consumption, smoking, sedentary behavior, stress and self-efficacy were also evaluated at these time points. RESULTS: Men in the intervention group relative to the control group demonstrated reductions in blood pressure, $\mathrm{BMI}$ and waist circumference. There were also greater increases in physical activity and self-efficacy. These finding were obtained at post-intervention, with further gains at 3 months follow-up. CONCLUSION: The program led to positive and sustainable lifestyle changes for men at midlife to reduce their risk of CVD. Manguard is an accessible, online program that can be utilized by men without having to visit a health care professional.

CORRESPONDING AUTHOR: Marita McCabe, Institute for Health and Ageing, marita.mccabe@acu.edu.au

\section{O16.2}

EFFECTS OF A MULTIDOMAIN LIFESTYLE INTERVENTION ON TELOMERE LENGTH: THE FINGER TRIAL

Shireen Sindi ${ }^{1}$, Ingemar Kåreholt ${ }^{1}$, Tiia Ngandu $^{2}$, Iiris Hovatta ${ }^{3}$, Dharma Singh $^{4}$, Benjamin Wolozin ${ }^{5}$, Jaakko Tuomilehto ${ }^{2}$, Hilkka Soininen ${ }^{6}$, Alina Soininen ${ }^{6}$

${ }^{1}$ Karolinska Institute- Sweden

${ }^{2}$ National Institute for Health and Welfare- Finland

${ }^{3}$ University of Helsinki- Finland

${ }^{4}$ Alzheimer's Research and Prevention Foundation- USA

${ }^{5}$ Boston University- USA

${ }^{6}$ University of Eastern- Finland

Introduction: Leukocyte telomere length (LTL) is a biomarker of aging and aging-related diseases. While LTL tends to decrease with age, various lifestyle factors are associated with LTL maintenance or decline. The goal was to assess the association between a 2-year multidomain lifestyle intervention, LTL change and cognition. Methods: The Finnish Geriatric Intervention Study to Prevent Cognitive Impairment and Disability (FINGER) was a 2-year randomized controlled trial among older adults at risk for cognitive decline $(\mathrm{N}=1260$, aged $60-77$ years). Participants were randomly assigned to the lifestyle intervention (diet, exercise, cognitive training and vascular risk management) and control (general health advice) groups. Telomere length measurements were available for 775 participants (392 control, 383 intervention). Primary outcome was change in cognition (neuropsychological test battery). Secondary outcomes were changes in specific cognitive domains: memory, executive functioning, and processing speed. Mixed effects regression models were used to analyse change in cognition as a function of randomization group, time, and LTL change. We also tested the associations between the FINGER intervention and cognition among individuals with or without LTL decline. All models adjusted for age and sex. Results: Less LTL decline was associated with significantly larger cognitive benefits from the intervention for the abbreviated memory score $(\beta=$ $0.253, \mathrm{p}=0.032)$ and executive functioning $(\beta=0.232, \mathrm{p}=0.008)$. Intervention effects were found among those without LTL decline for the total composite scores $(\beta=0.031, p=0.029)$, abbreviated memory score $(\beta=0.055, \mathrm{p}=0.025)$, and executive functioning $(\beta=0.051, \mathrm{p}=$ $0.005)$. In contrast, the intervention effect was found among those with LTL decline for processing speed $(\beta=0.056, p=0.025)$. Conclusion: For the first time, a multi-domain lifestyle intervention was shown to have significant impact on the associations between LTL change and cognitive improvement. Compared to controls, individuals without LTL decline showed larger cognitive benefits on the total composite score, abbreviated memory and executive functioning, while individuals with LTL decline showed the largest benefits for processing speed. No LTL decline may 
represent an important underlying mechanism for the associations between multidomain lifestyle interventions and cognitive improvement.

CORRESPONDING AUTHOR: Shireen Sindi, Karolinska Institute, shireen.sindi@ki.se

\section{O16.3}

EFFECTIVENESS OF A BUDDHIST TEMPLE-BASED LIFESTYLE INTERVENTION PROGRAMME TO CONTROL HIGH NORMAL BLOOD PRESSURE AND/OR HIGH NORMAL BLOOD GLUCOSE IN TEMPLE MEMBERS: A CLUSTER RANDOMIZED CONTROLLED TRIA

Supa Pengpid ${ }^{1}$, Karl Peltzer ${ }^{2}$

${ }^{1}$ ASEAN Institute for Health Development Mahidol- Thailand

${ }^{2}$ Human Sciences Research Council- South Africa

Background: Lifestyle intervention is recommended as the primary management for high normal blood pressure and/or high normal blood glucose. The aim of the study was to examine the effect of a community-based lifestyle intervention on glucose and blood pressure levels in Nakhon Pathom province, Thailand. Methods: We conducted a single-blinded, cluster randomized, community trial in 12 Buddhist temples, enrolling 443 members, aged 35-65 years with prediabetes and/or prehypertension. The temple (and their members) was randomized to a six two-hour group sessions lifestyle intervention (physical activity and nutrition) over six months guided by the Health Action Process Approach (HAPA) model or receiving a healthy lifestyle pamphlet. Physical outcomes (glucose, blood pressure, lipid profiles, and anthropometric measures) were assessed at 6 and/or 12 months following baseline assessments. The effects of the lifestyle intervention were assessed with generalized linear mixed models. Results: The study recruited 443 participants in 12 temples, 220 in the intervention and 223 in the control group, $89 \%$ completed the 12 months evaluation. At 12month follow-up mixed modelling found significant interaction effects on fasting plasma glucose $(\mathrm{P}=0.017)$ and diastolic blood pressure $(\mathrm{P}=0.002)$. Improvements were found for systolic blood pressure, but no significant interaction effect was detected. Conclusion: The lifestyle intervention was only partially effective. More research is needed to investigate lifestyle interventions in this community-based setting.

CORRESPONDING AUTHOR: Supa Peengpid, ASEAN Institute for Health Development Mahidol University, supaprom@yahoo.com

\section{O16.4}

THE INFLUENCE OF PSYCHOSOCIAL WORKING CONDITIONS ON LATE-LIFE PHYSICAL FUNCTIONING

Ingemar Kåreholt ${ }^{1}$, Ross Andel $^{2}$, Alexander Darin-Mattsson ${ }^{3,4,5}$, Charlotta Nilsen ${ }^{3,4,5}$

${ }^{1}$ Institute of Gerontology- Sweden

${ }^{2}$ University of South Florida and International Clinical Research Center- USA

${ }^{3}$ Aging Research Center- Sweden

${ }^{4}$ Karolinska Institutet- Sweden

${ }^{5}$ Stockholm University- Sweden

Introduction and purposes: In older adults, increasing age correlates with declining physical functioning. The growing challenge posed by an aging population makes finding predictors of physical functioning in old age increasingly important. Work dominates much of our adult lives. Active jobs (high psychological demands, high control) are considered to increase learning, which may reduce the perception of situations as stressful and instead be viewed as challenges and opportunities for personal growth. This will, in turn, lead to feelings of self-efficacy that may encourage an active leisure-time, such as physical activity. We investigated the long-term association between active jobs and late-life mobility. Method: Two individually linked Swedish surveys were used $(n=775)$.
A psychosocial job exposure matrix was used to measure active jobs four times in midlife (age 40-65). An active job is a job with high demands, but also high control. Jobs with low control OR low demands were considered as not active. Mobility was measured in 2014 as the self-reported ability to stand without support, walk up and down stairs, walk 100 meters fairly briskly, rise from a chair with arms crossed across the chest, and ability to balance indoors, summarized in a 5-item index (0-5). Data were analyzed with ordered logistic regressions. Results: People having a higher active job score had significantly better mobility in old age compared to people in non-active jobs. However, the accumulated score of active jobs over working life were not more strongly associated with mobility in old age than the active job score in 1991. This might indicate that the conditions of a person's most recent job has the strongest association to mobility in old age. Conclusions: Active job conditions in midlife are important predictors of mobility in old age. Promoting active job conditions at midlife may be used as an interventions aimed at preventing physical deterioration later in life.

CORRESPONDING AUTHOR: Ingemar Kåreholt, Institute of Gerontology, ingemar.kareholt@ju.se

\section{O16.5}

DEPRESSION, LONELINESS AND SOCIAL SUPPORT AMONG OLDER PEOPLE: STUDY IN INDIGENOUS RESIDING IN THE NORTH AND SOUTH OF CHILE

Lorena Gallardo-Peralta ${ }^{1}$, Esteban Sánchez-Moreno ${ }^{2}$, Cecilia MayorgaMuñoz ${ }^{3}$, Vicente Rodríguez-Rodríguez ${ }^{4}$, Abel Soto-Higuera ${ }^{3}$, Marcelo Piña-Morán ${ }^{5}$

${ }^{1}$ Universidad de Tarapacá- Chile

${ }^{2}$ Universidad Complutense de Madrid- Spain

${ }^{3}$ Universidad de La Frontera- Chile

${ }^{4}$ Consejo Superior de Investigaciones Científicas- Spain

${ }^{5}$ Universidad Católica del Maule- Chile

Introduction \& Purpose: Depression is a recurring disorder in old age, in Chile warns a high prevalence in people over 60 (Sandoval et al. 2016) Depression in older Chileans tends to be more frequent in women, in indigenous, in widowers, in who live alone and in with some disability. These dimensions, along with social support and loneliness will be analyzed in your association with depression. On the basis of the following questions: what social networks inhibit depressive symptoms? Which the weight of the feeling of loneliness in older Chileans depression? Methods: The study was quantitative and transversal, the sample consists of 800 elderly people living in north and south of Chile. The questionnaire was applied via personal interview by professionals from the fields of social work and psychology, during the months of August to October 2017. The measures used were: Geriatric Depression Scale (Brink et al. 1982), Loneliness Scale (De Jong Gierveld \& Van Tilburg, 2006), Barthel Index (Mahoney \& Barthel, 1965) and Perceived Social Support Questionnaire (Gracia et al. 2002). Hierarchical regression analysis is used. Results: The results show that the predictors of depression are: age (older, more depression), being indigenous, live alone, have some degree of dependence (avd) and feel alone. However, different sources of social support analyzed, only social support from childen is associated with less depression Conclusion: Currently gerontology is interested in reduce and intervention to preventing to the loneliness and insolation (Gardiner et al., 2018). Chile is no exception, the various changes in social and family structures; they show a new configuration of networks of support in old age and the tendency to live alone, not to have children, etc.. But there is another area that should be expanded in theorical and empirical: diversity aging, as it is the case of the ethnic minorities (native indigenous). These issues will be deepened in your debate.

CORRESPONDING AUTHOR: Lorena Gallardo-Peralta, Universidad de Tarapacá, lgallardo@uta.cl 
O17.1

INCORPORATING IF-THEN PLANNING INTO A SMARTPHONE APP TO REDUCE DRINKING: WHAT ARE THE LESSONS LEARNT?

Petra Staiger ${ }^{1}$, Paul Liknaitzky ${ }^{1}$, Renee O'Donnell ${ }^{1}$

${ }^{1}$ Deakin University- Australia

Introduction and purpose: Smartphone health-related apps may mitigate many barriers to help-seeking for those who wish to reduce their drinking. They are low cost, convenient, accessible, private, self-directed, and scalable across a range of populations. Over $80 \%$ of adults in developed countries currently use a smartphone. Most alcohol-related app interventions either focus on self-monitoring and goal setting, or offer a large range of intervention options which potentially overwhelm users and impedes upon engagement. Given the effectiveness of the IF-THEN PLANNING method to reduce alcohol consumption, we report on the process of adapting this method to a smartphone app. Methods: Through multiple iterations, we adopted an evidence-based approach to develop the IF-THEN planning component of the app. First, informed by research into putative mechanisms associated with IF-THEN PLANNING, we formulated PLANS that provided structural constraints yet tailored content. Second, we conducted a Delphi study comprised of clinicians and researchers to select and refine candidate IF and THEN statements. Third, we conducted a pilot study to evaluate the acceptability and feasibility of constructing personalised IF-THEN PLANS within an app environment. Results: Findings from the Delphi study $(\mathrm{N}=12)$ indicated that participants preferred short and highly specific statements, along with numerous specific adjustments to both content and wording. Results from the pilot evaluation of the app $(\mathrm{N}=11)$ reported a high usability score on a standard measure (within the top 10 percentile), however all participants indicated that the app did not make the IF-THEN PLANS prominent enough, nor did it provide any structured process to engage with, learn, and simulate these PLANS. Qualitative interviews provided additional insights that informed a revised version of the app with substantial adjustments and gaming features to increase engagement with the IF-THEN PLANS. Our prototype of this version will be described. Conclusions: Incorporating the IF-THEN PLANNING method into a smartphone app is not without its challenges. However, with good building blocks (the IF and THEN statement options), an effective balance between constrained structure and tailoring, and gaming and notification methods that increase regular engagement with the PLANS, an app that employs IF-THEN PLANS has great promise as a behaviour change intervention. The design of our proposed randomised controlled trial will be briefly discussed.

CORRESPONDING AUTHOR: Petra Staiger, Deakin University, petra.staiger@deakin.edu.au

\section{O17.2}

MYHEALTHPA: A MOBILE-BASED INTERVENTION TO REDUCE SUBSTANCE USE AND OTHER HEALTH RISK BEHAVIOURS IN PEOPLE WITH MENTAL HEALTH PROBLEMS

Louise Thornton ${ }^{1}$, Frances Kay-Lambkin ${ }^{2}$, Bree Tebbutt ${ }^{2}$, Tanya Hanstock $^{2}$, Amanda Baker ${ }^{2}$

${ }^{1}$ University of New South Wales- Australia

${ }^{2}$ The University of Newcastle- Australia

Introduction and Purpose: Substance use and other health risk behaviours (e.g. poor diet and physical inactivity) are much more common among people with mental health problems. Smoking, alcohol misuse, physical inactivity and poor diet are also consistently identified as the top four behavioural risk factors associated with chronic disease such as cardiovascular disease (CVD) which are responsible for more deaths in this population than suicide. However, due to time constraints, lack of awareness, training and resources, mental health services often confine their services to mental health issues therefore, neglecting these types of health risk behaviours. This presentation describes the development and initial evaluation of the first mobile-based intervention to target multiple key health risk behaviours (smoking, alcohol misuse, poor diet and physical inactivity) for people with mental health problems called MyHealthPA. Methods: The development of MyHealthPA occurred in four stages: 1) reviewing and addressing the literature; 2) a scoping survey with endusers ( $(=251) ; 3$ ) program development; and 4) pilot testing with young people with and without a previous diagnosis of a mental illness (MI) $(\mathrm{n}=$ 28). Results: Twenty-eight young people aged 19 to 25 years were recruited to the pilot trial. Of these, $43 \%$ had been previously diagnosed with a MI. Small improvements in fruit and vegetable consumption, level of physical activity, alcohol use, and mood were found between baseline and end-of-treatment and follow-up, particularly among people with experience of MI. Most participants reported the program had above average usability, however participants also identified a number of ways in which the program could be improved. Conclusions: The MyHealthPA program represents an innovative approach to chronic disease risk reduction among people with mental health problems. MyHealthPA appears to be an acceptable, easy to use, and potentially effective mHealth intervention to assist young people with mental illness to monitor risk factors for chronic disease. However, ways in which the program could be improved for future testing and dissemination were identified and will be discussed.

CORRESPONDING AUTHOR: Louise Thornton, University of New South Wales, L.Thornton@unsw.edu.au

\section{O17.3}

EFFICACY OF MOTIVATIONAL INTERVIEWING IN SMOKING GROUPS IN PRIMARY HEALTH CARE: A RANDOMIZED CLINICAL TRIAL

Daniel Demétrio Faustino-Silva ${ }^{1}$, Ricardo Melnick ${ }^{1}$, Roger Keller Celeste $^{2}$

${ }^{1}$ Grupo Hospitalar Conceição (GHC)- Brazil

${ }^{2}$ Graduate Dentistry Program of the Universidade Federal do Rio Grande do Sul (UFRGS)- Brazil.

Introduction: Smoking is a major risk factor in public health, with a strong association with chronic noncommunicable diseases and great socioeconomic damages. It is considered the leading cause of preventable death in the world. Primary health care is a strategic setting in the treatment of this problem, therefore it is necessary to evaluate new technologies for the management of this pathology in this context. Motivation is the main factor involved in the cessation of smoking. There is a need for evaluation and development of technologies to increase the degree of motivation. Motivational Interviewing (MI) is a person-centered, communicative method whose purpose is to work and increase the user's motivation for behavior change. Objectives: The objective of this study is to evaluate the efficacy of MI on the smoking cessation rates of the smoking groups performed by the primary care teams of the Conceição Hospitalar Group, and also whether there is an increase in the means of attending the sessions and the completion rate of the groups. Methodology: A randomized community trial was conducted in the 12 units of this service between July 2016 and May 2017, with randomization by cluster. In the intervention group, the coordinators of smoking groups in their units received an 8-hour training in $\mathrm{MI}$ in order to associate it with the conventional approach, while the control group units used only the conventional cognitive-behavioral approach. After 11 months, smoking cessation rates were compared between the two groups. Results: During this period, 44 groups were performed, totaling 329 patients (178 in the intervention group and 151 in the control group). The smoking cessation rate in the group that used $\mathrm{MI}$ was $61.8 \%$, with a $\mathrm{RR}=1.25(\mathrm{CI}=95 \% 1,01-1,54, \mathrm{p}=$ 0.043 ), and in the conventional group, $47.7 \%$. The mean number of sessions was 3.1 in the intervention group and 2.9 in the control group. The percentage of completion of the groups with the intervention was $65.2 \%$ and, in the control, $57.6 \%$. Conclusion: The motivational 
interviewing showed efficacy and superiority about the conventional approach. There was a statistically significant increase of $25 \%$ in smoking cessation rates. It is a light, easy-to-apply and appropriate technology for primary care and smoking groups. It is presented as a useful strategy in the face of the tobacco epidemic.

CORRESPONDING AUTHOR: Daniel Demétrio Faustino-Silva, Grupo Hospitalar Conceição (GHC), ddemetrio@gmail.com

\section{O17.4}

INCREASING ENGAGEMENT WITH, AND THE EFFECTIVENESS OF, A BRIEF ONLINE INTERVENTION TO REDUCE ALCOHOL CONSUMPTION

Paul Norman ${ }^{1}$, Thomas Webb ${ }^{1}$, Abigail Millings ${ }^{1}$, Laura Pechey ${ }^{2}$

${ }^{1}$ University of Sheffield- United Kingdom

${ }^{2}$ Haringey Advisory Group on Alcohol- United Kingdom

Introduction \& Purpose: Despite the potential reach of brief online alcohol interventions, their effectiveness may be compromised by low levels of engagement and by the inclusion of ineffective behaviour change techniques. This study assessed (i) whether a tunneled intervention in which the content is delivered in a pre-specified order would lead to greater engagement and effectiveness than a free-roam version in which the content can be viewed in any order and (ii) whether being instructed to form ifthen plans (i.e., implementation intentions) to cut down on drinking would lead to greater reductions in alcohol consumption than only choosing strategies to cut down. Methods: After completing baseline measures of alcohol consumption, participants ( $\mathrm{N}=286$ university staff and students) were randomly allocated to one of four versions of a brief online alcohol intervention (DontBottleItUp) in a 2 (structure: free-roam versus tunneled) $\mathrm{x} 2$ (planning: strategies versus if-then plans) factorial design. Measures of engagement with the online intervention (pages visited, time) were recorded automatically. Participants' $(n=236)$ alcohol consumption was assessed again at one-month follow-up. Results: The structure of the online intervention had a significant effect on engagement. Participants who viewed the tunneled version viewed significantly more pages $(d=1.36)$ and spent significantly more time on the website $(d=0.22)$ than participants who viewed the free-roam version. A significant reduction in alcohol consumption was observed between baseline and follow-up $(d=0.29)$; however, neither the structure of the intervention or type of planning had a significant effect on reductions in alcohol consumption. Conclusions: The present results indicate that brief online alcohol interventions should employ a tunneled structure in order to increase engagement. However, neither the structure of the intervention or the type of plan that users made influenced effectiveness, suggesting that future research should seek to identify the "active ingredients" (i.e., key behaviour change techniques) of brief online alcohol interventions using experimental/factorial designs.

CORRESPONDING AUTHOR: Paul Norman, University of Sheffield, p.norman@sheffield.ac.uk

\section{O17.5}

RESIDENT INVOLVEMENT IN HEALTH PROMOTION ACTIVITIES IS ASSOCIATED WITH HEALTH OUTCOMES: A CASE-STUDY EVALUATION IN 11 SOCIALLY VULNERABLE NEIGHBORHOODS IN DENMARK

Charlotte Demant Klinker ${ }^{1}$, Jens Aagaard-Hansen ${ }^{1}$, Nethe Katrine Jørgensen $^{2}$, Helle Terkildsen Maindal ${ }^{3}$

${ }^{1}$ Steno Diabetec Center Copenhagen- Denmark

${ }^{2}$ Independent

${ }^{3}$ University of Aarhus- Denmark

Introduction and purpose: This qualitative study explored how active involvement of residents in six phases of health promotion activities (theme selection, idea generation, planning, implementation, evaluation and continuation) influenced self-reported mental well-being, social relations and empowerment among residents in socially vulnerable neighborhoods. Methods: The Partnership for Health and Innovation (PHI) project was initiated to develop and implement health promotion activities in 11 socially vulnerable neighborhoods across three Danish municipalities. The activities should be based on active partnerships between residents, health promoters and social workers. Processes and self-reported health promoting outcomes were documented during a three-year case-study evaluation. Transcripts from focus groups with residents and semistructured interviews with professionals and descriptions of 22 activities were analyzed based on six pre-defined aspects of resident involvement and a logic model defining expected project outcomes (increased social relations, well-being and empowerment). Theory of social capital (bonding, bridging and linking) was applied. Results: A total of 44 residents and 34 professionals from the 11 neighborhoods took part in the evaluation. Degree of resident involvement varied across the six phases of health promotion activities and the three municipalities. The implementation phase had most often a high degree of involvement, whereas at low degree of involvement was seen in the idea generation phase. One municipality displayed a high degree of resident involvement across all activities and activity phases, whereas in the other two municipalities resident involvement was low in some phases. Comprehensive resident involvement seemed to be associated with increased mental well-being, social relations and empowerment as shown in the municipality with a high overall degree of resident involvement, whereas health outcomes were less evident in those with less involvement. Conclusions: Comprehensive involvement of residents in all phases of health promotion activities seems to play a critical role in the achievement of positive self-reported health outcomes among residents in socially vulnerable neighborhoods.

CORRESPONDING AUTHOR: Charlotte Demant Klinker, Steno Diabetec Center Copenhagen, charlotte.demant.klinker@ regionh.dk

\section{O17.6}

IMPROVING THE REPORTING OF BEHAVIOUR CHANGE INTERVENTIONS: INITIAL DEVELOPMENT OF AN ONTOLOGY TO UNDERPIN STUDY REPORTS

Ildiko Tombor ${ }^{1}$, Jamie Brown ${ }^{1}$, Lion Shahab ${ }^{1}$, Emma Beard ${ }^{1}$, Susan Michie $^{1}$, Robert West ${ }^{1}$

${ }^{1}$ University College London- England

Introduction and Purpose: In the field of behavioural science, understanding "what works, compared with what, how well, with what degree of exposure, for whom, in what settings, with what behaviours, and why" requires systematic knowledge and evidence syntheses. Ontologies can facilitate this by providing clear and consistent vocabularies organised in a coherent structure. We do not yet have suitable ontology in this domain. This paper describes the development of a pilot ontology structure for reporting a complex behaviour change intervention (BCI) evaluation. Methods: The evaluation of a smartphone app ('SmokeFree Baby') to aid smoking cessation in pregnancy provided the basis for this work. The prototype ontology structure and a controlled vocabulary with labels and definitions for all entities in the ontology were developed iteratively based on expert consensus. Entities included in the ontology structure complied with the CONSORT guideline for the reporting of randomised controlled trials, Behaviour Change Techniques Taxonomy v1, Mode of Delivery Ontology v0 and the Behaviour Change Intervention Ontology including the Populations and Setting Ontologies. Results: Previously identified upper-level entities in the Behaviour Change Intervention Ontology were elaborated so that entities represented all key information related to the intervention and its evaluation. This included lower-level entities relating to the i) 'BCI scenario' representing the $\mathrm{BCI}$ itself, the target behaviour and the factors that influence the effect of the $\mathrm{BCI}$ on the 
target behaviour; and ii) 'BCI evaluation' representing a comparison between two or more BCI scenarios. Conclusions: A controlled vocabulary and a prototype ontology structure provided a usable basis for reporting of a smoking cessation intervention evaluation to facilitate the standardised representation of information so that evidence can be more efficiently reported and synthesised in the future.

CORRESPONDING AUTHOR: Ildiko Tombor, University College London, ildiko.tombor@ucl.ac.uk

\section{O18.1}

INPATIENT SLEEP QUANTITY AND QUALITY AS PREDICTORS OF CLINICAL OUTCOMES AFTER CANCER SURGERY Carissa Low ${ }^{1}$, Dana Bovbjerg ${ }^{1}$, Haroon Choudry ${ }^{1}$, Matthew Holtzman ${ }^{1}$, Heather Jones ${ }^{1}$, James Pingpank ${ }^{1}$, Herbert Zeh ${ }^{1}$, Amer Zureikat ${ }^{1}$, David Bartlett $^{1}$

${ }^{1}$ University of Pittsburgh- USA

Introduction \& purpose: Sleep facilitates healing but is frequently disrupted by external (e.g., noise) and internal (e.g., pain) factors after surgery. Research on links between inpatient sleep disruption and postoperative clinical outcomes is scant. The goal of these analyses was to determine whether sleep quantity and quality during inpatient recovery are related to length of stay, 60-day readmission, or survival after cancer surgery. Methods: Patients with metastatic peritoneal cancer scheduled for surgical resection were enrolled at their preoperative clinic visit. Throughout inpatient recovery, Fitbit devices quantified daily sleep and physical activity, and patients rated the severity of their sleep disturbance on a scale from 0 (not present) to 10 (as bad as you can imagine) each day. Information on length of stay, readmissions, and survival was extracted from electronic medical records. Results: 53 patients participated and provided Fitbit sleep data (mean age $=58$, range 31-80; 43\% female). Mean time asleep per 24-hour period was calculated for each participant over the postoperative inpatient stay (mean stay $=13$ days; mean sleep $=$ 6.9 hours per night, range 2.5-12.9 hours). On average, patients rated the severity of their sleep disturbance as 4.7 out of 10 (range $0-8.8$ ), and worse patient-reported sleep disturbance was related to lower sleep duration $(r(52)=-.27, p=.056) .38 \%$ of patients were readmitted within 60 days of discharge, and $26 \%$ patients died during follow-up (median follow-up $=2$ years). After adjusting for prognostic covariates and physical activity, longer inpatient sleep duration was not significantly related to readmission risk, but was marginally related to shorter length of stay ( $\beta$ $=-.22, \mathrm{p}=.08)$ and significantly associated with longer survival (HR $0.72,95 \%$ CI $0.54-0.97, p=.03$ ). Similarly, patient-reported sleep disturbance was not significantly related to length of stay or readmission risk but was associated with survival, with patients reporting worse sleep at higher risk of mortality (HR 1.40, 95\% CI 1.03-1.90, $\mathrm{p}=.03$ ). Conclusions: Findings from this small preliminary study suggest that both objective quantity and subjective quality of sleep during postoperative recovery may be related to long-term survival after metastatic cancer surgery.

CORRESPONDING AUTHOR: Carissa Low, University of Pittsburgh, lowca@upmc.edu

\section{O18.2}

PREDICTING TOXICITY SEVERITY: THE LONGITUDINAL IMPACT OF RESPONSE EXPECTANCIES DURING RADIOTHERAPY FOR PROSTATE CANCER

Elise Devlin ${ }^{1}$, Hayley Whitford ${ }^{2}$, Linley Denson ${ }^{3}$, Andrew Potter ${ }^{4}$

${ }^{1}$ The University of Adelaide- Australia

${ }^{2}$ The University of South Australia- Australia

${ }^{3}$ The University of Adelaide- Australia

${ }^{4}$ Royal Adelaide Hospital- Australia
Introduction \& Purpose: Research has indicated patients' pre-treatment response expectancies of side effects moderately predict subsequent toxicity experiences. In oncology, research has focused on chemotherapy, a systematic therapy targeting all cells in the body. No research has directly considered expectancies during radiotherapy, which targets local cells, producing different toxicities at alternate phases. Furthermore, no homogenous male samples have been represented. Thus, we investigated associations between expectancies of radiotherapy side effects and subsequent experiences in male patients throughout therapy. Methods: Using a longitudinal, within-participants design, 35 patients scheduled for radiotherapy for prostate cancer (in 4-weeks) rated their expectancies for toxicity severity (T1). Standardized emotional state (Depression Anxiety and Stress Scale 21), and coping style (Mental Adjustment to Cancer) measures were also administered at T1, to be controlled in statistical analyses. Follow-up occurred on two occasions; two weeks into treatment (T2), when toxicities are not medically explicable but are often observed clinically, and seven weeks into treatment (T3) when side effects are medically expected to occur. During follow-ups, patients rated their future expectancies (T2) and toxicity experience (T2 and T3). Results: A series of multiple hierarchical linear regressions, controlling for related confounds, revealed expectancies significantly $(\mathrm{p}<0.05)$ and uniquely predicted 6 of 18 toxicities at T2 ( $\beta=0.39-0.67)$, explaining $12-30 \%$ of the variance. Expectancies measured at $\mathrm{T} 2$ significantly and uniquely $(\mathrm{p}<0.05)$ predicted 7 of 16 side effects at T3 $(\beta=0.49-0.91)$, explaining $23-50 \%$ of the variance. Throughout treatment, expectancies for inability to reach orgasm revealed the strongest associations with subsequent experiences ( $\beta=0.67$ at $\mathrm{T} 2 ; \beta=0.91$ at T3). Conclusions: In a homogenous group of men, and controlling for multiple confounds, patient expectancies predicted multiple experiences from early in radiotherapy, highlighting possible psychosomatic influences. These outcomes suggest toxicities for localised therapy appear to be influenced by patient expectancies in the same way as in systemic therapies. Thus, any interventions targeting expectancy reduction should be also considered across therapy types.

CORRESPONDING AUTHOR: Elise Devlin, The University of Adelaide, elise.devlin@ adelaide.edu.au

\section{O18.3}

ILLNESS PERCEPTIONS, TREATMENT BELIEFS AND SIDE EFFECTS PREDICT DEPRESSION AND ANXIETY AT 12 MONTHS IN WOMEN SURVIVORS OF BREAST CANCER WHO ARE PRESCRIBED TAMOXIFEN

Lyndsay Hughes ${ }^{1}$, Natasha Askaroff ${ }^{1}$, Myra Hunter ${ }^{1}$, Rona MossMorris $^{1}$, Zoe Moon ${ }^{1}$

${ }^{1}$ King's College London- United Kingdom

Introduction \& Purpose: Breast cancer survivorship is improving however, survivors remain at higher risk of depression than the general population with up to $48 \%$ of women within the first year of primary treatment experiencing high depression and/or anxiety. However, there is currently little understanding of the mechanisms contributing to psychological comorbidity. Methods: This is a prospective 12 month observational study of women within the first year of primary treatment. Questionnaires including the illness perceptions questionnaire for breast cancer survivors (IPQ-BCS), beliefs about medications and hospital anxiety and depression scale were completed at baseline and 12 months. Hierarchical multiple regression analyses were used to predict anxiety and depression. Results: 345 women were recruited at baseline and 258 (74.8\%) completed 12 month questionnaires. At baseline, $16 \%$ had depression scores of $\geq 8$ and $39 \%$ had anxiety scores of $\geq 8$, which did not change significantly over time. At baseline, the final regression model accounted for $55 \%$ of the overall variance in anxiety $(F(20,274)=16.454, p<0.001)$. Higher consequences and emotional representations scores as well as stronger beliefs in emotional causes of cancer were related to higher anxiety. At 12 months, the final regression model accounted for $58 \%$ of the variance 
in anxiety $(\mathrm{F}(16,224)=18.92, \mathrm{p}<0.001)$ with baseline anxiety and greater tamoxifen side effects predicting greater anxiety. At baseline, the final regression model explained $59 \%$ of the variance in depression $(\mathrm{F}(20,247)=17.96, \mathrm{p}<0.001)$. Higher consequences, identity and psychological causes scores; lower health behaviour causes and lower tamoxifen side effects scores were related to higher depression. At 12 months, the overall model explained $60 \%$ of the variance $(F(18,187)=15.82, p<0.001)$ with higher baseline depression, tamoxifen side effects and identity scores predicting higher depression. Conclusions: A significant proportion of women initiating tamoxifen experience high levels of depression and anxiety which are related to side effects, perceived consequences and emotional reactions. Depression and anxiety can have negative impacts on quality of life and adherence to treatment. These results suggest areas to target for intervention.

CORRESPONDING AUTHOR: Lyndsay Hughes, King's College London, lyndsay.hughes@kcl.ac.uk

\section{O18.4}

USING MOBILE AND SENSOR TECHNOLOGY TO IDENTIFY EARLY DEHYDRATION RISK IN HEAD AND NECK CANCER PATIENTS UNDERGOING RADIATION TREATMENT: IMPACT ON QUALITY OF LIFE

Susan Peterson $^{1}$, Beth Beadle ${ }^{2}$, Adam Garden ${ }^{1}$, Sanjay Shete ${ }^{1}$, Stephanie Martch $^{1}$, Emiilia Farcas ${ }^{3}$, Kai Lin ${ }^{3}$, Fred Raab ${ }^{3}$, Viswanath Nandigam ${ }^{3}$

${ }^{1}$ University of Texas MD Anderson Cancer Center- USA

${ }^{2}$ Stanford University- USA

${ }^{3}$ University of California-San Diego- USA

Introduction \& Purpose: Assessment and intervention using mobile and sensor technology have great potential to improve the early detection and mitigation of cancer treatment-related complications, and thus improve quality of life, reduce complications, and lower health care costs. We evaluated the efficacy of a home monitoring system, CYCORE (CYberinfrastructure for COmparative effectiveness Research). CYCORE engages head and neck cancer (HNC) patients undergoing radiation treatment (RT) in the use of sensor and mobile technology to assess daily weight, blood pressure (BP)/pulse, and patient-reported outcomes (PROs, e.g., pain, nausea, dizziness, medication use, food/fluid intake). Clinicians reviewed data daily to identify early risk of dehydration, with the goal of early intervention to mitigate hospitalization and emergency room visits. We evaluated longitudinal quality of life (QOL) outcomes in patients randomized to use CYCORE during RT versus those randomized to usual care. Methods: HNC patients $(n=357)$ completed the Functional Assessment of Cancer Therapy- Head \& Neck (FACT-HN), which assesses QOL across several domains (physical, social/family, emotional, functional well-being) as well as HNC-specific outcomes. FACT-HN was completed at RT initiation (baseline), completion of RT (6-7 weeks post-baseline), and 8 weeks post-RT completion. Time point and group differences in QOL scores were evaluated using repeated measures ANOVA. Results: Mean age was 60 years (range 25-86), 21\% were female, $85 \%$ were White, and $54 \%$ completed college. Baseline QOL mean scores were similar in patients randomized to CYCORE $(\mathrm{n}=169)$ or usual care $(\mathrm{n}=188)$. Mean scores on HNC-specific QOL outcomes and total scores were higher in the CYCORE group at 8 weeks post-RT completion ( 23.4 vs $22.1, \mathrm{p}=.023$; 61.8 vs $58.8, \mathrm{p}=.049)$. Conclusion: HNC patients randomized to the CYCORE system during RT self-reported better QOL over time compared to those randomized to usual care. Sensor and mobile technologies can monitor patients' symptoms and related outcomes during critical periods of outpatient cancer treatment, can provide timely information to facilitate rapid clinical decision making about care, and may ultimately result in better QOL outcomes.

CORRESPONDING AUTHOR: Susan Peterson, University of Texas MD Anderson Cancer Center, petersonsusank@gmail.com
O18.5

UNDERSTANDING THE INFLUENCES ON EARLY LUNG CANCER SYMPTOM PRESENTATION AND INTERVENTION PREFERENCES IN HIGH RISK GROUPS: A QUALITATIVE STUDY Grace McCutchan ${ }^{1}$, Julia Hiscock ${ }^{2}$, Peter Murchie ${ }^{3}$, Kerry Hood ${ }^{1}$, Richard Neal ${ }^{4}$, Sara Thomas ${ }^{5}$, Ann Maria Thomas ${ }^{1}$, Gareth Newton ${ }^{1}$

${ }^{1}$ Cardiff University- United Kingdom

${ }^{2}$ Bangor University- United Kingdom

${ }^{3}$ University of Aberdeen- United Kingdom

${ }^{4}$ University of Leeds- United Kingdom

${ }^{5}$ Public Health Wales- Wales

Background Lung cancer is the leading cause of cancer-related death worldwide. People at highest risk for lung cancer- current or former smokers, aged over 40 years, with serious lung comorbidity and living in areas of deprivation- are more likely to prolong presenting to a GP with symptoms, leading to advanced stage diagnosis. This qualitative study sought to understand the influences on early presentation with lung cancer symptoms in high risk individuals and intervention preferences. Methods Semi-structured interviews were conducted with 37 high risk individuals, recruited through GP practices in deprived areas of England, Scotland and Wales. A lung symptom attribution task was used to explore symptom interpretations, symptom presentation action planning, and beliefs surrounding lung cancer, underpinned by Leventhal's Common Sense model. Focus groups with members of the public $(n=2)$ and local stakeholders (healthcare professionals and community partners; $n=2$ ) were conducted to explore intervention preferences. Data were analysed using Framework. Results Interviews Participants perceived greater control over their short-term health (managing chest infections) than longer term health (preventing or detecting lung cancer). Chest infections were described as controllable through constant symptom monitoring leading to quick appraisal/detection and effective treatment with antibiotics. In contrast, lung cancer was considered inevitable but incurable, and participants anticipated refusing treatment. Focus groups Suggestions for intervention content included information to raise awareness of lung cancer symptoms, modify negative lung cancer beliefs and highlight the importance of earlier diagnosis. Multi-faceted interventions were suggested including talks and stands in community venues, led by a trained, nonjudgemental facilitator. Conclusions Attributing lung symptoms to a 'treatable' cause means that high risk individuals manage their lung health in the short term, but potentially avoid/deny symptoms related to 'incurable' lung cancer. A multi-faceted is being developed to highlight lung cancer symptoms and the importance of early diagnosis.

CORRESPONDING AUTHOR: Grace McCutchan, Cardiff University, mccutchanGM@cardiff.ac.uk

\section{O18.6}

DEVELOPMENT OF A SELF-MANAGEMENT INTERVENTION TO IMPROVE ADHERENCE RATES AND SUPPORT BREAST CANCER SURVIVORS TAKING TAMOXIFEN

Zoe Moon ${ }^{1}$, Rona Moss-Morris ${ }^{1}$, Myra Hunter ${ }^{1}$, Lyndsay Hughes ${ }^{1}$

${ }^{1}$ King's College London- United Kingdom

Introduction and Purpose: Non-adherence to tamoxifen is common in breast cancer survivors and is associated with increased risk of recurrence and mortality. Previous research has identified several psychosocial variables associated with non-adherence to tamoxifen, yet interventions to improve adherence are currently lacking. This study developed and tested a psychoeducational intervention to modify key psychological variables and improve adherence rates in breast cancer survivors taking tamoxifen. Methods: A series of studies were conducted to understand patients' experiences of tamoxifen and to identify potentially modifiable predictors of non-adherence. Following MRC guidelines and using the Intervention Mapping Framework, these findings informed the development of a 
guided self-management intervention manual, offering information on how tamoxifen works, how to take tamoxifen and managing side effects, with activities around goal setting, implementation intentions and symptom management. The intervention was tested in a small pre-post feasibility and acceptability study including 33 women prescribed tamoxifen. Pre- and post-intervention questionnaires were completed. Qualitative interviews assessed the acceptability of the intervention materials. Results: Recruitment and uptake for the feasibility study were good, with $87 \%$ of eligible participants agreeing to take part. Around two thirds of participants recruited to the study completed the follow up questionnaires. Paired samples t-tests showed small improvements in adherence over time, as well as improvements in necessity and concern beliefs, personal control, coherence, distress, symptom experience and self-efficacy for managing side effects. The qualitative interviews showed that the participants found the materials acceptable and helpful. Conclusions: This selfmanagement intervention has the potential to improve both adherence rates and quality of life in breast cancer survivors taking tamoxifen. The intervention booklet appears to be acceptable and feasible in this population. Larger scale trials are needed to establish the efficacy of the materials.

CORRESPONDING AUTHOR: Zoe Moon, King's College London, zoe.moon@kcl.ac.uk

O19.1

SUPPORTING MAINTENANCE OF SUGAR-SWEETENED BEVERAGE REDUCTION USING AUTOMATED VERSUS LIVE TELEPHONE SUPPORT: FINDINGS FROM A RANDOMIZED CONTROL TRIAL

Jamie Zoellner ${ }^{1}$, Wen You ${ }^{2}$, Paul Estabrooks ${ }^{3}$, Yvonnes Chen ${ }^{4}$, Brenda Davy $^{2}$, Kathleen Porter ${ }^{1}$, Valisa Hedrick ${ }^{2}$, Anglea Bailey ${ }^{5}$, Natalie Kružliaková ${ }^{2}$

${ }^{1}$ The University of Virginia- USA

${ }^{2}$ Virginia Tech- USA

${ }^{3}$ University of Nebraska Medical Center- USA

${ }^{4}$ University of Kansas- USA

${ }^{5}$ Bridgewater State University- USA

Background: Although reducing sugar-sweetened beverage (SSB) intake is an important behavioral strategy to improve health, no known SSBfocused behavioral trial has examined maintenance of SSB behaviors after an initial reduction. Guided by the RE-AIM framework, this study examines 6-18 month and 0-18 month individual-level maintenance outcomes from an SSB reduction trial conducted in a medically-underserved, rural Appalachia region of Virginia. Reach and implementation indicators are also reported. Methods: Following completion of a 6-month, multicomponent, behavioral RCT to reduce SSB intake (SIPsmartER condition vs. comparison condition), participants were further randomized to one of three 12-month maintenance conditions. Each condition included monthly telephone calls, but varied in mode and content: 1) interactive voice response (IVR) behavior support, 2) human-delivered behavior support, or 3) IVR control condition. Assessments included the BEVQ15 , weight, BMI, and quality of life. Call completion rates and costs were tracked. Analysis included descriptive statistics and multilevel mixedeffects linear regression models using intent-to-treat procedures. Results: Of 301 subjects enrolled in the 6-month RCT, 242 (80\%) were randomized into the maintenance phase and $235(78 \%)$ included in the analyses. SIPsmartER participants maintained significant 0-18 month decreases in SSB. For SSB, weight, BMI and quality of life, the 6-18 month changes were not significant among SIPsmartER participants, indicating post-program maintenance. The IVR-behavior participants reported greater improvement in SSB kcals/day during the 6-18 month maintenance phase, compared to the IVR control participants $(-98,95 \% \mathrm{CI}=$ $196,-0.55, \mathrm{p}<0.05$ ); yet the human-delivered behavior condition was not significantly different from either the IVR-behavior condition $(27,95 \%$
$\mathrm{CI}=-69,125)$ or davIVR control condition $(-70,95 \% \mathrm{CI}=-209$, 64). Call completion rates were similar across maintenance conditions (4.2-4.6 out of 11 calls); however, loss to follow-up was greatest in the IVR control condition. Approximated costs of IVR and human-delivered calls were remarkably similar (i.e., $\$ 3.15 /$ participant/month or $\$ 38 /$ participant total for the 12-month maintenance phase), yet implications for scalability and sustainability differ. Conclusion: Overall, SIPsmartER participants maintained improvements in SSB behaviors. Using IVR to support SSB behaviors is effective and may offer advantages as a scalable maintenance strategy for real-world systems in rural regions to address excessive SSB consumption. Clinicaltrials.gov; NCT02193009.

CORRESPONDING AUTHOR: Jamie Zoellner, The University of Virginia, jz9q@virginia.edu

\section{O19.2}

IMPACT OF CALORIE LABELLING IN WORKSITE CAFETERIAS: A STEPPED WEDGE RANDOMISED CONTROLLED PILOT TRIAL Milica Vasiljevic ${ }^{1}$, Emma Cartwright ${ }^{2}$, Mark Pilling ${ }^{1}$, Mei-Man Lee ${ }^{2}$, Giacomo Bignardi ${ }^{1}$, Rachel Pechey ${ }^{1}$, Gareth J. Hollands ${ }^{1}$, Susan A. $\mathrm{Jebb}^{2}$, Theresa M. Marteau ${ }^{1}$

${ }^{1}$ University of Cambridge- United Kingdom

${ }^{2}$ University of Oxford- United Kingdom

Introduction and Purpose: For working adults, about one-third of energy is consumed in the workplace making this an important context in which to reduce energy intake to tackle obesity. The aims of the current study were first, to identify barriers to the feasibility and acceptability of implementing calorie labelling in preparation for a larger trial, and second, to estimate the potential impact of calorie labelling on energy purchased in worksite cafeterias. Methods: Six worksite cafeterias were randomised to the intervention starting at one of six fortnightly periods, using a stepped wedge design. The intervention comprised labelling all cafeteria products for which such information was available with their calorie content (e.g. " 250 calories") displayed in the same font style and size as for price. A post-intervention survey with cafeteria patrons and interviews with managers and caterers were used to assess the feasibility and acceptability of the intervention. Intervention impact was assessed using generalised linear mixed modelling. The primary outcome was the total energy (kcal) purchased from intervention items in each cafeteria each day. Results: Recruitment and retention of worksite cafeterias proved feasible, with post-intervention feedback suggesting high levels of intervention acceptability. Several barriers to the implementation of calorie labelling were identified. There was no overall effect of the intervention: $-0.4 \%$ (95\% CI -3.8 to $2.9, \mathrm{p}=.803)$. One site showed a statistically significant effect of the intervention, with an estimated $6.6 \%$ reduction $(95 \% \mathrm{CI}-12.9$ to $-0.3, \mathrm{p}=.044)$ in energy purchased in the day following the introduction of calorie labelling, an effect that diminished over time. The remaining five sites did not show robust changes in energy purchased when calorie labelling was introduced. Conclusions: A calorie labelling intervention was acceptable to both cafeteria operators and customers. The predicted effect of labelling to reduce energy purchased was only evident at one out of six sites studied. Before progressing to a full trial, the calorie labelling intervention needs to be optimised, and a number of operational issues resolved. Trial Registration: ISRCTN52923504

CORRESPONDING AUTHOR: Milica Vasiljevic, University of Cambridge, milica.vasiljevic@medschl.cam.ac.uk

\section{O19.3}

THE HELPMEDOIT! WEIGHT LOSS TRIAL: MAIN RESULTS Sharon Simpson ${ }^{1}$, Lynsay Matthews ${ }^{1}$, Juliana Pugmire ${ }^{1}$, Laurence Moore $^{1}$

${ }^{1}$ University of Glasgow- United Kingdom 
Abstract: Introduction: Helpmedoit! is an innovative weight loss intervention using a website and app that harnesses social support by enabling participants to nominate friends and family to help them. The aim of the study is to test the feasibility of the intervention in supporting adults with obesity to achieve weight loss goals. Methods: Feasibility randomised controlled trial and process evaluation. Participants were adults interested in losing weight, BMI $\geq 30 \mathrm{~kg} / \mathrm{m} 2$. The intervention group (and their helpers) had access to the app/website. Participants nominated one or more helpers to support them. The control group received a leaflet on healthy lifestyle. Follow-up data were collected at 12 months, focussing on the progression criteria for the trial and the feasibility of delivering the intervention and the trial methods. The study assessed: (i) recruitment and retention; (ii) usability and acceptability of the app/website and (iii) three primary outcomes: BMI, physical activity (7 Day PAR and accelerometer) and diet (DINE and multiple-pass 24 hour recall) for use in a full trial. Statistical analyses focussed on feasibility outcomes and initial estimates of effects. Results: 109 participants were recruited and 84 (77\%) were retained at 12 months. Three quarters of the participants used the app at least once and over half used it three times or more. Technical problems with the app in the initial stages hampered engagement. However, qualitative data indicated that the intervention was acceptable and participants liked the intervention concept. The primary outcome measures were feasible to use apart from the multiple pass 24 hour dietary recall which was not collected at follow-up. Conclusions: The intervention was acceptable to participants and the trial methods feasible. The progression criteria were achieved which supports progression to a full trial. Social support is crucial in helping people achieve and sustain lifestyle behaviour change and this intervention is a novel way of mobilising that support.

CORRESPONDING AUTHOR: Sharon Simpson, University of Glasgow, sharon.simpson@glasgow.ac.uk

\section{O19.4}

IMPACT OF THE STATEWIDE HEALTHY INCENTIVES PROGRAM Tamara Calise ${ }^{1}$, . Laura Ruggiero ${ }^{1}$, Natalie Spitzer ${ }^{1}$, Steve Schaffer ${ }^{1}$, Chloe Wingerter ${ }^{1}$

JSI Research and Training Institute- United Kingdom

Introduction \& Purpose. The local food environment has been recognized as a determinant of food access and diet quality. Numerous strategies have been implemented to improve access to healthy foods including farmers market initiatives. Efforts to increase low-income consumers' purchasing power, such as incentive programs are promising in regards to individual behaviors. Moreover, increasing access to, and purchasing of, locally grown fruits and vegetables, has the potential to also impact the local economy. Farmers markets, farm stands, mobile markets (MM), and community supported agriculture programs (CSAs) may be particularly beneficial because they offer local healthy fruits and vegetables and are increasingly becoming more equipped to accept Supplemental Nutrition Assistance Program (SNAP) benefits. Massachusetts (MA) has a robust and growing local food economy anchored by a broad regional network of community partners, including "buy local" organizations, farm organizations, and non-profits. Although SNAP redemption at farmers markets has increased, it is still a small fraction of the overall $\$ 1.2$ billion dollars redeemed at MA retailers each year. Even a small increase in redemption at farmers markets, farm stands, MM, and CSAs could increase access to healthy foods for households receiving SNAP benefits and boost economic activity for local farms. Yet, limited capacity to conduct outreach, processing fees, and technology has made it difficult for these retailers to process SNAP benefits. The Healthy Incentives Program (HIP) is a statewide initiative that provides a $\$ 1$ to $\$ 1$ match to SNAP recipients when they purchase fruits and vegetables from Massachusetts' farmers at farmers markets, farm stands, MMs, and CSAs. The purpose of this study was to assess whether HIP improved access to local, healthy fruits and vegetables among SNAP recipients, increased sales at the targeted retail types, and impacted both HIP-and non-HIP authorized retailer. Methods. The evaluation included qualitative and quantitative methods across multiple sectors. At the individual-level, we analyzed SNAP household demographics. At the retailer-level, we analyzed SNAP sales, HIP earnings and redemptions, and surveyed both HIP-and non-HIP authorized retailers on their perceptions of HIP. We utilized Geographic Information Systems mapping to explore accessibility, population need and utilization of HIP. We geocoded and mapped the farmer's markets, farm stands, MMs, and CSA pick-up sites, sales data, and de-identified aggregate totals for SNAP household locations. In addition, we looked up hours and days of operation. Retailer survey data were cleaned and analyzed in SAS. Results. Saturdays were the most popular retail day resulting in an average of 604 available hours across all HIP-approved retailers. $48 \%$ of all SNAP clients lived within 1 mile of a HIP retailer in September 2017 up from just 5\% in April. A higher percentage of Hispanic and minority households lived closer to a HIPretailer compared to households who identify as White (.35 miles vs. .81 miles). Within the first 10 months of HIP, over $\$ 2.3$ million were given in incentives. The average value earned per transaction was $\$ 14.72 . .19 \%$ of the value of HIP earned was redeemed at a HIP-authorized retailer. While the roll out of HIP proved challenging, both HIP and non-HIP authorized retailers felt the HIP program had a positive impact.

CORRESPONDING AUTHOR: Tamara Calise, John Snow Inc., tcalise@jsi.com,

\section{O19.5}

A COMPARISON OF CARDIOVASCULAR RISK PROFILE, PHYSICAL ACTIVITY AND DIETARY BEHAVIOURS OF THE FOOD-INSECURE OBESE VERSUS FOOD SECURE NON-OBESE: LONGITUDINAL DATA FROM THE STOP-SA STUDY

Kufre Okop ${ }^{1}$, Naomi Levitt ${ }^{1}$, Thandi Puoane ${ }^{2}$, Feyisayo OdunitanWayas $^{1}$, Estelle V. Lambert ${ }^{1}$

${ }^{1}$ University of Cape Town- South Africa

${ }^{2}$ University of the Western- South Africa

Introduction and purpose: Two-thirds of the South African population is overweight, or obese, and half of these are food insecure. This study describes the CVD risk profiles of the food insecure obese, and their physical activity levels and dietary intake patterns in comparison to the food secure non-obese in economically disadvantaged communities. Methods: This is a longitudinal study involving 800 adults (212 men, 588 women) aged 29-78 year from the harmonized STOP-SA study cohort. Only participants with baseline and follow-up anthropometric measurements and relevant cardiovascular disease (CVD) risk factors we considered. The mean follow-up duration was 4.5 years. Data were collected using validated questionnaires and study protocols. Food insecure obese (FIO) was defined as those with BMI $>25 \mathrm{~kg} / \mathrm{m} 2$ who affirmed that they had not enough food to eat or meet their household nutrition needs at least once in the past 12 months. CVD risk factors considered included systolic blood pressure (SBP), body fat percentage (BF\%), body mass index (BMI), reported diabetes, hypertension and smoking. Absolute 10year CVD risk scores were determined based on non-laboratory Framingham's risk score equations. Dietary behaviours recorded include self-reported intake of sugar-sweetened beverages (SSBs) in servings/ week, patterns of intake of snacks/takeaway, fruits and vegetables, and meat obtained using food frequency questionnaires. Moderate-tovigorous physical activity (MVPA) was recorded using International Physical Activity Questionnaire and with an accelerometer device. Comparisons of the outcome variables were undertaken using ANOVA and student t-tests. Multivariate GLM was also used to determine the effects of exposure variables on SBP controlling for sex and age. Results: The FIO compared to the food secure non-obese persons have similar adverse CVD risk factors and absolute CVD risk scores $(15.1 \%$ vs. $16.3 \%)$; SBP (136.8 vs. $134.9 \mathrm{~mm} \mathrm{Hg}$ ) and DBP (88.6 vs. $88.2 \mathrm{~mm}$ 
$\mathrm{Hg}$ ) at follow-up, and significantly higher mean (SD) BMI (35.3 (8.5) vs 29.1 (9.4) p=0.001), BF\% (46.2 (10.7) vs. 37.9 (14.0), p=0.001). Significant proportions of FIO had lower Total MVPA quantiles levels, Total work MVPA, Total Leisure MVPA, daily intake of vegetable and fruits, but higher intake of SSBs (10.9 vs. 9.5 servings/week, $p=0.025$ ) and snacks/take-aways. Controlling for age and sex, daily fruits and vegetables intake separately was associated with low SBP in the FIO whereas SSBs was associated with high SBP levels at follow-up. Conclusion: The juxtaposition of obesity and food insecurity in poor South Africans could lead to a high disposition to cardiovascular disease risk factors including raised blood pressure levels.

CORRESPONDING AUTHOR: Kufre Okop, University of Cape Town, kufreokop@gmail.com

\section{O19.6}

DOCTORS CAN PROMOTE HEALTHY BEHAVIORS IN PATIENTS WITH OBESITY BY ENGAGING IN SUPPORTIVE DISCUSSIONS ABOUT WEIGHT

Lydia Hayward $^{1}$, Lenny Vartanian ${ }^{1}$, Sammantha Neang ${ }^{1}$

${ }^{1}$ University of New South Wales Sydney- Australia

People who are overweight and obese frequently report experiencing weight stigma and this is associated with a range of harmful health consequences. Little is known about how this plays out in health-care settings and how doctors' conversations with their patients about weight impact health motivation and compliance with recommendations to make lifestyle changes. We asked 334 people who are overweight or obese to imagine themselves as the patient in one of three scenarios describing a doctor-patient interaction. In one scenario, the doctor stigmatized the patient about their weight; in another, the doctor discussed weight in a supportive, empathetic manner. A control scenario described a conversation about a wrist injury. Significant ANOVAs were found for positive and negative affect, motivation to engage in health behaviors, positive perceptions of the doctor, willingness to comply with the doctor's recommendations, and willingness to visit the doctor again in future, Fs $>57.23$, ps $<.001, \eta 2>.25$. Participants who imagined a supportive interaction with the doctor rated the doctor more positively and reported greater desire to visit them again, as well as greater motivation to engage in health behaviors than control (all ps <.004). Participants in the supportive condition also reported just as much positive affect and were just as willing to comply with the doctor's recommendations as control participants. Participants who imagined a stigmatizing interaction reported more negative and less positive affect, rated the doctor less positively, and reported lower willingness to comply with the recommendations and visit the doctor again in future (all ps $<.001$ ). Mediation models revealed that experiencing a stigmatizing interaction led to harmful consequences by reducing positive affect (bs $<-.51,95 \%$ CIs [-29.62, -.38]). Overall, these findings suggest that doctors can have constructive conversations with their patients about weight, improving motivation to engage in health behaviors and encouraging high compliance, without damaging the doctor/patient relationship. However, a stigmatizing conversation about weight appears to harm the doctor/patient relationship and reduce the likelihood of making recommended lifestyle changes.

CORRESPONDING AUTHOR: Lydia Hayward, University of New South Wales Sydney, lydia.hayward@unsw.edu.au

\section{O20.1}

REASONS TO ABSTAIN FROM ALCOHOL USE AMONG MEDICALLY VULNERABLE YOUTH

Lauren Wisk $^{1,2}$, Kara Magane ${ }^{1}$, Sharon Levy ${ }^{1,2}$, Elissa Weitzman ${ }^{1,2}$

${ }^{1}$ Boston Children's Hospital

${ }^{2}$ Harvard Medical School
Abstract: Introduction \& Purpose: Increasing numbers of youth globally live with a chronic illness. These youth use alcohol at levels similar to their healthy peers but are at higher risk for adverse outcomes related to their condition. Little is known about motivating reasons to abstain or limit use among this group - important information for developing, tailoring and targeting preventive interventions. We examine patterns of reasons to abstain (RTA) from alcohol use among chronically ill youth and evaluate how RTA patterns impact use behaviours. Methods: Eligible youth with a chronic medical condition (YCMC) ages 9-18 years at one of 5 clinics completed a survey about their alcohol use behaviours and attitudes. YCMC rated the importance of each of 14 RTA items. Respondents' RTA were examined using cluster analysis, to discern similarities and differences among sub-groups of youth. Clusters were examined for differences in alcohol use using multivariate regression analyses controlling for demographics. Results: Among 515 participants $(76.7 \%$ response), $30.5 \%$ reported past year alcohol use, of which $38.9 \%$ consumed alcohol in the past month. Past year drinkers consumed 15.9 drinks during the past 3 months, on average. We identified an eight-cluster solution with dominating patterns of RTA ranging from high concern about health, concerns about addiction, to moderate concerns across multiple domains. Multivariate analyses revealed that compared to those in the "ambivalent" cluster, those with greater concern for the effects of drinking had lower total volume of alcohol consumption $(\mathrm{p}<0.05)$, while YCMC most concerned about their reputation had both lower alcohol consumption $(\mathrm{p}<0.05)$ and lower odds of past month use (OR:0.18; 95\%CI:0.03-0.97). Conclusions: Reasons to abstain from alcohol are multifactorial for YCMC. Strong beliefs regarding risks or harms may have a protective effect. Alternatively even modest concerns across several domains may be associated with lesser consumption and interventions that encourage youth to contemplate the intersection of alcohol use and health may be impactful.

CORRESPONDING AUTHOR: Lauren Wisk, Boston Children's Hospital and Harvard Medical School, lauren.wisk@childrens.harvard.edu

\section{$\mathrm{O} 20.2$}

REACTIONS TO THE RETURN OF AGGREGATE RESEARCH RESULTS - A CATALYST FOR ENGAGING CLINICAL COHORTS IN RESEARCH AND SHARED DECISION-MAKING Elissa R Weitzman $^{1,2}$, Kara Magane ${ }^{2}$, Lauren E Wisk ${ }^{1,2}$

${ }^{1}$ Harvard Medical School- USA

${ }^{2}$ Boston Children's Hospital- USA

Introduction \& Purpose: Interest is growing in engaging patients in sharing patient-reported outcomes (PRO) to improve understanding of disease and treatment. Yet questions remain about how to encourage and sustain PRO sharing. A novel strategy involves collecting PROs and returning them as cohort summaries or "aggregate research results" (ARR), which may be helpful and disquieting. We sought to understand the impacts of returning ARR on motivation for research participation, experience of harm/benefit, and planned actions. Methods. PROs were obtained about children in a national disease registry from parents, summarized as ARR in text and graphic displays and returned. Parents $(\mathrm{N}=111)$ were surveyed on their reactions to and the perceived value of ARR. Reaction patterns were summarized using principal components analysis and associations among patterns and interest in research participation and planned actions estimated using multivariate logistic regression. Results. Parents had children who were: average age $12.0(\mathrm{SD}=3.6)$, female $(79.7 \%)$, white/non-Hispanic $(75.2 \%)$, average disease duration 7.7 years $(\mathrm{SD}=3.5)$; most parents $(71.3 \%)$ had some college education. Endorsement of ARR for better understanding their child's condition and decision-making was high: the proportion rating ARR as "very valuable" was $44.2 \%$ to $77.3 \%$ across ten topics. Most (52.3\%) parents reported being more interested in participating in research after viewing ARR, with the remainder unchanged. Reactions to ARR reflected experiences of 
Validation/Affirmation and Information Burden, which varied with educational status. Parents with higher Validation/Affirmation scores had increased odds of reporting heightened interest in research participation (AOR: 1.97, 95\%CI: 1.18-3.30) while higher Information Burden scores were associated with decreased odds of planned discussions with their child (AOR: 0.59, 95\%CI: 0.36-0.95) and increased odds of planned discussions with providers (AOR: 1.75, 95\%CI: 1.02-3.00). Conclusions. Returning ARR may foster a "virtuous cycle" of research engagement and affect planned actions to share and discuss ARR in support of a child's disease care and treatment. Experience of ARR varied including by educational status, with implications for equipoise and disparities.

CORRESPONDING AUTHOR: Elissa R Weitzman, Harvard Medical School, Boston Children's Hospital, elissa.weitzman@ childrens.harvard.edu

\section{O20.3}

DO HEALTH ORIENTED SUMMER SCIENCE PROGRAMS INFLUENCE KNOWLEDGE, ATTITUDES, AND BEHAVIOR? Patrice Saab ${ }^{1}$, Mary Kate Clennan ${ }^{1}$, Daniella Carucci ${ }^{1}$, Maria Llabre ${ }^{1}$, Marissa Alert ${ }^{2}$, Sylvia Perez ${ }^{3}$, Judy Brown ${ }^{4}$

${ }^{1}$ University of Miami- USA

${ }^{2}$ Johns Hopkins University- USA

${ }^{3}$ New York Hall of Science- USA

${ }^{4}$ Patricia and Phillip Frost Museum of Science- USA

Although considerable effort has been directed at improving children's health behaviors, many children continue to be physically inactive and have poor eating habits. Innovative approaches to facilitate and encourage healthy lifestyles are warranted. Promoting interest in health by enhancing science engagement has the potential to give children a scientific understanding of the value of a healthy lifestyle for their own health. To this end, we examined the influence of health-oriented summer science enrichment programs (that incorporated interactive science and health activities) on knowledge, attitudes, and behavior in middle school-aged girls. The participants were 375 girls (mean age 11.8 years; $92.5 \%$ ethnic minority) randomized to 1 of 2 summer programs (GROOVE or VW+ GROOVE) that were identical in health and science content addressing nutrition, physical activity, and healthy lifestyle but differed in whether virtual world (VW) technology was used to reinforce concepts. Didactic lessons were interspersed with hands-on health-oriented activities, physical activity (e.g., dance), and group collaborations. Summer programs lasted 3 weeks and were conducted at science museums in Miami, FL and Queens, NY from 2013-2016. Girls completed self-report measures at baseline and at the summer program's end. Measures included science self-efficacy, health knowledge, eating behavior, physical activity, and sedentary activity. For the intention-to-treat-analyses examining the effects of condition (GROOVE, VW+GROOVE) and site (FL, NY), maternal education, parent marital status, age, ethnicity, program year, and percent attendance, and body mass index were covaried. Relative to the VW+GROOVE condition, the girls in the GROOVE condition reported greater increase in science-self efficacy $(\mathrm{F}(1,361)=7.298, \mathrm{p}=.007)$ and physical activity $(\mathrm{F}(1,360)=5.0736, \mathrm{p}=.025)$ but no change in sedentary activity. Girls in both conditions modestly increased their health knowledge $(\mathrm{t}(374)=8.63, \mathrm{p}<.001)$, and significantly decreased their unhealthy eating behavior $(\mathrm{t}(374)=-4.91, \mathrm{p}<.001)$. Secondary analyses underscored the importance of attendance on program outcomes. Controlling for aforementioned covariates and condition and site, greater percent attendance was associated with a greater increase in physical activity $(b=1.359)$ and health knowledge $(b=1.47)$. The findings support the view that engaging health-oriented summer science educational experiences can have a health benefit as well as an educational benefit. This work suggests that such programs have the capacity to increase understanding, influence attitudes, promote healthy lifestyle behaviors, and serve as prevention strategies in youth. As such, future efforts need to be directed at examining the potential of educational activities to ameliorate the risk for later cardiometabolic conditions and reduce health disparities.

CORRESPONDING AUTHOR: Patrice Saab, University of Miami, psaab@miami.edu

\section{O20.4}

BARRIERS AND ENABLERS TO STRUCTURED TYPE 1 DIABETES EDUCATION FOR ADOLESCENTS: THE TEAM T1 EXPERIENCE

Virginia Hagger ${ }^{1}$, Eileen Collins ${ }^{2}$, Kim Duggan-Larkin ${ }^{2}$, Christel Hendrieckx ${ }^{1}$, Jane Speight ${ }^{2}$

${ }^{1}$ Deakin University- Australia

${ }^{2}$ Diabetes Victoria- Australia

Introduction \& purpose: Teens Empowered to Actively Manage Type 1 diabetes (TEAM T1) is a structured type 1 diabetes education program, adapted from the adult DAFNE course (Dose Adjustment for Normal Eating) for adolescents aged $14-18$ years and their parents. As part of a national initiative, funding was provided to 10 diabetes centres (regional and metropolitan hospitals) to train facilitators (22 diabetes nurse educators and dietitians) and to deliver (in total) 40 TEAM T1 programs. Some centres achieved better uptake than others; three centres were unable to complete the expected number of programs while others were able to deliver more. The aim of this study was to investigate the barriers and enablers to implementing TEAM T1. Methods: Researchers invited TEAM T1 facilitators to take part in a semi-structured, in-depth interview exploring perceived benefits, challenges, barriers and enablers to implementing TEAM T1 in their centre. Interview transcripts were analysed thematically. Results: Eight facilitators, representing seven centres, were interviewed. Four main themes emerged regarding the perceived benefits of TEAM T1: learning about diabetes; peer support; empowerment; self-management. Facilitators identified professional development; practice change; and improved relationships as personal benefits. Three themes captured the perceived barriers and enablers to implementation of TEAM T1: - management and team support: facilitators were allowed time to administer programs, other team members reinforced TEAM T1 principles; $\bullet$ resources and administration: facilitators had access to funds and technology; • recruitment was most successful when: parents were engaged; paediatricians and other team members were supportive; TEAM T1 was incorporated into the care pathway (e.g. when management was sub-optimal, or pre-pump). Conclusions: Facilitators identified benefits of TEAM T1 for adolescents, parents and their own professional development. However, providing structured education in a hospital setting required redesign of the way self-management education was delivered and resourced. Successful implementation of TEAM T1 needed support from the healthcare team, in particular paediatricians and managers, and alignment with usual care.

CORRESPONDING AUTHOR: Virginia Hagger, Deakin University, virginia.hagger@deakin.edu.au

\section{O20.5}

EFFECTIVENESS OF THE HEALTHY HIGH SCHOOL STUDY ON PERCEIVED STRESS AMONG HIGH SCHOOL STUDENTS: A CLUSTER RANDOMIZED CONTROLLED TRIAL

Camilla Thørring Bonnesen ${ }^{1,2}$, Naja Hulvej Rod ${ }^{1,3}$, Stine Kjær Wehner ${ }^{1,2}$, Marie Pil Jensen ${ }^{1,2}$, Mette Toftager ${ }^{1,2}$, Rikke Fredenslund Krølner ${ }^{1,2}$

${ }^{1}$ National Institute of Public Health- Denmark

${ }^{2}$ University of Southern Denmark- Denmark

${ }^{3}$ University of Copenhagen- Denmark

Introduction \& Purpose: The prevalence of low well-being, perceived stress and unhealthy behaviors is high among Danish high school 
students, but few intervention trials have been aimed at reducing these problems. The Healthy High School Study (HHSS) was developed to promote well-being (primary outcome) by preventing 1) stress and improving 2) sleep, 3) sense of community, 4) regular movement and 5) regular meals among Danish high school students. The purpose of the present study was to assess the effect of the HHSS on self-reported stress among first-year students at the end of the school year in which the intervention was delivered. Methods: The HHSS employed a clusterrandomized controlled trial design. High schools were randomized into either the intervention $(n=15)$ or control group with no intervention $(n=15)$, and a total of 2,975 students were nested within these schools. The HHSS comprised two educational and two environmental components: 1) curricular activities, 2) the HHSS app, 3) compulsory organizational and environmental changes at the school level, and 4) a peer-led innovation workshop aiming at inspiring students to initiate and participate in a broad range of activities that focused on increasing movement and sense of community. Information on perceived stress was gathered through self-reported questionnaires at baseline (August 2016) and follow-up (May 2017) and measured by the 10 item Perceived Stress Scale. Data was analyzed by multilevel linear regression analyses and were adjusted for perceived stress at baseline, sex and family occupational class. Results: At baseline, the mean PSS-10 score was 13.9 and 14.1 for students at intervention and control schools, respectively. There were no statistically significant differences in perceived stress among students after the intervention in the two groups ( $\beta=0.54$, CI 95\%: 0.19-1.26). Adjusting for sociodemographic factors did not change the results. Conclusions: This study did not show any effect of the intervention on perceived stress. Future analyses will reveal the importance of missing data and implementation fidelity as well as the effect on other outcomes. More research is needed to identify effective interventions to prevent stress among high school students.

CORRESPONDING AUTHOR: Camilla Thørring Bonnesen, National Institute of Public Health, University of Southern Denmark, catb@ sdu.dk

\section{O20.6}

CHANGING PARENT'S INFANT FEEDING BEHAVIOURS TO PREVENT CHILDHOOD OBESITY: IDENTIFYING IMPORTANT OUTCOME MEASURES

Karen Matvienko-Sikar ${ }^{1}$, Molly Byrne ${ }^{2}$, Colette Kelly ${ }^{2}$, Elaine Toomey ${ }^{2}$, Marita Hennessy ${ }^{2}$, Declan Devane ${ }^{2}$, Caroline Heary ${ }^{2}$, Janas Harrington ${ }^{1}$, Niamh McGrath ${ }^{1}$

${ }^{1}$ University College Cork- Ireland

${ }^{2}$ National University of Ireland Galway- Ireland

Changing parent's infant feeding behaviours to prevent childhood obesity: Identifying important outcome measures K. Matvienko-Sikar1, M. Byrne2, C. Kelly3, E. Toomey2, M.Hennessy2, D. Devane4, C. Heary2, J. Harrington1, N. McGrath1, M. Queally5, P.M. Kearney1 1. School of Public Health, University College Cork 2. School of Psychology, National University of Ireland Galway 3. Discipline of Health Promotion, School of Health Sciences, National University of Ireland, Galway 4. School of Nursing and Midwifery, National University of Ireland Galway 5. Discipline of Economics, National University of Ireland, Galway Introduction and Purpose: Parent's infant feeding behaviours are implicated in the aetiology of childhood obesity. Lack of standardisation of behaviour change outcomes in trials of infant feeding interventions limits examination of intervention effects and mechanisms of change. Core outcome sets (COS) represent the agreedupon minimum outcomes that should be measured in trials of a specific health condition. The aim of this research is to identify core infant feeding outcomes for children $\leq 1$ year, to be evaluated in childhood obesity intervention trials. Methods: Identification of core infant feeding outcomes was conducted in the following stages: (1) systematic review identifying all infant feeding outcomes in the extant literature, (2) small group expert stakeholder meeting to clarify and discuss outcomes identified in the systematic review, (3) prioritisation of outcomes using the e-Delphi technique with an international panel of expert stakeholders. Stakeholders in stages 2 and 3 were: Researchers, Healthcare Professionals, Parents, and Childcare Professionals. Results: The systematic review identified 82 infant feeding outcomes. These were categorised into the following 9 outcome domains: 'breast and formula feeding', 'introduction of solids', 'parent feeding practices and styles', 'parent knowledge and beliefs', 'practical feeding', 'food environment', 'dietary intake', 'perceptions of infant behaviour and preferences', and 'child weight outcomes'. Outcomes were discussed and clarified with 13 stakeholders at the small group meeting and additional outcomes, not captured in the systematic review, were added at this stage. The e-Delphi study was completed by 169 international stakeholders. Thirty-three outcomes from 8 domains were identified as crucial for inclusion in the COS; no 'practical feeding' outcomes were considered crucial for inclusion. Conclusions: The COS development process facilitated identification of 33 outcomes considered essential by expert stakeholders for inclusion in trials of infant feeding interventions to prevent childhood obesity. Identification of core infant feeding outcomes is necessary to inform development and standardised evaluation of future trials of infant feeding interventions to prevent childhood obesity.

CORRESPONDING AUTHOR: Karen Matvienko-Sikar, University College Cork, karen.msikar@ucc.ie

\section{$\mathrm{O} 21.1$}

EFFORT-REWARD IMBALANCE AT WORK AND GLYCEMIC CHANGES IN THE BRAZILIAN LONGITUDINAL STUDY OF ADULT HEALTH (ELSA-BRASIL)

Raíla Santos ${ }^{1}$, Rosane Griep ${ }^{2}$, Maria de Jesus Fonseca ${ }^{1}$, Fabiola Eto ${ }^{1}$, Enirtes Melo ${ }^{1}$

Escola Nacional de Saúde Pública (ENSP) - FIOCRUZ- Brazil

${ }^{2}$ Oswaldo Cruz Institute- Brazil

Introduction \& Purpose This study intends to evaluate the association between psychosocial stress at work according to the Effort-Reward Imbalance model and changes in glycemic levels. The relationship between stressful working conditions and worker health has been increasingly demonstrated in the literature. Several theoretical models have been proposed to evaluate the deleterious effects of stress on the work environment on health, one of them is the Effort-Reward Imbalance model (Siegrist et al., 2009). According to this model, the individual's excessive effort to the demands imposed by the work and low rewards perceived to the dedicated effort provokes sustained stress reactions that, in the long run, can have serious consequences for health, such as cardiovascular diseases, changes in glycemic levels and type 2 diabetes ( $\mathrm{Li}$ et al., 2013; Siegrist et al., 2009). Methods The Brazilian Longitudinal Study of Adult Health (ELSA - Brasil) is a multicenter study. In this transversal cut, 10.665 active workers were selected at first follow-up visit. Job stress was evaluated through the Brazilian version of the Effort-Reward Imbalance model. Glycemic levels were assessed through glycosylated haemoglobin $(\mathrm{HbAlc})$. We calculated odds ratio (OR) with respective 95\% of confidence intervals (CI) and multiplicative interactions for overcommitment and education level. The study was approved by the National Research Ethics Commission. Result For women with overcommitment, the high effort is associated with altered glycemia (OR 1.72, 95\% CI 1.09-2.72) and women with overcommitment and low educational level, the low reward is associated with intermediate glycemia (OR $1.48,95 \%$ CI 1.15-1.9) and altered (OR 1.78, 95\% CI 1.03-3.08). Among women of overcommitment, there is an association between effort-reward imbalance at work and intermediate glycemic levels (OR $1.28,95 \%$ CI 1.04-1.57) and altered glycemic levels (OR 2.54, 95\% CI 1.42-4.54). For men, no significant associations were found. Conclusion The relationship between effort-reward imbalance at work and changes in 
glycemic levels was mediated by overcommitment that stands out as a determining factor for glycemic changes at intermediate (pre-diabetes) and elevated (diabetes) levels among women.

CORRESPONDING AUTHOR: Raíla Santos, Escola Nacional de Saúde Pública (ENSP) - FIOCRUZ, railappc@gmail.com

\section{O21.2}

PYSCHOSOCIAL JOB FACTORS ASSOCIATED WITH BURNOUT AND HEALTH IN INFORMAL STREET VENDORS IN MORELOS, MÉXICO

Arturo Juárez García ${ }^{1}$, Cinthya Anamia Flores -Jiménez ${ }^{1}$, Kimberly Sánchez-Santana ${ }^{1}$

${ }^{1}$ Universidad Autónoma del Estado de Morelos- Mexico

Introduction: Even though the workforce in Latin-American region is mainly characterized by informal work, few studies have been carried out in order to explore job psychosocial exposure and health-related issues. Whereas there are no standardized and validated questionnaires to explore specific psychosocial stressors in informal work, the objective of this study is to explore with a mixed method strategy (qualitative and quantitative) the psychosocial stressors and motivators in informal street vendors and to explore their associations with burnout and health outcomes. Methods: 332 informal street vendors from local markets and small shop stores in Cuernavaca Morelos México were recruited to obtain information about psychosocial factors and health. Open questions were used to explore psychosocial stressors and were qualitatively and quantitatively categorized. Association of this factors with burnout (MBI), mental health problems (GHQ) and diagnosed diseases (self-report) were tested. Results: The most common stressors were: poor physical environment, dealing with customers and low sales. The most common motivators were comfort and pleasure, autonomy, gratifying tasks, sharing with others and attending clients. $40 \%$ of informal workers had high levels of burnout and 26\% were "cases" of poor mental health. Digestive (30\%) and musculoskeletal $(28 \%)$ disorders were the most frequent diseases. Logistic regressions showed a significant association between "dealing with customers" and burnout and in the other hand, "gratifying tasks" and "autonomy" were protector variables for burnout. "Attending clients" was a protector for mental health problems and sharing with others with respiratory and digestive disorders. Conclusion: Given the prevalence of mental illnesses and diseases in this sample, it seems that urgent interventions are needed in this sector. Interestingly, many motivators had a substantial role as protective factors. Very specific stressors were associated to health problems, and these stressors are poorly included in standardized questionnaires of job stress. Further studies interested in developing psychosocial measures in informal workers must consider these findings. Also, more studies are needed to understand the relationship between psychosocial risks factors and health in informal workers.

CORRESPONDING AUTHOR: Arturo Juárez García, Universidad Autónoma del Estado de Morelos, arturojuarezg@hotmail.com

\section{$\mathrm{O} 21.3$}

UNEMPLOYMENT AND MENTAL HEALTH: THE ROLE OF SUBJECTIVE SOCIAL STATUS IN THE GERMAN POPULATION Marie Neubert ${ }^{1}$, Frank Euteneuer ${ }^{1}$, Winfried Rief ${ }^{1}$, Philipp Süssenbach ${ }^{2}$ ${ }^{1}$ Philipps University Marburg- Germany

${ }^{2}$ University of Applied Sciences- Germany

Subjective social status (SSS) reflects individuals' perceived position in a social hierarchy. Low SSS is associated several health impairments. The aim of this study was to test if unemployed individuals report lower SSS in Germany (national SSS) and lower SSS in their social community (local SSS). We further examined whether SSS is associated with mental health and employment status. We included 113 unemployed and 1117 employed individuals of working age from the innovation sample of the German Socio-Economic Panel survey (SOEP-IS). SSS was assessed with the German version of the MacArthur Scales. Objective status was defined by the net equivalent income. Mental health was measured using the mental component scale (MCS) of the SF-12. Unemployed individuals reported significantly lower national SSS, local SSS and mental health (all $\mathrm{p}<$ .001) compared with employed participants. Lower national SSS was associated with lower mental health in the unemployed sample, $r=.224, \mathrm{p}=$ .021. A conceptual model revealed that national SSS mediates the association between employment status $(B=-0.600,95 \%$ CI [-1.154, -0.245], p $=.008)$ and mental health. Results indicate that unemployment is associated with lower SSS and lower mental health. The perceived position relative to others in the country (i.e., national SSS) mediates the association between employment status and mental health. Future studies should investigate this mediating role of SSS in a long-term survey.

CORRESPONDING AUTHOR: Marie Neubert, Philipps University Marburg, marie.neubert@uni-marburg.de

\section{O21.4}

SAFETY BEHAVIOR DURING PATIENT TRANSFER AND RISK OF BACK INJURIES AMONG NURSES: PROSPECTIVE COHORT STUDY

Lars Andersen ${ }^{1}$,

${ }^{1}$ National Research Centre for the Working Environment- Denmark

The nurse occupation has a high proportion of back injuries during patient transfer. Consistent use of assistive device during patient transfer is considered a basic safety behavior, and may reduce the risk for back injuries. However, the protective effect of specific types of assistive devices is largely unknown. This prospective cohort study among 3000 Danish nurses investigates the association between use of different types of assistive devices and risk of back injury. Every other week during one year, the nurses fill in a questionnaire about use of patient transfers, use assistive devices, and back injuries. Prospective associations between use of assistive devices (predictor) and risk of back injury (outcome) will be modelled using logistic regression. At baseline, $11 \%$ of the nurses reported that a back injury had occurred during patient transfer during the last 12 months. Only $1 \%$ used assistive devices during every patient transfer, and $40 \%$ never used assistive devices. Data collection for the 1-year follow-up is finished in March 2018, and the prospective association between use of assistive devices and risk of back injury will be presented. The study will contribute with knowledge about good safety behavior during patient transfer and the risk of back injuries.

CORRESPONDING AUTHOR: Lars Andersen, National Research Centre for the Working Environment, 1la@nrcwe.dk

\section{O21.5}

INFORMATION EXCHANGE USING A PRESCRIBED FORM AND INVOLVEMENT OF OCCUPATIONAL HEALTH NURSES PROMOTES OCCUPATIONAL PHYSICIANS TO COLLABORATE WITH ATTENDING PHYSICIANS FOR SUPPORTING WORKERS WITH ILLNESS

Kazuhito Yokoyama ${ }^{1}$

${ }^{1}$ Juntendo University Faculty of Medicine- Japan

INTRODUCTION \& PURPOSE: The maintenance of a balance between work and disease treatment is an important issue in Japan. This study explored factors that affect collaboration between occupational physicians (OPs) and attending physicians (APs).

METHODS: A questionnaire was mailed to 1,102 OPs. The questionnaire assessed the demographic characteristics of OPs; their opinions and 
behaviors related to collaboration, including the exchange of medical information with APs; and the occupational health service system at their establishments.

RESULTS: In total, 275 OPs completed the questionnaire $(25.0 \%$ response rate). Over $80 \%$ of respondents believed OPs should collaborate with APs. After adjusting for company size, collaboration $>10$ times/year (with regard to both returning to work following sick leave and annual health check-ups for employees) was significantly associated with environmental factors, such as the presence of occupational health nurses (OR $=5.56$ and 5.01, respectively, $\mathrm{p}<0.05)$ and the use of prescribed forms for information exchange $(\mathrm{OR}=4.21$ and 3.63 , respectively, $\mathrm{p}<0.05)$ but not with the demographic characteristics of the OPs $(p>0.05)$.

CONCLUSIONS: The majority of OPs believed that collaboration with APs is important for supporting workers with illnesses. Support systems including prescribed forms of information exchange and occupational health nurses, play pivotal roles in promoting this collaboration.

CORRESPONDING AUTHOR: Kazuhito Yokoyama, Juntendo University Faculty of Medicine, kyokoya@juntendo.ac.jp

\section{O21.6}

OVERCOMMITMENT AS MEDIATOR BETWEEN EFFORT AND STRESS IN FACULTY PROFESSORS

Mariantonia Lemos ${ }^{1}$, Tatiana Roldan-Rojo ${ }^{1}$, Melissa Valencia ${ }^{1}$, Gabriela Calle-Gonzalez ${ }^{1}$, Isabel Goez ${ }^{1}$, Juan Pablo Román-Calderon ${ }^{1}$

${ }^{1}$ Universidad EAFIT- Colombia

Introduction: The effort-reward imbalance (ERI) model claims that a job with high levels of responsabilities for the employee and with low rewards could lead to stress, emotional symptoms and cardiovascular disease. In addition, overcommitment has been taken as a factor that moderates this relationship. Nowadays, working as faculty professor implies that more than an instructor, a professor has the responsibility to guide his students. This study looks forward to examine Siegrist's model in order to explain stress levels in faculty professors. Method: The study has been taken with 61 professors (34\% women) at a Colombian university. Professors completed the ERI questionnaire and the Perceived Stress Scale at the beginning (T1) and end (T2) of an academic period. Results: The structural equation modeling approach showed strong time-invariance for the stress measure, comparing average stress at two different time points, including the imbalance and the overcommitment scores as a covariates of stress in both time points, $\chi 2(79, \mathrm{~N}=62)=$ $106.30, p>.05 ; \mathrm{CFI}=.91 ; \mathrm{RMSEA}=.07$. Stress decreased with time $(\mathrm{Mt} 2=-.41, \mathrm{p}<.01)$ and both measures resulted to be positively correlated $(\mathrm{r}=.63, \mathrm{p}<.01)$. The results indicate that overcommitment mediates the effect of imbalance score on stress at both time points; and the imbalance score exerted a positive indirect effect on stress (bt1 $=.32, \mathrm{p}<.01$; $\mathrm{bt} 2=.33, \mathrm{p}<.01)$. The model explained $26 \%$ and $36 \%$ of the variance of stress at time 1 and 2, respectively. Conclusions: the ERI model is adequate to explain stress in faculty professors. Over commitment is a key variable to address in health interventions in order to reduce stress levels.

CORRESPONDING AUTHOR: Mariantonia Lemos, Universidad EAFIT, mlemosh@eafit.edu.co

\section{$\mathrm{O} 22.1$}

THE FIVE DIMENSIONS OF ADHERENCE TO TREATMENT: A LITERATURE REVIEW

Luisa Andrea Castro Díaz ${ }^{1}$

${ }^{1}$ Integridad Laboral- Colombia

Abstract Introduction: Health loss is one of the aspects that have the major impact on human well-being. Nowadays, it is striking that despite the fact that there is greater coverage and quality in health services, rates of diseases increase, generating significant repercussions at the individual and collective levels. In this context, an explanatory framework associated with the concept of adherence emerges, on which a considerable interest has recently been set. Objectives: Carry out a review focused on deepening and complementing conceptually, the adherence to treatment construct and its dimensions. Methodology: Having as conceptual basis the classification established by the World Health Organization (WHO), scientific literature between 2003 and 2017 was revised to address the core issues of this research. Results: The transversal elements of adherence to treatment and its dimensions were updated. Likewise, were complemented the notion of this concept with key aspects that hasn't been taken into account yet, Conclusions: This analysis added relevant content about the dimensions associated with modifiable factors, linked directly to the the field of action of health psychology and its fundamental role in different areas of health care. These findings allows to provide an input that leads to optimize programs aimed at promoting and intervening the adherent behaviors of people.

CORRESPONDING AUTHOR: Luisa Andrea Castro Díaz, Integridad Laboral,ps.luisa97@gmail.com

$\mathrm{O} 22.2$

COMPLIANCE TO TREATMENT OF DUTCH ASYMPTOMATIC INDIVIDUALS AFTER SCREENING FOR CARDIOVASCULAR DISEASE IN THE ROBINSCA-TRIAL

Sabine Denissen ${ }^{1}, \mathrm{CM}$ van der Aalst ${ }^{1}, \mathrm{MA}$ van Aerde $^{1}, \mathrm{M}_{\text {Vonder }}{ }^{2}, \mathrm{M}$ Oudkerk $^{2}$, HJ de Koning ${ }^{1}$

${ }^{1}$ Public Health department- Netherlands

${ }^{2}$ Centre for Medical Imaging North-East Netherlands

Introduction: Screening for cardiovascular diseases (CVD) is a promising strategy to identify potential high-risk individuals and consequently start preventive treatment to reduce CVD morbidity and mortality. The population-based Risk Or Benefit IN Screening for CArdiovascular disease (ROBINSCA)-trial investigates whether screening for a high risk for developing CVD will reduce coronary heart disease (CHD) by at least $15 \%$ compared to no screening. This study investigated the prevention-seeking behavior and compliance to treatment of ROBINSCA-participants. Methods: Asymptomatic Dutch individuals $(n>43,000)$, men aged $45-74$ years and women aged 55-74 years, were randomized $(1: 1: 1)$ to: 1) control arm (usual care), 2) intervention arm A (CVD screening by traditional risk assessment) and 3 ) intervention arm B (CVD screening by means of determining the amount of coronary artery calcification). A random sample $(\mathrm{n}=700 ; 100$ in each of the six risk stratification categories in both intervention arms and 100 in the control arm) of ROBINSCAparticipants received an online questionnaire to measure preventionseeking behavior after receiving the screening result (in intervention arms A and B) or the randomization result (in the control arm) and to measure compliance to subsequent preventive treatment. Results and conclusion: We expect all results to be ready in summer 2018. The overall response of participants was $71 \%$ (497/700). In intervention arm A, 86 of $137(62.8 \%)$ participants with an elevated CVD risk visited their general practitioner (GP) of which 39 (45.3\%) had the wish to reduce the CVD risk. There were 140 out of $149(94 \%)$ participants with an elevated CVD risk in intervention arm B that visited their GP and $86(61.4 \%)$ of them had the wish to reduce the CVD risk. Preventive CVD medication was prescribed for $38 / 86$ $(44.2 \%)$ and $108 / 140(77.1 \%)$ of the elevated-risk participants of intervention arms A and B respectively, and compliance was $97.4 \%$ and $97.2 \%$. Further analyses will provide more information on motives for (not) seeking prevention. Trial registration number: NTR6471

CORRESPONDING AUTHOR: Sabine Denissen, Public Health department, s.denissen@erasmusmc.nl 


\section{$\mathrm{O} 22.3$}

WHAT ARE THE EFFECTIVE ASPECTS OF ADHERENCE INTERVENTIONS FOR INHALED CORTICOSTEROIDS IN CHILDREN WITH ASTHMA?: A SYSTEMATIC REVIEW

Christina Joanne Pearce ${ }^{1}$, Amy Chan ${ }^{1}$, Rob Horne ${ }^{1}$, Louise Fleming ${ }^{2,3}$, Andrew Bush ${ }^{2,3}$, Tracy Jackson ${ }^{4}$

${ }^{1}$ University College London- United Kingdom

${ }^{2}$ The Royal Brompton Hospital- United Kingdom

${ }^{3}$ Imperial College London- United Kingdom

${ }^{4}$ The University of Edinburgh- United Kingdom

Introduction \& Purpose: Although asthma is often manageable by preventer medicines, such as inhaled corticosteroids (ICS), child asthma deaths still occur every day. Poor adherence to ICS contributes to poor control, severe exacerbations and hospitalisations in patients with asthma. This systematic review aimed to: 1 . Synthesise the literature focusing on interventions in children with asthma 2. Determine the behaviour change techniques (BCTs) which were used in effective interventions 3. Evaluate how well the interventions fitted with the Perceptions and Practicalities Approach (PAPA) to intervention design Methods: This systematic review was conducted by searching key databases including: PubMed, PsychINFO and Web of Science for randomised control trial (RCT) interventions in children with asthma which measured adherence in ICS. A narrative review was conducted due to high heterogeneity in the studies included. Results: Of 170 papers found, 18 were eligible and included in the review. Ten studies were effective and nine studies mapped fully with the PAPA. The BCTs used in effective interventions were: Pharmacological support as part of a complex intervention; Feedback and monitoring; Association-prompts; Natural consequences; Instruction on how to perform a behaviour; Goal setting and action planning, and Rewards when tailored to the patient. Conclusions: The systematic review found that interventions to influence adherence to ICS in children with asthma were more successful when interventions are tailored to address both perceptions and practical factors, and when using BCTs such as feedback and monitoring, association-prompts, and rewards.

CORRESPONDING AUTHOR: Christina Joanne Pearce, University College London, christina.pearce.15@ucl.ac.uk

\section{$\mathrm{O} 22.4$}

LONGITUDINAL EFFECT OF ANXIETY AND DEPRESSIVE SYMPTOMS ON ART-ADHERENCE IN NEWLY DIAGNOSED PEOPLE LIVING WITH HIV

Ivonne Nalliely Pérez-Sánchez ${ }^{1,2}$, Nancy Patricia Caballero-Suárez ${ }^{2}$, Evelyn Rodríguez Estrada ${ }^{2}$, Gustavo Reyes-Terán ${ }^{2}$

${ }^{1}$ CONACYT- Mexico

${ }^{2}$ INER: CIENI- Mexico

Longitudinal effect of anxiety and depressive symptoms on ART-adherence in newly diagnosed people living with HIV Authors. Ivonne Nalliely Pérez Sánchez1,2, Nancy Patricia Caballero Suárez1, Evelyn Rodríguez Estrada1, Gustavo Reyes-Terán1. Affiliations. 1. Departamento de Investigación de Enfermedades Infecciosas (CIENI) of Instituto Nacional de Enfermedades Respiratorias (INER) 2. Consejo Nacional de Ciencia y Tecnología (CONACYT) Background. Few studies have asses the relation of depression and anxiety symptoms with antiretroviral treatment (ART) adherence in newly diagnosed people living with HIV (PLWH). Our objective was to explore the effect of anxiety and depressive symptoms on ART-adherence in newly diagnosed PLWH. Methods. We interviewed newly diagnosed ( $<6$ months) PLWH on more than one month of ART at initial presentation and we reassessed to them 6 months later at the Center for Research in Infectious Diseases (CIENI) in Mexico City from February 2016 to August 2017. All participants completed Beck's Anxiety (BAI) and Depressive (BDI) Inventories and the Assessment of Adherence to Antiretroviral Therapy Questionnaire (CEAT-VIH). Written consent was provided. Sperman correlations and Wilcoxon test analyses were calculated using SPSS 20.0. Results.
114 PLWH were interview, 36 have started ART, 21 had more than month on ART and 14 completed both basal and 6-moth follow up evaluations. Of these 14 patients, most of them were male $(85.7 \%, \mathrm{n}=12)$, single $(64.2 \%$, $\mathrm{n}=9$ ), with median age of 33 (28.75-40.25) years. Depressive symptoms were present in $57.1 \%$ at baseline (50\% had mild and $7.1 \%$ had moderate intensity) vs $14.2 \%$ at 6 month evaluation (7.1\% with mild and $7.1 \%$ moderate intensity). Anxiety symptoms were reported by $21.4 \%$ at baseline $(14.2 \%$ with mild, $7.14 \%$ with moderate intensity) and were similar at 6 moths follow up. ART adherence was adequate to strict in $71.4 \%$ of patients, both at baseline and follow-up evaluation. There were not significant differences in Wilcoxon test analyses between baseline and six month-follow-up for anxiety, depression or ART-adherence scores. Both anxiety $(\mathrm{r}=-.666, \mathrm{p}=.036)$ and depression $(r=-.728, p=.017)$ were negatively correlated with ART-adherence at baseline but not at six month-follow-up. Conclusions. Depressive and anxiety symptoms were associated with ART-adherence only at baseline, while ART adherence did not change by the time. Our results could suggest the influence of different variables on ART adherence. But more studies with a larger sample size are needed to confirm these results.

CORRESPONDING AUTHOR: Ivonne Nalliely Pérez-Sánchez, CONACYT/ INER: CIENI, ivonne.perez@ cieni.org.mx

\section{O22.5}

PATIENT'S EXPERIENCE OF THE DOCTOR-PATIENT RELATIONSHIP: ARE YOU TALKING TO ME?

Susana Aronsohn ${ }^{1}$, Rodrigo Rojas ${ }^{1}$

${ }^{1}$ Universidad Academia de Humanismo Cristiano- Chile

The WHO (2003) has reported 50\% adherence of patients to medical prescriptions in chronic illnesses. Clues for understanding such results have been sought widely in the doctor-patient relationship with over 8000 publications. All studies report on the exploration of selected and objective aspects of the interaction. Specific factors have been addressed, communication skills, psychological aspects, patient and doctor characteristics and strategies and questionnaires have been developed. Reparatory measures have also been taken such as training doctors in specific skills. However, low compliance persists as a major problem. Information for non chronic illnesses is not available and will be addressed qualitatively in the present study. Subjective aspects have been much more neglected. Chalmers in 1994 refers to the subjective aspect as experience while Nagel (1974) had already highlighted the difficulty of understanding any objective meaning of experience. Drawing on the distinction between paradigmatic thought - logical characterization of the world - and narrative thought - meaning ascribed to experience in narrative, we addressed the later in our study. Narrative inquiry was used for in depth interviews in an intensive sample of 3 non chronic patients chosen for the salience of their adherence stories to a specific treatment, addressing the initial visit to a medical doctor or dentist. Our objective was to unravel the 1st person perspective of patients in a face-to-face interaction with their doctors. Results showed remarkably diverse and personal narrative plots deriving in final compliance or noncompliance, suggesting that standardized prescriptions for medical behavior in doctor-patient relationships cannot guarantee optimum outcomes.

CORRESPONDING AUTHOR: Susana Aronsohn, Universidad Academia de Humanismo Cristiano, capehuen@gmail.com

\section{O22.6}

FACTORS ASSOCIATED WITH MEDICATION ADHERENCE IN PATIENTS WITH MAJOR DEPRESSIVE DISORDER

Carolina Baeza-Velasco ${ }^{1,2}$, Emilie Olié ${ }^{2}$, Séverine Béziat ${ }^{2}$, Sebastien Guillaume $^{2}$, Philippe Courtet ${ }^{2}$

${ }^{1}$ University Paris Descartes-Sorbonne Paris Cité- France

${ }^{2}$ INSERM U1061- France 
Authors: Carolina Baeza-Velasco1,2, Emilie Olié 2,3, Séverine Béziat2, Sébastien Guillaume2,3, Philippe Courtet2,3 1 Laboratory of Psychopathology and Health Processes, University Paris Descartes Sorbonne Paris Cité, Boulogne Billancourt, France. 2 INSERM U1061, Neuropsychiatry: Epidemiological and Clinical Research, Department of Emergency Psychiatry and Acute Care, CHU Montpellier, France 3 University of Montpellier 1, Montpellier, France. Abstract Introduction/ Purpose: It is well known that in several cases Major Depressive Disorder is a chronic condition which requires a long-term pharmacologic treatment. Despite the efficacy of available antidepressants, the medication adherence in those affected is usually very poor. In this scenario, further research is needed concerning drivers of non-adherence in this highly prevalent condition. The aim of this work was to explore medication adherence in patients with Major Depressive Disorder, and identify sociodemographic, clinical (psychiatric antecedents, comorbidities, medication, pain), and psychosocial factors (negative life events, childhood trauma) related to low adherence. Method: Three hundred and seventyfour people diagnosed with Major Depressive Disorder responded to the Medication Adherence Rating Scale, and then were divided into two groups according to adherence levels: low and high. Sociodemographic, clinical and psychosocial variables were compared between the groups. Results: Seventy six percent of participants $(n=284)$ showed a low level of medical adherence. There were no differences concerning sociodemographic factors between high and low adherents. Selfreported depression severity, physical and psychological pain, consumption of anxiolytics, antipsychotics and mood stabilizers drugs, the number of medications, negative life events and antecedents of physical maltreatment in childhood were significantly higher in the low adherent group. Multivariate analyses showed that depression severity, physical pain, antipsychotic consumption and the number of medications are variables that increase the probability of belonging to the low adherent group. Conclusion: These results confirm previous reports showing a low level of medication adherence in patients with Major Depressive Disorder. Clinicians should be vigilant to identify patients with characteristics associated to poor medication adherence revealed by research, in order to propose them interventions aiming to improve this health behavior.

CORRESPONDING AUTHOR: Carolina Baeza-Velasco, University Paris Descartes-Sorbonne Paris Cité / INSERM U1061, baezacarolina@yahoo.com

O23.1

HEALTH AT A ONE-CLICK DISTANCE: SENIOR CITIZENS AND THE INTERNET

Maria Joao Figueiras ${ }^{1}$, David Dias Neto ${ }^{2}$

${ }^{1}$ Instituto Piaget / RECI- Portugal

${ }^{2}$ ISPA - Portugal

Introduction: Internet use is common in the search for health information in the general population. The present study aimed to investigate the extent to which senior citizens use the Internet to obtain health information. Method: 120 subjects aged 60-85 years were asked about sources of health information, how often they were used, what health topics they search and differences in use according to individual characteristics (eg, gender, age, literacy). Results: health professionals are still the main source of information for health topics, although the internet appears as an increasing well-qualified resource for health matters, both for the search, decision-making and for trustworthiness. There were differences in sex and age group in relation to the amount of information collected through television or books and the reliability of information through the internet. Conclusion: Although exploratory, this study contributes to characterize sources of health information and its reliability for senior citizens. The way available resources can facilitate access to information has relevant implications for behaviour and decision-making related to health care in aging.
CORRESPONDING AUTHOR: Maria Joao Figueiras, Instituto Piaget / RECI, figueirasmar@gmail.com

\section{$\mathrm{O} 23.2$}

ASSOCIATION BETWEEN MULTIMORBIDITY AND HEALTHRELATED QUALITY OF LIFE AMONG OLDER ADULTS: IMPACTS OF FUNCTIONAL DEPENDENCE AND DEPRESSIVE SYMPTOMS

Rui She ${ }^{1}$, Zhongrui Yan ${ }^{2}$, Joseph Lau ${ }^{1}$, Chengxuan Qiu ${ }^{3}$

${ }^{1}$ The Chinese University of Hong Kong- China

${ }^{2}$ Jining No. 1 People's Hospital- China

${ }^{3}$ Karolinska Institutet- Sweden

Introduction \& purpose: Multimorbidity, a common condition in older people, is associated with health-related qualify of life (HRQL). We examined the associations of multimorbidity patterns with HRQL among rural-dwelling older adults in China, and explored to what extent their associations were mediated by functional dependence and depressive symptoms. Methods: This population-based study included 1497 adults (age $\geq 60$ years, $66.4 \%$ women) who participated in the 2 nd wave examination of the Confucius Hometown Aging Project in Shandong (20142016). Data on demographics, lifestyles, and health history were collected through interviews, clinical examinations, and laboratory tests. We assessed functional status with basic activities of daily living (ADL) and HRQL with EQ-5D-3L. Data were analyzed using factor analysis and multiple logistic regression models. Results: Multimorbidity $(\geq 2$ chronic diseases) was present in over $80 \%$ of participants. We identified four patterns of multimorbidity, characterized by cardiovascular-degenerative, respiratory, neurological, and metabolic disorders. These patterns of multimorbidity had various impacts on HRQL and its dimensions. ADLdisability and depressive symptoms were significantly associated with EQ-5D-3L index. The association between multimorbidity and HRQL was diluted when taking into account ADL-disability and depressive symptoms. Conclusions: Multimorbidity is highly prevalent and is associated with poor HRQL in older adults, and their associations are partly mediated by functional dependence and geriatric depressive symptoms.

CORRESPONDING AUTHOR: Rui She, The Chinese University of Hong Kong, sherryshe0319@link.cuhk.edu.hk

\section{$\mathrm{O} 23.3$}

HEALTHY DIETARY CHANGES IN MIDLIFE ARE ASSOCIATED WITH A REDUCED DEMENTIA RISK LATER IN LIFE

Shireen Sindi ${ }^{1}$, Ingemar Kåreholt ${ }^{1}$, Marjo Eskelinen ${ }^{2}$, Babak Hooshmand $^{1}$, Hilkka Soininen ${ }^{2}$, Tiia Ngandu ${ }^{3}$, Miia Kivipelto ${ }^{1}$

${ }^{1}$ Karolinska Institute- Sweden

${ }^{2}$ University of Eastern- Finland

${ }^{3}$ National Institute for Health and Welfare- Finland

Background: Diet is an important modifiable lifestyle factor related to dementia risk. Specific dietary patterns or nutritional components are associated with a reduced risk for dementia. It is however unknown whether midlife healthy dietary changes are associated with dementia risk. Objective: To investigate whether healthy dietary changes in midlife are associated with dementia risk later in life, using a population-based cohort study. Methods: Data were collected from participants in The Cardiovascular Risk Factors, Aging, and Dementia (CAIDE) population-based cohort study $(n=2000)$ (baseline mean age 50 years). Participants returned for two re-examinations in late-life (mean age 70 and 78 years). 1511 subjects participated in at least one re-examination (mean total follow-up 22 years). Midlife diet was measured in a subsample $(n=535)$ using a food frequency questionnaire. Dietary change in the past year was measured in midlife using questions specific components (modifying quality of fats, increasing vegetables, decreasing sugar, 
decreasing salt). Detailed examinations were carried out for dementia diagnoses. Analyses adjusted for potential confounding factors. Results: Total midlife healthy dietary changes (modifying quality of fats, increasing vegetables, decreasing salts and sugars) were associated with a reduced risk for dementia (fully adjusted OR $0.42,95 \%$ confidence interval $(C I)=0.18-0.96)$. In contrast when each factor was assessed individually, they were not significantly associated with dementia risk. Conclusions: This study is the first to show that midlife dietary changes are associated with a reduced dementia risk later in life. The results highlight the importance of targeting dietary patterns, where various food items may have synergistic effects.

CORRESPONDING AUTHOR: Shireen Sindi, Karolinska Institute, shireen.sindi@ki.se

\section{O23.4}

\section{QUALITY OF LIFE IN CAREGIVERS OF ALZHEIMER DISEASE} PATIENTS

Maria da Graça Pereira ${ }^{1}$, Daniela Rego ${ }^{1}$, Sara $_{\text {Lima }}{ }^{1}$, Ana R. Abreu ${ }^{1}$ University of Minho- Portugal

Introduction: The present study assessed the relationship between family functioning, social support, spirituality, patient's behavior and memory problems and quality of life in caregivers of Alzheimer Disease patients. taking also in consideration whether the caregiver chose or not intentionally the caregiver's role. Method: The sample includes 102 caregivers who answered the following instruments: Depression, Anxiety and Stress Scale-21; Satisfaction with Social Support Scale, Revised Memory and Behavioral Problems Checklist, Family Adaptability and Cohesion Evaluation Scales; Spiritual and Religious Attitudes in Dealing with Illness and the Quality of Life in Alzeimer's Disease (Caregiver Version). Results: Caregivers who chose the caretaker's role were more satisfied with their family functioning, family communication, and showed high QoL compared to those who did not intentionally decide to caregive. Age, psychological morbidity, social support, family functioning and patient's behavior problems predicted caregiver's quality of life. Conclusion: Interventions should include family communication skills and spirituality given its moderator role in the promotion of caregiver's quality of life.

CORRESPONDING AUTHOR: Maria da Graça Pereira, University of Minho,gracep@psi.uminho.pt

\section{O23.5}

CORRELATES AND DETERMINANTS OF QUALITY OF LIFE IN CHILEAN PEOPLE WITH DEMENTIA

Claudia Miranda-Castillo ${ }^{1}$

${ }^{1}$ Pontificia Universidad Católica de Chile- Chile

Introduction and purpose: Assessing quality of life $(\mathrm{QoL})$ in dementia is important because it leads to a better understanding of the impact that dementia has on individuals at different stages of the disease (Black and Rabins, 2005). Since QoL per se has a subjective character, the basic principle must be to obtain the perception of the people with dementia (PWD) about their QoL whenever possible. Evidence shows that PWD are capable of rating their QoL with adequate reliability and validity (Thorgrimsen et al., 2003). This is true even for people with severe dementia (Hoe et al., 2005). There is lack of evidence regarding people with dementia's QoL in Latin America. Several studies from developed countries have already related PWD's quality of life with PWD's cognition and functional impairment (Ready et al., 2002, Gonzalez-Salvador et al., 2000; Woods et al., 2006), unmet needs (Miranda-Castillo et al., 2010), behavioral and psychological disturbances (Banerjee et al., 2006) and depressive symptoms (Thorgrimsen et al., 2003; Woods et al., 2006;
Hoe et al., 2006). The aim of this study was to identify correlates and determinants of QoL in Chilean older adults with dementia. Methods: This was a cross-sectional study. One-hundred and twenty-four PWD and their informal caregivers were interviewed. PWD was assessed about cognitive function, needs and QoL. Their caregivers answered both, instruments about PWD (functional status and behavioral and psychological symptoms) and also about themselves (burden, depression, anxiety and social support). A stepwise multiple linear regression analysis was performed to determine predictors of QoL of Chilean people with dementia. Results: People with dementia with a longer period since diagnosis $(\mathrm{rs}=.246 ; \mathrm{p}<.01)$, a better functional status $(\mathrm{rs}=.203 ; \mathrm{p}<.05)$ and those who have less unmet needs ( $r s=-.345 ; \mathrm{p}<.01)$ had a higher QoL. Also, PWD who were looked after by caregivers with high level of burden ( $\mathrm{rs}=-.255$; $\mathrm{p}<.01)$ showed a lower QoL. Finally, a low number of unmet needs $\mathrm{p}<.001$ and a good functional status $(\mathrm{p}<.01)$ explained $35 \%$ of PWD's quality of life $(\mathrm{F}=15.607 ; \mathrm{p}<.01)$. Conclusions: In order to improve PWD's quality of life, it is necessary to address their unmet needs. Within this sample, the most common unmet needs of PWD were located in psychological and social areas, such as "Daily activities" (39.2\%), "Company" (36.1\%), and "Memory" (34.9\%); followed by "Information about the disease" $(27.1 \%)$ and "Psychological distress" $(21.1 \%)$. In addition, the fact that PWD's quality of life was associated to caregiver's burden highlights the importance of considering both, PWD and their informal caregivers, as targets for institutional support. It is expected that national public policies, which are currently being piloted, decrease PWD's number of unmet needs ultimately improving PWD's quality of life. This research was supported by the National Fund for Scientific and Technological Development (FONDECYT) Project $\mathrm{N}^{\circ} 1141279$ and 11100457 and the Fund for Innovation and Competitiveness (FIC) of the Chilean Ministry for the Economy Development and Tourism, through the Millennium Scientific Initiative, grant number IS130005.

CORRESPONDING AUTHOR: Claudia Miranda-Castillo, Pontificia Universidad Católica de Chile, camirand@uc.cl

\section{O23.6}

CULTURAL ENGAGEMENT AS A HEALTH BEHAVIOUR: LONGITUDINAL ASSOCIATIONS BETWEEN VISITING MUSEUMS \& GALLERIES AND COGNITION \& DEMENTIA IN OLDER AGE

Daisy Fancourt $^{1}$, Andrew Steptoe ${ }^{1}$, Dorina Cadar ${ }^{1}$

${ }^{1}$ University College London- United Kingdom

Introduction and Purpose: Museums and art galleries ('museums') number c.40,000 in Europe, USA and Canada and reach diverse geographical populations and socioeconomic groups, however they are currently underutilised for public health interventions. Theories of cognitive reserve and disuse syndrome have suggested that activities that are mentally engaging, enjoyable, stress-reducing and socially interactive could be protective against cognitive decline and the development of dementia. However, it remains unknown whether visiting museums could be neuroprotective. Methods: This study used data from the English Longitudinal Study of Ageing assessed between 2004/5 and 2014/15. Phase A (n=3,468) used OLS regression models to explore associations between visiting museums and change in cognitive function over 10 years amongst adults aged 50+. Phase B $(n=3,946)$ used Poisson regression to calculate the Incidence Rate Ratio (IRR) of dementia incidence across the 10 years. Both phases controlled for baseline cognition as well as all identified demographic confounders, health confounders (including sensory impairment, depression, vascular conditions) and social confounders (including other forms of community engagement).

Results: For Phase A, independent of confounders, visiting museums once/twice a year or more was associated with a lesser decline in both memory $(\beta=0.60 \pm 0.15$, CI $0.30-0.90)$ and executive function $(\beta=1.06 \pm$ 
0.31, CI 0.47-1.66). For Phase B, independent of confounders, going to museums every few months or more was associated with lower dementia incidence (IRR $=0.56 \pm 0.12$, CI $0.37-0.87$ ). For both phases, sensitivity analyses confirmed effects were unaffected by considerations of mobility, attrition or pre-clinical symptoms of dementia.

Conclusions: In light of the results presented here, it is suggested that further behavioural intervention studies are designed to test further whether museum engagement can decrease the risk of developing dementia for older adults

CORRESPONDING AUTHOR: Daisy Fancourt, University College London, d.fancourt@ucl.ac.uk

O24.1

THE MEDIATING EFFECT OF SOCIAL SUPPORT ON THE ASSOCIATION OF PARTNER INTIMATE RELATIONSHIP AND RESILIENCE AMONG PEOPLE LIVING WITH HIV

Chengbo Zeng ${ }^{1}$, Hanxi Zhang ${ }^{1}$, Jiaying Qiao ${ }^{1}$, Zhimeng $\mathrm{Xu}^{1}$, Yan Alicia Hong $^{2}$, Weiping $\mathrm{Cai}^{3}$, Yan Guo ${ }^{1}$

${ }^{1}$ Sun Yat-Sen University- China

${ }^{2}$ Texas A\&M University- USA

${ }^{3}$ Guangzhou No.8 People's Hospital- China

Introduction \& Purpose: Resilience is an ability to positively cope with stress and recover from stress. Existing studies on partner intimate relationship (PIR), social support, and resilience have typically employed relational analyses demonstrating associations between these concepts, but few have examined the mediating effect and mechanisms among these concepts. Thus, the current study employed structural equation modelling (SEM) to examine the mediating effect of social support and mechanisms among PIR, social support, and resilience in PLWH. Methods: A Cross-sectional survey by convenience sampling was conducted among 411 PLWH between March and June, 2013 in Guangzhou, China. Participants were interviewed about their socio-demographic characteristics, sexual partners, social support (using the MOS-SSS Scale), and resilience (using the CDRISC Scale). PIR is the emotional closeness between PLWH and their sexual partners. SEM was performed to examine the direct and indirect associations between PIR and resilience. Results: Among the 411 PLWH, $55.0 \%$ (226/411) of the PLWH reported to have sexual partners in the past six months; among whom, 36.3\%(82/226) did not have intimate relationships with their sexual partners. The median scores of social support and resilience were 52.6 and 27, respectively. Results of SEM showed a good model fit (Chi-Square $=44.70$, RMSEA $=0.08, \mathrm{CFI}=0.98$, SRMR=0.06). Outcome of SEM indicated that the direct pathway from PIR to resilience was not significant $(\beta=0.07, p=0.33$ ), but indirect pathway between the two factors was significant $(\beta=0.05, p=0.03)$. The results indicated that there was a complete mediating effect of social support on the association between PIR and resilience. Conclusions: Many PLWH did not have intimate relationship with their sexual partners. PLWH who had intimate relationship with their sexual partners were more likely to report increased levels of social support, which in turn were associated with increased levels of resilience. Results of the current study have important implications for future intervention to improve mental health status of PLWH. Interventions to improve the relationship between PLWH and their sexual partners and social support of PLWH have the potential to enhance resilience in PLWH.

CORRESPONDING AUTHOR: Chengbo Zeng, Sun Yat-sen University, zengchb@yahoo.com

$\mathrm{O} 24.2$

COST-EFFECTIVENESS OF INTERVENTIONS TARGETING AT REDUCING HIV AND HCV INFECTIONS AMONG METHADONE MAINTENANCE TREATMENT IN CHINA: A SYSTEM DYNAMIC MODELLING STUDY
Yin Liu ${ }^{1}, \mathrm{Xia} \mathrm{Zou}^{1}$, Yong $\mathrm{Xu}^{1}$, Wen $\mathrm{Chen}^{1}$, Yinghua Xia ${ }^{1}$, Cheng Gong ${ }^{1}$, Li Ling ${ }^{1}$,

${ }^{1}$ Sun Yat-sen University- China

Abstract Aim: Health education, needle exchange program, condom promotion, psychological interventions like contingency management and psychological counseling, and treatment have not been integrated in methadone maintenance treatment (MMT) in China, leading to continuing high HIV and HCV infections among MMT participants. Currently, there is a lack of economic evaluation on these interventions in MMT. This study aims to conduct a modelling study to evaluate the cost-effectiveness of integrating these intervention in MMT to reduce HIV and HCV in China. Methods: This study developed a system dynamic model on MMT system using data from cohort study, cross-sectional survey and literature reviews. The model details have been published elsewhere. We collected the cost data from literatures and cross-sectional surveys. In this study, we hypothesis that all interventions were conducted by an extra clinic staff member. Cost-effectiveness was evaluated using incremental cost effectiveness ratio (ICER) for each intervention method. Results: The health education cost less (2,269.4 US dollars) than the other interventions for per HIV infection averted. Following by needle exchange program (26,294.0 US dollars), condom promotion (64,482.8 US dollars), contingency management (104,017.7 US dollars), and psychological counseling (180,113.6 US dollars) for per HIV averted in 2014-2020. The incremental cost of integrated ART in MMT was 135,147.8 US dollars per $\mathrm{HIV}$ averted. While the intervention for $\mathrm{HCV}$ infections were more costeffective than avert HIV infections. The incremental costs of implementing health education, needle exchange program and condom promotion were 93.9 US dollars, 125.2 US dollars, 422.6 US dollars per HCV averted. While the incremental cost of contingency management and psychological counseling were 3615.4 US dollars and 7246.5 US dollars per HCV averted. The incremental cost of implementing HCV treatment in MMT was 13,647.8 per $\mathrm{HCV}$ averted. Conclusion: Health education was the most cost-effective way to avoid HIV and HCV infections. We promote to implement routine health education targeting at reducing HIV and HCV infections among MMT participants in China.

CORRESPONDING AUTHOR: Yin Liu, Sun Yat-sen University, liuyin2016@126.com

\section{$\mathrm{O} 24.3$}

KNOWLEDGE, SELF-EFFICACY, AND DISCUSSION REGARDING PARENTAL HIV DISCLOSURE AMONG HEALTH CARE PROVIDERS (HCPS) CARING FOR PARENTS LIVING WITH HIV (PLHS) IN CHINA

Wendi $\mathrm{Da}^{1}$, Shan Qiao ${ }^{1}$, Xiaoming $\mathrm{Li}^{1}$, Yuejiao Zhou ${ }^{2}$, Zhiyong Shen ${ }^{2}$

${ }^{1}$ University of South Carolina- USA

${ }^{2}$ Guangxi Center for Disease Control and Prevention- China

Introduction \& Purpose Health care providers (HCPs) play an important role in parental HIV disclosure (i.e., parents disclose their HIV seropositive status to children), yet little is known about whether they're prepared to facilitate disclosure. This study aims to assess the level of knowledge about parental HIV disclosure, self-efficacy for facilitating parental disclosure, and provider-patient discussion regarding parental HIV disclosure among HCPs caring for parents living with HIV (PLHs) in China. Methods In 2013, 379 HCPs were recruited from 44 clinics across 16 cities/counties for an HIV parental disclosure intervention in Guangxi, China. Knowledge and self-efficacy were each measured by a 10-item 4-point Likert scale. Provider-patient discussion was measured using a single item asking whether HCPs had discussed parental HIV disclosure with patients in the past 6 months with a 5-point Likert scale. ANOVA and post-hoc tests with Bonferroni correction were used to compare the differences in mean knowledge and self-efficacy across discussion patterns. ANCOVA adjusting for demographic and occupational characteristics was further conducted. Results The average score was 29.5 ( $\mathrm{SD}=2.8,23-37)$ for 
knowledge and 27.5 ( $\mathrm{SD}=4.4,10-40$ ) for self-efficacy. More than half $(\mathrm{n}=208,56.2 \%)$ of HCPs reported never having parental HIV disclosure discussions, $106(28.6 \%)$ reported "seldom discussed", 38 (10.3\%) reported "discussed sometimes", and 18 (4.9\%) reported "discussed often". For knowledge, no significant difference was found across discussion patterns $(\mathrm{F}=1.60, \mathrm{p}=0.19)$. However, significant difference was found for selfefficacy $(\mathrm{F}=6.60, \mathrm{p}<0.001)$. Compared with HCPs who never discussed parental HIV disclosure issue, those who reported "seldom discussed", "discussed sometimes", or "discussed often" were 1.37 ( $\mathrm{p}=0.047), 2.55$ $(\mathrm{p}=0.005)$, and $2.95(\mathrm{p}=0.032)$ points higher in self-efficacy, respectively, yet no significant difference was found across these three groups. After adjusting for covariates, statistical significance persisted for the "discussed sometimes" $(\beta=2.35, p=0.036)$ and the "discussed often" $(\beta=3.16$, $\mathrm{p}=0.036)$ groups, but not for the "seldom discussed" group $(\beta=1.21$, $\mathrm{p}=0.22$ ). Conclusion Considering the relatively low levels of knowledge and self-efficacy among HCPs, further trainings specifically targeting HIV parental disclosure are needed. Compared with knowledge, self-efficacy might be a stronger facilitator for provider-patient discussion about parental HIV disclosure. Therefore, interventions with a focus on self-efficacy building may be particularly useful to improve such discussion.

CORRESPONDING AUTHOR: Wendi Da, University of South Carolina,dawendi.cool@gmail.com

\section{O24.4}

A STRUCTURAL EQUATION MODEL OF HIV-RELATED STIGMA, POSITIVE COPING, AND PERCEIVED STRESS AMONG PEOPLE LIVING WITH HIV AND DEPRESSION

Mengting Zhu ${ }^{1}$, Chengbo Zeng ${ }^{1}$, Hanxi Zhang ${ }^{1}$, Jiaying Qiao ${ }^{1}$, Zhimeng $\mathrm{Xu}^{1}$, Yiran $\mathrm{Li}^{1}$, Yan Alicia Hong ${ }^{2}$, Weiping $\mathrm{Cai}^{3}$, Linghua $\mathrm{Li}^{3}$

${ }^{1}$ Sun Yat-sen University- China

${ }^{2}$ Texas A\&M University- USA

${ }^{3}$ Guangzhou No.8 People's Hospital- China

Introduction \& Purpose: Studies indicated that perceived stress among people living with HIV (PLWH) was associated with severe mental health problems, reduced quality of life, and high risk behaviors. HIV-related stigma among PLWH is widespread in China. HIV-related stigma and positive coping are associated with perceived stress, yet few studies have examined the mediating effect of positive coping between HIV-related stigma and perceived stress. Baseline data from a randomized controlled trial using $\mathrm{mHealth}$ intervention were analyzed to examine the association between HIV-related stigma and perceived stress and the mediating effect of positive coping so as to provide evidence for future intervention. Methods: A randomized clinical trial is underway in the only designated HIV treatment hospital in Guangzhou, China. Of 1555 PLWH approached and screened for depression with the Center for Epidemiological Studies Depression Scale (CES-D), 300 people living with HIV and depression (PLWHD) with CES-D $\geq 16$ were recruited. The Perceived Stress Scale (PSS), the HIV Stigma Scale (HSS), and the Simplified Coping Style Questionnaire (SCSQ) were used to measure perceived stress, HIV-related stigma, and positive coping, respectively. Structural equation modeling (SEM) was used to examine the direct and indirect associations between HIV-related stigma and perceived stress. Mediating effect of positive coping was also examined in the SEM. Results: Of the 300 participants, 277(92.30\%) participants were male, and the average age of PLWHD was $28.21(\mathrm{SD}=5.83)$ years. The rates of low stress $(0 \leq \mathrm{PSS} \leq 13)$ and moderate stress $(14 \leq \mathrm{PSS} \leq 26)$ and high perceived stress $(27 \leq \mathrm{PSS} \leq 40)$ were $6.3 \%$, $85.3 \%$ and $8.3 \%$, respectively. As hypothesized, results indicated that HIVrelated stigma had significantly direct $(0.293$, C.I.: $0.19 \sim 0.39)$ and indirect effects (0.04, C.I.: 0.00 0.08) on perceived stress. Moreover, positive coping mediated the positive relationship between HIV-related stigma and perceived stress. Higher levels of HIV-related stigma were associated with lower levels of positive coping, which in turn were associated with higher levels of perceived stress. The model fit the data well after controlling for covariates of age and years of HIV infection (Chi-Square $=40.98$, RMSEA $=0.062, \mathrm{CFI}=0.971$,
TLI=0.959, SRMR=0.049). Conclusions: PLWHD experienced a higher level of perceived stress. The results suggest that positive coping can buffer the negative impact of HIV-related stigma on perceived stress. Findings highlight the need for multilevel interventions to reduce HIV-related stigma and enhance positive coping so as to improve psychological wellbeing of PLWHD.

CORRESPONDING AUTHOR: Mengting Zhu, Sun Yat-sen University, 13056751151@163.com

\section{O24.5}

EFFECT OF A MULTICOMPONENT BEHAVIOURAL PMTCT CLUSTER RANDOMIZED CONTROLLED TRIAL ON HIV STIGMA REDUCTION AMONG PERINATAL HIV POSITIVE WOMEN IN MPUMALANGA PROVINCE, SOUTH AFRICA

Karl Peltzer ${ }^{1}$, Suat Babayigit ${ }^{2}$, Violeta J Rodriguez ${ }^{2}$, Jenny Jean ${ }^{3}$, Sibisiso Sibisiso ${ }^{1}$, Deborah L Jones ${ }^{2}$

${ }^{1}$ Human Sciences Research Council- South Africa

${ }^{2}$ University of Miami- USA

${ }^{3}$ University of Rochester- USA

Background: We evaluate the impact a multicomponent, behavioural, prevention of mother to child transmission (PMTCT), cluster randomized controlled trial on HIV stigma reduction among perinatal HIV infected women in rural South Africa. Methods: In a cluster randomized controlled trial, twelve community health centers (CHCs) in Mpumalanga Province, South Africa, were randomized; pregnant women living with HIV enrolled received either: A Standard Care (SC) condition plus time-equivalent attention-control on disease prevention (SC; 6 CHCs; $\mathrm{n}=357$ ), or an Enhanced Intervention (EI) condition of SC PMTCT plus the "Protect Your Family" intervention (EI; 6 CHCs; $n$ =342). HIV-infected pregnant women in the SC attended four antenatal and two postnatal video sessions; those in the EI, four antenatal and two postnatal group PMTCT sessions, including stigma reduction, led by trained lay health workers. Maternal PMTCT, HIV knowledge and HIV related stigma were assessed. The impact of the EI was ascertained on stigma reduction (baseline, 12 months postnatally). A series of logistic regression and latent growth curve models were developed to test the impact of the intervention. Results: In all, 699 women living with HIV were recruited during pregnancy (8-24 weeks), and assessments were completed prenatally at baseline and at 12 months (59.5\%) postnatally. Baseline scores of overall HIV related stigma and the four scale factors decreased at follow-up in the intervention group, while baseline scores of overall stigma and three scale factors increased at follow-up in the control group. Using longitudinal analyses, Model 1, which included time-invariant predictors of stigma assessed over the two time periods of baseline and 12 months, increases in stigma from baseline to 12 months were associated with being unemployed, having been diagnosed with HIV before the current pregnancy, and alcohol use. In Model 2, which included time-varying predictors, lower stigma scores were associated with participation in the intervention, greater male partner involvement, and consistent condom use. Conclusion: The enhanced PMTCT intervention, including stigma reduction, administered by trained lay health workers had a significant effect on the reduction of HIV related stigma.

CORRESPONDING AUTHOR: Karl Peltzer, Human Sciences Research Council, kpeltzer@hsrc.ac.za

O24.6

A STRUCTURAL EQUATION MODEL OF HIV-RELATED STIGMA, PERCEIVED STRESS, AND SUICIDAL STATUS AMONG PEOPLE LIVING WITH HIV AND DEPRESSION IN CHINA

Yiran $\mathrm{Li}^{1}$, Chengbo Zeng ${ }^{1}$, Hanxi Zhang ${ }^{1}$, Jiaying Qiao ${ }^{1}$, Zhimeng $\mathrm{Xu}^{1}$, Mengting $\mathrm{Zhu}^{1}$, Yan Alicia Hong ${ }^{2}$, Weiping $\mathrm{Cai}^{3}$

${ }^{1}$ Sun Yat-Sen University- China

${ }^{2}$ Texas A\&M University- USA

${ }^{3}$ Guangzhou No.8 People's Hospital- China 
Introduction: People living with HIV (PLWH) suffer from severe HIVrelated stigma and face various stressors. Previous studies indicated that HIV-related stigma and perceived stress were positively associated with suicidal status (suicidal ideation and/or suicidal attempts), yet few studies explored the mechanisms among these three concepts. The current study examined the mediating effect of perceived stress from HIV-related stigma to suicidal status in people living with HIV and depression (PLWHD) in China using structural equation modeling (SEM). Methods: An ongoing randomized controlled trial using mHealth (mobile health) intervention to improve mental health of PLWHD started in August, 2017 in Guangzhou, China $(n=300)$. Participants were interviewed on their HIV-related stigma (HIV Stigma Scale), perceived stress (Perceived Stress Scale), suicidal status, and socio-demographic characteristics at baseline. SEM was performed to examine the pathways from HIVrelated stigma and perceived stress to suicidal status controlling for covariates (including employment, years of HIV infection, and affordability of daily or medical expenses) which were significantly associated with suicidal status. Results: At baseline, 44\% (132/300) PLWHD reported suicidal ideation and 9.6\% (29/300) PLWHD reported suicidal attempts in the last three months, resulting in 45\% (135/300) PLWHD in suicidal status. Results of SEM showed a good model fit (Chi-square=27.81, $\mathrm{CFI}=0.96$, RMSEA $=0.04$, WRMR=0.85). In the SEM, HIV-related stigma had significantly direct (pathway coefficient $=0.16$, C.I.: $0.00 \sim 0.32$ ) and indirect (pathway coefficient=0.08, C.I.:0.01 0.15) effects on suicidal status. Perceived stress partially mediated the positive relationship between HIV-related stigma and suicidal status (pathway coefficient $=0.21$, C.I.:0.01 0.24). Conclusion: The rate of suicidal status among PLWHD was high. PLWHD who experienced elevated HIV-related stigma and perceived stress were more likely to have suicidal ideation and attempts. To reduce suicidal status of PLWHD, targeted intervention to reduce HIVrelated stigma and perceived stress are warranted.

CORRESPONDING AUTHOR: Yiran Li, Sun Yat-Sen University, zengchb3@mail2.sysu.edu.cn

\section{O25.1}

MODERATE TO SEVERE DEPRESSION PREDICTS HIGHER RISK SEX IN MEN WHO HAVE SEX WITH MEN (MSM) IN HIGH INCOME COUNTRIES: THEORETICAL IMPLICATIONS FOR BEHAVIOURAL INTERVENTIONS

Jamie Frankis ${ }^{1}$,Paul Flowers ${ }^{1}$, Yvonne $\mathrm{Kerr}^{2}$, Nicky Coia ${ }^{3}$, Lisa $\mathrm{McDaid}^{4}$,

${ }^{1}$ Glasgow Caledonian University- United Kingdom

${ }^{2}$ NHS Lothian- United Kingdom- United Kingdom

${ }^{3}$ NHS Greater Glasgow \& Clyde- United Kingdom

${ }^{4}$ University of Glasgow- United Kingdom

In high income countries (HiC), HIV incidence remains highest amongst MSM. In addition, high levels of depression have also been observed amongst MSM. Meyer's minority stress theory explains how prejudice and discrimination may lead to depressive symptoms amongst MSM, which cognitive escape theory suggests may increase the likelihood of higher risk sex. This paper examines levels of depression amongst MSM in $4 \mathrm{HiC}$ to examine i) which issues are linked with depression and ii) the relationship between depression and higher risk sex. The SMMASH2 study was an online, crosssectional survey of MSM in Scotland, Wales, Northern and the Republic of Ireland, which examined; i) levels of moderate to severe depression (MSD; $P H Q \geq 10$ ), ii) higher risk sex in the last year and iii) sociodemographics. Herein we focus on $n=2399$ HIV-/untested men who were sexually active in the last year. Data were analysed using uni- and multi-variate regression. Overall $25.6 \%$ of men reported MSD and $35.7 \%$ reported higher risk sex. MSD was significantly higher amongst men who reported higher risk sex+, financial worries\#, hazardous drinking*, chemsex drug use+ and illicit drug use in the last year\#; younger ( $\leq 45$ years)\#, students/unemployed\# and single men\#; and men with lower educational qualifications\#. It was not related to recruitment country, ethnicity, sexual orientation or chemsex in the last year. Higher risk sex was significantly higher amongst men who reported financial worries*, hazardous drinking+, chemsex\#, chemsex drug use\# and illicit drug use\# in the last year; younger $(\leq 35 \text { years })^{*}$ and gay identified men\#; and men who were in a relationship\# or out to most people\#. It was not related to recruitment country, ethnicity, qualifications or employment status. In the multivariate model, higher risk sex was significantly predicted by reporting $\mathrm{MSD}(\mathrm{OR}=1.3, \mathrm{CI}=1.1-1.7)^{*}$, being single $(\mathrm{OR}=1.4$, $\mathrm{CI}=1.1-1.7)+$ or being out to most people $(\mathrm{OR}=1.42, \mathrm{CI}=1.1-1.7)+$. In $\mathrm{HiC}$, depression is higher amongst MSM than the general population and related to higher risk sex. This relationship may be mediated by syndemic health issues including hazardous drinking, illicit and sexualized drug use. As such, theory based interventions to address depression amongst MSM are urgently warranted. *0.05 $+0.01 \# 0.001$

CORRESPONDING AUTHOR: Jamie Frankis, Glasgow Caledonian University, j.frankis@gcu.ac.uk

\section{$\mathrm{O} 25.2$}

ASSOCIATIONS BETWEEN SYNDEMIC FACTORS AND THE HIV CONTINUUM OF CARE AMONG YOUNG GAY/BISEXUAL MEN AND TRANSGENDER WOMEN

Brian Mustanski ${ }^{1}$, Daniel Ryan ${ }^{1}$, Michael Newcomb ${ }^{1}$, Richard D'Aquila ${ }^{1}$

${ }^{1}$ Northwestern University- USA

Introduction and purpose: The HIV care cascade begins with testing, and for those who test HIV positive: linkage to HIV medical care, engagement in care, initiation and adherence to antiretroviral therapy (ART), and ultimately achieving viral suppression. Suppressing HIV viral load through daily ART substantially reduces the risk of HIV transmission and simultaneously reduces the harmful health effects of ongoing HIV replication. Prior research has shown that multiple co-occuring psychosocial epidemics ("syndemics") increase risk for HIV infection, but little research has examined the association between syndemics and the HIV care cascade. We study these associations prospectively in a longitudinal cohort study of young gay/bisexual men and transgender women (YGBT). Methods: Participants were 224 HIV-infected YGBT from a large cohort study $(\mathrm{N}=1,200)$ in the Chicago area. $88 \%$ identified as male, $64 \%$ Black and 23\% Hispanic/Latino. Every 6 months participants completed study visits including interviews, surveys, and laboratory tests. Syndemic factors included alcohol disorder (AUDIT), cannabis disorder (CUDIT), Depression (PROMIS), microaggressions, and victimization. Longitudinal analyses were performed using generalized estimating equation (GEE). Results: $87 \%$ of HIV positive YGBT in the cohort knew their HIV status prior to the study visit. $68 \%$ were taking ART, and $49 \%$ had achieved viral suppression to an undetectable level $(<200$ copies $/ \mathrm{mL})$ in study-specific laboratory testing. In longitudinal GEE analyses, problematic marijuana use $(\mathrm{p}<.01)$, microagressions $(\mathrm{p}<.05)$, and barriers to HIV care $(\mathrm{p}<.01)$ were significantly related to having an detectable viral load after controlling for time on ART. Conclusions: Approximately 50\% of HIV positive YGBT achieved viral suppression to undetectable levels, suggesting the need for more research to understand how to improve HIV medical care engagement and ART adherence. Some syndemic indicators were related to viral suppression (e.g., marijuana, microagressions), whereas others were not (e.g., depression, alcohol problems). Future research should examine the mechanisms linking heavy marijuana use to poor viral control and how microagressions may affect healthcare engagement for this population.

CORRESPONDING AUTHOR: Brian Mustanski, Northwestern University, brian@northwestern.edu 
O25.3

PREDICTORS OF PRE-EXPOSURE PROPHYLAXIS NONADHERENCE AND DISCONTINUATION IN A COHORT OF YOUNG MEN WHO HAVE SEX WITH MEN

Michael Newcomb ${ }^{1}$, Daniel Ryan ${ }^{1}$, Brian Mustanski ${ }^{1}$

${ }^{1}$ Northwestern University- USA

Background: Men who have sex with men (MSM) accounted for $70 \%$ of HIV diagnoses in the United States in 2015. Pre-exposure prophylaxis (PrEP) is a daily oral medication that is highly effective at reducing HIV transmission. PrEP efficacy is dependent upon high levels of adherence, and PrEP adherence varies substantially among MSM. Further, PrEP discontinuation may increase HIV risk in the absence of another prevention strategy (e.g., condom use). The current study used longitudinal data on PrEP use from a cohort of young MSM (YMSM) to evaluate demographic, psychosocial and sexual behavior predictors of PrEP nonadherence and discontinuation. Method: Data were collected between 2015 and 2017 as part of RADAR, an ongoing longitudinal cohort of YMSM in Chicago ( $N=1,125,80.1 \%$ HIV-negative). Participants completed study visits every 6 months, at which time we assessed PrEP outcomes (past 6 month PrEP use and discontinuation, 7-day adherence), psychosocial factors, sexual behavior and demographics. Generalized estimating equations were used to fit repeated measures logistic regression models using SAS 9.4. Results: Using data from participants' most recent study visit, $22.8 \%$ of HIV-negative YMSM reported taking PrEP during the past 6 months; $38.7 \%$ of these had discontinued use by the study visit. Among current PrEP users (14\% of HIV-negative YMSM), $9.8 \%$ reported $2+$ missed doses in the past 7 days $(3.6 \%$ reported $4+$ missed doses). In longitudinal analyses, living with one's parents $(\mathrm{OR}=3.52, \mathrm{p}<.05)$, elevated depression $(\mathrm{OR}=1.06, \mathrm{p}<.05)$ and a higher number of condomless male sex partners $(\mathrm{OR}=1.05, \mathrm{p}<.01)$ each predicted PrEP non-adherence (2+ missed doses in the past 7 days). Meeting a new serious romantic partner predicted discontinuation $(\mathrm{OR}=2.49$, $\mathrm{p}<.01$ ), regardless of sexual behavior with outside partners. Conclusion: Various demographic, psychosocial and behavioral factors predicted PrEP non-adherence. Of particular concern, having more condomless anal sex partners predicted non-adherence, which may potentiate new HIV infections. YMSM likely discontinue PrEP when in serious relationships due to lower perceived HIV risk. Future research should examine the accuracy of this perception in the context of continued sex with outside partners or non-monogamy arrangements.

CORRESPONDING AUTHOR: Michael Newcomb, Northwestern University, newcomb@northwestern.edu

\section{$\mathrm{O} 25.4$}

SUBSTANCE-RELATED EXPECTANCIES MEDIATE RELATIONSHIPS BETWEEN SUBSTANCE USE (STIMULANTS, ALCOHOL) AND CONDOMLESS ANAL SEX AMONG MSM

Amy Mullens ${ }^{1}$, Erich Fein ${ }^{12}$, Madeleine Ray, Ross Young ${ }^{3}$

${ }^{1}$ University of Southern Queensland- Australia

${ }^{2}$ School of Psychology \& Counselling- Australia

${ }^{3}$ Faculty of Health, Queensland University of Technology- Austraia.

Introduction \& Purpose We investigated effects of stimulant frequency (SF) and alcohol frequency (AF) on condomless anal sex (CAS), and the potential moderation effects of substance-related expectancies (i.e., beliefs about the effects of substances post-use). Three expectancy factors ['enhanced sexual activity' (ESA), 'social and emotional facilitation (SEF)', and 'cognitive impairment' (CI)] from the 'Stimulant Expectancies Questionnaire for MSM' (SEQ-MSM) and the 'Drinking Expectancies Questionnaire for MSM' (DEQ-MSM) were investigated, along with potential moderation associated with HIV status and use of HIV pre-exposure prophylaxis (PreP)-among MSM. Methods Involving a community sample of 654 adult men who have sex with men (MSM), in partnership with a GLBTIQ-health lead agency, we recruited via advertisements in gay media for MSM to complete an online survey [sociodemographic characteristics, HIV/sexual health (e.g., HIV status, viral load, prep use, STIs), substance use, and sexual activity (including 'chemsex')]. Results All three SEQ-MSM factors displayed a moderation effect, where the negative effect from SF to CAS was conditional to the effect of the expectancies. Namely, at high levels of ESA and moderate/ high levels of SEF there was a conditional positive direct effect of SF to CAS. At moderate/high levels of CI there was a conditional negative direct effect of SF to CAS. Similarly, all three DEQ-MSM factors displayed a moderation effect, where the negative effect from AF to CAS was conditional to the effect of the expectancies. Namely, at moderate/high levels of ESA and CI there was a conditional negative direct effect of DF to CAS. At low/moderate levels of SEF there was a conditional negative direct effect of DF to CAS. Further, when HIV Status was known (versus unknown) there was a moderate conditional positive direct effect of AF and SF to CAS. Conclusions Beliefs about the effects of substance use (expectancies) represent a valuable and modifiable construct influencing substance use patterns and subsequent sexual activity associated with STI and HIV transmission. Expectancies are a useful target for continued health promotion to reduce harms associated with both substance use and sexual activity.

CORRESPONDING AUTHOR: Amy Mullens, University of Southern Queensland, amy.mullens@usq.edu.au

\section{$\mathrm{O} 25.5$}

EXPLORING EMOTIONAL COMPETENCY AND SEXUAL BEHAVIOUR ASSOCIATIONS IN MEN WHO HAVE SEX WITH MEN

Dimitra Eleftheria Strongylou ${ }^{1}$, Jamie Frankis ${ }^{1}$, Paul Flowers ${ }^{1}$, Lawrie Elliott ${ }^{1}$

${ }^{1}$ Glasgow Caledonian University

Introduction \& Purpose: Homophobia, heterosexism, and heteronormativity may lead to emotional dysregulation that might predict risky sexual behaviour (RSB) among men who have sex with men (MSM), although this is not yet substantiated by research. This study examines: 1) whether MSM have lower emotional competency (EC) than the general population, 2) whether EC relates to RSB and 3) the perceptions of EC and RSB among MSM and sexual health clinicians. Methods: This study follows a mixed method approach with qualitative and quantitative findings being integrated. 4690 MSM across Scotland, Wales, Northern Ireland and the Republic of Ireland participated in an online survey which assessed EC (TEIQue-SF questionnaire) and sexual behaviour. Data were analysed using univariate statistics. Eight focus groups with $21 \mathrm{MSM}$ and 19 sexual health clinicians were analysed with thematic analysis. Results: MSM overall reported significantly lower EC $(\mathrm{M}=3.26, \mathrm{SD}=0.88)$ than the general population $(\mathrm{M}=4.95, \mathrm{SD}=0.61)$ $(\mathrm{t}=49.3, \mathrm{p}<0.001)$. Men who reported condomless anal intercourse (CAI) in the last year, a key measure of high-risk sex, scored significantly higher $(\mathrm{t}(2344)=-2.169, \mathrm{p}=0.03)$ only for the self-control facet of the TEIQue-SF ( $M=21.6, S D=6.1)$ than men who reported no CAI $(M=21$, $\mathrm{SD}=6.1$ ). EC was significantly lower for MSM who were more sexually active, participated in 'chemsex' parties, and were willing to stop using condoms in the heat of the moment. The thematic analysis showed that MSM and clinicians believed that some MSM knowingly engage in RSB to deal with the emotional impact of distal stressors such as homophobia and heteronormativity. RSB was understood as a complex term; encompassing STI/HIV transmission but also feelings of regret and "self-harm". Conclusions: Sexual risk definitions should be reformed to include emotional aspects of sexual behaviour. Multilevel interventions addressing the distal and proximal determinants of ill health are needed. Tackling the determinants of, and increasing skills in relation to EC may contribute to the design of effective interventions targeting improved 
sexual health among MSM.

CORRESPONDING AUTHOR: Dimitra Eleftheria Strongylou, Glasgow Caledonian University, dimitra.strongylou@gcu.ac.uk

O25.6

ELIMINATION OF HIV INFECTION AMONG MEN WHO HAVE SEX WITH MEN IN GUANGDONG, CHINA USING COMBINED HIV BIOMEDICAL INTERVENTIONS: A MATHEMATICAL MODELLING STUDY

Jinghua Li ${ }^{1}$, Keru Wang ${ }^{1}$, Liping Peng ${ }^{1}$, Joseph Tak-Fai Lau ${ }^{2}$

${ }^{1}$ Sun Yat-sen University

${ }^{2}$ The Chinese University of Hong Kong

Introduction and Purpose: HIV prevalence among men who have sex with men (MSM) has reached over 10\% in 2015 in Guangdong. Significant gaps still exist towards the $90-90-90$ targets by 2020 . Additional prevention measures (e.g., enhanced testing and treatment, and Pre-exposure prophylaxis, PrEP) are needed. The objective of this study is to investigate the long-term impact of PrEP and combined HIV interventions and the possibility of elimination of HIV among MSM. Methods: A deterministic mathematical model was developed and calibrated to reflect the dynamics of disease progression and transmission among MSM (aged 15-64 years) in Guangdong, China during 2018-2037. We examined several scenarios with differing combination of HIV testing, ART, and PrEP (with various coverages and adherence). Sensitivity analyses were conducted for PrEP adherence and risk compensation. An elimination status is defined as an incidence of below one new infection per 1,000 person-years. Results: With the current policy implementation level, HIV epidemic would continue to grow among MSM in Guangdong, and a total of 58,500 new HIV infections were estimated to occur over the next 20 years. Under the 90-90-90 strategy, HIV incidence at 2037 is estimated to be 2.8 per 1000 person-years. With the combined strategies of $90-90-90$ and PrEP among $25 \%, 50 \%$, or $75 \%$ of high-risk MSM (with annual sexual partnerships of 15), it is possible to achieve a $65.1 \% \sim 79.2 \%$ reduction in HIV incidence within 20 years, leading to an elimination status. In our sensitivity analysis, with poor PrEP adherence, even the aggressive strategy (i.e., joint strategy of 90-90-90 and PrEP $75 \%$ high-risk MSM) will not achieve HIV elimination in the next 20 years. With an assumed PrEP adherence of $90 \%$, the total number of new infections prevented and HIV epidemic situation were not sensitive to sexual risk compensation. Conclusion: In combination with enhanced implementation of current testing and treatment guidelines, the novel strategies could move Guangdong towards the elimination of HIV within the next 20 years. Efforts to improve adherence will be important in practical implementation of PrEP in China. Risk compensation could be a significant hindrance to the impact of PrEP only in situations where PrEP adherence is low.

CORRESPONDING AUTHOR: Jinghua Li, Sun Yat-sen University, lijinghua3@mail.sysu.edu.cn

\section{O26.1}

SCREENING AND STEPPED CARE TARGETING PSYCHOLOGICAL DISTRESS IN PATIENTS WITH METASTATIC COLORECTAL CANCER: THE TES CLUSTER RANDOMIZED TRIAL

Joost Dekker $^{1}$, Claudia Schuurhuizen ${ }^{1}$, Annemarie Braamse ${ }^{2}$, Aartjan Beekman $^{1}$, Pim Cuijpers ${ }^{1}$, Mecheline van der Linden ${ }^{1}$, Adriaan Hoogendoorn $^{1}$, Inge Konings ${ }^{1}$, Henk Verheul ${ }^{1}$

${ }^{1}$ VU University Medical Center- Netherlands

${ }^{2}$ Academic Medical Center Amsterdam- Netherlands

Background Psychological distress occurs frequently in patients with cancer. Literature suggests that effective management of psychological distress requires targeted selection of patients $(\mathrm{T})$, followed by enhanced care (E), and the application of stepped care for psychological distress (S). This study aimed to evaluate the effectiveness of a screening and stepped care program (the TES program) in reducing psychological distress compared to care as usual (CAU), in patients with metastatic colorectal cancer starting with first line systemic treatment. Patients and methods In this cluster randomized trial, 16 hospitals were assigned to either the TES program or CAU. Patients in the TES arm were screened for distress with the Hospital Anxiety and Depression Scale (HADS) and the Distress Thermometer/Problem List (at baseline, 10 and 18 weeks). Stepped care was offered to those patients with high distress. Stepped care consisted of watchful waiting, guided self-help, face-to-face problem-solving therapy, or referral to specialized mental health care. The primary outcome was change in psychological distress over time (HADS); secondary outcomes were quality of life (QOL), satisfaction of care, and actual recognition and referral of distressed patients by clinicians. Measures were assessed at baseline, after 3, 10, 24 and 48 weeks of follow-up. Linear mixed models were used to assess the effectiveness of the TES program. Results A total of 349 patients were included; 184 in the TES program and 165 in CAU. In the TES arm, $60.3 \%$ of the patients screened positive for psychological distress; $26.1 \%$ of patients entered the stepped care program (14.7\% only used watchful waiting, and $11.4 \%$ used at least one of the following steps). There was no difference in course of psychological distress over time between treatment groups ( $p>0.05$ ). The TES group reported higher satisfaction of received treatment and better QOL scores (all p's $<0.05$ ). Conclusion Screening and subsequent treatment for psychological distress does not improve psychological distress. Our results suggest that enhanced discussion of psychosocial concerns may improve aspects of patient's well-being.

CORRESPONDING AUTHOR: Joost Dekker, VU University Medical Center, j.dekker@vumc.nl

\section{O26.2}

IMPACT OF LONG-TERM DISORDERS ON CANCER SCREENING UPTAKE: A MIXED METHODS APPROACH

Katie Robb ${ }^{1}$, Marie Kotzur ${ }^{1}$, Richard Papworth ${ }^{1}$, Paula McSkimming ${ }^{1}$, Christine Campbell ${ }^{2}$, Emilia Crighton ${ }^{3}$, Sara Macdonald ${ }^{1}$, Bob Steele ${ }^{4}$, David Weller ${ }^{2}$

${ }^{1}$ University of Glasgow- United Kingdom

${ }^{2}$ University of Edinburgh- United Kingdom

${ }^{3}$ NHS Greater Glasgow and Clyde- United kingdom

${ }^{4}$ University of Dundee- United kingdom

Introduction \& Purpose: Worldwide there are disparities in cancer screening uptake, even among countries such as the UK where cancer screening is offered at no cost to the individual. While socioeconomic disparities have been well-described, little research has considered disparities in cancer screening uptake associated with long-term disorders and co-morbidity. The purpose of this work was to examine the impact of comorbidity and long-term disorders on cancer screening uptake. Methods: Phase 1 - data on breast, cervical and colorectal screening invitations and attendances for women aged 50-60 in the Greater Glasgow and Clyde Health Board during 2009-2013 were linked to demographic data, hospital discharge records, prescribing data, and GP Local Enhanced Service data. Co-morbidity was assessed using a Charlson Index of co-morbidity and long term antidepressant use was assessed by prescribing data. Data were analysed using logistic regression. Phase 2 examined why women $(n=59)$ chose to participate in none, some or all screening programmes including the impact of long-term disorders using qualitative interviews with a purposive sample of women identified through the Phase 1 dataset. Framework analysis was used. Results: Phase 1 - 68,234 women were eligible for all three cancer screening programmes. Uptake between $2009-2013$ was $75.8 \%$ for breast, $83.8 \%$ for cervical and $64.8 \%$ for colorectal. Among women with long term antidepressant use uptake was lower at $70.6 \%, 65.8 \%$ and $57.5 \%$, 
respectively. Women with multi-morbid illness had lower uptake across all three cancer screening programmes. Phase 2 - women described that existing long-term physical disorders meant they felt unable to cope with the prospect of additional treatment should an abnormality be detected at screening. Anxiety prevented some from deciding for or against cancer screening. Among a few, depression and suicidality let cancer seem to be an easier or blameless way to die, which negated any perceived need for cancer screening. Conclusions: Co-morbidity and long-term disorders were associated with lower uptake of breast, cervical and colorectal cancer screening. Combining the quantitative and qualitative methods provided a greater understanding which can inform the development of future interventions.

CORRESPONDING AUTHOR: Katie Robb, University of Glasgow, katie.robb@glasgow.ac.uk

\section{O26.3}

PSYCHOLOGICAL IMPLICATIONS OF ROUTINE HPV PRIMARY TESTING IN CERVICAL SCREENING: A LARGE CROSSSECTIONAL SURVEY ASSESSING ANXIETY AND DISTRESS Emily McBride ${ }^{1}$, Laura Marlow ${ }^{1}$, Alice Forster ${ }^{1}$, Jo Waller ${ }^{1}$

${ }^{1}$ University College London- United Kingdom

Introduction and purpose Cervical screening in England will move to human papillomavirus (HPV) primary testing from 2019, meaning all screened women will receive an HPV result. Testing HPV positive and/ or receiving abnormal cytology results can trigger adverse psychological consequences for some women; however, the impact of integrating routine communication of HPV status into cervical screening is unknown. This study was commissioned by Public Health England to examine differences in anxiety and distress between women receiving different test results at HPV primary screening. Method Women $(n=1132)$ were recruited from 5 National Health Service (NHS) sites in England where HPV primary screening is being used. They completed a postal survey approximately 2 weeks after receiving their screening results. Anxiety, distress, reaction to results (concern and reassurance), worry about developing cervical cancer and perceived risk of cervical cancer were compared across six groups with different HPV and cytology results using ANOVAs and Games-Howell post-hoc tests. Results Anxiety was higher in women with any HPV+ result, compared to women testing HPV- (Welch's F(5, $359)=9.35, \mathrm{p}<.001)$; anxiety was similar across HPV+ groups regardless of whether HPV was accompanied by normal or abnormal cytology ( $>$.05). Distress was higher in women who tested HPV+ with abnormal cytology, or HPV+ with normal cytology at their first test (Welch's F(5, $394)=3.41, \mathrm{p}<.001)$. Worry and concern were higher, and reassurance lower, in HPV+ groups $(\mathrm{p}<.001)$. Despite the fact that the different HPV+ groups would vary in their clinical risk of cervical cancer, perceived risk did not differ between them ( $p>$.05). Conclusions Testing positive for HPV with any cytology result appears to raise anxiety, worry and concern at the population level. Distress was slightly elevated in some HPV positive groups, indicating that for some women an HPV diagnosis can disrupt everyday functioning. Inaccuracies in perceived risk of cervical cancer may, in part, drive unnecessary anxiety and distress. Rigorous behavioural science input is needed to inform the wording of test result letters and information materials to minimise unnecessary anxiety and mitigate possible adverse effects on screening re-attendance rates.

CORRESPONDING AUTHOR: Emily McBride, University College London, e.mcbride@ucl.ac.uk

\section{O26.4}

DOES MANIPULATING ANTICIPATED REGRET INCREASE COLORECTAL CANCER SCREENING INTENTIONS? A RANDOMISED CONTROLLED TRIAL
Ian Zajac ${ }^{1}$, Deborah Turnbull ${ }^{2}$, Hugh Hunkin ${ }^{3}$

${ }^{1}$ Commonwealth Scientific and Industrial Research Organisation- Australia.

${ }^{2}$ University of Adelaide- Australia

${ }^{3}$ CSIRO

OBJECTIVE: Colorectal cancer (CRC) is the second most commonly diagnosed cancer in Australia, but regular screening can substantially improve disease outcomes. Despite this, screening participation rates remain low at around $35 \%$ in Australia, with similar rates globally. The notion of invoking emotional responses to improve screening uptake has been considered, most notably in the context of Anticipated Regret (i.e., how one might feel in the future if they did not partake in a screening opportunity). Although AR has been shown to be positively related to a variety of health behaviours and intentions, questions remain regarding the extent to which interventions which incorporate its measurement actually lead to stronger intentions and subsequent behavioural participation. The present study investigated whether measuring one's Anticipated Regret from failing to screen for bowel cancer might lead to stronger screening intentions and the extent to which Faecal Aversion moderates this impact (in addition to several other moderators). METHODS: A 2 (AR measured pre/post intention-to-screen) x 2 (FA measured pre/post intention) single blind parallel randomised controlled trial was employed. Participants aged 45 and over completed an online survey measuring demographic variables, AR, FA, intentions-to-screen, theory of planned behaviour variables and other potential moderators. RESULTS: $\mathrm{N}=666$ participants were randomised and analysed. Consistent with prior studies, higher AR was as strong predictor of future to intentions-to-screen. However, measuring AR in the pre-condition resulted in a significantly lower intention to screen $(\mathrm{d}=0.18,95 \% \mathrm{CI}[0.03 ., 0.33])$ which is contrary to expectations. Trait reactance predicted a significantly lower intention when it was at least $0.52 \mathrm{SD}$ above the mean, but no other moderators were supported. CONCLUSIONS: The results replicate the strong association between Anticipated Regret and future intentions-to-screen for colorectal cancer. However, this study suggests that measuring ones AR may in fact decrease intentions-to-screen, calling into question its relevance as an interventional approach to improving screening uptake.

CORRESPONDING AUTHOR: Ian Zajac, Commonwealth Scientific and Industrial Research Organisation ian.zajac@csiro.au

\section{O26.5}

FEASIBILITY RANDOMIZED TRIAL OF RISK COMMUNICATION INTERVENTIONS FOR PROMOTING CANCER GENETIC RISK ASSESSMENT

Anita Kinney $^{1}$, Anita Kinney ${ }^{1}{ }^{2}$, Rachel Howell ${ }^{1}$, Belinda Vicuna ${ }^{1}$, Rachel Ruckman ${ }^{2}$, Scott Walters 34

${ }^{1}$ University of New Mexico- USA

${ }^{2}$ Department of Internal Medicine,

${ }^{3}$ Department of Health Behavior and Health Systems

${ }^{4}$ University of North Texas

Introduction and Purpose: Cancer Genetic Risk Assessment (CGRA) is a precision medicine strategy that facilitates informed decision- making for effective health management among women at increased risk for inherited breast and ovarian cancer. However, less than one third of high-risk women have accessed CGRA. In preparation for a large, definitive trial $(\mathrm{n}=927)$, we conducted a pilot randomized controlled trial to assess preliminary efficacy and test the hypothesis that a personalized risk communication and navigation (TCN) intervention will have greater effects in promoting CGRA over usual care (UC) and mailed generic/targeted print (TP). Methods: Thirty-four high-risk women were recruited through a statewide cancer registry and randomized to UC, TP, or TCN. The TCN intervention was guided by evidence based behavior change strategies, the Extended Parallel Process Model, and the Health Action Process Approach to bridge the intention-behavior gap. Telephone surveys 
elicited perceptions about CGRA and the intervention. Results: Women in the TP $(\mathrm{M}=4.10, \mathrm{SD}=0.15)$ and $\mathrm{TCN}$ arms $(\mathrm{M}=4.38, \mathrm{SD}=0.28)$ reported high satisfaction with the interventions with no observed differences between the two study arms. CGRA uptake at six months post-intervention was observed for $30 \%$ of women in the TCN arm, $8 \%$ in the UC arm, and $0 \%$ in the TP arm $(\mathrm{P}<0.05)$. Financial and insurance concerns were the most frequently reported barriers to obtaining CGRA. Conclusion: Findings suggest high acceptability and feasibility with the target population and that a personalized intervention is more effective than a targeted intervention in motivating CGRA uptake. Better addressing women's barriers to accessing CGRA services through patient navigation may increase the intervention's potency in our ongoing definitive RCT.

CORRESPONDING AUTHOR: Anita Kinney, University of New Mexico, aykinney@salud.unm.edu

\section{O26.6}

THE IMPACT OF DESCRIPTIVE NORMS ON MOTIVATION TO PARTICIPATE IN CANCER SCREENING

Sandro Tiziano Stoffel ${ }^{1}$, Alex Ghanouni ${ }^{1}$, Yasemin Hirst ${ }^{1}$, Jo Waller ${ }^{1}$, Christian von Wagner

${ }^{1}$ University College London- United Kingdom

Introduction There is a strong relationship between descriptive norms and cancer screening participation. Thus informing people that uptake is high could motivate them to attend. The current study presented uptake information as part of a hypothetical vignette about Bowel Scope Screening (BSS). The aim of this study was to test whether manipulating normative beliefs increases intention to take part among a group of previously disinclined individuals. Methods We recruited 7,912 men and women aged 3554 living in England from an online panel. After an explanation of BSS, $1,733(21.8 \%)$ stated that they did not intend to participate. Of these 1,494 $(86.2 \%)$ estimated uptake as less than 6 out of $10(\mathrm{x}<6)$ and were randomised to one of four social norm conditions: (1) Echo and confirm ('you guessed uptake is $x$, uptake is x'), (2) Echo with proportional augmentation ('you guessed $x$; uptake is $x+3$ '); (3) Echo with standard augmentation (you guessed $\mathrm{x}$; uptake is 8 out of 10); (4) standard augmentation alone ('uptake is 8 out of 10 '). We used multivariable logistic regression adjusting for sociodemographic variables to predict intention by condition and report adjusted odds ratio (aOR) and 95\% confidence intervals (CI). Results 1,432 of 1,494 (95.9\%) respondents completed all comprehension and manipulation checks. Most were female (62.2\%), WhiteBritish $(80.5 \%)$, married or living with someone $(59.0 \%)$ and working $(70.4 \%)$. The three augmented messages led to a significantly greater proportion of BSS intenders $(17.8 \%, 27.7 \%$ and $28.2 \%$ for conditions 2,3 and 4 respectively) compared with 'Echo and Confirm, Condition1' (10.5\%); (aOR 1.91; CI 1.23-2.96; aOR 3.42; 2.25-5.20; aOR 2.27; CI 2.27-5.31). Conditions 3 and 4 were also associated with a significantly greater proportion of intenders compared with Condition 2 (aORs 3.42 vs 1.91; $\mathrm{p}<0.01$; aORs 3.47 vs $1.91 ; \mathrm{p}<0.01$ ). Conclusion High uptake messages are positively associated with screening intention. Future randomised controlled trials should evaluate its impact on screening attendance.

CORRESPONDING AUTHOR: Sandro Tiziano Stoffel, University College London, s.stoffel@ucl.ac.uk

\section{O27.1}

BELIEFS AND FACTORS DETERMINING BINGE DRINKING AMONG MEDICAL STUDENTS IN VIET NAM

Huong Nguyen ${ }^{1}$, Thi Thu Huong Nguyen ${ }^{2}{ }^{3}$, Katherine White ${ }^{2}{ }^{3}$, Marguerite Sendall ${ }^{2}{ }^{3}$, Ross McD Young ${ }^{3}$.

${ }^{1}$ Queensland University of Technology- Australia

${ }^{2}$ School of Psychology and Counselling, Faculty of Health

${ }^{3}$ Queensland University of Technology
Introduction and purpose: Risky drinking behaviours among medical students and physicians may influence their attitudes and practice for consultation with patients about alcohol problems. The Theory of Planned Behaviour (TPB) has demonstrated its effectiveness in understanding binge drinking intentions and behaviour among student populations. This study aims to elicit salient TPB beliefs and examine factors underlying binge drinking among Vietnamese medical students. Methods: This study adopted a mixed methods approach. The qualitative study included focus groups/interviews and a brief written survey $(\mathrm{N}=19)$ to explore salient beliefs underlying binge drinking. Results from this qualitative study informed the content of the quantitative survey. In the quantitative study, 206 medical students completed the TPB measures (intention, attitude, subjective norm, perceived behavioural control, and underlying beliefs) and group norm, role identity, and drinking culture at time 1. At time 2 ( 2 weeks later), 156 students reported their drinking behaviour for the previous fortnight. Results: The qualitative study found that medical students believed they were not binge drinkers while reporting experience of binge drinking occasions among medical students. Alcohol use was considered as culturally acceptable in Vietnamese society and a way for medical students to create and improve relationships with others. Group affiliation and peer pressure to drink excessive alcohol were identified among medical students. The quantitative study found few students reported binge drinking occasions. Perceived behavioural control, friends' group norm, and role identity as future doctors predicted binge drinking intentions and key beliefs determining intention were identified. Conclusions: Limited engagement in binge drinking was reported in surveys among this cohort despite qualitative descriptions of its occurrence. The culture of drinking behaviour and key factors predicting binge drinking intentions among Vietnamese medical students were identified. Overall, these findings highlight the importance of friends' group norm and role identity as future doctors for those with more risky drinking intentions, offering theory-informed strategies for future interventions among this cohort.

CORRESPONDING AUTHOR: Huong Nguyen, Queensland University of Technology, thuhuong1611@gmail.com.

\section{O27.2}

A SYSTEMATIC REVIEW EXAMINING THE EXTENT TO WHICH DRINKING MOTIVES AND CONTEXT PREDICT RISKY DRINKING IN YOUNG ADULTS

Renee O'Donnell ${ }^{1}$, Ben Richardson ${ }^{2}$, Petra Staiger

${ }^{1}$ Deakin University- Australia

${ }^{2}$ School of Psychology- Australia

${ }^{3}$ Deakin University, Geelong, Australia

Behaviour-change interventions that target drinking motives as a way to reduce problematic drinking among young adults, has received mixed success. A possible explanation for the lack of success of these interventions could be due to the lack of understanding of the interaction between drinking motives and situational factors being the most precise predictor of problematic drinking outcomes. No study to date has synthesised this literature. Reviewing these studies will provide important insights into effective development of behaviour-change interventions that target both drinking motivations and contextual factors. The aim of this systematic review is to analyse the extent to which drinking motives (i.e., social, enhance, cope and conform) and contextual factors (i.e., location of use, who the person is with and the affect they experienced) interact to predict problematic drinking among young adults. Method: A total of 31 studies were identified that examined the relationship between motives, contextual factors and alcohol use among young adults. Results: Results consistently found that young adults who endorsed enhancement motives (i.e., drink to enhance positive affect) were more likely to engage in heavy drinking if they were exposed to any of the following situations; surrounded by others' who were drinking, in a social type setting or 
indicated positive affect. In contrast, young adults who endorsed coping motives (i.e., drink to manage negative affect) were consistently shown to experience drinking-related problems if they consumed alcohol in any of the following situations; whilst at home, when alone or during times of negative affect. Less consistent findings were found for social and conformity motives. Conclusion: These results indicate that behaviourchange interventions designed to address young adults' problematic drinking behaviours should be focused on managing their drinking motivations and desires, and equally, the temptations and urges that arise within specific drinking situations. Indeed, educating young people on understanding what their drinking motivation is and the profile of situations that are likely to trigger their alcohol use, could be a feasible and effective way to prevent alcohol use before it is initiated.

CORRESPONDING AUTHOR: Renee O'Donnell, Deakin University, Renee.odonnell@deakin.edu.au

\section{$\mathrm{O} 27.3$}

MEDICATION ADHERENCE AMONG STROKE SURVIVORS IN RURAL CHINA: A MIXED METHODS STUDY

Wanbing $\mathrm{Gu}^{1}$, Wanbing $\mathrm{Gu}^{1}$, Enying Gong ${ }^{1}$, Lijing Yan ${ }^{1}$

${ }^{1}$ Duke Kunshan University- China

Introduction Stroke is the leading cause of deaths and disabilities in China with about 11.1 million stroke survivors. Despite the growing burden from stroke in rural China, effective secondary prevention of stroke is far below guideline recommendations. Good medication adherence is one of the most important components of secondary prevention, which is associated with better health outcome and lower risk of recurrence and mortality. The objective of this study is to describe the current situation of medication adherence among stroke survivors in Nanhe County, rural China, and to explore factors influencing medication adherence. Methods Stroke survivors from 50 villages of five townships (ten villages in each township) in Nanhe County were recruited through a multistage cluster sampling. Information on history of stroke, use of and adherence to medication was obtained through a standard questionnaire by trained interviewers. Medication adherence were measured by using four-item Morisky Green Levine Scale with score 0-1 represents a good adherence. Stroke survivors were also invited to participated in semi-structured interviews during the intervention. The interview questions include patients' attitude toward medication use and adherence. Descriptive analysis was performed on survey data by using STATA and thematic content analysis was performed for interview data by using Nvivo. Results In total, 1299 stroke survivors in Nanhe County, rural China participated in the survey (57.4\% males, average age: 65.7 years old). Among these participants, 1030 were taking anti-hypertensive medicines, while only $797(77.4 \%)$ adhered to the anti-hypertensive medicines. 852 were taking aspirin with $691(81.1 \%)$ had good adherence. Only 340 patients were taking statins with $274(80.6 \%)$ stroke survivors adhere to the prescription. The qualitative data showed that forgetting taking medicine, economic difficulty in purchasing medicine, asymptomatic conditions and lack of knowledge of medicine are the most common reasons of poor adherence. In addition, the severity of diseases, having a caregiver and the expertise and authority of healthcare providers are facilitating factors in promoting medication adherence among stroke survivors. Conclusion Improving medication adherence among stroke survivors is essential to promote the secondary prevention of stroke in rural China. In addition to factors related to patients, factors related to caregivers and healthcare providers, which are always overlooked, also have impacts on patients' medication adherence. Future intervention programs should target on patients, caregivers and healthcare providers to promote medication adherence.

CORRESPONDING AUTHOR: Wanbing Gu, Duke Kunshan University, wanbing.gu@duke.edu
O27.5

TRAIT SELF-CONTROL AND SOCIAL COGNITIVE PREDICTORS OF HEALTH BEHAVIOR: MEDIATION AND MODERATION EFFECTS

Martin Hagger ${ }^{1}$, Nelli Hankonen ${ }^{2}$, Taru Lintunen ${ }^{3}$, Juho Polet ${ }^{3}$

${ }^{1}$ Curtin University- Australia

${ }^{2}$ University of Helsinki- Australia

${ }^{3}$ University of Jyvaskyla- Australia

Introduction and Purpose. Trait self-control is consistently related to participation in health-related behavior. However, few studies have explored the processes by which self-control relates to health behavior. In the current study, we tested two candidate processes: an indirect effect mediated by constructs from social cognitive theories, and a moderation effect in which self-control interacts with intentions in the prediction of behavior. The effects were tested in six data sets for ten health-related behaviors. Methods. Using prospective designs, participants completed measures of constructs from social cognitive theories (theory of planned behavior, health action process approach) and trait self-control at an initial time point and self-reported their behavior at a later point in time. Results. Results revealed indirect effects of trait self-control on health-related behavior through social cognitive constructs and intentions in seven behaviors: eating fruit and vegetables, avoiding fast food, dietary restrictions, binge drinking, physical activity, out-of-school physical activity, and predrinking. There were also direct effects of self-control on behavior. Interactive effects of self-control and intentions on behavior were found for three behaviors: dietary restriction, binge drinking, and alcohol consumption. Conclusions. Current findings suggest trait self-control may serve as a source of information for beliefs about future health behavior participation, and direct and indirect effects imply separate conscious and non-conscious processes. Moderation of the intention-behavior relationship by self-control may depend on the extent to which the behavior is driven by impulse control. Findings suggest that training self-control and shaping environments to minimize contingencies that may derail goaldirected action may be effective intervention strategies.

CORRESPONDING AUTHOR: Martin Hagger, Curtin University, martin.hagger@curtin.edu.au

\section{O27.6}

OLDER ADULTS' UNDERSTANDING OF THE CONCEPT OF SEDENTARY BEHAVIOUR: A QUALITATIVE STUDY

Laura McGowan ${ }^{1}$, David French ${ }^{1}$, Rachael Powell ${ }^{1}$

${ }^{1}$ University of Manchester- United Kingdom

Introduction and Purpose: Sedentary behaviour is associated with negative health outcomes for older adults and this age group is the most sedentary portion of society. There is currently a limited understanding of how older adults perceive sedentary behaviour, and this has important implications for interventions seeking to reduce sedentary behaviour in this population. This study aimed to explore older adults' understanding of the concept of sedentary behaviour. Methods: Face-to-face, semistructured interviews were conducted with a purposive sample of 22 community-dwelling older adults. Participants were selected to be diverse in socio-economic background and current activity levels. Interviews were recorded and transcribed verbatim. A thematic analysis was conducted structured using the Framework approach. Results: Participants often thought of sedentary behaviour as synonymous with a lack of physical activity, and perceived reducing sedentary behaviour and increasing physical activity as one and the same thing. Participants perceived the term 'sedentary' to have negative connotations and were often judgemental of people who engaged in high levels of sedentary behaviour. Many older adults seemed to be reluctant to identify as being 'sedentary'. Most participants considered reducing sedentary behaviour to be of value, though more active individuals were unconvinced of the value 
of reducing sedentary behaviour above that of being physically active. Conclusions: The findings of this qualitative study offer an in-depth understanding of how older adults think about sedentary behaviour. This has important implications for the way in which messages regarding sedentary behaviour should be delivered and how the design of interventions to reduce sedentary behaviour in this population should be approached.

CORRESPONDING AUTHOR: Laura McGowan, University of Manchester, laura.mcgowan@manchester.ac.uk

\section{O28.1}

SETTING THE AGENDA IN CHILDHOOD OBESITY PREVENTION: CO-PRODUCTION OF RESEARCH PRIORITIES AND FACILITATORS AND BARRIERS TO KNOWLEDGE TRANSLATION Marita Hennessy ${ }^{12} 3$, Molly Byrne ${ }^{123}$, Rachel Laws ${ }^{4}$, Jenny Mc Sharry $^{12}$, Caroline Heary ${ }^{3}$

${ }^{1}$ National University of Ireland Galway

${ }^{2}$ Health Behaviour Change Research Group

${ }^{3}$ School of Psychology

${ }^{4}$ Institute for Physical Activity and Nutrition

${ }^{5}$ Deakin University

Introduction \& Purpose: Childhood obesity is a public health challenge, yet research priorities are not established. Co-production of priorities leads to research which may be more translatable to the domains of policy and practice. The aim of this study was to co-create priorities for childhood obesity prevention research and knowledge about facilitators and barriers to knowledge translation. Methods: Nominal group technique was conducted during multi-stakeholder workshops hosted during a two-day national obesity conference. Participants contributed, to varying degrees, to three rounds of priority identification and consensus building. Results: Seventy-seven delegates participated in ten nominal groups on day one ( $91 \%$ response rate). Each group generated areas for future research and their top three priorities were identified. On day two, a group of 14 stakeholders, comprising national and international researchers, policymakers, health service managers and practitioners discussed, clarified and amended the 26 knowledge gaps identified, and participated in two further rounds of voting to rank their top five priorities. Barriers and facilitators to knowledge translation were also generated and ranked during nominal groups on day one. Key themes emerging from this study were the importance of funding and resources, co-production of research, and a focus on both implementation research and social determinants within the field of childhood obesity prevention. The top-five research priorities identified were: (1) Evaluate (including economic evaluation) current programmes to inform practice and policy; (2) How to change culture towards addressing the determinants of health; (3) Implementation science: process; (4) How to integrate obesity prevention into existing service structures; (5) How to enhance opportunities for habitual physical activity, including free play and active travel. Conclusion: A list of policyrelevant priorities for future childhood obesity prevention research was co-produced systematically with a range of stakeholders. These may help to inform the research agendas of funders and researchers, stimulate multi-disciplinary collaboration, aid in the conduct of policy-relevant research and the translation of research into practice.

CORRESPONDING AUTHOR: Marita Hennessy, National University of Ireland Galway, m.hennessy11 @ nuigalway.ie

$\mathrm{O} 28.2$

CLUSTERING PATTERNS OF EATING BEHAVIORS, PHYSICAL ACTIVITY AND SEDENTARY ACTIVITY AND THE ASSOCIATION WITH OVERWEIGHT OR OBESITY AMONG CHINESE CHILDREN AND ADOLESCENTS: FINDINGS FROM A CROSS-SECTIONAL
Juan ZHANG ${ }^{1}$, Kaiyuan MIN ${ }^{1}$, Wei LIAO ${ }^{1}$, Shuya CAI ${ }^{1}$, Yang LIU $^{1}$

${ }^{1}$ Peking Union Medical College \& Chinese Academy of Medical Sciences- China

BACKGROUND: Childhood obesity is becoming a public health challenge in China. Beijing, the capital city of People's Republic of China, ranks top in terms of the prevalence of childhood obesity. Poor dietary habits, physical inactivity and a sedentary lifestyle are important modifiable contributors to overweight or obesity. Evidence suggests possible synergetic effects of multiple behaviors on overweight or obesity. Scant research has been done on the clustering and correlations of obesityrelated behaviors in Chinese childhood and adolescence. This study examined the clustering patterns of eating behaviors, physical activity and sedentary activity among Chinese children and adolescents in Dongcheng District of Beijing, and investigated the association of clustering patterns with overweight or obesity. METHODS: This study was conducted in a representative sample of 1372 children and adolescents (49.8\% male) from primary schools through high schools in Dongcheng District of Beijing. Eating behaviors was investigated by a 23 -item food and beverage frequency questionnaire, physical activity and sedentary activity were measured using self-reported questionnaires. Exploratory factor analysis (EFA) was used to identify patterns of eating behaviors; cluster analysis (CA) was performed to examine the clustering patterns of eating behaviors, physical activity and sedentary activity; and multiple logistic regression analysis was conducted to analyze the association of clustering patterns of obesity-related behaviors with overweight or obesity. Overweight and obesity was defined by using the references developed by WHO. RESULTS: The rate of overweight was $12.28 \%$ for boys and $7.26 \%$ for girls, and the rate of obesity was $6.14 \%$ for boys and $3.17 \%$ for girls; boys had higher odds of being overweight or obese compared to girls (Adjusted $\mathrm{OR}=2.03,95 \% \mathrm{CI}=1.35-3.05, \mathrm{P}<0.001$ ). Three patterns of eating behaviors were identified. Pattern 1 was associated with fried food, snack and Western fast-food; Pattern 2 was associated with vegetable and fruit; and Pattern 3 was associated with sugar-sweetened beverages (SSBs). A following CA identified three meaningful clustering patterns labeled "low energy-dense (ED) food/ low physical activity/ low sedentary activity", "high snack and fast-food/ high sedentary activity" and "high vegetable and fruit/ high SSBs/ high physical activity/ high sedentary activity". Clusters varied by age and gender. Multiple logistic regression analysis showed that "high vegetable and fruit/ high SSBs/ high physical activity/ high sedentary activity"cluster was a risk factor for overweight or obesity (Adjusted $\mathrm{OR}=4.55,95 \% \mathrm{CI}=2.69-7.70$, $\mathrm{P}<0.001)$. CONCLUSION: Clustering of obesity-related behaviors are common among Chinese children and adolescents. This has important implications for public health because understanding clustering of behaviors can be used to assist in the development of targeted obesity prevention initiatives to bring about multiple behavior change.

CORRESPONDING AUTHOR: Juan ZHANG, Peking Union Medical College \& Chinese Academy of Medical Sciences, zhangjuan@sph.pumc.edu.cn.

O28.3

DECLINED CALORIC INTAKE AND INCREASED CHILD OBESITY IN CHINA: WHAT ROLE DOES SUGAR SWEETENED BEVERAGE PLAY?

Yang $\mathrm{Wu}^{1}$

${ }^{1}$ Jiangxi University of Finance and Economics- China.

Introduction \& Purpose The childhood obesity rate in China has risen dramatically during the past two decades. However, Chinese children's total caloric intake has declined simultaneously. This study tries to explore whether this discrepancy could be possibly explained by an increase in sugar sweetened beverage intake. Methods Dietary data of 3,319 Chinese children and adolescents aged 7 to 18 was collected using both 
consecutive 3-day 24- hour dietary recalls and food frequency questionnaires from a nation- wide prospective cohort study, the China Health and Nutrition Survey in years 2004, 2006, 2009 and 2011. Anthropometric measures were taken by trained staff, and weight status was obtained using the International Obesity Task Force cutoffs. Random- effect models clustered at the individual and community levels were used to estimate the relationship between sugar sweetened beverage intake and the risk of being overweight or obese, after adjusting for socio- demographic, urbanicity, and caloric intake factors. Results That rate of overweight or obesity increased slightly, from $8.9 \%$ to $12.0 \%$ over six years. After adjusting for energy intake, sugar sweetened beverage intake appeared not to be related to children's risks of being overweight or obese $(\mathrm{OR}=0.88,95 \% \mathrm{CI}: 0.50 \sim 1.55)$. Gender plays a more critical role in predicting the risks of overweight or obesity $(\mathrm{OR}=0.61,95 \% \mathrm{CI}$ : $0.42 \sim 0.87$ ). Conclusions The increasing childhood obesity rate in China could not be explained by either total energy intake or increased sugarsweetened beverage intake. Other mechanisms, such as fat intake, physical activity levels and sedentary behaviors shall be taken into consideration.

CORRESPONDING AUTHOR: Juan ZHANG, Peking Union Medical College \& Chinese Academy of Medical Sciences, zhangjuan@sph.pumc.edu.cn

\section{O28.4}

LIFESTYLE BEHAVIOURS AND THE PROSPECTIVE RELATIONSHIP BETWEEN DEPRESSION DURING ADOLESCENCE AND OBESITY, HYPERTENSION AND DIABETES IN ADULTHOOD

Erin Hoare ${ }^{1}$, Erin Hoare ${ }^{1}$, Sarah Dash ${ }^{2}$

${ }^{1}$ Deakin University- Australia.

${ }^{2}$ Baker Heart and Diabetes Institute- Australia

Introduction \& Purpose The evidence for the dietary and physical activity correlates of common mental disorders is consistent and compelling. However, further longitudinal research is needed to support the causal relationships between lifestyle behaviours, mental health, and chronic disease outcomes. The purpose of this research was to investigate the extent to which adolescent depressive symptoms were predictive of early adulthood biomedical status in a large representative sample of US individuals, controlling for diet and physical activity. Methods The US National Longitudinal Study of Adolescent to Adult Health was the largest and most comprehensive longitudinal study of adolescents ever undertaken. The data analysed here were Wave I in-school questionnaires when participants were in grades 7-12 in $1995(\mathrm{n}=3932)$. Wave IV data included questionnaire and biomedical measures to assess chronic disease and other health outcomes in adulthood when participants were aged 2432 years in 2008. Logistic regression models were used to assess the likelihood of objectively measured obesity, hypertension and diabetes determined by adolescent depressive symptoms, measured by the Centre for Epidemiological Studies Depression Scale. Results Female adolescents were at greater risk for experiencing higher levels of depressive symptoms compared to males. Among females, depressive symptoms during adolescents were significantly associated to greater likelihood of obesity (OR 1.02, 95\% CI 1.00-1.03, p<0.05), hypertension (OR $1.01,95 \% \mathrm{CI} 1.00,1.02,0<0.05)$ and diabetes $(\mathrm{OR} 1.03,95 \% \mathrm{CI}$ $1.00,1.05, \mathrm{p}<0.05)$ in adulthood, after controlling for ethinicity and level of education. In the fully adjusted model (further controlling for adolescent lifestyle behaviour risks and adult depression) females who reported higher levels of depressive symptoms during adolescence were at greater risk of hypertension (OR 1.02, 95\% CI 1.00, 1.03, p<0.05) in adulthood. Conclusions The mental health experiences of young people hold implications not only during younger years, but also for future health outcomes. Sex-specific associations are consistent with the literature, and warrant further investigation of female and male specific experiences of psychosocial, physiological, environmental factors driving health and disease. High systolic blood pressure was the leading risk factor attributable to disability-adjusted life years among females and the second leading risk factor among males. Our findings point support the need for a dual mental and physical health approach across the lifespan in investigating health outcomes with mental health symptoms suggesting future hypertension risk among females.

CORRESPONDING AUTHOR: Erin Hoare, Deakin University, erin.hoare1@deakin.edu.au

O28.5

OFF THE COUCH, INTO THE KITCHEN: LEVERAGING PERSONAL NORMS TO PROMOTE HOME COOKING AND HEALTHY EATING

Ana Isabel Almeida $\operatorname{Costa}^{1}$, Cláudia Simão ${ }^{1}$

${ }^{1}$ Católica-Lisbon School of Business and Economics, Universidade Católica Portuguesa- Portugal

Objectives: Poor diets and obesity are often associated to high consumption of away-from-home foods. Yet, little is known about how to motivate people to cook more regularly. This research explores the role of Personal Norms(PN) in self-regulating home cooking. Methods: Study 1) Nationally representative, postal survey of Dutch households (482 adult meal preparers, $77 \%$ female, mean age 43 ). Meal preparation/consumption habits were assessed. Awareness of Benefits(AB), Self-Accountability(SA), Chronic $\mathrm{PN}(\mathrm{PNChr})$, Social Norm(SN), Affect and Habit regarding the preparation of Home Cooked Meals(HMC) were measured. Study 2) Lab experiment (162 adults, $77 \%$ regular home cooks, $52 \%$ female, $71 \%$ Portuguese, mean age 23). SA, SN and Affect were assessed. PN was activated by exposing participants to information about benefits of HCM (high vs. low AB). PN activation(PNAct) was subsequently measured. Study 3 ) Lab experiment $(65$ adults, $62 \%$ regular home cooks, $34 \%$ female, $82 \%$ Portuguese, mean age 23). Meal preparation/consumption habits and SA were assessed. Participants completed a Scrambled Sentence Test (PN prime vs. control). Personal obligation to prepare $\mathrm{HCM}(\mathrm{PNPrm})$ was subsequently measured. Participants finally evaluated information about benefits of $\mathrm{HCM}$ (high $\mathrm{AB}$ ). Results: Study 1) Best fitting model independently regressed PNChr, Affect and Habit on frequency of preparing $\operatorname{HCM}(\mathrm{R} 2=0.25 ; \mathrm{F}(3,478)=53.84, \mathrm{p}<.001)$, with $\mathrm{PNChr}$ making the highest contribution $(\Omega=0.34 ; \mathrm{t}=7.54, \mathrm{p}<.001)$ and fully mediating the effect of $\mathrm{AB}$ (but not SA) [Sobel $\mathrm{t}(2$, $479)=4.88, \mathrm{p}<.001 ; \mathrm{R} 2=0.20 ; \mathrm{F}(2,479)=61.29, \mathrm{p}<.001]$. Study 2) PNAct was higher in the high $\mathrm{AB}$ condition [3.5 \pm 1.9 vs.2.6 $\pm 1.6 ; \mathrm{F}(1,160)=10.244, \mathrm{p}<$ .001], while SA, SN and Affect did not co-vary. Study 3) PNPrm was higher in primed participants $[3.2 \pm 1.2$ vs. $2.7 \pm 1.1 ; F(1,63)=3.506, p<.10]$. A general linear model with treatment as factor and PNPrm as co-variate $[\mathrm{F}(2$, $62)=4.969, p<.05]$ positively associated these variables with evaluations of information about the health benefits of $\mathrm{HCM}$ ( $\mathrm{p}<.1$ and $\mathrm{p}<.05$, respectively). Conclusions: PN motivates people to prepare $\mathrm{HCM}$ more often and increases affect for messages about their health benefits. Policies designed to leverage these self-regulatory effects may be effective in promoting healthier diets.

CORRESPONDING AUTHOR: Ana Isabel Almeida Costa, CatólicaLisbon School of Business and Economics, Universidade Católica Portuguesa, anacosta@ucp.pt

\section{O28.6}

CHRONIC STRESS AND GENETIC SUSCEPTIBILITY TO OBESITY IN U.S. HISPANIC/LATINOS. PRELIMINARY RESULTS FROM THE HISPANIC COMMUNITY HEALTH STUDY/STUDY OF LATINOS (HCHS/SOL)

Carmen Isasi ${ }^{1}$, Jee-Young Moon ${ }^{1}$, Qibin $\mathrm{Qi}^{1}$, Tao Wang ${ }^{1}$, Linda C Gallo ${ }^{2}$, Daniela Sotres-Alvarez ${ }^{3}$, Maria M Llabre ${ }^{4}$, Tasneem Khambaty ${ }^{4}$

${ }^{1}$ Albert Einstein College of Medicine- USA 
${ }^{2}$ San Diego State University

${ }^{3}$ University of North Carolina at Chapel Hill

${ }^{4}$ University of Miami

Introduction" Prior research shows associations between psychosocial stress and obesity, but few studies take account for underlying genetic susceptibility to obesity. Genome-wide association studies (GWAS) have identified several single nucleotide polymorphisms (SNPs) associated with BMI and obesity; however, gene-environment interactions of psychosocial factors have been less explored. In this study, we examined the relative contributions of chronic stress and obesity genetic risk score, as well as their interaction, in relation to BMI and obesity in a diverse sample of Hispanic/Latino adults. Methods: The study includes 7162 participants (56\% female; mean age 50 years) from the second examination of HCHS/ SOL, a population-based cohort conducted in four US cities (Bronx, NY; Chicago, IL; Miami, FL; San Diego, CA) that included participants of Mexican, Cuban, Puerto Rican, Dominican, Central and South American ancestries. Chronic stress was assessed by the chronic stress burden scale, which asks about stressors lasting more than 6 months in major life domains (financial, health, work). Genetic risk score (GRS) was derived by summing the risk alleles of 97 known BMI-associated SNPs previously identified. Analyses were adjusted for the complex sampling design, site, ancestry, relatedness, age and sex. Results: Only $25 \%$ of participants reported no chronic stressors, $45 \%$ had $1-2$, and $30 \%$ had $\geq 3$ stressors. Mean $\mathrm{BMI}$ was $30 \mathrm{~kg} / \mathrm{m} 2$ ( $\mathrm{SE}=.12$ ). Both chronic stress and genetic risk score were associated with higher BMI and obesity. The adjusted regression model showed that each unit increase in chronic stress was related to $0.35(0.04)$ increase in BMI $(\mathrm{p}<0.001)$ and each unit increase in GRS was associated with a 0.69 increase in BMI $(p<0.001)$. However, the interaction between stress and GRS was not significant. Chronic stress explained $1.37 \%$ of the BMI variance and GRS explained $0.97 \%$. Participants with a higher number of chronic stressors had higher odds of obesity $(\mathrm{OR}=1.11 ; 95 \% \mathrm{CI} 1.08,1.14)$ and higher GRS was also associated with obesity ( $\mathrm{OR}=1.24,95 \%$ CI 1.18,1.3). Conclusions: Although associations were small, chronic stress significantly related to BMI and obesity in Hispanic/Latinos adults, even after controlling for underlying genetic susceptibility.

CORRESPONDING AUTHOR: Dr. Carmen Isasi, Albert Einstein College of Medicine, carmen.isasi@ einstein.yu.edu

O29.1

USING A CAMPAIGN STRATEGY TO INFLUENCE THE PURCHASE OF HEALTHY FOODS BEARING THE HEART MARK LOGO

Pamela Naidoo ${ }^{1}$

${ }^{1}$ University of the Western Cape- South Africa

Introduction and Purpose: The Heart Mark (HM) is an endorsement programme of the Heart and Stroke Foundation South Africa (HSFSA). Products bearing the HM logo on p signifies that the HSFSA based on evidence has endorsed the product as a healthy food choice for cardiovascular disease (CVD) prevention. SA has a high CVD burden. To ascertain the shift in the knowledge, attitudes, beliefs, purchasing behaviour, and awareness of the HM logo, the HSFSA conducted a pre-post campaign survey with the purpose of influencing the purchase of HM products and promote the HM endorsement programme, approved by the Department of Health. Methods: The 3-province campaign strategy targeted primarily women in the lower socio-economic groups, and men and young individuals. The strategy pillars were: creating awareness of the HM through radio, digital/print media, and in-store promotions. A pre-post survey was conducted to assess the impact of the campaign on the level of awareness and brand recognition of the HM and to assess the overall impact of the HM campaign. The sample size for the precampaign was 300 and post-campaign was 184 adults with a $75 \%$ response rate. Results: The 4 categories of responses were: knowledge, attitudes and beliefs, purchasing behaviour of healthy foods and communication platforms for HM exposure. Pre-Post survey results for the knowledge component showed an increase in the recognition of the HM logo on food items in the fats and oils category (39.7\%vs $56.6 \%$ ), the association of the HM with HSFSA (19.4\% vs $40.2 \%)$ and trusting the logo $(79.3 \%$ vs $98.4 \%)$. Attitudes and beliefs post campaign showed an increase in the belief that food labels are important in order to know the nutritional value of the product and that the HM logo helps in making healthy food choices easier. There was an increase in the purchase of foods with the HM logo (16.3\% vs $30.4 \%$ ) and eating healthily $(33.2 \%$ vs $37.5 \%$ ). Finally, the majority of participants heard or read healthrelated messaging through radio, television, or print media respectively. Conclusion: Descriptive results indicate that campaigns encouraging healthy food choices to reduce unhealthy eating behaviour can reduce risk for CVD and be effective as one component of a multi-pronged approach to disease prevention.

CORRESPONDING AUTHOR: Pamela Naidoo, University of the Western Cape, pamela.naidoo63@gmail.com

\section{$\mathrm{O} 29.2$}

SOCIO-COGNITIVE ASPECTS OF THE UPTAKE OF INFLUENZA VACCINATION AMONG JAPANESE ADULTS

Mika Omori ${ }^{1}$, Naomi Yoshitake ${ }^{2}$, Masumi Sugawara ${ }^{3}$, Kiko Akishinonomiya $^{3}$, Sachiko Shimada ${ }^{3}$

${ }^{1}$ Ochanomizu University- Japan.

${ }^{2}$ Juntendo University- Japan

${ }^{3}$ Ochanomizu University- Japan

Background: Seasonal influenza is a highly prevalent and incapacitating disease that results in various public and individual health challenges, including pandemics, absenteeism, and death. Despite public health efforts to promote vaccination uptake to prevent the infection, global vaccination rates remain lower than the ideal. A survey conducted with a working age population in Japan, for example, indicated that only $24.2 \%$ of male and $27.6 \%$ of female respondents reported that they had received vaccination (Iwasa et al., 2013). Therefore, it was considered critical to identify psychological factors associated with vaccination behaviors to facilitate individual decisions to take the influenza vaccine. Purpose: To investigate socio-cognitive factors associated with influenza vaccination in the general population of Japan by considering interpersonal concerns regarding vaccination uptake as a unique attribute of a collectivistic culture. Methods: A population-based survey was conducted using a stratified sampling method to obtain nationally representative data. Responded to the survey were Japanese adults $(\mathrm{N}=911$, Mean Age $=15.24, \mathrm{SD}=2.05 ; 374$ men and 401 women). Questionnaires inquired about perceptions regarding seasonal influenza and vaccination uptake, including perceived vulnerability, adverse feelings, perceived threats, perceived prevalence of vaccination uptake, cues to action, self-efficacy of vaccination, interpersonal concerns, and vaccination uptake. Results: The proportion of individuals that took the vaccine was $46.1 \%$ (women, $51 \%$; men $41 \%$ ). Binary logistic regression analyses were conducted to identify socio-cognitive factors contributing to vaccination uptake. Perceived vulnerability $(\mathrm{OR}=.97$, $\mathrm{p}<.05)$, threat $(\mathrm{OR}=1.05, \mathrm{p}<.05)$, cues to action $(\mathrm{OR}=.83, \mathrm{p}<.01)$, self-efficacy $(\mathrm{OR}=.90, \mathrm{p}<.01)$, and perceived prevalence $(\mathrm{OR}=.98$, $\mathrm{p}<.01)$ significantly predicted vaccination uptake. Interestingly, no significant impact of interpersonal concerns on vaccination uptake was observed. Discussions: Results support previous findings by suggesting the need for integrating socio-cognitive aspects of vaccination uptake in future preventive efforts.

CORRESPONDING AUTHOR; Mika Omori, Ochanomizu University, omori.mika@ocha.ac.jp 
O29.3

LEVERAGING CITIZEN SCIENCE TO CREATE HEALTHPROMOTING ENVIRONMENTS WORLDWIDE: FINDINGS FROM THE OUR VOICE GLOBAL CITIZEN SCIENCE RESEARCH NETWORK

Abby King ${ }^{1}$, Ann Banchoff ${ }^{1}$, Sandra Winter ${ }^{1}$, Jenna Hua ${ }^{1}$, Olga Lucia Sarmiento $^{2}$, NIcolas Aguilar-Farias ${ }^{3}$, Sebastien Chastin ${ }^{4}$, Estelle V. Lambert

${ }^{1}$ Stanford University School of Medicine- USA

${ }^{2}$ Universidad de los Andes, Bogotá- Colombia

${ }^{3}$ Universidad de La Frontera- Chile.

${ }^{4}$ Glasgow Caledonian University- United Kingdom

${ }^{5}$ University of Capetown- Sudafrica

Introduction/Purpose: Sustained community engagement is recognized as a key component in building healthier environments, yet few systematic methods exist for activating resident engagement over time, particularly in under-resourced communities. We describe the development and initial results of a global research network, called Our Voice, aimed at systematically engaging diverse residents as citizen scientists in enacting local healthy environment changes. METHOD: Researchers from 14 countries (6 continents) contributed to building a systematic, empirically-driven citizen science approach where residents, regardless of circumstances, learn to use a simple, geo-tagged mobile app to capture local barriers to healthy behaviors, build consensus around high priority issues, and activate realistic changes with key stakeholders. The process of Network development and early project results have been captured via online surveys and a web portal. RESULTS: During the first year, Network target populations and problem areas have been diverse, including transforming underutilized public spaces for low-income seniors (Taiwan); pioneering methods for engaging homeless residents in environmental audits of living conditions (Colombia); building peer-to-peer leadership and civic participation among school-aged children (S. Africa); developing hands-free audit tools to document and address barriers to cycling to work in low-income workers (Chile); and addressing environmental degradation and social inequities among residents living in proximity to inland waterways (Scotland). Network member ratings were generally strong for local stakeholder and resident receptivity to citizen science engagement (means $=7.2$ and 8.1 out of 10 , respectively), though Network member self-efficacy (SE) ratings for completing a 1-yr citizen science project were more variable (mean $=5.7$ out of 10 , range $=1-10$ ). Of note, 7 of 9 sites that rated their SE as at least 7 out of 10 were able to successfully conduct a project during the initial 1 -year period. CONCLUSION: Network results thus far indicate the acceptability and promise of Our Voice in engaging residents across diverse locales and cultures. Goals include fostering increased researcher SE for conducting each step of the citizen science approach.

CORRESPONDING AUTHOR; Abby King, Stanford University School of Medicine, king@stanford.edu

\section{O29.4}

IMPACT OF EMBEDDING A HEALTHY LIFESTYLE CLINICIAN IN A COMMUNITY MENTAL HEALTH SERVICE TO ADDRESS THE HEALTH RISK BEHAVIOURS OF CLIENTS

Caitlin Fehily ${ }^{1}$, Kate Bartlem ${ }^{2}$, John Wiggers ${ }^{2}$, Paula Wye ${ }^{2}$, Richard Clancy $^{2}$, David Castle ${ }^{3}$, Sonia Wutzke ${ }^{4}$, Chris Rissel $^{5}$, Andrew Wilson ${ }^{4}$ ${ }^{1}$ University of Newcastle- Australia

${ }^{2}$ The University of Newcastle- Australia

${ }^{3}$ The University of Melbourne- Australia

${ }^{4}$ The Australian Prevention Partnership Centre- Australia

${ }^{5}$ The University of Sydney. Australia

Introduction \& purpose: Compared to the general population, persons with a mental illness experience higher morbidity and mortality from chronic diseases, contributed to by a higher prevalence of health risk behaviours, including: tobacco smoking, poor nutrition, harmful alcohol consumption and physical inactivity. Clinical practice guidelines recommend that mental health services routinely address these risk behaviours by assessing level of risk, providing brief advice to improve behaviours and referring at-risk clients to specialist behaviour change supports, such as telephone based support services. However, provision of this recommended preventive care is consistently reported to be low. This study aims to assess the impact of allocating a clinician within one community mental health service to the specific role of addressing clients' risk behaviours. Methods: A randomised controlled trial was conducted within one community mental health service in New South Wales (NSW), Australia. Clients $(n=811)$ were randomised to receive either usual care (control group) or usual care plus the offer of an additional face-to-face appointment and follow-up telephone call with a 'healthy lifestyle clinician' (intervention group). Telephone interviews were undertaken at baseline and a one-month follow-up to assess participants' receipt of preventive care from the mental health service (assessment, advice \& referral/s). Results: One third of clients allocated to the intervention attended the additional appointment with the healthy lifestyle clinician. Preliminary intention to treat analyses indicate significantly greater improvements between time points for the intervention group for the receipt of assessment (for nutrition, $\mathrm{p}=.026$; for alcohol, $\mathrm{p}=.045$ ), advice (for nutrition, $\mathrm{p}=.006$ ) and referral to the NSW Get Healthy Information and Coaching service $(p=.001)$. Conclusions: These results represent preliminary evidence for the effectiveness of the healthy lifestyle clinician role and suggest a model of preventive care provision which might be successfully implemented within mental health services.

CORRESPONDING AUTHOR; Caitlin Fehily, University of Newcastle, caitlin.fehily@uon.edu.au

\section{O29.5}

ASTHMA CONTROL INTERVENTION THROUGH COMMUNITY HEALTH WORKERS IN THE CALIFORNIA CENTRAL VALLEY EFFECTIVENESS AND PROGRAM EVALUATION

Gracy Mantoan ${ }^{1}$

${ }^{1}$ University of California Merced- USA

Introduction and Purpose: Asthma is the leading chronic illness on children in the USA, resulting in a great cost to the health care system and society. The California Central Valley has one of the highest rates of asthma in the nation, due to geo-demographic conditions. As the local efforts introduce a Community Health Worker Educational Intervention for the first time, what effects are observed in the community's asthma health outcomes? Would Central Valley participants accept peer support intervention? Methods: Using data from the Central California Asthma Collaborative Asthma Education Intervention, we evaluate its effectiveness on asthma outcomes (medication use, frequency of symptoms, need for emergency visits or hospital admissions, quality of life, etc.).Asthma Management measured by ACT-Asthma Control Test, and patient evaluation measured by MPQOLS (Mini Pediatric Asthma Quality of Life Survey). We also use a survey to evaluate patient satisfaction with the program, obtain patients' suggestions for improvement and inquire about acceptance of peer support. Results: Preliminary results of RCT with initial 37 participants show statistically significant improvement of asthma control test, and in quality of life survey. Self-reported asthma related hospitalizations decreased by $70 \%$, emergency visits decreased by $81 \%$, and outpatient visits decreased by $53 \%$. The cost-benefit analysis shows net-savings of $\$ 2,214$ per participant over 24 months, and ROI of $\$ 3.63$ per dollar. Patient Satisfaction Survey and analysis of new additional patients is ongoing. Conclusion: Educating patients and caregivers on environmental control of allergens and use of medication is vital for asthma control. Community Health Workers' Intervention add an aspect of community support that can greatly influence underserved 
communities in behavior change. Adding peer support can potentially increase adoption of new behaviors without increasing cost.

CORRESPONDING AUTHOR: Gracy Mantoan, University of California Merced,gmantoan@ucmerced.edu

\section{O29.6}

EFFECTIVENESS OF A DRAMA-AND-SONG-BASED HEALTH EDUCATION IN INCREASING KNOWLEDGE ON HOUSEHOLD WASTE MANAGEMENT IN MANCHU ETHNIC MINORITYBASED DACAO VILLAGE

Dawnie Ho Hei Lau ${ }^{1}$, Emily Ying Yang Chan ${ }^{1}$, Carol Ka Po Wong ${ }^{2}$

${ }^{1}$ The Chinese University of Hong Kong- Hong Kong

${ }^{2}$ Collaborating Centre for Oxford University and CUHK for Disaster and Medical Humanitarian Response

Introduction: Dioxin emissions from burning plastic waste are known to contain human carcinogens detrimental to health, yet indoor burning of household waste is a common practice in rural China. As part of the Ethnic Minority Health Project (EMHP), a drama-and-song-based health education on household waste management was conducted at Dacao village, to improve individual and community health through primary prevention. This study aims to evaluate the effectiveness of the health education at increasing knowledge in the Manchu ethnic minority-based village in China. Methods: A cross-sectional study was conducted in June 2017, in Dacao village in Jilin Province, using quantitative methods of pre-intervention and post-intervention household surveys $(\mathrm{N}=43)$. All data were double-entered and all calculations were made through Microsoft Excel and SPSS, with significance level defined at $p<0.05$. Results: Prior to the health education, $63.2 \%$ of the villagers did not know about the harmful effects of burning plastic waste, particularly for indoor household waste burning. After the intervention, results showed that villagers who attended the intervention were 5 times more likely to have improved knowledge in waste burning practice $(\mathrm{OR}=5.3 ; \mathrm{p}<0.01)$, and 7 times more likely to demonstrate knowledge improvement in the 4Rs of waste management $(\mathrm{OR}=7.5 ; \mathrm{p}<0.01)$. These findings indicate that participants show increased knowledge retention on household waste management. While there is no association between intervention success and sex, education, occupation or previous waste practice, those below the age of 60 are 10 times more likely to have correct knowledge on proper waste burning location after the intervention $(\mathrm{OR}=10.0 ; \mathrm{p}<$ 0.01). Conclusion: In the current era of globalisation, household waste management is becoming a growing concern to many countries. A bottom-up approach is key to the improvement of community health, especially in rural areas that lack proper waste treatment infrastructure. For ethnic minority-based remote villages with substandard basic health knowledge, a drama-and-song-based health education seems to be a good starting point for primary prevention, with further needs to transform knowledge into belief, and goals into sustained action.

CORRESPONDING AUTHOR: Dawnie Ho Hei Lau, The Chinese University of Hong Kong, dawnielau2020@gmail.com

\section{O30.1}

ANALYSIS \& DEVELOPMENT OF A PREDICTIVE MODEL OF VISITING BEHAVIOR TO PHYSICIANS IN HIGH-RISK INDIVIDUALS OF LIFESTYLE-RELATED DISEASES: A LONGITUDINAL STUDY USING CLAIMS \& ANNUAL HEALTH CHECK-UP DATA

Go Muto ${ }^{1}$, Atsushi Goto ${ }^{2}{ }^{4}$, Ryoko Katagiri ${ }^{4}{ }^{6}$, Motoki Endo ${ }^{3}$, Kazuhito Yokoyama $^{78}$

${ }^{1}$ Harvard T.H.Chan School of Public Health- USA

${ }^{2}$ Epidemiology and Prevention Group- USA

${ }^{3}$ Center for Public Health Sciences- Australia
${ }^{4}$ National Cancer Center- Japan

${ }^{5}$ Harvard T.H.Chan School of Public Health - USA

${ }^{6}$ Departments of Nutrition, Boston, USA \& Epidemiology and Prevention Group

${ }^{7}$ Graduate school of Medicine, Juntendo University- Japan

${ }^{8}$ Department of Epidemiology and Environmental health

[Objectives] To clarify and develop a prediction model of the initial visiting behavior to physicians in high-risk individuals of hypertension (HT), diabetes mellitus (DM), and dyslipidemia (DL) after annual health checkups. [Methods] Using both claim and health check-up data (JMDC) of beneficiaries aged 20 to 74 years who were covered by health insurance societies between 2008 and 2016, we identified high risk 533,955 examinees of health checkups who met the following criteria and had no claim data on the corresponding items 4 months prior to the health checkups. The criteria was either blood pressure, blood glucose level, or blood lipid level provided by Japanese government. We assessed examinees first visit to medical institutions, calculated cumulative non-visiting rates by Kaplan-Meier estimates, and evaluated associations between characteristics of high-risk individuals and their visiting behavior using multivariable logistic regression models. [Results] The cumulative non-visiting rates at 3, 6, 9, and 12 months after health check-ups were 91.4, 88.2, 86.2 , and $84.4 \%$, respectively. As for HT, DM, and DL, the cumulative non-visiting rates at 12 months were $84.3,67.9$, and $86.1 \%$, respectively. When we focus on the extremely high risk group, the rate was $74.0 \%$, since the rate for extremely high risk of DM ( $\mathrm{HbA} 1 \mathrm{c} \geq 8.4 \%$ ) was $51.9 \%$. Furthermore, there was no difference between the tendency of the rate of the previous period (2008-12) and that of the latter period (2012-16) despite a slight improvement of rate for individuals with extremely high risk DM. [Conclusion] Over 80\% high risk examinees of noncommunicable diseases did not visit to medical institutions during 1 year period after health check-ups, although approximately half of the individuals with extremely high risk of DM visited to physicians at least one time. From the analysis stratified, the factors associated with higher tendency of visiting behavior were identified. Finally, we developed a predictive scoring model for visiting to medical institutions, and it was well validated from both discrimination and calibration viewpoints. This model enable public/occupational health staffs to individualize population for strong intervention.

CORRESPONDING AUTHOR: Go Muto, Harvard T.H.Chan School of Public Health, g-muto@juntendo.ac.jp

\section{O30.2}

EFFECTS OF A PREDIABETES DIAGNOSIS ON PATIENT ENGAGEMENT IN STRATEGIES TO PREVENT DIABETES

Jeffrey Kullgren ${ }^{1}$, Angela Fagerlin ${ }^{2}$, Caroline Richardson ${ }^{1}$, Bradley Youles $^{3}$, Dina Hafez ${ }^{3}$, Michele Heisler ${ }^{3}$

${ }^{1}$ University of Michigan- USA

${ }^{2}$ Salt Lake City VA Medical Center and University of Utah- USA

${ }^{3}$ VA Ann Arbor Healthcare System- USA

Introduction \& Purpose: A window of opportunity to engage at-risk patients in strategies to prevent type 2 diabetes mellitus (T2DM) is when they are screened for T2DM and found to have prediabetes. Yet, little is known about the effects of a prediabetes diagnosis on patient engagement in preventive behaviors and potential mediators of this engagement. Methods: In a parallel-design randomized controlled trial we recruited 315 non-diabetic patients from the Veterans Affairs Ann Arbor Healthcare System who had at least 1 risk factor for T2DM but had not had a hemoglobin A1c (HbA1c) test to screen for T2DM in the last year. Patients completed a baseline survey and then using 4:1 allocation were randomly assigned to, at their next primary care visit, (1) undergo an $\mathrm{HbA} 1 \mathrm{c}$ test to screen for T2DM and receive brief standardized counseling about their $\mathrm{HbA} 1 \mathrm{c}$ results or (2) review a brochure about recommended 
clinical preventive services. Three months later patients were surveyed about their perception of their risk for developing T2DM (on a 0-100 scale), level of motivation to prevent T2DM (on a 0-10 scale), weight status, and physical activity. We used difference-in-differences analyses adjusted for age, gender, and body mass index to compare 3-month changes in outcomes between patients in the HbA1c test arm who were found to have prediabetes and patients in the brochure arm. Results: The 106 patients in the $\mathrm{Hb} 1 \mathrm{Ac}$ test arm who were found to have prediabetes (out of 252 patients in that arm) had a greater increase in their 3-month level of motivation to prevent T2DM (mean $0.8 ; \mathrm{P}=0.004$ ) than the 63 patients in the brochure arm, but there were no significant differences between these groups in changes in perception of risk for developing T2DM, self-reported weight loss, or physical activity. Conclusions: Using an $\mathrm{HbAlc}$ test to identify patients with prediabetes and providing them with brief counseling may increase their short-term level of motivation to prevent T2DM, but may not influence perception of risk for developing T2DM or engagement in key preventive behaviors. New strategies may be needed to help patients found to have prediabetes through screening translate their heightened motivation to prevent T2DM into engagement in preventive behaviors.

CORRESPONDING AUTHOR: Jeffrey Kullgren, University of Michigan,jkullgre@med.umich.edu

\section{O30.3}

ASSOCIATIONS BETWEEN HEALTH LITERACY AND HEALTHCARE UTILIZATION IN PATIENTS WITH COEXISTING DIABETES AND END STAGE RENAL DISEASE

Konstadina Griva ${ }^{1}$, Russell K1 Yoong ${ }^{2}$, Mooppil Nandakumar ${ }^{3}$, Eric $\mathrm{Khoo}^{4}$, Moothathamby Rajeswari ${ }^{3}$, Augustine Wc Knag ${ }^{2}$, Stanton P Newman.

${ }^{1}$ Lee Kong Chian School Of Medicine Nanyang Technological University- Singapore

${ }^{2}$ National University- Singapore

${ }^{3}$ National Kidney Foundation- Singapore

${ }^{4}$ National University Hospital

${ }^{5}$ City University of London- United Kingdom.

BACKGROUND: Patients with comorbid diabetes and end stage renal disease (DM ESRD) represent the fastest growing and most frail segment of dialysis population, with elevated rates of hospitalization and mortality. It is important to examine psychosocial factors that may compromise health outcomes. Health literacy has become an increasingly important concept in public health with recent advances in measurement allowing the profiling of personal and social dimensions of the concept. The aim of this study was to use a multi-dimensional tool to profile health literacy domains of DM-ESRD patients and to evaluate their associations with hospitalization and mortality in this multi-morbid population. METHODS: Depression and Health Literacy was assessed in a prospective outpatient cohort of patients with DM ESRD on the Hospital Anxiety and Depression Scale (HADS) and the Health Literacy Questionnaire (HLQ). Primary outcomes were all-cause admission (number of events; days of hospitalization) and mortality over 15 to 24 months. Negative binomial or Cox regressions were used to model risk factors for hospitalization and mortality respectively. RESULTS: Of 221 participants [median age 59 years, $39.4 \%$ women, $54.8 \%$ Chinese], 95 (43.0\%) screened positive for depression. Risk for depression was higher for Chinese patients (OR 2.499, 95\% CI $1.436-4.349, \mathrm{P}=.001)$ compared to nonChinese respondents. Depression, older age, Chinese Ethnicity, Nonmarried status, lower education were all associated with lower scores in most health literacy domains. Lower hospitalisation rates were independently associated with home-ownership (IRR=0.639) and higher Actively Managing Health HLQ scores (IRR=0.674). Cumulative hospitalisation days were independently associated with serum albumin (IRR=0.914), HbA1c $(\operatorname{IRR}=1.190)$, Charson Comorbidity Index $(I R R=1.171)$ and depression ( $I R R=1.061)$. Mortality was also significantly associated with Actively Managing Health (Hazard Ratio $=0.382$ ) after controlling for other risk factors. CONCLUSIONS: These findings suggest that strategies for improving health outcomes and reducing hospitalization and mortality risk in multi-morbid patients may benefit from adopting a stronger focus on critical and communicative domains of Health Literacy as part of patient education, and addressing emotional distress.

CORRESPONDING AUTHOR: Konstadina Griva, Lee Kong Chian School Of Medicine Nanyang Technological University, konstadina.griva@ntu.edu.sg

\section{O30.4}

OPTIMIZING EXPECTATIONS ABOUT ENDOCRINE TREATMENT FOR BREAST CANCER: RESULTS OF THE RANDOMIZED CONTROLLED PSY-BREAST TRIAL

Meike Shedden-Mora ${ }^{1}$, Yiqi $\mathrm{Pan}^{2}{ }^{3}$, Sarah Heisig ${ }^{2}{ }^{3}$, Pia von Blanckenburg $^{2}{ }^{3}$, Winfried Rief ${ }^{2}{ }^{3}$, Isabell Witzel ${ }^{4}{ }^{5}$, Ute-Susann Albert $^{67}$, Yvonne Nestoriuc ${ }^{2} 3$

${ }^{1}$ University Medical Center Hamburg-Eppendorf- Germany.

${ }^{2}$ Department of Psychosomatic Medicine and Psychotherapy- Germany

${ }^{3}$ University Medical Center Hamburg-Eppendorf, Hamburg- Germany

${ }^{4}$ Department of Gynecology- Germany

${ }^{5}$ University Medical Center Hamburg-Eppendorf, Hamburg, Germany

${ }^{6}$ AWMF-Institute for Medical Knowledge Management, Philipps

${ }^{7}$ University Marburg, Germany

Objective: Medication side effects are strongly determined by non-pharmacological, "nocebo" mechanisms, particularly patients' expectations. Optimizing expectations could minimize side effect burden, increase adherence and enhance quality of life. This study evaluated whether a brief expectation management training (EXPECT) optimizes medicationrelated expectations in women starting adjuvant endocrine therapy (AET) for breast cancer. Methods: In a multisite randomized controlled design, 197 women with breast cancer were randomized to EXPECT, supportive therapy (SUPPORT) as psychological control condition, or treatment as usual (TAU). The three-session cognitive-behavioral EXPECT employs psychoeducation, guided imagery, and side effect management training. Outcomes were necessity-concern beliefs about AET (BMQ), expected side effects (GASE-expect), expected coping ability (GASE-coping), treatment control expectations (B-IPQ), and adherence intention. Results: Both interventions were well accepted and feasible, treatment adherence and therapist allegiance were high. Patients' necessity-concern beliefs were optimized in EXPECT compared to both TAU and SUPPORT, $\mathrm{d}=.41, \mathrm{p}<.001 ; \mathrm{d}=.40, \mathrm{p}<.001$. Expected coping ability and treatment control expectations were optimized compared to TAU, $\mathrm{d}=.35, \mathrm{p}=.02 ; \mathrm{d}=.42, \mathrm{p}<001$, but not to SUPPORT. Adherence intention was optimized compared to SUPPORT, $\mathrm{d}=.29, \mathrm{p}=.02$, but not to TAU. Expected side effects did not change significantly. Conclusions: Expectation management effectively and partly specifically modified medication-related expectations in women starting AET. Given the influence of expectations on long-term treatment outcome, psychological interventions like EXPECT might provide potential pathways to reduce side effect burden and improve quality of life during medication intake.

CORRESPONDING AUTHOR: Meike Shedden-Mora, University Medical Center Hamburg-Eppendorf, m.shedden-mora@uke.de

\section{O30.5}

PREGNANCY INTENTIONS, HEALTH-RELATED LIFESTYLE BEHAVIOURS, AND PSYCHOLOGICAL WELLBEING: A SYSTEMATIC MAPPING REVIEW OF THE LITERATURE

Briony Hill ${ }^{1}$, Emily J. Kothe ${ }^{2}$, Sinéad Currie ${ }^{3}$, Meaghan Danby ${ }^{2}$, Madelon North $^{2}$, Adina Lang ${ }^{1}$, Cate Bailey ${ }^{1}$, Lisa J. Moran ${ }^{1}$, Helena Teede ${ }^{1}$ 
${ }^{1}$ Monash Centre for Health Research and Implementation- Australia ${ }^{2}$ School of Psychology, Deakin University- Australia

${ }^{3}$ Psychology, Faculty of Natural Sciences, University of Stirling, United Kingdom

Introduction and Purpose: Suboptimal lifestyle behaviours and psychological wellbeing before and during pregnancy are associated with a host of adverse maternal and infant outcomes, including obesity. Pregnancy intentions are an important consideration in understanding motivations for maternal behaviour change. The aim of this study was to conduct a systematic mapping review of the literature that explored associations of pregnancy intentions with health-related lifestyle behaviours and psychological wellbeing before and during pregnancy. Methods: In May 2017, six medical/health databases were searched for quantitative studies exploring the association between pregnancy intention and psychological wellbeing and/or health-related lifestyle behaviours preconception or during pregnancy. Results: The search retrieved 19,430 articles, 818 full-text articles were assessed for eligibility. 312 articles were included, involving $2,656,228$ participants; median sample size was 913 . Preconception pregnancy intentions: Only 25 studies prospectively measured pregnancy intentions during the preconception period. 108 studies considered associations between preconception pregnancy intention and behaviours/ psychological wellbeing, including supplement use $(\mathrm{k}=62)$, alcohol use $(\mathrm{k}=37)$ and smoking $(\mathrm{k}=32)$. Preconception weight-related lifestyle behaviours were assessed in relation to pregnancy intention in only 16 studies; 8 studies assessed psychological wellbeing. Pregnancy intentions when already pregnant: 240 studies considered associations between pregnancy intention in women already pregnant and behaviours/ psychological wellbeing, including prenatal care $(\mathrm{k}=81)$, antenatal depression ( $\mathrm{k}=62)$, smoking $(\mathrm{k}=58)$, and alcohol use $(\mathrm{k}=44)$. Weightrelated lifestyle behaviours were uncommon (exercise/physical activity $\mathrm{k}=2$; nutrition $\mathrm{k}=5$ ). Conclusions: This literature is hampered by the limitations associated with retrospective assessment of pregnancy intention. Despite the large volume of studies, there are obvious gaps in the literature. Future research should focus on prospectively assessed pregnancy intentions in addition to preconception weight-related lifestyle behaviours such as dietary and physical activity behaviours, as these are key modifiable risk factors for maternal obesity.

CORRESPONDING AUTHOR: Briony Hill, Monash Centre for Health Research and Implementation, briony.hill@monash.edu

\section{O30.6}

EFFECTS OF A CHILDBIRTH EDUCATION IN SIMULATED DELIVERY ROOM ON FEAR OF CHILDBIRTH AND OBSTETRIC OUTCOMES

Yu Mengting ${ }^{1}$, Wang Leyuan ${ }^{2}$, Diao Guanwei ${ }^{3}$, Du Yaping ${ }^{4}$, Ma Liangkun ${ }^{3}$

${ }^{1}$ Zhejiang University School of Public Health- China

${ }^{2}$ Beijing Chao-Yang Hospital- China

${ }^{3}$ Peking Union Medical College Hospital- China

${ }^{4}$ Zhejiang University School of Public Health- China

Introduction \& Purpose: Fear of childbirth (FOC) can impair mental and physical health of pregnant women and lead to undesirable obstetric outcomes. The aim of this study was to evaluate effects of a childbirth education with simulation teaching method in a simulated delivery room on reducing FOC and improving obstetric outcomes. The research questions are as follows: 1 . What is the effect of childbirth education with simulation teaching method on fear of childbirth? 2. What is the effect of childbirth education with simulation teaching method on obstetric outcomes? Methods: A quasi-experimental pre- and post-test comparative design. The course consisted of two parts, part 1 imparting basic knowledge of childbirth with theory teaching method in a health education classroom, part 2 demonstrating the process of labor and birth, practicing skills of pain management and pushing for childbirth with simulation teaching method in a simulated delivery room. 86 primiparae in third trimester were enrolled and allocated randomly into experimental group $(n=42)$ receiving both parts of the course, or into control group $(n=44)$ receiving part 1 of the course. Primary outcomes were reduction of FOC and improvement in obstetric outcomes. Data was collected with demographic, psychosocial \& obstetric characteristics questionnaire, WijmaDelivery Expectancy Questionnaire-version A(WDEQ-A) and obstetric records. Statistical methods included analysis of covariance(ANCOVA), Student's t-test, Mann-Whitney U test and chi-squared test. Results: Statistically significant differences were found between groups on posteducation scores for $\mathrm{FOC}(52.00$ vs. $58.00, \mathrm{p}=0.033)$, change scores(preeducation minus post-education) for $\mathrm{FOC}(14.10 \mathrm{vs}$. 5.44, $\mathrm{p}=0.008)$, vaginal birth frequency $(90.48 \%$ vs. $72.73 \%, \mathrm{p}=0.034)$ and episiotomy frequency $(50.00 \%$ vs. $75.00 \%, p=0.032)$. Two groups didn't differ evidently in perineal tear frequency, analgesic pain management frequency, duration of labor and amount of postpartum haemorrhage $(\mathrm{P}>0.05)$. Conclusion: Childbirth education with simulation teaching method in a simulated delivery room was more effective in reducing fear of childbirth and improving obstetric outcomes compared with traditional one. It's strongly advocated to apply simulation teaching method and setting into childbirth education.

CORRESPONDING AUTHOR: Yu Mengting, Zhejiang University School of Public Health, ymt856@163.com

\section{O31.1}

DO MUSCULOSKELETAL CONDITIONS INCREASE THE RISK OF CHRONIC DISEASE: A SYSTEMATIC REVIEW AND METAANALYSIS OF COHORT STUDIES

Amanda Williams ${ }^{1}$, Steven Kamper ${ }^{2}$, John Wiggers ${ }^{3}$, Kate O'Brien ${ }^{3}$, Hopin Lee ${ }^{4}$, Luke Wolfenden ${ }^{3}$, Serene Yoong ${ }^{3}$, Emma Robson ${ }^{3}$, James McAuley $^{3}$

${ }^{1}$ University of Newcastle- Australia

${ }^{2}$ University of Sydney- Australia

${ }^{3}$ University of Newcastle- Australia

${ }^{4}$ University of Oxford

Introduction \& Purpose: A growing body of research shows that musculoskeletal conditions are associated with chronic diseases. A suggested mechanism for this relationship is that musculoskeletal conditions contribute to the development of other chronic diseases. For example, pain and disability may limit physical activity and subsequently increase the risk of obesity, cardiovascular disease, diabetes, or cancer. We undertook a systematic review to investigate the causal relationship between common musculoskeletal conditions (neck and back pain, and osteoarthritis of the knee or hip) and chronic diseases (cardiovascular disease, cancer, diabetes, chronic respiratory disease and obesity). Methods: We searched relevant databases up to November 2016 and identified longitudinal cohort studies that estimated a temporal association between the prevalence of musculoskeletal conditions (neck or back pain, and osteoarthritis of the knee or hip) at baseline and chronic disease (cardiovascular disease, cancer, diabetes, chronic respiratory disease and obesity) throughout follow up. Risk of bias was assessed using a modified version of the QUIPS tools. We pooled adjusted hazard ratios and incidence rate ratios, using random-effects meta-analyses. We conducted subgroup analyses separated by musculoskeletal conditions and by outcome where possible. Results: We identified 13,736 articles of which 16 articles reporting on 11 studies met the inclusion criteria. The primary meta-analysis included data from 10 studies and 2,669,751 persons. Cardiovascular disease was the outcome in 9 studies and cancer in 1 study. Two studies measured back pain as the exposure and eight measured osteoarthritis. The primary meta-analysis revealed a statistically significant increase in the risk of developing 'chronic disease' in those reporting musculoskeletal conditions at baseline (HR=1.18; 95\% CI, 1.13 to 1.22 ; I2 57\%). Subgroup 
analysis by condition revealed a similar result for osteoarthritis and chronic disease. No meta-analysis was performed for back pain. The two studies of back pain found people with back pain had an increased risk of cardiovascular disease $(\mathrm{HR}=2.13$; 95\% CI, 1.32 to 3.44$)$ and 10 -year incidence of cancer (IRR $=1.25 ; 95 \% \mathrm{CI}, 1.19$ to 1.32 ). Conclusions: Patients with musculoskeletal conditions have an increased risk of subsequent chronic disease. Effective treatment of musculoskeletal conditions and comorbid lifestyle risks, such as inadequate physical activity, may play a role in preventing other chronic diseases.

CORRESPONDING AUTHOR: Amanda Williams, University of Newcastle, amanda.j.williams@hnehealth.nsw.gov.au

\section{O31.2}

POSITIVE AND NEGATIVE AFFECT INSTABILITY CAN EXACERBATE CHRONIC PAIN OUTCOMES: AN ECOLOGICAL MOMENTARY ASSESSMENT STUDY

Alissa Beath ${ }^{1}$, Danielle Blaydon ${ }^{1}$, Michael Jones ${ }^{1}$

${ }^{1}$ Macquarie University- Australia

Introduction \& Purpose Chronic pain is highly prevalent and substantially impacts individuals' daily life. Improving the outcomes of individuals with chronic pain requires identifying factors that influence the pain experience. While research supports the role of emotion in pain experience, little attention has been given to emotional dynamics, particularly intense fluctuations in positive affect (PA) or negative affect (NA), labelled affective instability. The current study aimed to explore the role of affective instability on within-day reports of pain outcomes using an ecological momentary assessment (EMA) methodology, specifically investigating whether high affective instability enhanced the detrimental effect of pain intensity on pain outcomes. Methods Individuals with chronic pain $(\mathrm{N}=67$ final sample, 54 women, mean age $=21, \mathrm{SD}=6)$ recruited from Macquarie University and surrounding areas reported a comprehensive pain history and demographics at baseline. Participants then received five random within-day notifications for seven consecutive days, administered via a smartphone app, reporting PA and NA (6 adjectives respectively), pain intensity (1 item), pain-related disability (1 item), and cognitive complaints ( 3 items). Affective instability was calculated using the mean square of successive differences of reports of PA and NA respectively. Multi-level models were used to analyse the hierarchical (nested) data with bootstrapped standard errors. Results Both PA and NA instability moderated the effect of pain intensity on pain outcomes. As affective instability increased, the effect of increasing pain intensity on pain outcomes became stronger (more detrimental) (PA instability x pain intensity on disability: $\mathrm{b}=0.38, \mathrm{SE}=0.11, \mathrm{p}=.001 ; \mathrm{PA}$ instability $\mathrm{x}$ pain intensity on cognitive complaints: $\mathrm{b}=0.34, \mathrm{SE}=0.10, \mathrm{p}=.001$; NA instability x pain intensity on cognitive complaints: $\mathrm{b}=0.48, \mathrm{SE}=0.19, \mathrm{p}=.006$ ). There was also evidence that for lower-than-average pain intensity, greater PA instability predicted lower cognitive complaints $(b=-1.02, \mathrm{SE}=0.22$, $\mathrm{p}=.001$ ), whereas this was not the case for NA instability. Conclusions This study is the first of its kind to demonstrate the relationships of within-day fluctuations in affect and chronic pain outcomes. We have shown that while extreme affective instability, both positive and negative, is detrimental when coupled with high pain, there may be a beneficial role of positive affect instability when pain is lower, potentially via emotion regulation mechanisms such as distraction.

CORRESPONDING AUTHOR :Alissa Beath, Macquarie University, alissa.beath@mq.edu.au

\section{O31.3}

"THE PATIENT IN CHARGE": PATIENTS' EXPERIENCES OF MULTIDISCIPLINARY PAIN REHABILITATION AND ITS IMPACT ON THEIR DAILY LIFE
Anke Samulowitz ${ }^{1}$, Pia Nordström ${ }^{2}$, Malin Wiklund ${ }^{1}$, Nenad Stankovic ${ }^{2}$, Gunnel Hensing ${ }^{1}$.

${ }^{1}$ University of Gothenburg- SWEDEN

${ }^{2}$ Region Västra Götaland- SWEDEN

Introduction \& Purpose Chronic pain is often a lifelong condition with negative effects on physical health, mental health, quality of life, work capacity, family relations, household duties, and leisure activities. However, the role of pain rehabilitation for how men and women with pain handle their everyday life needs further investigation. The aim of this study was to examine how men and women with pain experience the impact of multimodal pain rehabilitation on their everyday life. Methods Individual semi-structured interviews of five women and three men, which have participated in a pain rehabilitation program. Results An overall theme developed as "the patient in charge". The sense of control was described by the participants as a key for their quality of life, and illustrated by three categories: The importance of the patient-provider relationship, knowledge gained, and pain in a social context. Interesting patterns appeared, like the importance of trust in health care's expertise, combined with the surprisingly low significance of social support. The role of gender was also discussed. A pattern showed, illustrating that both men and women seemed to experience gendered norms in health care, which affected them in different ways. Conclusions The data generated several hypothesis. A trustful patient-provider relationship that supports patients' autonomy, and confidence in the provider's professionality, could be a prerequisite for acceptance of pain and improved quality of life. It is possible that the importance of social support by friends or relatives has been overvalued by earlier research, especially for women. Gendered norms, held by health care providers may have a negative impact on both men and women, in different ways. The results from this study should be explored in further research and translated into clinical practise.

CORRESPONDING AUTHOR: Anke Samulowitz, University of Gothenburg, anke.samulowitz@vgregion.se

\section{O31.4}

SELF-EFFICACY AND DISABILITY AMONG PEOPLE WITH SPINAL CORD INJURY: A LONGITUDINAL EXPLORATION

Amy Richardson ${ }^{1}$, Ari Samaranayaka ${ }^{1}$, Sarah Derrett ${ }^{1}$

${ }^{1}$ University of Otago- New Zealand

Introduction \& Purpose: The experience of disability among people with spinal cord injury (SCI) has been related to a diverse range of factors. However, few studies have investigated whether psychological factors prior to SCI are related to subsequent function. The purpose of this longitudinal investigation was to examine whether pre-SCI self-efficacy was associated with the experience of disability in a sample of New Zealanders living with SCI. Methods: Between 2007 and 2009, 118 people admitted to one of New Zealand's two spinal units for an initial SCI were recruited to take part in three telephone interviews. Interviews were conducted approximately 6,18 , and 30 months post-SCI. Participants provided comprehensive health and well-being information in response to a range of standardised questionnaires. The General Self-Efficacy Scale (GSE) questionnaire was used to assess pre-SCI self-efficacy and the World Health Organization Disability Assessment Schedule 2.0 (12item) was used to measure disability at each assessment point. Results: Complete data was available for 118 participants at 6 months, 103 participants at 18 months, and 91 participants at 30 months. Average disability scores were found to increase over time, with lower disability reported at 6 months $(\mathrm{M}=19.66, \mathrm{SD}=10.03)$, compared to $18(\mathrm{M}=27.65, \mathrm{SD}=$ $10.09)$ and 30 months $(M=26.17, S D=9.79)$. No difference in disability was observed between low and high self-efficacy groups at 6 and 18 months. However, high self-efficacy participants reported lower disability scores at 30 months $(\mathrm{M}=24.78, \mathrm{SD}=9.12)$ compared to low self- 
efficacy participants $(M=27.42, \mathrm{SD}=10.30)$, and this difference approached statistical significance $(t(d f)=1.293(89), p=0.09)$. Results did not differ when restricting the analyses to those who completed all interviews. Conclusions: These preliminary results suggest that selfefficacy prior to SCI may be related to future disability among New Zealanders with SCI. Further longitudinal research is needed to confirm whether self-efficacy promotes lower levels of disability, particularly when controlling for injury-related characteristics. This would help to clarify whether self-efficacy is an important target for intervention in people with SCI.

CORRESPONDING AUTHOR: Amy Richardson, University of Otago, amy.richardson110@gmail.com

\section{O31.5}

PAIN PREVALENCE, CHARACTERISTICS AND IMPACT AMONG PEOPLE WITH HAEMOPHILIA: INSIGHTS FROM THE FIRST PORTUGUESE SURVEY AND CONTRIBUTIONS TO IMPROVE PAIN ASSESSMENT AND MANAGEMENT

Patrícia R Pinto $^{1}$, Ana Cristina Paredes ${ }^{123}$, Armando Almeida ${ }^{1} 23$

${ }^{1}$ University of Minho- Portugal

${ }^{2}$ Life and Health Sciences Research Institute (ICVS)- Partugal

${ }^{3}$ School of Health Sciences

Introduction \& Purpose: Haemophilia is a rare genetic bleeding disorder characterized by spontaneous bleeding mostly affecting the joints. People with haemophilia (PWH) experience acute pain during joint bleeds, but also chronic pain due to hemophilic arthropathy. Pain is therefore a critical aspect of life among PWH, but a systematic assessment of haemophilia-related pain in Portugal was never conducted. This study aimed to understand pain experience among Portuguese $\mathrm{PWH}$, by describing its prevalence, characteristics and impact, and unveiling intervention needs in the realm of haemophiliarelated pain care. Methods: This was a cross-sectional study wherein participants ( $\mathrm{N}=144)$, with haemophilia $\mathrm{A}$ or $\mathrm{B}$ of all severities, answered questions on socio-demographic and clinical data, pain, emotional distress and quality of life. Results: Pain in the previous year was reported by $82(78.8 \%)$ adults, $16(76.2 \%)$ children/teenagers (age 10-17) and 13 (68.4\%) children (age 1-9). Mean number of pain locations was $5.23(\mathrm{SD}=3.95)$ for adults, 4.13 $(\mathrm{SD}=3.48)$ for children/teenagers and $3.15(\mathrm{SD}=1.99)$ for children between 1-9 years, with pain in the lower limbs causing the greatest negative impact. Pain duration varied from 1 month to 51 years in adults and from 1 month to 9 years in children/teenagers. Moreover, adults highlighted physical efforts and/or movements as the most often pain triggering factor $(61 ; 74.4 \%)$ and children and teenagers most frequently pointed bleeds as a pain trigger [age 10-17: 14 (87.5\%); age 1-9: 9 (69.2\%)]. Adult participants with pain had a higher prevalence of anxiety $(\mathrm{OR}=1.698 ; \mathrm{p}=0.003)$ and depression $(\mathrm{OR}=1.961 ; \mathrm{p}=0.025)$ symptoms, and significantly lower quality of life $(\mathrm{OR}=0.928 ; \mathrm{p}=0.001)$. Conclusions: Current findings highlight that pain is a critical aspect of haemophilia, by revealing its high prevalence and frequency at all age ranges, its potentially simultaneous acute and chronic nature, along with the likelihood to affect multiple locations in the same individual. The results also show the detrimental impact of pain on peoples' lives, namely on well-being and quality of life. Important insights concerning intervention needs can be drawn from these findings, ultimately contributing to improve pain management and patients' care among $\mathrm{PWH}$.

CORRESPONDING AUTHOR: Patrícia R Pinto, University of Minho, patipinto@gmail.com

O31.6

THE ASSOCIATIONS BETWEEN FATIGUE AND COPING SELFEFFICACY IN PATIENTS WITH RHEUMATOID ARTHRITIS

Alexandra Husivargova $^{12}{ }^{2}$, Zelmira Macejova $^{12}{ }^{3}$, Jozef Benka $^{123}$, Dagmar Breznoscakova ${ }^{2} 4_{4}$, Iveta Nagyova ${ }^{1} 23$
${ }^{1}$ Department of Social and Behavioural Medicine- Slovakia

${ }^{2}$ Faculty of Medicine- Slovakia

${ }^{3}$ PJ Safarik University, Kosice- Slovakia

${ }^{4} 1$ st Department of Psychiatry -Slovakia

Introduction \& Purpose The prevalence of fatigue in patients with rheumatoid arthritis (RA) is $80-93 \%$. Despite being one of the most prevalent symptoms, it is still rather neglected. In relation to RA, self-efficacy expectations seem to be of major importance. Studies have shown that there are associations between coping self-efficacy and health status in RA patients, but associations with fatigue are less clear. Therefore, the aim of this study was to unravel the associations between fatigue and coping self-efficacy in RA patients. Methods The sample consisted of 297 RA patients ( $80.8 \%$ female; mean age $56.03 \pm 11.57$ years). All patients completed the Visual Analogue Scale-Fatigue (VAS), 36-Item Short Form Health Survey Vitality Scale (SF-36) and the Coping SelfEfficacy Scale (CSE) with 3 subscales: Problem Focussed Coping (PFC), Getting Support from Family and Friends (GSFF) and Stopping Unpleasant Emotions and Thoughts (SUET). Multiple linear regressions were used for statistical analyses. The first regression model (Model 1) comprised sociodemographic variables: age, gender and education. In Model 2 clinical variables (DAS28crp, ESR, disease duration) and functional status (HAQ) were added. In Model 3, due to high collinearity, one of the three CSE subscales (PFC, GSFF, SUET) was added, respectively. Results In final regression models significantly associated with VASfatigue and SF-36 vitality were HAQ and all three CSE subscales. After controlling for relevant sociodemographic and clinical variables in VASfatigue CSE subscale PFC contributed with $6 \%$ to the total explained variance, GSFF added $7 \%$ and added $9.5 \%$. In SF-36 Vitality Scale, PFC contributed with $4.8 \%$ to the total explained variance, GSFF with $5 \%$ and SUET with $5.3 \%$. The total explained variances ranged between $18.5 \%$ and $24.8 \%$. Conclusions Our results confirm that coping selfefficacy is significantly associated with fatigue. Caregivers and physicians have to find a way to help RA patients to learn how to manage chronic diseases, especially enhancing their coping self-efficacy skills. This can be efficiently done in disease self-management programmes. [Grant support: APVV-15-0719]

CORRESPONDING AUTHOR: Alexandra Husivargova, Department of Social and Behavioural Medicine, Faculty of Medicine, PJ Safarik University, Kosice, Slovakia, alexandra.husivargova@upjs.sk

\section{O32.1}

STIGMA AND DISCRIMINATION INFLUENCE HIV CARE ENGAGEMENT AMONG LATINO IMMIGRANTS LIVING IN THE U.S.

Janet Myers $^{1}$, Andres Maiorana ${ }^{1}$, Sophia Zamudio-Haas ${ }^{1}$, Remi Frazier ${ }^{1}$, Starley Shade ${ }^{1}$

${ }^{1}$ University of California, San Francisco-USA

Introduction \& Purpose: Migrants face complex barriers to accessing HIV care and Latinos living in the continental U.S., but born elsewhere, may be particularly vulnerable. Intersectional issues of stigmatization based on a person's HIV status, sexual orientation, gender identity, ethnicity and/or race may impact care engagement. In this study, we examined reports of self-perceived stigma and reports of discrimination and their impact on HIV care experiences among Mexicans and Puerto Ricans living in the U.S. Methods We used quantitative survey data to assess internalized stigma and experiences of discrimination based on HIV-, gay- and ethnicity-related identities to explore how 893 study participants' perceptions of stigma were associated with measures of HIV care engagement. We also used qualitative interview data from 48 clients to describe the intersectionality of stigma in participants' lives and its impact on engagement in care. Results Individuals with higher HIV stigma scores were: less likely to have ever been in HIV care $(\mathrm{OR}=0.88 ; 95 \% \mathrm{CI}=0.82,0.92)$ 
and less likely to be on antiretroviral therapy (ART; OR=0.84; 95\% $\mathrm{CI}=0.80,0.90)$. Individuals with higher ethnicity-related stigma scores were also less likely to have ever been in HIV care $(\mathrm{OR}=0.90 ; 95 \%$ $\mathrm{CI}=0.84-0.97)$, and less likely to be on ART $(\mathrm{OR}=0.91$; $95 \% \mathrm{CI}=0.85$ 0.97). MSM stigma was not associated with engagement in care or use of ART in the quantitative data. However, in interviews, participants reported having experienced discrimination due to their sexuality or gender presentation. Participants shared stories of discrimination during medical care attributed to their accent or their ethnicity. Most recounted internalized HIV-related stigma, expressed through a sense of shame about their diagnosis. Some felt that HIV was a punishment for their sexual behavior, suggesting internalized homophobia combined with internalized HIV related stigma. The sense of shame around diagnosis and, for a few, negative experiences with medical providers contributed to a reluctance to ask questions during appointments or a general avoidance of care. Conclusions: Interventions for Latino immigrants should prioritize addressing intersectional stigma and its influence on HIV care seeking and engagement.

CORRESPONDING AUTHOR: Janet Myers, University of California, San Francisco, janet.myers@ucsf.edu

\section{O32.2}

SOULS IN A FOREIGN CITY: PSYCHOSOCIAL CORRELATES OF DISTRESS AMONG ENGLISH-SPEAKING FOREIGN DOMESTIC WORKERS IN HONG KONG

Nelson C. Y. Yeung ${ }^{1}$, Karon K. Y. Kan ${ }^{1}$, Joseph T. F. Lau ${ }^{1}$

${ }^{1}$ The Chinese University- Hong Kong

Introduction and Purpose. In Hong Kong, foreign domestic helpers make up nearly $5 \%$ of the total population. Subject to work-related and immigrant-related stressors, these foreign domestic workers are at-risk of having poor mental health. Studies have found that stressors, coping resources and strategies (e.g., social support), and social/environment factors (e.g., perceived discrimination, satisfaction with working environment) are associated with mental health among migrant workers in other countries. Given the limited research on foreign domestic workers in Hong Kong, this study applied the Stress and Coping model to examine the psychosocial correlates of distress among those domestic workers. Methods. English-speaking domestic workers $(\mathrm{N}=453)$ living in the 18 major districts in Hong Kong recruited through respondent-driven sampling. Participants' demographic characteristics, coping resources, coping strategies, and their experience working and living in Hong Kong were measured in a survey. Results. After controlling for covariates, hierarchical regression results showed that higher acculturative stress $(\beta=0.22, p$ $<.001)$, higher perceived discrimination $(\beta=0.15, \mathrm{p}<.01)$, lower satisfaction of working environment $(\beta=-0.18, p<.001)$, lower social support ( $\beta=-0.17, p<.01)$, less positive reframing $(\beta=-0.24, p<.001)$, and less religious coping $(\beta=-0.11, \mathrm{p}<.05)$ were associated with higher distress. We also found that perceived discrimination moderated the relationship between humor coping and distress $(\beta=0.20, p<.001)$, such that perceived discrimination was associated with higher distress only among those using more humor coping ( $\beta=0.31, p<.001$ ), suggesting that humor coping may exacerbate the negative effect of perceived discrimination on distress. Conclusions. Our findings shed light on the important strategies to improve foreign domestic workers' distress in future interventions. Interventions helping the foreign domestic helpers reduce acculturative stress, perceived discrimination, providing social support, and encourage the use of positive reframing and religious coping may decrease their distress. However, humor coping is not recommended for foreign domestic helpers who perceived higher levels of perceived discrimination.

CORRESPONDING AUTHOR: Nelson C. Y. Yeung, The Chinese University of Hong Kong, nelson.cuhk@gmail.com
O32.3

ACCESS TO HEALTHCARE AMONG IMMIGRANTS AND CHILEANS: REPEATED ANALYSIS OF POPULATION SURVEY CASEN 2009, 2011, 2013 AND 2015

Baltica Cabieses ${ }^{1}$, Marcela Oyarte ${ }^{1}$, Macarena Chepo ${ }^{1}$, Daniel Larenas ${ }^{1}$, Sofia Astorga ${ }^{1}$, Margarita Bernales ${ }^{1}$

${ }^{1}$ Universidad del Desarrollo- Chile.

Introduction \& Purpose International migration represents a global phenomenon. There are some studies about healthcare provision entitlement among immigrants and Chileans, but they have not been updated since 2013. The purpose of this study was update the healthcare provision entitlement of immigrants and Chileans, based on a repeated analysis of a population representative survey from 2009 to 2015. Methods Exploratory secondary analysis of nationally representative, anonymous Chilean survey CASEN 2009, 2011, 2013 and 2015 (participants belonging to about 70000 households each time). We divided the sample into self-reported immigrants (about 3500) and Chilean-born (about 210000 ). We did not include in the analysis those who preferred not to report their migration status (missing values about 2000 each time). We estimated self-reported healthcare provision entitlement of immigrants and Chileans separately, as follows: public healthcare (Fonasa A,B,C,D), private healthcare, armed force, other, none. Results The public system brought together the bulk of the population, both national and international, reaching by $201577.7 \%$ coverage for Chileans and $62 \%$ for immigrants. A worrisome situation is the proportion of international migrants without healthcare entitlement across all years of study (up to $15,7 \%$ in 2015). There were variations in healthcare entitlement to different categories of Fonasa over time among immigrants (higher proportions of A in 2009 and 2011 than Chileand and higher proportions of B in other years). Also noteworthy is the amount of immigrant population that ignore their healthcare provision belonging (12,2\% in 2015) compared to those born in Chile (5.7\% in 2015). Conclusions Based on a repeated population survey analysis, international migrants reported consistently higher proportions of no healthcare and ignored healthcare provision belonging than Chileans. Both groups tend to concentrate in the public healthcare sector, with variations in the type categories (based on payment) over time. Based on a human rights in health perspective, we need urgent action in the protection of vulnerable migrants that are not accessing the healthcare system.

CORRESPONDING AUTHOR: Baltica Cabieses, Universidad del Desarrollo,

\section{O32.4}

HEALTH-RELATED PRACTICES OF URBAN MIGRANT WORKERS IN METRO MANILA: DETERMINANTS AND OUTCOMES

Kenneth Jim Joseph Jimeno ${ }^{1}$

${ }^{1}$ Department of Health-Health Policy Development and Planning Bureau- Philippines

The rapid urbanization in developing countries presents new opportunities and challenges especially to migrants who face multiple health vulnerabilities. This cross-sectional study aims to describe the health-related practices of urban migrant workers in Metro Manila and their associated personal characteristics. It also ascertains the correlation of their health condition with related practices and work performance level. Filipino migrant workers $(n=119)$ were mainly selected from peri-urban areas of Bulacan, Cavite, and Rizal through purposive sampling. Main inclusion criteria were (1) working regularly in Metro Manila and (2) living outside Metro Manila or returning weekly to place of origin. The mean age of the respondents is $25(\mathrm{SD}=3)$ ranging from 18 to 41 years. Majority were males (57\%), not married (93\%), college-educated (79\%), white-collar workers (58\%), living with family (76\%), and earning median monthly 
income of 16000 PHP ( 320 USD). A 53-item self-administered survey questionnaire was developed and distributed for a period of two weeks in October 2016. The primary outcome was score rating on a five-point Likert scale indicating frequency of engagement. Cramer's V and Spearman's rank correlation coefficient (rs) were utilized in the analysis of data. Education and occupation are major determinants of healthrelated practices among urban migrant workers in Metro Manila. Sleep tends to have the most impact on health while interpersonal relationships affect work performance. Healthy migrant hypothesis appears true for this population and the results can be attributed to resiliency, family-oriented values, and importance of harmonious relationships in Filipino culture. The findings of this study can be used in developing migrant-sensitive health system in cities and further inquiry in this emerging research field.

CORRESPONDING AUTHOR: Kenneth Jim Joseph Jimeno, Department of Health-Health Policy Development and Planning Bureau, kennethjimeno.doh@gmail.com

\section{O32.5}

HEALTH AND WELLBEING IN THE CONTEXT OF MIGRATION: VULNERABILITIES AND THREATS AFFECTING WOMEN AND GENDER AND SEXUALITY DIVERSE PEOPLE IN MIGRANT AND HOST COUNTRY COMMUNITIES

John de Wit $^{1}$, Berteke Waaldijk ${ }^{1}$, Marjolein van den Brink ${ }^{1}$, Mara Yerkes $^{1}$

${ }^{1}$ Utrecht University- Netherlands

Introduction \& Purpose: The recent migration crisis in Europe has focused attention on the health and wellbeing of people who seek physical, social and economic safety away from home. Prominent incidents have foregrounded vulnerabilities and threats affecting women and gender and sexuality diverse people in migrant as well as host country communities. We undertook a mixed method, interdisciplinary scoping study to identify key health and wellbeing issues affecting women and gender and sexual minority migrants, which can inform a behavioral medicine research agenda and programmatic responses. Methods: Reviews were conducted of law, humanities and social sciences scholarly literatures. Interviews were undertaken with 23 professionals working in refugee and/or migrant health at national and local levels in The Netherlands. A one day face-to-face meeting was held to discuss findings and obtain feedback from 27 national and international research, policy and program experts. Results: Gender was found to be a key theme in relation to health and wellbeing in the context of migration, mostly addressed in relation to human trafficking and gender relations, notably in law and humanities research. Social science research regarding health/wellbeing in relation to gender and migration is limited. Health and wellbeing of sexual minority migrants was a key emerging theme across law, humanities and social sciences literatures and the main theme emerging from expert interviews. Specific issues in relation to the health and wellbeing of sexual minority migrants include their safety and experiences of violence, cultural differences regarding sexuality and identity that may affect perceived credibility of sexual minority refugees, and inclusion/exclusion in host country migrant, sexual minority and general communities. Conclusions: Women and gender and sexual minorities are at increased risk of adverse health and wellbeing outcomes in the context of migration, as noted in practice settings and law and humanities research. There is an opportunity and need for more social science and behavioural medicine engagement to increase understanding of the nature, drivers and responses to protect and promote the health and wellbeing of women and gender and sexuality diverse migrants.

CORRESPONDING AUTHOR: John de Wit, Utrecht University, j.dewit@uu.nl
O32.6

EFFECTS OF SLEEPING QUALITY AND FAMILY FACTORS ON HEALTH AND MENTAL HEALTH OUTCOMES: A COMPARISON BETWEEN CROSS-BORDER AND LOCAL CHILDREN IN HONG KONG

Qiaobing $\mathrm{WU}^{1}$

${ }^{1}$ The Hong Kong Polytechnic University

Introduction and Purpose: The increasing economic and social interaction between the Chinese mainland and Hong Kong has witnessed the growth of a unique population - children born in Hong Kong thus holding HK citizenship but to parents who are non-HK citizens. A considerable proportion of these children live in the adjacent city of HK and have to take long hours of transportation to attend school in HK every day, thus being called cross-border children. This living and learning arrangements have posed many threats to their health and mental health. The present study aims to investigate how the sleeping quality and other factors in the family sphere contribute to the health and mental health outcomes of cross-border children, as compared to their local counterparts in HK. Methods: Data came from a cross-sectional survey with 2,028 4th-9th grade students from twelve schools in Hong Kong. Sleeping quality was measured by the average sleeping hours per night and the self-rated sleeping quality ranging from very poor (1) to very good (5). Health was also assessed by a self-rated item on the same 5-point scale. Mental health was assessed by the Warwick-Edinburgh Mental Well-being Scale. And other family factors assessed included household size and sibling size, socioeconomic status, parent-child interaction and the child's relationship with mother and father respectively. Results: Sleeping quality was found to have significantly strong effects on health and mental health, with cross-border children reporting poorer sleeping quality and consequently poorer health and mental health. Among the family correlates, higher socioeconomic status, better quality of parent-child interaction and relationships were protective for children's health and mental health outcomes. Conclusions: The research findings revealed the significance of sleeping quality and family support for the health and mental health of cross-border children in dealing with the unique challenges they are faced with. Health interventions may be designed accordingly to promote children's health outcomes.

CORRESPONDING AUTHOR: Qiaobing WU, The Hong Kong Polytechnic University, qiaobing.wu@gmail.com

O33.1

IMPACT OF INCREASING THE RELATIVE AVAILABILITY OF HEALTHIER OVER LESS HEALTHY FOODS ON ENERGY PURCHASED IN WORKSITE CAFETERIAS: A STEPPED WEDGE RANDOMISED CONTROLLED PILOT TRIAL

Rachel Pechey ${ }^{1}$, Emma Cartwright ${ }^{1}$, Mark Pilling ${ }^{1}$, Gareth J Hollands ${ }^{1}$, Milica Vasiljevic ${ }^{1}$, Susan A Jebb ${ }^{2}$, Theresa M Marteau ${ }^{1}$

${ }^{1}$ University of Cambridge

${ }^{2}$ University of Oxford

Increasing the proportion of healthier foods available in food outlets could encourage healthier food consumption but the evidence underpinning this is limited in scope and quality. This study aims to: (a) examine the feasibility and acceptability of intervening upon product availability in worksite cafeterias; and (b) estimate the effect of increasing the availability of a broad range of healthier foods on energy purchased. Methods. Six worksite cafeterias in England increased the proportion of healthier foods available, in a stepped wedge randomised controlled pilot trial conducted between January and May 2017 (Trial Registration: ISRCTN52923504). Each site was randomised to an implementation date, staggered fortnightly, following a four-week baseline period. After implementation, sites maintained changes until the study end. The outcome was daily energy (kcal) purchased from targeted food categories 
(cooked meals, snacks, cold drinks, sandwiches). Generalised linear mixed models controlled for cafeteria usage and time trends, with random effects for worksite. Results. Feasibility and Acceptability: Changes to pre-packaged products were mostly adhered to across sites, but changes to cooked meals were only implemented at sites where menus were preplanned. Very few customer surveys were completed, suggesting $78 \%$ either favoured or did not object to the intervention remaining in place post-study. Impact: The average effect of the intervention across all sites was a reduction of $6.9 \%(95 \% \mathrm{CI}$ : $-11.7 \%,-1.7 \%, \mathrm{p}=0.044)$ in energy purchased from targeted food categories. At the level of individual sites, energy purchased from targeted categories was significantly reduced in two sites (-10.7\% (95\%CI: $-18.1 \%$ to $-2.6 \%, \mathrm{p}=0.046)$; $-18.4 \%$ (95\%CI: $26.9 \%$ to $-8.8 \%, \mathrm{p}=0.013)$ ), while no significant differences were seen in the other four sites. Conclusions. Increasing the proportion of healthier food options in worksite cafeterias can reduce energy purchased. Impact varied by site, perhaps relating to degree of implementation, food categories targeted (e.g. cooked meals) or site characteristics. Further work should try to establish reasons for this differential impact, and thereby the most effective ways of implementing this promising intervention.

CORRESPONDING AUTHOR: Rachel Pechey, University of Cambridge, rachel.pechey@medschl.cam.ac.uk

\section{O33.2}

INTERVENTION TO SUBSTITUTE SUGAR-SWEETENED BEVERAGES FOR HEALTHIER ALTERNATIVES

Elizaveta Novoradovskaya ${ }^{1}$, Monica Yee ${ }^{2}$, Gaby Judah ${ }^{2}$, Barbara Mullan $^{1}$, Lina Johansson ${ }^{2}$

1. Curtin University - Australia

2. Imperial College London - United Kingdom

Obesity and diseases associated with it, such as diabetes and cancer, are on the increase globally. While cases of obesity are multifaceted, one of the contributors is the increased consumption of energy dense nutrient poor food, including sugar-sweetened beverages (SSB). One strategy that has been shown to successfully lead to behaviour change in habitual behaviours, such as this, is implementation intentions, where SSB consumption is substituted with an alternative behaviour, such as drinking water. However, as SSB have a hedonic component, substituting them with diet drinks may result in larger behaviour change. The aim of the current study was to develop an online implementation intention based intervention to substitute SSB consumption for diet drinks or water consumption. Methods: Participants $(n=136)$ were randomly assigned to one of two intervention conditions. They were asked to develop implementation intentions for consuming diet drinks in the first condition and water in the second condition. Measures of consumption of SSB, diet drinks and water, along with habit strength and hedonic reward were taken at baseline and after two months. Results: Both groups significantly decreased SSB consumption $(\lambda=0.31, \mathrm{~F}(1,134)=294.55, \mathrm{p}<0.001, \eta \mathrm{p} 2=0.69)$ and SSB habit strength $(\lambda=0.57, \mathrm{~F}(1,134)=102.08, \mathrm{p}<0.001, \eta \mathrm{p} 2=0.43)$, however, no group differences were observed $(\lambda=1.00, F(1,134)=0.02, p=0.88$, $\eta \mathrm{p} 2=0.0002)$. Diet drink $(\mathrm{t}(56)=6.17, \mathrm{p}<0.01, \mathrm{~d}=0.82)$ and water $(\mathrm{t}$ $(78)=2.07, p=0.04, d=0.23$ ) habit strengths increased, with the former experiencing the larger increase. SSB consumption and diet drink habit strength were not associated $(r=0.11, n=57, p=0.4)$, with drinking water habit strength showing association with SSB consumption $(\mathrm{r}=0.23, \mathrm{n}=79$, $\mathrm{p}=0.04)$. Hedonism did not differ between diet drink and water consumption $(\mathrm{t}(134)=1.80, \mathrm{p}=0.07, \mathrm{~d}=0.31)$. Conclusions: The results of the current study demonstrated that a simple implementation intention intervention can be successful in reducing SSB consumption and habit strength and substituting it with consumption of diet drink or water and future research can build on this promising start.

CORRESPONDING AUTHOR: Elizaveta Novoradovskaya, Curtin University, elizaveta.novoradovskaya@postgrad.curtin.edu.au
O33.3

A SCHOOL-BASED GAMIFICATION STRATEGY TO REDUCE OBESITY: RESULTS FROM A PILOT STUDY

Sebastián Peña ${ }^{1}$, Macarena Carranza ${ }^{2}$, Pau Espinoza ${ }^{2}$, Valeska Müller ${ }^{2}$, Ricardo Cerda ${ }^{3}$, Pedro Zitko ${ }^{3}$, Andrea Cortinez ${ }^{4}$, Nicolás Loira ${ }^{5}$, Cristobal Cuadrado ${ }^{6}$

${ }^{1}$ National Institute for Health and Welfare - Finland

${ }^{2}$ Municipality of Santiago - Chile

${ }^{3}$ University of Chile - Chile

${ }^{4}$ Pontifical Catholic University - Chile

${ }^{5}$ Independent - Chile

${ }^{6}$ University of Chile - Chile

School-based interventions have shown mixed results. Most studies have lacked enough statistical power and have been carried out in North America and Europe. Multidimensional interventions have often used education to address nutrition and physical activity, without structural components and weak community participation. In this study, we report the results of the gamification strategy and effectiveness of a pilot study in Santiago de Chile. Methods: The Juntos Santiago cluster-randomized trial uses a gamification strategy (i.e. elements of a game, points, levels and rewards) with a strong community participation component. Participants of the pilot study were 381 children in 5th and 6th grade of three schools in Santiago selected by convenience. Children voted their enrolment and collectively chose the activity reward they were playing for. The intervention consisted of a healthy snacks challenge and a steps challenge. The reward for the pilot was a fun day at the City swimming pool. The primary outcome was change in BMI zscore and waist circumference. Results: Of enrolled students, $88 \%$ was examined at baseline and followup. Unhealthy snacks brought from home reduced by 6.5 percentage points. Data from steps challenge was not available due to encryption of activity trackers. We observed statistically significant reductions in zBMI (-0.09) and waist circumference $(-0.79 \mathrm{cms})$. No difference was observed for BMI. Systolic blood pressure reduced on average 5.19 $\mathrm{mmHg}$ and diastolic blood pressure increased by $1.48 \mathrm{mmHg}$. The latter changes are likely due to equipment change. Results of the 2018 trial in 4000 participants will also be presented. The trial in 2018 will incorporate a points system and structural rewards (improvements in school infrastructure for physical activity and nutrition). Conclusions: Despite the short duration of the pilot study, results are promising. The gamification strategy appears to foster community engagement and motivation. Attention should be given to data extraction and quality assurance prior the beginning of the trial in 2018 .

CORRESPONDING AUTHOR: Sebastian Peña, National Institute for Health and Welfare, sebastian.pena.fajuri@thl.fi

\section{O33.4}

DEVELOPING THE LOGIC MODEL OF CHANGE FOR A HEALTH PROMOTION INTERVENTION AIMING TO REDUCE SOCIOECONOMIC DIFFERENCES IN PRESCHOOLERS' HEALTH BEHAVIORS AND WELLBEING- THE DAGIS STUDY Carola Ray ${ }^{1}$, Riikka Kaukonen ${ }^{1}$, Elviira Lehto ${ }^{1}$, Henna Vepsäläinen ${ }^{2}$, Reetta Lehto ${ }^{1}$, Nina Sajaniemi ${ }^{3}$, Maijaliisa Erkkola ${ }^{4}$, Eva Roos ${ }^{1}$

${ }^{1}$ Folkhälsan Research Center - Finland

${ }^{2}$ Department of Food and Environmental Sciences, University of Helsinki - Finland

${ }^{3}$ Division of Early Education, University of Helsinki - Finland

${ }^{4}$ Department of Food and Environmental Sciences, University of Helsinki - Finland

Socioeconomic status (SES) differences in children's energy balancerelated behaviors (EBRBs) exist already at preschool age. When planning health promotion interventions targeting all, it is important to pay special attention to those who most need it. The aim is to present the process of 
planning the logic model of change for the Increased Health and Wellbeing in preschool (DAGIS) intervention. The first steps in the Intervention Mapping (IM) framework were used as a guiding tool in the process. Methods: The first steps in IM; needs assessment and the definition of behavioral outcomes were guiding the process. The needs assessment was conducted as literature review, focus group interviews, and an extensive survey among parents and preschool personnel. Mediation analysis of the survey data detected the most relevant determinants for SES differences in children's EBRBs. Additionally, behavior change theories were used in the development of the logic model. Results: The needs assessment showed the largest SES differences in following EBRBs; children's screen time, intake of sugary foods and drinks, and children's self-regulation. These formed the secondary outcomes. The mediator analysis yielded, together with behavior change theories, following determinants for children's EBRBs; parents and preschool personnel as role models for EBRB's, the availability and accessibility of screens and sugary food. These formed the intermediate outcomes in the intervention. Additional determinants formed the short term outcomes; norms about suitable amounts of screen time, food consumption, and physical activity (PA), importance of PA, restrictions about screen time and food consumption, knowledge about children's self-regulation and EBRBs recommendations, awareness of the EBRBs environment at home and preschool, parents and preschool personnel self-efficacy in promoting children's self-regulation and EBRBs. Conclusions: The development of the logic model of change for the preschool family involved intervention was preceded by an extensive needs assessment in accordance with IM. The final model includes preschool and home components which will influence short time-, intermediate-, and secondary outcomes.

CORRESPONDING AUTHOR: Carola Ray, Folkhälsan Research Center, carola.ray@folkhalsan.fi

\section{O33.5}

A RANDOMIZED CONTROLLED TRIAL EVALUATING EFFICACY OF AN INTERVENTION WHICH ENHANCES SOCIAL SUPPORT AND POSITIVE AFFECT THROUGH ONLINE SOCIAL NETWORKING IN SMOKING CESSATIONS

Xue Yang ${ }^{1}$, Joseph Lau ${ }^{1}$, Zixin Wang ${ }^{1}$, Yuan Fang ${ }^{1}$, Mary Ip ${ }^{1}$

${ }^{1}$ The Chinese University of Hong Kong - China

The study aims to develop an intervention which enhances social support and positive affect through online social networking in smoking cessations and test its efficacy with a randomized controlled trial (RCT). The proposed online social networking (WeChat) cessation intervention involves three evidence-based components: i) involving and enhancing social (peer) support toward smoking cessation, ii) improving positive affect through positive psychology intervention (PPI), and iii) providing immediate preventive cue of action to smokers through online social networking. It is supplemented by basic health education information on cognitions (perceived severity of smoking and benefits of cessation) and tips on resisting situational temptations for relapse prevention which will also be sent to the control group. It is hypothesized that the quit rate of the intervention group would be higher than that of the control group at 6-month follow-up. Methods We recruited 408 (59.1\% male) Chinese adults in Hong Kong. They were randomly assigned into either the intervention group $(\mathrm{n}=205)$ or control group (receiving health education messages via WeChat; $n=203$ ). The primary outcome is self-reported 7-day point prevalence (pp) quit rate of smokers at 6 months post end-of-intervention. As a validation of the primary outcome, all participants who report quitting at 6 months post end-of-intervention will be invited to visit the PWH for bio-chemical validation (exhale carbon monoxide test and/ or saliva cotinine test). Other variables including positive affect, negative affect, self-efficacy, perceived behavioral control, attitudes toward smoking, perceived subjective norm and perceived stress were also assessed. Results The self-reported 7-days point prevalence (pp) quit rates were $29.0 \%$ in the intervention group and $27.3 \%$ in the control group $(\mathrm{RR}[95 \% \mathrm{CI}]=1.06[0.74-1.53], \mathrm{ARR}[95 \% \mathrm{CI}]=1.6[-8.6-11.9])$. There was no significant difference between the intervention group and control group. There was no significant difference in positive affect, negative affect, self-efficacy, perceived behavioral control, attitudes toward smoking, perceived subjective norm or perceived stress. Conclusions Both intervention group and control group seem to be effective for smoking cessation. The efficacy of intervention group was not significantly better than that of the control group. The potential explanations and future directions to improve the intervention were discussed.

CORRESPONDING AUTHOR: Xue Yang, The Chinese University of Hong Kong, yangxuemail8@gmail.com

\section{O33.6}

THE LIVESTOCK MART AS A SETTING TO ENGAGE MALE FARMERS IN A CARDIOVASCULAR HEALTH INTERVENTION: "FARMERS HAVE HEARTS'

Diana van Doorn ${ }^{1}$

${ }^{1}$ National Centre for Men's Health IT Carlow - Ireland

Context Irish farmers have been found to be a 'high risk' group for CVD mortality and a 'hard to reach' group with regard to preventative health intervention. 'Farmers Have Hearts' (FHH) targeted farmers with a health screening programme at their workplace, i.e. the mart. The programme aimed to detect risk factors for CVD as well as to prompt health behaviour change. Study aim The study explored what factors prompt a so-called hard to reach group (male livestock farmers) to engage in a workplace prevention health screening programme and if participation in a health check contributes to health behavior change. Research methods Data was collected from a convenience sample $(n=310)$ of male farmers. Baseline information provided insight in the prevalence of risk factors for CVD. Consent at baseline also included permission to participate in two follow up questionnaires by phone at Week $1(n=224)$ and Week $12(n=172)$, which measured engagement in the FHH and health behavior change. All data were entered into the SPSS (v22) for both in-depth descriptive and inferential statistical analysis. Results This study found a high prevalence of anthropometric measurements (36\% obesity and 38\% 'high risk' waist circumference) and multiple (four or more) risk factors for CVD (83\%) among participating farmers. Majority of farmers (79\%) were referred to their GP. By Week 12, 32\% of farmers had acted on this advice. At Week 12 , at least one behaviour change was reported by $48 \%$ of farmers. Paradoxically, those farmers most in need of health behaviour change based on adverse screening outcomes were found to be least likely to change health behaviour. Notably, $42 \%(n=72)$ of farmers reported that they would not have gone for a health screening were it not for FHH. Conclusion This study found that the high prevalence of obesity and multiple risk factors for CVD among this study-group is a cause of concern. Workplace health screenings can be a catalyst for health behaviour change for some Irish farmers; however, more follow-up prompts and support networks are needed to prompt increased behaviour change.

CORRESPONDING AUTHOR: Diana van Doorn, National Centre for Men's Health IT, diana.vandoorn@itcarlow.ie

\section{O34.1}

WHY PEOPLE WITH MIGRAINE DELAY TAKING MIGRAINESPECIFIC MEDICATION: A PROSPECTIVE OBSERVATIONAL STUDY

Elizabeth Seng ${ }^{1}$, Nicole Butler ${ }^{2}$, Robert Nicholson ${ }^{3}$

${ }^{1}$ Albert Einstein College of Medicine - USA

${ }^{2}$ Yeshiva University - USA

${ }^{3}$ Mercy Health System - USA 
Introduction and Purpose Migraine is a common, disabling disease with recurrent attacks of severe head pain and neurological symptoms. Migraine-specific medications (MSMs: triptans and ergots) are the firstline treatment for migraine and are most effective if taken when migraine attack pain is mild. Extant research suggests many people with migraine delay using MSMs until pain is moderate or severe. This study aims to prospectively evaluate why people with migraine delay taking MSMs during individual migraine attacks. Methods Two-hundred nineteen adults with a physician diagnosis of migraine ( $\geq 4$ headache days/month) were recruited from a large health system in the United States. Participants completed baseline demographic surveys and three months of daily diary evaluating headache symptoms and medication-taking [type and pain at dosing (mild, moderate or severe)]. If a participant delayed taking MSM during an attack (pain at dosing $=$ moderate or severe), they endorsed reasons for delaying MSM from a bank of 12 items developed through patient and provider feedback. Descriptive analyses and exploratory factor analysis using Horn's Parallel Analysis and varimax rotation evaluated rates and reasons for delaying MSM. Results Participants (92.6\% women, 91.1\% White, Non-Hispanic, $64.6 \%$ with a college degree or higher, $\mathrm{M}$ age $=41.7, \mathrm{SD}=11.3$ ) recorded an average of $82.2(\mathrm{SD}=28.2)$ diary days, with an average of $29.0(\mathrm{SD}=$ $22.5)$ headache days and 16.8 (14.7) migraine days over the three month study period. Fifty $(22.8 \%)$ participants met criteria for chronic migraine ( $\geq 15$ headache days/month). Participants recorded a total of 5,027 migraine days. An MSM was the first acute medication used on 2,901 migraine days $(57.7 \%)$. Participants delayed MSM on 1,013 migraine days $(34.9 \%)$. Insurance limits $(39.4 \%)$ and desire to ensure the attack was a migraine $(34.4 \%)$ were the most common reasons for delaying taking MSM. Factor analysis revealed 4 factors that explained $51.0 \%$ of the variance in reasons for delaying MSM during a migraine attack: 1) Symptoms/Side Effects (eigenvalue $=2.28$; "I wanted to make sure it was a migraine"); 2) Cost (eigenvalue $=1.60$; "I did not take it because of the limits insurance puts on this medication"); 3) Concern About Overuse (eigenvalue $=1.15$; "I was worried about the effects of using this medication too often") 4) Non-Pharmacologic Choice (eigenvalue = 1.09; "I tried using a non-medication approach"). Conclusions Reasons for delaying taking MSM differ across people and attacks, and are grouped by Symptoms/Side Effects, Cost, Concern About Overuse, and NonPharmacologic Choice. Patient decision aids for acute migraine attack treatment should consider these reasons for delaying MSM.

CORRESPONDING AUTHOR: Elizabeth Seng, Albert Einstein College of Medicine, seng.elizabeth@gmail.com

\section{O34.2}

COGNITIVE FACTORS PREDICTIVE OF THERAPEUTIC ADHERENCE IN PEOPLE WITH CHRONIC DISEASES

Gerardo Leija Alva ${ }^{1}$, Viridiana Peláez Hernández ${ }^{2}$, Benjamín Domínguez Trejo $^{3}$, Arturo Orea Tejeda ${ }^{2}$, Elisa Pérez Cabañas ${ }^{3}$, Ruth Pablo Santiago ${ }^{3}$, Dulce González Islas ${ }^{2}$

${ }^{1}$ Centro Interdisciplinario en Ciencias de la Salud, Unidad Santo Tomas Del Intituto Politécnco Nacional - Mexico

${ }^{2}$ Clínica de Insuficiencia Cardiaca Del Instituto Nacional de Enfermedades Respiratorias "Ismael Cosío Villegas" - Mexico

${ }^{3}$ Facultad de Psicología, UNAM - Mexico

Sackett and Haynes mention more than 200 variables that are related to adherence to some treatment. Currently, WHO and other researchers have determined these as the factors that most influence this process: a) interaction with the health professional, b) communication between the patient and health personnel, c) the therapeutic regimen, d) the complexity of the treatment, e) the cost-benefit relationship offered by the treatment, f) the characteristics of the disease, g) the patient's beliefs and h) social-family support. The present work focuses on describing cognitive phenomena (beliefs) that relate to the patient and adherence. Objective: This study attempts to identify a structure among the factors of 3 cognitive models (Health Beliefs, Planned Behavior, Wallston model) that could predict adherence to treatment in patients with chronic diseases. The data were managed using a. Results: Of all the 14 factors of the four models only 4 predict adherence to treatment: value for health, behavioral control, attitudes and behavioral intention, these explain 13\% of therapeutic adherence in this population Conclusion: This provides information on the weight of the factors of 4 models used in health psychology to explain adherence behaviors, and does not help to understand that it is necessary to elaborate or rethink such models since not all factors fulfill that function.

CORRESPONDING AUTHOR: Gerardo Leija Alva, Centro Interdisciplinario en Ciencias de la Salud, Unidad Santo Tomas Del Intituto Politécnco Nacional, gelealipn@hotmail.com

\section{O34.3}

VALIDATION OF A SCALE FOR MULTIDIMENSIONAL TREATMENT ADHERENCE FOR ARTERIAL HYPERTENSION IN CHILE: INTEGRATION OF USERS, EXPERTS AND CLINICAL GUIDELINE EVALUATIONS

Daniela Nicoletti ${ }^{1}$, Ricardo Cerda ${ }^{1}$, Valentina Muñoz ${ }^{1}$

${ }^{1}$ Universidad de Chile - Chile

This summary presents the content validation process of the Arterial Hypertension multidimensional treatment adherence scale, integrating data from three sources: expert judgment, application of a preliminary instrument through a cognitive interview to a sample of users of the Cardiovascular Health Program (CVHP) from Primary Health Care (PHC) and the revision of different international clinical guidelines, including the chilean one.

CORRESPONDING AUTHOR: Daniela Nicoletti, Universidad de Chile, dnicolettirojas@gmail.com

\section{O34.4}

EXACTLY WHAT IT SAYS ON THE TIN? A QUALITATIVE STUDY ON MEDICATION ADHERENCE FOR RESISTANT HYPERTENSION IN PRIMARY CARE

Hannah Durand ${ }^{1}$, Monica Casey $^{2}$, Peter Hayes ${ }^{2}$, Andrew Murphy ${ }^{2}$, Gerry Molloy ${ }^{3}$

${ }^{1}$ National University of Ireland - Galway

${ }^{2}$ Discipline of General Practice, School of Medicine, National University of Ireland - Galway

${ }^{3}$ School of Psychology, National University of Ireland - Galway

Non-adherence to antihypertensive medication is a leading cause of apparent treatment resistance. Previous quantitative findings suggest that habit strength plays a greater role in predicting long-term adherence to antihypertensive medications for people with apparent treatment resistant hypertension (aTRH) than treatment-related beliefs. This study aimed to corroborate previous findings regarding predictors of long-term antihypertensive adherence using qualitative methods. Methods: Participants were patients with aTRH who previously participated in a prevalence study of resistant hypertension in primary care. Patients were recruited into this study based on self-reported adherence scores (i.e., highest and lowest adherence scores were included). Thirteen semi-structured face-toface or phone interviews were conducted. Data were analysed using thematic analysis. Results: Two main themes emerged: habit and routine, and beliefs about treatment. High adherers reported taking medication as part of their daily routine, and using both time and place cues to help them to remember to take their medication. High adherers also reported receiving support from family and their GPs. They also had a realistic understanding of the consequences of non-adherence, and believed their medicine 
did more good than harm. Low adherers reported forgetting their medication, and scepticism about the effectiveness of medication for controlling their blood pressure. They also expressed greater desire to be taken off medications in the future. Few participants reported intentionally not taking their medication. Conclusions: Current findings suggest that stable medication routines, good doctor-patient communication, and appropriate medication and illness beliefs are helpful in achieving optimal medication adherence for aTRH in primary care. Interventions to enhance antihypertensive adherence should take into account both intentional and nonintentional adherence to optimise outcomes.

CORRESPONDING AUTHOR: Hannah Durand, National University of Ireland, h.durand1@nuigalway.ie

\section{O34.5}

THE DEVELOPMENT, FEASIBILITY AND PILOTING OF AN INTERVENTION TO SUPPORT MEDICATION ADHERENCE FOLLOWING ACUTE CORONARY SYNDROME (ACS)

Jacob Crawshaw $^{1}$, John Weinman ${ }^{1}$, Duncan McRobbie ${ }^{2}$, Vivian Auyeung ${ }^{1}$

${ }^{1}$ King's College London - United Kingdom

${ }^{2}$ Guy's and St Thomas' NHS Foundation Trust - United Kingdom

Introduction and Purpose Previous research suggests that approximately a third of patients do not adhere to medications following acute coronary syndrome (ACS). We recently undertook two systematic reviews with meta-analysis to identify: i) the psychosocial predictors of medication adherence following ACS; ii) the effectiveness of healthcare provider-led interventions to support adherence post-ACS. A qualitative study was also conducted to provide insight into the experiences of patients hospitalised with ACS. We used data from these preliminary studies to develop an intervention to support medication adherence following ACS. We report the findings from a feasibility and acceptability intervention study. Methods We conducted a single-arm intervention study with ACS patients recruited from an NHS hospital in London, UK. The intervention consisted of two face-to-face sessions delivered pre-discharge to target both intentional (Session 1) and unintentional barriers (Session 2) to adherence. Patients' beliefs about medications were measured using the BMQspecific (necessity, concerns, necessity-concerns differential). Pre-post intervention comparisons were made. Study acceptability (setting, timing, content, delivery) was measured quantitatively and qualitatively. Results A total of 15 patients completed the intervention (39\% recruitment) (14 male, 1 female). There was a significant difference in pre-intervention $(\mathrm{M}=$ $20.50, \mathrm{SD}=3.67)$ and post-intervention $(\mathrm{M}=22.36, \mathrm{SD}=2.24)$ necessity scores, $\mathrm{t}(13)=-3.36(95 \% \mathrm{CI}-3.05--0.66), \mathrm{p}=.005$. There was also a significant difference in pre-intervention (median $=5$ ) and postintervention (median $=7$ ) necessity-concerns differential scores, $Z=-$ $2.94, \mathrm{p}=.003$. Study acceptability was rated highly (mean $=9.5, \mathrm{SD}=$ 1.0). Conclusions Patients elicited stronger beliefs about treatment necessity after receiving the intervention. The intervention was deemed acceptable by patients. Relatively low recruitment rate suggests some feasibility issues. Since this study was completed, we have been undertaking a nonrandomised pilot study to determine whether our intervention can be successfully delivered by hospital pharmacists. Treatment beliefs and selfreported medication adherence are the main outcomes of this pilot study.

CORRESPONDING AUTHOR: Jacob Crawshaw, King's College London, jacob.crawshaw@kcl.ac.uk

\section{O34.6}

"IT'S LIKE TRYING TO FIT A PIECE INTO AN ALREADY NOT WORKING PUZZLE": NON ADHERENCE TO INHALED CORTICOSTEROIDS IN YOUNG PEOPLE WITH PROBLEMATIC ASTHMA: A QUALITATIVE STUDY
Christina Pearce ${ }^{1}$, Amy Chan ${ }^{1}$, Rob Horne ${ }^{1}$, Louise Fleming ${ }^{2}$, Andrew Bush $^{2}$, Angela Jamalzadeh ${ }^{3}$

${ }^{1}$ University College London - United Kingdom

${ }^{2}$ The Royal Brompton Hospital and Imperial College London - United Kingdom

${ }^{3}$ The Royal Brompton Hospital - United Kingdom

Sub-optimal adherence to inhaled corticosteroids (ICS) leads to poor asthma control and exacerbations. Even while adherence is electronically monitored, over half of patients have adherence to ICS of $<80 \%$. No previously published research has used objective electronic monitoring device (EMD) data to qualitatively investigate nonadherence. This study aimed to investigate determinants of nonadherence using EMDs. Methods: Patients aged 12-17 years old with poor adherence to ICS $(<80 \%)$ attending the Royal Brompton Hospital, took part in semistructured interviews using their EMD data. The interview discussion guide was based on relevant adherence frameworks including the Perceptions and Practicality Approach. Interviews were transcribed and thematic analysis was conducted. Results: 20 patients were recruited, mean age of 14 years (range=12-17). Using thematic analysis five key themes emerged: Importance of parental encouragement and reminders; Trivialisation of the seriousness of asthma; Poor understanding of asthma as a long-term condition; Low necessity beliefs for ICS use daily in the absence of symptoms; and Difficulties in maintaining routine. Conclusion: Young people with PSA have complex beliefs related to the use of ICS. They and their families need to recognise asthma as severe and as a long-term condition. Interventions should focus on effective ways of communicating the nature of asthma and the need for daily preventer usage in the absence of daily symptoms and on shifting the reliance from the reliever to the preventer inhaler. Adherence support should be tailored to address both the motivation and the ability of the patient.

CORRESPONDING AUTHOR: Christina Pearce, University College London, christina.pearce.15@ucl.ac.uk

O35.1

DYADIC COPING AND INFLAMMATION: EXAMINING THE MODERATING ROLE OF CHRONIC PARENTING STRESS

Jean-Philippe Gouin ${ }^{1}$, Erin Barker ${ }^{1}$, Carsten Wrosch ${ }^{1}$, Linda Booij ${ }^{1}$, Jennifer McGrath ${ }^{1}$

${ }^{1}$ Concordia University - Canada

Chronic parenting stress has been associated with greater risk for poor health. Mothers caring for a child with a neurodevelopmental disorder report more physical health symptoms and increased systemic inflammation, compared to mothers of typically developing adolescents. Dyadic coping, the extent to which partnered individuals work together to deal with the stress that either or both partners are facing, is an interpersonal process that may promote adjustment to chronic parenting stress. In a small pilot study, positive dyadic coping has been associated with lower inflammation among parents of children with a neurodevelopmental disorder. The goals of the present study were to replicate past findings showing an association between dyadic coping and inflammation in a larger sample and to test whether this association is stronger in the context of chronic parenting stress. Methods: Partnered mothers of adolescents with autism or intellectual disability $(\mathrm{n}=105)$ as well as mothers of typically developing adolescents $(n=72)$ completed the Dyadic Coping Inventory to indicate to extent to which they deal with stress jointly with their romantic partner. They also provided blood samples for circulating inflammatory markers analysis. Interleukin-6 (IL-6), tumor necrosis factor(TNF- ), and C-reactive protein (CRP) were assayed using multiplex arrays. Results: In a model adjusting for age and body mass index, more dyadic coping was associated with lower IL-6, R2=.019, p = .04, and marginally related to lower TNF-, R2 $=.017, \mathrm{p}=.07$, and CRP, R2=.013, 
$\mathrm{p}=.08$. Mothers of adolescents with a neurodevelopmental disorder had higher levels of TNF- , R2 =.044, $\mathrm{p}=.003$, but similar levels of IL-6, $\mathrm{R} 2=.005, \mathrm{p}=.25$, and CRP, R2 $=.001, \mathrm{p}=.61$, compared to mothers of typically developing adolescents. None of the caregiving by dyadic coping interactions were significant (all p's $>.49$ ), indicating that the association of dyadic coping with circulating inflammatory markers was similar across mothers of adolescents with neurodevelopmental disorders and mothers of typically developing adolescents. Conclusion: Greater dyadic coping is related to lower levels of circulating inflammatory markers. Dyadic coping may be one interpersonal process underlying the protective effects of close relationships on health.

CORRESPONDING AUTHOR: Jean-Philippe Gouin, Concordia University, jpgouin@gmail.com

\section{O35.2}

PLACEBO EFFECTS AND CONDITIONING OF THE NEUROENDOCRINE SYSTEM: LEARNED OXYTOCIN RESPONSES Andrea Evers ${ }^{1}$, Aleksandrina Skvrotsova ${ }^{1}$, Judy Veldhuijzen ${ }^{1}$, Henriet van Middendorp ${ }^{1}$, Omer van den Bergh ${ }^{2}$

${ }^{1}$ Leiden University - Netherlands

${ }^{2}$ University of Leuven - Netherlands

Preliminary evidence in the area of placebo research suggests that endogenous hormone secretion, such as cortisol or insulin, might be behaviourally conditionable in humans. Whether other neuroendocrine hormones such as oxytocin can be conditioned as well is currently unclear. We report here the results of the first randomized controlled trial that investigated the effect of conditioning with oxytocin on endogenous oxytocin release. Secondly, we studied the effects of oxytocin conditioning on pain sensitivity and trust, two parameters that were previously demonstrated to be affected by oxytocin administration. Ninety-nine female participants were randomly allocated to one of three groups: 1) conditioned group, 2) placebo group, or 3) a drug-control group. A validated two-phase randomized placebo-controlled conditioning paradigm was applied. In the acquisition phase, a distinctive odor (conditioned stimulus, CS) was associated with the administration of exogenous oxytocin (unconditioned stimulus, US) in the experimental and drug-control groups or placebo in the placebo group on three consecutive days. During the evocation phase, participants in the conditioned and placebo groups were given a placebo in combination with the CS during three consecutive days, whereas the drug-control group received oxytocin without the CS. Salivary oxytocin levels, pain sensitivity and responses to a facial trustworthiness task were measured during the evocation sessions. Preliminary data demonstrated increased oxytocin levels in the conditioned group in comparison to the placebo group, but only during the first evocation sessions. No effects of conditioning or oxytocin administration on pain sensitivity or trust were found. The final results of the full sample will be presented at the conference. Current findings demonstrate preliminary evidence that it might be possible to condition endogenous oxytocin release by pairing exogenous oxytocin administration with a distinctive odor. Conditioned hormonal responses might be one of the underlying mechanisms of the placebo effect and might be used in clinical practice for reducing medication side effects and costs of hormone based treatments.

CORRESPONDING AUTHOR: Andrea Evers, Leiden University, a.evers@fsw.leidenuniv.nl

O35.3

GOAL DISENGAGEMENT CAPACITIES PREDICT INFLAMMATORY CYTOKINES (IL-6) AMONG PARENTS OF CHILDREN WITH AUTISM SPECTRUM DISORDER

Heather Herriot ${ }^{1}$, Carsten Wrosch ${ }^{1}$, Erin Barker ${ }^{1}$, Jean-Philippe Gouin ${ }^{1}$

${ }^{1}$ Concordia University
Chronic parenting stress experienced by parents of children with Autism Spectrum Disorder (ASD) is associated with poor health. Persistently elevated stress may "get under the skin" by elevating levels of inflammation in the body. However, research suggests that individual difference factors involved in stress regulation can influence the degree to which stress can perturb biological dysregulation. To this end, goal adjustment theory posits that the ability to disengage from unattainable goals and reengage in new goals can promote more adaptive biological responses in the context of stressful experiences. The buffering impact of goal regulation strategies may thus be greater in the context of chronic parenting stress. Purpose: To examine whether goal adjustment capacities serve as self-regulation factors that protect chronically stressed mothers from experiencing high levels of inflammation. Methods: This cross-sectional study examined 211 mothers for their levels of goal adjustment capacities and an indicator of proinflammatory cytokines (IL-6). The study included 124 mothers of children with ASD whose child was transitioning out of high school, and a comparison group of 87 demographically similar mothers with typically developing adolescents. Analyses controlled for relevant covariates (e.g., age and BMI). Results: Regression analyses revealed a significant interaction between goal disengagement capacities and chronic parenting stress, $\beta=-.03, \mathrm{p}<.05$. Goal disengagement capacities (but not goal reengagement capacities) were negatively associated with IL- 6 among mothers of children with $\operatorname{ASD}(\beta=-.02, p<.04)$, such that mothers who reported higher levels of goal disengagement had lower levels of IL-6. In contrast, no significant association was observed between goal disengagement and IL- 6 among control mothers $(\beta=.01, p$ $=.38$ ). Conclusions: The results of this study suggest that goal disengagement capacities represent an adaptive self-regulation factor that may prevent inflammation among chronically stressed parents. Interventions targeting goal disengagement should be developed and implemented for this population experiencing lifelong parenting challenges.

CORRESPONDING AUTHOR: Heather Herriot, Concordia University, heatherherriot@gmail.com

\section{O35.4}

EFFECTS OF CHILDHOOD TRAUMA, DAILY STRESS AND EMOTIONS ON DIURNAL CORTISOL LEVELS IN INDIVIDUALS VULNERABLE TO SUICIDE

Daryl O'Connor ${ }^{1}$, Dawn Branley ${ }^{1}$, Jessica Green ${ }^{1}$, Eamonn Ferguson ${ }^{2}$, Ronan $\mathrm{O}^{\prime} \mathrm{Carroll}^{3}$, Rory O'Connor ${ }^{4}$

${ }^{1}$ University of Leeds - United Kingdom

${ }^{2}$ University of Nottingham - United Kingdom

${ }^{3}$ University of Stirling - United Kingdom

${ }^{4}$ University of Glasgow - United Kingdom

Introduction \& purpose Suicide is a global health issue. Dysregulated hypothalamic-pituitary-adrenal (HPA) axis activity, as measured by cortisol levels, has been identified as one potential risk factor for suicide. Recent evidence has indicated that blunted cortisol reactivity to stress is associated with childhood trauma. The current study investigated whether childhood trauma and daily stressors and emotions were associated with diurnal cortisol levels over a 7-day study. Methods: 154 participants were recruited and grouped according to history of previous suicidal attempt, suicidal ideation or as control participants. Participants completed background questionnaires including the Childhood Trauma Questionnaire before commencing at 7-day daily diary study. Cortisol samples were provided immediately upon waking, +15 mins after waking, +30 mins, +45 mins, +3 hours, +6 hours, +9 hours and +12 hours on 7 consecutive days. Measures of daily stressors, mood and defeat and entrapment were completed at the end of each day. Diurnal cortisol slope was calculated as the change in cortisol from the peak following waking to late evening. Data were analysed using multi-level modelling. Results: Participants who had made a previous suicide attempt exhibited significantly flatter diurnal cortisol slopes across the 7 days compared to participants in the 
control group; suicide ideators were intermediate to both groups. Childhood trauma was found to be associated with flatter diurnal cortisol slopes, such that individuals who had experienced higher levels of trauma exhibited flatter cortisol slopes across the day. In particular, physical abuse was identified as being the most important subscale. Higher levels of daily defeat/entrapment were found also to be associated with a flatter diurnal cortisol slope. Conclusions: These results extend other findings from the laboratory into naturalistic settings and indicate that dysregulated hypothalamic-pituitary-adrenal (HPA) axis activity is associated with suicidal behaviour. Childhood trauma and perceptions of defeat/ entrapment may be important proximal and distal factors associated with the development of HPA axis dysregulation. The challenge for researchers is to elucidate the precise causal mechanisms linking trauma, cortisol and suicide risk in order to develop interventions to help build resilience in vulnerable populations.

CORRESPONDING AUTHOR: Daryl O'Connor, University of Leeds, d.b.oconnor@leeds.ac.uk

\section{O35.5}

A MANUALISED INTERVENTION TO REDUCE PSYCHOLOGICAL DISTRESS IN INFLAMMATORY BOWEL DISEASE: A RANDOMISED CONTROLLED FEASIBILITY TRIAL Lyndsay Hughes $^{1}$, Anja Fischer ${ }^{2}$, Peter Irving ${ }^{3}$, Rona Moss-Morris ${ }^{1}$

${ }^{1}$ King's College London - United Kingdom

${ }^{2}$ University of Central Lancashire - United Kingdom

${ }^{3}$ Guy's and St Thomas NHS Foundation Trust - United Kingdom

Depression and anxiety rates are higher in Inflammatory Bowel Disease (IBD) than the general population. To address this, a novel IBD specific self-management manual (QOLITI) with minimal therapist telephone support was developed. The manual was grounded in evidence based interventions together with patient and professional input. This study assessed the feasibility and acceptability of the QOLITI. Methods: A two-arm pilot randomised control trial (NCT02707068) was conducted. Recruitment was through hospital clinics and the Crohn's and Colitis UK website. Participants were eligible if they had a confirmed diagnosis of IBD and scored in the moderate to high range of depression on the PHQ-9. Patients were randomised into either the intervention arm of QOLITI manual plus three telephone support sessions $(n=32)$ or waitlist control arm of usual care $(n=31)$. Questionnaires were administered pre- and post-intervention (8 weeks). Results: $97 \%$ of patients approached in clinics were eligible and $50 \%$ subsequently consented. $85 \%$ of patients who began the trial were retained in the study. $80 \%$ of those in the treatment arm completed at least 1 telephone session. Semi-structured interviews indicated a high level of acceptability with willingness to actively engage with the manual, despite challenging tasks. Conclusions: Good feasibility and acceptability was shown for a self-directed manual targeting distress. It is feasible to recruit participants from clinics and retain them through an 8 week intervention. Telephone support was helpful to guide progress through the manual. Feedback on the manual was positive and further testing following MRC guidelines will continue to test the effectiveness, costeffectiveness and implementation of the QOLITI.

CORRESPONDING AUTHOR: Lyndsay Hughes, King's College London, lyndsay.hughes@kcl.ac.uk

\section{O35.6}

FROM PSYCHOLGICAL TO BIOBEHAVIORAL: A MODEL OF ADJUSTMENT TO CHRONIC ILLNESS

Michael Hoyt ${ }^{1}$

${ }^{1}$ Hunter College, City University of New York - USA

Most people are affected by chronic illness. Chronic health conditions can cause profound life disruption across life domains and this greatly contributes to public health burden. This talk will focus on presenting an integrated model of adjustment to chronic illness that has evolved from a purely psychological model to one that integrates social, psychological, and biological processes that exert influence on clinical health outcomes. Traditional models have relied on the role of emotional, behavioral, and motivational factors in predicting trajectories of adjustment to chronic illness. At the same time, great progress over the past decade has called attention to the role of biobehavioral processes such as depression, sleep quality, and chronic stress on disease progression and physical health. This has included a focus on biological mechanisms, such as alterations in immune and neuroendocrine function, that are associated with both disease progression as well as indicators of psychological adjustment following chronic illness. Examples from work in cancer, HIV, and cardiovascular disease have demonstrated such relationships. However, no conceptual framework has unified these related areas of research.

Dr. Hoyt will call upon examples from his own research with cancer survivors, as well as evidence from psychosomatic medicine and health psychology, to articulate a biobehavioral model of adjustment. A focus will be on understanding the role of coping in the process of adjustment and the potential biological mechanisms for which evidence is building. Attention will be given to contextual factors, such as age, gender, and interpersonal relationships; as well as the associations of biobehavioral processes with health-related quality of life. Implications for intervention development and future research directions will be discussed.

CORRESPONDING AUTHOR: Michael Hoyt, Hunter College, mhoyt44@gmail.com

O36.1

A NORMALISATION PROCESS THEORY PERSPECTIVE ON GP ATTITUDES TOWARDS A PROPOSED TYPE 2 DIABETES APP: AN INTERVIEW STUDY

Julie Ayre $^{1}$, Carissa Bonner ${ }^{1}$, Rajini Jayaballa ${ }^{2}$, Sian Bramwell ${ }^{2}$, Don Nutbeam $^{1}$, Glen Maberly ${ }^{2}$, Kirsten McCaffery ${ }^{1}$

${ }^{1}$ University of Sydney- Australia

2Western Sydney Diabetes- Australia

Introduction This study explored general practitioners' (GPs) attitudes towards a proposed type 2 diabetes self-management app for patients with lower health literacy. Interviews were conducted during a period of transition in Australia towards a more integrated primary care model called 'Health Care Homes'. Methods 25 semi-structured interviews explored GP attitudes about the feasibility of diabetes apps for patients with lower health literacy. The interview schedule discussed an app design that would integrate the GPs' health care plans and services. Interviews were audio-recorded, transcribed and coded using Framework Analysis to ensure rigour, guided by Normalisation Process Theory. Results Three overarching themes were identified: 1) role of face-to-face care; 2) concerns about delineating GP/patient responsibilties; 3) attitudes towards workload changes. Theme 1 identified beliefs underpinning a commonly expressed preference for face-to-face care. GPs perceived information was more tailored, salient and better understood, and presented with greater empathy when delivered face-to-face, rather than online. Theme 2 reflected perceptions of how an app might increase responsibility for the GP. Concerns about patient safety, liability and workload influenced attitudes towards to the app. GPs also discussed strategies to overcome these concerns, such as monitoring patients' use of the app, limiting the use of synched data, and maintaining patient autonomy in prompts and advice. Theme 3 focussed on attitudes towards app implementation, particularly in the context of transitioning to Health Care Homes. Most GPs anticipated an initial burden whilst learning to use a new clinical tool. Some GPs accepted this burden on the basis that the change was inevitable as healthcare became more integrated. Others reported the potential benefits were outweighed by effort to implement the app. Conclusions This study provided insight into GP attitudes towards a diabetes app integrated with 
primary care services. This was observed as more than just a technological change; GPs were concerned about changes in liability, workload, and the nature of consultations. This research highlighted potential facilitators and barriers to engaging GPs in the implementation process.

CORRESPONDING AUTHOR: Julie Ayre, The University of Sydney, julie.ayre@sydney.edu.au

\section{O36.2}

OUTCOMES OF ONLINE CBT FOR DEPRESSION AND ANXIETY TAILORED FOR EARLY STAGE CANCER SURVIVORS (ICANADAPT EARLY)

Jill Newby ${ }^{1}$, Michael Murphy ${ }^{2}$, Phyllis Butow ${ }^{3}$, Joanne Shaw ${ }^{3}$, Heather Shepherd ${ }^{3}$, Melanie Price ${ }^{3}$, Laura Kirsten ${ }^{4}$, Jessica Cuddy ${ }^{3}$, Gavin Andrews

${ }^{1}$ University of New South Wales, Sydney- Australia

${ }^{2}$ St Vincent's Hospital, Sydney- Australia

${ }^{3}$ University of Sydney- Australia

${ }^{4}$ Nepean Cancer Care Centre, Sydney- Australia

Introduction \& Purpose: This is the first trial of internet delivered cognitive behavioural therapy (iCBT) as an intervention for people with early stage cancer who have clinical depression and/or anxiety. 'iCanADAPT Early' is an iCBT course based on 8 lessons, delivered over 16 weeks, which focuses on both core iCBT skills and cancer specific issues such as the fear of cancer recurrence. Clinician supervision and guidance is provided over the course. Methods*: Participants were recruited from a variety of sources including cancer research databases. After applying online, they underwent a structured diagnostic interview to assess for a clinical diagnosis of a major depressive disorder (MDD) and/or an anxiety disorder. Eligible participants were randomised to the iCBT intervention up front, or to treatment as usual (TAU). Results: Of the 113 participants that partook in the trial, the mean age was 53.3 yo, and the majority of participants were female $(88 \%, \mathrm{n}=100)$, with breast cancer $(66 \%, \mathrm{n}=$ 75 ), with $12 \%$ of the cohort receiving their cancer diagnosis in the year preceding. On clinical interview, participants meet criteria for 1.76 disorders with the most common disorder being Generalised Anxiety Disorder (GAD), followed by MDD. The primary outcome measure is the Hospital Anxiety and Depression Scale, HADS. There were statistically significant improvements in depressive and anxious symptoms as demonstrated by decreases in the HADS total score. The iCBT group improved from baseline $(M=21.28 ; S D=5.31)$ to post treatment $(M=13.15 ; S D=6.23)$ vs- TAU group which had smaller improvement from baseline $(\mathrm{M}=$ 19.68; $\mathrm{SD}=5.97)$ to post treatment $(\mathrm{M}=17.36 ; \mathrm{SD}=5.62), \mathrm{t}(100)=-$ $3.578, \mathrm{p}=.001$. Conclusions: iCBT for depression and anxiety is an effective treatment for depression and anxiety in early stage cancer, and outperforms usual care, with improvements in symptoms maintained up to 3-months follow-up. Expanded details of the trial, measures and potential future implications will be discussed.

CORRESPONDING AUTHOR: Jill Newby, University of New South Wales, Sydney, j.newby@unsw.edu.au

\section{O36.3}

ENHANCING SELF-CARE SELF-EFFICACY AND REDUCING SYMPTOM DISTRESS IN WOMEN WITH BREAST CANCERRELATED LYMPHOEDEMA

Kerry Sherman ${ }^{1}$, Carla Sullivan-Myers ${ }^{1}$, John Boyages ${ }^{2}$, Louise Koelmeyer $^{2}$, Helen Mackie ${ }^{2}$

${ }^{1}$ Macquarie University- Australia

${ }^{2}$ ALERT, Macquarie University- Australia

Introduction and Purpose Breast cancer-related lymphoedema (BCRL) is a chronic condition affecting more than $20 \%$ of breast cancer survivors.
Physical symptoms, functional limitations and associated psychosocial concerns adversely impact women with this condition. Management of BCRL involves the enactment of self-care behaviours that are critical for successful lymphoedema management. We have developed a web-based intervention (LYSCA - LYmphoedema Self-Care) to enhance lymphoedema self-care behaviours and lymphoedema symptom management via a goal setting process. We aimed to pilot the LYSCA intervention to determine its effect on self-care self-efficacy, lymphoedema symptom distress, and cognitive and affective lymphoedema-related illness representations in women with BCRL. Methods Applying a pre-post design, 97 women diagnosed with BCRL were recruited from the lymphoedema treatment clinic at Macquarie University Hospital and a private lymphoedema clinic (LK). Following consent, participants completed online study questionnaires including self-care self-efficacy, lymphoedema symptom intensity and distress, and adherence to selfcare behaviours. Secondary outcomes included a range of cognitive and affective illness representations of lymphoedema measured by the IPQ-R. Participants were then given unlimited access to the LYSCA website, and an accompanying daily diary for goal setting and tracking of goal progress. Follow-up online assessments were undertaken at 1- and 2-months. Results Multilevel linear mixed model analyses indicated significant increases over time in self-care self-efficacy $(\mathrm{p}=.016)$, and reductions in lymphoedema-related symptom intensity and distress $(\mathrm{p}=.001)$. Adherence to self-care behaviours did not change $(\mathrm{p}=.210)$. Illness coherence improved $(\mathrm{p}=.041)$ and emotional representations regarding lymphoedema diminished $(\mathrm{p}=.009)$ significantly following intervention exposure. Conclusions These findings provide strong preliminary support for the use of the LYSCA intervention to reduce symptom intensity and enhance self-care management of lymphoedema. Future research utilising a randomised controlled design is needed to investigate the full potential of the LYSCA intervention for facilitating lymphoedema self-care.

CORRESPONDING AUTHOR: Kerry Sherman, Macquarie University, kerry.sherman@mq.edu.au

\section{O36.4}

HIGHER USE OF MOBILE DEVICES BY INPATIENTS IS ASSOCIATED WITH BETTER PSYCHOLOGICAL WELLBEING Elizabeth Broadbent ${ }^{1}$, Aryanah Paul $^{1}$, Chris Ellis $^{2}$

${ }^{1}$ The University of Auckland- New Zealand

${ }^{2}$ Auckland District Health Board- New ealand

Introduction \& Purpose It is common to see hospital patients with mobile phones and computer tablets by their bedsides. Little research has investigated what inpatients are using the devices for, when, and the effects these devices have on patients' wellbeing. This study aimed to explore inpatients' use of mobile devices in a hospital setting. Hypotheses were that patients would use mobile devices primarily for communicating with friends and family, and that increased use of mobile devices would be associated with higher perceived social support and better psychological outcomes. Methods A cross-sectional study was conducted with 120 patients admitted to the cardiology ward and coronary care unit at a large Public hospital in New Zealand. Participants completed a questionnaire about their use of mobile devices in hospital, as well as stress, social support, anxiety, depression, loneliness, pain, and perceived control. Results Over $80 \%$ of inpatients used at least one mobile device in hospital, most often a smart-phone. The top uses were texting friends and family (over $90 \%$ of users), calling friends and family (over $70 \%$ ), searching for information (50\%; searching for information on illnesss specifically $26 \%$ ), and reading (41\%). Higher mobile device use was significantly associated with lower perceived stress $(r=-.22, p=.017)$, higher perceived social support $(\mathrm{r}=.42, \mathrm{p}<.001)$, lower anxiety $(\mathrm{r}=-.35$, $\mathrm{p}<.001)$, lower depression $(\mathrm{r}=-.41, \mathrm{p}<.001)$, lower loneliness $(\mathrm{r}=-.38$, $\mathrm{p}=.026)$, lower pain $(\mathrm{r}=-.27, \mathrm{p}=.003)$, and higher personal control $(\mathrm{r}=$ $.28, \mathrm{p}=.002)$. Associations held after controlling for demographic 
factors. Conclusions This study suggests that the use of mobile devices is associated with better psychological outcomes for hospitalised patients. The direction of the association cannot be determined, and future research needs to replicate this study with other patient populations with longitudinal and experimental designs. Mobile devices may have considerable utility in improving the psychological health and well-being of hospitalised patients predominantly via improved contact with friends and family.

CORRESPONDING AUTHOR: Elizabeth Broadbent, The University of Auckland, e.broadbent@auckland.ac.nz

\section{O36.5}

THE PAIN COURSE: EVIDENCE OF EFFECTIVENESS OF AN ONLINE CBT PAIN MANAGEMENT PROGRAMME IN A RANDOMIZED TRIAL IN IRELAND

Brian McGuire $^{12}$, Catherine Navin ${ }^{12}$, Jonathan $\operatorname{Egan}^{1}$, Brian Slattery ${ }^{1}$, Monika Pilch ${ }^{1}$, Laura O'Connor ${ }^{1}$, Stephanie Haugh ${ }^{1}$, Bevin Malone ${ }^{1}$, Cristina Perez ${ }^{12}$

${ }^{1}$ School of Psychology \& Centre for Pain Research- Ireland

${ }^{2}$ National University of Ireland- Ireland

Background. Despite the demonstrated efficacy of cognitive behavioural therapy (CBT) in the treatment of people with chronic pain, access to these effective, evidence-based interventions is limited. Internet-based CBT (iCBT) has emerged as an innovative approach to address these treatment barriers. One such internet-delivered psychological pain management programme, the Pain Course, was developed and empirically tested in Australia. The Pain Course is a clinician guided iCBT programme for adults with chronic pain and the aim of the current study was to evaluate the efficacy of this intervention in a sample of Irish adults with chronic pain. Methods. Following a pilot study to confirm the cultural suitability of the program, 133 individuals were randomly assigned to either a Treatment Group or wait-list Control Group. The Pain Course programme was a cognitive behaviour therapy intervention comprising five internetdelivered lessons, released sequentially over an 8 week period. The programme also included homework tasks, additional resources, weekly contact with a clinician via e-mail or telephone, and automated e-mails. The primary outcome was pain-related disability as measured by the Roland Morris Disability Questionnaire (RMDQ). The secondary outcomes were depression, anxiety, pain severity, self-efficacy, fear of movement, pain impact, pain acceptance, and treatment satisfaction. The online measurements were conducted prior to the commencement of the Course and nine weeks after the start of the Course. Results. In terms of lesson completion, $80 \%$ of the Treatment Group completed all five lessons. Improvements were significantly greater for Treatment Group participants compared to Control Group participants in levels of disability, anxiety, depression, and average pain levels at posttreatment. Over $80 \%$ of participants rated the programme as highly acceptable. Overall, the clinician spent a total mean time of 83.4 minutes contact time per patient throughout the programme. Conclusion. The findings of the study suggest that a brief iCBT intervention is clinically effective, acceptable to patients, and efficient in terms of therapist contact time. The findings add to the existing literature and are broadly in line with previous trials of the Pain Course.

CORRESPONDING AUTHOR: Brian McGuire, School of Psychology \& Centre for Pain Research, National University of Ireland, brian.mcguire@nuigalway.ie

\section{O36.6}

INTERNET TREATMENT FOR ALCOHOL USE DISORDERS Christopher Sundström ${ }^{1}$, Niels Eék ${ }^{2}$, Martin Kraepelien ${ }^{1}$, Miriam Jakobson $^{3}$, Mikael Gajecki ${ }^{1}$, Maria Beckman ${ }^{1}$, Claudia Fahlke ${ }^{2}$, Viktor Kaldo $^{1}$, Anne H Berman ${ }^{1}$
${ }^{1}$ Karolinska Institutet- SWEDEN

${ }^{2}$ University of Gothenburg- SWEDEN

${ }^{3}$ Stockholm Center for Dependency Disorders- SWEDEN

Introduction and purpose: A large proportion of individuals with alcohol use disorders (AUD) do not seek psychological treatment, but access to such treatment could potentially be increased by delivering it digitally. Cognitive behavioural therapy (CBT) is widely recognized as one of the psychological treatment methods for alcohol problems for which evidence is most robust. Most internet interventions evaluated have been low-intensity, either brief intervention or briefer CBT modules without therapist contact. This study tested a high-intensity therapist-guided CBT treatment with therapist guidance against a previously evaluated lowintensity CBT treatment without therapist guidance and a waitlist control group. Methods: Participants in the study $(n=168)$ were recruited through Google Adwords and, after initial internet screening, diagnostically assessed by telephone. They were then randomized to a) high-intensity internet-based treatment, b) low-intensity internet treatment or c) a waitlist control group (WLC). Primary outcomes were number of drinks consumed and number of heavy drinking days (HDD) preceding week, measured with the self-rated Timeline Followback. Participant data were collected at screening (T0), immediately pre-treatment (T1), posttreatment (T2), three months post-treatment (T3) and one year posttreatment (T4). Results: Of the included participants, $87 \%$ completed outcome measures post-treatment and $78 \%$ completed outcome measures at three-month follow-up. An intent-to-treat analysis showed that participants in the high-intensity treatment consumed significantly fewer standard drinks compared to WLC (-10.11 drinks per week) and had significantly fewer HDD compared to both WLC and low-intensity treatment ($1.30 /-0.61 \mathrm{HDD} /$ week) at post-treatment. At the three-month follow up, no significant differences were observed between the two treatment groups. One-year follow-ups are currently being collected and will be presented at the conference. Conclusion: These results indicate that high-intensity internet treatment may be more effective in short-term reduction of heavy drinking compared to low-intensity treatment. Future research should aim to optimize the additional effects of therapist guidance

CORRESPONDING AUTHOR: PhD. Christopher Sundström, Karolinska Institutet, christopher.sundstrom@ki.se

O37.1

THE RELATIONSHIP BETWEEN POSTTRAUMATIC STRESS DISORDER AND POSTTRAUMATIC GROWTH AMONG MEN LIVING WITH HIV/AIDS: THE MEDIATING ROLE OF COPING STRATEGIES

Danhua Lin ${ }^{1}$, Zhi Ye ${ }^{1}$, Lihua Chen ${ }^{1}$

${ }^{1}$ Institute of Developmental Psychology, Faculty of Psychology, Beijing Normal University- China

Introduction It was reported that there were 660,000 people living with HIV/AIDS at the end of 2016 in China and sexual transmission has become the driving force of HIV infection since 2007. Traumatic experience of HIV/AIDS may product a myriad of mental health problems, especially posttraumatic stress disorder (PTSD) and also bring positive changes which was conceptualized as posttraumatic growth (PTG). Growing research has explored the relationship between PTSD and PTG with mixed findings. While some researchers suggested that PTSD is negatively related to PTG, others argued that to some extent PTSD is a prerequisite for the occurrence of PTG. In addition, growing research suggests that psychosocial and cognitive factors may mediate the development of PTG after trauma exposure. Specifically, individuals with traumatic experience may have fewer psychological symptoms and better mental health through the utilization of adaptive coping strategies. Furthermore, problem-focused coping strategy is used to resolve the 
stressful event or situation whereas emotion-focused coping strategies is used to manage the emotion distress in response to severe stress from jobs, family, and interpersonal relationships. Although men living with HIV/AIDS constitute a newly emerged high-risk group in China, little research is available on the relationship and mechanism of PTSD and PTG among this vulnerable population. Therefore, the present study aimed to investigate the relationship between PTSD and PTG, and moreover, the potential mediating effect of coping strategies through which PTSD contribute to PTG among men living with HIV/AIDS in Beijing, China. Methods A sample of 140 men living with HIV/AIDS (Mean=26.60, SD=6.60) was recruited from Beijing Center for Disease Prevention and Control. Participants were asked to complete a battery of self-administered questionnaires including sociodemographic and HIVrelated characteristics, coping strategies (i.e., problem-focused coping strategy and emotion-focused coping strategy), PTSD (i.e., intrusion and avoidance), and PTG (i.e., relating to others, new possibilities, personal strength and appreciation of life). Hierarchical regression and structural equational modeling were conducted to analyze the relationships among PTSD, coping strategies, and PTG. Results Regression analyses showed that, after controlling for sociodemographic and HIV-related variables, PTSD was negatively related to PTG $(\beta=-0.18, p<0.05)$. Mediational model revealed that problem-focused coping strategy played mediating role in the association between PTSD and PTG (indirect effect $=-0.24, \mathrm{p}<0.01,95 \% \mathrm{CI}=[-0.36,-0.08])$. Although emotion-focused coping strategy was found to be significantly associated with both PTSD and PTG, the mediating effect of emotion-focused coping strategy on the PTSD and PTG link was nonsignificant (indirect effect $=-0.08, \mathrm{p}=0.08$, $95 \% \mathrm{CI}=[-0.17,0.01])$. Thus, the current study indicated that problemfocused coping strategy served as a crucial pathway through which PTSD was associated with PTG among Chinese men living with HIV/AIDS. Conclusions The present study contributes to an understanding of the association between PTSD and PTG as well as underscores the role of problem-focused coping strategy in linking PTSD to PTG among young men living with HIV/AIDS. On the basis of these findings, psychological interventions focusing on enhancing problem-solving skills may be particularly beneficial for HIV-positive populations to achieve positive changes after traumatic experience.

CORRESPONDING AUTHOR: Danhua Lin, Institute of Developmental Psychology, Faculty of Psychology, Beijing Normal University, danhualin@bnu.edu.cn

\section{O37.2}

THE INCIDENCE OF RETROSPECTIVE AND CURRENT MENTAL HEALTH PROBLEMS IN PEOPLE LIVING WITH HIV LOW ANTIRETROVIRAL TREATMENT ADHERENCE AND LOST TO FOLLOW-UP

Lydia Edith Perrusquia Ortiz ${ }^{1}$, Lydia E. Perrusquia Ortiz ${ }^{1}$, Ivon N. Sanchez Perez ${ }^{1}$, Evelyn Rodriguez Estrada ${ }^{1}$, Gustavo Reyes Teran ${ }^{1}$

${ }^{1}$ Departamento de Investigación de Enfermedades Infecciosas (CIENI) of Instituto Nacional de Enfermedades Respiratorias (INER)

The incidence of retrospective and current mental health problems in people living with HIV low antiretroviral treatment adherence and lost to follow-up Authors. Lydia E. Perrusquía-Ortiz;1 Ivonne N. PérezSánchez, 1,2 Evelyn Rodriguez-Estrada; 1 Gustavo Reyes-Terán. 1 Affiliations. 1. Departamento de Investigación de Enfermedades Infecciosas (CIENI) of Instituto Nacional de Enfermedades Respiratorias (INER) 2. Consejo Nacional de Ciencia y Tecnología (CONACYT) Background. Psychiatric and psychological conditions are highly prevalent in people living with HIV (PLWH) and have been related with low ART adherence, increased sexual risk behaviors, failure of ART, the progression of HIV infection and higher mortality rates. Objectives of this study were 1) to describe the incidence of retrospective and current mental health problems (psychiatric and/or psychological problems), 2) To describe the association between retrospective and current mental health problems (MHP) with ART adherence and lost to follow-up (LTFU). Methods. A cross-sectional study was carried out from May 2016 to October 2017 in the Center for Research in Infectious Diseases (CIENI) in Mexico. PLWH who attendance for mental health evaluation were included. All participants completed the Assessment of Adherence to Antiretroviral Therapy Questionnaire (CEAT-VIH) and a 45 minutes interview for assessing psychological problems, the incidence of retrospective and current psychiatry diagnostic was obtained from patients medical records. Written consent was provided. Chi-square test and OR analysis were done using SPSS 20.0. Significant p-value was $<.05$. Results: Participated in the study 113 PLWH. Most of them were male $81.4 \%$, single $72.6 \%$, with pre-university educational level $33.6 \%$, and a median age of $37.5, \mathrm{~S} . \mathrm{D}=10.3$ years. The $24.8 \%$ of the sample had a history of MHP ( 24 had psychological problems, 4 had psychiatry problems and 5 had both psychological and psychiatry). History of MHP was not related with ART-adherence or history of LTFU. Current MHP were found in $33.8 \%$ of patients ( 24 had psychological problems, 13 have psychiatry problems and 8 had both of them). PLWH with a current MHP were three times more prone to have low ART-adherence (Low adherence and current MHP 54.34\% vs Low adherence without MHP 45.6\%, Chi2=7.39, gl=1; $\mathrm{p}=.006 ; \mathrm{OR}=2.97(1.34,6.60))$ and more than three times more prone to have been in LTFU (LTFU in patients with current MHP $80.7 \%$ vs LTFU in patients without MHP $19.23 \%$, Chi2=5.97, gl=1; $\mathrm{p}=.012 ; \mathrm{OR}=3.57(1.23,10.34))$ and Conclusión: This study highlights the importance of screen for MHP in PLWH due to their relationship with low adherence and LTFU.

CORRESPONDING AUTHOR: Lydia Edith Perrusquia Ortiz, ${ }^{1}$ Departamento de Investigación de Enfermedades Infecciosas (CIENI) of Instituto Nacional de Enfermedades Respiratorias (INER), lydia.perrusquia@cieni.org.mx

\section{O37.3}

ADDRESSING DRUG USE, MEDICATION ADHERENCE, AND MENTAL HEALTH IN OLDER ADULTS LIVING WITH HIV: FINDINGS FROM A RANDOMIZED CONTROLLED TRIAL

Jeffrey Parsons ${ }^{1}$, Brett Millar ${ }^{2}$, Tyrel Starks ${ }^{1}$, Jorge Cienfuegos Szalay ${ }^{3}$, David Marcotte ${ }^{4}$,

${ }^{1}$ Hunter College - CUNY- United States of America

${ }^{2}$ Center for HIV/AIDS Educational Studies and Training- United States of America

${ }^{3}$ Graduate Center - CUNY- United States of America

${ }^{4}$ Fordham University- United States of America

Introduction \& Purpose: To improve the overall health of older adults living with HIV (OALWH), we tested the effectiveness of a 12-session cognitive self-schema therapy intervention (3S) in reducing drug use and improving medication adherence among substance-using OALWH in New York City. We hypothesized that, compared to both a 12-session Education and a 1-session LifeSteps condition, the $3 \mathrm{~S}$ treatment would yield greater reductions in drug use and improvements in medication adherence, immune functioning, and mental health. Methods: Of 120 OALWH, 60 were randomized to the $3 \mathrm{~S}$ treatment, 30 to Education, and 30 to LifeSteps. Eligibility criteria included being prescribed antiretroviral (ART) medication, missing medication on at least three days (in past month), and meeting dependence for a drug which the participant has used in the past year. Changes in drug use and ART adherence (measured in a 30-day Timeline Follow Back), CD4 count and viral load, and depression at 3-month and 6-month follow-up were compared between conditions. Results: Most of the sample identified as Black (75\%) and as male (67.5), 57\% of whom were gay or bisexual. At baseline, the sample reported a mean of 11.5 days of drug use (out of 30 days). Postintervention, those in the treatment condition reported significantly fewer days of drug use (Mean $=6.4$ days), compared to Education (Mean $=10.2$ 
days) and LifeSteps (Mean $=9.8$. days), both $p<.01$. These differences were sustained at 6-months. No significant reduction in missed medication days were observed at follow-up and no differences emerged between conditions. All conditions improved on CD4 and viral load, however no between-condition differences were observed. Post-intervention, depression in the treatment condition was lowest of all three groups, and was significantly lower than for the LifeSteps condition $(p<.01)$. Conclusions: The 12-session $3 \mathrm{~S}$ therapy achieved significant reductions in drug use (almost halving drug use days) and depression among our sample of OALWH. Although improvements in medication adherence were not observed in this trial, it is anticipated that reductions in drug use and depression may produce longer-term beneficial effects on global physical health. Implications for improving health behaviors and wellbeing in OALWH will be discussed.

CORRESPONDING AUTHOR: Jeffrey Parsons, Hunter College CUNY,jparsons@chestnyc.org

\section{O37.4}

FINDINGS FROM A CULTURALLY-TAILORED SOCIO-CENTRIC NETWORK INTERVENTION FOR INCREASING HIV PREVENTIVE BEHAVIORAL ADHERENCE

Mariano Kanamori ${ }^{1}$, Mario De La Rosa ${ }^{2}$, Stephanie Diez ${ }^{2}$, Jessica Weissman ${ }^{2}$, Mary Jo Trepka ${ }^{2}$, Patria Rojas ${ }^{2}$

${ }^{1}$ University of Miami Miller School of Medicine- United States of America

${ }^{2}$ Florida International University, United States of America

Introduction \& Purpose HIV remains a serious public health issue for Latinas in the US. Social network translational programs are a promising approach for improving adherence to behavioral interventions. The main purpose of this talk is to present findings from a twogroup randomized-design program with a culturally-tailored socio-centric network intervention, Progreso en Salud ("Progreso"), as the test condition and a standard health promotion intervention as the control. Research question: Is Progreso superior to the control in increasing HIV preventive behavioral adherence (i.e., condom use, female condom use, having conversations with friends about condom use and having HIV communication with partner)? Methods We recruited 261 participants grouped in 20 socio-centric networks. They were interviewed at baseline, 6- and 12-months after the intervention. Analyses included non-parametric related-samples Friedman's test and repeated measures general linear models. The program was implemented from June 2015 to February 2017 in South Florida, U.S. Results Only the Progreso intervention resulted in significant changes in HIV preventive behavioral adherence over time. As such, we are not reporting results from the control intervention. Progreso increased condom use $(p=0.042)$ and female condom use $(p=0.029)$ over time. Compared to baseline, a higher percentage of women at 6 and 12 months used condoms every time they had vaginal and/or anal sex $(12.4 \%$ vs. $15.6 \%$ vs. $15.9 \%)$ and a lower percentage never used condoms $(64.8 \%$ vs. $46.7 \%$ vs. $47.7 \%)$ when they had sex. Compared to baseline, a higher percentage of women at 6 and 12months used female condoms (9.1\% vs. $21.3 \%$ vs. $20.9 \%)$. Scores increased significantly between baseline and 6 months and between baseline and 12-month for "Having conversations with friends about condom use" scores $[\mathrm{F}(2)=16.21, \mathrm{p}<0.001, \mathrm{hp} 2.182]$ and between baseline to 6 months for "HIV communication with partner" scores. Conclusions This study demonstrates that a socio-centric network intervention, culturally-tailored for underserved Latinas, resulted in positive effects on HIV preventive behavioral adherence, and these effects were sustained 12-months after implementation of the intervention.

CORRESPONDING AUTHOR: Dr. Mariano Kanamori, University of Miami Miller School of Medicine, mkanamori@med.miami.edu
O37.5

SEPA A CULTURALLY APPROPIATE HIV PREVENTION INTERVENTION IMPACTS HISPANIC BEHAVIORS

Nena Peragallo-Montano ${ }^{1}$, Nilda Peragallo-Montano ${ }^{1}$, Natalia Villegas ${ }^{1}$, Rosina Cianelli ${ }^{1}$, Lilian Ferrer ${ }^{2}$

${ }^{1}$ School of Nursing, University of North Carolina- United States of America ${ }^{2}$ Escuela de enfermería, Pontificia Universidad Catolica de Chile- Chile

In the United States, the incidence of HIV is increasing among minorities, representing more than $72 \%$ of new HIV cases and $21 \%$ of general population living with HIV. The counties of Miami-Dade and Broward have the highest number of AIDS cases in Florida and although statistics show a trend of transmission to Hispanic men and women, there are no HIV prevention programs available to them. There is need for culturally adapted and accessible community programs for Hispanics between 18 and 50 years of age. Methodology: A mixed design was used to identify information on HIV risk factors and SEPA's adaptation needs. Sexually active participants in the last 3 months $(n=71)$ answered a questionnaire about risk factors and preferences for an intervention, in order to determine the feasibility of using SEPA for Hispanic men and women in group sessions and with a base in the cognitive-social theory and Freirean pedagogy model. SPSS was used for the descriptive data analysis, together with the focus groups' content analysis. Results: the distribution by sex was 36 men (50.7\%) and 35 women (49.3\%). The preferred language was Spanish $(n=55)$, the average number of children was $2.01 \pm 1.9($ range $=$ $0-7)$; and regarding the country of origin, the majority were from Mexico $(n=28,39.4 \%)$ and other countries of Latin America $(n=7,9.8 \%)$. The average level of education was $10.34 \pm 4.2$ years and the majority had a job $(\mathrm{n}=38,53.5 \%)$. In relation to the risk of HIV, almost half of the participants $(\mathrm{n}=30,42.3 \%)$ did not feel at risk of contracting HIV and most of them ( $\mathrm{n}=56,78.9 \%)$ had taken the test. The use of condoms among Hispanic women with a stable partner was low $(\mathrm{n}=11,31.42 \%)$ and even lower for men. Participants report the risk of contracting HIV and lack of interventions adapted for them, moreover, considered that SEPA was appropriate and accessible. Conclusion: culturally specific interventions promise to prevent HIV in Hispanics in USA. SEPA is perceived as appropriate and should be used as a preventive strategy.

CORRESPONDING AUTHOR: Nena Peragallo-Montano, School of Nursing, University of North Carolina, Npm@email.unc.edu

\section{O37.6}

IS IT POSSIBLE FOR TRANSGENDER MEN TO CONTRACT STIS? A RETROSPECTIVE REVIEW OF LABORATORY TESTED STIS AND ASSOCIATED FACTORS OF TRANSGENDER MEN FROM SOUTHERN BRAZIL

Angelo Brandelli Costa ${ }^{1}$

${ }^{1}$ Pontifícia Universidade Católica do Rio Grande do Sul- Brazil

Objectives: The present study assessed the prevalence of sexually transmitted infections (STIs) and associated factors in 94 transgender men from southern Brazil. Material and Method: A retrospective review of the medical records of all transgender men who visited an outpatient clinic in Rio Grande do Sul from 1998 to 2017 was performed. Results: Although the sample had a high prevalence of risk factors, such as drug use, one-third of the participants had never been tested for STIs and, when checked, it was mostly for HIV, but not for syphilis or other STIs. Based only on laboratory-tested transgender men, the prevalence of syphilis and hepatitis $\mathrm{C}$ was $3.4 \%$ and $1.6 \%$, respectively. These are very significant percentages when compared to the general population, and could be used to justify required screening exams for all transgender men. Conclusion: It is clear that health professionals need to broaden their understanding of transgender men and take into account the existence of transgender men who have sex with men (TMSM) and possible changes in sexual behavior after gender affirmation surgery (GAS). 
CORRESPONDING AUTHOR: Angelo Brandelli Costa, Pontifícia Universidade Católica do Rio Grande do Sul, angelobrandellicosta@gmail.com

O38.1

GENDER-BASED ANALYSIS OF PROBLEMATIC DRINKING AMONG CANADIAN MILITARY PERSONNEL

Isabelle Richer ${ }^{1}$, Michel Gauthier ${ }^{1}$, Francois Theriault ${ }^{1}$, Barbara Strauss ${ }^{1}$

${ }^{1}$ Department of National Defence / Government of Canada

Almost $20 \%$ of members of the Canadian Armed Forces (CAF) engage in harmful drinking. Several individual factors are known to be linked to risky alcohol use. Conversely, studies focusing on the effect of environmental factors are still sparse. As such, this study aims to better understand individual and environmental correlates of problematic alcohol use among military personnel of the CAF by gender. Secondary analyses were conducted on a representative sample of the 2013/14 CAF Regular Force population. Outcomes examined were hazardous drinking and binge drinking, and correlates were: rurality of serving base, age, marital status, rank, element, psychological distress, symptoms of posttraumatic stress disorder (PTSD), and self-rated level of physical health. Results of multivariate regression models suggest that, among women, being single (odds ratio $[\mathrm{OR}]=1.77, \mathrm{p} \leq 0.05$ ), being younger $(\mathrm{OR}=5.86$, p_ $\leq 0.0001)$, and exhibiting higher scores of PTSD symptoms $(\mathrm{OR}=1.26, \mathrm{p} \leq 0.05)$ are related to hazardous drinking. Similarly, being single $(O R=1.93, p \leq 0.05)$, being younger $(O R=3.3, \quad p \leq 0.05)$, and exhibiting psychological distress $\left(\mathrm{OR}=3.03, \mathrm{p}_{-} \leq 0.05\right)$ are related to binge drinking. Among men, being younger $(\mathrm{OR}=3.77, \mathrm{p} \leq 0.001)$ and exhibiting poorer levels of self-reported physical health $(\mathrm{OR}=1.31$, $\ldots$ p_ $\leq 0.05)$ are related to hazardous drinking. Binge drinking among men is related to rurality of serving base $(\mathrm{OR}=1.98, \quad \mathrm{p} \leq 0.001)$, rank $\left(\mathrm{OR}=1.6, \mathrm{p}_{-} \leq 0.05\right)$, being single $\left(\mathrm{OR}=2.1, \mathrm{p}_{-} \leq 0.001\right)$, and being younger $(\mathrm{OR}=3.8, \mathrm{p} \leq 0.001)$. Findings highlight differences in correlates of problem drinking across gender. Beyond socio-demographic factors, mental health symptoms seem to be specifically related to problematic drinking among women. For men, physical health and serving base environment seem to be more important factors. These findings deepen our understanding of problematic drinking among military personnel and provide evidence for gender specific prevention and intervention strategies.

CORRESPONDING AUTHOR: Isabelle Richez, Department of National Defence / Government of Canadaisabelle.richer@ forces.gc.ca

\section{O38.2}

MALADAPTIVE EMOTION REGULATION AND PSYCHOPATHOLOGY IN YOUNG PEOPLE ACCESSING YOUTH DRUG TREATMENT IN AUSTRALIA

Elise Sloan ${ }^{1}$, Elise Sloan ${ }^{1}$, Kate Hall ${ }^{1}$, George Youssef ${ }^{1}$, Richard Moulding ${ }^{1}$, Helen Mildred, ${ }^{1}$ Petra Staiger ${ }^{1}$

${ }^{1}$ Deakin University- Australia

Background: Individuals accessing treatment in the youth drug sector represent a highly vulnerable population who present with complex patterns of substance use and mental health comorbidity. Deficits in Emotion Regulation (ER) have been identified as a core construct underlying both of these difficulties, and represent a promising treatment target. However, ER is a broad construct, and little is known about what particular pattern of ER strategy use is associated with the most severe mental health outcomes in this cohort of vulnerable young people. This is essential in informing the development of targeted and effective psychological interventions in this cohort. Aim: To examine what pattern of ER strategy use is associated with the highest and lowest levels of psychopathology in this cohort of young people. Method: Participants were young people $(\mathrm{N}=306, \mathrm{M}=20.8$ years) accessing youth drug and alcohol treatment services in Victoria, Australia. They completed an online survey which assessed their use of 14 ER strategies, including both maladaptive (i.e. rumination, avoidance, self-harm, binge/purge) and adaptive (problem solving, reappraisal, physical activity) in response to a recent emotional event. Symptoms of anxiety, depression, substance use, disordered eating and borderline personality disorder were also assessed. Results: Latent Class Analysis (LCA) identified that young people $(n=76)$ who engaged in a pattern of emotion regulation characterised by heightened rumination and avoidance in the absence of adaptive ER strategies exhibited the most significant symptoms psychopathology across all diagnostic categories. In contrast, the class of young people $(n=129)$ who engaged in very low use of maladaptive ER (i.e. self harm, binge/purge) had the lowest levels of psychopathology. Conclusions: Our findings highlight that it is the presence or absence of maladaptive ER strategies (i.e. rumination, avoidance, self harm) that has the greatest impact on subsequent psychopathology. These findings can inform the development of targeted behaviour change interventions that focus on the reduction of rumination and avoidance as maladaptive ways of managing distress in this cohort of vulnerable young people.

CORRESPONDING AUTHOR: Elise Sloan, Deakin University, elise.sloan@deakin.edu.au

\section{O38.3}

WHAT MAKES ADOLESCENTS BINGE DRINK SO OFTEN? RESEARCH EVIDENCE FROM A POPULATION SCHOOL SURVEY IN CHILE

Maria Francisca Roman ${ }^{1}$, Noriko Cable ${ }^{2}$

${ }^{1}$ Universidad de La Frontera- Chile

${ }^{2}$ University College London- England

Binge drinking is known to cause alcohol-related harm among young people. Although the link between adolescent binge drinking and adolescents' own, parental or peer factors were established, little is known about the factors that associate with the frequency of binge drinking in the school contexts. The aim of this study is to examine the contextual associations between parental, peer and school factors and the frequency of binge drinking among Chilean school children aged 13 to 18 . We hypothesised that severe school level deprivation would be associated with an increased number of binge drinking events as well as lower levels of parental supervision, and parental and peer drinking. Information on frequency of binge drinking in the past month, parental supervision, paternal and maternal drinking and peer drinking was extracted from the Tenth Chilean School Population National Substance Use Survey conducted in 2013. Frequency of binge drinking was analysed among those reporting alcohol use. The individual-level information was linked to school-level information (percentage of free school meal children) obtained from the Ministry of Education. Hierarchical data, individuals $(\mathrm{N}=41,146)$ nested within schools $(\mathrm{N}=1,687)$, were analysed using multilevel zero-inflated Poisson regression. Coefficients from the Poisson part were exponentiated to obtain Incidence Rate Ratios (IRR). Estimates were adjusted for parental education, child's age and school type and boys and girls were analysed separately. Results from the Poisson part in the final model showed significant associations between lower levels of parental supervision and increased binge drinking frequencies in boys and girls. For girls, maternal drinking during weekends increased their binge drinking episodes by $10 \%(\mathrm{p}=0.005)$, while mother's daily drinking habits increased it by $24 \%(\mathrm{p}<0.0001)$. Maternal daily drinking also showed increases in boy's binging episodes by average episode by $22 \%(\mathrm{p}<0.0001)$. Having at least half of friends that consumed alcohol increased the average number of binge drinking episodes by $21 \%(p<0.0001)$ for boys, but by $60 \%(p<0.0001)$ for girls. School deprivation was positively associated with the number of events of binge drinking only for girls only (IRR $=1.0034, \mathrm{p}<0.0001$ ). Norms, especially mother's supporting alcohol use and peer influences were major contributor for frequent binge drinking among Chilean adolescents. 
Girls are likely to be more vulnerable to frequent binge drinking, influenced by their peer groups and school environment.

CORRESPONDING AUTHOR: Maria Francisca Roman, Universidad de La Frontera, maria.roman.13@ucl.ac.uk

\section{O38.4}

TREATMENT OUTCOMES FOR PATIENTS WITH ALCOHOL USE DISORDERS ADMINISTERED LONG-ACTING NALTREXONE

Desiree Crevecoeur-MacPhail ${ }^{1}$, Desiree Crevecoeur-MacPhail ${ }^{1}$, Sarah Cousins $^{1}$

${ }^{1}$ UCLA ISAP- United States of America

A pilot study was conducted with patients enrolled in substance use disorder treatment programs. Patients were medically cleared to take extended-release naltrexone (XR-NTX) and the medication was provided free of charge, while the patients were in treatment. Data on urges to drink and side effects were collected in addition to treatment outcomes (engagement, retention, changes in alcohol use and treatment compliance). Treatment outcome data from the XR-NTX group was compared to a post-hoc comparison group who were matched on several demographic and treatment variables. In addition to Chi-square analyses, binary logistic regression analyses with propensity score adjustments, were used to determine if the inclusion of XR-NTX as part of psychosocial treatment improved outcomes (engagement, retention, reductions in primary drug use, treatment compliance) for the XR-NTX group over the post-hoc matched comparison group. Results indicate that the XR-NTX group was significantly more likely to be engaged and retained in treatment and to be discharged from treatment with positive compliance. There were no significant differences between the XR-NTX group and the post-hoc comparison group with regards to reductions in substance use; both groups reduced their substance use by the same number of days. These results indicate that XR-NTX may be an effective tool to help keep patients in treatment longer and to improve treatment compliance while in treatment. Further studies could examine if similar results are found with opiate users and if there are gender or other group differences in outcomes for those who use this medication as part of the treatment plan.

CORRESPONDING AUTHOR: Desiree Crevecoeur-MacPhail, UCLA ISAP, desireec@ucla.edu

\section{O38.5}

BEING MINDFUL OR FLEXIBLE? INVESTIGATION OF ASSOCIATED FACTORS WITH PROBLEMATIC ONLINE GAMING AMONG ASIAN UNIVERSITY STUDENTS

Anise M.S. Wu ${ }^{1}$, Mark H.C. Lai ${ }^{2}$, Masao Yogo ${ }^{3}$, Shu $\mathrm{Yu}^{1}$

${ }^{1}$ University of Macau- China

${ }^{2}$ University of Cincinnati- United States of America

${ }^{3}$ Doshisha University- Japan

Introduction \& Purpose: People with problematic online gaming report symptoms similar to substance dependence, and the literature shows that this behavioral addiction is prevalent in Asian countries. Positive personality traits such as coping flexibility may protect ones from problematic gaming but the overall scientific evidence was limited and inconsistent. This study aims to test if mindfulness and coping flexibility world lower Asian university students' vulnerability to problematic online gaming. Methods: We recruited over 1,000 online gamers among university students who aged 18-25 years, in China and Japan. After giving their consent for voluntary participation, the students received an anonymous questionnaire, which was composed of validated inventories such as the 9-item scale of problematic gaming, 15-item mindfulness scale, 10-item coping scale, and 21-item scale of mental distress. The measurement invariance across gender and cultural groups was examined before hypothesis testing.
Results: As hypothesized, mindfulness was negatively, while mental distress (particularly depression) was positively, correlated with problematic gaming $(\mathrm{p}<.05)$. Mindfulness was also found to moderate the effect of mental distress on problematic gaming in two cultural groups $(\mathrm{p}<.05)$. However, coping flexibility showed no significant correlation with problematic gaming or moderating effect on the relationship between mental distress and problematic gaming.

Conclusions: Some positive personality traits may provide better protective effects against the development of problematic gaming. In both Chinese and Japanese university students, our findings suggest that mindfulness trait, but not coping flexibility, may decrease one's vulnerability to problematic gaming by both direct and buffering effect (against the adverse effect of mental distress). Mindfulness promotion programs hence can be incorporated into school-based preventive interventions for problematic gaming.

CORRESPONDING AUTHOR: Anise M.S. Wu, University of Macau, anisewu@umac.mo

\section{O38.6}

INVESTIGATING PROTECTIVE AND RISK FACTORS OF INTERNET GAMING DISORDER AMONG SPANISH UNIVERSITY STUDENTS

Shu Yu ${ }^{1}$, José Carlos Sánchez Prieto ${ }^{2}$, Anise M. S. Wu ${ }^{1}$

${ }^{1}$ University of Macau- China

${ }^{2}$ University of Salamanca- Spain

Introduction \& Purpose High prevalence of Internet gaming disorder (IGD) has been reported among Spanish youth in recent years. This study aimed to investigate the protective and risk factors of IGD among Spanish university students within the theoretical framework of a needcognitive-behavioral model. The association of psychological need satisfaction, maladaptive cognitions, and cognitive-behavioral skills and their effect on IGD were tested. Methods Data were collected in a public university in Salamanca, Spain. Participants $(N=397$, Mage $=21.2$ ) voluntarily completed an anonymous paper-and-pencil questionnaire, which consisted of self-report measures of IGD tendency, satisfaction for needs (e.g., relatedness), maladaptive cognitions (e.g., gaming perfectionism), task duration estimation skills, and demographic information. Results As hypothesized, results of correlation analysis showed that satisfaction of psychological need (relatedness) and cognitive-behavioral skill (task duration estimation skill) were protective factors against IGD tendency ( $\mathrm{rs}=-.46$ and -.32 respectively, $\mathrm{ps}<$ .001 ), whilst maladaptive cognitions (negative outcome expectancies of reducing game use and gaming perfectionism) were risk factors ( $\mathrm{rs}=$ .65 and .59 respectively, $\mathrm{ps}<.001$ ). Path analysis results showed that, with demographic variables controlled, relatedness satisfaction and the two maladaptive cognitions had direct effect on IGD tendency. The effect of both relatedness satisfaction and gaming perfectionism on IGD tendency were partially mediated by negative outcome expectancies $(\mathrm{ps}<.001)$. Task duration estimation skills buffered the effect of gaming perfectionism on IGD tendency $(\mathrm{p}<.05)$. The total variance in IGD tendency explained was about $57 \%$. Conclusions This study provided empirical findings of the protective and risk psychosocial factors of IGD tendency, which can contribute to the understanding of IGD development. Theoretically, it may give insights for future studies on the effect of psychological needs, maladaptive cognitions, and cognitive-behavioral skills, and their interactions; Practically, the findings can guide professionals and service providers of prevention programs for IGD. For instance, school-based intervention programs for IGD may consider modifying students' maladaptive cognitions and promoting cognitive-behavioral skills regarding task duration estimation.

CORRESPONDING AUTHOR: Shu Yu, University of Macau, mogu.yus@gmail.com 
O39.1

UNDERSTANDING WHY A COGNITIVE PATIENT EDUCATION INTERVENTION FOR LOW BACK PAIN FAILED TO REDUCE DISABILITY COMPARED TO USUAL CARE

Margreth Grotle $^{1}$, Kjersti Storheim ${ }^{2}$, Ida Löchting ${ }^{2}$, Erik Werner ${ }^{3}$, Gemma Mansell ${ }^{4}$

${ }^{1}$ OsloMet / Oslo Metropolitan University- Norway

${ }^{2}$ Formi, Oslo University Hospital- Norway

${ }^{3}$ Institute of Health and Society, University of Oslo- Norway

${ }^{4}$ Keele University, London- England

Introduction \& Purpose: Many interventions for the treatment of low back pain (LBP) exist, but the mechanisms through which such treatments work are not always clear. This is especially true for biopsychosocial interventions which incorporate several different components and methods of delivery. The purpose of this study was therefore to examine the indirect effects of the Cognitive Patient Education (COPE) intervention via illness perceptions, back pain myths and pain catastrophising to influence disability outcome. Methods: Secondary analysis of the COPE randomised controlled trial. Mediation analysis techniques were employed to examine the indirect effect of the COPE intervention via residualised change (baseline - post-treatment) in the three variables hypothesised to be targeted by the COPE intervention on post-treatment disability outcome. Pain intensity at baseline, pain duration and a treatment-mediator interaction term were controlled for in the analysis. Results: Preliminary analyses confirmed that change in pain catastrophising and illness perceptions (not back pain myths) was related to both allocation to the intervention arm and post-treatment disability score. The treatment exerted statistically significant indirect effects via changes in illness perceptions and pain catastrophising on post-treatment disability score (illness perceptions standardised indirect effect $0.08,95 \%$ CI 0.03 to 0.15 ; pain catastrophising standardised indirect effect 0.05 , $95 \%$ CI 0.01 to 0.12 ). However, the inclusion of an interaction term led to the indirect effects being significantly reduced, with the effects no longer being statistically significant. Conclusions: The finding that a small indirect effect of the COPE intervention via changes in illness perceptions and pain catastrophising on post-treatment disability could be estimated indicates that these variables may be viable treatment targets for biopsychosocial interventions, but this finding must be viewed in light of the adjusted analyses which showed that the indirect effect was significantly reduced through the inclusion of a treatment-mediator interaction term.

CORRESPONDING AUTHOR: Margreth Grotle, OsloMet - Oslo Metropolitan University, margreth.grotle@hioa.no

\section{O39.2}

PHYSIOTHERAPY INFORMED BY ACCEPTANCE AND COMMITMENT THERAPY (PACT): A RANDOMISED CONTROLLED TRIAL OF PACT VERSUS USUAL CARE PHYSIOTHERAPY FOR ADULTS WITH CHRONIC LOW BACK PAIN

Emma Godfrey ${ }^{1}$, Vari Wileman ${ }^{1}$, Melissa Galea Holmes ${ }^{1}$, Sam Norton ${ }^{1}$, Duncan Critchley ${ }^{1}$, Lance McCracken ${ }^{1}$

${ }^{1}$ King's College London- United Kingdom

Introduction Chronic low back pain (CLBP) is a common condition and the largest single cause of years lived with disability. It is associated with comorbidities, such as sleep disorders, anxiety and depression and is a frequent reason for absence from work. CLBP is thus debilitating for patients, challenging for health care systems and costly for society. Many patients receive physiotherapy that is moderately effective and might be improved by incorporating psychological models and techniques. Acceptance and Commitment Therapy (ACT) has a good evidence base in chronic pain but has not been adapted for or delivered by physiotherapists. This trial aimed to test the efficacy of physiotherapy informed by ACT (PACT) against usual care (UC) physiotherapy for CLBP. Methods Phase II, assessor blind, multi-centre randomised controlled trial (RCT). PACT consisted of two individual, 60-minute, face-toface sessions two weeks apart, plus one 20-minute telephone call one month later. UC included individual physiotherapy and/or back classes, exercises, manual therapy and hydrotherapy. PACT physiotherapists had training and supervision provided and separate groups of clinicians delivered PACT and UC. Inclusion criteria: non-specific CLBP $\geq 12$ weeks, $\geq 3$ points on Roland-Morris Disability Questionnaire (RMDQ). Primary outcome was functioning, assessed with RMDQ, at 3 months follow-up. Secondary outcomes included: pain, mood, quality of life, satisfaction, acceptance, committed action and self-efficacy. Estimates of treatment effect at follow-up used linear-mixed models following the intention-totreat principle. Results RCT recruited 248 participants $(59 \%$ female, age $=48$ ) with CLBP from physiotherapy clinics in NHS hospitals in the UK and 219 (88.3\%) completed measures at follow-up. PACT participants had significantly better functioning at 3 months compared to UC (1.07, $\mathrm{p}=0.037, \mathrm{~d}=0.2$ ). They also had a clinically important reduction of $\geq 3$ points on the RMDQ and were more satisfied with their treatment. At 12 months, there were no significant differences between groups for RMDQ scores (mean difference $-0.38, \mathrm{p}=0.52$ ) or for other outcomes. PACT was feasible and delivered with high $(85 \%)$ treatment fidelity. Conclusions PACT improved functioning in the short but not long term compared to UC physiotherapy. PACT was a feasible and credible treatment for patients with CLBP. The model of delivery enabled physiotherapists to incorporate psychological techniques successfully. PACT requires further testing in a larger effectiveness trial but may inform the delivery of physiotherapy services for CLBP, with potential benefits for both patients and the NHS.

CORRESPONDING AUTHOR: Emma Godfrey, King's College London, emma.1.godfrey@kcl.ac.uk

\section{O39.3}

EFFECTS OF MINDFULNESS YOGA VERSUS CONVENTIONAL STRETCHING AND RESISTANCE TRAINING EXERCISES ON PSYCHOLOGICAL DISTRESS IN PEOPLE WITH PARKINSON'S DISEASE: A RANDOMIZED CONTROLLED TRIAL

JoJo Yan Yan Kwok ${ }^{1}$, Jackie Cheuk Yin Kwan ${ }^{2}$, Man Auyeung ${ }^{3}$, Vincent Chung Tong Mok ${ }^{4}$, Ka Yee Lau ${ }^{4}$, Helen Yue Lai Chan ${ }^{5}$

${ }^{1}$ The Nethersole School of Nursing, Faculty of Medicine, The Chinese University of Hong Kong

${ }^{2}$ The Hong Kong Society for Rehabilitation

${ }^{3}$ Department of Medicine, Pamela Youde Nethersole Eastern Hospital

${ }^{4}$ Department of Medicine and Therapeutics, Therese Pei Fong Chow Research Center for Prevention of Dementia, Gerald Choa Neuroscience Centre, Faculty of Medicine, The Chinese University of Hong Kong

${ }^{5}$ Prince of Wales Hospital, The Chinese University of Hong Kong

Abstract: Introduction:Psychological distress, which is prevalent among people with Parkinson's disease (PD),aggravates their motor symptoms, thereby leads to increased disability, high healthcare burden, and poor health-related quality of life (HRQOL). This study aimed to compare the effects of mindfulness yoga versus stretching and resistance training exercises(SRTE) on psychological, physical, and spiritual wellbeing, and HRQOL among people with mild-to-moderate PD. Methods:A community-based, single-blind, randomized trial was conducted. A total of 138 people with idiopathic $\mathrm{PD}(\mathrm{H} \& \mathrm{Y}$ stage I-III) were recruited and randomized into yoga $(\mathrm{n}=71)$ or $\operatorname{SRTE}(\mathrm{n}=67)$ groups. For 8 weeks, the interventional group received a weekly 90 -min session of mindfulness yoga, while control group received a weekly 60 -min session of SRTE. Outcome measures included psychological distress in terms of anxiety and depression, severity of motor symptoms, mobility,spiritual wellbeing 
in terms of affliction and equanimity, and HRQOL. Assessments were done at baseline, immediate(T1) and three-month(T2) after the completion of intervention. Results:The mean age of participants was 63.7 years, nearly half were male and the majority suffered from moderate PD. The participation rate was $86.8 \%$ with an adherence of $78.3 \%$.Both groups appeared to reduce motor symptoms. The generalized estimating equations analyses revealed yoga group generally had significant improvement in various outcomes than control group; particularly, on anxiety(time by group interaction, T $1: \beta=-1.79, \mathrm{p}=0.001 ; \mathrm{T} 2: \beta=-$ $1.97, \mathrm{p}<0.001)$, depression $(\mathrm{T} 1: \beta=-3.05, \mathrm{p}<0.001 ; \mathrm{T} 2: \beta=-$ $2.64, \mathrm{p}<0.001)$, perceived affliction $(\mathrm{T} 1: \beta=-0.87, \mathrm{p}<0.001 ; \mathrm{T} 2: \beta=-$ $0.75, \mathrm{p}<0.001)$, perceived equanimity $(\mathrm{T} 1: \beta=1.14, \mathrm{p}<0.001 ; \mathrm{T} 2: \beta=$ $1.11, \mathrm{p}<0.001)$ and HRQOL $(\mathrm{T} 1: \beta=-2.48, \mathrm{p}<0.001 ; \mathrm{T} 2: \beta=-$ $2.55, \mathrm{p}<0.001)$.Conclusions:The mindfulness yoga programme was well received in patients with mild-to-moderate PD. Yoga demonstrated addtional benefits of improved psychological wellbeing, sipritual wellbeing and HRQOL, when compared to conventional SRTE. Considering that PD is chronic in nature, rehabilitation programmes should not just to improve physical, but also their psychological wellbeing.

CORRESPONDING AUTHOR: JoJo Yan Yan Kwok, The Nethersole School of Nursing, Faculty of Medicine, The Chinese University of Hong Kong, jojokwok@link.cuhk.edu.hk

\section{O39.4}

TARGETING DYSFUNCTIONAL BEHAVIOURAL COGNITIONS IN A TWO-WEEK PAIN MANAGEMENT PROGRAM IMPROVES FUNCTIONAL OUTCOMES IN LONGSTANDING CHRONIC LOW BACK PAIN

Johanna Vriezekolk ${ }^{1}$, Miranda van Hooff ${ }^{2}$, John O'Dowd ${ }^{4}$. Maarten Spruit $^{3}$

${ }^{1}$ Sint Maartenskliniek- Holland

${ }^{2}$ Research Department, Sint Maartenskliniek, Nijmegen, The Netherlands

${ }^{3}$ Orthopaedic Department, Sint Maartenskliniek, Nijmegen, The Netherlands

${ }^{4}$ Orthopaedic Department, Hampshire Hospitals NHS Foundation Trust, Basingstoke, United Kingdom; The RealHealth Institute, London, United Kingdom

Introduction \& purpose: The Fear Avoidance Model is used to explain why some patients with acute low back pain develop chronic low back pain (CLBP). Cognitive behavioral therapy (CBT) targeting dysfunctional behavioral cognitions (i.e. pain catastrophizing and fear of movement) are recommended for these patients. The purpose of this study is to investigate whether a two-week CBT-based pain management program results in improvement in dysfunctional behavioral cognitions and, whether these improved cognitions improve functional outcomes (i.e. functional disability and pain severity). Methods: A cohort of 524 consecutive CLBP-patients was followed. Patients completed self-reported questionnaires pre- and post-treatment, and at one and 12-months followup. Main outcome was functional disability (ODI), secondary outcomes were pain severity (NRS), pain catastrophizing (PCS) and fear of movement (TSK). Improvement over time was analysed with repeated measures ANOVA. The influence of pain catastrophizing and fear of movement on functional disability and pain severity was examined using path analyses. Multiple imputation was used to complete missing data. Results: Participants with incomplete data $(12.8 \%)$ were not significantly different from those with complete data $(n=457)$. The mean age was 46 $( \pm 9.5)$ years, $59 \%$ were female, and mean CLBP duration was $12( \pm 10.8)$ years. All outcomes significantly improved at post-treatment; a slight significant improvement was observed between post-treatment and 12 months follow-up. Path analyses showed, after correction for pretreatment scores, a significant direct effect for pain catastrophizing on post-treatment functional disability. An indirect effect was observed for pain catastrophizing through fear of movement on post-treatment functional disability. Comparable results were found with pain severity as outcome measure. Conclusion: A two-week CBT-based pain management program improved the dysfunctional behavioral cognitions and functional outcomes in patients with longstanding CLBP. Targeting both pain catastrophizing and fear of movement during the program resulted in improved functional outcomes. These results were maintained for one year.

CORRESPONDING AUTHOR: Dr. Johanna Vriezekolk, Sint Maartenskliniek,j.vriezekolk@maartenskliniek.nl

\section{O39.5}

PSYCHO-PHYSIOLOGICAL DATA INFORMS HEALTH PSYCHOLOGY TREATMENTS: AN APPROACH FOR COMORBID PAIN AND OPIOID ADDICTION

Amy Wachholtz ${ }^{1}$, Gerado Gonzalez ${ }^{2}$, Douglas Ziedonis ${ }^{3}$

${ }^{1}$ University of Colorado Denver

${ }^{2}$ University of Massachusetts Medical School

${ }^{3}$ University of California San Diego

INTRODUCTION: Patients with opioid use disorder (OUD) and pain are 5 times more likely to relapse compared to patients with OUD alone. This study series used psychophysiological data to develop a psychotherapy treatment for comorbid pain and opioid addiction(COAP). Study 1 examined psychophysiological differences in pain sensitivity and tolerance among OUD patients on Medication Assisted Treatment. Study 2 developed a 12-week psychotherapy treatment from the psychophysiological data and piloted Self-regulation Therapy for Opioid addiction and Pain(STOP). METHOD: STudy \#1-chronic pain patients were recruited in 4 groups $(\mathrm{N}=120 ; n=30)$ : 1 -current methadone for COAP; 2 -current buprenorphine for COAP; 3-history of opioid treatment for COAP but with prolonged opioid abstinence (M=121 weeks; $\mathrm{SD}=23.3)$; and 4-opioid-naïe. They completed psychopsychological assessments during a pain task, recording time to first pain (sensitivity) and time to disengagement (tolerance). In the second study, 14 participants completed the intervention, pre-post-3 month followup psychophysiological testing, with weekly craving, and pain levels. RESULTS: Kaplan-Meier analyses showed group differences for pain sensitivity and tolerance ( $\mathrm{Log}$ rank=20.11;p<.001). Opioid treatment groups showed sensitivity differences compared to opioid-naïve(p's $<.01)$. Tolerance improved across methadone-use to prolonged abstinence to opioid-naïve participants ( $p$ 's<.001). Prolonged abstinence group had increased tolerance as abstinence length increased $(\mathrm{R}=.37 ; \mathrm{p}<.05)$; but abstinence did not alter sensitivity. Using this data, the resulting STOP psychotherapy intervention While not a fully powered study, from pre-intervention to 3 month followup did show significant functional improvement $(\mathrm{F}(1$, $12)=45.82 ; \mathrm{p}<.001)$ and decreased pain severity levels $(\mathrm{F}(1,12)=37.62$; $\mathrm{p}<.01$ ) along with improved pain tolerance, increased daily activity, decreased cravings, and no substance abuse after week 8. DISCUSSION: Elevated pain sensitivity does not resolve with opioid treatment discontinuation. However, pain tolerance increases after opioid cessation which has implications for treating COAP. The STOP pilot may improve COAP treatment. Discussion will include how this methodology can be used improve other psychological treatments.

CORRESPONDING AUTHOR: Amy Wachhol, University of Colorado Denver, amy.wachholtz@ucdenver.edu

\section{O39.6}

ATTENTIONAL CONTROL INFLUENCES IMMERSION IN VIRTUAL REALITY

Susanne Blokzij1 ${ }^{1}$, Lamberts, J.M. Spikman ${ }^{2}$

${ }^{1}$ Martini Hospital- Netherlands

${ }^{2}$ University Medical Centre Groningen 
Background: The use of Virtual Reality (VR) in addition to or as alternative for pharmacological pain relief in medical procedures is rising. However, not every patient benefits from VR as pain relief. We try to get insight in the application and working mechanism of VR in a medical setting. Our main hypothesis is that the capacity for attentional control influences the extent to which someone is immersed in the virtual environment, leading to more or less pain reduction. In the present study, we experimentally investigated this in healthy subjects being exposed to cold pressor pain while distracted by VR with higher or lesser degree of immersion (game vs. video respectively). Method: 52 healthy subjects participated: 23 men $(44.2 \%)$, mean age 41.2 year $(S D=17.4)$ and mean educational level on a 7 points scale $6.1(\mathrm{SD}=0.7)$. Subjects were randomly allocated to one of the 2 distraction conditions: 1) VR Game or 2) VR Video. They underwent a Cold Pressor Task (CPT) with the distraction technique. The time subjects kept their hand in the cold water $(\mathrm{M}=$ 2.8 degrees; $\mathrm{SD}=0.9$ degrees) was measured (tolerance time: TT). This was maximally 180 seconds. Prior to the CPT, participants performed four attentional control tasks intended to measure inhibition (Colour Word Test: CWT), mental flexibility (Trailmaking Test: TMT), working memory (Digit Span: DS) and attentional switching (Visual Elevator: $\mathrm{VE}$ ). Afterwards, the pain experience (PE) and extent of immersion (EI) were rated on a 10-point scale. Results: a higher EI was related to a longer TT $(\rho=0.45 ; p=0.001)$, but not to a lower PE. The game-group had a higher EI than the Video-group $(\chi 2(1)=8.520,6.00 ; p=0.014)$. Furthermore, a higher EI was related to a better score on inhibition/ switching ( $\rho=0.42 ; p=0.002)$ and a lower accuracy score on attentional switching ( $\rho=-0.28 ; p=0.044)$. Conclusion: Immersion makes the procedure more tolerable, but not less painful. The ability to be immersed is related to interactive VR-content and to a better ability to suppress distracting information but a lower ability to switch attention flexibly. In order to maximize the VR effect in medical procedures immersion should be maximised especially in those patients with less attentional control.

CORRESPONDING AUTHOR: Susanne Blokzijl, Martini Hospital, s.blokzijl@mzh.nl

\section{O40.1}

DOES WEIGHT STATUS MEDIATE THE INFLUENCE OF CARDIORESPIRATORY FITNESS ON CLUSTERED CARDIOVASCULAR DISEASE RISK FACTORS IN YOUTH? A MEDIATION ANALYSIS STUDY

José Castro Piñero ${ }^{1}$, Alejandro Pérez-Bey ${ }^{1}$, Jorge del Rosario FernándezSantos ${ }^{1}$, Irene Esteban-Cornejo ${ }^{2}$, Sonia Gómez-Martínez ${ }^{3}$, Oscar L. Veiga $^{4}$, Ascensión Marcos ${ }^{3}$, Francisco B. Ortega ${ }^{2}$, Fernando RodríguezRodríguez ${ }^{5}$

${ }^{1}$ University of Cádiz

${ }^{2}$ University of Granada

${ }^{3}$ Spanish National Research Council

${ }^{4}$ Autonomous University of Madrid

${ }^{5}$ Pontificia Universidad Católica de Valparaíso

Introduction and Purpose: The precursors of cardiovascular disease (CVD) are already present from an early age and the detection of risk factors in this stage of life might help to prevent the development of CVD in adulthood. High levels of cardiorespiratory fitness (CRF) in youth are associated with a cardiovascular health profile and it is a strong predictor of CVD risk factors later in life. However, whether CRF levels exert a direct effect on CVD risk factors, or if this association is mediated by weight status in youth, has not been fully defined. We examined the individual and combined associations of CRF and body mass index (BMI) with clustered CVD risk factors, and the mediator role of BMI in the association between CRF and clustered CVD risk factors in youth. Methods: 497 youth (231 girls) aged 6-17.9 years were included in this cross-sectional study. Height and weight were assessed and BMI was calculated. A CVD risk factor index (CVDRF-I) was computed using the standardized values of: waist circumference, systolic blood pressure, triglycerides, high-density lipoprotein cholesterol and glucose. CRF was assessed using the 20-m shuttle run test. Mediation hypothesis was tested through Baron and Kenny procedures.

Results: CRF was negatively associated with CVDRF-I (all $\mathrm{p}<0.05$ ); however after adjusting for BMI the associations were no longer significant either in children or adolescents of both sex groups. In contrast, the association between BMI and CVDRF-I was independent of CRF (all p $<0.001)$. The effect of CRF on CVDRF-I was mediated by BMI. The percentage of the total effect of CRF on CVDRF-I mediated by BMI for children boys and girls and adolescents boys and girls were $79.5 \%, 100 \%$, $81.2 \%$ and $55.7 \%$, respectively. Similar results were found when we performed analyses removing waist circumference from CVDRF-I. We also repeated all analyzes using the percentage of body fat instead of BMI as a measure of adiposity and the results persisted.

Conclusions: BMI is an independent predictor of CVDRF-I and a mediator of the association between CRF and CVDRF-I in youth. Exercise programs aiming at preventing future CVD should focus on maintaining an optimal weight status, what might be attained by increasing CRF levels.

CORRESPONDING AUTHOR: José Castro Piñero, University of Cádiz, jose.castro@uca.es

\section{O40.2}

DOES ENGAGING IN A GENDER-SENSITISED COMMUNITYBASED PHYSICAL ACTIVITY REDUCE THE RISKS ASSOCIATED WITH CARDIOVASCULAR DISEASE?

Liam Kelly ${ }^{1}$, Liam Kelly ${ }^{2}$, Noel Richardson ${ }^{2}$, Paula Carroll ${ }^{3}$, Michael Harrison $^{3}$, Alex Donohoe ${ }^{3}$, Aisling Keohane ${ }^{3}$, Steve Robertson ${ }^{4}$

${ }^{1}$ National Centre for Men's Health / Institute of Technology Carlow

${ }^{2}$ National Centre for Men's Health / Institute of Technology CarlowIreland

${ }^{3}$ Centre for Health Behaviour Research, Waterford Institute of Technology, Ireland.

${ }^{4}$ Leeds Beckett University, Leeds, UK.

The main outcome results were not available at the time of abstract submission so while this is written as an outcomes paper (and outcomes will be presented) it is difficult to accurately critique, particularly with regard to the significance which I have marked down due to unknown results Comment2: The intervention itself is interesting, however, I think just presenting the baseline descriptive data does not justify an oral presentation.

Abstract: Background: Cardiovascular diseases (CVDs) are the leading cause of death globally, and are particularly prevalent in middle-aged men in Ireland. Most CVDs can be prevented by addressing behavioural risk factors. However, creating suitable physical activity (PA) interventions in the right environments that can attract and support men in changing health practices has proved difficult. Evidence suggests that gender-specific strategies related to community-engagement are necessary in creating sustainable health-promotion programmes that appeal to men. Purpose: This study reports on the impact of a community-based PA intervention (Men on the Move) on the CVD profile of participants. Methods: Inactive males $(n=927)$ were recruited across 8 counties ( 4 intervention $[n=501] ; 4$ comparison-in-waiting [ $\mathrm{n}=426]$ ). Self-administered questionnaires combined with recorded outcome measures were used to gather data on participants' at baseline, 12, 26 and 52 weeks. Data were computed in accordance with defined protocols, with descriptive and comparative means analysed. Results: Based on international guidelines, six self-reported CVD risk factors were identified; $102 \mathrm{~cm}$, a smoker, alcohol consumption $>14$ units, blood-pressure medication, and cholesterol medication. Comparative data examining incidence/prevalence of CVD risk factors at each time-point will be presented (data analysis in progress). At 
baseline, the majority $(85.5 \%)$ of participants' presented with at least one risk factor, with $53.1 \%$ presenting with two or more. Conclusions: Baseline findings indicate that the programme succeeded in reaching a 'high-risk' population group, and that there is an urgent need for more targeted gender-specific programmes that support service providers to effectively engage inactive men in PA.

CORRESPONDING AUTHOR: Liam Kelly, National Centre for Men's Health / Institute of Technology Carlow, Liam.Kelly@itcarlow.ie

\section{O40.3}

EXERCISE ATTENUATES METHAMPHETAMINE-INDUCED BBB DISRUPTION, ABERRANT NEUROGENESIS, AND BEHAVIORAL ALTERATIONS

Michal Toborek ${ }^{1}$

${ }^{1}$ University of Miami- United States of America

Introduction \& Purpose. There is no effective therapeutic intervention developed targeting cerebrovascular toxicity of drugs of abuse, including methamphetamine (METH). We hypothesize that exercise can protect against METH-induced neurotoxicity by enhancing the blood-brain barrier (BBB) integrity and protection against aberrant hippocampal neurogenesis. Methods. Mice were administered with METH for 5 days with an escalating dose regimen, followed by voluntary wheel running for 2 weeks resembling the voluntary pattern of human exercise. The frequency, duration, and intensity of each running session were monitored for each mouse via a direct data link to a computer and the running data are analyzed by Clocklab ${ }^{\mathrm{TM}}$ Analysis software. Controls included sedentary mice that did not have access to running wheels and/or injections with saline. Results. METH administration disrupted hippocampal neurogenesis by inducing aberrant differentiation of neural progenitor cells to both immature and mature neurons. In addition, METH increased hippocampal BBB permeability by disrupting expression and localization of tight junction proteins. These changes were preserved in sedentary mice; however, exercise significantly attenuated METH-induced aberrant neurogenesis and disruption of the BBB integrity. Importantly, abnormal neurogenesis observed in METH-treated mice was associated with alternations of short term memory as assessed by novel object recognition and spatial memory as evaluated by Morris water maze. These behavioral changes were also attenuated by exercise. Conclusions. Results indicate that physical exercise can protect against METH-induced alterations in neurogenesis presumably by stabilization of the BBB. This work was supported by the NIH, grants DA039576, DA044579, HL126559, MH098891, MH072567, and by the NSC grant 2015/17/B/NZ7/02985.

CORRESPONDING AUTHOR: Michal Toborek, University of Miami, mtoborek@med.miami.edu

\section{O40.4}

EFFECTS OF ACUTE EXERCISE AND DIETARY QUALITY ON ACUTE PSYCHOSOCIAL STRESS RESPONSES

Jana Strahler ${ }^{1}$, Ramona Wurst ${ }^{2}$, Reinhard Fuchs ${ }^{2}$, Kathrin Wunsch ${ }^{3}$

${ }^{1}$ Justus Liebig University Giessen- Germany

${ }^{2}$ Sportpsychology Unit, Department of Sport Science, University of Freiburg, Germany

${ }^{3}$ Institute of Sport Science, Karlsruhe Institute of Technology, Germany

Background: Acute bouts of exercise are known to reduce distress, anxiety and physiological stress reactivity. Likewise, healthy diet shows mental and physical health benefits. To date, the actual effect of performing a healthy diet on acute psychobiological stress responses and whether effects multiply with a single bout of exercise before stressor onset remains unknown. Thus, the present study aimed to investigate effects of habitual diet and acute exercise on biological parameters during acute psychosocial stress. Methods: 84 male subjects were randomized to (1) an acute exercise group (i.e. $30 \mathrm{~min}$ indoor-cycling at $70 \%$ of individual maximum load, $\mathrm{n}=42$ ) or (2) a placebo exercise group (30 min light stretching, $n=42$ ). Following the exercise intervention, all men were exposed to the Trier Social Stress Test for Groups (TSST-G). Salivary alpha amylase (sAA) and cortisol (sCort) were repeatedly assessed to examine stress-induced activity of the autonomic nervous system (ANS) and the hypothalamus pituitary adrenal (HPA) axis, respectively. Subjects completed the Food Frequency Questionnaire and a median split was applied according to subjects' dietary quality. Results: A 2 (acute exercise vs. placebo) x 2 (unhealthy vs. healthy diet) x 5 (measurement time points) ANOVA revealed pronounced TSST-G-induced changes in both stress markers (all $\mathrm{p}<0.001)$ with diminished sCort changes in the acute exercise group ( $\mathrm{p}<0.001)$. Moreover, subjects in the unhealthy diet group showed exaggerated sCort responses to the TSST-G ( $\mathrm{p}=0.010$ ). Despite lowest sCort responses in the acute exercise - healthy diet group, the interaction effect did not reach significance $(\mathrm{p}=0.284)$. There was neither an effect of intervention $(\mathrm{p}=0.246)$, diet $(\mathrm{p}=0.249)$ nor an intervention by diet effect $(\mathrm{p}=0.160)$ on sAA. Of note, intervention and diet groups did not differ regarding physical activity and fitness. Conclusions: Exercising just before psychosocial stress exposure and performing a rather healthy diet reduces acute endocrine but not autonomic stress reactivity. However, effects did not multiply. While present results comply with the assumption of cross-stressor adaptation, findings do not provide evidence for a specific mechanism for producing the autonomic effects related to dietary quality.

CORRESPONDING AUTHOR: Jana Strahler, Justus Liebig University Giessen, jana.strahler@psychol.uni-giessen.de

\section{O40.5}

ACTIVE TRAVEL, EMPLOYMENT STATUS AND PHYSICAL ACTIVITY LEVELS: A CROSS-SECTIONAL ANALYSES IN A POPULATION BASED SAMPLE OF US ADULTS

Lee Smith ${ }^{1}$, Liang $\mathrm{Hu}^{2}$, Graham Colditz ${ }^{3}$, Lin Yang ${ }^{4}$

${ }^{1}$ Anglia Ruskin University- United Kingdom

${ }^{2}$ Zhejiang University- China

${ }^{3}$ Washington University School of Medicine- United States

${ }^{4}$ Medical University of Vienna

Abstract: Introduction and Purpose: Previous studies investigating the association between active travel and physical activity have produced mixed results, probably due to lack of considering employment status and lack of sufficient sample in both gender groups. In addition, most studies have used self-reported measures for physical activity assessment. The aim of the present study was to investigate associations between active transport, employment status and objectively measured moderateto-vigorous physical activity (MVPA) in a representative sample of US adults. Methods: Cross-sectional analyses of data from the National Health and Nutrition Examination Survey. A total of 5180 adults $(50.2$ years old, $49.0 \%$ men) were classified by levels of active transportation and employment status. Outcome measure was weekly time spent in MVPA as recorded by the Actigraph accelerometer. Associations between active transport, employment status and objectively measured MVPA were examined using multivariable linear regression models adjusted for age, BMI, race and ethnicity, education level, marital status, smoking status, working hour duration (among the employed only), and self-reported leisure time physical activity. Results: Patterns of active transport were similar between the employed $(n=2,897)$ and unemployed $(n=2,283)$, such that $76.0 \%$ employed and $77.5 \%$ unemployed engaged in no active transport. For employed adults, those engaging in high levels of active transport ( $\geq 90 \mathrm{~min} /$ week) had higher amount of MVPA than those who did not engage in active transport. This translated to 40.8 (95\% CI: 15.7, 65.9) additional minutes MVPA per week in men and 57.9 (95\% CI: $32.1,83.7)$ additional minutes MVPA per week in women. Among the 
unemployed adults, higher levels of active transport were associated with more MVPA among men (44.8 min/week MVPA, 95\% CI: 9.2, 80.5), only. Conclusions: Findings from the present study support interventions to promote active transport to increase population level physical activity. Additional strategies are likely required to promote physical activity among unemployed women.

CORRESPONDING AUTHOR: Lee Smith, Anglia Ruskin University, lee.smith@anglia.ac.uk

\section{O40.6}

DID YOU EXERCISE TODAY FOR 30 MINUTES? SELF-REPORT VS ACTIGRAPHY

Joseph Schwartz ${ }^{1}$, Keith Diaz ${ }^{2}$, Matthew Burg ${ }^{3}$, Karina Davidson ${ }^{2}$

${ }^{1}$ Academy of Behavioral Medicine Research- United States

${ }^{2}$ Columbia University Medical Center- United States

${ }^{3}$ Yale University- United States

Introduction/Purpose. We sought to quantify responses to the question, "Did you exercise today for 30 minutes or more at a moderate or vigorous level?" by conducting both nomothetic and ideographic comparisons of responses to concurrently collected Fitbit data. Methods. 79 individuals enrolled in a study designed to assess the bi-directional relationship between stress and physical exercise. After providing informed consent, each participant was given a Fitbit Flex device to wear and asked to complete daily ecological momentary assessments (EMA) every day for a year. The end-of-day EMA included the above question. Days in which participants wore the Fitbit for 10+ hours and completed the end-of-day assessment were analyzed $(\mathrm{N}=12783$ person-days, median $=164$, quartiles: 89, 246). Previously, we defined a 30 -min bout of moderate/ vigorous physical activity (MVPA) as the occurrence of any 30 consecutive minutes in which 24+ minutes were classified as MVPA by the Fitbit software. Here, for every consecutive 30 -min period, we computed the harmonic mean (Hmean) of the 24 highest step counts, and then treated the maximum value for the day as a measure of maximum 30min physical activity for that day. We then compared these measures to participants' self-reports of having exercised for the sample as a whole, and separately for each individual, identifying the cutpoint that maximized agreement with the self-report. Results. Overall, participants reported having exercised on $41.9 \%$ of days. The cutpoint that maximized agreement with self-reported exercise was Hmean $=73$ steps with an agreement rate of $69.4 \%$. When we identified a separate cutpoint for each participant, the median optimal cutpoint was 73 (quartiles: 35,102 ); the overall agreement was $80.9 \%$ with individual agreement rates having a median of $81.0 \%$ (quartiles: $74.1 \%, 88.5 \%$ ). Conclusions. On average, reporting that one exercised today was most consistent with the Hmean for the best 24 minutes of a consecutive 30 -min period being $>=73$. However, there are large individual differences, with self-reported exercise being associated with a Hmean 106 steps/min for another $25 \%$. Clearly, self-reports of exercise correspond to markedly different levels of activity for different people.

CORRESPONDING AUTHOR: Joseph Schwartz, Academy of Behavioral Medicine Research, Joeph.Schwartz@StonyBrookMedicine.edu

\section{O41.1}

COGNITIVE PREDICTORS OF TREATMENT OUTCOMES IN CHRONIC FATIGUE SYNDROME: ATTENTIONAL BIAS, ATTENTIONAL MALLEABILITY AND INTERPRETATION BIAS Alicia Hughes ${ }^{1}$, Trudie Chalder ${ }^{1}$, Rona Moss-Morris ${ }^{1}$, Colette Hirsch ${ }^{1}$ ${ }^{1}$ King's College London- United Kingdom

Introduction \& Purpose: This study investigates whether cognitive processes of attentional bias, attentional malleability and interpretation bias, predicts response to treatments for Chronic Fatigue Syndrome (CFS). Method: Patients with CFS who received either Cognitive Behavioural Therapy (CBT) or Graded Exercise Therapy (GET) for CFS completed measures of fatigue and physical functioning pre- and post-treatment. Patients also completed experimental tasks to assess attentional bias towards CFS information, (visual-probe task), malleability of attentional bias (assessed via extent of change on attentional bias over a brief attentional bias training) and interpretation bias of ambiguous material (recognition task), pre-treatment. Cognitive processing variables were entered as predictors in regression analyses, with post-treatment fatigue or functioning scores as the outcome variables, and pre-treatment scores as covariates. Results: Fatigue and functioning significantly improved after both CBT and GET. Pre-treatment attentional bias and an increased attentional malleability predicted better functioning, but not fatigue, posttreatment. Interpretation bias did not predict either fatigue or functioning outcomes. Conclusions: These findings suggest both attentional biases towards CFS material, and attentional malleability, are important factors in predicting treatment outcomes in CFS. This knowledge can help us understand the cognitive characteristics of those most likely to benefit from current treatment protocols for CFS and guide further research to tailor treatments.

CORRESPONDING AUTHOR: Alicia Hughes, King's College London, alicia.hughes@kcl.ac.uk

\section{O41.2}

COGNITIVE AND BEHAVIOURAL DIFFERENCES IN IRRITABLE BOWEL SYNDROME SUBGROUPS

Sula Windgassen ${ }^{1}$, Rona Moss-Morris ${ }^{1}$, Trudie Chalder $^{1}$, Kimberley Goldsmith $^{1}$, Alice Sibelli ${ }^{1}$, Hazel Everitt ${ }^{2}$

${ }^{1}$ King's College London- United Kingdom

${ }^{2}$ University of Southampton- United Kingdom

Introduction and purpose Irritable bowel syndrome (IBS) is a functional gastrointestinal syndrome consisting of four bowel pattern subtypes: diarrhoea predominant, (IBS-D) constipation predominant (IBS-C), alternating (IBS-A) or unclassified (IBS-U). This study aimed to identify whether there were significant associations between bowel pattern subtypes in irritable bowel syndrome and psychological factors or outcome variables. Methods Oneway ANOVAs were conducted to assess differences between IBS-related cognitions, behaviours, general anxiety, depression, symptom severity and work and social adjustment. Analysis was conducted on baseline data of 557 individuals with refractory IBS recruited into the assessing cognitive therapy in irritable bowel (ACTIB) randomised controlled trial. Bowel pattern subtype was classified according to the ROME III criteria. Due to the small number of individuals with IBS-U $(n=16)$, this group was excluded from analysis. Results The most predominant bowel pattern subtype was IBS-A $(n=287)$. IBS-D $(n=178)$ was more predominant than IBS-C $(n=76)$. Results indicated that individuals with IBS-D had significantly higher levels of unhelpful cognitions than those with IBS-C. Those with IBS-D and IBS-A engaged in more unhelpful IBS-related avoidance behaviours than those with IBS-C. In contrast both IBS-C and IBS-A engaged in more unhelpful safety behaviours than those with IBS-D. Conclusion The results may be important for informing assessment and formulation in psychological therapies for IBS.

CORRESPONDING AUTHOR: Sula Windgassen, King's College London, sula.1.windgassen@kcl.ac.uk

\section{O41.3}

THE MODERATING IMPACT OF PAIN AETIOLOGY ON THERAPEUTIC CHANGE IN COGNITIVE-BEHAVIOURAL THERAPY FOR CHRONIC PAIN

David McNaughton ${ }^{1}$

${ }^{1}$ Macquarie University- Australia 
Background: The role of aetiology in chronic pain is not well understood. One approach to classify chronic pain disorders is to consider whether the condition has an identifiable pathophysiology or not. Such a distinction has led to the use of terms organic and functional conditions, respectively. In addition, the contemporary treatment of chronic pain has highlighted the importance of psychological therapies, such as Cognitive Behavioural Therapy (CBT). However, variability of its therapeutic effect exists. The aim of the present study is to determine whether pain-related treatment outcomes are moderated (altered) by a functional or organic pain aetiology, of those undergoing CBT for chronic pain. Methods: The present study is a secondary analysis of data collected from an internetdelivered pain management program, known as The Pain Course. Participants pain aetiology was identified as functional or organic, via interview and self-reported data provided. A formal test of moderation was undertaken via analysis of covariance in which all dependent variables (pain disability, pain intensity, pain self-efficacy, pain acceptance, fear avoidance, depression and anxiety). The interaction between treatment group (active CBT or Control) and pain aetiology (organic or functional), were used as the independent variables. Results: $\mathrm{N}=471$ participants engaged in a 5 week trail of CBT for chronic pain. Between group differences of both the active CBT and waitlist control, for functional or organic status, age, gender, mean pain duration and average number of painful sites were all non-significant. All dependent variables were not moderated by a functional or organic status (pain disability: $\mathrm{F}(1,417)=$ $0.012, p=0.91$, pain intensity: $F(1,417)=3.68, p=0.06$, pain selfefficacy: $F(1,417)=0.10, p=0.75$, pain acceptance: $F(1,417)=3.14$, $\mathrm{p}=0.08$, fear avoidance: $\mathrm{F}(1,416)=0.02, \mathrm{p}=.89$, depression: $\mathrm{F}(1,417)=$ $3.14, p=0.08$ and anxiety: $F(1,417)=1.75, p=0.19)$. Discussion/ Conclusion: The present study identifies that the therapeutic change experienced by participants undergoing CBT for chronic pain is not altered by the presenting aetiology being organically driven. This reinforces a biopsychosocial formulation of those experiencing persistent pain, independent of the driving stimuli. Future research should identify further predictors of treatment responses which optimize outcomes and reduce the overall financial costs associated with chronic pain.

CORRESPONDING AUTHOR: David McNaughton, Macquarie University, david.mcnaughton@hdr.mq.edu.au

\section{O41.4}

INTEROCEPTION OF RESPIRATORY SENSATIONS IN PATIENTS WITH SOMATIC SYMPTOM DISORDER

Omer Van den Bergh ${ }^{1}$, Nadia Zacharioudakis ${ }^{1}$, Elke Vlemincx ${ }^{2}$

${ }^{1}$ KU Leuven - University of Leuven- Belgium

${ }^{2}$ Queen Mary University London- United Kingdom

Introduction and purpose: Patients with somatic symptom disorder (SSD) have been shown to report symptoms that less related to experimentally induced physiological dysfunction, and more influenced by contextual cues. This suggests that they are more influenced by categorical priors and less sensory-perceptual evidence in determining their somatic state (see Van den Bergh et al., Neurobiological and Biobehavioral Reviews, 2017). In the present study, we investigated interoception of respiratory resistances and the effect of imposing categorical priors. Methods: Patients SSD $(\mathrm{N}=60)$ with dyspnea complaints unrelated to physiological dysfunction and healthy controls $(\mathrm{N}=30$, matched for age, gender and educational level) were presented in random order 8 inspiratory resistances increasing in intensity with a constant factor $(\Delta=0.32$; POWERbreathe, International Ltd., Southham, UK). In phase 1, all resistances were presented 6 times. They were either allocated to 2 categories: A (A1.. A4, 4 low intensity resistances) and B (B1.. B4, 4 high intensity resistances) or presented in a dimensional way (S1..S8). After each presentation, ratings on intensity and unpleasantness were collected. In phase 2 , resistances were presented 8 times each without additional information, and participants had to identify resistances with a category or dimensional label and rate certainty about this decision. Results: Partial results $(\mathrm{N}=36$ patients; 17 in categorical, 19 in dimensional condition) show a significant condition $\mathrm{x}$ resistance effect for intensity (partial eta: .132) and unpleasantness (partial eta: .198): In the categorical condition, low intensity resistances are perceived as more intense and unpleasant, and highintensity resistances as less intense and unpleasant compared to the dimensional condition. We expect this effect to be larger in patients compared to healthy controls. We also expect more misclassification (with higher certainty) of low intensity resistances into a high category in patients compared to controls (phase 2 data). Conclusion: Preliminary analyses show an effect of categorical priors on the experience of intensity and unpleasantness of respiratory resistances in patients with SSD. Comparisons with controls and more in-depth analysis of classification behaviour will be presented.

CORRESPONDING AUTHOR:Omer Van den Bergh, KU Leuven University of Leuven, omer.vandenbergh@ppw.kuleuven.be

\section{O41.5}

CHANGES IN PSYCHOLOGICAL PROCESSES THROUGHOUT ANORECTAL BIOFEEDBACK THERAPY: ASSOCIATIONS WITH PATIENT-REPORTED OUTCOMES

Alissa Beath ${ }^{2}$, Allison Malcolm ${ }^{1}$, Yoav Mazor ${ }^{1}$, John Kellow ${ }^{1}$, Michael Jones $^{2}$

${ }^{1}$ Macquarie University- Australia

${ }^{2}$ Royal North Shore Hospital, St Leonards, Sydney- Australia

Introduction \& Purpose Anorectal biofeedback (BF) therapy has established efficacy for both fecal incontinence and dyssynergic defecation. The BF program involves education on toileting behaviour, manometric-based biofeedback, and sensory and balloon expulsion retraining. Limited patient psychological characteristics have been shown to predict anorectal BF efficacy, and research in other types of BF have shown psychological improvements (e.g. executive function, cognitive flexibility). Our aims were to determine whether psychological traits change as a result of anorectal $\mathrm{BF}$, and whether changes in psychological traits correlate with changes in patient outcomes, in order to better understand their role in treatment efficacy. Methods 67 patients (93\% female, Mage $=57, \mathrm{SD}=17$ ) presenting to the Neurogastroenterology Unit at the Royal North Shore Hospital (Sydney, Australia) with fecal incontinence (FI) $(n=39)$ or dyssenergic defecation $(n=28)$ underwent a 6-visit instrumented anorectal BF program. Measures of executive function, cognitive flexibility, perceived stress, emotion regulation and self-efficacy were recorded prior to and at the end of therapy. Patient reported outcomes were symptom severity and quality of life $(\mathrm{QoL})$ for FI and dyssenergic defecation patients as appropriate. Spearman's correlations above .3 were considered clinically meaningful. Results Patients' perceived stress, executive function, cognitive flexibility, self-efficacy, and adaptive healthfocused emotion regulation significantly improved throughout BF. Change in psychological traits correlated more strongly with changes in QoL, as opposed to symptom severity. Improvement in constipation QoL was moderately correlated with greater decrease in stress $(\mathrm{rs}=.39, \mathrm{p}=.09)$ and executive function impairment ( $\mathrm{rs}=.37$, $\mathrm{p}=.11$ ); improvement in FI QoL correlated with greater reduction in self-blame ( $\mathrm{rs}=-.38, \mathrm{p}=.03$ ) and catastrophizing ( $\mathrm{rs}=-.35, \mathrm{p}=.05)$, and increases in cognitive flexibility $(\mathrm{rs}=.53, \mathrm{p}<.01)$ and selfefficacy ( $\mathrm{rs}=.35, \mathrm{p}=.07$ ). Conclusions Psychological traits of emotion regulation, cognitive functioning, and stress improve from start to end of BF, and correlate with improvements in patient reported outcomes. These results open the possibility of mechanisms in BF efficacy, with potentially different mechanisms for dyssenergic defecation and FI.

CORRESPONDING AUTHOR: Alissa Beath, Macquarie University, alissa.beath@mq.edu.au 
O41.6

WARNING SIGN? THE RECIPROCAL RELATIONSHIP OF STRESS AND TINNITUS IN AN AMBULATORY ASSESSMENT STUDY Cornelia Weise ${ }^{1}$, Urs M. Nater ${ }^{2}$, Jana Strahler ${ }^{3}$

${ }^{1}$ Philipps-University Marburg- Germany

${ }^{2}$ University of Vienna- Austria

${ }^{3}$ Justus-Liebig University Giessen- Germany

Introduction: Although many tinnitus sufferers report stress as a precipitating factor for tinnitus distress, only few studies prospectively investigated the relation between stress and the onset and progression of tinnitus. As one of the major stress responsive systems, the hypothalamuspituitary-adrenal (HPA) axis shows irregularities in basal levels as well as in response to experimental stress in chronic tinnitus. The aim of the current pilot study was to explore the relation between stress in day-today-life and tinnitus-related distress in acute tinnitus patients, utilizing an ecological momentary assessment (EMA) approach. Given the diverse results of previous studies, both directions, i.e. the influence of stress on tinnitus and vice versa, were investigated. Methods: 14 male participants with recent onset tinnitus (tinnitus duration $<3$ months) were assessed over seven consecutive days. At the beginning of the test period, participants answered a survey on general tinnitus distress, chronic stress and further variables. Perceived stress and tinnitus-related distress in day-today-life were assessed six times a day with a pre-programmed electronic diary. In parallel to each entry, participants provided a saliva sample via passive drool to examine the possible mediating role of HPA activity by means of salivary cortisol (sCort). Results: Hierarchical linear modelling showed perceived stress and sCort levels to predict subsequent tinnitusrelated distress, however only by trend. A comparatively stronger effect was found for tinnitus-related distress on perceived stress. By contrast, sCort was unrelated to tinnitus-related distress in the preceding time frame. Additionally, sCort levels only partially mediated the relationship between stress and tinnitus-related distress. Conclusions: In summary, there is a reciprocal relationship between stress and tinnitus-related distress. Given the small sample size, the reciprocity of stress and distress as well as findings on HPA axis functioning as a mediator of this relation can only be considered preliminary. Yet, these pilot findings suggest that therapeutic approaches for tinnitus sufferers should particularly take into account the relationship of stress and tinnitus distress.

CORRESPONDING AUTHOR: Cornelia Weise, Philipps-University Marburg,weise@uni-marburg.de

\section{O42.1}

HEALTHY LIVING AFTER CANCER: QUALITY OF LIFE AND HEALTH BEHAVIOR AMONG BREAST CANCER SURVIVORS IN COLOMBIA AND CHILE

Irene Tami-Maury ${ }^{1}$, Fernando Cadiz ${ }^{2}$, Hector Garcia ${ }^{3}$, Leticia Gatus ${ }^{4}$, Lizzet Rangel ${ }^{4}$, Banu Arun ${ }^{4}$, Susan Peterson ${ }^{4}$, Liang $\mathrm{Li}^{4}$, Karen BasenEngquist ${ }^{4}$

${ }^{1}$ The University of Texas MD Anderson Cancer Center- United States of America

${ }^{2}$ Clinica Alemana- Chile

${ }^{3}$ Instituto de Cancerología Las Américas- Colombia

${ }^{4}$ UT MD Anderson Cancer Center- United States of America

Introduction \& Purpose: Studies in the US indicate that breast cancer survivors suffer from a range of after effects of breast cancer, including declines in physical functioning, fatigue, pain, lymphedema, and psychological distress. Also, US reports indicate that exercise, weight management, and improved nutrition are beneficial for breast cancer survivors, improving their quality of life and overall health. Despite these advances, there has been relatively little emphasis on addressing the needs of posttreatment cancer survivors in Latin America. This study is aiming to fill the data gap on health behaviors and quality of life in breast cancer survivors in Chile and Colombia by understanding their quality of life issues, health behaviors and weight gain. Methods: We will conduct a cross-sectional survey among female breast cancer survivors who are still living and between one and five years from cancer diagnosis who are seeking care at two major cancer centers in Chile and Colombia. Survivors with any stage breast cancer will be eligible to participate. The data collection abstraction forms will be electronically entered by the local research teams in Chile and Colombia into a REDCap database, developed centrally at MD Anderson Cancer Center (USA). Data on physical activity, current weight, diet, tobacco use, alcohol use, quality of life, physical functioning, and symptoms, as well as information about survivors' preferences for receiving additional survivorship information and/or services will be recorded. Results: Four hundred female breast cancer survivors will be recruited for the purpose of this study (200 in Chile and 200 in Colombia). Data collection process is expected to start in April 2018 and finalized by June 2018 at each study site. Data analysis will be conducted at MD Anderson Cancer Center in collaboration with Clinica Alemana (Chile) and Instituto de Cancerologia Las Americas (Colombia). Conclusion: Findings of this pioneering study will assist in the development of programs that improve the health and quality of life of cancer survivors in Chile and Colombia, with the ultimate goal of replicating the model to other cancer center in Latin America where physical activity and weight management are not yet included as an integral component of cancer treatment and survivorship.

CORRESPONDING AUTHOR: Irene Tami-Maury, The University of Texas MD Anderson Cancer Center, itami@mdanderson.org

\section{O42.2}

PARTICIPANT PERSPECTIVES ON AN ADAPTED EATINGFOCUSED LIFESTYLE INTERVENTION FOR WOMEN WITH TYPE 2 DIABETES

Alyssa Vela ${ }^{1}$, Brooke Palmer ${ }^{1}$, Alyssa Minnick ${ }^{1}$, Virginia Gil-Rivas ${ }^{1}$, Fary Cachelin $^{2}$

${ }^{1}$ The University of North Carolina at Charlotte- United States of America ${ }^{2}$ University of East London- United Kingdom

Introduction \& Purpose: Pandemic rates of type 2 (T2) diabetes mellitus are largely attributed to health behaviors such as poor diet, overeating, and sedentary lifestyle. T2 diabetes management is demanding, requiring daily effort, and eating is often the most challenging aspect of management. Disordered eating (i.e., binge, emotional, external, and restrictive behaviors), occurs more often in populations diagnosed with T2 diabetes than in the general population and is associated with increased risk for diabetes-related complications, higher $\mathrm{HbA} 1 \mathrm{c}$ and poor diabetes management. Thus, there is a need for further development of low-cost and accessible treatment modalities for $\mathrm{T} 2$ diabetes and related health problems, including disordered eating. Methods: The current pilot study assessed the preliminary efficacy and acceptability of a CBT- based guided self-help (CBTgsh) intervention for women with $\mathrm{T} 2$ diabetes who endorsed disordered eating behaviors. Ten women completed the 12week intervention (one drop out) that included an emphasis on establishing a regular pattern of eating, food monitoring, adjusting thinking related to eating, and increasing physical activity. Results: Overall, the intervention was effective in reducing disordered eating (EDE-Q $t(9)=7.85$, $\mathrm{p}<0.00$; DEBQ $\mathrm{t}(9)=7.87, \mathrm{p}<0.00)$ and improving diabetes-related outcomes $(\mathrm{HbAl} \mathrm{ct}(8)=3.40, \mathrm{p}<0.00$; diabetes distress $\mathrm{t}(9)=0.59, \mathrm{p}<0.00)$. Following completion of the intervention, participants reported on the acceptability of the study and provided feedback for further development of the intervention. Constant-comparison analysis was conducted by a two-person coding team and reveled seven themes: including those that fell into categories of program likes, program dislikes, recommendations, and suggestions for future modifications. Conclusions: Participant feedback suggests that including diabetes-specific language, such as that utilized by the American Diabetes Association, inclusion of blood-glucose 
monitoring, and the inclusion of general diabetes management information within the manual would be beneficial. We will discuss how the feedback provided has been used to adapt the intervention

CORRESPONDING AUTHOR: Alyssa Vela, The University of North Carolina at Charlotte, avela@uncc.edu

\section{O42.3}

FAMILY-BASED GROUP TREATMENT FOR OVERWEIGHT/ OBESE CHILDREN

Ana Bogdanic ${ }^{1}$, Marina Grubic ${ }^{1}$, Petra Matkovic ${ }^{1}$, Anita Spehar-Uroic ${ }^{1}$ University Hospital Centre Zagreb, Department of Pediatrics- Croatia

Objective: To evaluate the efficacy of outpatient family-based group treatment program for overweight children. Methods: The treatment program is based on a multidisciplinary approach, structured treatment, education and monitoring of children with excessive body weight over 2 years. Both children and parents were separately included in small age-homogenous groups from 6 to 10 patients in which they went through one week program that included workshops about obesity and its consequences, healthy diet, physical activity and habits related to obesity. In the first year follow-up meetings were conducted every 2 months, and in a second year every 3 months. The multidisciplinary team consisted of psychologists, pediatric endocrinologists, social pedagogue, nutritionists and physiotherapists who separately educated children and their parents. The intervention was delivered at the University Hospital Centre Zagreb with 79 overweight and obese children aged 6$18 \mathrm{yrs}$ (45girls, 34boys) and their parents. The efficacy of the treatment was measured through change in BMI z-score from baseline after one and two years, through $\%$ of children that lost weight and by comparison with a control group (age 6-17yrs, 39girls, 42boys) that undergone standard individual physician counseling. Participants were randomly assigned to intervention and control group. Results: One year after the baseline $90 \%$ of intervention group and $60 \%$ of controls lost weight. $2 \mathrm{yrs}$ after the baseline $86 \%$ of intervention group and $70 \%$ controls maintained weight loss. Significant loss in $\mathrm{zBMI}$ has been found in both intervention and control group 1yr after the baseline $(\Delta \mathrm{zBMIint}=0.5, \mathrm{t}=9.26, \mathrm{p}<0,001 ; \Delta \mathrm{zBMIcontr}=0.2, \mathrm{t}=3.23$, $\mathrm{p}<0.01)$, as well as at the $2 \mathrm{yr}$ folow-up meeting $(\Delta \mathrm{zBMIint}=0.6, \mathrm{t}=6.43$, $\mathrm{p}<0.001 ; \Delta \mathrm{zBMIcontr}=0.2, \mathrm{t}=4.2, \mathrm{p}<0.001)$. The intervention group had significantly higher zBMI reduction than controll group at both times $(\mathrm{t} 1=4.27, \mathrm{p}<0,001 ; \mathrm{t} 2=3.27, \mathrm{p}<0,01)$. Conclusion: Our family-based outpatient group treatment has been shown effective in reducing obesity in children and more efficient than standard physician weight loss counseling. These results imply that an multidisciplinary family-based group program is a promising treatment option for childhood obesity.

CORRESPONDING AUTHOR: Ana Bogdanic, University Hospital Centre Zagreb, Department of Pediatrics, abogdani@gmail.com

\section{O42.4}

CAN RE-POSITIONING ONLINE MENU ITEMS INCREASE FRUIT AND VEGETABLE SALES FROM AUSTRALIAN PRIMARY SCHOOL CANTEENS? A CLUSTER RANDOMISED TRIAL

Rebecca Wyse ${ }^{1}$, David Just ${ }^{2}$, Gnel Gabrielyan ${ }^{2}$, Jeff Swigert ${ }^{3}$, Tessa Delaney $^{1}$, Jia Ooi ${ }^{1}$, Luke Wolfenden ${ }^{1}$, Serene Yoong ${ }^{1}$

${ }^{1}$ School of Medicine and Public Health, University of NewcastleAustralia

${ }^{2}$ Charles H. Dyson School of Applied Economics and Health, Cornell University- United States

${ }^{3}$ College of Policy Analysis \& Management, Cornell University- United States

INTRODUCTION AND PURPOSE: School canteens are a key setting for implementing healthy eating initiatives for children. Online canteen ordering systems, where users pre-order their lunch online, are increasingly popular in Australian schools, and provide an opportunity to implement public health initiatives at scale and at relatively low cost. Evidence from paper-based menus suggests that manipulating the order in which menu items are listed influences the frequency of their selection. However, this has not been tested within an online canteen setting. As such, the study purpose was to determine whether re-positioning fruit and vegetable items within an online school canteen ordering system increases sales of those items. METHODS: Six Australian primary schools participated in a clustered RCT and were randomised to receive either: no change to their online menu (control); or a redesigned online menu (intervention). On each intervention school menu, all fruit and vegetable snack items (target items) were grouped together in a single category, named "Fruit and Veggie Snacks", which was positioned prominently at two places, top and bottom of the online menu). Intervention fidelity was assessed by checking the online menu weekly to ensure the position of target items remained the same. The online ordering system automatically recorded all sales data from lunchtime orders. Sales data will be analysed to determine the proportion of all online lunch orders that contained at least one target item (i.e. fruit and vegetable snack item). (Registration ACTRN12616001520426). RESULTS: Over 30,000 items were ordered throughout the trial, with 2136 and 1742 children placing orders during the 4-week baseline and intervention periods respectively. Between group differences in the proportion of online lunch orders at follow-up that contain at least one target item (i.e. fruit and vegetable snack item) will be analysed using linear mixed models adjusted for clustering, repeated measures and controlled for baseline values. CONCLUSION: If proven effective, the redesign of online canteen menus to position healthier items (such as fruit and vegetable snacks) in positions of greater prominence, may increase sales of these items, and may in turn yield public health benefits.

CORRESPONDING AUTHOR: Rebecca Wyse, The University of Newcastle, rebecca.wyse@hnehealth.nsw.gov.au

\section{O42.5}

OVERWEIGHT AND OBESITY AMONG YOUTH: AN EXPLORATION OF GENDER, INDIVIDUAL AND REGIONAL INFLUENCES

Tingzhong Yang ${ }^{1}$, Xiaozhao Yang ${ }^{2}$

${ }^{1}$ Zhejiang University- China

${ }^{2}$ Murray State University- United States of America

Background While many studies have examined the factors associated with overweight and obesity, no study has yet compared both and regional variants of overweight and obesity in men and women. The present study examines gender differences of individual and regional variants in overweight and obesity between men and women. Methods Participants were 11,942 college students identified through a multistage survey sampling process conducted in 50 universities in China. The data collection instrument was a self-administered questionnaire. Multilevel logistic regression models were used to examine individual and regional influences on overweight and obesity. Results The prevalence of overweight and obesity was 9.5\% (95\% CI: $7.7 \%, 11.3 \%$ ) in the overall study sample, $13.9 \%$ (95\% CI: $11.5 \%, 16.7 \%)$ in males and $6.1 \%(95 \%$ CI: $4.1 \%, 8.1 \%)$ in females, respectively. The final multiple level logistic model showed that family income and higher original GDP, higher perceived life stress and regional unemployment were associated with higher overweight and obesity in males. However, unlike male students, only regional unemployment was associated with overweight and obesity in the opposite direction among females. Conclusions Our research underscores the importance of individual and environmental influences in understanding gender differences in overweight and obesity in China. This information should be considered in formulating gender sensitive strategies in chronic disease nursing.

CORRESPONDING AUTHOR: Tingzhong Yang, Zhejiang University, Tingzhongyang@zju.edu.cn 
O42.6

PSYCHOLOGICAL AND BEHAVIORAL PATHWAYS BETWEEN PERCEIVED STRESS AND WEIGHT CHANGE IN A BEHAVIORAL WEIGHT LOSS INTERVENTION

Tiffany Carson ${ }^{1}$, Kristine Molina ${ }^{2}$, Monica Baskin ${ }^{1}$

${ }^{1}$ University of Alabama at Birmingham- United States of America

${ }^{2}$ University of Illinois at Chicago- United States of America

Introduction \& Purpose: Black women have the highest prevalence of obesity and lose less weight in behavioral weight loss programs than any other demographic group in the United States. Stress has been implicated as a contributor to weight management challenges but research findings are mixed with some studies showing a positive association between stress and weight while others show no association. These inconsistencies suggest that the relationship between stress and weight may be mediated by other factors that are associated with both variables such as depression and/or diet. The purpose of this study was to systematically examine pathways between stress, depressive symptoms, diet and weight among a group of black women participating in an evidence-based behavioral weight loss program. Methods: Overweight and obese black women in a behavioral weight loss program $(n=409)$ were measured for height, weight, and completed validated surveys to measure stress [Perceived Stress Scale-10 (PSS-10)], depressive symptoms [Center for Epidemiologic Studies Depression 10 (CES-D 10)], and dietary intake [NCI Automated Self-Administered 24-hour (ASA-24) Dietary Assessment Tool] at baseline and 6-months. PSS-10, CES-D 10, and dietary intake scores were calculated. Participants also completed demographic and general health history surveys. A parallel mediator model was tested, with 6-month dietary intake (i.e., total fats, sugars, sodium in grams) and depressive symptoms as mediators between baseline perceived stress (PSS-10) and 6-month BMI score. Results: Results of our parallel mediator model indicated that baseline PSS-10 was not directly associated with 6-month BMI. Further, although PSS-10 was associated with depressive symptoms $(b=.14, p<.05)$ and higher levels of depressive symptoms were associated with higher BMI $(b=.06, p<.001)$, the indirect effect of PSS-10 on BMI was only marginally significant $[\mathrm{I}=.008$, S.E. $=.004,95 \% \mathrm{CI}=.000, .017, \mathrm{p}=.061)$ after adjusting for sociodemographic factors, overall health, and baseline depressive symptoms and baseline BMI. Lastly, PSS-10 was not associated with dietary intake $(\mathrm{b}=-.07, \mathrm{p}=.129)$, but poorer dietary intake score was associated with higher BMI $(b=.02, p<.01)$; the indirect effect from PSS-10 to BMI via dietary intake was non-significant $(\mathrm{I}=-.002$, S.E. $=.002,95 \% \mathrm{CI}=$ $-.005, .001, \mathrm{p}=.217)$. Conclusions: Our findings show that there was no direct relationship between PSS and BMI. Consistent with previous literature, higher levels of depression and less healthy dietary patterns were associated with higher BMI. Additional research is needed to understand the interplay between psychological and behavioral contributors to weight change among black women.

CORRESPONDING AUTHOR: Tiffany Carson, University of Alabama at Birmingham, tiffanycarson@Uabmc.edu

\section{O43.1}

SLEEP AND QUALITY OF LIFE IN OLDER ADULTS: INFLUENCE OF HEALTH STATUS AND SOCIAL NETWORK

Neha Gothe ${ }^{1}$, Diane Ehlers ${ }^{2}$, Susan Aguinaga ${ }^{1}$, Arthur Kramer $^{3}$, Edward McAuley ${ }^{1}$

${ }^{1}$ Kinesiology and Community Health, College of Applied Health Sciences, University of Illinois at Urbana Champaign, Urbana, IL- United States

${ }^{2}$ Exercise Science, Arnold School of Public Health, University of South Carolina, Columbia, SC- United States

${ }^{3}$ Office of the Provost, Northeastern University, Boston, MA- United States

Introduction: Quality of sleep has been consistently associated with quality of life (QOL) in a number of clinical and non-clinical populations.
However, mechanisms underlying this relationship are not well understood. The purpose of this study was to longitudinally test a model examining the role of physical and mental health status and social network as potential mediators of the relationship between sleep and QOL in a sample of healthy older adults. Methods: Participants $(\mathrm{N}=247$, mean age $=65.4 \pm 4.6$ years) completed measures of sleep (Pittsburgh Sleep Quality Index), physical and mental health status (SF-12 Health Survey), social ties (Social Network Index) and QOL (Flourishing Scale) at baseline and following a 6-month physical activity intervention. Relationships among model constructs were examined over the 6-month period using panel analysis within a covariance-modeling framework. Age, sex, exercise group and accelerometer measured physical activity at the respective time-points were included as covariates. Results: The hypothesized model provided a good model-data fit $(\chi=62.05$, $\mathrm{d}=41, \mathrm{p}<.02$; $\mathrm{CFI}=0.98$; $\mathrm{SRMR}=0.04$; RMSEA $=0.05$ ) when controlling for covariates. At baseline, sleep indirectly influenced QOL via physical health status, mental health status and social network (QOL R square $=.48, \mathrm{p}<.001)$. These relationships were also supported across time at month 6 (QOL $\mathrm{R}$ square $=.49, \mathrm{p}<.001)$. Among covariates, age was inversely associated with mental health status and females reported higher social ties at baseline. Physical activity at baseline was significantly associated with sleep quality however, at month 6 it was associated with social ties, which may be a function of the group-based social nature of the 6-month physical activity intervention. Conclusion: Our results support a novel sleep and QOL model that can be used as a framework to design health interventions to promote sleep quality thereby influencing QOL by targeting modifiable mediators of physical-mental health status and social network. Our findings have significant implications for older adult as well as clinical populations that report compromised sleep, QOL and impaired health.

CORRESPONDING AUTHOR: Neha Gothe, University of Illinois at Urbana Champaign,npg@illinois.edu

\section{O43.2}

A DOUBLE-EDGED SWORD: THE RELATIONSHIP BETWEEN DAYTIME NAPPING DURATION AND METABOLISM-RELATED DISEASES

Xueyin Zhao ${ }^{1}$, Lin Cheng ${ }^{2}$, Chaonan Zhu ${ }^{1}$, Shankuan Zhu ${ }^{1}$

${ }^{1}$ Zhejiang University- China

${ }^{2}$ The University of New South Wales- Australia

Introduction and Purpose: Some studies have suggested that daytime napping, a common practice in many countries, may increase the risk of type 2 diabetes for some age and gender groups under certain conditions. However, limited data has revealed the association between nap duration and metabolic diseases other than diabetes. Data from the baseline survey of Lanxi Cohort Study, a population-based study of the general health condition of natural residents in Zhejiang Province, China, were used to investigate the relationship between nap duration and metabolic abnormalities. To be specific, how long a nap can decrease or increase the risk of a specific kind of disease. Methods: A total of 3,236 participants underwent a physical examination, laboratory tests, and face to face interview. They were categorized into four groups according to nap duration (no napping, $\leq 30,30$ to60 min). Logistic regression models were used to examine the odds ratios (ORs) of napping duration with four metabolism-related diseases. Stratified analysis was further used to explore the interaction effects of gender and age on results. Results: Compared to the no daytime napping group, people who napped during the daytime for more than one hour were independently associated with a greater prevalence of diabetes (OR 1.56, 95\% CI, 1.02-2.39). Those who napped during the daytime within a half hour have showed a lower prevalence of fatty liver, dyslipidemia, and central obesity (fatty liver: OR 0.78, 95\% CI, 0.65-0.95; dyslipidemia: OR 0.81, 95\% CI, 0.67-0.97; central obesity: OR $0.82,95 \% \mathrm{CI}, 0.68-0.99)$. To be more specific, those 
who habitually napped during the daytime for more than one hour exhibited an increasing prevalence of diabetes among female older than 50 years old. Those who habitually napped during the daytime within a half hour exhibited a decreasing prevalence of fatty liver and dyslipidemia among male less than 50 years old, a decreasing prevalence of central obesity among female less than 50 years old. Conclusions: Short daytime napping duration (within a half hour) protect people from metabolism related diseases, whereas long daytime napping duration (more than one hour) may increase the risk of diabetes, which then can be harmful for health.

CORRESPONDING AUTHOR: Xueyin Zhao, Zhejiang University, xueyin129@126.com

\section{O43.3}

PREVALENCE AND PREDICTORS OF SLEEP DISTURBANCE IN PSORIASIS PATIENTS

Robert Zachariae ${ }^{1}$, Peter Jensen ${ }^{2}$, Lone Skov ${ }^{2}$, Claus Zachariae ${ }^{2}$

${ }^{1}$ Aarhus University Hospital- Denmark

${ }^{2}$ Copenhagen University Hospital, Gentofte- Denmark

INTRODUCTION AND PURPOSE: Psoriasis is associated with potentially sleep disrupting symptoms, e.g., pain and itch, and a higher prevalence of sleep disturbance could be expected among psoriasis patients. However, the available findings are unclear. We therefore examined the prevalence of sleep disturbance in psoriasis patients, compared the results with those of healthy controls, and examined possible demographic and clinical predictors.

METHODS: In a cross-sectional, case-controlled design, 179 consecutively recruited psoriasis patients attending a university hospital outpatient clinic were compared to 105 age and gender matched controls from the general population. Measures included psoriasis severity (Psoriasis Area and Severity index [PASI]), HRQoL (Dermatology Life Quality Index [DLQI]), insomnia severity (Insomnia Severity Index [ISI]), sleep quality (Pittsburgh Sleep Quality Index [PSQI]), stress (Perceived Stress Scale [PSS]), Itch (Itch Severity Scale [ISS]), and depressive symptoms (Beck Depression Inventory [BDI]). Analyses included group comparisons and multiple regression analyses to identify possible predictors of sleep disturbance.

RESULTS: Twenty-five percent of patients with psoriasis reported clinical insomnia (ISI>15), compared with $10.5 \%$ of controls. In all, $53.9 \%$ of psoriasis patients were characterized as poor sleepers (PSQI $>5$ ), compared with $21.9 \%$ of controls. Itch emerged as a statistically significant $(\mathrm{p}<0.05)$ predictor across all sleep-related outcomes.

CONCLUSIONS: A higher proportion of psoriasis patients experienced poor sleep compared with controls from the general population. Sleep is essential for daytime functioning and health, and to improve sleep in psoriasis patients, effective control of itch is needed in this patient group. When this is not possible or insufficient, cognitive-behavioral therapy for insomnia may be an option.

CORRESPONDING AUTHOR: Robert Zachariae, Aarhus University Hospital, bzach@aarhus.rm.dk

\section{O43.4}

IMPACT OF CHRONIC INSUFFICIENT SLEEP IN ADOLESCENCE ON CIGARETTE SMOKING AND ALCOHOL USE IN YOUNG ADULTHOOD: A MARGINAL STRUCTURAL MODEL ANALYSIS Carol Strong ${ }^{1}$, Chia-Yi Ho ${ }^{1}$, Meng-Che Tsai ${ }^{1}$, Yi-Ching Lin ${ }^{1}$, ChungYing Lin $^{2}$

${ }^{1}$ National Cheng Kung University- Taiwan

${ }^{2}$ Hong Kong Polytechnic University- Hong Kong

INTRODUCTION Insufficient sleep in adolescent samples exhibit a bidirectional relationship with substance use, including cigarette smoking and alcohol drinking. It is further complicated considering that the confounders vary over time and are often in response to adolescents' previous condition, which can be addressed by a counterfactual approach using a marginal structural model. PURPOSE To identify the impact of chronic insufficient sleep in adolescence on cigarette smoking and alcohol drinking in young adulthood. METHODS Data in this study are from the Taiwan Youth Project, a longitudinal study that started in 2000 (wave 1) and surveyed 2,690 7th grade students in Northern Taiwan. Data from waves 1, 2, 3, 6, 8 and 9 were used. Outcomes include frequent cigarette smoking and alcohol drinking at age 21 (wave 9). Chronic insufficient sleep was a sum of insufficient sleep (less than 7 hours) from wave 2 to 9 . Time invariant confounders included gender, area of residence and pubertal timing. A marginal structural model with stabilized inverse probability-of-treatment weights was used to address time-varying confounders including depression, and the adolescents' alcohol and smoking use in each wave. RESULTS A total sample of 1,039 adolescents with complete information were used for this study. The proportion of insufficient sleep increased from $14.73 \%$ in wave 2 to $84.41 \%$ in wave 9 . We did not find significant association between chronic insufficient sleep in adolescence on the odds of cigarette smoking ( $\mathrm{OR}=0.94,95 \% \mathrm{CI}$ : 0.77 , $1.15)$ or alcohol use $(\mathrm{OR}=1.15,95 \% \mathrm{CI}: 0.89-1.49)$ at age 21. CONCLUSIONS With adjustment of time varying confounders and considering the changes of smoking and alcohol use behavior in each wave, chronic insufficient sleep was not associated with smoking or alcohol use in young adulthood. Further analysis is needed to examine whether gender differences exist, or whether other aspects of sleep quality are associated with later substance use.

CORRESPONDING AUTHOR: Carol Strong, National Cheng Kung University, carol.chiajung@gmail.com

\section{O43.5}

DETERMINANTS OF HEALTHY SLEEP PRACTICES AMONG BELGIAN UNIVERSITY STUDENTS: AN APPLICATION OF THE THEORY OF PLANNED BEHAVIOR

Stephan Van den Broucke ${ }^{1}$, Sarah Fakroune ${ }^{1}$

${ }^{1}$ Université Catholique de Louvain- Belgium

Introduction and purpose. University students often show unhealthy sleep practices, including irregular sleep habits, daytime napping, alcohol use, or using internet before going to sleep. Poor sleep hygiene is related to a variety of ill-health effects, including metabolism dysregulation, cardiovascular problems, memory and attention problems and poor academic performance. However, research on sleep practices mostly focuses on the consequences of poor sleep behavior, and seldom addresses its determinants. The purpose of this study was to test if the constructs of the Theory of Planned Behavior (TPB) predicted (un)healthy sleep behaviors of Belgian university students. Method. An online questionnaire was used to assess the frequency of irregular sleep times, daytime napping, alcohol use before sleep, and engaging in internet or video watching before bedtime, as well as the attitudes, perceived norms, perceived control and intentions towards these behaviors, amongst 1006 undergraduate students enrolled at a French-speaking university (70\% females). Principal Component analysis (PCA) and internal consistency analysis (Cronbach alpha) was used to establish the validity and reliability of the scales representing the dimensions of the TCP for reach behavior Results. Multiple regression analyses showed that perceived advantage and disadvantage, perceived norms and perceived control significantly predicted $21 \%$ of the intention to avoid irregular sleep patterns, $74 \%$ of the intention to take daytime naps, $27 \%$ of the intention to use internet or watch videos, and $45 \%$ of the intention to consume alcohol before bedtime. Intentions and perceived behavioral significantly predicted self-reported irregular sleeping times $\left(\mathrm{R}^{2}=.15\right)$, daytime napping $\left(\mathrm{R}^{2}=.36\right)$, internet use or video watching before bedtime $\left(\mathrm{R}^{2}=.23\right)$, and alcohol use before bedtime $\left(\mathrm{R}^{2}=.51\right)$. Multivariate analysis of variance revealed significant for the 
four sleep hygiene behaviors with regard to gender, study type and type of residence. Conclusion The TPB dimensions provide a useful framework to predict healthy sleep intentions and behaviors of undergraduate students. Their role can be taken into account when developing healthy sleep promotion campaigns for students.

CORRESPONDING AUTHOR: Stephan Van den Broucke, Université catholique de Louvain, stephan.vandenbroucke@uclouvain.be

\section{O43.6}

QUALITY AND QUANTITY OF SLEEPING AND GLYCEMIC CONTROL IN TYPE 2 DIABETES

Solange Campos ${ }^{1}$, Silvia Barrios ${ }^{1}$, Patricia Masalan ${ }^{1}$, Viviana Guajardo ${ }^{1}$, Nelson Arias $^{2}$, Lucas Bobadilla ${ }^{1}$

${ }^{1}$ Pontificia Universidad Católica de Chile

${ }^{2}$ Universidad de Caldas- Colombia

Introduction: In Chile type 2 diabetes (DM2) have duplicated its prevalence from $4,2 \%$ to $9,4 \%$, overmore, only $32.4 \%$ of people diagnosed with DM2 have a Hb1 AC less than 7\%. Different studies have shown outside of Chile that the quality and amount of sleeping correlates with glycemic control. This association has not been explored in Chile. Methodology: Cross-sectional study with a sample of 223 individuals living with DM2 aged 20 to 64 who attend a family community health care center. Quality and quantity of sleeping was assessed using a survey and its association with $\mathrm{Hb} 1 \mathrm{AC}$ was assessed with SPSS. Results: $66.4 \%$ were women with a mean age of 54,7, and 8,4 years of school. They have been diagnosed in average for 5,8 years with DM2 de 5,8. Pittsburg index indicated that $40,81 \%$ of the sample have problems with sleeping and requires attention and treatment. In addition, 7,16\% have severe sleep disorders and $33 \%$ of them sleep between 7 and 9 hours. In relation to metabolic compensation, $57,4 \%$ have and $\mathrm{Hb} 1 \mathrm{Ac}$ over $7 \%$. No significant statistical correlation was found between quality of sleep and $\mathrm{Hb} 1 \mathrm{Ac}$ levels. Still there was a tendency and $65 \%$ of good sleepers have more chances to have better levels of Hb1 AC. Conclusions: No statistical significance was found, this could relate to high levels of sleep problems and $\mathrm{Hb} 1 \mathrm{AC}$, originating a homogenous sample. Nonetheless, these findings support the need to assess sleeping behaviors and patterns in order to support behavioral changes.

CORRESPONDING AUTHOR: Solange Campos, Pontificia Universidad Católica de Chile, Scamposr@uc.cl

O44.1

ARTIFICIAL INTELLIGENCE FOR DEMENTIA SCREENING

Kelvin K.F. Tsoi ${ }^{1-2}$ Max WY Lam ${ }^{2}$, Christopher T.K. $\mathrm{Chu}^{2}$, Samuel Y.S. Wong $^{3}$

${ }^{1}$ Chinese University of Hong Kong

${ }^{2}$ Stanley Ho Big Data Decision Analytics Research Centre, The Chinese University of Hong Kong

${ }^{3}$ The Jockey Club School of Public Health and Primary Care, Faculty of Medicine, The Chinese University of Hong Kong

Introduction: Many current dementia screening tests are in paper-andpencil form and evaluated subjectively by healthcare professionals where human bias is inevitable. To eliminate human bias, we developed a digital drawing platform to capture drawing behaviours and apply machine learning algorithm to distinguish the patients with or without dementia. Methods: Patients with clinically diagnosed Alzheimer's disease (AD) were recruited from dementia clinics. People without dementia and with MoCA score at least 22 were recruited from elderly centres. All participants were asked to draw two interlocking pentagons in a tablet with reference to a sample figure. The platform captured drawing speed and motion. Markov chains of higher order and Gaussian process regression were applied to predict the chance of having dementia. The performance of the platform was compared to the scoring method in the Mini-Mental State Examination (MMSE). Results: A total of 465 participates was recruited, and 194 (41.7\%) of them were labelled with $\mathrm{AD}$. The average age of $\mathrm{AD}$ patients was 79.6 years $(\mathrm{SD}=6.2)$, and with average MoCA scores of $12.0(\mathrm{SD}=4.8)$. The median drawing time was 17.5 seconds. In the 271 healthy subjects, the average age was 75.6 years $(\mathrm{SD}=7.7)$, and with average MoCA scores of $25.0(\mathrm{SD}=2.1)$. The median drawing time was 12.6 seconds. Diagnostic performance with the machine learning algorithm on $\mathrm{AD}$ was sensitivity of $77.7 \%$ and specificity of $71.4 \%$. Conclusion: Machine learning methods can improve the screening accuracy for dementia. Other behavioral tests on memory, attention, and executive abilities can be further developed for cognitive screening.

CORRESPONDING AUTHOR: Prof. KKF Tsoi, Stanley Ho Big Data Decision Analytics Research Centre, The Chinese University of Hong Kong kelvintsoi@cuhk.edu.hk

CORRESPONDING AUTHOR:Kelvin K.F. Tsoi, Chinese University of Hong Kong, kelvintsoi@cuhk.edu.hk

\section{O44.2}

EFFECT OF HEALTH-LITERATE DESIGN FOR AN ONLINE PLANNING TOOL ON UNHEALTHY SNACKING BEHAVIOUR: AN EXPERIMENTAL STUDY

Julie Ayre ${ }^{1}$, Carissa Bonner ${ }^{1}$, Erin Cvejic $^{1}$, Don Nutbeam ${ }^{1}$, Kirsten McCaffery ${ }^{1}$

${ }^{1}$ The University of Sydney- Australia

Introduction To date, health literacy interventions have focused on education and motivation rather than volitional processes (e.g. planning and self-monitoring). This is surprising given their central role in behaviour change research. This study aimed to address this gap by investigating whether health-literate design for volitional processes can increase the effectiveness of an online planning tool to reduce unhealthy snacking for low health literate users. Methods 440 participants completed baseline measures of self-reported unhealthy snacking and health literacy (NVS: Newest Vital Sign). They were randomised to 3 online planning interventions: 1) health-literate design (one snacking situation paired with a specific behaviour change); 2) instructions to create a detailed plan; or 3) a healthy snacking fact sheet. Baseline snacking, intervention, NVS, age, education and home language were entered into a multiple regression model to predict unhealthy snacking at 4-week follow-up. Results 373 participants $(84.8 \%)$ completed the follow-up survey. On the NVS, $24 \%$ had high and $25 \%$ had possible likelihood of limited health literacy; $52 \%$ had adequate health literacy. Participants ate 16.2 serves of unhealthy snacks per week at baseline. Unhealthy snacking at follow-up was associated with lower health literacy $(b=-1.7, p=0.015)$. There was no difference across intervention groups. However, participants with lower NVS scores who used the health-literate tool ate fewer unhealthy snacks compared to those who used the detailed planning tool; whereas those with higher NVS scores ate fewer unhealthy snacks using the detailed planning tool $(b=1.7$, $\mathrm{p}=0.027)$. The model explained $18 \%$ of variance in snack scores $(\mathrm{F}=9.0, \mathrm{p}<0.001)$ and estimated that people scoring 2 points below the mean NVS $(M=3.4, S D=2.0)$ ate 8 fewer serves of unhealthy snacks if they used the health-literate tool, whereas people scoring 2 above the mean NVS ate 6 more serves of unhealthy snacks using the same tool. Conclusion The online planning tool with health-literate design was beneficial for people with lower health literacy but not for those with higher health literacy. Targeted health literacy interventions could be improved by using volitional strategies that have lower health literacy demands.

CORRESPONDING AUTHOR: Julie Ayre, The University of Sydney, julie.ayre@sydney.edu.au 
O44.3

USING THE HELPMEDOIT! APP AND WEBSITE TO SET AND MONITOR WEIGHT LOSS GOALS AND MOBILISE SOCIAL SUPPORT: QUALITATIVE FINDINGS

Lynsay Matthews ${ }^{1}$, Juliana Pugmire ${ }^{2}$, Laurence Moore ${ }^{2}$, Sharon Simpson $^{2}$

${ }^{1}$ University of Glasgow- United Kingdom

${ }^{2} \mathrm{MRC} / \mathrm{CSO}$ Social and Public Health Sciences Unit, Institute of Health and Wellbeing, University of Glasgow- United Kingdom

Introduction and purpose: HelpMeDoIt! is an app/website that harnesses social support for weight loss by enabling participants to nominate friends/family to help them with their weight loss goals. The intervention focused on goal setting, self-monitoring and social support, guided by elements of control theory, social cognitive theory, self-determination theory and social support theories. Aim: To explore the views and experiences of trial participants and their helpers and explore whether the intervention theory was supported. Methods: Qualitative interviews were conducted at $6 \mathrm{mth}$ follow-up, via telephone, with intervention participants $(n=22)$ and their helpers $(n=9)$. The sample included individuals who had and had not engaged regularly with the app/website. Interviews were recorded, transcribed verbatim and analysed thematically. Results: Insights were gained on various aspects of the intervention, including: feasibility of using an app to set and monitor weight loss goals; the social support process; barriers to engagement; and suggested improvements for a future trial. Key findings indicated that participants and helpers engaged with the concept of the intervention but their participation was hindered to some extent by technical problems. Helpers often provided social support without using the app, e.g. face-to-face or by text. Findings supported several aspects of the intervention theory including: helpers providing instrumental, emotional and informational social support; increased knowledge and skills, sometimes via support from helpers e.g. cooking skills; increased action planning and problem solving via effective goalsetting; and increased motivation either via successful self-monitoring or goal accomplishment. Participant/helper insight provided several suggestions to enhance the intervention, including, providing a community of peer support. Conclusions: Participants were enthusiastic about having helpers to support them as well as using an app/website for lifestyle behaviour change. The technology needs to be free of technical issues to avoid unnecessary barriers to engagement. Findings will be used to help inform a future full trial.

CORRESPONDING AUTHOR: Lynsay Matthews, University of Glasgow, lynsay.matthews@glasgow.ac.uk

\section{O44.4}

IMMERSION IN A HEALTH-FOCUSED VIRTUAL WORLD PREDICTS HEALTHY BEHAVIOR IN MIDDLE SCHOOL AGE GIRLS

Mary Kate Clennan ${ }^{1}$, Daniella Carucci ${ }^{1}$, Maria Llabre ${ }^{1}$, Judy Brown ${ }^{2}$, Patrice $\mathrm{Saab}^{1}$

${ }^{1}$ University of Miami- United States

${ }^{2}$ Patricia and Phillip Frost Museum of Science- United States

Introduction \& Purpose: Research with adults suggests that healthy behaviors that occur in a virtual world may lead to healthy behaviors in the real world. This study investigated the effect of immersion in a virtual world on healthy behaviors in middle school aged girls. Methods: 127 middle school aged $(M=11.8)$ girls from ethnically diverse backgrounds explored the GROOVE Island 3-D virtual world during a 3-week summer program that emphasized the importance of a healthy lifestyle. GROOVE Island provided the girls with an opportunity to create a realistic avatar, learn about health, engage in virtual healthy behaviors, and socialize with other healthy avatars. Participants completed the 11-item GROOVE Presence scale (influenced by the work of Fox et al., 2009), which assessed how present the participant feels in the 3-D virtual world. Three of the 11 items (e.g., "I would like to spend more time on GROOVE Island,") were included as indicators of a latent variable 'Immerse,' which measured level of immersion in the virtual world. Items were rated on a 5 -point Likert scale $(1=$ "disagree a lot" to $5=$ "agree a lot"). Healthy eating and physical activity were assessed at week 1 and 3 by self-report measures. Healthy eating was operationalized as the frequency of healthy food consumption the day prior to completing the measure. Physical activity was operationalized as the number of days per week the child was physically active for at least 60 minutes. Covariates included cohort, participant ethnicity, age, body mass index, percent program attendance, parent marital status, and maternal education. Results: A confirmatory factor analysis indicated that the 3 Presence items share a common variance that can be explained by the latent factor, 'Immerse'. This latent factor was used as a predictor in two separate regression equations with 1) healthy eating and 2) physical activity as the dependent variables. Being immersed in the virtual world during the first week of the program predicted healthy eating and more physical activity at the end of the program. For every 1-point increase in 'Immerse' at week 1, healthy eating increased by 1.9 units at week $3(\mathrm{p}=.006)$, controlling for baseline healthy eating and all covariates. Further, for every 1-point increase in 'Immerse' at week 1 , week 3 physical activity increased by .33 days $(\mathrm{p}=$ .033 ), controlling for baseline physical activity and all covariates. Discussion: These findings suggest that healthy behaviors explored by girls in an engaging and absorbing virtual world can generalize to the real world. The availability of the virtual world provides girls with additional opportunities to learn about health, rehearse health behaviors, collaborate with healthy peer models in a virtual environment, and develop selfefficacy for healthy behaviors. Virtual world technologies may be particularly beneficial for children who have limited access to health information and/or healthy role models in their environment.

CORRESPONDING AUTHOR: Mary Kate Clennan, University of Miami, marykateclennan@gmail.com

\section{O44.5}

FACEBOOK USE, SOCIAL SUPPORT, AND DEPRESSION IN MEDICAL STUDENTS

Anna Serlachius $^{1}$, Bianca Brown ${ }^{1}$

${ }^{1}$ The University of Auckland- New Zealand

Introduction \& Purpose: Facebook is the world's largest and most pervasive social networking site, with young adults making up the largest proportion of users. Research is mixed as to whether Facebook use is associated with better or worse mental and physical health outcomes. Medical students are a highly stressed population who are also likely to use Facebook. Examining the association between Facebook use, health behaviours, and mental health outcomes may provide more insight into whether Facebook is a beneficial tool for increasing social support for vulnerable groups. Methods: Seventy-seven medical students filled out questionnaires at the beginning of semester and again during the following exam period. Questions examined Facebook use, perceived social support, health behaviours, stress, depression, and anxiety. Pearson's correlations were used to assess the relationships between the different outcome measures, and paired samples t-tests were used to examine differences in the outcome measures between the two time-points. Hierarchical linear regression was used to assess whether the demographic variables, health behaviours, Facebook use, or Facebook social support predicted depression. Linear regression was also used to examine whether an interaction existed between Facebook social support and stress when predicting depression during the exam period. Results: Stress significantly increased in medical students between the beginning of the semester and during the exam period $(\mathrm{p}=.037)$, but no changes in depression, anxiety, and health behaviours were observed. Higher levels of Facebook social support at the beginning of semester was associated with lower 
depression during the following exam period $(\mathrm{r}=-.252, \mathrm{p}=.027)$. Facebook use at time 1 (specifically photo-tagging behaviours) was associated with lower depression during the exam period $(\mathrm{r}=-0.256$, $\mathrm{p}=.025)$. The interaction between stress and Facebook social support was significantly and negatively associated with depression $(\beta=-$ $1.889, \mathrm{p}=.018$ ), demonstrating the buffering effects of Facebook social support on depression. Conclusions: Facebook may have the potential to positively influence its users through the provision of online social support. However, the relationship between Facebook and health behaviours remains unclear. Furthermore, Facebook may only be a helpful platform for social support for those who are more socially integrated. Future research is needed to explore this nuanced relationship further.

CORRESPONDING AUTHOR: Anna Serlachius, The University of Auckland, a.serlachius@auckland.ac.nz

\section{O44.6}

THERE ARE MANY PHYSICAL ACTIVITY APPS BUT ONLY FEW INCLUDE BEHAVIOR CHANGE TECHNIQUES: RESULTS OF A CONTENT-ANALYSIS

Claudia Pischke ${ }^{1}$, Berit Steenbock ${ }^{1}$, Stefanie Helmer ${ }^{1}$, Janna Sill ${ }^{1}$, Tobias Moellers $^{2}$, Mihiretu Kebede ${ }^{1}$

${ }^{1}$ Leibniz-Institute for Prevention Research and Epidemiology University of Bremen, Health Sciences - Germany

${ }^{2}$ Network Aging Research, University of Heidelberg- Germany

INTRODUCTION AND PURPOSE: The market for physical activity (PA) applications (apps) has grown rapidly in recent years. This study aimed to identify German and English PA apps and evidence-based behavior change techniques (BCTs) employed in those apps. METHODS: PA apps were identified in a systematic search using 25 predefined search terms in the Google Play Store. Two reviewers independently screened app descriptions using predefined inclusion and exclusion criteria. Apps were included if the description contained information about PA promotion, they were available in English or German, PA recommendations of the World Health Organization or the American College of Sport Medicine were mentioned, and PA was objectively assessed. Two researchers downloaded and tested apps matching these criteria for two weeks and coded their content according to the BCT Taxonomy v1 (BCTTv1). RESULTS: The initial screening in the Google Play Store yielded 6018 apps, 4108 of which were neither focused on PA nor in German nor English. Descriptions of 1910 apps were further screened for eligibility. Duplicate apps $(n=694)$ and those requiring additional equipment for objectively assessing PA or not mentioning any PA guideline $(\mathrm{n}=1184)$ were excluded. The remaining 32 apps were downloaded: four were no longer available, 22 apps were retrospectively deemed ineligible during testing because the description differed from the actual content, and six apps were rated. Of the 93 BCTs of the BCTTv1, on average, nine (SD: 5) were identified in these six apps (min: 4, max: 18). Of the 16 hierarchical clusters, on average, five (SD: 3 ) were addressed (min: 2, max: 10). Only BCTs of two hierarchical clusters (i.e., 'goals and planning' and 'feedback and monitoring') were addressed in all apps. CONCLUSIONS: Descriptions of PA apps often do not accurately reflect content. Only few PA apps available for Android platforms mention internationally recognized recommendations in their description and use BCTs.

CORRESPONDING AUTHOR: Claudia Pischke, Leibniz-Institute for Prevention Research and Epidemiology, pischke@1eibniz-bips.de

\section{O45.1}

STAGES OF BEHAVIOR CHANGE RELATED TO PHYSICAL ACTIVITY ASSOCIATED WITH SLEEP QUALITY AND SLEEP DURATION IN ADOLESCENTS
Margarethe Knebel ${ }^{1}$, Adriano Borgatto ${ }^{1}$, Marcus Lopes ${ }^{1}$, Alexsandra Bandeira ${ }^{1}$, Kelly Silva ${ }^{1}$

${ }^{1}$ Universidade Federal de Santa Catarina- Brazil

Introduction \& Purpose: Poor sleep quality and insufficient sleep duration have consequences for adolescents' health. Physical activity (PA) has been evidenced as a protective factor for sleep disturbances. It is hypothesized that stages of behavior change for habitual PA practice present distinct associations with adolescents' sleep behavior. This study aimed to examine the associations of the stages of behavior change related to PA with sleep quality and sleep duration in adolescents. Methods: The study included an analysis of the baseline data from the "Movimente" program (2017) with students from six schools in a southern city of Brazil. The sample consisted of 1,259 students (mean age $13.2 \pm 01.1$ years, $50.5 \%$ female). All variables were measured by questionnaire. Sleep quality (frequently/occasionally/rarely sleeping well) and sleep duration on schooldays $(\geq 8 \mathrm{hs} /$ day=sufficient; $<8 \mathrm{hs} /$ day=insufficient $)$ were the dependent variables. The independent variable stages of behavior change related to PA was used in three categories: maintenance, action and initial stages (grouped pre-contemplation, contemplation, preparation). Binary and multinomial logistic regressions adjusted for age and gender were used. Results: The proportion of students who reported occasionally or rarely sleeping well were $25.1 \%$ (95\% CI $22.8-27.6)$ and $9.0 \%(95 \% \mathrm{CI}$ 7.5-10.7), respectively. Moreover, 46.6\% (95\%CI 43.8-49.3) reported insufficient sleep duration on schooldays. Students in the action stage were less likely to report occasionally sleeping well (OR $0.58 ; 95 \% \mathrm{CI}$ 0.39-0.86) when compared to those in the initial stages. Those in the maintenance stage had lower odds to report occasionally (OR 0.66 ; $95 \%$ CI $0.49-0.90$ ) or rarely sleeping well (OR $0.41 ; 95 \%$ CI $0.24-0.69$ ). The stages of behavior change related to PA were not associated with insufficient sleep duration on schooldays. Conclusions: Students in the maintenance stage were less likely to report occasionally or rarely sleeping well, whereas those in the action stage had lower odds only to report occasionally sleeping well. The stages of behavior change related to PA were not associated with sleep duration. PA practices over a long period of time appear to offer major improvements in adolescents' sleep quality and should be encouraged.

CORRESPONDING AUTHOR: Margarethe Knebel, Universidade Federal de Santa Catarina, margk429@hotmail.com

\section{O45.2}

ASSOCIATION BETWEEN PARENT'S PERCEPTIONS OF THE NEIGHBORHOOD ENVIRONMENT AND CHILDREN'S OUTDOOR TIME, NUTRITIONAL STATUS AND PHYSICAL ACTIVITY GUIDELINES COMPLIANCE IN CARAHUE, CHILE Sebastian Miranda Marquez ${ }^{1}$, Camila Mella-García ${ }^{1}$, Damian ChandíaPoblete $^{1}$, Nicolas Aguilar-Farias ${ }^{1}$

${ }^{1}$ Universidad de La Frontera- Chile

BACKGROUND: Children's outdoor time (OT) has been associated with higher probabilities to meet physical activity (PA) guidelines (1 hour of moderate-to-vigorous intensity PA per day). OT may be influenced by different external factors, but parent's perceptions of the neighborhood characteristics may play a key role in this behavior. PURPOSE: To examine the association between children's OT, PA guidelines compliance and body mass index (BMI) with parent's perceptions of the physical and social neighborhood environment according to sociodemographic characteristics in Carahue, Chile (third poorest town in the country). METHODS: A cross-sectional study was conducted in children between 8 to 13 years old from six primary schools $(n=237)$. Sociodemographic characteristics, OT on week and weekends days, PA guidelines compliance and neighborhood perceptions were obtained through self-report using questionnaires completed by parents and children independently. Neighborhood characteristics reported by parents were grouped into 
subscales (i.e. traffic safety, distance to PA facilities, etc.) and global scores, in which higher values represent better perceptions. Height and weight were measured to obtain BMI. Multilevel Regression models were used to determine the association between parent's perception of neighborhood and OT, PA guidelines compliance and BMI. Analyses were adjusted by sociodemographic characteristics. RESULTS: High score on global perception of the neighborhood was associated with more time spent outdoor on weekend days $(\beta=0.04 ; p=0.001)$. Better perceived traffic security was associated with lower probability of being overweight/obese $(\beta=0.08 ; p=0.039)$. After school OT was associated with meeting PA guidelines $(\beta=0.41 ; p=0.029)$ for both genders. Children with more educated mothers had higher chances to meet PA guidelines $(\beta=0.15 ; p=0.021)$. CONCLUSIONS: Parent's perceptions about the neighborhood characteristics may play a key role in defining not only OT, but also nutritional status due to the interaction between these variables. Efforts and investments for improving the built and social environment may promote better health in children from low-income areas.

CORRESPONDING AUTHOR: Sebastian Miranda Marquez, Universidad de La Frontera, sebastian.miranda.marquez@gmail.com

\section{O45.3}

CLUSTERING OF PHYSICAL ACTIVITY, DIET AND SEDENTARY BEHAVIOR AND ITS SOCIODEMOGRAPHIC ASSOCIATIONS AMONG BRAZILIAN ADOLESCENTS: NATIONAL SCHOOLBASED HEALTH SURVEY (PENSE)

Gabrielli Mello ${ }^{1}$, Adriano Borgatto ${ }^{1}$, Thiago Matias ${ }^{1}$, Jaqueline Silva ${ }^{1}$, Kelly Silva ${ }^{1}$

${ }^{1}$ Federal University of Santa Catarina- Brazil

Introduction \& Purpose: Clustering of health behaviors has gained further attention since it helps to understand the co-occurrence of physical activity (PA), sedentary behavior (SB), and dietary patterns. This study aimed to identify clustering of Leisure-time PA (LTPA), diet and SB and its sociodemographic associations among Brazilian adolescents. Methods: Data from the National School based Health Survey (PeNSE) (2015) of 102.0729 th grade students were used (females: 52.8\%). LTPA, healthy (green salads/vegetables and fruits) and unhealthy diet (deep-fried empanadas, candies, soda, ultra-processed and fast foods) SB and sociodemographic variables were obtained using a questionnaire. Twostep cluster analysis and multinomial logistic regression were performed (reference:health-risk cluster). Results: Three clusters were identified: (1) health-promoting SB and diet (32.6\%):0.68 days/week of LTPA, 1.60 and 2.68 days/week of unhealthy and healthy food, respectively, and 2.59 hours/day in SB; (2) health-promoting LTPA and diet (44.9\%):4.56 days/week of LTPA, 2.87 and 4.10 days/week of unhealthy and healthy food, respectively, and 3.85 hours/day in SB; (3) health-risk (22.6\%): 0.86 days/week in LTPA, 3.24 and 2.58 days/week of unhealthy and healthy food, respectively and 7.78 hours/day in SB. Girls were more likely not to be in the cluster $1(\mathrm{OR}=0.85 ; 95 \% \mathrm{CI}=0.78-0.93)$ and cluster $2(\mathrm{OR}=0.43 ; 95 \% \mathrm{CI}=0.40-0.46)$. Age was positively associated with cluster $1(\mathrm{OR}=1.07 ; 95 \% \mathrm{CI}=1.03-1.12)$. Adolescents with the highest level of maternal education were more likely to be in the cluster 2 $(\mathrm{OR}=1.23 ; 95 \% \mathrm{CI}=1.10-1.38)$. Maternal education was associated with cluster 1 (ORcollege $=0.63 ; 95 \% \mathrm{CI}=0.56-0.72$; ORhigh school $=0.65$; $95 \% \mathrm{CI}=0.58-0.71$; and ORelementary $=0.76 ; 95 \% \mathrm{CI}=0.68-0.85$ ). Students from private schools were more likely not to be in the cluster $1(\mathrm{OR}=0.71 ; 95 \% \mathrm{CI}=0.63-0.79)$ than those from public schools. Conclusions: Three clusters were identified, which more than one quarter of the adolescents being at the unhealthy cluster. Besides, all clusters were composed by at least one unhealthy behavior. There were associations among sociodemographic indicators and clusters, which should be considered during the development of health promotion programs among Brazilian adolescents.
CORRESPONDING AUTHOR: Gabrielli Mello, Federal University of Santa Catarina, gabi.tmello@hotmail.com

O45.4

COMPLIANCE OF PHYSICAL ACTIVITY, SEDENTARY BEHAVIOUR AND SLEEPING TIME RECOMMENDATIONS ACCORDING TO SOCIODEMOGRAPHIC CHARACTERISTICS IN CHILEAN CHILDREN FROM A LOW-INCOME TOWN

Marcelo Toledo ${ }^{1}$, Patricio Pérez-Contreras ${ }^{1}$, Damián Chandia-Poblete ${ }^{1}$, Nicolás Aguilar-Farias ${ }^{1}$

${ }^{1}$ UFRO Actívate- Chile

Background: Meeting recommendations of physical activity (PA), sedentary behaviour (SB) and sleeping time (ST), have begun to be studied together in the 24-Hour Movement Guidelines for Children and Youth (24MGCY) developed in Canada, to try to explain, prevent and improve health indicators. The purpose of this study was to determine the prevalence of compliance of the recommendations of movement in 24-hrs that considering their three components according to sociodemographic variables in preadolescents of the Carahue, Chile. Materials and methods: A cross-sectional study of 222 children from third to sixth grade was conducted in Carahue, Chile. PA, SB and ST were measured through selfreport. Meeting 24MGCY has been defined as meeting all three behavioural recommendations: 1) doing at least $60 \mathrm{~min} /$ day of moderate-tovigorous PA (MVPA), 2) $\leq 2 \mathrm{~h} /$ day of SB and 3) 9 to $11 \mathrm{~h} / \mathrm{night}$ of ST. Prevalence of these behaviours were described according to sociodemographic variables (independently and combined). Age, nutritional status, unhealthy diet and parental education were included as effect modifier covariates in multivariate analyses. Results: The mean age of the sample was 10.2 (SD 1.0) years of which $50.5 \%$ were boys. $64.0 \%$ of children were overweight or obese. The prevalence of children meeting $24 \mathrm{MGCY}$ was $3.2 \%$. When analyzing a combination of movement behaviours, a low proportion of children met PA+SB (4.1\%), PA+ST (5.9\%) and $\mathrm{SB}+\mathrm{ST}(14.9 \%)$ recommendations. Independently, the highest prevalence of compliance was for ST (53.2\%), followed by SB (27.0\%) and PA $(13.1 \%)$. Girls with mothers that completed primary education had significantly higher compliance of SB recommendation compared with boys with mothers with the same level of education $(75.0 \%$ vs $25.0 \%$, $\mathrm{p}=0.044)$. Normal-weight girls showed significantly higher prevalence of SB compliance than normal weight boys $(70.0 \%$ vs $30.0 \%, p=0.031)$. No other differences were observed according to gender. Conclusion: An extremely low percent of Chilean children met the $24 \mathrm{MGCY}$ when comparing these results with international data. More initiatives for promoting PA, reduction of SB and better sleep must be implemented, especially in low socioeconomic communities in Chile.

CORRESPONDING AUTHOR: Marcelo Toledo, UFRO Activate, Toledov.marcelo@gmail.com

\section{O45.5}

IMPACT OF INTEGRAL SPORTS SCHOOLS PROGRAM ON THE PHYSICAL ACTIVITY LEVEL IN SCHOOL DAY

Fernando Rodríguez-Rodríguez ${ }^{1}$, Carlos Cristi-Montero ${ }^{1}$, José CastroPiñero $^{2}$, Luis Espinoza Oteiza ${ }^{1}$

${ }^{1}$ IRyS Group, School of Physical Education, Pontificia Universidad Católica de Valparaíso, Viña del Mar- Chile

${ }^{2}$ Department of Physical Education, Faculty of Education Sciences, University of Cádiz, Puerto Real- Spain.

Introduction and Purpose: Children are expected to accumulate activities $>3$ METs as long as possible throughout the day, which will reduce the risk of diseases caused by physical inactivity. Regular physical activity (PA) programs in children have an important impact on the PA levels in the school day. The National Sports Institute (NSI) of Chile, created the 
Integral Sports Schools program (ISS), to add physical activity in public schools for 3 times/week, in addition to physical education classes (PE). We studied the effect of the ISS, on PA level during the school day and compliance with the daily recommendations. Methods: 147 girls and boys aged 8-11 years were included in intervention study. ISS $(n=67)$ and 80 children control group (CON) were randomly chosen in 7 regions of Chile, who carried an Actigraph wGTX3 accelerometer during $8 \mathrm{~h}$ /day including ISS session (60 min) and PE (90 min). Descriptive statistics were performed and the results were compared with the Chi-Square test, with a statistical significance of $\mathrm{p}<0.05$ using SPSS v21 software. Results: not statistical differences on the PA levels during the whole day between ISS and CON, although it tends to be higher in ISS $(12.8 \%$ and $11.3 \%)$. When separating by schedules ISS reaches 3.1 METs vs. 2.1 of CON at $10-11 \mathrm{am} ; 2.4$ vs 1.8 at $14-15 \mathrm{pm}$ and $2.1 \mathrm{vs}$ 1.6 at $15-16 \mathrm{pm}(\mathrm{p}<0.000)$. The moderate-to-vigorous physical activity (MVPA) in ISS it accumulates mainly in the first $3 \mathrm{~h}$ and the steps in the first $4 \mathrm{~h}(\mathrm{p}<0.000)$. ISS session and PE of CON, present significant differences in METs (3.1 vs 2.5), MVPA\% (22,9\% vs $16.2 \%$ ) and steps (1954 vs 1348) respectively. Conclusions: the ISS program increase the PA levels and helps meet the daily recommendations of MVPA, unkile the PE class of CON.

CORRESPONDING AUTHOR: Fernando Rodriguez, Pontificia Universidad Católica de Valparaíso, fernando.rodriguez@pucv.cl

\section{O45.6}

WEIGHT FLUCTUATION AND DIET CONCERN NEGATIVELY AFFECT FOOD-RELATED LIFE SATISFACTION IN MALE AND FEMALE ADOLESCENTS IN CHILE

Berta Schnettler ${ }^{1}$, Edgardo Miranda-Zapata ${ }^{1}$, Klaus Grunert ${ }^{2}$, Germán Lobos $^{3}$, Marianela Denegri ${ }^{1}$, Clementina Hueche ${ }^{1}$

${ }^{1}$ Universidad de La Frontera- Chile

${ }^{2}$ Universidad de Aarhus

${ }^{3}$ Universidad de Talca

Introduction \& Purpose: Eating problems and obesity are frequent problems encountered during adolescence that can potentially cause psychological, social and physical consequences that may interfere with adolescent development and well-being. The present study evaluates the relationship between satisfaction with food-related life and satisfaction with family life, and their relationship with life satisfaction in male and female adolescents. We explored the relationships between both subscales of the Revised Restraint Scale (RRS), Diet Concern (DC) and Weight Fluctuation (WF) and adolescent life satisfaction as well as satisfaction with food-related life and family life. Method: A questionnaire was applied to a non-probabilistic sample of 470 adolescents (mean age 13.2 years, $52.3 \%$ female) in Temuco, Chile, including the RRS, Satisfaction with Life Scale (SWLS), Satisfaction with Food-related Life (SWFoL) scale and Satisfaction with Family Life (SWFaL) scale. Results: Using structural equation modeling, we found that adolescent life satisfaction was related to satisfaction with family life and food-related life, regardless of gender. In male adolescents, a negative relationship was identified between WF and satisfaction with food-related life, which could serve a mediating role between WF and life satisfaction. On the contrary, a negative relationship was identified in female adolescents between DC and food-related life satisfaction, which could serve a mediating role between DC and life satisfaction. DC and WF were not directly related to life satisfaction nor to satisfaction with family life in both genders. Conclusions: These findings suggest that reducing diet concern in female adolescents and reducing weight fluctuation in male adolescents may improve their satisfaction with food-related life and, indirectly, their life satisfaction.

CORRESPONDING AUTHOR: Berta Schnettler, Universidad de La Frontera, berta.schnettler@ufrontera.cl
O46.1

ILLNESS EXPERIENCES OF WOMEN WITH ORAL DRYNESS AS A RESULT OF SJÖGREN SYNDROME

Gonzalo Rojas-Alcayaga ${ }^{1}$, Andrea Herrera ${ }^{1}$, Pamela Wurmann ${ }^{2}$, Nailah Shakthur $^{3}$, Rinie Geenen ${ }^{4}$, Loreto Leiva ${ }^{1}$

${ }^{1}$ Universidad de Chile- Chile

${ }^{2}$ Clinical Hospital Universidad de Chile- Chile

${ }^{3}$ Sjögren Chile Association

${ }^{4}$ Utrecht University. Department of Psychology-

Introduction and purpose Illness experiences of women with oral dryness and health-related behaviors as a result of Sjögren's syndrome (SS) could be important for oral health care. SS is a rheumatic autoimmune disease that impairs different bodily organs and tissues, particularly the lacrimal and salivary glands. Xerostomia and damage to oral tissues are the main problems affecting patients and they could be important contributing factors to impair daily life. The aim of this study was to give an indepth account of the life experiences and health-related behaviors in women with oral dryness as a result of Sjögren Syndrome. Methods. The investigation was an exploratory relational study employing qualitative methodology based on in-depth interview. Interviews were carried out with women with SS who regularly attended the Clinical Hospital of the University of Chile or who are member of the Sjögren Chile Association. All participants were adult Chilean women aged over 45 and diagnosed with SS according to established criteria. The size of the sample was determined by the principle of theoretical saturation, which means that data collection is finished at the point when additional data from interviews seems redundant in the light of the emergent model. The data were analyzed using methods based on the principles of grounded theory. The constant comparative method was used. It is a general approach to analyze qualitative data using explicit coding and analytic procedures to generate and suggest categories, properties and hypotheses. The Ethics Committee of the Faculty of Dentistry of the University of Chile approved the study. Results. Oral symptoms are frequently associated with limitations in daily life (household, social and work activities) which patients live with varying degrees of difficulty and experience as a loss of their usual capabilities. (However,) Oral dryness is not recognized as a symptom of disease and it is a cause of delayed SS diagnosis and poor dental care. Withdrawal from social activities is a result of the discomfort or embarrassment that patients may experience for presenting certain symptoms, oral functional limitations or dental aesthetic defects. Household limitations and inability to work are associated with oral functional difficulties and general symptoms, mainly fatigue. Conclusions. Oral functional difficulties (speech, swallowing and taste), oral tissue damage (caries, fungal infections) and general symptoms of SS (pain and fatigue) affect self-perception and self-esteem. Interference with social role and consequently social isolation are experienced by many patients. This finding is consistent with the results of studies that used measures such as the SF-36 to evaluate Health Related Quality of Life (HRQOL). A better health status and appropriated oral care of women with SS requires careful consideration of psychosocial aspects.

CORRESPONDING AUTHOR : Gonzalo Rojas-Alcayaga, Universidad de Chile, grojasalcayaga@yahoo.es

\section{O46.2}

DEVELOPMENT OF A SCALE OF MEASUREMENT OF CULTURAL COMPETENCE IN HEALTH WORKERS IN CHILE: VALIDITY AND RELIABILITY OF THE PRELIMINARY VERSION Victor Pedrero ${ }^{1}$, Margarita Bernales ${ }^{1}$, Miguel Perez ${ }^{2}$

${ }^{1}$ Universidad del Desarrollo- Chile

${ }^{2}$ Fresno State University- USA

Background Cultural competence (CC) has been recognized as a tool to address health inequities. There's agreement about presence of three main 
dimensions of CC, sensitivity - awareness of own values and beliefs and the effect they have on health care, knowledge -understanding of the way of seeing the world of patients in the context in which it develops, and skill -ability to adapt care strategies according to patients' values and beliefs. Currently there is no instrument for the measurement of CC appropriate to the Chilean context. Aim To analyze the validity and reliability of a new scale to measure $\mathrm{CC}$ in health workers in Chile. Methods Literature review was carried out that included theoretical aspects of CC and several scales developed to measure this construct. Later, an instrument with 31 items divided into the three theoretical dimensions of the $\mathrm{CC}$, each with Likert type response, was elaborated. These were evaluated by experts and health workers (focus groups). The present investigation realizes the initial psychometric evaluation of this scale in a sample of 91 health workers in Chile. For reliability estimation, Cronbach's alpha was performed, while exploratory structural equation modeling (ESEM) was used to evaluate the internal structure (validity). The estimates were made in MPLUS 8 and R. Results The evaluation of experts and health workers were useful in creating the new scale. ESEM model showed an adequate fit $($ Chi-Square $=0.14$, RMSEA $=0.042, \mathrm{CFI}=0.984, \mathrm{TLI}=$ 0.976 ) with 17 of them divided into the three theoretical dimensions of the CC. The reliability of the scale was acceptable $($ alpha $=0.65)$. Conclusions The results support that the new scale is a valid and reliable measure of cultural competence for primary care health workers in Chile, Analysis shows that the three theoretical dimensions are present in the new CC scale. However, few items had to be eliminated to improve the model's fit.

CORRESPONDING AUTHOR: Victor Pedrero, Universidad del Desarrollo,vpedrero@udd.cl

\section{O46.3}

PERCEPTIONS AND EXPERIENCES OF PATIENTS BELONGING TO PATIENTS ORGANIZATIONS IN CHILE

Francisca Manríquez Bergenfreid ${ }^{1}$, Margarita Bernales ${ }^{2}$, Manuel Espinoza $^{1}$

${ }^{1}$ Pontificia Universidad Católica de Chile- Chile

${ }^{2}$ Universidad del Desarrollo

Background. The high costs of treatment and diagnosis for certain oncological, immunological, rare and chronic diseases represent a challenge for health systems. In Chile, the Ricarte Soto Law was born in 2015, as a response to a citizen demand to finance high-cost treatments and diagnoses. In this context, groups of patients are given a formal space for participation in decision-making for the first time. Aim. To describe the perception of the roles, dynamics and participation in the process of decision-making in health of patients belonging to patients organizations. Method. A qualitative study of narrative approach was carried out with 20 patients belonging to patients organizations. Semistructured interviews were delivered, achieving the saturation of the information. Results. The findings were grouped into two thematic axes: 1 . Roles and dynamics in decision-making in health and, 2. Knowledge, Needs and Demands around the decision-making process in health. According to the patients belonging to patients organizations, even though it has been established citizen participation from representatives of these groups as part of the Law, it hast not been binding and the process of decision of the pathologies that where included in the first decrees has not been transparent. At the same time, patients declare not knowing clearly how the participation process will take place and what will be the decision policy. Additionally, patients are in a constant crisis due to their health condition, which often makes them difficult to participate. Conclusions. The findings put into discussion the new health challenges that Chile is facing to achieve universal coverage. The needs faced by patients belonging to patients organizations respond to the access and costs associated with the pathologies they present and the lack of psychological support in the health-disease process. Funding source: Fonis Project SA1610248, "Participation of
Patient Groups in Decision Making Processes on Health Coverage in Chile: Exploring Challenges and Opportunities."

CORRESPONDING AUTHOR: Francisca Manríquez Bergenfreid, Pontificia Universidad Católica de Chile, fnmanriquez@uc.cl

\section{O46.4}

MEASURES OF MENTAL HEALTH AND SUBJECTIVE WELLBEING IN A SAMPLE OF GAY MEN AND LESBIAN WOMEN IN CHILE

Mónica Guzmán ${ }^{1}$

${ }^{1}$ Universidad Católica del Norte

There are scarce data about the mental health and well-being of sexual minorities in Chile; while data from other contexts put emphasis on negative health indicators and the prevalence of risky behavior. This paper deals with the levels of mental health and subjective well-being in a sample of gay men and lesbian women in Chile. To recruit the sample, snowball-type chain sampling was used in four Chilean cities (Antofagasta, Santiago, Valparaiso, and Concepcion). The sample consists of 467 individuals defining themselves as gay (57.4\%) or lesbians (42.6\%), aging from 18 to 67 years $(M=27.9$ and $\mathrm{DE}=7.9)$. More than $98 \%$ of the subjects revealed their sexual orientation. Main findings show that lesbian women report higher levels of satisfaction with life and better adjustment in their close personal relations, as compared to gay men. In addition, $7.9 \%$ subjects reported attempts to take their lives in their hands in a certain moment in their life. The findings and their scope are discussed in this paper.

CORRESPONDING AUTHOR: Mónica Guzmán, Universidad Católica del Norte, moguzman@ucn.cl

\section{O46.5}

THE INFLUENCE OF ACCULTURATION STRATEGIES IN QUALITY OF LIFE BY IMMIGRANTS IN NORTHERN CHILE Alfonso Úrzua ${ }^{1}$

${ }^{1}$ Universidad Católica del Norte- Chile

Purpose: to establish the influence of acculturation strategies on Quality of Life, henceforth QoL. Methods: using a cross-sectional design, two questionnaires were applied, the WHOQoL Bref from the World Health Organization (WHO) and Basabe's Acculturation Strategies. The questionnaires were applied to 853 Colombian and Peruvian immigrants living in Northern Chilean cities of Arica, Antofagasta and Santiago de Chile. To assess the effects of acculturation strategies on the domains of QOL, factorial ANOVAs were performed. Additionally, considering the restrictions of the criteria forming strategies of acculturation, structural models and multi-group comparisons were conducted widespread using the method of least squares estimation. Since according to the multivariate Mardia Kurtosis it was not possible to assume multivariate normality, as this method fails to be robust with large samples and models of low complexity. Results: in the psychological and social domains, as well as in the overall assessment of QoL, the most beneficial strategies are those where customs are maintained from the homeland, where "integration" then "separation" are the most beneficial. On the contrary, when the strategy for maintaining homeland customs is low, the QoL tends to be lower. The strategy of "marginalized" (people who neither identify with their heritage culture nor with the new culture), is associated with a lower QoL. Conclusions: acculturation strategies mildly or moderately affect the psychological and social domains of Quality of Life as well as the overall assessment of QoL.

CORRESPONDING AUTHOR: Alfonso Úrzua, Universidad Católica del Norte, alurzua@ucn.cl 


\section{O47.1}

UNDERSTANDING THE SOCIAL GRADIENT IN HEALTH AND WELLBEING: AN INTERDISCIPLINARY SCOPING STUDY

Michèlle Bal ${ }^{1}$, John de $\mathrm{Wit}^{1}{ }^{2}$, Kees van den Bos ${ }^{1}$, Tanja van der Lippe ${ }^{1}$

${ }^{3}$, Carlijn Kamphuis ${ }^{3}$, Maartje Poelman ${ }^{4}$, Jet Smit ${ }^{5}$

${ }^{1}$ Utrecht University- Netherlands

${ }^{2}$ Health and Organizational Psychology- Netherlands

${ }^{3}$ Interdisciplinary Social Science: Public Health- Netherlands

${ }^{4}$ Geosciences: Human Geography and Planning- Netherlands

5 Julius Center: Public Health, Healthcare Innovation and Medical Humanities

Introduction \& Purpose Socioeconomic inequities in health and wellbeing pose significant challenges for social policy and reflect the influence of social determinants of health. Despite increased attention in research and policy-making, inequities persist and may even be growing. Explanations of the link between SES and health and wellbeing vary across disciplines, but have largely been studied in isolation. Moreover, while extant hypotheses explain differences between the worst-off and the well-off, they mostly do not adequately account for the social gradient in health, that is, differences between every rung on the social ladder, including the well-off and very well-off, and not just between the poor and the rest of the population. In this study, we specifically synthesize evidence regarding main explanations across disciplines for this social gradient. Methods We conducted a systematic scoping study and narrative synthesis of English-language research publications on the social gradient in health and well-being, using a variety of search engines (e.g., PubMed, Scopus, CINAHL) covering the fields of public health, human geography, social sciences and behavioral sciences. Results Three main types of explanations could be distinguished in the literature: those focusing on individual-behavioral explanations (differences in lifestyles), structuralinstitutional explanations (differences in living conditions, exposure to risk factors, and access to resources), and psychosocial explanations (aversive effects of relative disadvantage). Relative deprivation is a key factor linking SES to differential health outcomes. Specifically, feelings of relative deprivation elicit psychological stress and influence health directly, through cardiovascular responses, and indirectly, through risky behaviors to cope with distress (e.g., tobacco and alcohol use). Conclusions Our scoping study underscores the importance of psychosocial factors, especially relative deprivation, in understanding the social gradient in health and wellbeing. This extends the traditional focus on individual-behavioral and structuralinstitutional factors, and underscores the importance of addressing lifestyles, systems as well as social processes in interventions promoting health and wellbeing for all.

\section{CORRESPONDING AUTHOR: Michèlle Bal, Utrecht University}

\section{O47.3}

EXAMINING PARTNERSHIP-HEALTH ASSOCIATIONS IN U.S. SEXUAL AND GENDER MINORITIES, USING POPULATIONLEVEL DATA

Steve Du Bois ${ }^{1}$, Nicole Legate ${ }^{1}$, Ashley D. Kendall ${ }^{2}$

${ }^{1}$ Illinois Institute of Technology- USA

${ }^{2}$ University of Illinois at Chicago- USA

Introduction \& Purpose: The partnership-health association states that on average, partnered individuals are healthier than unpartnered ones. Some research indicates a positive, linear association between partnership status and health: The divorced, separated, and widowed are less healthy than singles; singles are less healthy than the unmarried partnered; and, the unmarried partnered are less healthy than the married, who report the best health of all partnership status levels. This research has been limited to heterosexuals. Here we ask if partnership-health associations apply to sexual and gender minorities (SGM). We provide the first, broad assessment of partnership-health associations across partnership statuses (married, partnered, single, divorced/separated/widowed) among a national sample of U.S. SGM. Methods: Population-level data (N=7,089 aggregated SGM) came from the 2016 U.S. Behavioral Risk Factor Surveillance System (BRFSS). We compared SGM of different partnership statuses on global health, psychological health, health behaviors, and healthcare access. Results: Married SGM reported greatest health across domains, with some exceptions; followed generally by partnered, single, and then divorced/separated/widowed SGM. Conclusions: Continuing to collect population-level data on SGM and health is useful, and necessary. Here we found the well-established partnership-health associations among heterosexuals apply to SGM in terms of psychological health, health behaviors, and healthcare access. Our findings demonstrate the positive health correlates of same-sex marriage legislation among SGM, and lay a foundation for more nuanced investigation into the temporal links between such legislation and health outcomes. More broadly, the present report provides an early indication that additional legislation providing equal rights to SGM, for instance legalization of hospital visits for same-sex partners, could similarly be related to health outcomes. Additional research in this area is warranted, and could help address health disparities among SGM.

CORRESPONDING AUTHOR: Steve Du Bois, Illinois Institute of Technology, sdubois@iit.edu

\section{O47.4}

SOCIAL CONSTRUCTION OF DISABILITY FROM THE PERSPECTIVE OF PRIMARY HEALTH CARE OFFICIALS IN CHILE

Francisca Valdebenito $^{1}$, Julio Hasbún ${ }^{1}$, Javier García ${ }^{1}$

${ }^{1}$ Symbolon- Chile

An exploratory-descriptive investigation was conducted to study the gaps in Primary Health Care (PHC) for people with disabilities in Chile. The objective of the research was to identify the social construction of disability from the perspective of PHC officials. The research question was: what are the attitudes toward disability that officials have? The questionnaire "Attitudes towards disabled people (ATDP)", in its O form, was applied to 436 PHC officials of 7 health establishments in 5 local governments in 3 regions of the country. The sample had an intentional selection in order to observe rural and urban realities, under municipal administration and the Ministry of Health, in local governments of the Center, North and South of the country. The data collected was analyzed based on the Principal Components Analysis (PCA). From the analysis of the principal components, the results indicate the existence of 7 components that explain $70 \%$ of the variance. A total of 5 components report statistically significant values $(0.5>)$, accumulating a $57 \%$ explanation of the variety of evidence collected. The statistically significant components allow the grouping of the variables in the optimal phases for the evaluation of attitudes around a disability situation. The dimensions identified are linked to the social construction of disability in the field such as: cognitive/emotional $(23.81 \%$, total variance explained), perceptions about health care $(12.76 \%)$, expectations $(8.793 \%)$ and sociability $(6,485)$. Based on the results, it is possible to understand the aspects linked to the social construction of disability from low expectations, lack of visibility and stigmas associated with a disability situation. The model that underlies this construction is the biomedical based on functional rehabilitation, reinforced by public campaigns from the assistencialist model in the media in Chile. These attitudes are the greatest barrier to the inclusion of people in a situation of disability in society, especially when health professionals are used by families to guide them, reproducing these negative attitudes.

CORRESPONDING AUTHOR: Francisca Valdebenito, SymbolonChile, fvaldebenito@symbolon.cl 
O47.5

CULTURAL COMPETENCE IN HEALTH: A NECESSARY SKILL TO MANAGE THE EDUCATIONAL LEVEL GAP AMONG HEALTH WORKERS AND PATIENTS

Claudia Perez ${ }^{1}$, Victor Pedrero ${ }^{1}$, Margarita Bernales ${ }^{1}$, Miguel Perez ${ }^{2}$

${ }^{1}$ Universidad del Desarrollo- Chile

${ }^{2}$ Fresno State University- USA

Introduction: Cultural competence in health has been used as a strategy to reduce inequities in health, and a vast amount of studies on cultural competence have been focused on racial and ethnic differences. However, the relevance between the educational level gap among health workers and patients has been less studied as a phenomenon that affects interactions and communication in health care settings. Aim: to explore the perception of health professionals about how patients' education level impacts on health interactions. Methodology: From a constructivist paradigm a case study was realized in an economic disadvantage community in Santiago, Chile. Data collection includes three focus groups with health workers at primary health level, the total number of participants was 29 people who join the study prior informed consent process. The information from focus group was transcribed and analysed using thematic analysis approach trough NVivo 11. The study was approved by the Ethic Committee of Universidad del Desarrollo, Chile. Results: health workers refer that patients educational level usually becomes a barrier to health interaction: "education that they have made the gap between us and them as a big abyss", one of the examples referred by health workers relates to child-raising: "you works with moms and you give them some guidelines to the children and they don't understand how to do things, it is very disappointing". In general, all health workers acknowledge this barrier: "our work is to be educators and you have to deal with any kind of patient without considering maybe his/her educational level... I think that that should not be a problem, but it is a problem". Conclusion: The low educational level of some patients is considered an issue by health workers since it affects communication between them. It is important to recognize this barrier and to take actions to overcome it. Training in cultural competence for health workers with a focus on educational level, could enabling them to cope with an increasingly diverse population in health care in effective ways.

CORRESPONDING AUTHOR : claudia Perez, Universidad del desarrollo, claudiaperez@udd.cl

\section{O47.6}

HOW DO HEALTH WORKERS IN CHILE ASSESS THEIR OWN LEVEL OF CULTURAL COMPETENCE AND THE LEVEL FROM OTHERS IN THE HEALTHCARE TEAM?: IMPLICATIONS FOR INTERDISCIPLINARY WORK

Paulina Fernández ${ }^{1}$, Víctor Pedrero ${ }^{2}$

${ }^{1}$ Pontificia Universidad Católica de Chile- Chile

${ }^{2}$ Universidad del Desarrollo

Background The multidisciplinary work in health has proven to be an effective strategy to deliver high quality health care. Confidence in professional competencies among team members is relevant to a successful collaboration. A competence that has been documented as necessary in healthcare workers (HW) is Cultural Competence (CC), especially because it has been associated with better health outcomes in minority and socially disadvantaged groups. However, the degree of confidence that HW has in his own level of CC and levels of other members of health team has been poorly studied. Aim To describe the confidence that $\mathrm{HW}$ of different professions have in their own level of cultural competence and in the levels of other members of the healthcare team. Method An instrument developed by the authors was used to assess CC, which has adequate validity and reliability. In addition, the perception that the HW had of its own level of cultural competence was evaluated, as well as its perception on the level of CC in other HW (Likert scale 5 points). It was considered a sample of 91 primary care HW from an area of Santiago. Statistical analysis included descriptive data analysis and ANOVA to assess score differences. Results The total CC scores measured with the instrument did not show significant differences when purchasing the different TS groups $(\mathrm{F}(4,85)=1, \mathrm{p}=0.34)$. However, it is noted that the different HW self-rated their level of CC better than their own group in general, and better than the other professionals. Thus, $53.8 \%$ of physicians consider that they are fully prepared to care for patients with different customs and values (high level of CC), but only $32 \%$ believe that doctors in general are fully prepared and less than $20 \%$ belive that other professions are adequately prepared for this. Conclusions Although there are no differences in the scores of the instrument, different HW rely more on their level of CC than in other TS. These findings may be influenced by expectations and beliefs (stereotypes) about members of the health team. These beliefs could affect the coordinated team work and the delivery of care with $\mathrm{CC}$. The promotion of interdisciplinary work in health is relevant in this context, especially in the early stages of training.

CORRESPONDING AUTHOR: Paulina Fernández, Pontificia Universidad Católica de Chile, pdfernan@uc.cl

O48.1

HOW DO YOU THINK ABOUT STRESS? A QUALITATIVE INVESTIGATION OF OUR BELIEFS ABOUT STRESS

Christopher Kilby ${ }^{1}$, Kerry Sherman ${ }^{2}$, Viviana Wuthrich ${ }^{2}$

${ }^{1}$ Macquarie University

${ }^{2}$ Centre for Emotional Health, Department of Psychology, Macquarie University

Introduction and Purpose: There is evidence that beliefs about stress relate to health and behavior outcomes such as coping, mental health, and risk of heart disease and death. These beliefs may exert their influence by biasing how information is interpreted from stressful situations. However, to date there is no empirical verification of these effects. This may stem from prior research using individual beliefs (e.g., coping with, or the cause/consequence of stress) or using limited sets of beliefs (e.g., the control over and nature of stress) derived from health models that may not measure a broad enough range of beliefs to demonstrate this beliefinterpretation-behavior relationship. As such, current beliefs about stress measures may not accurately measure this construct. This study aimed to gain a qualitative understanding of beliefs about stress as a first step to develop a new measure. Method: 35 psychology students completed 1hour semi-structured interviews. Questions were modelled from the Common-sense Model of Health and Illness Representations and the $\mathrm{ABC}$ Model of Behavior providing a wide range of topics on stress, including the sensation, cause, purpose, valence, consequence, control, and timeline of stress. Double coded interviews were analyzed via thematic analysis. Results: 5 broad themes (and 17 subthemes) were identified: Cognition (focus on the self, working memory, understanding of stress, negative thoughts, time, control, insight, uncertainty), Emotion (positive/negative emotions, mood at the time of being stressed), Physical health (internal symptoms, external symptoms), Social (support networks, other people), and Behavior (performance, confidence, coping). Often, the causes and consequences of stress were interchangeable (e.g., feelings of anxiety caused stress for some but were a consequence of stress for others). Conclusions: People hold a wide variety of beliefs about stress. This study not only replicated previously identified beliefs but identified additional beliefs (such as Social and Cognition beliefs) that may be important to the beliefs about stress literature. Accurately measuring these beliefs in future work may provide greater insight into the importance and role of beliefs about stress in health and behavior.

CORRESPONDING AUTHOR: Christopher Kilby, Macquarie University, christopher.kilby@mq.edu.au 
O48.2

THE TREATMENT EXPECTATION QUESTIONNAIRE (TEX-Q) DEVELOPMENT OF A GENERIC MULTIDIMENSIONAL MEASURE ASSESSING PATIENT EXPECTATIONS OF MEDICAL AND PSYCHOLOGICAL TREATMENTS

Meike Shedden-Mora ${ }^{1}$, Jannis Alberts ${ }^{2}$, Keith Petrie ${ }^{2}$, Johannes Laferton $^{2}$, Yvonne Nestoriuc ${ }^{2}$, Bernd Löwe ${ }^{2}$

${ }^{1}$ University Medical Center Hamburg-Eppendorf

${ }^{2}$ Department of Psychosomatic Medicine and Psychotherapy, University Medical Center Hamburg-Eppendorf, Hamburg, Germany

Introduction \& Purpose: Patient expectations - as a central mechanism of placebo and nocebo effects - are an important predictor for health outcomes. However, conceptual heterogeneity and the lack of generic assessment tools impede an integrated understanding of expectations and their role for outcome across medical and psychological treatments. The aim of this study was to develop a generic, multidimensional measure assessing patient expectations of medical and psychological treatments. Methods: Based on the integrative model of expectations (Laferton, Kube, Salzmann, Auer, \& Shedden-Mora, 2017) we developed the 30-item Treatment Expectation Questionnaire (TEX-Q). The multidimensional structure of the TEX-Q is based on assessing three expectation constructs (probabilistic expectations, value-based expectations, process expectations) across three outcome domains (benefit, adverse events, impact). Relevant steps include literature review, the development of a representative item pool, expert ratings, and cognitive interviews with patients undergoing treatment. Patients in different in- and out-patient settings complete the TEX-Q prior to receiving different medical and psychological treatments. Additionally, they complete measures to assess convergent validity with other expectation scales, and divergent validity with generalized expectation constructs such as optimism, and general anxiety. Results: In the process of scale development, 10 experts rate a pool of 63 items according to item fit, clarity, and comprehensibility. After selecting the most suitable 45 items, 15 patients prior to treatment are interviewed using "think aloud"-technique to evaluate items regarding comprehensibility, phrasing preference, acceptability, and consistence of understanding. Item characteristics, factor structure, internal consistency and construct validity are examined in 300 individuals before receiving different medical and psychological treatments. Conclusions: The TEX-Q is a generic, multidimensional self-report measure to assess patient expectations of medical and psychological treatments. Overcoming the constraints of ad-hoc scales and disease-specific assessment methods for expectations, it allows comparing the impact of expectations across different conditions,

CORRESPONDING AUTHOR: PhD. Meike Shedden-MoraUniversity Medical Center Hamburg-Eppendorf, m.shedden-mora@uke.de

\section{O48.3}

ADVERSE LIFE EVENTS - A CROSS-CULTURAL VALIDATION OF THE CUMULATIVE LIFETIME ADVERSITY MEASURE IN THE GENERAL POPULATION

Tina Carstensen ${ }^{1}$, Eva Ørnbøl ${ }^{1}$, Torben Jørgensen², Thomas Dantoft ${ }^{2}$, Cæcilie Buhmann ${ }^{3}$, Per Fink ${ }^{1}$

${ }^{1}$ The Research Clinic for Functional Disorders and Psychosomatics, Aarhus University Hospital, Aarhus University- Denmark

${ }^{2}$ Centre for Clinical Research and Prevention, Bispebjerg and Frederiksberg Hospital, Capital Region of Denmark- Denmark

\footnotetext{
${ }^{3}$ Mental Health Center Nordsjaelland, Mental Health Services- Denmark Introduction \& Purpose Negative life events have been shown to predict mental illness, however focusing on childhood or recent adversity, selected areas or traumatic events only. The Cumulative Lifetime Adversity Measure developed in the US examines the cumulative effect of a wide range of events including number of events within the same event. It
}

assesses lifelong adversity, checklist approach to avoid confounding from appraisal, and has the advantage of age specific information that usually has to be retrieved by interviews. This not yet validated measure gives opportunity to collect detailed data on lifetime adversity in large cohort studies. We aim to cross-culturally validate the Cumulative Lifetime Adversity Measure in the general population. Methods Translation has been conducted in accordance with established guidelines and validation process in accordance with the COSMIN procedure for formative models including pilot test interviews. Content and construct validity are evaluated including hypothesis testing of accumulated lifetime adversity having a quadratic pattern (replication of findings of the US measure). That is participants with low levels of cumulated lifetime adversity as opposed to no or high levels have statistical significantly lower mental distress, functional impairment, and pain impairment. Field testing sample was the DanFunD part 2 cohort $(n=7493$, aged $18-72)$ randomly drawn in Denmark 2012-2015. Results 10 interviews show that questions are confronting but not offensive, straight forward and easy to fill in. Acceptability is evaluated as good. Back-translation showed similar item content. Field testing showed an error rate of $2 \%$ of the total registered life events, evaluated as acceptable as is for missing scores less than $3 \%$. Quadratic pattern between accumulated lifetime adversity and mental distress $(\beta(95 \% \mathrm{CI}) 0.005(0.003 ; 0.008), \mathrm{p}<0.001)$, functional impairment $(\beta(95 \% \mathrm{CI})-0.001(-0.002 ;-0.001), \mathrm{p}<0.001)$, and pain impairment $(\beta(95 \% \mathrm{CI}) 0.002(0.001 ; 0.003), \mathrm{p}=0.002)$ was found. Conclusions In this cross-cultural validation of the lifetime adversity measure we replicated results. The measure can be evaluated as comprehensively translated, construct validity is high. Acceptability is good and error rate is sufficient, reflecting good content validity.

CORRESPONDING AUTHOR: Tina Carstensen The Research Clinic for Functional Disorders and Psychosomatics, tinacars@ @rm.dk

O48.4

THE HEALTH INFORMATION PREFERENCE INDEX (HIPI): A NEW TOOL FOR MEASURING PATIENT PREFERENCES FOR VISUAL VERSUS WRITTEN HEALTH INFORMATION FORMATS Annie Jones ${ }^{1}$, Maria Kleinstauber ${ }^{2}$, Leslie Martin², Justin Fernandez ${ }^{3}$, Keith Petrie ${ }^{2}$

${ }^{1}$ University of Auckland- New Zealand

${ }^{2}$ Department of Psychological Medicine, University of Auckland, Auckland- New Zealand

${ }^{3}$ Auckland Bioengineering Institute, University of Auckland, AucklandNew Zealand

Introduction. Effectively delivering health information to patients is essential for ensuring understanding and treatment compliance. Patients are likely to have individual preferences regarding modalities for learning about health, including whether information should be delivered in a written or visual format. Delivering health information to patients in their preferred format may promote better understanding and health outcomes, yet no scale exists to measure this. Purpose. This study examines the psychometric properties of the Health Information Preference Index (HIPI), a new, brief measure for assessing patient preferences for visual versus written health information. Methods. 54 patients undergoing elective colorectal surgery and 103 healthy university undergraduates completed the 9-item HIPI. The Style of Processing Scale and BRIEF Health literacy Screening Tool were used to assess convergent and discriminant validity respectively. The student sample completed the HIPI twice, approximately two weeks apart, to assess test re-test reliability. Exploratory factor analyses and internal consistency analyses were conducted on the two samples. Results. The factor analysis revealed the HIPI has a onefactor structure. The HIPI demonstrated good internal consistency in both the student $(\alpha=.70-.80)$ and patient samples $(\alpha=.79)$. Means for the scale items were positively skewed, likely representing a general preference in the community for visual health information. The scale demonstrated 
convergent and discriminant validity, and good test re-test reliability ( $\mathrm{r}=$ $.63, \mathrm{p}<.001)$. Conclusions. These results provide initial support for the psychometric properties of the HIPI. This tool could be utilised to identify patients who would benefit from additional visual aids when receiving health information.

CORRESPONDING AUTHOR: Annie Jones, University of Auckland, annie.jones@auckland.ac.nz

\section{O48.5}

THE HIGHER THE DROP-OUT THE MORE SELECTED THE RESPONDENTS IN TERM OF HEALTH BEHAVIOUR? FINDINGS FROM FOUR FOLLOW UP POSTAL HEALTH SURVEYS WITH VARYING RATES OF ATTRITION

Sakari Suominen ${ }^{1}$, Pekka Virtanen ${ }^{2}$, Olli Kiviruusu ${ }^{3}$, Noora Berg ${ }^{3}$, Urban Janlert ${ }^{2}$

${ }^{1}$ University of Skövde- Sweden

${ }^{2}$ University of Umeå- Sweden

${ }^{3}$ National Institute for Health and Welfare- Finland

Introduction \& Aim It is unclear how drop-out in follow up health surveys is associated with the strength of association between variables being studied. The aim of this study was to explore whether a higher drop-out more strongly selected respondents in terms of health behaviour in four postal follow up health surveys with varying rates of attrition. Methods The 'Northern Sweden Cohort (NoSCo65)' consists of all pupils $(n=1$ 083) who in 1981 attended the last year of compulsory school (age 16 years) in the city of Luleå, Sweden. At the follow-up at the age of 43 years 93\% of the cohort at the age of 21 years participated. The 'Younger Northern Sweden Cohort (NoSCo73)' is a similar sample from the same town $(n=897)$ in 1989 . At the follow-up of 39 years of age $86 \%$ of the cohort at the age of 21 years participated. The 'Stress, development and mental health study (TAM)' consisted of 16 year old pupils in the 9th grade of compulsory school in the city of Tampere, Finland in $1983(\mathrm{n}=2$ 269). At the follow-up of 42 years of age $70 \%$ of the cohort at the age of 22 years participated. 'Health and Social Support (HeSSup)' is an ongoing population based follow up survey initiated in 1998 of a random sample of 64797 individuals in Finland representing the working aged population. Of the respondents born 1977 40\% participated in their 35 year of age follow up. Social background was described by sex and labour market status (gainful employment, unemployed, student, else) and health behaviour by smoking status (current vs. rest), alcohol consumption (heavy episodic drinking monthly or more often vs. rest), and BMI ( $>25$ vs. rest). The data were pooled together and analyzed with multivariate logistic regression. The outcome was adherence from one wave to another vs. becoming a drop-out. Explanatory variables were sex, social background, health behaviour, and study cohort representing varying drop-out. Results Health behaviour did not differentiate adherers from drop-outs with the only exception of heavy drinking adherers in NoSCo73. No significant interaction between any explanatory variable and study cohort was detected with the only exception of heavy drinking. Conclusions Attrition rate did not seem to increase selection of respondents in terms of health behaviour.

CORRESPONDING AUTHOR: Sakari Suominen University of Skövde, sakari.suominen@utu.fi
O48.6

ADAPTATION AND VALIDATION OF A QUESTIONNAIRE TO MEASURE STIGMA TOWARD MENTAL ILLNESS AMONG HEALTH PROFESSIONALS WORKING IN PRIMARY CARE IN CHILE

Jaime Camilo Sapag Muñoz de la Peña ${ }^{1}$, Paola Velasco ${ }^{1}$, Claudia Parra ${ }^{1}$, Samanta Anríquez ${ }^{1}$, Luis Villarroel ${ }^{1}$, Ruben Alvarado ${ }^{2}$, Fernando Poblete $^{1}$, Ana Jofré ${ }^{1}$

${ }^{1}$ Departarments of Public Health and Familiy Medicine, School of Medicine, Faculty of Medicine, Pontificia Universidad Catolica de Chile ${ }^{2}$ School of Public Health, Universidad de Chile \& Instituto de Ciencias de la Salud, Universidad de O'Higgins- Chile

Introduction \& Purpose: Stigma toward the mentally ill is a global problem and one of the main obstacles to the better integration of mental health $(\mathrm{MH})$ into primary health care $(\mathrm{PHC})$. The purpose of this study is to adapt and validate an instrument to measure stigma among PHC providers in Chile. Given there are no well validated instruments to measure stigma among PHC in Chile, an alternative is to adapt measures from other countries with special attention to sociocultural aspects. Methods: The first step was the translation and backtranslation of a questionnaire that was specially built to measure stigma in PHC workers in Canada (Kassam et al, 2012): the Opening Minds Scale for Health Care Providers. This instrument was selected because its focus coincides with the objectives of this project, for ease of application and its good psychometric properties. A standard procedure was followed, based on WHO (2014) recommendations for this kind of need. The translated version was revised by a group of experts, for the analysis of its content and context validity. Subsequently, it was applied to a sample of 20 PHC professionals for semantic analysis of their validity. Finally, the adapted version was conducted among 830 PHC professionals in 32 PHC Centres across the country (North, Centre and South of the country; urban and rural areas). A psychometric analysis of the instrument was performed, including a study of its dimensions (factorial analysis of principal components) of its internal consistency (Cronbach's alpha for its size) and its concurrent validity (evaluating the correlation of scores with another widely used questionnaire for stigma among health providers: Mental illness: clinicians' attitudes (MICA) scale (Kassam et al., 2010)). Results: The final Spanish version of the instrument is presented. Main aspects of the translation and adaptation process are shared focused on five types of equivalence between original and translated instruments (Flaherty et al. , 1988): (1) Content ; (2) Semantic; (3) Technical; (4) Criterion; and (5) Conceptual. Finally, the results of the psychometric analysis of the instrument, including a study of its dimensions (factorial analysis of principal components) of its internal consistency and its concurrent validity are presented. Conclusions: This is the first National study to validate a questionnaire to measure stigma toward people with mental illness in PHC in Chile. It represents a fundamental step in order to (1) assess the stigma characteristics toward mental illness among PHC professionals in the Chilean public health system, and (2) to measure the effectiveness of interventions reduce stigma in this context.

CORRESPONDING AUTHOR: Jaime Camilo Sapag Muñoz de la Peña, Departarments of Public Health and Familiy Medicine, School of Medicine, Faculty of Medicine, Pontificia Universidad Catolica de Chile, jsapag@med.puc.cl 